WSRC-TR-94-0615

Unclassified

\title{
METALLURGICAL LABORATORY HAZARDOUS WASTE MANAGEMENT FACILITY GROUNDWATER MONITORING REPORT (U)
}

FOURTH QUARTER 1994

AND 1994 SUMMARY

Publication Date: March 1995

Authorized Derivative Classifier and Reviewing Official:

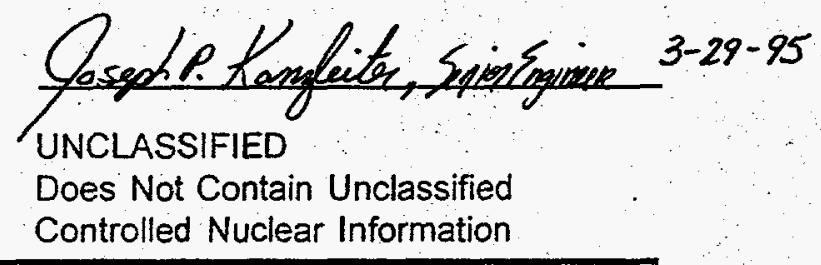

Westinghouse Savannah River Company

Savannah River Site

Aiken, SC 29808 
WSRC-TR-94-0615

Unclassified

\section{METALLURGICAL LABORATORY HAZARDOUS WASTE MANAGEMENT FACILITY GROUNDWATER MONITORING REPORT (U)}

\section{FOURTH QUARTER 1994 AND 1994 SUMMARY}

Publication Date: March 1995

Authorized Derivative Classifier and Reviewing Official:

UNCLASSIFIED

Does Not Contain Unclassified Controlled Nuclear Information

Westinghouse Savannah River Company Savannah River Site

Aiken, SC 29808 


\section{DISCLAIMER}

This report was prepared as an account of work sponsored by an agency of the United States Government. Neither the United States Government nor any agency thereof, nor any of their employees, makes any warranty, express or implied, or assumes any legal liability or responsibility for the accuracy, completeness, or usefulness of any information, apparatus, product, or process disclosed, or represents that its use would not infringe privately owned rights. Reference herein to any specific commercial product, process, or service by trade name, trademark. manufacturer, or otherwise does not necessarily constitute or imply its endorsement, recommendation, or favoring by the United States Government or any agency thereof. The views and opiaions of authors expressed herein do not necessarily state or reflect those of the United States Govemment or any agency thereof.

This report has been reproduced directly from the best available copy.

Available to DOE and DOE contractors from the Office of Scientific and Technical Information, P. O. Box 62. Oak Ridge, TN 37831; prices available from (615) $576-8401$.

Available to the public from the National Technical Information Service, U. S. Department of Commerce, 5285 Port Royal Rd., Springfield, VA 22161 


\section{DISCLAIMER}

Portions of this document may be illegible in electronic image products. Images are produced from the best available original document. 
WSRC-TR-94-0615

Unclassified

\section{METALLURGICAL LABORATORY HAZARDOUS WASTE MANAGEMENT FACILITY GROUNDWATER MONITORING REPORT (U)}

\section{FOURTH QUARTER 1994 \\ AND 1994 SUMMARY}

Publication Date: March 1995

Key Words

AMB wells

pH

tetrachloroethylene

total organic halogens

trichloroethylene

Westinghouse Savannah River Company

Savannah River Site

Aiken, SC 29808 
WSRC-TR-94-0615

Unclassified

THIS PAGE LEFT BLANK INTENTIONALLY. 


\section{Abstract}

During fourth quarter 1994, samples from AMB groundwater monitoring wells at the Metallurgical Laboratory Hazardous Waste Management Facility (Met Lab HWMF) were analyzed for selected heavy metals, indicator parameters, radionuclides, volatile organic compounds, and other constituents. Five parameters exceeded standards during the quarter.

As in previous quarters, tetrachloroethylene and trichloroethylene exceeded final Primary Drinking Water Standards (PDWS). Bis(2-ethylhexyl) phthalate, which exceeded final PDWS in one well during third quarter 1994, was not analyzed for during fourth quarter 1994. Field measurements for pH and specific conductance and laboratory analyses for total organic halogens exceeded the Savannah River Site (SRS) Flag 2 criteria. Aluminum, iron, manganese, and tin, all of which exceeded Flag 2 criteria in at least one well each during third quarter 1994, were not analyzed for during fourth quarter 1994.

Groundwater flow direction and rate in the M-Area Aquifer Zone were similar to previous quarters. Conditions affecting determination of groundwater flow directions and rates in the Upper Lost Lake Aquifer Zone, Lower Lost Lake Aquifer Zone, and the Middle Sand Aquifer Zone of the Crouch Branch Confining Unit were also similar to previous quarters.

During second quarter 1994, SRS received South Carolina Department of Health and Environmental Control approval for constructing five point-of-compliance wells and two plume definition wells near the Met Lab HWMF. This project began in July 1994 and is complete; however, analytical data from these wells are not yet available. 
WSRC-TR-94-0615

Unclassified

THIS PAGE LEFT BLANK INTENTIONALLY. 


\section{Contents}

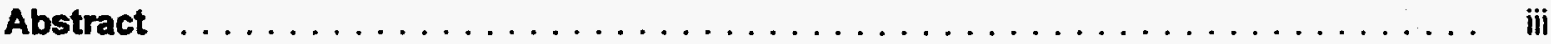

List of Figures $\ldots \ldots \ldots \ldots \ldots \ldots \ldots \ldots \ldots \ldots \ldots \ldots \ldots \ldots \ldots \ldots$

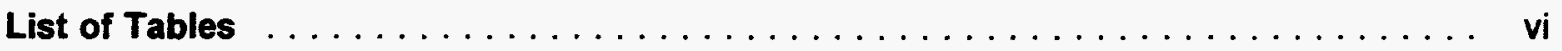

Executive Summary $\ldots \ldots \ldots \ldots \ldots \ldots \ldots \ldots \ldots \ldots \ldots \ldots \ldots \ldots$

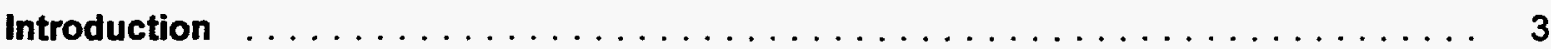

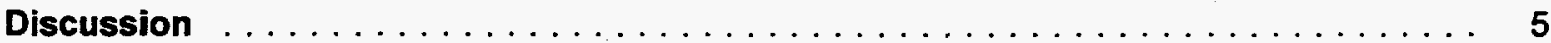

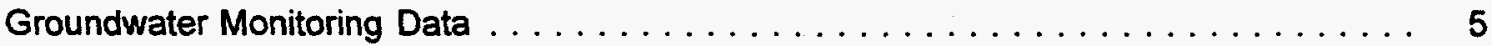

Integrity of the Monitoring Well Network $\ldots \ldots \ldots \ldots \ldots \ldots \ldots \ldots \ldots \ldots$

Analytical Results Exceeding Standards $\ldots \ldots \ldots \ldots \ldots \ldots \ldots \ldots \ldots \ldots \ldots$

Time Series Results . . . . . . . . . . . . . . . . . . . . . 7

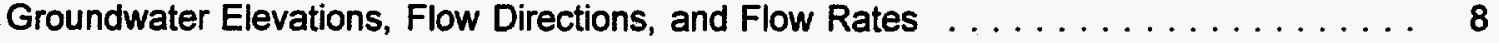

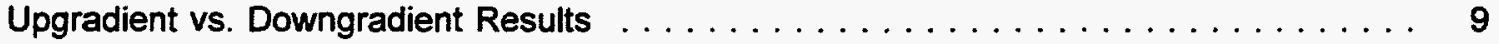

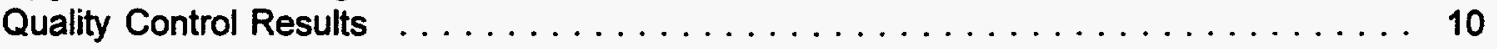

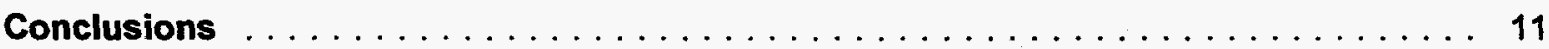

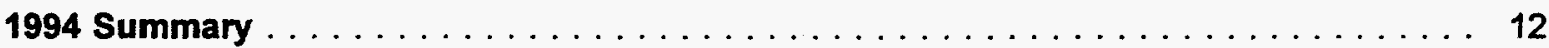

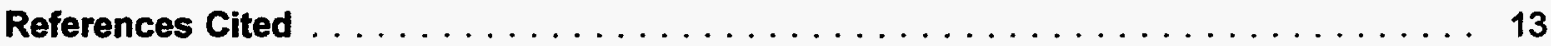

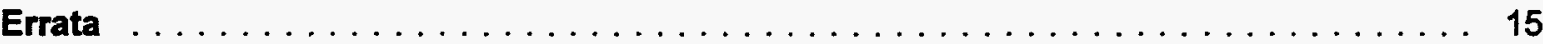

Appendix A-Final Primary Drinking Water Standards $\ldots \ldots \ldots \ldots \ldots \ldots \ldots \ldots$

Appendix B-Flagging Criteria $\ldots \ldots \ldots \ldots \ldots \ldots \ldots \ldots \ldots \ldots \ldots \ldots \ldots \ldots \ldots \ldots$

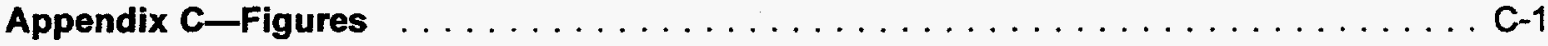

Appendix D-Groundwater Monitoring Results Tables $\ldots \ldots \ldots \ldots \ldots \ldots \ldots \ldots$

Appendix E-Data Quality/Usability Assessment $\ldots \ldots \ldots \ldots \ldots \ldots \ldots \ldots \ldots$

Appendix F-Time Series Plots $\ldots \ldots \ldots \ldots \ldots \ldots \ldots \ldots \ldots \ldots \ldots \ldots \ldots \ldots \ldots \ldots$

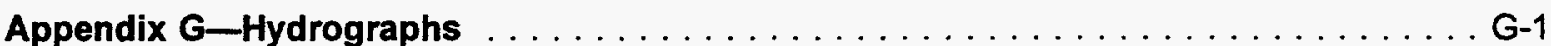

Appendix $\mathrm{H}-$ M-Area Water-Elevation Maps $\ldots \ldots \ldots \ldots \ldots \ldots \ldots \ldots \ldots \ldots \ldots$ 


\section{List of Figures}

Page

1. Location of the Metallurgical Laboratory HWMF at the Savannah River Site $\ldots \ldots$ C-3

2. Piezometric Surface Map of the M-Area Aquifer Zone in A/M Areas $\ldots \ldots \ldots \ldots$

3. Piezometric Surface Map and Location of the M-Area Aquifer Zone Wells at the Metallurgical Laboratory HWMF . . . . . . . . . . . . . . . . . . C-5

4. Water-Elevation Map and Location of Monitoring Wells in the Upper Lost Lake Aquifer Zone at the Metallurgical Laboratory HWMF . . . . . . . . . . . . . . C-6

5. Water-Elevation Map and Location of Monitoring Wells in the Lower Lost Lake Aquifer Zone at the Metallurgical Laboratory HWMF

6. Water-Elevation Map and Location of Monitoring Wells in the Middle Sand Aquifer Zone of the Crouch Branch Confining Unit at the Metallurgical Laboratory HWMF

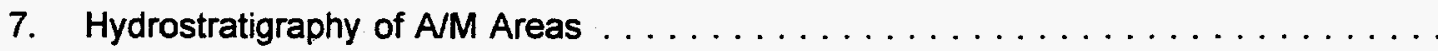

\section{List of Tables}

1. Maximum Levels of Constituents Exceeding the Final Primary Drinking

Water Standards

D-7

2. Maximum Levels of Constituents Exceeding Other Flag 2 Criteria $\ldots \ldots \ldots \ldots$ D-8

3. Groundwater Monitoring Results for Individual Wells $\ldots \ldots \ldots \ldots \ldots \ldots \ldots$ 
WSRC-TR-94-0615

Unclassifjed

\section{Executive Summary}

The 18 AMB wells at the Metallurgical Laboratory Hazardous Waste Management Facility (Met Lab HWMF) are monitored for selected constituents to comply with the Natural Resources Defense Council et al. Consent Decree of May 1988 that identifies the Met Lab HWMF as subject to the Resource Conservation and Recovery Act. In addition, the wells are monitored as requested for other constituents as part of the Savannah River Site (SRS) Groundwater Monitoring Program.

During fourth quarter 1994, all 18 wells were monitored at the facility. Well screen zone nomenclature uses the following hydrostratigraphic assignments: wells AMB 4D, 5, 6, 7, 8D, 9D, 10D, $11 \mathrm{D}$, and $12 \mathrm{D}$ monitor the M-Area Aquifer Zone; well AMB 11B monitors the Upper Lost Lake Aquifer Zone; wells AMB 4B, 7B, and 10B monitor the Lower Lost Lake Aquifer Zone; wells AMB 4A, 7A, 10A, and 13AR monitor the Middle Sand Aquifer Zone of the Crouch Branch Confining Unit (CBCU); and well AMB 10DD monitors a perched groundwater zone beneath the Met Lab HWMF.

Samples from these wells were analyzed for alkalinity, heavy metals, $\mathrm{pH}$, total alpha-emitting radium, specific conductance, volatile organic compounds, and other constituents. The results of the analyses of these constituents are listed in Table 3. This report describes the results that exceeded final Primary Drinking Water Standards (PDWS) and SRS flagging criteria.

As in previous quarters, tetrachloroethylene and trichloroethylene exceeded the final PDWS. Tetrachloroethylene exceeded standards in wells AMB 4A, 4D, and 7A; trichloroethylene exceeded standards in wells AMB 4A, 4D, 5, 6, and 7A. Total organic halogens exceeded the Flag 2 criteria in wells $A M B$ 4A, 4D, and 7A. No constituents exceeded either the final PDWS or the Flag 2 criteria in upgradient wells AMB $11 \mathrm{D}$ and 12D or in downgradient wells AMB 4B, 7, 7B, 8D, 9D, 10A, 10B, 10D, 10DD, 11B, and 13AR. Dichloromethane, a common laboratory contaminant, has not exceeded the final PDWS in any AMB well since fourth quarter 1993.

Groundwater flow direction and rate in the M-Area Aquifer Zone were similar to previous quarters; the flow rate estimate was $130 \mathrm{ft} /$ year during fourth quarter 1994. Reliable estimates of flow directions and rates in the Upper Lost Lake Aquifer Zone could not be determined because data were insufficient. Reliable estimates of flow directions and rates in the Lower Lost Lake Aquifer Zone and in the Middle Sand Aquifer Zone of the CBCU could not be calculated because of the low horizontal gradient and the near-linear distribution of the monitoring wells.

In response to the January 28,1994, Notice of Deficiency issued by SCDHEC on Revision 0 of the 1992 Met Lab HWMF Part B permit renewal application, SRS plans to obtain additional hydrogeologic and groundwater quality data to further define the groundwater contaminant plume near the Met Lab HWMF and has constructed additional point-of-compliance and plume definition wells. During second quarter 1994, SRS received approval for and constructed the proposed Met Lab HWMF monitoring wells. Analytical data from these wells are not yet available. 
THIS PAGE LEFT BLANK INTENTIONALLY. 


\section{Introduction}

The Metallurgical Laboratory Hazardous Waste Management Facility (Met Lab HWMF) is located in the eastern section of AMM Areas at the Savannah River Site (SRS) (Figures 1 and 2, Appendix C). The facility consists of the process sewer line leading to the Metallurgical Laboratory Basin, the Metallurgical Laboratory Basin, the drainage outfall to a nearby Carolina bay, and the Carolina bay. The following description outlines important events at the facility:

- The Metallurgical Laboratory Basin was constructed and began receiving waste water effluent from the Metallurgical Laboratory Building in 1956 (WSRC, 1992a; WSRC, 1992b). Waste water released to the basin consisted of laboratory wastes from metallographic sample preparation and corrosion testing of stainless steel-based and nickel-based alloys. The quantity of waste water discharged to the basin was small, averaging approximately 1,000 gallons per day (Heffner and Exploration Resources, 1991).

- Release of hazardous waste from the Metallurgical Laboratory Building to the basin was discontinued in 1983 (WSRC, 1992a; WSRC, 1992b).

- Quarterly groundwater sampling began first quarter 1984 (WSRC, 1992a; WSRC, 1992b).

- Waste water flow to the Met Lab HWMF was terminated November 8, 1985 (WSRC, 1992a; WSRC, 1992b).

- The Met Lab HWMF was named in Section III of the Natural Resources Defense Council et al. Consent Decree, May 26, 1988 (Civil Action 1:85-2583-6, U.S. District Court, District of South Carolina, Aiken Division), thus becoming subject to the requirements of Subtitle $C$ of the Resource Conservation and Recovery Act (RCRA).

- Revision 0 of the facility's RCRA Part B post-closure care permit application (WSRC, 1989) was submitted to the South Carolina Department of Health and Environmental Control (SCDHEC) August 18, 1989.

- A groundwater quality assessment plan (Jerome, 1990) was submitted to SCDHEC in October 1990.

- A revised groundwater quality assessment plan was submitted to SCDHEC in March 1991 and approved by SCDHEC in June 1991 (Jerome, 1991). The plan lists the selected heavy metals, indicator parameters, radionuclides, volatile organic compounds, and other constituents that currently require monitoring.

- In September 1991, a phased closure plan for the Met Lab HWMF was approved by SCDHEC, and construction to close the basin began (WSRC, 1992b).

- Revision 1 of the RCRA Part B post-closure care permit application (WSRC, 1991) was submitted to SCDHEC December 16, 1991.

- Closure construction of the basin was completed May 1,1992; official closure for the basin was completed May 11, 1992; and closure certification of the basin was submitted to 
SCDHEC July 10, 1992 (letter from J. Gray, Environmental Restoration Closures (ERC), to R. Sentelle, ERC, June 11, 1992). No decision has been made on closure of the Carolina bay.

- Revision 0 of the 1992 RCRA Part B post-closure permit renewal application (WSRC, 1992b) was submitted to SCDHEC in September 1992 in accordance with the regulatory requirement to update and resubmit permit applications every five years.

- Revision 1 of the 1992 RCRA Part B post-closure permit renewal application (WSRC, 1993) was completed in February 1993 and resubmitted to SCDHEC. Revision 1 incorporated changes requested by SCDHEC.

- Revision 3 of the 1992 RCRA Part B post-closure permit renewal application (WSRC, 1994) was submitted to SCDHEC in July 1994. Revision 3 incorporated changes requested by SCDHEC.

Presently, the Environmental Protection Department/Environmental Monitoring Section (EPD/ EMS) samples the $18 \mathrm{AMB}$ wells each quarter, and the Environmental Restoration Department reports the results of this sampling to SCDHEC to meet the requirements of the South Carolina Hazardous Waste Management Regulations (SCDHEC, 1990). 


\section{Discussion}

\section{Groundwater Monitoring Data}

The sampling procedure (WSRC, 1992c) requires evacuation of a minimum of two well volumes and stabilization of $\mathrm{pH}$, specific conductance, and turbidity prior to sample collection. Stability is established when a minimum of three successive measurements, taken within a given time period, are within a specified tolerance range. If a well pumps dry before two well volumes are purged or before stabilization is achieved, it must be revisited within 24 hours for the data to be considered from a single sampling event. On the second visit within 24 hours, samples are taken without purging or stability measurements; thus, these samples may not be representative of groundwater quality.

During fourth quarter 1994, the groundwater samples were analyzed by General Engineering Laboratories of Charleston, South Carolina, and Roy F. Weston, Inc., of Lionville, Pennsylvania, for selected heavy metals, indicator parameters, total alpha-emitting radium, volatile organic compounds, and other constituents. This report describes the monitoring results that equaled or exceeded the following standards:

- Safe Drinking Water Act final Primary Drinking Water Standards (PDWS) or drinking water screening levels, as established by the U.S. Environmental Protection Agency (EPA) (Appen$\operatorname{dix}$ A)

- South Carolina final PDWS for lead (Appendix A)

- SRS flagging criteria that are based on final PDWS, Secondary Drinking Water Standards, and method detection limits (Appendix B)

For simplicity, results that equaled or exceeded final PDWS or SRS Flag 2 criteria are described as exceeding standards, above standards, or as elevated. The final PDWS for individual analytes provided in Appendix A may not always match the SRS flagging criteria provided in Appendix $B$. The final PDWS are used as guidelines in this compliance report to meet regulatory requirements; the flagging criteria are used by EPD/EMS to identify relative levels of constituents in the groundwater and to guide scheduling of groundwater sampling.

\section{Integrity of the Monitoring Well Network}

The groundwater monitoring well network at the Met Lab HWMF has developed during the past decade as described below (EPD/EMS, 1994):

- Groundwater monitoring wells AMB 1, 2, and 3 were installed in 1983.

- Wells AMB 1 and 3 were abandoned and replaced by wells AMB $1 A$ and $3 A$ in 1984 .

- In 1988, wells AMB 4, 5, 6, and 7 were installed, and wells AMB 1A, 2, and $3 A$ were abandoned. 
- In 1989, wells AMB 8, 8D, 9, 9D, 10, 10D, 10DD, 11D, and 12D were installed. Subsequently that year, wells $A M B 8,9$, and 10 were abandoned.

- in 1991, wells AMB 4A, 4B, 4D, 7A, 7B, 10A, 10B, 11B, and 13AR were installed, and well AMB 4 was abandoned.

In response to the January 28,1994 , Notice of Deficiency issued by SCDHEC on Revision 0 of the 1992 Met Lab HWMF Part B permit renewal application, SRS plans to obtain additional hydrogeologic and groundwater quality data to further define the groundwater contaminant plume near the Met Lab HWMF. During second quarter 1994, SRS received approval to construct seven new Met Lab HWMF monitoring wells. This project began in July 1994 and is complete. The monitoring wells installed under the Phase $X$ Drilling Project are as follows:

- M-Area Aquifer Zone: 1 Point-of-Compliance (POC) and 2 plume definition wells

- Lost Lake Aquifer Zone: 2 POC wells

- Middle Sand Aquifer Zone of the Crouch Branch Confining Unit (CBCU): 2 POC wells

The positions of the wells are based on present groundwater flow information in the respective aquifers. The locations will provide three additional downgradient M-Area Aquifer Zone monitoring wells for the Met Lab Basin; one additional well downgradient to the Basin; one downgradient well immediately south of the Carolina bay in the Lost Lake Aquifer Zone; and two additional downgradient wells to the Met Lab Basin in the Middle Sand Aquifer Zone of the CBCU. Cone penetrometer technology (e.g., hydrocone sampling) was used to determine where to place the plume definition wells. Analytical data from these wells will be presented in this report when it is available.

Well designations: Hydrostratigraphic nomenclature and well screen zone assignments (Lewis and Aadland, 1993) were revised fourth quarter 1993. Appendix D provides previous and current nomenclature and definitions for the screen zone abbreviations, and Table 3 (Appendix D) indicates the screen zone assignment for each well.

According to the revised nomenclature, the current groundwater monitoring well network at the Met Lab HWMF (Figures 3, 4, 5, and 6, Appendix C) is comprised of the following: wells AMB 4D, 5, 6, 7, 8D, 9D, 10D, 11D, and 12D screened within the M-Area Aquifer Zone; well AMB 11B screened within the Upper Lost Lake Aquifer Zone; wells AMB 4B, 7B, and 10B screened within the Lower Lost Lake Aquifer Zone; wells AMB 4A, 7A, 10A, and 13AR screened within the Middle Sand Aquifer Zone of the CBCU; and well AMB 10DD screened within a perched groundwater zone beneath the Met Lab HWMF. Figure 7 (Appendix C) (Lewis and Aadland, 1993) illustrates the hydrostratigraphy of A/M Areas.

Background wells for the facility were proposed in the recent Part B Met Lab HWMF post-closure permit renewal application (WSRC, 1992b).

Well purging: Table 3 (Appendix D) lists the number of well volumes purged from each well during fourth quarter 1994 and provides sampling statements that describe incomplete or unsuccessful sampling events. Wells AMB 4D, 6, 7, 10A, and 13AR went dry during purging and were sampled after they recovered. Thus, the samples from these wells may not be representative of the groundwater quality at the Met Lab HWMF. 


\section{Analytical Results Exceeding Standards}

Results for analytes that exceeded the final PDWS (see Appendix A) during fourth quarter 1994 are provided in Table 1 (Appendix D). Tetrachloroethylene exceeded the final PDWS in wells AMB $4 A, 4 D$, and $7 A$, with a maximum concentration of $43 \mu \mathrm{g} / \mathrm{L}$ in well AMB $4 A$. Trichloroethylene was elevated in wells $A M B \quad 4 A, 4 D, 5,6$, and $7 A$, with a maximum concentration of $523 \mu \mathrm{g} / \mathrm{L}$ in well AMB $4 \mathrm{~A}$.

Constituents that exceeded other Flag 2 criteria (see Appendix B) during fourth quarter 1994 are summarized in Table 2 (Appendix D). Total organic halogens exceeded the Flag 2 criterion in wells AMB 4A, 4D, and 7A, with a maximum concentration of $156 \mu \mathrm{g} / \mathrm{L}$ in well AMB 4A.

Table 3 (Appendix D) presents all the results for individual wells and indicates the analytical laboratories that conducted the analyses and those analyses that received modifiers (which help identify laboratory accuracy and precision) or that exceeded the EPA-approved holding times during fourth quarter 1994. Constituent results in Table 3 that appear to equal the final PDWS, but are not marked in the ST column (exceeded final PDWS or screening level), are below the final PDWS in the database. Database results, the results that are compared to the final PDWS, are entered with more significant digits than the results given in this report. Apparent discrepancies are the result of the rounding of reported results.

Appendix $\mathrm{D}$ also provides definitions of the abbreviations and the modifiers used in the results tables as well as descriptions of holding times, data rounding, and data qualification practices. Appendix $E$ provides a general assessment of the quality and usability of the data provided by EPD/EMS.

\section{Time Series Results}

Time series plots of field $\mathrm{pH}$, field specific conductance, tetrachloroethylene, total radium, total organic carbon, total organic halogens, and trichloroethylene for the $18 \mathrm{AMB}$ wells are provided in Appendix F. Trends for these indicator parameters are as follows:

pH: Field $\mathrm{pH}$ values for most of the AMB wells have ranged between approximately $\mathrm{pH} 5$ and 7 since sampling began. However, the pH in well AMB 10A consistently has exceeded the alkaline Flag 2 criterion of $\mathrm{pH} 10$; during fourth quarter 1994, it was pH 9.8. The pH in well AMB 11D has usually fluctuated around neutral, but occasionally it has risen to greater than pH 9; during 1994, pH did not exceed standards. The pH level in well AMB 13AR has exceeded the alkaline Flag 2 criterion during recent quarters; during fourth quarter 1994, it was $\mathrm{pH} 10.2$.

Specific conductance: With few exceptions, field specific conductance for well AMB 10A consistently has exceeded the Flag 2 criterion of $500 \mu \mathrm{S} / \mathrm{cm}$ since sampling began; during fourth quarter 1994, specific conductance in well AMB 10A was $703 \mu \mathrm{S} / \mathrm{cm}$. Specific conductance has ranged between approximately 22 and $300 \mu \mathrm{S} / \mathrm{cm}$ in the remaining wells in recent years.

Tetrachloroethylene: Tetrachloroethylene concentrations for most of the AMB wells have been near or below the final PDWS of $5 \mu \mathrm{g} / \mathrm{L}$ since sampling began. Concentrations in well AMB 5 have been above the final PDWS since mid-1989, except during third quarter 1993 when the tetrachloroethylene concentration was less than the detection limit. During third quarter 1994, the tetrachloroethylene concentration in well AMB 5 was also below the detection limit. Concentrations above the final PDWS have been found in well AMB 4D during 
recent quarters, but during third quarter 1994 the tetrachloroethylene concentration was below the detection limit. Concentrations in wells AMB 4A and 7A have consistently exceeded the final PDWS. During fourth quarter 1994, the tetrachloroethylene concentrations in these wells were 43 and $16 \mu \mathrm{g} / \mathrm{L}$, respectively.

Total alpha-emitting radium: All AMB wells have consistently exhibited total alpha-emitting radium activities below the Flag 2 criterion of $20 \mathrm{pCi} / \mathrm{L}$, as the wells did during fourth quarter 1994.

Total organic carbon: Total organic carbon concentrations in the AMB well series have been consistently less than the Flag 2 criterion of $10,000 \mu \mathrm{g} / \mathrm{L}$ since shortly after sampling began, except for a few isolated analyses in different wells during late 1989 through early 1990.

Total organic halogens: Most AMB wells have exhibited total organic halogen concentrations less than the Flag 2 criterion of $50 \mu \mathrm{g} / \mathrm{L}$ since sampling began. However, concentrations in wells $A M B 4 A, 5$, and $7 A$ have exceeded this standard during most quarters that samples were analyzed. During fourth quarter 1994, concentrations exceeded standards in wells AMB 4A, 4D, and $7 A$, which had concentrations of 156,96 , and $144 \mu \mathrm{g} / \mathrm{L}$, respectively.

Trichloroethylene: Trichloroethylene concentrations in wells AMB 4A, 4D, 5, and 7A have consistently exceeded the final PDWS of $5 \mu \mathrm{g} / \mathrm{L}$ since sampling began. During fourth quarter 1994 , these wells had trichloroethylene concentrations of $523,135,40$, and $200 \mu g / L$, respectively. Concentrations in wells $A M B 6,7,7 B$, and $8 D$ have fluctuated around the final PDWS; during 1994, trichloroethylene was elevated in two wells. During second quarter 1994, well AMB 7 had a trichloroethylene concentration of $6.6 \mu \mathrm{g} / \mathrm{L}$. Trichloroethylene concentrations in well AMB 6 have been consistently elevated since fourth quarter 1993; during fourth quarter 1994, the concentration in well AMB 6 was $7.2 \mu \mathrm{g} / \mathrm{L}$. Concentrations in the remaining AMB wells have consistently been less than the final PDWS or the detection limit.

\section{Groundwater Elevations, Flow Directions, and Flow Rates}

Figure 2 (Appendix $C$ ) provides water-elevation contours for the M-Area Aquifer Zone in AM Areas. Figures 3 through 6 (Appendix $C$ ) present water-elevation data for the M-Area Aquifer Zone, the Upper Lost Lake Aquifer Zone, the Lower Lost Lake Aquifer Zone, and the Middle Sand Aquifer Zone of the CBCU beneath the Met Lab HWMF. Hydrographs for the AMB wells are in Appendix G, and water elevation and potentiometric maps of the M-Area Aquifer Zone, the Upper Lost Lake Aquifer Zone, the Lower Lost Lake Aquifer Zone, and the Middle Sand Aquifer Zone of the CBCU are in Appendix $\mathrm{H}$. Water-elevation figures in Appendix $\mathrm{C}$ are oriented to true north using universal transverse Mercator (UTM) coordinates, while figures in Appendix $\mathrm{H}$ are oriented to true north using latitude-longitude coordinates.

Historically, using UTM coordinates, the horizontal groundwater flow in the M-Area Aquifer Zone beneath the Met Lab HWMF is to the west-northwest, the flow in the Upper Lost Lake Aquifer Zone is to the south, the flow in the Lower Lost Lake Aquifer Zone is to the south-southwest, and the flow in the Middle Sand Aquifer Zone of the CBCU is to the southwest. During fourth quarter 1994, horizontal flow in the M-Area Aquifer Zone was west to northwest (Figure 3, Appendix C). The Upper Lost Lake Aquifer Zone was not contoured because it is monitored by only one well. Potentiometric surfaces in the Lower Lost Lake Aquifer Zone and in the Middle Sand Aquifer Zone of the CBCU were not contoured because of the low horizontal gradient or the near-linear distribution of the monitoring wells. 
Estimated horizontal flow rates for the hydrostratigraphic divisions during the past four quarters are provided below.

Horizontal Groundwater Flow Rates (ft/year) in the Hydrostratigraphic Divisions beneath the Met Lab HWMF

\begin{tabular}{|lllll|}
\hline Division & 1Q94 & 2Q94 & 3 Q94 & 4Q94 \\
\hline M-Area Aquifer Zone & 130 & 150 & 120 & 130 \\
Upper Lost Lake Aquifer Zone & $\mathrm{N}^{\mathrm{a}}$ & $\mathrm{N}$ & $\mathrm{N}$ & $\mathrm{N}$ \\
Lower Lost Lake Aquifer Zone & $\mathrm{N}$ & $\mathrm{N}$ & $\mathrm{N}$ & $\mathrm{N}$ \\
Middle Sand Aquifer Zone of the CBCU & $\mathrm{N}$ & $\mathrm{N}$ & $\mathrm{N}$ & $\mathrm{N}$ \\
\hline
\end{tabular}

a $N=$ Horizontal gradient too low, well orientation too linear, or not enough data points to determine the flow rate.

The groundwater flow rate beneath the Met Lab HWMF is estimated using the following equation:

$$
\text { Flow }(\mathrm{ft} / \text { day })=\frac{\text { Hydraulic Conductivity }(\mathrm{ft} / \text { day) }}{\text { Porosity (unitless) }} \times \frac{d h(\mathrm{ft})}{d l(\mathrm{ft})}
$$

Hydraulic conductivity constants estimated for the M-Area Aquifer Zone, the Upper Lost Lake Aquifer Zone, the Lower Lost Lake Aquifer Zone, and the Middle Sand Aquifer Zone of the CBCU are $27,45,45$, and $45 \mathrm{ft}$ day, respectively; the effective porosity value for each is 20 percent (Lewis and Aadland, 1992). The value $d h$ is the difference in head, and $d l$ is the length of the flow path.

Flow path length is calculated to the nearest $50 \mathrm{ft}$ for each hydrostratigraphic unit. Flow rate per day is calculated to two significant digits, then multiplied by 365 and rounded to two significant digits for the flow rate per year. Flow rate estimates vary depending on the vertical gradient between wells, the size of the area under consideration, and the number of data points. The values presented here are useful as order of magnitude estimates only.

The flow rate estimate for groundwater in the M-Area Aquifer Zone beneath the Met Lab HWMF during fourth quarter 1994 is as follows (Figure 3, Appendix C):

$$
\begin{aligned}
& \frac{27}{0.20} \times \frac{2.0}{750}=0.36 \mathrm{ft} / \text { day } \\
& 0.36 \mathrm{ft} \text { day } \times 365 \text { days } \approx 130 \mathrm{ft} / \text { year }
\end{aligned}
$$

\section{Upgradient vs. Downgradient Results}

M-Area Aquifer Zone wells AMB 11D and 12D are upgradient relative to the Met Lab HWMF. No AMB wells monitor upgradient groundwater quality in the Upper and Lower Lost Lake Aquifer Zones or the Middle Sand Aquifer Zone of the CBCU. The 1992 RCRA Part B post-closure permit renewal application (WSRC, 1992b) proposes to identify upgradient wells for these units (WSRC, 1992b) after data are received from the Phase X Drilling Project.

During fourth quarter 1994, no elevated constituents were found in the upgradient wells in the M-Area Aquifer Zone. Downgradient M-Area Aquifer Zone well AMB 4D contained elevated lev- 
els of tetrachloroethylene, total organic halogens, and trichloroethylene; wells AMB 5 and 6 contained elevated levels of trichloroethylene.

Aluminum was not analyzed for during second and fourth quarters; downgradient well AMB 11B, in the Upper Lost Lake Aquifer Zone, contained an elevated level of aluminum during first quarter 1994. In the Lower Lost Lake Aquifer Zone, downgradient well AMB 4B did not contain elevated levels of trichloroethylene, unlike second quarter 1994. Downgradient wells AMB 4A and 7A in the Middle Sand Aquifer Zone of the CBCU contained elevated levels of tetrachloroethylene, total organic halogens, and trichloroethylene.

\section{Quality Control Results}

Well AMB 10B was selected to receive blind replicate analyses during fourth quarter 1994 . Blind replicate analyses, representing approximately 5 percent of the quarter's total groundwater samples, are performed by the analytical laboratories each quarter for wells selected by EPD/EMS as part of the EPD/EMS quality assurance program (see Appendix E). The results of the analyses are used for both intralaboratory and interlaboratory comparisons. The Savannah River Site's Groundwater Monitoring Program, Fourth Quarter 1994 (U) (EPD/EMS, 1995, in preparation) provides full replicate results and statistical comparisons of both blind replicate and duplicate results. As a part of intralaboratory quality assurance procedures, certain analyses were duplicated by the laboratory. These results are reported in Table 3 (Appendix D). 
WSRC-TR-94-0615

Unclassified

\section{Conclusions}

During fourth quarter 1994, as in previous quarters, tetrachloroethylene and trichloroethylene exceeded the final PDWS in several AMB wells. Tetrachloroethylene exceeded standards in wells AMB $4 A, 4 D$, and $7 A$, with values of 43,10 , and $16 \mu \mathrm{g} / \mathrm{L}$, respectively. Trichloroethylene exceeded standards in wells $A M B 4 A, 4 D, 5,6$, and $7 A$, with values of $523,135,40,7.2$, and $200 \mu \mathrm{g} / \mathrm{L}$, respectively. Bis(2-ethylhexyl) phthalate, gross alpha, and lead, all of which exceeded standards in previous quarters, were not analyzed for during fourth quarter 1994. Dichloromethane (a common laboratory contaminant) has not exceeded the final PDWS since fourth quarter 1993. The RCRA Part B post-closure care permit application for the Met Lab HWMF (WSRC, 1991) indicates that a small, isolated plume of tetrachloroethylene and trichloroethylene, located in the M-Area Aquifer Zone near the Met Lab HWMF, is from the Metallurgical Laboratory Basin. The permit application also indicates that the primary source of the contamination in the deeper units near the Met Lab HWMF is the extensive organic halogens plume resulting from M-Area operations. SRS is addressing the effects of organic halogens near the Met Lab HWMF under the provisions of the corrective action program of the M-Area HWMF Part B post-closure permit renewal application (WSRC, 1992b; WSRC, 1993; WSRC, 1994).

Similar to past quarters, field measurements for $\mathrm{pH}$ and specific conductance, and laboratory analyses for total organic halogens, exceeded the Flag 2 criteria in one or more of the AMB wells during fourth quarter 1994. Field pH exceeded standards in well AMB 13AR, with a value of pH 10.2. Field specific conductance exceeded standards with a value of $703 \mu \mathrm{S} / \mathrm{cm}$ in well AMB 10A. Total organic halogens exceeded standards in wells AMB 4A, 4D, and 7A, with values of 156, 96, and $144 \mu \mathrm{g} / \mathrm{L}$, respectively. Aluminum, iron, manganese, and tin, all of which exceeded Flag 2 criteria in several wells during third quarter 1994, were not analyzed for during fourth quarter 1994.

During fourth quarter 1994, no elevated constituents were detected in upgradient wells AMB 11D and 12D. Also, elevated constituents were not found in downgradient wells $A M B 4 B, 7,7 B, 8 D$, 9D, 10A, 10B, 10D, 10DD, 11B, and 13AR.

During second quarter 1994, SRS received SCDHEC approval to install five POC wells and two plume definition wells near the Met Lab HWMF. This project began in July 1994 and is complete. The data from these wells will be presented in this report when available.

Historically and currently, the horizontal flow direction in the M-Area Aquifer Zone beneath the Met Lab HWMF is west to northwest (UTM coordinates). Historically, the flow direction in the Upper Lost Lake Aquifer Zone is to the south-southeast, the flow in the Lower Lost Lake Aquifer Zone is to the south-southwest, and the flow in the Middle Sand Aquifer Zone of the CBCU is to the southwest. During fourth quarter 1994, data were insufficient to determine the flow direction and rate in the Upper Lost Lake Aquifer Zone. The low horizontal gradient or near-linear arrangement of the monitoring wells in the Lower Lost Lake Aquifer Zone and in the Middle Sand Aquifer Zone of the CBCU prevented determination of reliable flow directions and rates in these hydrostratigraphic divisions. The flow rate estimate for groundwater in the M-Area Aquifer Zone during fourth quarter was $130 \mathrm{ft} / \mathrm{year}$. 
WSRC-TR-94-0615

Unclassified

\section{Summary}

During 1994, 18 groundwater monitoring wells of the AMB series at the Met Lab HWMF were sampled quarterly to comply with the Natural Resources Defense Council et al. Consent Decree of May 1988 and as part of the Savannah River Site Groundwater Monitoring Program. SRS has completed construction of five POC and two plume definition wells near the Met Lab HWMF, as approved by SCDHEC during second quarter 1994. Analytical data from these wells will be included in this report when available.

During the year, samples from seven of the wells $(39 \%)$ exhibited levels of bis(2-ethylhexyl) phthalate, gross alpha, lead, tetrachloroethylene, and trichloroethylene that exceeded the final PDWS. In addition, aluminum, field $\mathrm{pH}$, field specific conductance, iron, manganese, tin, and total organic halogens exceeded Flag 2 criteria in one or more of the wells each quarter these constituents were analyzed.

The results for 1994 are similar to the results for 1993, except for dichloromethane and lead. Dichloromethane, a common laboratory contaminant, did not exceed the final PDWS in 1994 as it had done in 1993. The Flag 2 criterion for lead changed from 15 to $50 \mu \mathrm{g} / \mathrm{L}$ between fourth quarter 1993 and first quarter 1994; consequently, the third quarter result of $24 \mu \mathrm{g} / \mathrm{L}$ for well AMB 6 was not flagged as elevated.

Groundwater flow directions and rates at the Met Lab HWMF were similiar to flow directions and rates in 1993. 
WSRC-TR-94-0615

Unclassified

\section{References Cited}

EPD/EMS (Environmental Protection Department/Environmental Monitoring Section), 1994. Environmental Protection Department's Well Inventory (through the second quarter of 1994), ESH-EMS-940518. Westinghouse Savannah River Company, Savannah River Site, Aiken, SC.

EPD/EMS (Environmental Protection Department/Environmental Monitoring Section), 1995. The Savannah River Site's Groundwater Monitoring Program, Fourth Quarter 1994 (U), ESHEMS-940517, in preparation. Westinghouse Savannah River Company, Savannah River Site, Aiken, SC.

Heffner, J. D., and Exploration Resources, Inc., 1991. Technical Summary of Groundwater Quality Protection Program at the Savannah River Site (1952-1986), Volume I-Site Geohydrology and Waste Sites, DPSP-88-1002. Westinghouse Savannah River Company, Savannah River Site, Aiken, SC.

Jerome, K. M., 1990. Groundwater Quality Assessment Plan for the Metallurgical Laboratory Hazardous Waste Management Facility, WSRC-RP-90-1185, October 1990. Westinghouse Savannah River Company, Savannah River Site, Aiken, SC.

Jerome, K. M., 1991. Groundwater Quality Assessment Plan for the Metallurgical Laboratory Hazardous Waste Management Facility, WSRC-RP-90-1185, Rev. 1, March 1991. Westinghouse Savannah River Company, Savannah River Site, Aiken, SC.

Lewis, S. E., and R. K. Aadland, 1992. Hydrogeologic Setting of AM Area: Framework for Groundwater Transport, WSRC-TR-92-355. Westinghouse Savannah River Company, Savannah River Site, Aiken, SC.

Lewis, S. E., and R. K. Aadland, 1993. Hydrogeologic Setting of the AM Area: Framework for Groundwater Transport, WSRC-TR-92-355 (December 10, 1993, revision). Westinghouse Savannah River Company, Savannah River Site, Aiken, SC.

SCDHEC (South Carolina Department of Health and Environmental Control), 1990. South Carolina Hazardous Waste Management Regulations, R.61-79.124, .260 through .266, .268, and .270; November 1990. Columbia, SC.

WSRC (Westinghouse Savannah River Company), 1989. Application for a RCRA Part B PostClosure Care Permit, Vol. XIV, Metallurgical Laboratory Hazardous Waste Management Facility, Rev. O, June 1989. Savannah River Site, Aiken, SC.

WSRC (Westinghouse Savannah River Company), 1991. Application for a Hazardous Waste Part B Post-Closure Care Permit, Vol. XIV [Metallurgical Laboratory Hazardous Waste Management Facility], Rev. 1, December 1991. Savannah River Site, Aiken, SC.

WSRC (Westinghouse Savannah River Company), 1992a. Metallurgical Laboratory Hazardous Waste Management Facility Basin Closure Plan, Vol. I, Rev. 9, April 1992. Savannah River Site, Aiken, SC. 
WSRC (Westinghouse Savannah River Company), 1992b. 1992 RCRA Part B Permit Renewal Application, Volume XIV, Metallurgical Laboratory Hazardous Waste Management FacilityPost-Closure, WSRC-IM-91-53, Book 1, Rev. 0, September 1992. Savannah River Site, Aiken, SC.

WSRC (Westinghouse Savannah River Company), 1992c. Sampling Groundwater Monitoring Wells, Hydrogeologic Data Collection Procedures and Specifications, Manual 3Q5, Rev. 1 , Chapter 15. Savannah River Site, Aiken, SC.

WSRC (Westinghouse Savannah River Company), 1993. 1992 RCRA Part B Permit Renewal Application, Volume III, M-Area Hazardous Waste Management Facility-Post-Closure, WSRC-IM-91-53, Book 1, Rev. 1, February 1993. Savannah River Site, Aiken, SC.

WSRC (Westinghouse Savannah River Company), 1994. M-Area Hazardous Waste Management Facility 1992 Renewal Application for a RCRA Part B Permit, WSRC-IM-91-53, Vol. III, Rev. 3, July 1994. Savannah River Site, Aiken, SC. 


\section{Errata}

In tables with four quarters of data (Table 1, Appendix D), some values for earlier quarters may differ from values for those same quarters presented in earlier reports because some reanalyses may have been performed by the laboratories after the reports were printed. Also, samples for field data are collected once each quarter, but samples for analytical data may be collected more than once each quarter. Thus, because the results tables present the highest analytical results for the quarter, a reported analytical result may be from a sample collected on a different date than the date the field data sample was collected.

Fourth Quarter 1993:

- Page 5, Discussion, Groundwater Monitoring Data: Roy F. Weston, Inc., was omitted from the paragraph discussing the laboratories that analyzed groundwater samples for the quarter.

First Quarter 1994 through Third Quarter 1994:

- No errata have been reported. 
WSRC-TR-94-0615 Unclassified

THIS PAGE LEFT BLANK INTENTIONALLY, 
WSRC-TR-94-0615

Unclassified

\section{Appendix A}

Final Primary Drinking Water Standards 
WSRC-TR-94-0615

Unclassified

THIS PAGE LEFT BLANK INTENTIONALLY. 
Final Primary Drinking Water Standards

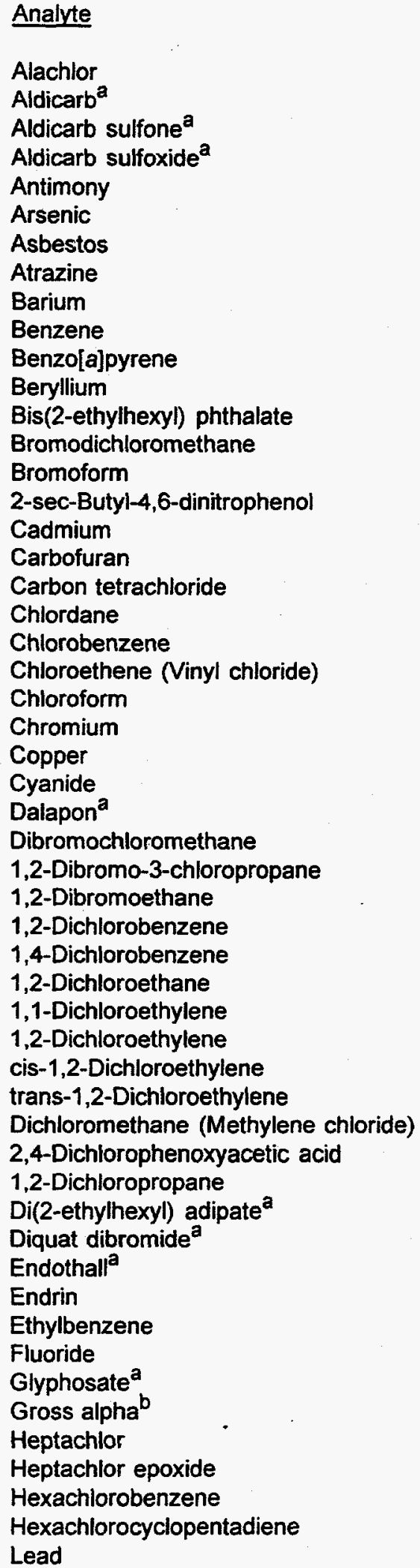

\begin{tabular}{|c|c|c|c|}
\hline Unit & Level & $\underline{\text { Status }}$ & Source \\
\hline$\mu g / L$ & 2 & Final & EPA, 1993 \\
\hline$\mu g / L$ & 3 & Final & EPA, 1993 \\
\hline$\mu g / L$ & 2 & Final & EPA, 1993 \\
\hline$\mu g / L$ & 4 & Final & EPA, 1993 \\
\hline$\mu g / L$ & 6 & Final & EPA, 1993 \\
\hline$\mu g / L$ & 50 & Final & EPA, 1993 \\
\hline Fibers $/ L$ & $7,000,000$ & Final & EPA, 1993 \\
\hline$\mu g / L$ & 3 & Final & EPA, 1993 \\
\hline$\mu g / L$ & 2,000 & Final & EPA, 1993 \\
\hline $\boldsymbol{\mu g} / \mathrm{L}$ & 5 & Final & EPA, 1993 \\
\hline$\mu g / L$ & 0.2 & Final & EPA, 1993 \\
\hline$\mu g / L$ & 4 & Final & EPA, 1993 \\
\hline$\mu \mathrm{gg} / \mathrm{L}$ & 6 & Final & EPA, 1993 \\
\hline$\mu g / L$ & 100 & Final & EPA, 1993 \\
\hline$\mu g / L$ & 100 & Final & EPA, 1993 \\
\hline$\mu g / L$ & 7 & Final & EPA, 1993 \\
\hline$\mu g / L$ & 5 & Final & EPA, 1993 \\
\hline$\rho g / L$ & 40 & Final & EPA, 1993 \\
\hline$\mu g / L$ & 5 & Final & EPA, 1993 \\
\hline$\mu g / L$ & 2 & Final & EPA, 1993 \\
\hline$\mu g / L$ & 100 & Final & EPA, 1993 \\
\hline$\mu g / L$ & 2 & Final & EPA, 1993 \\
\hline$\mu \mathrm{g} / \mathrm{L}$ & 100 & Final & EPA, 1993 \\
\hline$\mu g / L$ & 100 & Final & EPA, 1993 \\
\hline$\mu g / L$ & 1,300 & Final & EPA, 1993 \\
\hline$\mu g / L$ & 200 & Final & EPA, 1993 \\
\hline$\mu g / L$ & 200 & Final & EPA, 1993 \\
\hline$\rho g / L$ & 100 & Final & EPA, 1993 \\
\hline$\mu \mathrm{g} / \mathrm{L}$ & 0.2 & Final & EPA, 1993 \\
\hline$\mu \mathrm{g} / \mathrm{L}$ & 0.05 & Final & EPA, 1993 \\
\hline$\mu g / L$ & 600 & Final & EPA, 1993 \\
\hline$\mu g / L$ & 75 & Final & EPA, 1993 \\
\hline$\mu g / L$ & 5 & Final & EPA, 1993 \\
\hline$\mu \mathrm{rg} / \mathrm{L}$ & 7 & Final & EPA, 1993 \\
\hline$\mu g / L$ & 50 & Final & EPA, 1993 \\
\hline$\mu g / L$ & 70 & Final & EPA, 1993 \\
\hline$\mu g / L$ & 100 & Final & EPA, 1993 \\
\hline$\mu g / L$ & 5 & Final & EPA, 1993 \\
\hline$\mu g / L$ & 70 & Final & EPA, 1993 \\
\hline$\mu g / L$ & 5 & Final & EPA, 1993 \\
\hline$\mu g / L$ & 400 & Final & EPA, 1993 \\
\hline$\mu g / L$ & 20 & Final & EPA, 1993 \\
\hline$\mu g / L$ & 100 & Final & EPA, 1993 \\
\hline$\mu g / L$ & 2 & Final & EPA, 1993 \\
\hline$\mu g / L$ & 700 & Final & EPA, 1993 \\
\hline$\mu g / L$ & 4,000 & Final & EPA, 1993 \\
\hline$\mu g / L$ & 700 & Final & EPA, 1993 \\
\hline $\mathrm{pCi} / \mathrm{L}$ & $1.5 E+01$ & Final & EPA, 1993 \\
\hline$\mu g / L$ & 0.4 & Final & EPA, 1993 \\
\hline$\mu g / L$ & 0.2 & Final & EPA, 1993 \\
\hline$\mu g / L$ & 1 & Final & EPA, 1993 \\
\hline$\mu g / L$ & 50 & Final & EPA, 1993 \\
\hline$\mu g / L$ & 50 & Final & SCDHEC, 198 \\
\hline
\end{tabular}




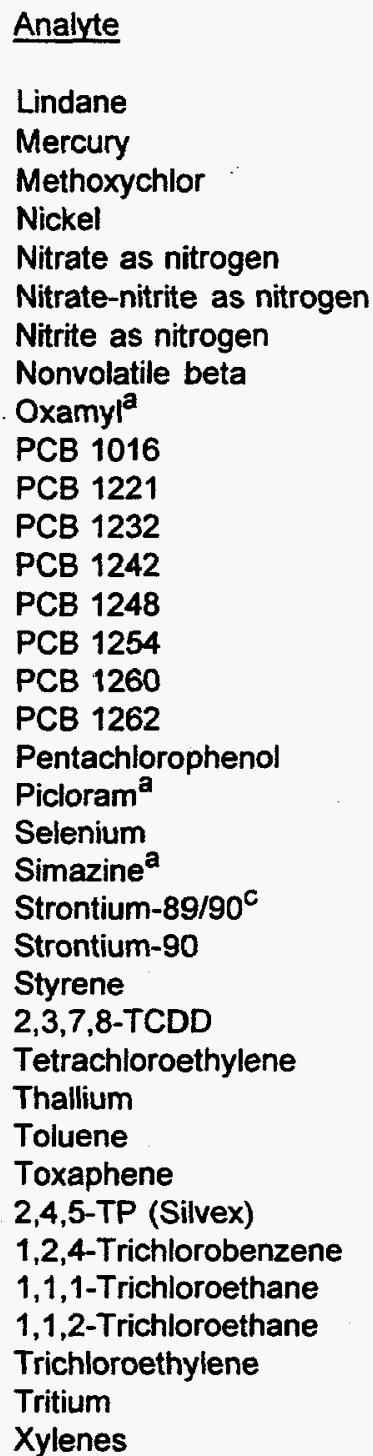

\begin{tabular}{|c|c|c|c|}
\hline Unit & Level & Status & Source \\
\hline$\mu g / L$ & 0.2 & Final & EPA, 1993 \\
\hline$\mu g / L$ & 2 & Final & EPA, 1993 \\
\hline$\mu g / L$ & 40 & Final & EPA, 1993 \\
\hline$\mu g / L$ & 100 & Final & EPA, 1993 \\
\hline$\mu g / L$ & 10,000 & Final & EPA, 1993 \\
\hline$\mu g / L$ & 10,000 & Final & EPA, 1993 \\
\hline$\mu g / L$ & 1,000 & Final & EPA, 1993 \\
\hline $\mathrm{pCi} / \mathrm{L}$ & $5 E+01$ & Interim Final & EPA, 1977 \\
\hline$\mu g / L$ & 200 & Final & EPA, 1993 \\
\hline$\mu g / L$ & 0.5 & Final & EPA, 1993 \\
\hline$\mu g / L$ & 0.5 & Final & EPA, 1993 \\
\hline$\mu g / L$ & 0.5 & Final & EPA, 1993 \\
\hline$\mu g / L$ & 0.5 & Final & EPA, 1993 \\
\hline$\mu g / L$ & 0.5 & Final & EPA, 1993 \\
\hline$\mu g / L$ & 0.5 & Final & EPA, 1993 \\
\hline$\mu g / L$ & 0.5 & Final & EPA, 1993 \\
\hline$\mu g / L$ & 0.5 & Final & EPA, 1993 \\
\hline$\mu g / L$ & 1 & Final & EPA, 1993 \\
\hline$\mu g / L$ & 500 & Final & EPA, 1993 \\
\hline$\mu g / L$ & 50 & Final & EPA, 1993 \\
\hline$\mu \mathrm{g} / \mathrm{L}$ & 4 & Final & EPA, 1993 \\
\hline pCi/L & $8 E+00$ & Final & EPA, 1993 \\
\hline $\mathrm{pCi} / \mathrm{L}$ & $8 E+00$ & Final & EPA, 1993 \\
\hline$\mu g / L$ & 100 & Final & EPA, 1993 \\
\hline$\mu g / L$ & 0.00003 & Final & EPA, 1993 \\
\hline$\mu g / L$ & 5 & Final & EPA, 1993 \\
\hline$\mu g / L$ & 2 & Final & EPA, 1993 \\
\hline$\mu g / L$ & 1,000 & Final & EPA, 1993 \\
\hline$\mu g / L$ & 3 & Final & EPA, 1993 \\
\hline$\mu g / L$ & 50 & Final & EPA, 1993 \\
\hline$\mu g / L$ & 70 & Final & EPA, 1993 \\
\hline$\mu g / L$ & 200 & Final & EPA, 1993 \\
\hline$\mu g / L$ & 5 & Final & EPA, 1993 \\
\hline$\mu g / L$ & 5 & Final & EPA, 1993 \\
\hline $\mathrm{pCi} / \mathrm{mL}$ & $2 E+01$. & Final & EPA, 1993 \\
\hline$\mu g / L$ & 10,000 & Final & EPA, 1993 \\
\hline
\end{tabular}

Note: Final PDWS were assigned to alachlor, aldicarb, aldicarb sulfone, aldicarb sulfoxide, atrazine, carbofuran, dalapon, di(2-ethylhexyl) adipate, diquat dibromide, endothall, glyphosate, oxamyl. picloram, and simazine in the SRS Groundwater Monitoring Program for the first time beginning first quarter 1994.

a At present, EMS does not perform this analysis because the constituent is not in the current contract.

b The standard given is for gross alpha including radium-226 but excluding radon and uranium.

c For double radionuclide analyses where each separate radionuclide has its own standard, the more stringent standard is used. 


\section{References}

EPA (U.S. Environmental Protection Agency), 1977. National Interim Primary Drinking Water Regulations, EPA-570/9-76-003. Washington, DC.

EPA (U.S. Environmental Protection Agency), 1993. National Primary Drinking Water Regulations, Code of Federal Regulations, Title 40, Part 141, pp. 592-732. Washington, DC.

SCDHEC (South Carolina Department of Health and Environmental Control), 1981. State Primary Drinking Water Regulations, R.61-58.5. Columbia, SC. 
WSRC-TR-94-0615

Unclassified

THIS PAGE LEFT BLANK INTENTIONALLY. 
WSRC-TR-94-0615

Unclassified

\section{Appendix B}

\section{Flagging Criteria}


WSRC-TR-94-0615

Unclassified

THIS PAGE LEFT BLANK INTENTIONALLY. 


\section{Flagging Criteria}

The Savannah River Site Environmental Protection Department/Environmental Monitoring Section (EPD/EMS) flagging criteria are as follows:

- Flag 2 criteria for constituents equal the Safe Drinking Water Act (SDWA) final Primary Drinking Water Standards (PDWS), the SDWA proposed PDWS, or the SDWA Secondary Drinking Water Standards (SDWS). If a constituent does not have a drinking water standard, the Flag 2 criterion equals 10 times the method detection limit (MDL) calculated as the 90th percentile detection limit obtained recently by one of the primary analytical laboratories.

- Flag 1 criteria for constituents equal one-half of the final PDWS, one-half the proposed PDWS, or one-half the SDWS. If a constituent does not have a drinking water standard, the Flag 1 criterion equals 5 times the MDL calculated as the 90th percentile detection limit obtained recently by one of the primary analytical laboratories.

- Flag 0 criteria are assigned to constituent levels below Flag 1 criteria, constituent levels below the sample detection limits, or constituents having no flagging criteria.

The following parameters are exceptions to the flagging rules:

- EPD/EMS sets flagging criteria for $\mathrm{pH}$ and specific conductance. No flags are set for alkalinity, calcium, carbonate, magnesium, potassium, silica, sodium, total dissolved solids, total phosphates (as P), and total phosphorus. Analyses for these parameters are conducted as part of the biennial comprehensive analyses or by special request.

- Aesthetic parameters such as color, corrosivity, Eh, odor, surfactants, and turbidity are not assigned flagging criteria but are analyzed by special request.

- Common laboratory contaminants and cleaners such as dichloromethane (methylene chloride), ketones, phthalates, and toluene are not assigned flagging criteria unless they have primary drinking water standards. These constituents are analyzed by special request.

\begin{tabular}{|c|c|c|c|c|}
\hline Analyte & Unit & Flag 1 & Flag 2 & Source $^{a}$ \\
\hline Acenaphthene & $\mu g / L$ & 50 & 100 & EPA Method 8270 \\
\hline Acenaphthylene & $\mu g / L$ & 50 & 100 & EPA Method 8270 \\
\hline Acetone & $\mu g / L$ & 500 & 1,000 & EPA Method 8240 \\
\hline Acetonitrile (Methyl cyanide) & $\mu g / L$ & 500 & 1,000 & EPA Method 8240 \\
\hline Acetophenone & $\mu g / L$ & 50 & 100 & EPA Method 8270 \\
\hline 2-Acetylaminofluorene & $\mu g / L$ & 50 & 100 & EPA Method 8270 \\
\hline Acrolein & $\mu g / L$ & 100 & 200 & EPA Method 8240 \\
\hline Acrylonitrile & $\mu g / L$ & 100 & 200 & EPA Method 8240 \\
\hline Actinium-228 & $\mathrm{pCi} / \mathrm{L}$ & $1.64 E+03$ & $3.27 E+03$ & Proposed PDWS (EPA, 1991) \\
\hline Alachlor & $\mu g / L$ & 1 & 2 & Final PDWS (EPA, 1993a) \\
\hline Aldicarb $^{b}$ & $\mu g / L$ & 1.5 & 3 & Final PDWS (EPA, 1993a) \\
\hline Aldicarb sulfone $e^{b}$ & $\mu g / L$ & 1 & 2 & Final PDWS (EPA, 1993a) \\
\hline Aldicarb sulfoxide $b$ & $\mu g / L$ & 2 & 4 & Final PDWS (EPA, 1993a) \\
\hline Aldrin & $\mu g / L$ & 0.25 & 0.5 & EPA Method 8080 \\
\hline Alkalinity (as $\mathrm{CaCO}_{3}$ ) & & No flag & No flag & Set by EPD/EMS \\
\hline Allyl chloride & $\mu g / L$ & 250 & 500 & EPA Method 8240 \\
\hline Aluminum & $\mu g / L$ & 25 & 50 & SDWS (EPA, 1993b) \\
\hline Aluminum, dissolved & $\mu g / L$ & 25 & 50 & SDWS (EPA, 1993b) \\
\hline Aluminum, total recoverable & $\mu g / L$ & 25 & 50 & SDWS (EPA, 1993b) \\
\hline
\end{tabular}


Analyte

Americium-241

Americium-243

4-Aminobiphenyl

Ammonia

Ammonia nitrogen

Aniline

Anthracene

Antimony

Antimony, dissolved

Antimony, total recoverable

Antimony-125

Aramite

Arsenic

Arsenic, dissolved

Arsenic, total recoverable

Asbestos

Atrazine

Azobenzene

Barium

Barium, dissolved

Barium, total recoverable

Barium-140 ${ }^{C}$

Benzene

alpha-Benzene hexachloride

beta-Benzene hexachloride

delta-Benzene hexachloride

Benzidine

Benzo[a]anthracene

Benzo[b]fluoranthene

Benzo[ $k]$ fluoranthene

Benzoic acid

Benzo[g, $h$, ]perylene

Benzo[a]pyrene

1,4-Benzoquinone

Benzyl alcohol

Beryllium

Beryllium, dissolved

Beryllium, total recoverable

Beryllium-7

Bis(2-chloroethoxy) methane

Bis(2-chloroethyl) ether

Bis(2-chloroisopropyl) ether

Bis(chloromethyl) ether

Bis(2-ethylhexyl) phthalate

Bismuth-214

Boron

Boron, dissolved

Boron, total recoverable

Bromide

Bromodichloromethane

Bromoform

Bromomethane (Methyl bromide)

4-Bromophenyl phenyl ether

Butylbenzyl phthalate

2-sec-Butyl-4,6-dinitrophenol

\begin{tabular}{|c|c|c|c|}
\hline Unit & Flag 1 & Flag 2 & Source \\
\hline ill & $3.17 \mathrm{E}+00$ & $6.34 E+00$ & Proposed PDWS (EPA, 1991) \\
\hline $\mathrm{Ci} / \mathrm{L}$ & $3.19 E+00$ & $6.37 E+00$ & Proposed PDWS (EPA, 1991) \\
\hline$\mu g / L$ & 50 & 100 & EPA Method 8270 \\
\hline$g / L$ & 500 & 1,000 & APHA Method 417B \\
\hline$\mu \mathrm{rg} / \mathrm{L}$ & 500 & 1,000 & EPA Method 350.1 \\
\hline$\mu g / L$ & 50 & 100 & EPA Method 8270 \\
\hline$\mu g / L$ & 50 & 100 & EPA Method 8270 \\
\hline$\mu g / L$ & 3 & 6 & Final PDWS (EPA, 1993a) \\
\hline$\mu g / L$ & 3 & 6 & Final PDWS (EPA, 1993a) \\
\hline$\mu g / L$ & 3 & 6 & Final PDWS (EPA, 1993a) \\
\hline pCill & $1.5 E+02$ & $3 E+02$ & Interim Final PDWS (EPA, 1977) \\
\hline$\mu g / L$ & 50 & 100 & EPA Method 8270 \\
\hline$\mu g / L$ & 25 & 50 & Final PDWS (EPA, 1993a) \\
\hline$\mu g / L$ & 25 & 50 & Final PDWS (EPA, 1993a) \\
\hline$\mu g / L$ & 25 & 50 & Final PDWS (EPA, 1993a) \\
\hline Fibers/L & $3,500,000$ & $7,000,000$ & Final PDWS (EPA, 1993a) \\
\hline$\mu \mathrm{g} / \mathrm{L}$ & 1.5 & 3 & Final PDWS (EPA, 1993a) \\
\hline$\mu g / L$ & 50 & 100 & EPA Method 625 \\
\hline$\mu g / L$ & 1,000 & 2,000 & Final PDWS (EPA, 1993a) \\
\hline$\mu g / L$ & 1,000 & 2,000 & Final PDWS (EPA, 1993a) \\
\hline$\mu g / L$ & 1,000 & 2,000 & Final PDWS (EPA, 1993a) \\
\hline $\mathrm{pCi} / \mathrm{L}$ & $4.5 E+01$ & $9 E+01$ & Interim Final PDWS (EPA, 1977) \\
\hline$\mu \mathrm{g} / \mathrm{L}$ & 2.5 & 5 & Final PDWS (EPA, 1993a) \\
\hline$\mu g / L$ & 0.25 & 0.5 & EPA Method 8080 \\
\hline$\mu g / L$ & 0.25 & 0.5 & EPA Method 8080 \\
\hline$\mu g / L$ & 0.25 & 0.5 & EPA Method 8080 \\
\hline$\mu g / L$ & 250 & 500 & EPA Method 8270 \\
\hline$\mu g / L$ & 0.05 & 0.1 & Proposed PDWS (EPA, 1990) \\
\hline$\mu g / L$ & 0.1 & 0.2 & Proposed PDWS (EPA, 1990) \\
\hline$\mu g / L$ & 0.1 & 0.2 & Proposed PDWS (EPA, 1990) \\
\hline$\mu g / L$ & 250 & 500 & EPA Method 8270 \\
\hline$\mu g / L$ & 50 & 100 & EPA Method 8270 \\
\hline$\mu g / L$ & 0.1 & 0.2 & Final PDWS (EPA, 1993a) \\
\hline$\mu g / L$ & 50 & 100 & EPA Method 8270 \\
\hline$\mu g / L$ & 50 & 100 & EPA Method 8270 \\
\hline$\mu g / L$ & 2 & 4 & Final PDWS (EPA, 1993a) \\
\hline$\mu g / L$ & 2 & 4 & Final PDWS (EPA, 1993a) \\
\hline$\mu g / L$ & 2 & 4 & Final PDWS (EPA, 1993a) \\
\hline $\mathrm{pCi} / \mathrm{L}$ & $3 E+03$ & $6 E+03$ & Interim Final PDWS (EPA, 1977) \\
\hline$\mu g / L$ & 50 & 100 & EPA Method 8270 \\
\hline$\mu g / L$ & 50 & 100 & EPA Method 8270 \\
\hline$\mu g / L$ & 50 & 100 & EPA Method 8270 \\
\hline$\mu g / L$ & 50 & 100 & EPA Method 8270 \\
\hline$\mu g / L$ & 3 & 6 & Final PDWS (EPA, 1993a) \\
\hline $\mathrm{pCi} / \mathrm{L}$ & $9.4 E+03$ & $1.89 E+04$ & Proposed PDWS (EPA, 1991) \\
\hline$\mu g / L$ & 150 & 300 & EPA Method 6010 \\
\hline$\mu g / L$ & 150 & 300 & EPA Method 6010 \\
\hline$\mu g / L$ & 150 & 300 & EPA Method 6010 \\
\hline$\mu g / L$ & 5,000 & 10,000 & EPA Method 300.0 \\
\hline$\mu g / L$ & 50 & 100 & Final PDWS (EPA, 1993a) \\
\hline$\mu \mathrm{g} / \mathrm{L}$ & 50 & 100 & Final PDWS (EPA, 1993a) \\
\hline$\mu g / L$ & 5 & 10 & EPA Method 8240 \\
\hline$\mu g / L$ & 50 & 100 & EPA Method 8270 \\
\hline & No flag & No flag & Set by EPD/EMS \\
\hline$\mu g / L$ & 3.5 & 7 & Final PDWS (EPA, 1993a) \\
\hline
\end{tabular}




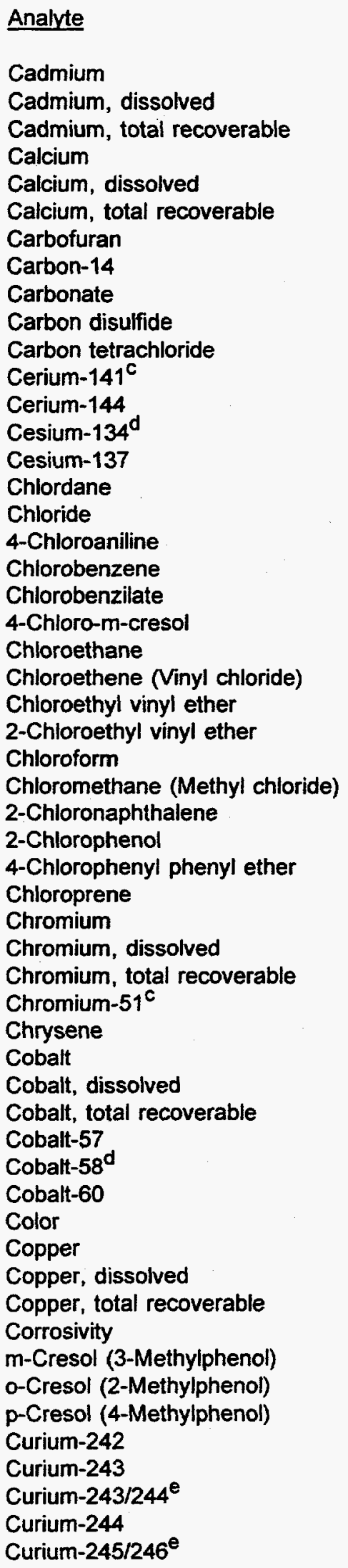

\begin{tabular}{|c|c|c|c|}
\hline Unit & Flag 1 & Flag 2 & Source \\
\hline$\mu g / L$ & 2.5 & 5 & Final PDWS (EPA, 1993a) \\
\hline$\mu g / L$ & 2.5 & 5 & Final PDWS (EPA, 1993a) \\
\hline$\mu \mathrm{g} / \mathrm{L}$ & 2.5 & 5 & Final PDWS (EPA, 1993a) \\
\hline & No flag & No flag & Set by EPD/EMS \\
\hline & No flag & No flag & Set by EPD/EMS \\
\hline & No flag & No flag & Set by EPD/EMS \\
\hline$\mu g / L$ & 20 & 40 & Final PDWS (EPA, 1993a) \\
\hline $\mathrm{pCi} / \mathrm{L}$ & $1 E+03$ & $2 \mathrm{E}+03$ & Interim Final PDWS (EPA, 1977) \\
\hline & No flag & No flag & Set by EPD/EMS \\
\hline$\mu g / L$ & 5 & 10 & EPA Method 8240 \\
\hline$\mu g / L$ & 2.5 & 5 & Final PDWS (EPA, 1993a) \\
\hline $\mathrm{pCi} / \mathrm{L}$ & $1.5 E+02$ & $3 E+02$ & Interim Final PDWS (EPA, 1977) \\
\hline $\mathrm{pCi} / \mathrm{L}$ & $1.31 E+02$ & 2.61E+02 & Proposed PDWS (EPA, 1991) \\
\hline $\mathrm{pCi} / \mathrm{L}$ & 4.07E+01 & 8.13E+01 & Proposed PDWS (EPA, 1991) \\
\hline pCill & $1 E+02$ & $2 E+02$ & Interim Final PDWS (EPA, 1977) \\
\hline$\mu g / L$ & 1 & 2 & Final PDWS (EPA, 1993a) \\
\hline$\mu \mathrm{g} / \mathrm{L}$ & 125,000 & 250,000 & SDWS (EPA, 1993b) \\
\hline$\mu \mathrm{rg} / \mathrm{L}$ & 50 & 100 & EPA Method 8270 \\
\hline$\mu g / L$ & 50 & 100 & Final PDWS (EPA, 1993a) \\
\hline$\mu g / L$ & 50 & 100 & EPA Method 8270 \\
\hline$\mu g / L$ & 50 & 100 & EPA Method 8270 \\
\hline$\mu g / L$ & 5 & 10 & EPA Method 8240 \\
\hline$\mu g / L$ & 1 & 2 & Final PDWS (EPA, 1993a) \\
\hline$\mu g / L$ & 5 & 10 & EPA Method 8240 \\
\hline$\mu g / L$ & 5 & 10 & EPA Method 8240 \\
\hline$\mu g / L$ & 50 & 100 & Final PDWS (EPA, 1993a) \\
\hline$\mu g / L$ & 5 & 10 & EPA Method 8240 \\
\hline$\mu g / L$ & 50 & 100 & EPA Method 8240 \\
\hline$\mu g / L$ & 50 & 100 & EPA Method 8270 \\
\hline$\mu g / L$ & 50 & 100 & EPA Method 8270 \\
\hline$\mu g / L$ & 1,000 & 2,000 & EPA Method 8240 \\
\hline$\mu g / L$ & 50 & 100 & Final PDWS (EPA, 1993a) \\
\hline$\mu g / L$ & 50 & 100 & Final PDWS (EPA, 1993a) \\
\hline$\mu g / L$ & 50 & 100 & Final PDWS (EPA, 1993a) \\
\hline pCill & $3 E+03$ & $6 E+03$ & Interim Final PDWS (EPA, 1977) \\
\hline$\mu g / L$ & 0.1 & 0.2 & Proposed PDWS (EPA, 1990) \\
\hline$\mu g / L$ & 20 & 40 & EPA Method 6010 \\
\hline $\operatorname{mg} / \mathrm{h}$ & 20 & 40 & EPA Method 6010 \\
\hline$\mu g / L$ & 20 & 40 & EPA Method 6010 \\
\hline $\mathrm{pCi} / \mathrm{L}$ & $5 E+02$ & $1 E+03$ & Interim Final PDWS (EPA, 1977) \\
\hline pCi/L & $4.5 E+03$ & $9 E+03$ & Interim Final PDWS (EPA, 1977) \\
\hline pC & $\begin{array}{l}5 E+01 \\
\text { No flag }\end{array}$ & $\begin{array}{l}1 E+02 \\
\text { No flaa }\end{array}$ & Interim Final PDWS (EPA, 1977) \\
\hline$\mu g / L$ & 500 & 1,000 & Final PDWS (SCDHEC, 1981) \\
\hline$\mu g / L$ & 500 & 1,000 & Final PDWS (SCDHEC, 1981) \\
\hline$\mu g / L$ & 500 & 1,000 & Final PDWS (SCDHEC, 1981) \\
\hline & No flag & No flag & Set by EPD/EMS \\
\hline$\mu g / L$ & 50 & 100 & EPA Method 8270 \\
\hline$\mu g / L$ & 50 & 100 & EPA Method 8270 \\
\hline$\mu g / L$ & 50 & 100 & EPA Method 8270 \\
\hline $\mathrm{pCi} / \mathrm{L}$ & $6.65 E+01$ & $1.33 E+02$ & Proposed PDWS (EPA, 1991) \\
\hline $\mathrm{pCi} / \mathrm{L}$ & 4.15E+00 & $8.3 E+00$ & Proposed PDWS (EPA, 1991) \\
\hline $\mathrm{pCi} / \mathrm{L}$ & $4.15 E+00$ & $8.3 E+00$ & Proposed PDWS (EPA, 1991) \\
\hline & $4.92 \mathrm{E}+00$ & $9.84 \mathrm{E}+00$ & Proposed PDWS (EPA, 1991) \\
\hline pCilL & $3.12 E+00$ & $6.23 E+00$ & Proposed PDWS (EPA, 1991) \\
\hline
\end{tabular}




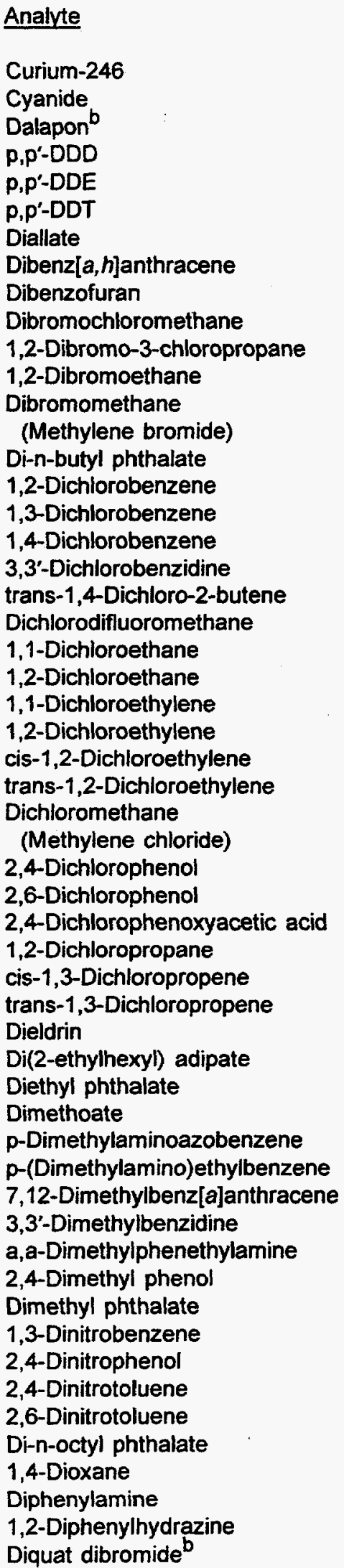

\begin{tabular}{|c|c|c|c|}
\hline$\underline{\text { Unit }}$ & Flag 1 & Flag 2 & Source \\
\hline pCill & $3.14 E+00$ & $6.27 E+00$ & Proposed PDWS (EPA, 1991) \\
\hline$\mu g / L$ & 100 & 200 & Final PDWS (EPA, 1993a) \\
\hline$\mu g / L$ & 100 & 200 & Final PDWS (EPA, 1993a) \\
\hline$\mu g / L$ & 0.5 & 1 & EPA Method 8080 \\
\hline$\mu g / L$ & 0.5 & 1 & EPA Method 8080 \\
\hline$\mu g / L$ & 0.5 & 1 & EPA Method 8080 \\
\hline$\mu g / L$ & 50 & 100 & EPA Method 8270 \\
\hline$\mu g / L$ & 0.15 & 0.3 & Proposed PDWS (EPA, 1990) \\
\hline$\mu \mathrm{g} / \mathrm{L}$ & 50 & 100 & EPA Method 8270 \\
\hline$\mu g / L$ & 50 & 100 & Final PDWS (EPA, 1993a) \\
\hline$\mu g / L$ & 0.1 & 0.2 & Final PDWS (EPA, 1993a) \\
\hline$\mu \mathrm{g} / \mathrm{L}$ & 0.025 & 0.05 & Final PDWS (EPA, 1993a) \\
\hline$\mu g / L$ & 5 & 10 & EPA Method 8240 \\
\hline & No flag & No flag & Set by EPD/EMS \\
\hline$\mu g / L$ & 300 & 600 & Final PDWS (EPA, 1993a) \\
\hline$\mu g / L$ & 50 & 100 & EPA Method 8270 \\
\hline$\mu g / L$ & 37.5 & 75 & Final PDWS (EPA, 1993a) \\
\hline$\mu g / L$ & 50 & 100 & EPA Method 8270 \\
\hline$\mu g / L$ & 150 & 300 & EPA Method 8240 \\
\hline$\mu g / L$ & 5 & 10 & EPA Method 8240 \\
\hline$\mu g / L$ & 5 & 10 & EPA Method 8240 \\
\hline$\mu g / L$ & 2.5 & 5 & Final PDWS (EPA, 1993a) \\
\hline$\mu g / L$ & 3.5 & 7 & Final PDWS (EPA, 1993a) \\
\hline$\mu g / L$ & 25 & 50 & Final PDWS (EPA, 1993a) \\
\hline$\mu g / L$ & 35 & 70 & Final PDWS (EPA, 1993a) \\
\hline$\mu g / L$ & 50 & 100 & Final PDWS (EPA, 1993a) \\
\hline$\mu g / L$ & 2.5 & 5 & Final PDWS (EPA, 1993a) \\
\hline$\mu g / L$ & 50 & 100 & EPA Method 8270 \\
\hline$\mu g / L$ & 50 & 100 & EPA Method 8270 \\
\hline$\mu g / L$ & 35 & 70 & Final PDWS (EPA, 1993a) \\
\hline$\mu g / L$ & 2.5 & 5 & Final PDWS (EPA, 1993a) \\
\hline$\mu g / L$ & 5 & 10 & EPA Method 8240 \\
\hline$\mu g / L$ & 5 & 10 & EPA Method 8240 \\
\hline$\mu g / L$ & 2.5 & 5 & EPA Method 8080 \\
\hline$\mu g / L$ & 200 & 400 & Final PDWS (EPA, 1993a) \\
\hline & No flag & No flag & Set by EPD/EMS \\
\hline$\mu g / L$ & 50 & 100 & EPA Method 8270 \\
\hline$\mu g / L$ & 50 & 100 & EPA Method 8270 \\
\hline$\mu g / L$ & 50 & 100 & EPA Method 8270 \\
\hline$\mu g / L$ & 50 & 100 & EPA Method 8270 \\
\hline$\mu g / L$ & 50 & 100 & EPA Method 8270 \\
\hline$\mu g / L$ & 50 & 100 & EPA Method 8270 \\
\hline$\mu g / L$ & 50 & 100 & EPA Method 8270 \\
\hline & No flag & No flag & Set by EPD/EMS \\
\hline$\mu g / L$ & 50 & 100 & EPA Method 8270 \\
\hline$\mu g / L$ & 250 & 500 & EPA Method 8270 \\
\hline$\mu g / L$ & 50 & 100 & EPA Method 8270 \\
\hline$\mu g / L$ & 50 & 100 & EPA Method 8270 \\
\hline & No flag & No flag & Set by EPD/EMS \\
\hline$\mu g / L$ & 50 & 100 & EPA Method 8270 \\
\hline$\mu g / L$ & 50 & 100 & EPA Method 8270 \\
\hline$\mu g / L$ & 50 & 100 & EPA Method 8270 \\
\hline$\mu \mathrm{g} / \mathrm{L}$ & 10 & 20 & Final PDWS (EPA, 1993a) \\
\hline
\end{tabular}




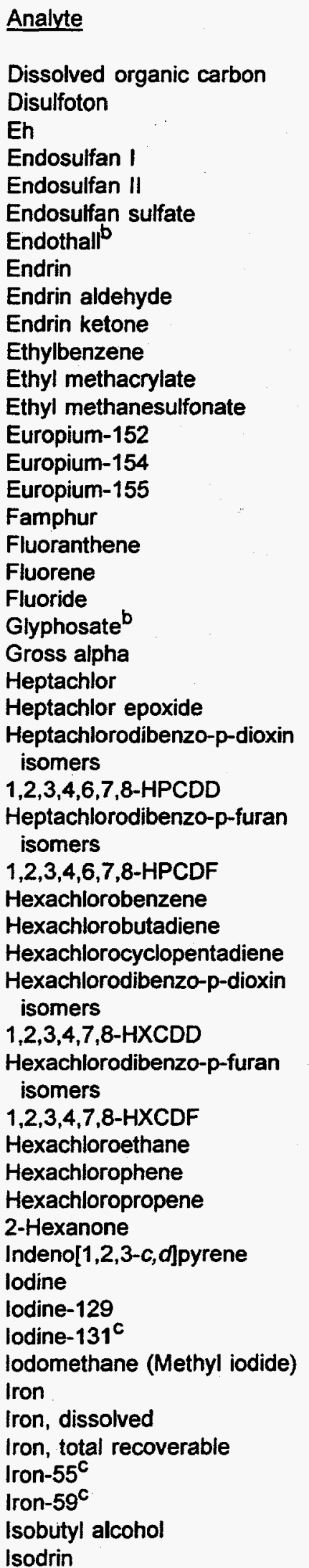

\begin{tabular}{|c|c|c|c|}
\hline Unit & Flaq 1 & Flag 2 & Source \\
\hline$\mu g / L$ & 5,000 & 10,000 & EPA Method 9060 \\
\hline$\mu g / L$ & 50 & 100 & EPA Method 8270 \\
\hline & No flag & No flag & Set by EPD/EMS \\
\hline$\mu g / L$ & 0.5 & 1 & EPA Method 8080 \\
\hline$\mu g / L$ & 0.5 & 1 & EPA Method 8080 \\
\hline$\mu g / L$ & 0.5 & 1 & EPA Method 8080 \\
\hline$\mu g / L$ & 50 & 100 & Final PDWS (EPA, 1993a) \\
\hline$\mu g / L$ & 1 & 2 & Final PDWS (EPA, 1993a) \\
\hline$\mu \mathrm{gg} / \mathrm{L}$ & 0.5 & 1 & EPA Method 8080 \\
\hline & No flag & No flag & Set by EPD/EMS \\
\hline$\mu g / L$ & 350 & 700 & Final PDWS (EPA, 1993a) \\
\hline$\mu g / L$ & 50 & 100 & EPA Method 8270 \\
\hline$\mu g / L$ & 50 & 100 & EPA Method 8270 \\
\hline pCill & $3 E+01$ & $6 E+01$ & Interim Final PDWS (EPA, 1977) \\
\hline $\mathrm{pCi} / \mathrm{L}$ & $1 E+02$ & $2 E+02$ & Interim Final PDWS (EPA, 1977) \\
\hline $\mathrm{pCi} / \mathrm{L}$ & $3 E+02$ & $6 E+02$ & Interim Final PDWS (EPA, 1977) \\
\hline$\mu g / L$ & 50 & 100 & EPA Method 8270 \\
\hline$\mu g / L$ & 50 & 100 & EPA Method 8270 \\
\hline$\mu g / L$ & 50 & 100 & EPA Method 8270 \\
\hline$\mu g / L$ & 2,000 & 4,000 & Final PDWS (EPA, 1993a) \\
\hline$\mu g / L$ & 350 & 700 & Final PDWS (EPA, 1993a) \\
\hline pCi/L & $7.5 E+00$ & $1.5 E+01$ & Final PDWS (EPA, 1993a) \\
\hline$\mu g / L$ & 0.2 & 0.4 & Final PDWS (EPA, 1993a) \\
\hline$\mu g / L$ & 0.1 & 0.2 & Final PDWS (EPA, 1993a) \\
\hline$\mu g / L$ & 0.00325 & 0.0065 & EPA Method 8280 \\
\hline$\mu g / L$ & 0.00325 & 0.0065 & EPA Method 8280 \\
\hline$\mu \mathrm{rg} / \mathrm{L}$ & 0.00225 & 0.0045 & EPA Method 8280 \\
\hline$\mu \mathrm{g} / \mathrm{L}$ & 0.00225 & 0.0045 & EPA Method 8280 \\
\hline$\mu g / L$ & 0.5 & 1 & Final PDWS (EPA, 1993a) \\
\hline$\mu g / L$ & 50 & 100 & EPA Method 8270 \\
\hline$\mu g / L$ & 25 & 50 & Final PDWS (EPA, 1993a) \\
\hline$\mu g / L$ & 0.00225 & 0.0045 & EPA Method 8280 \\
\hline$\mu g / L$ & 0.00225 & 0.0045 & EPA Method 8280 \\
\hline$\mu g / L$ & 0.002 & 0.004 & EPA Method 8280 \\
\hline$\mu g / L$ & 0.002 & 0.004 & EPA Method 8280 \\
\hline$\mu \mathrm{g} / \mathrm{L}$ & 50 & 100 & EPA Method 8270 \\
\hline$\mu \mathrm{g} / \mathrm{L}$ & 250 & 500 & EPA Method 8270 \\
\hline$\mu g / L$ & 50 & 100 & EPA Method 8270 \\
\hline$\mu g / L$ & 50 & 100 & EPA Method 8240 \\
\hline$\mu g / L$ & 50 & 100 & EPA Method 8270 \\
\hline$\mu g / L$ & 250 & 500 & APHA Method 415A \\
\hline $\mathrm{pCi} / \mathrm{L}$ & $5 E-01$ & $1 E+00$ & Interim Final PDWS (EPA, 1977) \\
\hline $\mathrm{pCi} / \mathrm{L}$ & $1.5 E+00$ & $3 E+00$ & Interim Final PDWS (EPA, 1977) \\
\hline$\mu g / L$ & 75 & 150 & EPA Method 8240 \\
\hline $\mathrm{\mu g} / \mathrm{L}$ & 150 & 300 & SDWS (EPA, 1993b) \\
\hline$\mu g / L$ & 150 & 300 & SDWS (EPA, 1993b) \\
\hline$\mu g / L$ & 150 & 300 & SDWS (EPA, 1993b) \\
\hline $\mathrm{pCi} / \mathrm{L}$ & $1 E+03$ & $2 E+03$ & Interim Final PDWS (EPA, 1977) \\
\hline $\mathrm{pCi} / \mathrm{L}$ & $1 E+02$ & $2 E+02$ & Interim Final PDWS (EPA, 1977) \\
\hline$\mu g / L$ & 500 & 1,000 & EPA Method 8240 \\
\hline$\mu g / L$ & 50 & 100 & EPA Method 8270 \\
\hline
\end{tabular}


Analyte

Isophorone

Isosafrole

Kepone

Lanthanum- $140^{\circ}$

Lead

Lead, dissolved

Lead, total recoverable

Lead-212

Lindane

Lithium

Lithium, dissolved

Lithium, total recoverable

Magnesium

Magnesium, dissolved

Magnesium, total recoverable

Manganese

Manganese, dissolved

Manganese, total recoverable

Manganese-54

Mercury

Mercury, dissolved

Mercury, total recoverable

Methacrylonitrile

Methapyrilene

Methoxychlor

3-Methylcholanthrene

2-Methyl-4,6-dinitrophenol

Methyl ethyl ketone

Methyl isobutyl ketone

Methyl methacrylate

Methyl methanesulfonate

2-Methylnaphthalene

Molybdenum

Molybdenum, dissolved

Molybdenum, total recoverable

Naphthalene

1,4-Naphthoquinone

1-Naphthylamine

2-Naphthylamine

Neptunium-237

Nickel

Nickel, dissolved

Nickel, total recoverable

Nickel-59 ${ }^{\mathrm{C}}$

Nickel-63 ${ }^{\mathrm{C}}$

Niobium-95 ${ }^{\mathrm{C}}$

Nitrate as nitrogen

Nitrate-nitrite as nitrogen

Nitrite as nitrogen

$m$-Nitroaniline

o-Nitroaniline

p-Nitroaniline

Nitrobenzene

Nitrogen by Kjeldahl method

2-Nitrophenol

\begin{tabular}{|c|c|c|}
\hline Unit & Flag 1 & Flag 2 \\
\hline$\mu g / L$ & 50 & 100 \\
\hline$\mu \mathrm{g} / \mathrm{L}$ & 50 & 100 \\
\hline$\mu g / L$ & 50 & 100 \\
\hline pCill & $3 E+01$ & $6 E+01$ \\
\hline$\mu g / L$ & 25 & 50 \\
\hline$\mu \mathrm{g} / \mathrm{L}$ & 25 & 50 \\
\hline$\mu g / L$ & 25 & 50 \\
\hline pCill & $6.2 E+01$ & $1.23 E+02$ \\
\hline$\mu g / L$ & 0.1 & 0.2 \\
\hline$\mu g / L$ & 25 & 50 \\
\hline$\mu g / L$ & 25 & 50 \\
\hline & 25 & 50 \\
\hline & No flag & No flag \\
\hline & $\begin{array}{l}\text { No flag } \\
\text { No flag }\end{array}$ & $\begin{array}{l}\text { No flag } \\
\text { No flag }\end{array}$ \\
\hline$\mu g / L$ & 25 & \\
\hline$\mu g / L$ & 25 & 50 \\
\hline$\mu g / L$ & 25 & 50 \\
\hline pCill & $1.5 E+02$ & $3 E+02$ \\
\hline$\mu g / L$ & 1 & 2 \\
\hline$\mu g / L$ & 1 & 2 \\
\hline$\mu g / L$ & 1 & 2 \\
\hline$\mu g / L$ & 250 & 500 \\
\hline$\mu g / L$ & 50 & 100 \\
\hline$\mu \mathrm{g} / \mathrm{L}$ & 20 & 40 \\
\hline$\mu g / L$ & 50 & 100 \\
\hline$\mu g / L$ & 250 & 500 \\
\hline & No flag & No flag \\
\hline & No flag & No flag \\
\hline$\mu g h$ & 50 & 100 \\
\hline$\mu g / L$ & 50 & 100 \\
\hline$\mu g / L$ & 50 & 100 \\
\hline$\mu g / L$ & 250 & 500 \\
\hline$\mu g / L$ & 250 & 500 \\
\hline$\mu g / L$ & 250 & 500 \\
\hline$\mu g / L$ & 50 & 100 \\
\hline$\mu g / L$ & 50 & 100 \\
\hline$\mu g / L$ & 50 & 100 \\
\hline$\mu \mathrm{gg} / \mathrm{L}$ & 50 & 100 \\
\hline $\mathrm{pCi} / \mathrm{L}$ & $3.53 E+00$ & $7.06 E+00$ \\
\hline$\mu \mathrm{g} / \mathrm{L}$ & 50 & 100 \\
\hline$\mu \mathrm{g} / \mathrm{L}$ & 50 & 100 \\
\hline$\mu g / L$ & 50 & 100 \\
\hline $\mathrm{pCi} / \mathrm{L}$ & $1.5 E+02$ & $3 E+02$ \\
\hline pCi/L & $2.5 E+01$ & $5 E+01$ \\
\hline pCi/l & $1.5 E+02$ & $3 . E+02$ \\
\hline$\mu g / L$ & 5,000 & 10,000 \\
\hline$\mu \mathrm{g} / \mathrm{L}$ & 5,000 & 10,000 \\
\hline$\mu g / L$ & 500 & 1,000 \\
\hline$\mu g / L$ & 50 & 100 \\
\hline$\mu g / L$ & 50 & 100 \\
\hline$\mu \mathrm{g} / \mathrm{L}$ & 50 & 100 \\
\hline$\mu \mathrm{g} / \mathrm{L}$ & 50 & 100 \\
\hline$\mu \mathrm{g} / \mathrm{h}$ & 500 & 1,000 \\
\hline$\mu \mathrm{g} / \mathrm{L}$ & 50 & 100 \\
\hline
\end{tabular}

Source

EPA Method 8270

EPA Method 8270

EPA Method 8270

Interim Final PDWS (EPA, 1977)

Final PDWS (SCDHEC, 1981)

Final PDWS (SCDHEC, 1981)

Final PDWS (SCDHEC, 1981)

Proposed PDWS (EPA, 1991)

Final PDWS (EPA, 1993a)

EPA Method 6010

EPA Method 6010

EPA Method 6010

Set by EPD/EMS

Set by EPD/EMS

Set by EPD/EMS

SDWS (EPA, 1993b)

SDWS (EPA, 1993b)

SDWS (EPA, 1993b)

Interim Final PDWS (EPA, 1977)

Final PDWS (EPA, 1993a)

Final PDWS (EPA, 1993a)

Final PDWS (EPA, 1993a)

EPA Method 8240

EPA Method 8270

Final PDWS (EPA, 1993a)

EPA Method 8270

EPA Method 8270

Set by EPD/EMS

Set by EPD/EMS

EPA Method 8270

EPA Method 8270

EPA Method 8270

EPA Method 6010

EPA Method 6010

EPA Method 6010

EPA Method 8270

EPA Method 8270

EPA Method 8270

EPA Method 8270

Proposed PDWS (EPA, 1991)

Final PDWS (EPA, 1993a)

Final PDWS (EPA, 1993a)

Final PDWS (EPA, 1993a)

Interim Final PDWS (EPA, 1977)

Interim Final PDWS (EPA, 1977)

Interim Final PDWS (EPA, 1977)

Final PDWS (EPA, 1993a)

Final PDWS (EPA, 1993a)

Final PDWS (EPA, 1993a)

EPA Method 8270

EPA Method 8270

EPA Method 8270

EPA Method 8270

EPA Method 351.2

EPA Method 8270 
Analyte

4-Nitrophenol

4-Nitroquinoline-1-oxide

$\mathrm{N}$-Nitrosodi-n-butylamine

$\mathrm{N}$-Nitrosodiethylamine

$\mathrm{N}$-Nitrosodimethylamine

$\mathrm{N}$-Nitrosodiphenylamine

$\mathrm{N}$-Nitrosodipropylamine

$\mathrm{N}$-Nitrosomethylethylamine

$\mathrm{N}$-Nitrosomorpholine

N-Nitrosopiperidine

N-Nitrosopyrrolidine

5-Nitro-o-toluidine

Nonvolatile beta

Octachlorodibenzo-p-dioxin isomers

Octachlorodibenzo-p-furan isomers

Odor

Oil \& Grease

Oxamyl

Parathion

Parathion methyl

PCB 1016

PCB 1221

PCB 1232

PCB 1242

PCB 1248

PCB 1254

PCB 1260

PCB 1262

Pentachlorobenzene

Pentachlorodibenzo-p-dioxin

isomers

1,2,3,7,8-PCDD

Pentachlorodibenzo-p-furan isomers

1,2,3,7,8-PCDF

Pentachloroethane

Pentachloronitrobenzene

Pentachlorophenol

$\mathrm{pH}$

$\mathrm{pH}$

Phenacetin

Phenanthrene

Phenol

Phenols

p-Phenylenediamine

Phorate

Picloram ${ }^{b}$

2-Picoline

Plutonium-238

Plutonium-239

Plutonium-239/240

Plutonium-240

Plutonium-241 ${ }^{\mathrm{C}}$

\begin{tabular}{|c|c|c|}
\hline Unit & Flag 1 & Flag 2 \\
\hline$\mu g / L$ & 50 & 100 \\
\hline$\mu g / L$ & 50 & 100 \\
\hline$\mu g / L$ & 50 & 100 \\
\hline$\mu g / L$ & 50 & 100 \\
\hline$\mu g / L$ & 50 & 100 \\
\hline$\mu \mathrm{g} / \mathrm{L}$ & 50 & 100 \\
\hline$\mu \mathrm{g} / \mathrm{L}$ & 50 & 100 \\
\hline$\mu \mathrm{g} / \mathrm{L}$ & 50 & 100 \\
\hline$\mu g / L$ & 50 & 100 \\
\hline$\mu g / L$ & 50 & 100 \\
\hline$\mu g / L$ & 50 & 100 \\
\hline$\mu g / L$ & 50 & 100 \\
\hline $\mathrm{pCi} / \mathrm{L}$ & 2.5E+01 & $5 E+01$ \\
\hline$\mu g / L$ & 0.005 & 0.01 \\
\hline \multirow[t]{2}{*}{$\mu g / L$} & 0.005 & 0.01 \\
\hline & No flag & No flag \\
\hline$\mu g / L$ & 5,000 & 10,000 \\
\hline$\mu g / L$ & 100 & 200 \\
\hline$\mu g / L$ & 0.25 & 0.5 \\
\hline$\mu g / L$ & 0.25 & 0.5 \\
\hline$\mu \mathrm{g} / \mathrm{L}$ & 0.25 & 0.5 \\
\hline$\mu g / L$ & 0.25 & 0.5 \\
\hline$\mu g / L$ & 0.25 & 0.5 \\
\hline$\mu \mathrm{g} / \mathrm{L}$ & 0.25 & 0.5 \\
\hline$\mu g / L$ & 0.25 & 0.5 \\
\hline$\mu g / L$ & 0.25 & 0.5 \\
\hline$\mu \mathrm{g} / \mathrm{L}$ & 0.25 & 0.5 \\
\hline$\mu g / L$ & 0.25 & 0.5 \\
\hline$\mu g / L$ & 50 & 100 \\
\hline$\mu g / L$ & 0.00275 & 0.0055 \\
\hline$\mu g / L$ & 0.00275 & 0.0055 \\
\hline$\mu g / L$ & 0.00275 & 0.0055 \\
\hline$\mu g / L$ & 0.00275 & 0.0055 \\
\hline$\mu g / L$ & 50 & 100 \\
\hline$\mu g / L$ & 50 & 100 \\
\hline$\mu \mathrm{g} / \mathrm{L}$ & 0.5 & 1 \\
\hline $\mathrm{pH}$ & 8 & 10 \\
\hline pH & 4 & 3 \\
\hline$\mu g / L$ & 50 & 100 \\
\hline$\mu g / L$ & 50 & 100 \\
\hline$\mu g / L$ & 50 & 100 \\
\hline$\mu g / L$ & 25 & 50 \\
\hline$\mu g / L$ & 50 & 100 \\
\hline$\mu g / L$ & 0.5 & 1 \\
\hline$\mu g / L$ & 250 & 500 \\
\hline$\mu g / L$ & 50 & 100 \\
\hline $\mathrm{pCi} / \mathrm{L}$ & $3.51 E+00$ & $7.02 E+00$ \\
\hline $\mathrm{pCi} / \mathrm{L}$ & $3.11 E+01$ & $6.21 E+01$ \\
\hline $\mathrm{pCi} / \mathrm{L}$ & $3.11 E+01$ & $6.21 E+01$ \\
\hline $\mathrm{pCi} / \mathrm{L}$ & $3.11 E+01$ & $6.22 E+01$ \\
\hline $\mathrm{pCi} / \mathrm{L}$ & $3.13 E+01$ & $6.26 E+01$ \\
\hline
\end{tabular}

Source

EPA Method 8270

EPA Method 8270

EPA Method 8270

EPA Method 8270

EPA Method 8270

EPA Method 8270

EPA Method 8270

EPA Method 8270

EPA Method 8270

EPA Method 8270

EPA Method 8270

EPA Method 8270

Interim Final PDWS (EPA, 1977)

EPA Method 8280

EPA Method 8280

Set by EPD/EMS

EPA Method 413.1

Final PDWS (EPA, 1993a)

EPA Method 8080

EPA Method 8080

Final PDWS (EPA, 1993a)

Final PDWS (EPA, 1993a)

Final PDWS (EPA, 1993a)

Final PDWS (EPA, 1993a)

Final PDWS (EPA, 1993a)

Final PDWS (EPA, 1993a)

Final PDWS (EPA, 1993a)

Final PDWS (EPA, 1993a)

EPA Method 8270

EPA Method 8280

EPA Method 8280

EPA Method 8280

EPA Method 8280

EPA Method 8270

EPA Method 8270

Final PDWS (EPA, 1993a)

Set by EPD/EMS

Set by EPD/EMS

EPA Method 8270

EPA Method 8270

EPA Method 8270

EPA Method 420.1

EPA Method 8270

EPA Method 8080

Final PDWS (EPA, 1993a)

EPA Method 8270

Proposed PDWS (EPA, 1991)

Proposed PDWS (EPA, 1991)

Proposed PDWS (EPA, 1991)

Proposed PDWS (EPA, 1991)

Proposed PDWS (EPA, 1991) 
Analyte

Plutonium-242 ${ }^{\mathrm{C}}$

Potassium

Potassium, dissolved

Potassium, total recoverable

Potassium-40

Promethium-144

Promethium-146

Promethium-147

Pronamid

Propionitrile

Pyrene

Pyridine

Radium (alpha-emitting) ${ }^{f}$

Radium-226

Radium-228

Radon-222

Ruthenium-103 ${ }^{\mathrm{C}}$

Ruthenium-106

Safrole

Selenium

Selenium, dissolved

Selenium, total recoverable

Silica

Silica, dissolved

Silica, total recoverable

Silver

Silver, dissolved

Silver, total recoverable

Simazine ${ }^{b}$

Sodium

Sodium, dissolved

Sodium, total recoverable

Sodium-22

Specific conductance

Strontium-89

Strontium-89/90 ${ }^{\mathrm{e}}$

Strontium-90

Styrene

Sulfate

Sulfide

Sulfotepp

Surfactants

2,3,7,8-TCDD

$2,3,7,8-T C D F$

Technetium-99

1,2,4,5-Tetrachlorobenzene

Tetrachlorodibenzo-p-dioxin isomers

Tetrachlorodibenzo-p-furan isomers

1,1,1,2-Tetrachloroethane

1,1,2,2-Tetrachloroethane

Tetrachloroethylene

2,3,4,6-Tetrachlorophenol

Thallium

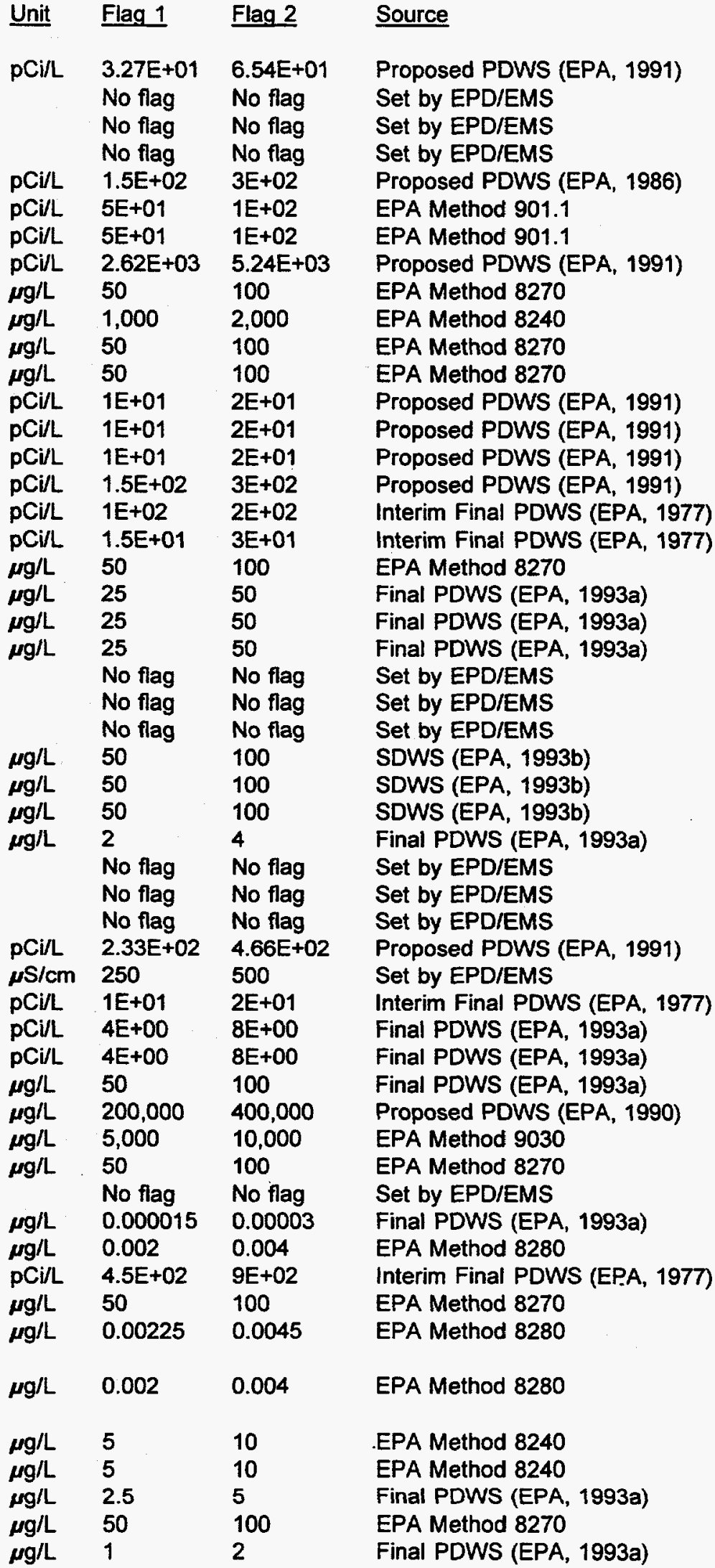

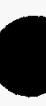


Analyte

Thallium, dissolved

Thallium, total recoverable

Thionażin

Thorium-228

Thorium-230

Thorium-232

Thorium-234

Tin

Tin, dissolved

Tin, total recoverable

Tin-113

Toluene

o-Toluidine

Total carbon

Total coliform

Total dissolved solids

Total hydrocarbons

Total inorganic carbon

Total organic carbon

Total organic halogens

Total organic nitrogen

Total petroleum hydrocarbons

Total phosphates (as P)

Total phosphorus

Toxaphene

2,4,5-TP (Silvex)

Tributyl phosphate

1,2,4-Trichlorobenzene

1,1,1-Trichloroethane

1,1,2-Trichloroethane

Trichloroethylene

Trichlorofluoromethane

2,4,5-Trichlorophenol

2,4,6-Trichlorophenol

2,4,5-Trichlorophenoxyacetic acid

1,2,3-Trichloropropane

$0,0,0$-Triethyl phosphorothioate

1,3,5-Trinitrobenzene

Tritium

Turbidity 9

Uranium

Uranium, dissolved

Uranium, total recoverable

Uranium alpha activity

Uranium-233/234

Uranium-234

Uranium-235

Uranium-238

Vanadium

Vanadium, dissolved

Vanadium, total recoverable

Vinyl acetate

\begin{tabular}{|c|c|c|}
\hline Unit & Flag 1 & Flag 2 \\
\hline$\mu g / L$ & 1 & 2 \\
\hline$\mu g / L$ & 1 & 2 \\
\hline$\mu g / L$ & 50 & 100 \\
\hline pCi/L & $6.25 E+01$ & $1.25 E+02$ \\
\hline $\mathrm{pCi} / \mathrm{L}$ & $3.96 E+01$ & $7.92 E+01$ \\
\hline pCi/L & $4.4 E+01$ & $8.8 E+01$ \\
\hline pCill & $2 E+02$ & $4.01 E+02$ \\
\hline$\mu g / L$ & 10 & 20 \\
\hline$\mu g / L$ & 10 & 20 \\
\hline $\boldsymbol{\mu} g / \mathrm{L}$ & 10 & 20 \\
\hline pCi/L & $1.5 E+02$ & $3 E+02$ \\
\hline$\mu g / L$ & 500 & 1,000 \\
\hline$\mu g / L$ & 50 & 100 \\
\hline$\mu g / L$ & 5,000 & 10,000 \\
\hline & $\begin{array}{l}0 \\
\text { No flag }\end{array}$ & 0 \\
\hline$\mu g / L$ & 5,000 & 10,000 \\
\hline$\mu g / L$ & 5,000 & 10,000 \\
\hline$\mu g / L$ & 5,000 & 10,000 \\
\hline$\mu g / L$ & 25 & 50 \\
\hline$\mu g / L$ & 500 & 1,000 \\
\hline$\mu g / L$ & 5,000 & 10,000 \\
\hline & No flag & No flag \\
\hline & No flag & No flag \\
\hline$\mu g / L$ & 1.5 & 3 \\
\hline$\mu g / L$ & 25 & 50 \\
\hline$\mu g / L$ & 50 & 100 \\
\hline$\mu g / L$ & 35 & 70 \\
\hline$\mu g / L$ & 100 & 200 \\
\hline$\mu g / L$ & 2.5 & 5 \\
\hline$\mu g / L$ & 2.5 & 5 \\
\hline$\mu g / L$ & 5 & 10 \\
\hline$\mu g / L$ & 50 & 100 \\
\hline$\mu g / L$ & 50 & 100 \\
\hline$\mu g / L$ & 2.5 & 5 \\
\hline$\mu g / L$ & 5 & 10 \\
\hline$\mu g / L$ & 50 & 100 \\
\hline$\mu g / L$ & 50 & 100 \\
\hline $\mathrm{pCi} / \mathrm{mL}$ & $1 E+01$ & $2 E+01$ \\
\hline & No flag & No flag \\
\hline$\mu g / L$ & 10 & 20 \\
\hline$\mu g / L$ & 10 & 20 \\
\hline$\mu g / L$ & 10 & 20 \\
\hline $\mathrm{pCi} / \mathrm{L}$ & 1.5E+01 & $3 E+01$ \\
\hline pCi/L & $6.9 E+00$ & $1.38 E+01$ \\
\hline $\mathrm{pCi} / \mathrm{L}$ & $6.95 E+00$ & $1.39 E+01$ \\
\hline pCi/L & $7.25 E+00$ & $1.45 E+01$ \\
\hline $\mathrm{pCi} / \mathrm{L}$ & $7.3 E+00$ & $1.46 E+01$ \\
\hline$\mu g / L$ & 40 & 80 \\
\hline$\mu g / L$ & 40 & 80 \\
\hline$\mu g / L$ & 40 & 80 \\
\hline$\mu g / L$ & 5 & 10 \\
\hline
\end{tabular}

Source

Final PDWS (EPA, 1993a)

Final PDWS (EPA, 1993a)

EPA Method 8270

Proposed PDWS (EPA, 1991)

Proposed PDWS (EPA, 1991)

Proposed PDWS (EPA, 1991)

Proposed PDWS (EPA, 1991)

EPA Method 282.2

EPA Method 282.2

EPA Method 282.2

Interim Final PDWS (EPA, 1977)

Final PDWS (EPA, 1993a)

EPA Method 8270

EPA Method 9060

Final PDWS (EPA, 1993a)

Set by EPD/EMS

EPA Method 418.1

EPA Method 9060

EPA Method 9060

EPA Method 9020

APHA Method 420

EPA Method 418.1

Set by EPD/EMS

Set by EPD/EMS

Final PDWS (EPA, 1993a)

Final PDWS (EPA, 1993a)

EPA Method 8270

Final PDWS (EPA, 1993a)

Final PDWS (EPA, 1993a)

Final PDWS (EPA, 1993a)

Final PDWS (EPA, 1993a)

EPA Method 8240

EPA Method 8270

EPA Method 8270

EPA Method 8150

EPA Method 8240

EPA Method 8270

EPA Method 8270

Final PDWS (EPA, 1993a)

Set by EPD/EMS

Proposed PDWS (EPA, 1991)

Proposed PDWS (EPA, 1991)

Proposed PDWS (EPA, 1991)

Proposed PDWS (EPA, 1991)

Proposed PDWS (EPA, 1991)

Proposed PDWS (EPA, 1991)

Proposed PDWS (EPA, 1991)

Proposed PDWS (EPA, 1991)

EPA Method 6010

EPA Method 6010

EPA Method 6010

EPA Method 8240 


\begin{tabular}{|c|c|c|c|c|}
\hline Analvie & Unit & Flag 1 & Flag 2 & Source \\
\hline Xylenes & $\mu g / L$ & 5,000 & 10,000 & Final PDWS (EPA, 1993a) \\
\hline Yttrium-88 & $\mathrm{pCi} / \mathrm{L}$ & $5 E+01$ & $1 E+02$ & EPA Method 901.1 \\
\hline Zinc & $\mu \mathrm{rg} / \mathrm{L}$ & 2,500 & 5,000 & SDWS (EPA, 1993b) \\
\hline Zinc, dissolved & $\mu \mathrm{g} / \mathrm{L}$ & 2,500 & 5,000 & SDWS (EPA, 1993b) \\
\hline Zinc, total recoverable & $\mu \mathrm{g} / \mathrm{L}$ & 2,500 & 5,000 & SDWS (EPA, 1993b) \\
\hline Zinc-65 & pCill & $1.5 \mathrm{E}+02$ & $3 E+02$ & Interim Final PDWS (EPA, 1977) \\
\hline Zirconium-95 & pCi/L & $1 E+02$ & $2 E+02$ & Interim Final PDWS (EPA, 1977) \\
\hline Zirconium/Niobium-95 ${ }^{\mathrm{C}}$ & $\mathrm{pCi} /$ & $1 E+02$ & $2 E+02$ & Interim Final PDWS (EPA, 1977) \\
\hline
\end{tabular}

a References for methods are in Appendix E; references for dated sources are at the end of this appendix.

b EMS is currently unable to perform this analysis.

c EMS discontinued monitoring this radionuclide because it is inappropriate for the SRS Groundwater Monitoring Program.

d EPD/EMS set this flagging criterion using the 1991 proposed PDWS because the final PDWS in 1977 may have been in error.

e For double radionuclide analyses where each separate radionuclide has its own standard, the more stringent standard is used.

$f$ The applied standard is for radium-226.

9 The primary maximum contaminant level range for turbidity is 1-5 NTU, which is inappropriate for the SRS Groundwater Monitoring Program.

\section{References Cited}

EPA (U.S. Environmental Protection Agency), 1977. National Interim Primary Drinking Water Regulations, EPA-570/9-76-003. Washington, DC.

EPA (U.S. Environmental Protection Agency), 1986. Water Pollution Control; National Primary Drinking Water Regulations, Radionuclides (Proposed). Federal Register, September 30, 1986, pp. 34835-34862. Washington, DC.

EPA (U.S. Environmental Protection Agency), 1990. National Primary and Secondary Drinking Water Regulations; Synthetic Organic Chemicals and Inorganic Chemicals (Proposed Rule).

Federal Register, July 25, 1990, pp. 30369-30448. Washington, DC.

EPA (U.S. Environmental Protection Agency), 1991. National Primary Drinking Water Regulations; Radionuclides; Proposed Rule. Federal Register, July 18, 1991, pp. 33052-33127. Washington, DC.

EPA (U.S. Environmental Protection Agency), 1993a. National Primary Drinking Water Regulations. Code of Federal Regulations, Title 40, Part 141, pp. 592-732. Washington, DC.

EPA (U.S. Environmental Protection Agency), 1993b. National Secondary Drinking Water Regulations. Code of Federal Regulations, Title 40, Part 143, pp. 774-777. Washington, DC.

SCDHEC (South Carolina Department of Health and Environmental Control), 1981. State Primary Drinking Water Regulations, R.61-58.5. Columbia, SC. 
WSRC-TR-94-0615

Unclassified

\section{Appendix C}

Figures 
WSRC-TR-94-0615

Unclassified

THIS PAGE LEFT BLANK INTENTIONALLY. 


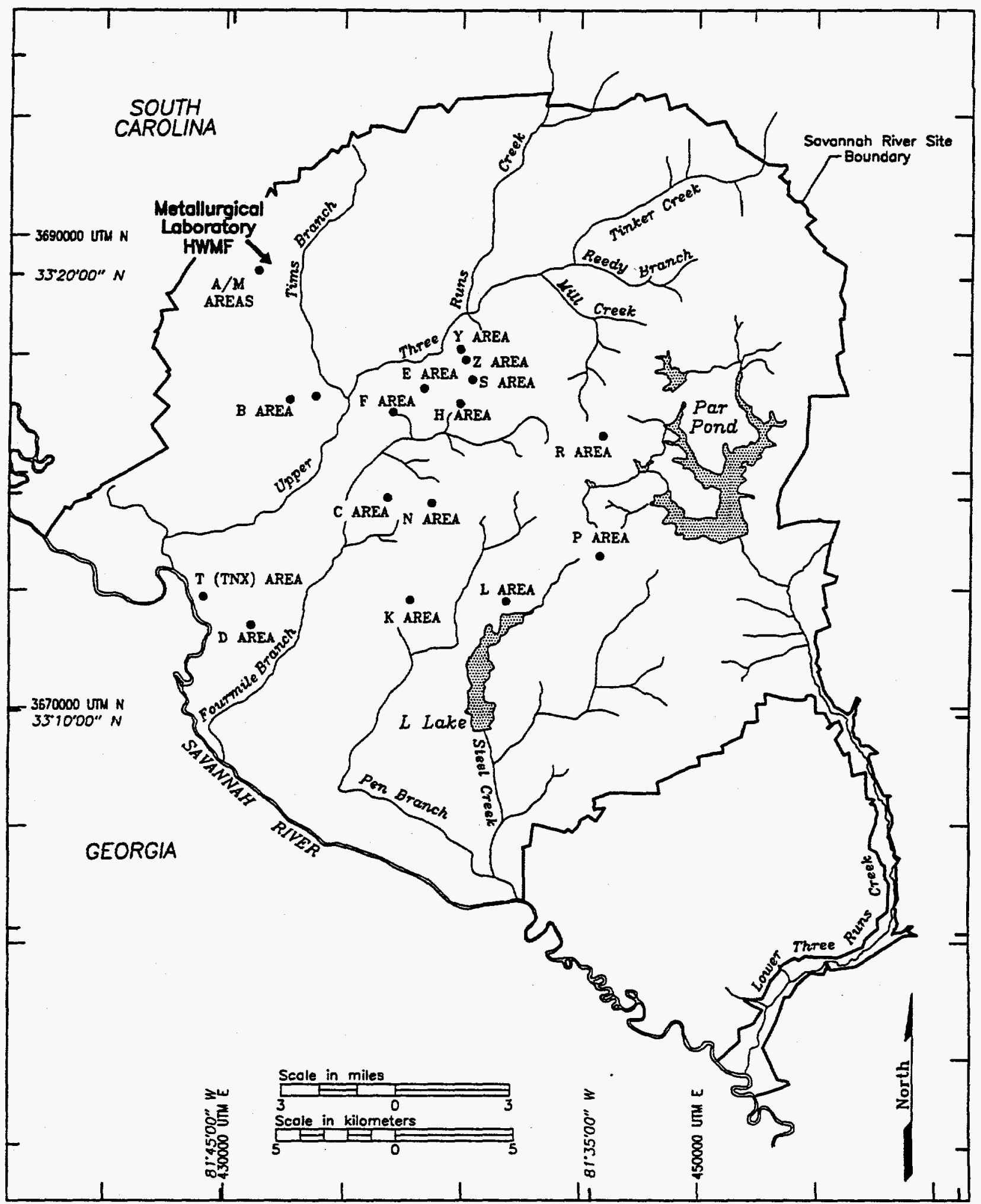

Figure 1. Location of the Metallurgical Laboratory HWMF at the Savannah River Site 


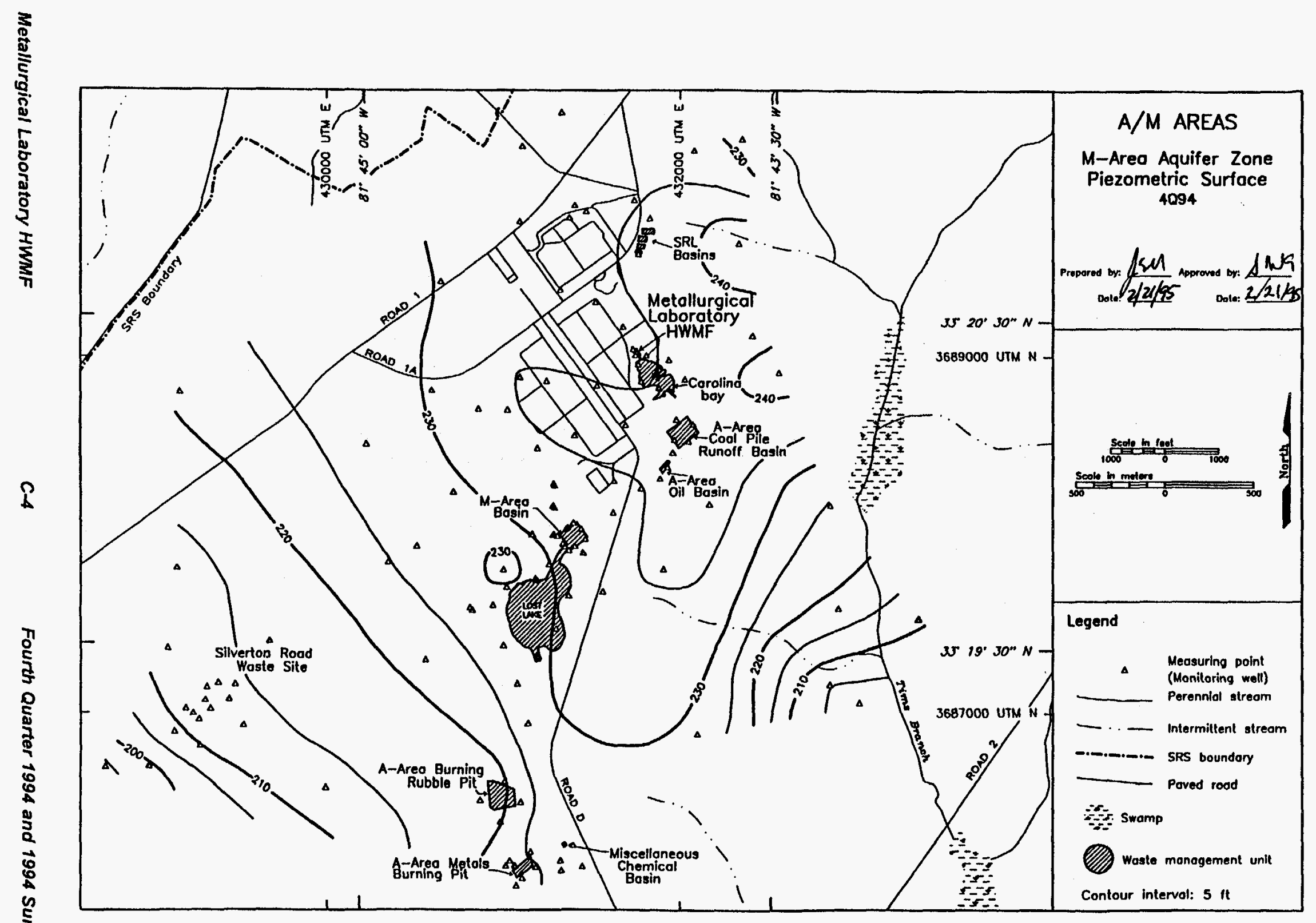

Figure 2. Piezometric Surface Map of the M-Area Aquifer Zone in A/M Areas 


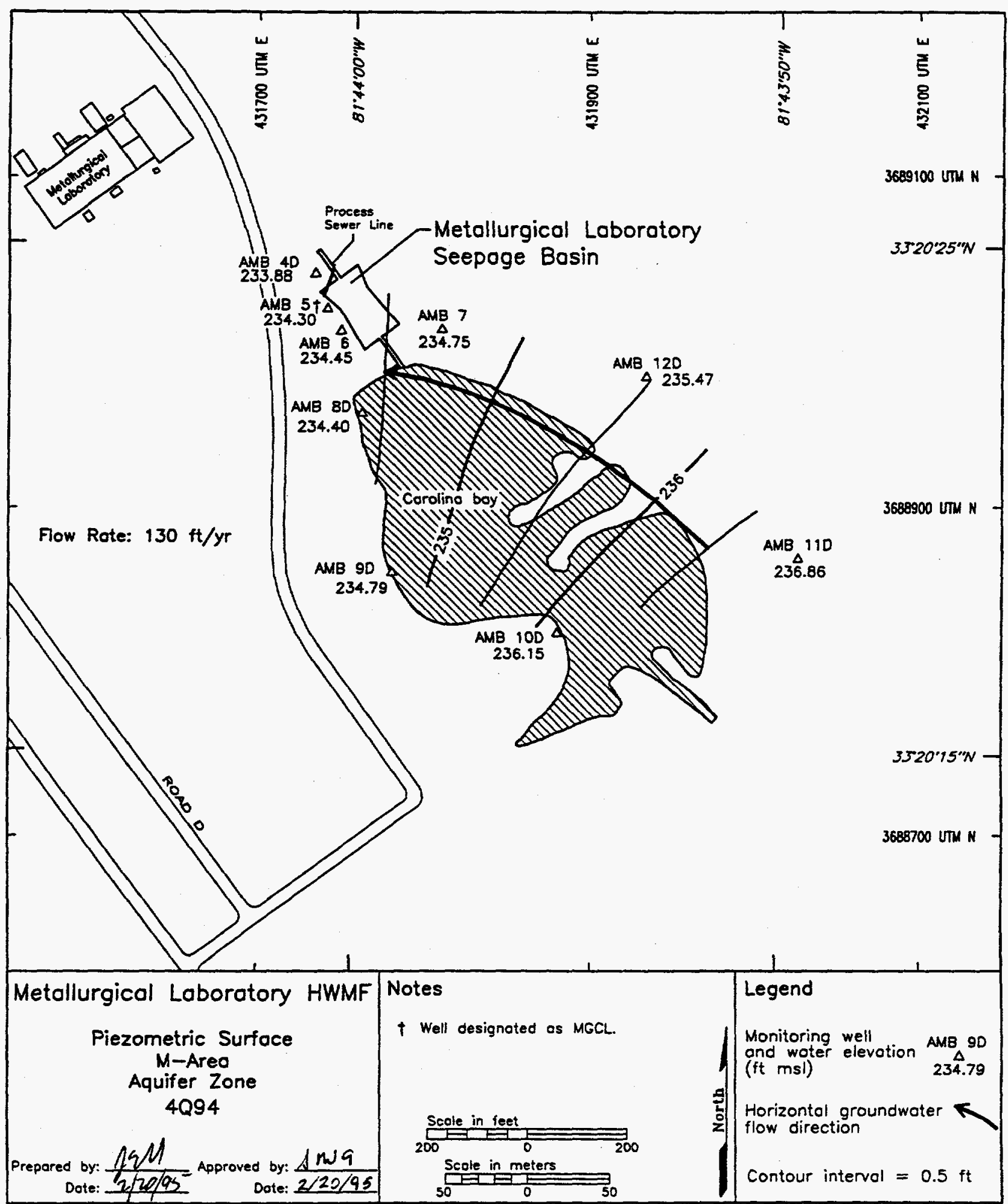

Figure 3. Piezometric Surface Map and Location of the M-Area Aquifer Zone Wells at the Metallurgical Laboratory HWMF 


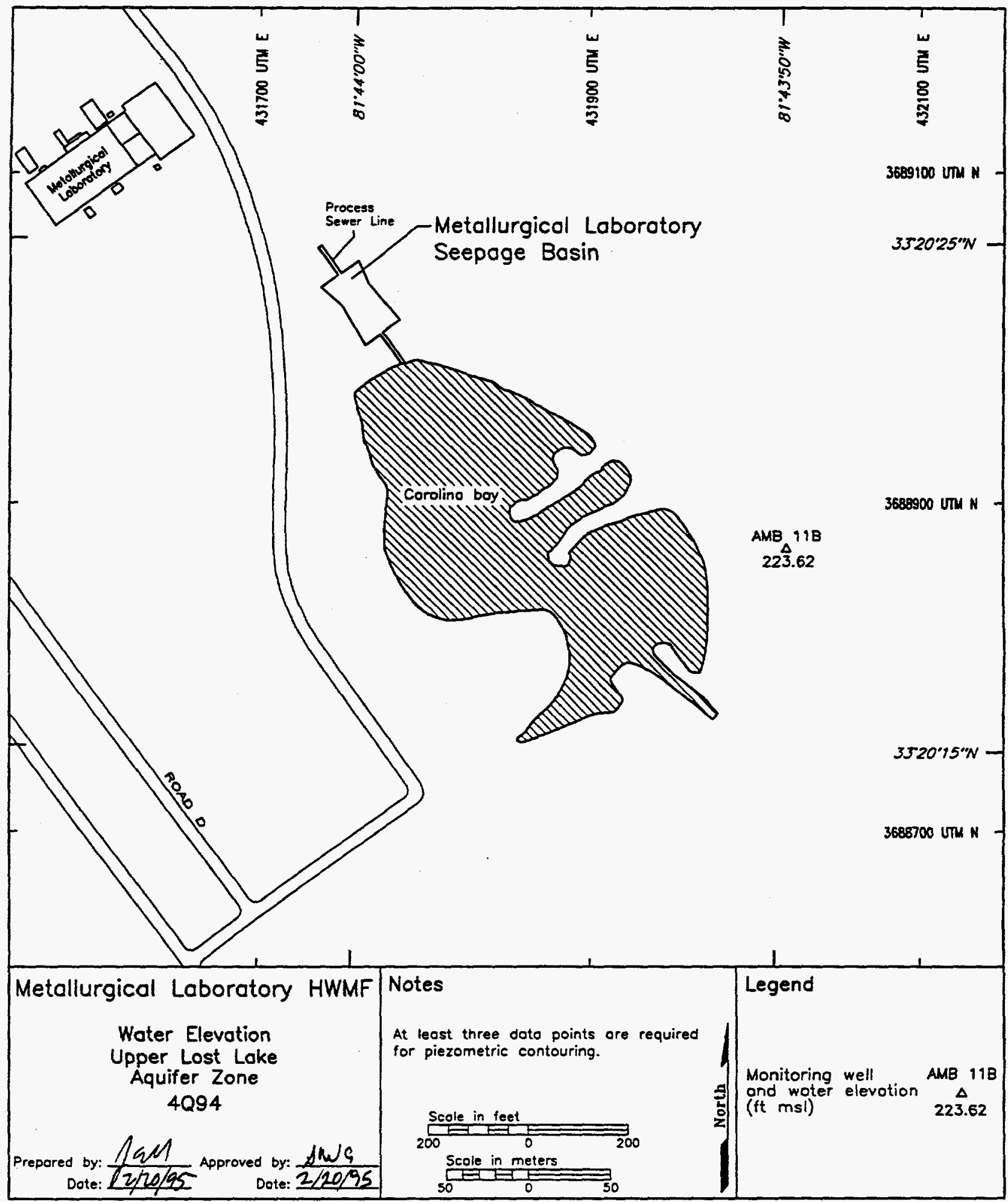

Figure 4. Water-Elevation Map and Location of Monitoring Wells in the Upper Lost Lake Aquifer Zone at the Metallurgical Laboratory HWMF 


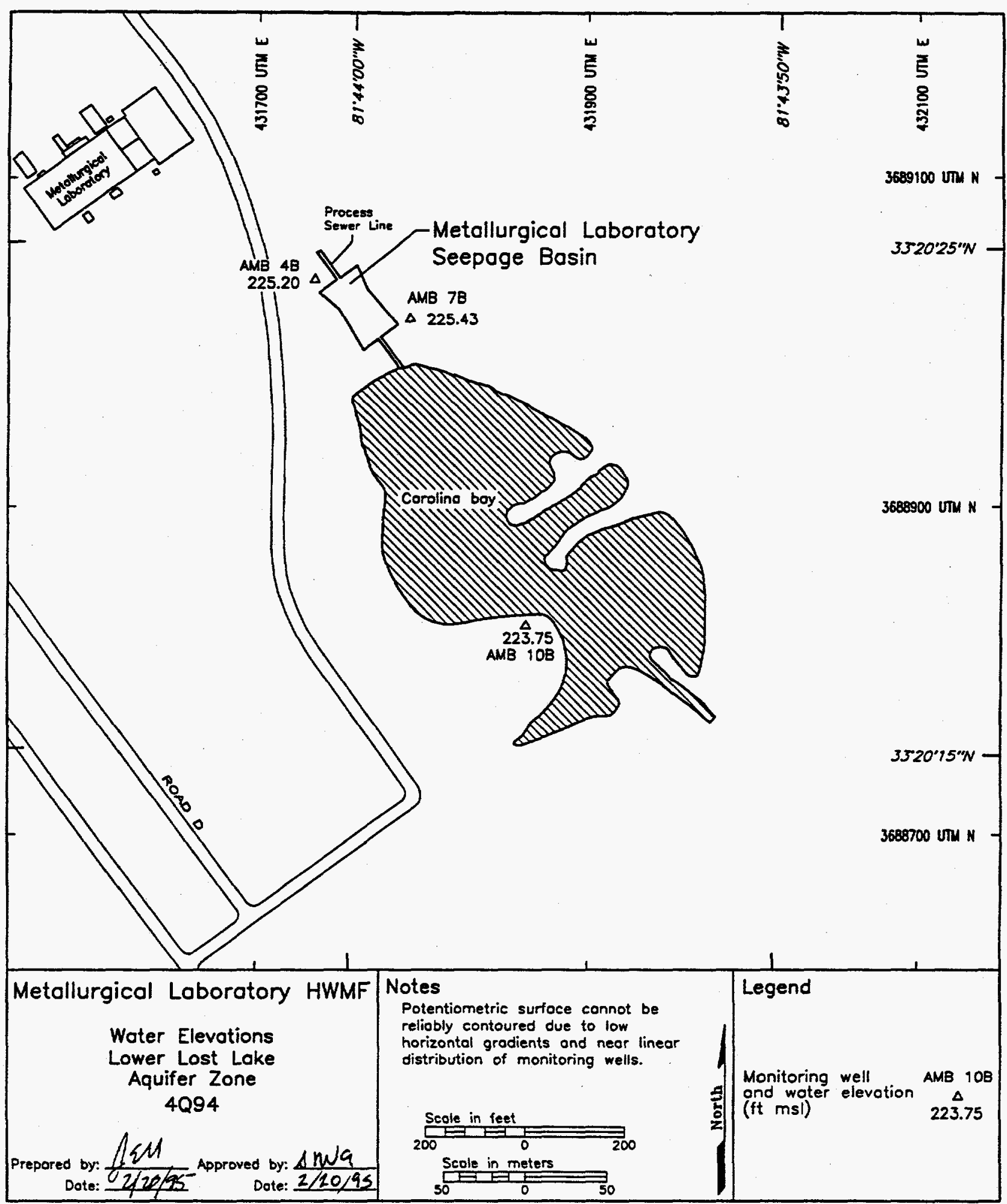

Figure 5. Water-Elevation Map and Location of Monitoring Wells in the Lower Lost Lake Aquifer Zone at the Metallurgical Laboratory HWMF 


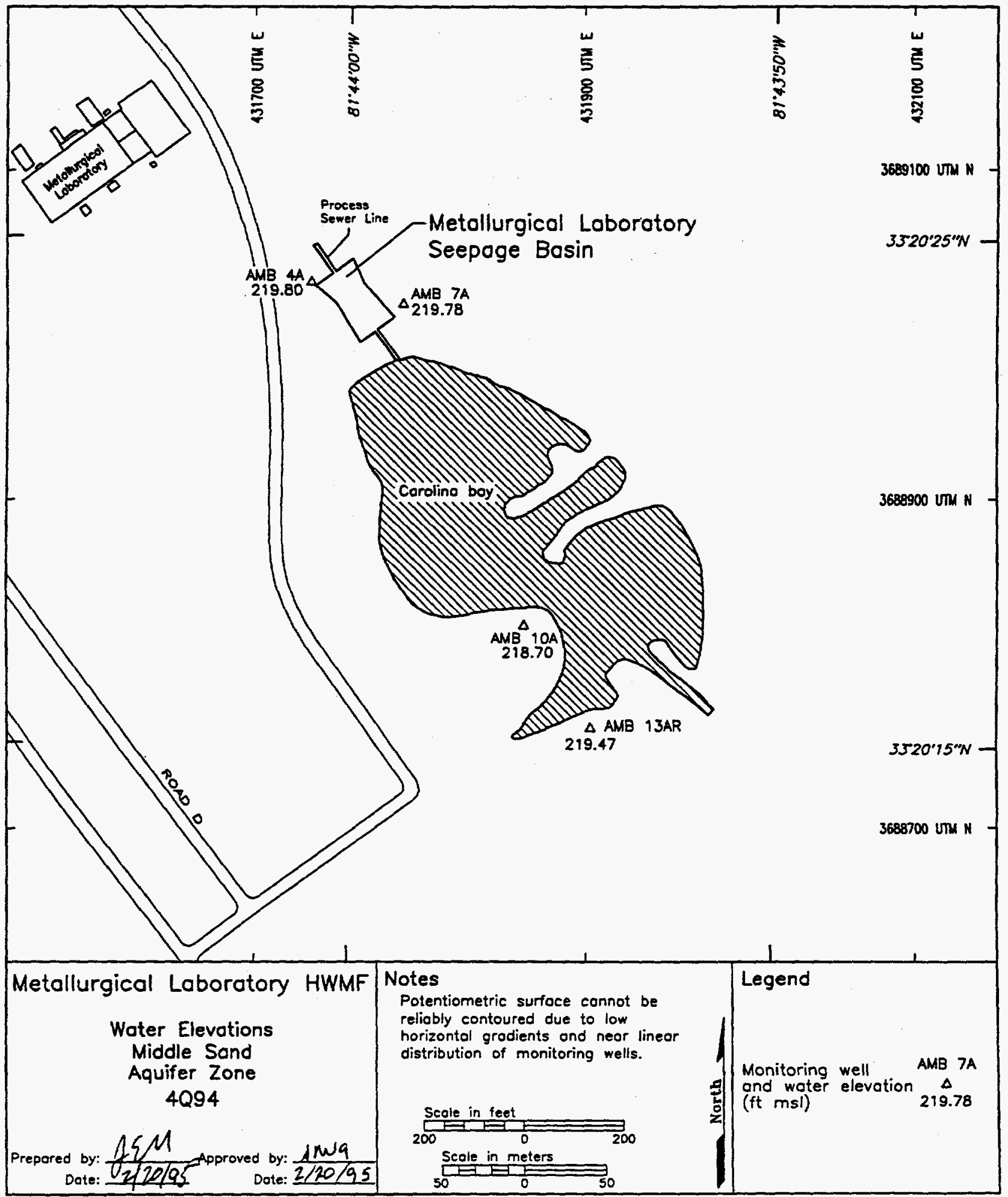

Figure 6. Water-Elevation Map and Location of Monitoring Wells in the Middle Sand Aquifer Zone of the Crouch Branch Confining Unit at the Metallurgical Laboratory HWMF 


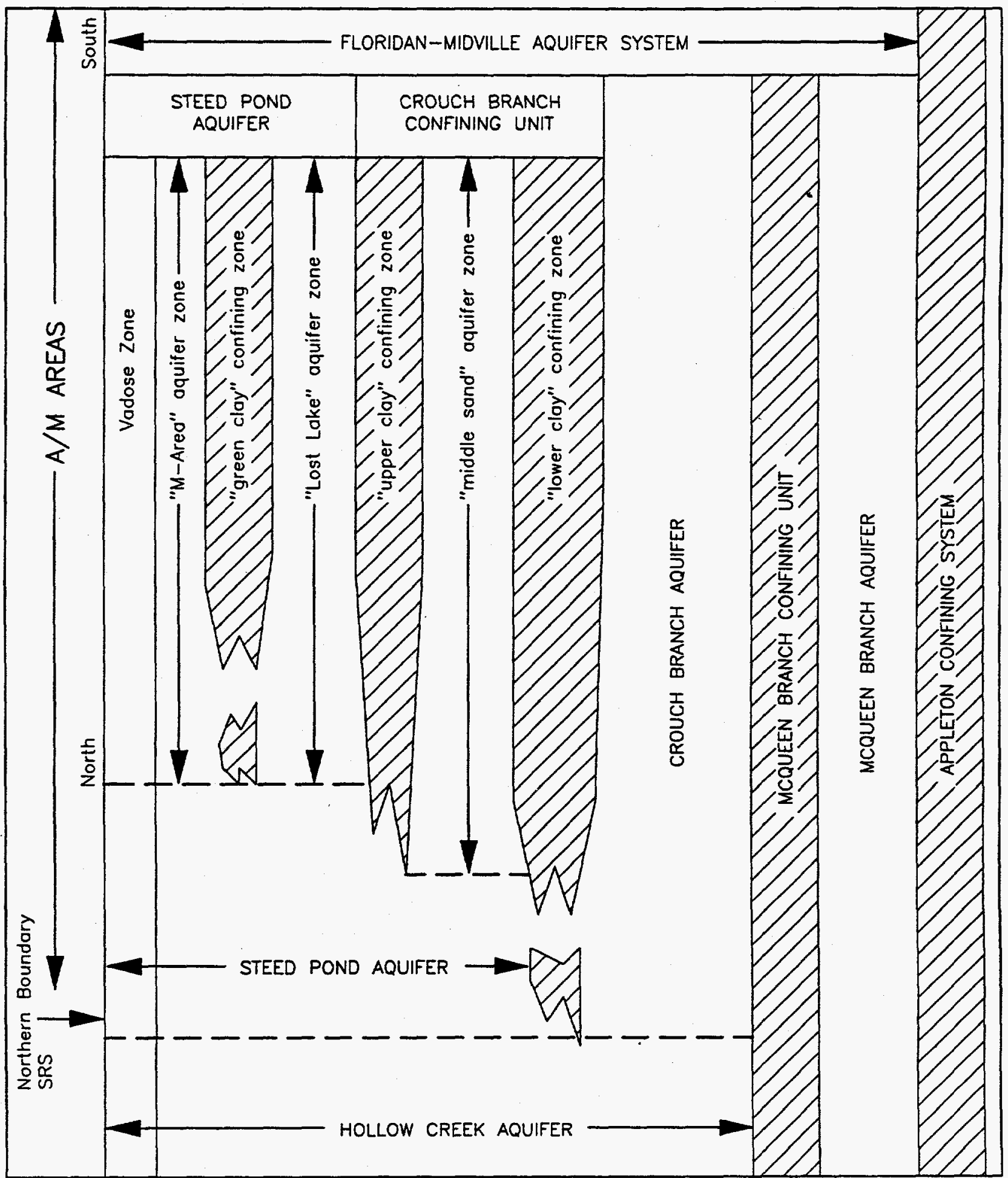

Figure 7. Hydrostratigraphy of A/M Areas 
WSRC-TR-94-0615

Unclassified

THIS PAGE LEFT BLANK INTENTIONALLY. 
WSRC-TR-94-0615

Unclassified

\section{Appendix D}

Groundwater Monitoring Results Tables 
WSRC-TR-94-0615

Unclassified

THIS PAGE LEFT BLANK INTENTIONALLY. 


\section{Key to Reading the Tables}

The following abbreviations may appear in the data tables:

\section{Constituents}

\author{
1,2,3,4,6,7,8-HPCDD \\ $1,2,3,4,6,7,8$-HPCDF \\ $1,2,3,4,7,8-H \times C D D$ \\ $1,2,3,4,7,8-\mathrm{HXCDF}$ \\ Lindane \\ PCB \\ $1,2,3,7,8-P C D D$ \\ $1,2,3,7,8$-PCDF \\ Sp. conductance \\ TCDD \\ TCDF
}

\section{Laboratories}

CN
EM
GE and GP
SC
SP
TM
WA and WS

Miscellaneous

CS
DF
H
Mod
PDWS
PVC
ST
SZ
TOC

\section{Nomenclature}

\author{
$A Z$ \\ Crouch Branch $A Z$ \\ - CBA \\ - $\mathrm{CBC}$ or $\mathrm{CBCU}$ \\ - LCBC \\ $\mathrm{CZ}$
}

\author{
1,2,3,4,6,7,8-heptachlorodibenzo-p-dioxin \\ 1,2,3,4,6,7,8-heptachlorodibenzo-p-furan \\ $1,2,3,4,7,8$-hexachlorodibenzo-p-dioxin \\ $1,2,3,4,7,8$-hexachlorodibenzo-p-furan \\ gamma-benzene hexachloride \\ polychlorinated biphenyl \\ 1,2,3,7,8-pentachlorodibenzo-p-dioxin \\ 1,2,3,7,8-pentachlorodibenzo-p-furan \\ specific conductance \\ tetrachlorodibenzo-p-dioxin \\ tetrachlorodibenzo-p-furan
}

Clemson Technical Center, Inc.

Environmental Protection Department/Environmental

Monitoring Section (EPD/EMS) Laboratory

General Engineering Laboratories

Savannah River Technology Center

Spencer Testing Services, Inc.

TMAVEberline

Roy F. Weston, Inc.

carbon steel

dilution factor column in data tables

holding time column in data tables

modifier column in data tables

primary drinking water standard

polyvinyl chloride

exceeded standard column in data tables

screen zone column in data tables

top of casing

Aquifer Zone

previously Black Creek

Crouch Branch Aquifer

Crouch Branch Confining Unit

Lost Lake AZ - CBCU

Confining Zone 
Nomenclature (cont.)

$\begin{aligned} \text { Lower Lost Lake AZ } & \text { - L } \\ & \text { - LL } \\ & \text { (LL)L/MCBC }\end{aligned}$

M-Area $A Z$

- GC

- $\mathrm{GCL}$

- $M$

- MGC

Middle Sand $A Z$ of the CBCU

- MCBC

Upper Lost Lake AZ

- UL

Miscellaneous Nomenclature

- $\mathrm{MGCL}$

- UD

- VZ

Sampling Codes

B

C

D

E

1

L

$\mathbf{P}$

$S$

$\mathrm{X}$

\section{Sampling Methods}

B

P

S

V

Units

E

$\mathrm{mg} / \mathrm{L}$

msI

MSL

NTU previously Lower Congaree

Lost Lake (Undifferentiated) AZ

Lower Lost Lake AZ

Lower Lost Lake/Middle Sand AZ of the CBCU

previously Water Table

Green Clay CZ

Green Clay - Lost Lake

M-Area AZ

M-Area AZ - Green Clay CZ

previously Ellenton Sand

Middle Sand AZ of the CBCU

previously Upper Congaree

Upper Lost Lake AZ

M-Area AZ - Green Clay CZ - Lost Lake AZ

Undifferentiated

Vadose Zone

blank sample was collected

well was pumping continuously

well was dry

equipment blank was collected

well went dry during sampling; insufficient water to collect all samples

well went dry before sampling began; only depth to water can be determined

inaccessibility or mechanical failure prevented sample

collection and field analysis of the water

no water in standpipe; for water-level events only

well went dry during purging; samples collected after well recovered

sample collected using an open-bucket bailer

sample collected using a bladder pump

sample collected using a single-speed centrifugal

downhole pump

sample collected using a variable-speed pump

exponential notation (e.g., $1.1 \mathrm{E}-09=1.1 \times 10^{-9}=$

0.0000000011 )

milligrams per liter

mean sea level

million structures per liter

nephelometric turbidity unit 
Units (cont.)

$\begin{array}{ll}\mathrm{pCi} / \mathrm{L} & \text { picocuries per liter } \\ \mathrm{pCi} / \mathrm{mL} & \text { picocuries per milliliter } \\ \mathrm{pH} & \text { pH unit } \\ \mu \mathrm{g} / \mathrm{L} & \text { micrograms per liter } \\ \mu \mathrm{S} / \mathrm{cm} & \text { microsiemens per centimeter }\end{array}$

\section{Holding Times}

Standard analytical methods include a limit, called holding time, on the maximum elapsed time between sample collection and extraction or analysis by the laboratory. In the data tables, a large bullet $(\bullet)$ in the $H$ (holding time) column indicates that holding time was exceeded. Analyses performed beyond holding times may not yield valid results.

The South Carolina Department of Health and Environmental Control allows only 15 minutes to elapse between sampling and analysis for $\mathrm{pH}$. Thus, only field $\mathrm{pH}$ measurements can meet the holding time criterion; laboratory $\mathrm{pH}$ analyses always will exceed it.

The laboratory procedure used for the determination of specific conductance allows one day to elapse between sampling and analysis. Thus, laboratory specific conductance measurements may exceed the holding time criterion.

\section{Data Rounding}

Constituent results in analytical results tables that appear to equal the final PDWS but are not marked in the ST (exceeded the final PDWS or screening level) column are below the final PDWS in the database. Values stored in the database contain more significant digits than the reported results. Apparent discrepancies in the tables are due to the rounding of reported results.

\section{Data Qualification}

The contract laboratories continually assess their own accuracy and precision according to U.S. Environmental Protection Agency (EPA) guidelines. They submit sample- or batch-specific quality assurance/quality control information either at the same time as analytical results or in a quarterly summary. Properly defined and used result modifiers (also referred to as qualifiers) can be a key component in assessing data usability. Result modifiers designed by the EPD/EMS and provided to the primary laboratories are defined below. These modifiers appear in the data tables under the column Mod. The lettered modifiers are based on EPA's STORET codes.

\section{Result Modifiers}

Data are not qualified. Numbers should be interpreted exactly as reported.

1

$J$
The value in the result field is the instrument reading, not the sample quantification limit. Always used with the result qualifier $U$.

Value is estimated because quantitation in the sample or in associated quality control samples did not meet specifications. 


\section{Result Modifiers}

$\mathbf{L}$

M

$\mathbf{R}$

T

U

V

Y
Value is off-scale high. The actual value is not known but is known to be greater than the value shown.

Presence of the analyte is verified but not quantified.

Result was rejected because performance requirements in the sample analysis or associated quality control analyses were not met.

Analyte was not detected; if present, it was below the criteria for detection.

Material analyzed for but not detected. Analytical result reported is less than the sample quantitation limit.

Analyte was detected in an associated method blank.

Result was obtained from an unpreserved or improperly preserved sample. Data may not be accurate.

Result may be an underestimation of the true value due to analytical bias.

Result may be an overestimation of the true value due to analytical bias.

The associated result may be of poor precision (high variability) due to analytical bias.

Result is associated with $Q A$ results indicating matrix interference.

The associated result is from a reanalysis performed out of holding time due to problems with an earlier analysis. 
Table 1. Maximum Levels of Constituents Exceeding the Final Primary Drinking Water Standards

M-Area Aquifer Zone

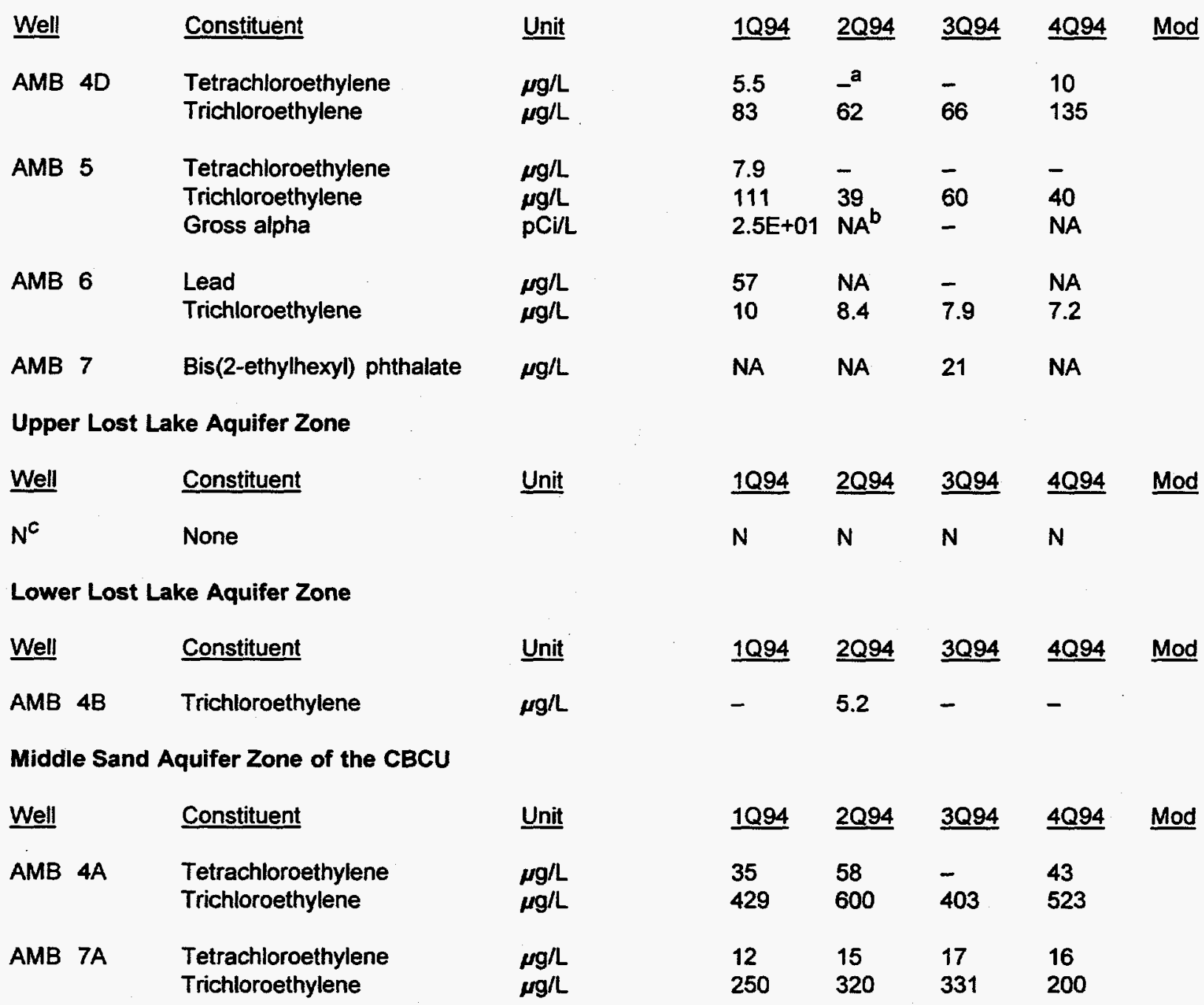

Notes: The modifier column applies to fourth quarter 1994 data only.

The groundwater samples are unfiltered. Thus, the results for metals are for total recoverable metals.

a $-=$ analyzed but not above the final PDWS.

b $N A=$ not analyzed.

c $N=$ not applicable. 
Table 2. Maximum Levels of Constituents Exceeding Other Flag 2 Criteria

M-Area Aquifer Zone

Well

Constituent

Unit

4Q94

Mod

AMB 4D

Total organic halogens

$\mu g / L$

96

Upper Lost Lake Aquifer Zone

Well Constituent

Unit

$\underline{4 Q 94}$

Mod

$\mathrm{N}^{\mathrm{a}}$

None

N

Lower Lost Lake Aquifer Zone

Well Constituent

$\underline{\text { Unit }}$

4Q94

Mod

N

None

N

Middle Sand Aquifer Zone of the CBCU

Well

Constituent

Unit

4Q94

Mod

AMB 4A

Total organic halogens

$\mu g / L$

156

AMB 7A

Total organic halogens

$\mu g / L$

144

Notes: These results do not include field data.

The groundwater samples are unfiltered. Thus, the results for metals are for total recoverable metals.

Flags are established by EPD/EMS and are based on final PDWS, Secondary Drinking Water Standards, or method detection limits (see Appendix B).

a $\mathbf{N}=$ not applicable. 
Table 3. Groundwater Monitoring Results for Individual Wells

\section{WELL AMB 4A}

\begin{tabular}{|c|c|c|c|c|c|c|}
\hline SRS Coord. & Lat/Longitude & Screen Zone Elevation & Top of Casing & Casing & Pump & Screen Zone \\
\hline $\begin{array}{l}\text { N104131.6 } \\
\text { E51469.8 }\end{array}$ & $\begin{array}{l}33.340041^{\circ} \mathrm{N} \\
81.733589^{\circ} \mathrm{W}\end{array}$ & $126.3-121.3 \mathrm{ft} \mathrm{msl}$ & $380.5 \mathrm{ft} \mathrm{msl}$ & 4" PVC & s & MCBC \\
\hline AMPLE D & & $02 / 01 / 94$ & $05 / 04 / 94$ & & & \\
\hline
\end{tabular}

FIELD DATA

Analyte
Water elevation
Depth to water
pH
Sp. conductance
Water temperature
Alkalinity as $\mathrm{CaCO}_{3}$
Turbidity
Volume purged
Sampling code

ANALYTICAL DATA

H. ST Analyte
Aluminum, total recoverable
Barium, total recoverable
Benzene
Bromodichloromethane
Bromoform
Bromomethane (Methyl bromide)
Cadmium, total recoverable
Carbon tetrachloride
Chloride
Chlorobenzene
Chloroethane
Chloroethene (Vinyl chloride)
2-Chloroethyl vinyl ether
Chloroform
Chloromethane (Methyl chloride)
Cobalt, total recoverable
Copper, total recoverable
Cyanide
Dibromochloromethane
1,1-Dichloroethane
1,2-Dichloroethane
1,1-Dichloroethylene
trans-1,2-Dichloroethylene
Dichloromethane
1,2-Dichloropropane
cis-1,3-Dichloropropene
trans-1,3-Dichloropropene
Ethylbenzene
Fluoride
Gross alpha
lron, total recoverable
Lead, total recoverable
Manganese, total recoverable
Nickel, total recoverable
Nitrate-nitrite as nitrogen
Nonvolatile beta
Radium, total alpha-emitting
Selenium, total recoverable
Silver, total recoverable
1,1,2,2-Tetrachloroethane

1094
219.1
161.4
6.6
48
18.1
12
1
2.6

$\underline{2094}$
220.2
160.3
6.6
48
18.9
7
1
2.9

1094

$\underline{2094}$

$<20$

$<10$

$<10$

$<10$

$<10$

$<2.0$

$<10$

$<10$

$<10$

$<10$

$<10$

$<10$

$<10$

$\begin{array}{ll} & \\ <10 & <5.0 \\ <10 & <10 \\ <10 & <10 \\ <10 & <10 \\ <10 & <10 \\ <10 & <10 \\ <10 & <10 \\ <10 & <10 \\ <10 & <10 \\ <10 & <10 \\ <10 & <10 \\ & <100\end{array}$

$\begin{aligned} & 5.1 E-01 \\ & 5.7< \\ &< 3.0 \\ & 7.4\end{aligned}$

$<10$

$<10$

$<10$

$<10$

$<10$

1,950

$<10$

$<10$
$<10$

$<10$

$<10$

$<10$

$<10$
$<10$

\begin{tabular}{|c|c|}
\hline $6.0 E-01$ & $<0.0 E+00$ \\
\hline 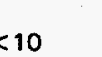 & $\begin{array}{l}<2.0 \\
<10\end{array}$ \\
\hline
\end{tabular}

3094
220.2
160.3
6.1
49
19.5
8
2
2.8

$\underline{4094}$
220.7
159.8
6.4
44
19.2
12
2
2.7

Unit

ft msl

ft

pH

$\mu \mathrm{S} / \mathrm{cm}$

$\mathrm{mg} / \mathrm{L}$

NTU

well vol.

msl
S/cm
mg/L
NTU
well vol.

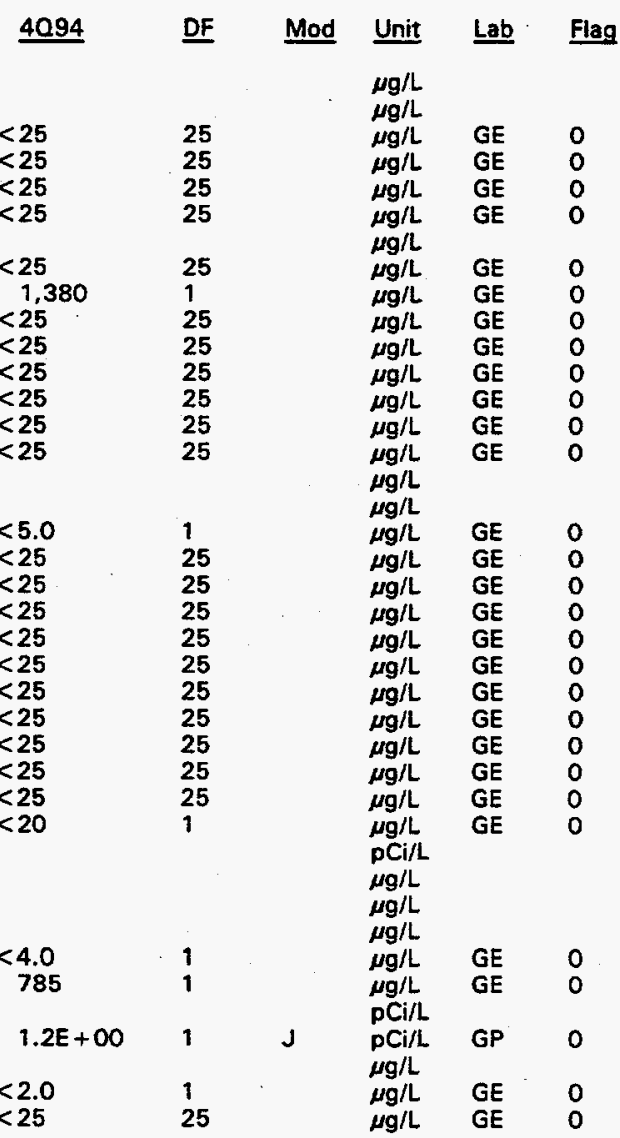

Note: Flagging, dilution factors, modifiers, and laboratories are for fourth quarter 1994 data only. See Appendix B for flagging criteria. - = exceeded holding time for fourth quarter 1994 .

- = exceeded screening level or final PDWS for fourth quarter 1994. 
H ST Analyte

- Tetrachloroethylene Toluene

Total organic carbon Total organic halogens 1,1,1-Trichloroethane 1,1,2-Trichloroethane

- Trichloroethylene

Trichlorofluoromethane

Zinc, total recoverable

\begin{tabular}{|c|c|c|}
\hline 1094 & 2094 & 3094 \\
\hline $\begin{aligned} & 35 \\
< & 10 \\
< & 1,000 \\
& 190 \\
< & 10 \\
< & 10 \\
& 429 \\
< & 10\end{aligned}$ & $\begin{aligned} & 58 \\
< & 10 \\
< & 1,000 \\
& 190 \\
< & 10 \\
< & 10 \\
& 600 \\
< & 10\end{aligned}$ & $\begin{array}{c}<25 \\
<25 \\
<1,000 \\
160 \\
<25 \\
<25 \\
403 \\
<25 \\
14\end{array}$ \\
\hline
\end{tabular}

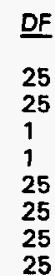

Mod

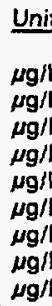

$\begin{array}{ll}\text { Lab } & \text { Flag } \\ \text { GE } & 2 \\ \text { GE } & 0 \\ \text { GE } & 0 \\ \text { GE } & 2 \\ \text { GE } & 0 \\ \text { GE } & 0 \\ \text { GE } & 2 \\ \text { GE } & 0\end{array}$

\section{WELL AMB 4B}

$\begin{array}{ll}\text { SRS Coord. } & \text { Lat/Longitude } \\ \text { N104145.6 } & 33.340093 \circ \mathrm{N} \\ \text { E51482.7 } & 81.733582^{\circ} \mathrm{W}\end{array}$

\section{Screen Zone Elevation}

157.3-152.3 ft msl

SAMPLE DATE

$02 / 12 / 94$

\section{Top of Casing \\ $380.4 \mathrm{ft} \mathrm{msl}$}

$\begin{array}{ll}\text { Casing } & \text { Pump } \\ \text { 4" PVC } & \mathrm{s}\end{array}$

Screen Zone

LL

FIELO DATA

Analyte
Water elevation
Depth to water
pH
Sp. conductance
Water temperature
Alkalinity as $\mathrm{CaCO}_{3}$
Turbidity
Volume purged
Sampling code

ANALYTICAL DATA

프 ST Analyte

Aluminum, total recoverable Barium, total recoverable Benzene

Bromodichioromethane

Bromofarm

Bromomethane (Methyl bromide)

Cadmium, total recoverable

Carbon tetrachloride

Chloride

Chlorobenzene

Chloroethane

Chioroethene (Vinyl chloride)

2.Chloroethyl vinyl ether

Chloroform

Chloromethane (Methyl chloride)

Cobalt, total recoverable

Copper, total recoverable Cyanide

Dibromochloromethane

1,1-Dichloroethane

1,2-Dichloroethane

1,1-Dichloroethylene

trans-1,2-Dichloroethylene

Dichloromethane

1,2-Dichloropropane

cis-1,3-Dichloropropene

\begin{tabular}{ll}
1094 & $\underline{2094}$ \\
\hline 225.1 & 225.2 \\
155.3 & 155.2 \\
5.1 & 4.8 \\
31 & 33 \\
18.4 & 18.9 \\
1 & 0 \\
0 & 0 \\
3.7 & 4.0
\end{tabular}

3094
226.2
154.2
4.7
32
20.5
0
1
3.0

$\begin{array}{ll}1094 & 2094 \\ 31 & \\ <1.0 & \\ <1.0 & <1.0 \\ <1.0 & <1.0 \\ <1.0 & <1.0 \\ <2.0 & <1.0 \\ <1.0 & <1.0 \\ <1.0 & <1.060 \\ <1.0 & <1.0 \\ <1.0 & <1.0 \\ <1.0 & <1.0 \\ <1.0 & <1.0 \\ <1.0 & <1.0 \\ & \\ & \\ <1.0 & <5.0 \\ <1.0 & <1.0 \\ <1.0 & <1.0 \\ <1.0 & <1.0 \\ <1.0 & <1.0 \\ <1.0 & <1.0 \\ <1.0 & <1.0 \\ <1.0 & <1.0 \\ <1.0 & <1.0\end{array}$

\begin{tabular}{ll}
$\quad 3094$ & \multicolumn{1}{r}{4094} \\
32 & \\
6.7 & \\
$<1.0$ & $<1.0$ \\
$<1.0$ & $<1.0$ \\
$<1.0$ & $<1.0$ \\
$<1.0$ & $<1.0$ \\
$<1.0$ & $<1.0$ \\
$<1.0$ & $<1.0$ \\
$<1.0$ & $<1.0$ \\
$<1.0$ & $<1.0$ \\
$<1.0$ & $<1.0$ \\
$<1.0$ & $<1.0$ \\
$<1.0$ & $<1.0$ \\
$<4.0$ & \\
$<4.0$ & $<5.0$ \\
$<5.0$ & $<1.0$ \\
$<1.0$ & $<1.0$ \\
$<1.0$ & $<1.0$ \\
$<1.0$ & $<1.0$ \\
$<1.0$ & $<1.0$ \\
$<1.0$ & $<1.0$ \\
$<1.0$ & $<1.0$ \\
$<1.0$ & $<1.0$ \\
$<1.0$ &
\end{tabular}

$10 / 27 / 94$

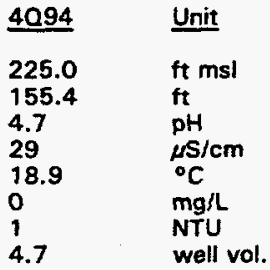

DF
1
1
1
1
1
1
1
1
1
1
1
1
1
1
1
1
1
1
1
1
1

\section{Mod}

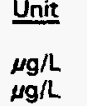

$\mu g / L$

$\mu g / L$ GE 0

$\mu g / L$

$\mu g / L$

$\mu g / L$

$\mu \mathrm{g} / \mathrm{L}$

$\mu \mathrm{g} / \mathrm{L}$

$\mu g / L$

$\mu \mathrm{g} / \mathrm{h}$

eng/L

$\mu g / L$

$\mu g / L$

$\mu g / L$

$\mu g / L$

$\mu g / \mathrm{h}$.

$\mu g / L$

$\mu \mathrm{g} / \mathrm{h}$

$\mu g / L$

$\mu \mathrm{g} / \mathrm{L}$

$\mu g / L$

$\mu g / L$

Lab Flag

GE $O$

GE

GE

GE

GE

GE

GE

GE

GE

GE $\quad 0$

GE

GE

GE

GE

GE 
Well AMB $4 B$ continued

ANALYTICAL DATA

H ST Analyte

trans-1,3-Dichloropropene

Ethylbenzene

Fluoride

Gross alpha

Iron, total recoverable

Lead, total recoverable

Manganese, total recoverable

Nickel, total recoverable

Nitrate-nitrite as nitrogen

Nonvolatile beta

Radium, total alpha-emitting

Selenium, total recoverable

Silver, total recoverable

1,1,2,2-Tetrachloroethane

Tetrachloroethylene

Toluene

Total organic carbon

Total organic halogens

1,1,1-Trichloroethane

1,1,2-Trichloroethane

Trichloroethylene

Trichlorofluoromethane

Zinc, total recoverable

$\begin{array}{lcc}1094 & \underline{2094} & \underline{3094} \\ <1.0 & <1.0 & <1.0 \\ <1.0 & <1.0 & <1.0 \\ 1.9 E+00 & <100 & 1.1 E+00 \\ 4.3 & & 12 \\ <3.0 & & <3.0 \\ 6.6 & & 6.6 \\ & <4.0 & <4.0 \\ & 680 & 682 \\ 1.7 E+00 & 5.0 E-01 & 1.4 E+00 \\ & & 1.0 E+00 \\ <1.0 & <2.0 & <2.0 \\ <1.0 & <1.0 & <1.0 \\ <1.0 & <1.0 & <1.0 \\ <1.000 & <1.0 & <1.0 \\ <5.0 & <1.000 & <1.000 \\ <1.0 & 5.1 & <5.0 \\ <1.0 & <1.0 & <1.0 \\ 4.1 & <1.0 & <1.0 \\ <1.0 & <1.0 & 4.4 \\ & & <1.0 \\ & & 17\end{array}$

$\begin{aligned} & 4094 \\ &< 1.0 \\ &<1.0 \\ & 36 \\ & \\ & \\ &<4.0 \\ & 584 \\ & \\ & 9.0 E-01 \\ &<2.0 \\ &<1.0 \\ &<1.0 \\ &<1.0 \\ & \\ & 1.260 \\ & 15 \\ &<1.0 \\ &<1.0 \\ & 3.6 \\ &<1.0\end{aligned}$

\section{DF Mod}

Unit Lab Flag

$\mu \mathrm{g} / \mathrm{L} \quad \mathrm{GE} \quad 0$

$\mu g / L \quad G E \quad O$

$\mu \mathrm{pCi} / \mathrm{L}$

$\mu g / 2$

$\mu g / L$

$\mu g / L$

$\mu g / L$

$\mu \mathrm{g} / \mathrm{L}$

pCi/L

$\mu g / L$

$\mu \mathrm{g} / \mathrm{L}$

$\mu g / L$

$\mu g / L$

$\mu g / L$

J

$\mu g / L$

$\mu \mathrm{g} / \mathrm{L}$

$\mu g / L$

$\mu g / L$

$\mu \mathrm{g} / \mathrm{L}$

$\mu \mathrm{g} / \mathrm{L}$

\section{WELL AMB 4D}

\begin{tabular}{ll} 
SRS Coord. & Lat/Longitude \\
N104154.7 & $\begin{array}{l}33.340124^{\circ} \mathrm{N} \\
\text { E51489.0 }\end{array}$ \\
\hline $81.733584^{\circ} \mathrm{W}$
\end{tabular}

\section{Screen Zone Elevation \\ 233.4-213.4 $\mathrm{tt} \mathrm{ms}$}

$02 / 12 / 94$

$04 / 22 / 94$

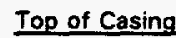

$380.3 \mathrm{ft} \mathrm{ms}$

Casing

4" PVC
Pump

S

SAMPLE DATE

FIELD DATA

Analyte
Water elevation
Depth to water
pH
Sp. conductance
Water temperature
Alkalinity as $\mathrm{CaCO}_{3}$
Turbidity
Volume purged
Sampling code

ANALYTICAL DATA

Acenaphthene Acenaphthylene

Acetone

Acetonitrile (Methyl cyanide)

Acetophenone

2-Acetylaminofluorene

Acrolein

Acrylonitrile

Aldrin

Allyl chloride

Aluminum, total recoverable

4-Aminobiphenyi

$\begin{array}{ll}2094 & \text { 3094 } \\ 234.1 & 234.0 \\ 146.2 & 146.4 \\ 5.2 & 5.3 \\ 38 & 39 \\ 19.2 & 20.7 \\ 6 & 1 \\ 2 & 1 \\ 4.7 & 6.8\end{array}$

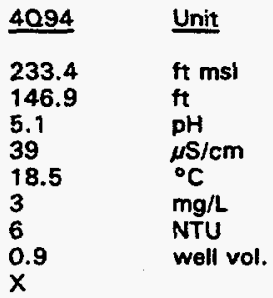

1094

$\underline{2094}$

$\quad \underline{3094}$
$<10$
$<10$
$<500$
$<25$
$<10$
$<10$
$<100$
$<100$
$<0.050$
$<250$
23
$<10$

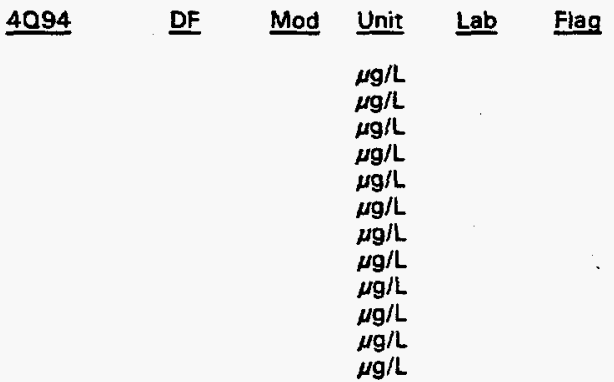

Note: Flagging, dilution factors, modifiers, and laboratories are for fourth quarter 1994 data only. See Appendix B for flagging criteria. - = exceeded holding time for fourth quarter 1994.

- = exceeded screening level or final PDWS for fourth quarter 1994. 


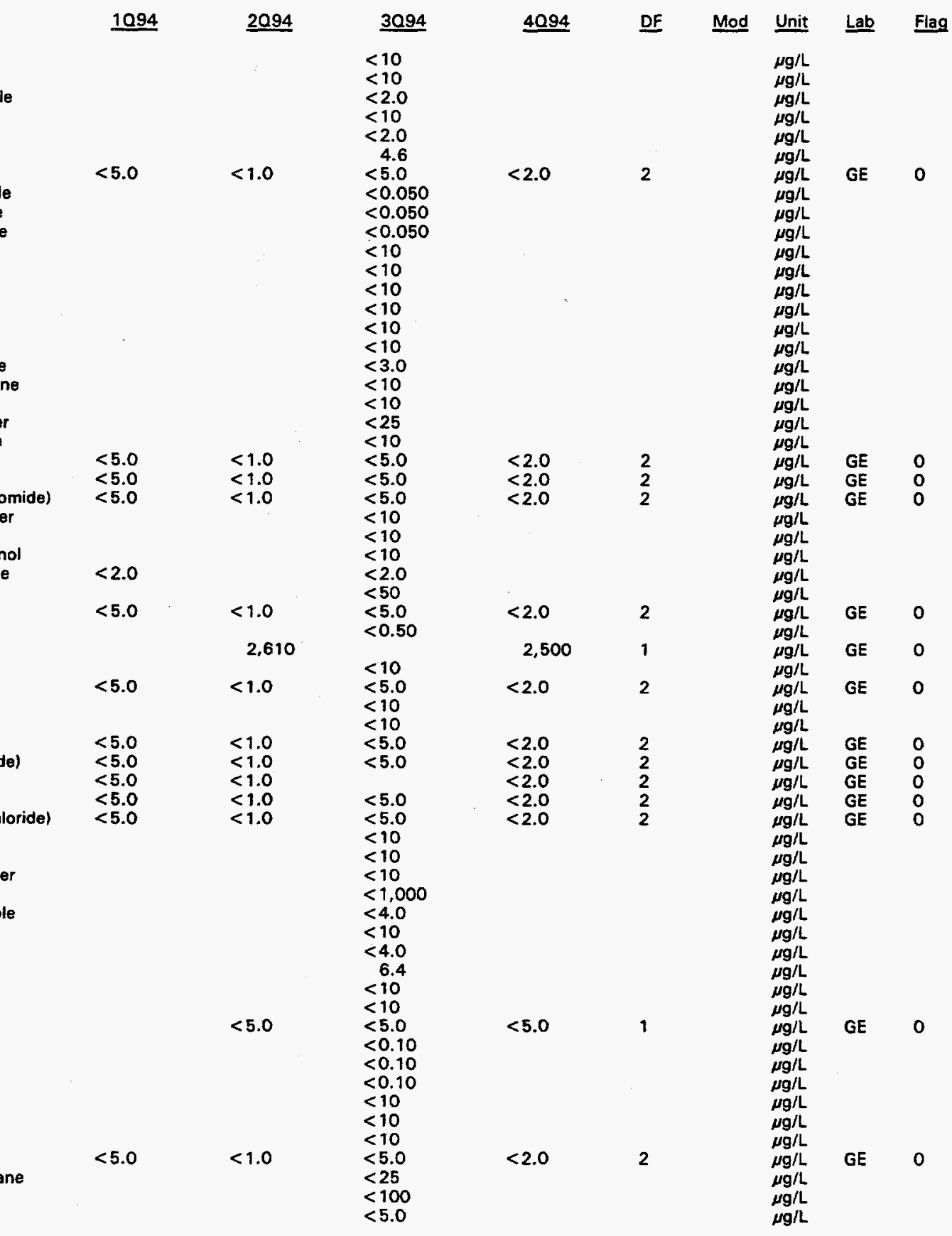

Aniline

Anthracene

Antimony, total recoverable

Aramite

Arsenic, total recoverable

Barium, total recoverable

Benzene

alpha-Benzene hexachloride

beta-Benzene hexachloride

delta-Benzene hexachloride

Benzo[a]anthracene

Benzolb]tluoranthene

Benzolk]fluoranthene

Benzolg,h, Iperylene

Benzolalpyrene

Benzyl alcohol

Beryllium, total recoverable

Bis(2-chloroethoxy) methane

Bis (2-chloroethyl) ether

Bis(2-chloroisopropyl) ether

Bis (2-ethylhexyl) phthalate

Bromodichloromethane

Bromoform

Bromomethane (Methyl bromide)

4-Bromophenyl phenyl ether

Butylbenzyl phthalate

2-sec-Butyl-4, 6-dinitrophenol

Cadmium, total recoverable

Carbon disulfide

Carbon tetrachloride

Chlordane

Chloride

4-Chloroaniline

Chlorobenzene

Chlorobenzilate

4-Chloro-m-cresol

Chloroethane

Chloroethene (Vinyl chloride)

2-Chloroethyl vinyl ether

Chloroform

Chloromethane (Methyl chloride)

2-Chloronaphthalene

2-Chlorophenol

4-Chlorophenyl phenyl ether

Chloroprene

Chromium, total recoverable

Chrysene

Cobalt, total recoverable

Copper, total recoverable

o-Cresol (2-Methylphenol)

m,p-Cresol

Cyanide

$p, p^{\prime}-D D D$

$p, p^{\prime}-D D E$

$p, p^{\prime}$-DDT

Diallate

Dibenz $[a, h]$ anthracene

Dibenzofuran

Dibromochloromethane

1,2-Dibromo-3-chloropropane

1,2-Dibromoethane

Dibromomethane

Note: Flagging, dilution factors, modifiers, and laboratories are for fourth quarter 1994 data only. See Appendix B for flagging criteria. - = exceeded holding time for fourth quarter 1994.

- = exceeded screening level or final PDWS for fourth quarter 1994. 
Well AMB 4D continued

ANALYTICAL DATA

H ST Analyte

Di-n-butyl phthalate

1,2-Dichlorobenzene

1,3-Dichlorobenzene

1,4-Dichlorobenzene

3,3'-Dichlorobenzidine

trans-1,4-Dichloro-2-butene

Dichlorodifluoromethane

1.1-Dichloroethane

1,2-Dichloroethane

1,1-Dichloroethylene

trans-1,2-Dichloroethylene

Dichloromethane

2,4-Dichlorophenol

2.6-Dichlorophenol

2,4-Dichlorophenoxyacetic acid

1,2-Dichloropropane

cis-1,3-Dichloropropene

trans-1,3-Dichloropropene

Dieldrin

Diethyl phthatate

Dimethoate

2,4-Dimethyl pheno

Dimethyl phthalate

p-Dimethylaminoazobenzene

7,12-Dimethylbenz[a]anthracene

3,3'-Dimethylbenzidine

a,a-Dimethylphenethylamine

1,3-Dinitrobenzene

2,4-Dinitrophenol

2,4-Dinitrotoluene

2,6-Dinitrotoluene

Di-n-octyl phthalate

1,4-Dioxane

Diphenylamine

Disulfoton

Endosulfan

Endosulfan II

Endosulfan sulfate

Endrin

Endrin aldehyde

Ethyl methacrylate

Ethyi methanesulfonate

Ethylbenzene

Famphur

Fluoranthene

Fluorene

Fluoride

Gross alpha

Heptachlor

Heptachior epoxide

1,2,3,4,6,7,8-HPCDD

Heptachlorodibenzo-p-dioxin isomers

$1,2,3,4,6,7,8$-HPCDF

Heptachlorodibenzo-p-furan isomers

Hexachlorobenzene

Hexachlorobutadiene

Hexachlorocyclopentadiene

$1,2,3,4,7,8-H X C D D$

Hexachlorodibenzo-p-dioxin isomers

$1,2,3,4,7,8-\mathrm{H} \times \mathrm{CDF}$

Hexachlorodibenzo-p-furan isomers

Hexachloroethane
1094

2094

$\underline{3094}$

$<10$

$<10$

$<10$
$<10$

$<10$

$<150$

$<5.0$

$<5.0$

$<5.0$

$<5.0$

$<5.0$

$<5.0$

$<5.0$

$<10$

$<0.0015$

$<5.0<1.0$

$<5.0$

$<1.0$

$<1.0$

$<5.0$

$<5.0$

$<5.0$

$<0.025$

$<10$

$<10$

$<10$

$<10$

$<10$

$<10$

$<10$

$<10$

$<10$

$<45$

$<10$

$<10$

$<10$

$<10$

$<10$

$<10$

$<0.10$

$<0.10$

$<0.10$

$<50$

$<0.10$

$<10$

$<10$

$<5.0$

$<10$

$<10$

$<10$

$6.1 E+00$

$<100$

$3.3 E+00$

$<2.0$

$<0.050$

$<0.065$

$<0.065$

$<0.045$

$<0.045$

$<10$

$<10$

$<10$

$<0.045$

$<0.045$

$<0.040$

$<0.040$

$<10$
4099

DF

Mod

Unir

$<2.0$

$<2.0$

$<2.0$

$<2.0$

$<2.0$

$<2.0$

$<2.0$

$\begin{array}{ll} & \\ & \\ & \\ & \\ & \\ 2.0 & \\ 2.0 & \\ 2.0 & \\ 2.0 & \\ 2 & \\ & \end{array}$

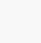

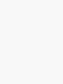

Lab

Flag

$\mu \mathrm{g} / \mathrm{L}$

$\mu \mathrm{g} / L$

$\mu \mathrm{g} / \mathrm{L}$

$\mu \mathrm{g} / \mathrm{L}$

$\mu g / L$

$\mu \mathrm{g} / \mathrm{L}$

$\mu g / L$

$\mu g / L$

$\mu g / L$

$\mu g / L$

$\mu g / L$

$\mu \mathrm{g} / \mathrm{L}$

$\mu g / L$

$\mu g / L$

$\mu g / L$

$\mu g / L$

$\mu g / L$

$\mu g / L$

$\mu \mathrm{g} / \mathrm{L}$

$\mu g / k$

$\mu g / L$

$\mu g / \mathrm{L}$

$\mu g / L$

$\mu g / L$

$\mu \mathrm{g} / \mathrm{L}$

$\mu g / L$

(IL

$\mu g / L$

$\mu g / L$

$\mu g / L$

$\mu g / L$

$\mu g h$

$\mu \mathrm{g} / \mathrm{L}$

$\mu g / L$

$\mu \mathrm{g} / \mathrm{L}$

$\mu g / L$

$\mu \mathrm{g} / \mathrm{L}$

$\mu \mathrm{g} / \mathrm{L}$

$<2.0$

2

$<20$

$\mu g / L$

$\mu g / L$

$\mu \mathrm{g} / \mathrm{L}$

$\mathrm{pCi} / \mathrm{L}$

$\mu g / L$

$\mu g / L$

$\mu g / L$

$\mu \mathrm{g} / \mathrm{L}$

$\mu \mathrm{g} / \mathrm{L}$

$\mu g / L$

$\mu g / L$

$\mu g / L$

$\mu g / L$

$\mu g / L$

$\mu g / L$

$\mu \mathrm{g} / \mathrm{L}$

$\mu g / L$ $\begin{array}{cl}\text { GE } & 0 \\ \text { GE } & 0 \\ \text { GE } & 0 \\ \text { GE } & 0 \\ \text { GE } & 0\end{array}$

GE $\quad 0$

GE $\quad 0$

GE

0

GE

0

Note: Flagging, dilution factors, modifiers, and laboratories are for fourth quarter 1994 data only. See Appendix B for flagging criteria. - = exceeded holding time for fourth quarter 1994.

- = exceeded screening level or final PDWS for fourth quarter 1994. 
WSRC-TR-94-0615

Unclassified

Well AMB 4D continued

ANALYTICAL DATA

H ST Analyte

Hexachlorophene

Hexachloropropene

2-Hexanone

Indeno[ $7,2,3-c, d]$ pyrene

lodomethane (Methyl iodide)

Iron, total recoverable

Isobutyl alcohol

Isodrin

isophorone

Isosafrole

Kepone

Lead, total recoverable

Lindane

Manganese, total recoverable

Mercury, total recoverabie

Methacrylonitrile

Methapyrilene

Methoxychlor

2-Methyl-4,6-dinitrophenol

Methyl ethyl ketone

Methyl isobutyl ketone

Methyl methacrylate

Methyl methanesulfonate

3-Methylcholanthrene

2-Methylnaphthalene

Naphthalene

1,4-Naphthoquinone

1-Naphthylamine

2-Naphthylamine

Nickel, total recoverable

Nitrate-nitrite as nitrogen

o-Nitroaniline

m-Nitroaniline

p-Nitroaniline

Nitrobenzene

2-Nitrophenol

4-Nitrophenol

4-Nitroquinoline-1-axide

$\mathrm{N}$-Nitrosodi-n-butylamine

$\mathrm{N}$-Nitrosodiethylamine

N-Nitrosodimethylamine

N-Nitrosodiphenylamine

$\mathrm{N}$-Nitrosodipropylamine

$N$-Nitrosomethylethylamine

$\mathrm{N}$-Nitrosomorpholine

$\mathrm{N}$-Nitrosopiperidine

N-Nitrosopyrrolidine

5-Nitro-0-toluidine

Nonvolatile beta

$0,0,0$-Triethyl phosphorothioate

1094

17

Octachlorodibenzo-p-dioxin

Octachlorodibenzo-p-furan

Parathion

Parathion methyl

PCB 1016

PCB 1221

PCB 1232

PCB 1242

PCB 1248

PCB 1254

PCB 1260

Pentachlorobenzene

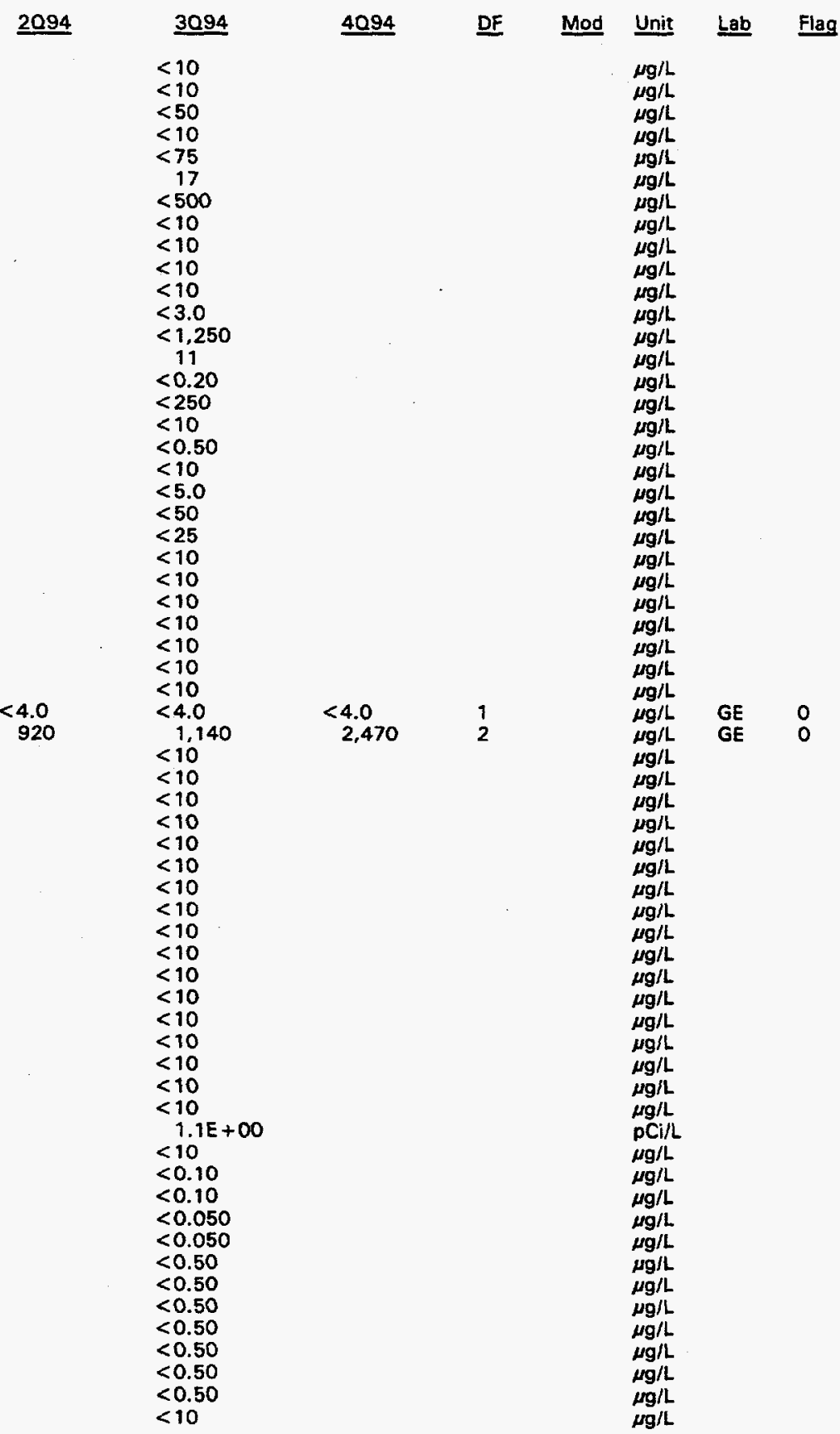

Note: Flagging, dilution factors, modifiers, and laboratories are for fourth quarter 1994 data only. See Appendix B for flagging criteria. - = exceeded holding time for fourth quarter 1994.

- = exceeded screening level or final PDWS for fourth quarter 1994. 
ANALYTICAL DATA

H ST Analvte

1,2,3,7,8-PCDD

Pentachlorodibenzo-p-dioxin isomers

$1,2,3,7,8$-PCDF

Pentachloroethane

Pentachloronitrobenzene

Pentachlorophenol

Phenacetin

Phenanthrene

Phenol

p-Phenylenediamine

Phorate

2-Picoline

Pronamid

Propionitrile

Pyrene

Pyridine

Radium, total alpha-emitting

Satrole

Selenium, total recoverable

Silver, total recoverable

Styrene

Sulfide

Sulfotepp

1,2,4,5-Tetrachlorobenzene

2,3,7,8-TCDD

$2,3,7,8$-TCDF

Tetrachlorodibenzo-p-dioxin isomers

Tetrachlorodibenzo-p-furan isomers

1,1,1,2-Tetrachloroethane

1,1,2,2-Tetrachloroethane

- Tetrachloroethylene

2,3,4,6-Tetrachlorophenol

Thallium, total recoverable

Thionazin

Tin, total recoverable

Toluene

o-Toluidine

Total organic carbon

Total organic halogens

Toxaphene

2,4,5-TP (Silvex)

1,2,4-Trichlorobenzene

1,1,1-Trichloroethane

1,1,2-Trichloroethane

- Trichloroethylene

Trichiorofluoromethane

2,4,5-Trichlorophenol

2,4,6-Trichlorophenol

2,4,5-T

1,2,3-Trichloropropane

1,3,5-Trinitrobenzene

Vanadium, total recoverable

Vinyl acetate

Xylenes

Zinc, total recoverable $\underline{2094}$

$\underline{3094}$

$\underline{4094}$

$\underline{\text { DF }}$

Mod

Unir

$<0.055$

$<0.055$

$<10$

$<10$

$<10$

$<10$

$<10$

$<10$

$<10$

$<0.10$

$<10$

$<10$

$<1,000$

$<10$

$4.8 E+00 \quad 2.9 E+\infty 0$

$2.6 \mathrm{E}+00$

$<10$

$<2.0$

$<2.0$

$<2.0$

$<50$

$<1,000$

$<10$

$<10$

$<0.045$

$<0.040$

$<0.045$

$<0.040$

$<5.0$
5.5

$<1.0$

$<5.0$

$<5.0$

$<5.0$

$<10$

$<2.0$

$<10$

$<2.0$

$<5.0$

$<5.0$

$<1.0$

$<1,000$

24

$<10$

$<1,000$

45

$<1,000$

25

$<0.00045$

$<10$

$<5.0$

$<5.0$

$<5.0$

$<1.0$

62
$<1.0$

$<5.0$

66

$<5.0$

$<10$

$<10$

$<0.00045$

$<5.0$

$<10$

$<8.0$

$<50$

$<10$

$5.7 E+\infty \quad 1$
$<2.0 \quad 1$

$<2.0$
10

$<2.0$

1,200

96

$\mu g / L$

$\mu g / L$

$\mu \mathrm{g} / \mathrm{L}$

$\mu g / L$

$\mu \mathrm{g} / \mathrm{h}$

$\mu \mathrm{g} / \mathrm{L}$

$\mu g / L$

$\mu g / L$

$\mu g / L$

$\mu \mathrm{g} / \mathrm{h}$

$\underset{\mu g / L}{\mu g / L}$

$\mu \mathrm{g} / \mathrm{L}$

DCi/L GP 0

$\mu \mathrm{g} / \mathrm{L}$

$\mu g / L$

$\mu g / L$

$\mu \mathrm{g} / \mathrm{L}$

$\mu g /$

$\mu g / L$

$\mu g / L$

$\underset{\mu g / L}{\mu} / L$

$\mu g / L$

$\mu g / L$ GE $O$

$\mu g / L \quad G E \quad 2$

$\mu \mathrm{g} / \mathrm{L}$

$\mu g / L$

$\mu g / L$

$\mu \mathrm{g} / \mathrm{L}$

$\mu \mathrm{rg} / \mathrm{L}$

$\mu \mathrm{g}$

GE $\quad 0$

GE $\quad 0$

$\mu \mathrm{g} / \mathrm{L}$

$\mu g / L$

$\mu \mathrm{g} / \mathrm{L}$

$\mu \mathrm{gg} / \mathrm{L}$

$\mu g / L$

$\mu g / L$

$\mu \mathrm{g} / \mathrm{L}$

$\mu g / L$

$\mu g / L$

$\mu g / L$

$\mu g / L$

$\mu g / L$

$\mu g / L$

$\mu g / L$

$\mu g / L$

Note: Flagging, dilution factors, modifiers, and laboratories are for fourth quarter 1994 data only. See Appendix B for flagging criteria. - = exceeded holding time for fourth quarter 1994.

- = exceeded screening level or final PDWS for fourth quarter 1994. 
WELL AMB 5

\section{SRS Coord. Lat/Longitude \\ N104083.4 $33.339930^{\circ} \mathrm{N}$ \\ $\begin{array}{ll}\mathrm{N} 51467.2 & 81.733502{ }^{\circ} \mathrm{W}\end{array}$}

SAMPLE DATE

FIELD DATA

Analve

Water elevation

Depth to water

$\mathrm{pH}$

Sp. conductance

Water temperature

Alkalinity as $\mathrm{CaCO}_{3}$

Turbidity

Volume purged

Sampling code

\section{ANALYTICAL DATA}

H ST Analve

Acenaphthene

Acenaphthylene

Acetone

Acetonitrile (Methyl cyanide)

Acetophenone

2-Acetylaminofluorene

Acrolein

Acrylonitrile

Aldrin

Allyl chloride

4-Aminobiphenyl

Aniline

Anthracene

Antimony, total recoverable

Aramite

Arsenic, total recoverable

Barium, total recoverabie

Benzene

alpha-Benzene hexachloride

beta-Benzene hexachloride

delta-Benzene hexachloride

Benzo[s]anthracene

Benzo[b]fluoranthene

Benzolk]fluoranthene

Benzolg, $h, \lambda$ perylene

Benzolajpyrene

Benzyl alcohol

Beryllium, total recoverable

Bis(2-chloroethoxy) methane

Bis(2-chloroethyl) ether

Bis(2-chioroisopropyl) ether

Bis(2-ethylhexyl) phthalate

Bromodichloromethane

Bromoform

Bromomethane (Methyl bromide)

4-Bromophenyl phenyl ether

Butylbenzyl phthalate

2-sec-Butyl-4,6-dinitrophenol

\section{Screen Zone Elevation}

242.1-222.1 ft ms!

$02 / 13 / 94$

$04 / 25 / 94$

2094

234.4

5.3

5.3

19.7

4

21.4

98
0.6

$x$

1094

$\underline{2094}$

3094

$<10$

$<10$
$<500$

$<25$

$<10$

$<10$

$<100$

$<100$

$<0.050$

$556<250$

556

$\begin{array}{r}159 \\ \hline\end{array}$

$<10$

$<10$

$<10$

$<2.0$

$<10$

$<2.0$

4.2

$<1.0$

$<1.0$

$<5.0$

$<0.050$

$<0.050$

$<10$
$<10$
$<10$

$<10$

$<10$

$<10$

$<10$

$<3.0$

$<10$

$<10$

$<25$

$<10$

$<5.0$

$<5.0$

$<5.0$

$<10$

$<10$

$<10$

$\begin{array}{lll}\text { Casing } & \text { Pump } & \text { Screen Zone } \\ 4^{n \text { PVC }} & \mathrm{S} & \mathrm{MGCL}\end{array}$

$10 / 28 / 94$

$\begin{array}{ll}4094 & \text { Unit } \\ 234.3 & \mathrm{ft} \mathrm{msl} \\ 145.3 & \mathrm{ft} \\ 5.3 & \mathrm{pH} \\ 47 & \mu \mathrm{S} / \mathrm{cm} \\ 18.8 & { }^{\circ} \mathrm{C} \\ 5 & \mathrm{mg} / \mathrm{L} \\ 27 & \text { NTU } \\ 9.9 & \text { well vol. }\end{array}$

4094 DF Mod Unit Lab Flag

$\mu g / L$

$\mu g / L$

$\mu \mathrm{g} / \mathrm{L}$

$\mu g h$

$\mu \mathrm{g} / \mathrm{L}$

$\mu \mathrm{g} / \mathrm{L}$

$\mu g h$

$\mu \mathrm{g} / \mathrm{L}$

$\mu g / L$

$\mu g / L$

$\mu g / L$

$\mu \mathrm{g} / \mathrm{L}$

$\mu g / L$

$\mu g / L$

$\mu g / L$

$\mu g / L$ GE 0

$\mu g / L$

$\mu g / L$

$\mu \mathrm{g} / \mathrm{L}$

$\mu g / L$

$\mu g / L$

$\mu \mathrm{g} / \mathrm{L}$

$\mu g / L$

$\mu g / L$

$\mu g / L$

$\mu g / L$

$\mu g / L$

$\mu g / L$

$\mu \mathrm{g} / \mathrm{L}$

$\mu \mathrm{g} / \mathrm{L}$

$\mu \mathrm{g} / \mathrm{L}$

$\mu g / L$

$\mu g / L$

Note: Flagging, dilution factors, modifiers, and laboratories are for fourth quarter 1994 data only. See Appendix B for flagging criteria. - = exceeded holding time for fourth quarter 1994.

- = exceeded screening level or final PDWS for fourth quarter 1994. 
Well AMB 5 continued

ANALYTICAL DATA

\section{H ST Analvte}

Cadmium, total recoverable

Carbon disulfide

Carbon tetrachloride

Chlordane

Chloride

4-Chloroaniline

Chlorobenzene

Chlorobenzilate

4-Chioro-m-cresol

Chloroethane

Chloroethene (Vinyl chloride)

2-Chloroethyl vinyl ether

Chloroform

Chloromethane (Methyl chloride)

2-Chloronaphthalene

2-Chlorophenol

4-Chiorophenyl phenyl ether

Chloroprene

Chromium, total recoverable

Chrysene

Cobalt, total recoverable

Copper, total recoverable

o-Cresol (2-Methyiphenol)

m,p-Cresol

Cyanide

$p, p^{\prime}-D D D$

P.P'-DDE

$p, p^{\prime}-D D T$

Diallate

Dibenzle,hlanthracene

Dibenzofuran

Dibromochloromethane

1,2-Dibromo-3-chloropropane

1,2-Dibromoethane

Dibromomethane

Di-n-butyl phthalate

1.2-Dichlorobenzene

1.3-Dichlorobenzene

1,4-Dichlorobenzene

3,3'-Dichlorobenzidine

trans-1,4-Dichloro-2-butene

Dichlorodifluoromethane

1, 1-Dichloroethane

1,2-Dichloroethane

1,1-Dichloroethylene

trans-1,2-Dichioroethylene

Dichloromethane

2,4-Dichlorophenol

2,6-Dichlorophenol

2,4-Dichlorophenoxyacetic acid

1,2-Dichloropropane

cis-1,3-Dichloropropen

trans-1,3-Dichloropropene

Dieldrin

Diethyl phthalate

Dimethoate

2.4-Dimethyl phenol

Dimethyl phthalate

p-Dimethylaminoazobenzene

7,12-Dimethylbenzlalanthracene

3,3'-Dimethylbenzidine

a,a-Dimethylphenethylamine

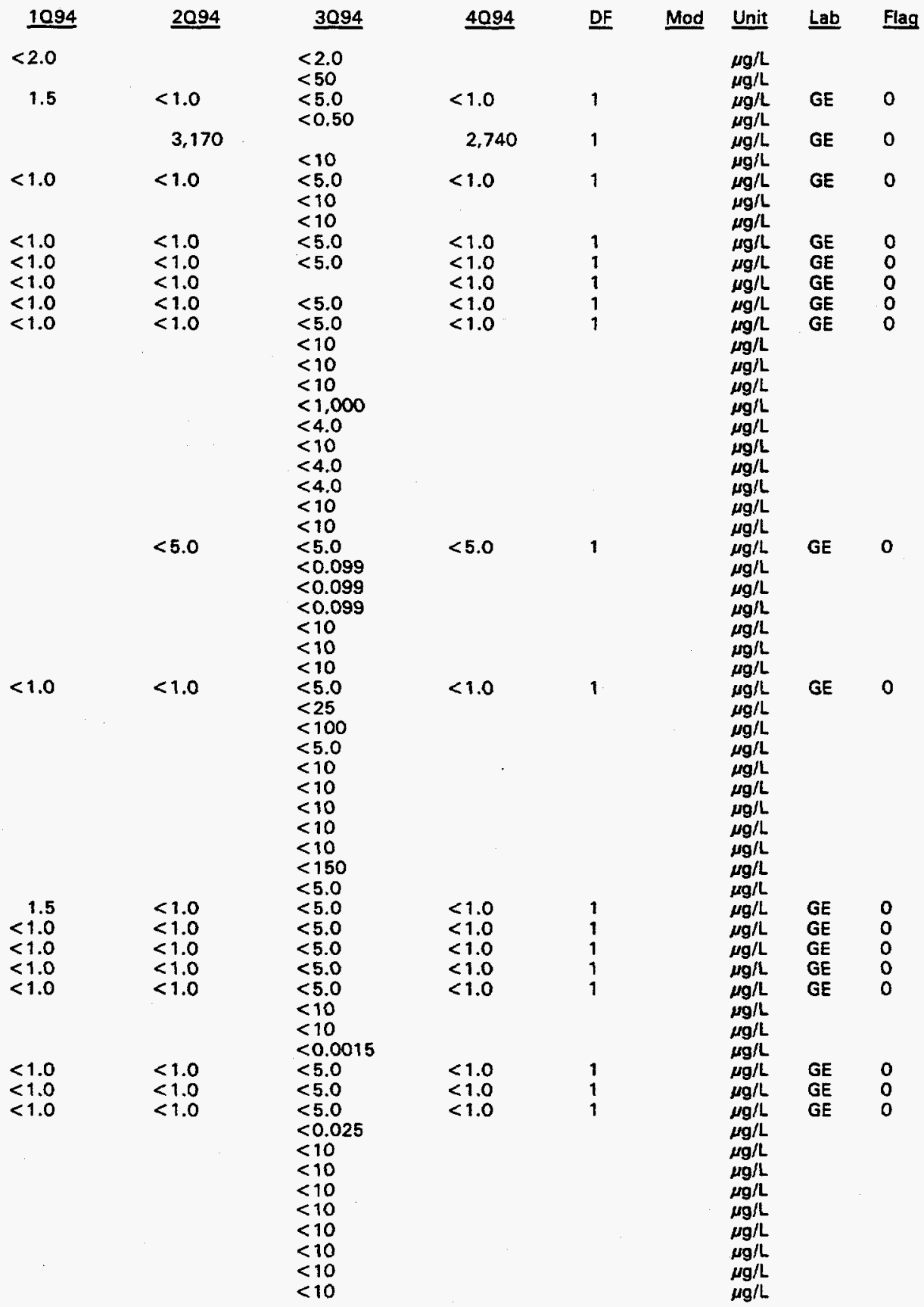

Note: Flagging, dilution factors, modifiers, and laboratories are for fourth quarter 1994 data only. See Appendix B for flagging criteria.

- = exceeded holding time for fourth quarter 1994.

- = exceeded screening level or final PDWS for fourth quarter 1994. 
H ST Analyte

1,3-Dinitrobenzen

2,4-Dinitrophenol

2,4-Dinitrotoluene

2,6-Dinitrotoluene

Di-n-octyl phthalate

1,4-Dioxane

Diphenylamine

Disulfoton

Endosultan I

Endosulfan II

Endosulfan sulfate

Endrin

Endrin aldehyde

Ethyl methacrylate

Ethyl methanesulfonate

Ethylbenzene

Famphur

Fluoranthene

Fluorene

Fluoride

Gross alpha

Heptachlor

Heptachlor epoxide

1,2,3,4,6,7,8-HPCDD

Heptachlorodibenzo-p-dioxin isomers

$1,2,3,4,6,7,8$-HPCDF

Heptachlorodibenzo-p-furan isomers

Hexachlorobenzene

Hexachlorobutadiene

Hexachlorocyclopentadiene

$1,2,3,4,7,8-\mathrm{HXCDD}$

Hexachlorodibenzo-p-dioxin isomers

$1,2,3,4,7,8-\mathrm{HXCDF}$

Hexachlorodibenzo-p-furan isomers

Hexachloroethane

Hexachlorophene

Hexachloropropene

2-Hexanone

Indenol $t, 2,3-c, d]$ pyrene

lodomethane (Methyl iodide)

Iron, total recoverable

isobutyl alcohol

Isodrin

isophorone

isosafrole

Kepone

Lead, total recoverable

Lindane

Manganese, total recoverable

Mercury, total recoverable

Methacrylonitrile

Methapyrilene

Methoxychior

2-Methyl-4,6-dinitrophenol

Methyl ethyl ketone

Methyl isobutyl ketone

Methyl methacrylate

Methyl methanesulfonate

3-Methylcholanthrene

2-Methylnaphthalene

Naphthalene

1,4-Naphthoquinone

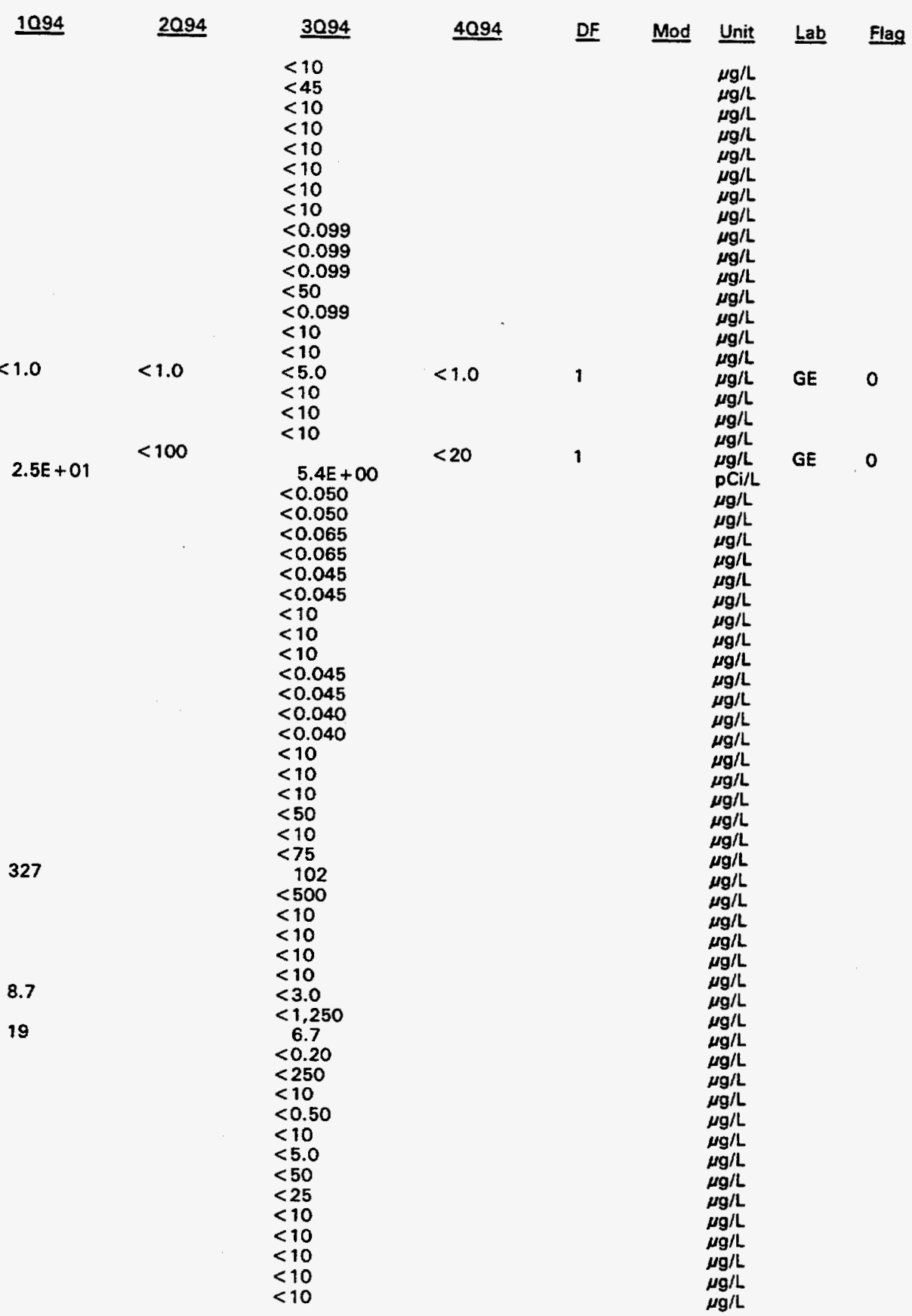

Note: Flagging, dilution factors, modifiers, and laboratories are for fourth quarter 1994 data only. See Appendix B for flagging criteria. - = exceeded holding time for fourth quarter 1994.

- = exceeded screening level or final PDWS for fourth quarter 1994. 
Well AMB 5 continued

ANALYTICAL DATA

H ST Analyte

1-Naphthylamine

2-Naphthylamine

Nickel, total recoverable

Nitrate-nitrite as nitrogen

o-Nitroaniline

m-Nitroaniline

p-Nitroaniline

Nitrobenzene

2-Nitrophenol

4-Nitrophenol

4-Nitroquinoline-1-oxide

$\mathrm{N}$-Nitrosodi-n-butylamine

N-Nitrosodiethylamine

$\mathrm{N}$-Nitrosodimethylamine

$\mathrm{N}$-Nitrosodiphenylamine

$\mathrm{N}$-Nitrosodipropylamine

$\mathrm{N}$-Nitrosomethylethylamine

N-Nitrosomorpholine

$\mathrm{N}$-Nitrosopiperidine

N-Nitrosopyrrolidine

5-Nitro-0-toluidine

Nonvolatile beta

$0,0,0$-Triethyl phosphorothioate

Octachlorodibenzo-p-dioxin

Octachlorodibenzo-p-furan

Parathion

Parathion methyl

PCB 1016

PCB 1221

PCB 1232

PCB 1242

PCB 1248

PCB 1254

PCB 1260

Pentachlorobenzene

1,2,3,7,8-PCDD

Pentachlorodibenzo-p-dioxin isomers

$1,2,3,7,8$-PCDF

Pentachloroethane

Pentachloronitrobenzene

Pentachlorophenol

Phenacetin

Phenanthrene

Phenol

p-Phenylenediamine

Phorate

2-Picoline

Pronamid

Propionitrile

Pyrene

Pyridine

Radium, total alpha-emitting

Safrole

Selenium, total recoverable

Silver, total recoverable

Styrene

Sulfide

Sulfotepp

1,2,4,5-Tetrachlorobenzene

$2,3,7,8$-TCOD

2,3,7,8-TCDF

Tetrachlorodibenzo-p-dioxin isomers

Note: Flagging, dilution factors, modifiers, and laboratories are for fourth quarter 1994 data only. See Appendix B for flagging criteria. - = exceeded holding time for fourth quarter 1994.

- = exceeded screening level or final PDWS for fourth quarter 1994. 
ANALYTICAL DATA

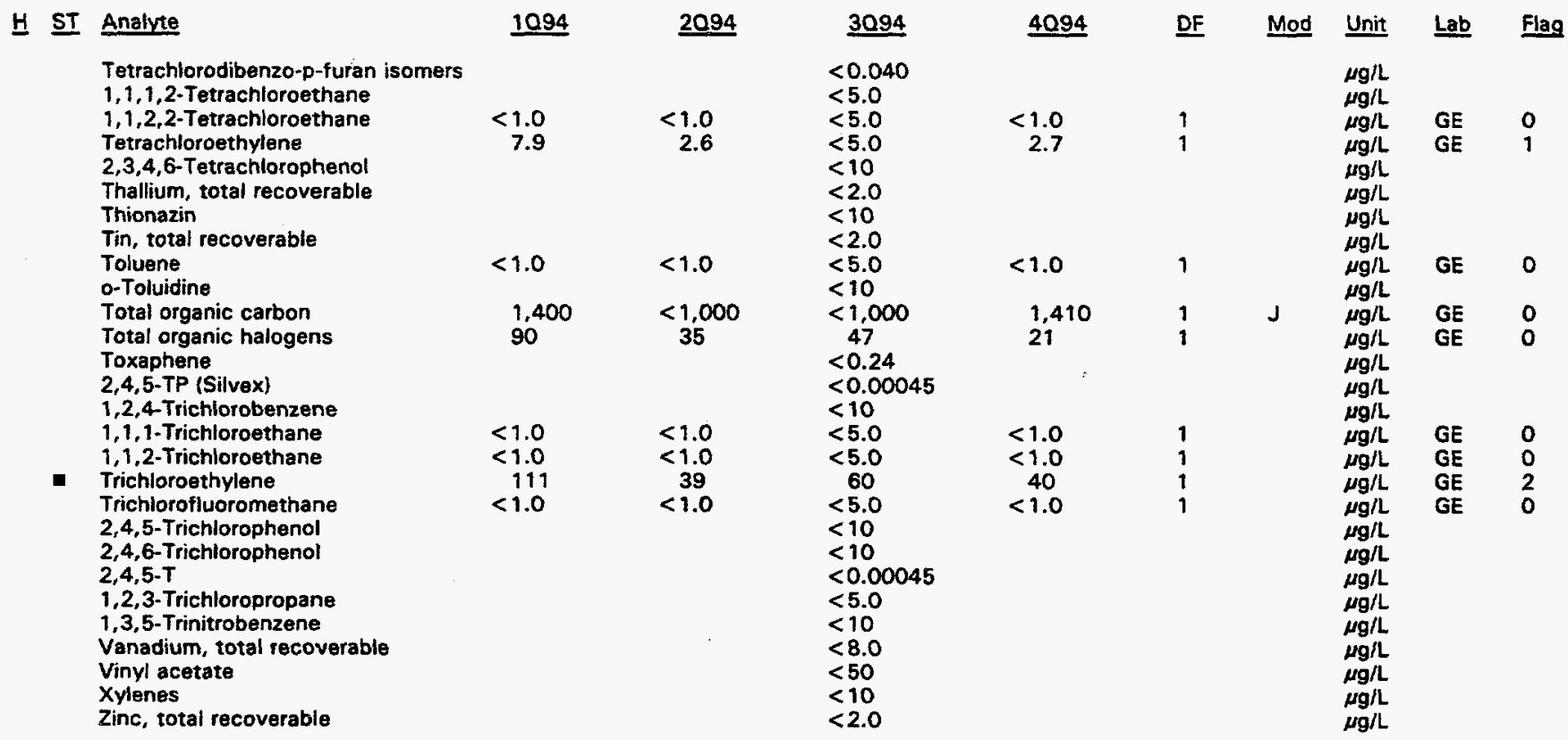

\section{WELL AMB 6}

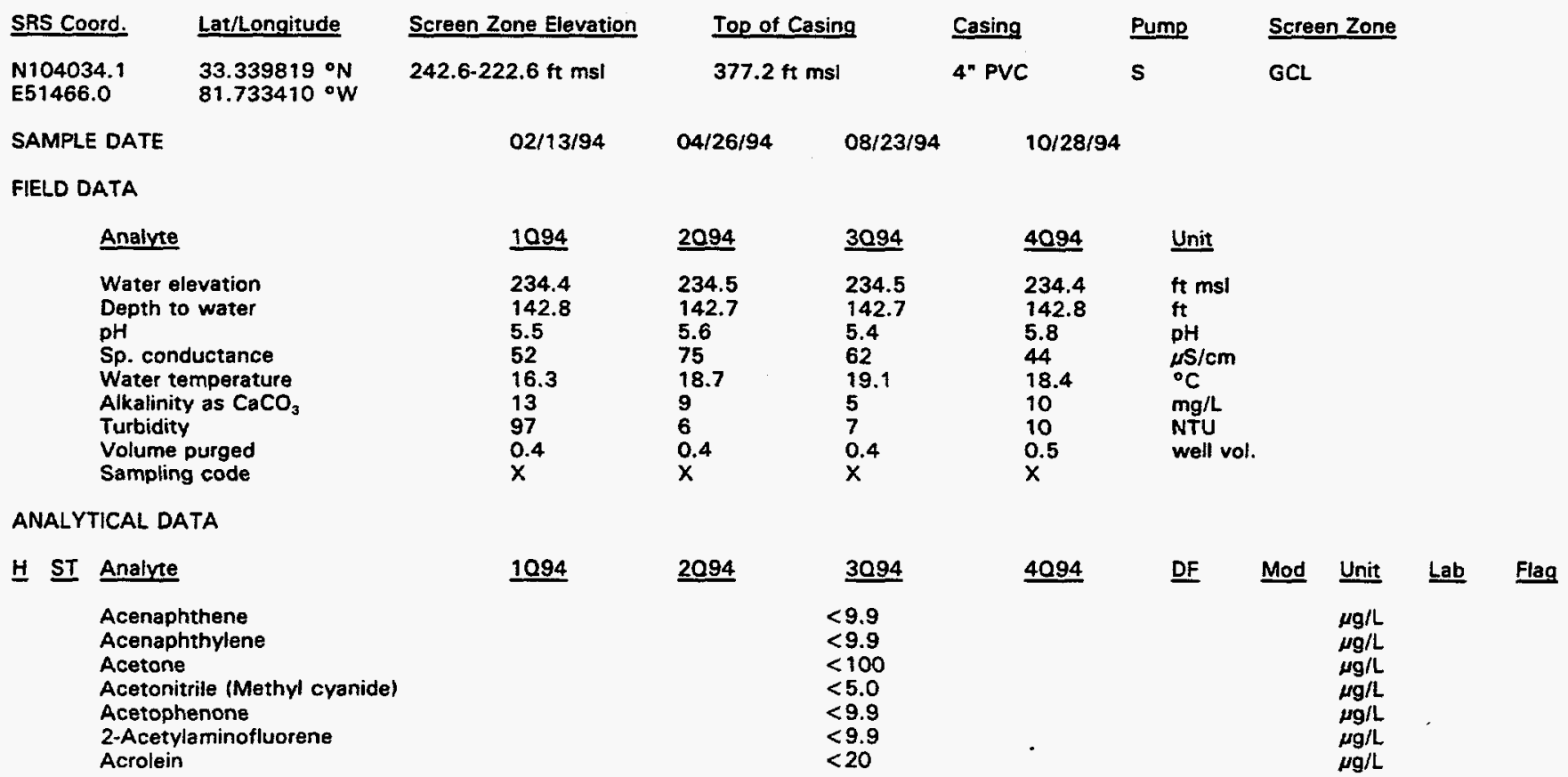

Note: Flagging, dilution factors, modifiers, and laboratories are for fourth quarter 1994 data only. See Appendix B for flagging criteria. - = exceeded holding time for fourth quarter 1994.

- = exceeded screening level or final PDWS for fourth quarter 1994. 
Well AMB 6 continued

ANALYTICAL DATA

\section{H ST Analyte}

Acrylonitrile

Aldrin

Allyl chloride

Aluminum, total recoverable

4-Aminobipheny!

Aniline

Anthracene

Antimony, total recoverable

Aramite

Arsenic, total recoverable

Barium, total recoverable

Benzene

alpha-Benzene hexachloride

beta-Benzene hexachloride

delta-Benzene hexachloride

Benzolajanthracene

Benzolb]fluoranthene

Benzo[k]fluoranthene

Benzo[g,h,i]perylene

Benzo[a]pyrene

Benzyl alcohol

Berylium, total recoverable

Bis(2-chloroethoxy) methane

Bis(2-chloroethyl) ether

Bis(2-chloroisopropyl) ether

Bis (2-ethylhexyl\} phthalate

Bromodichloromethane

Bromoform

Bromomethane (Methyl bromide)

4-Bromophenyl phenyl ether

Butylbenzyl phthalate

2-sec-Butyl-4,6-dinitrophenol

Cadmium, total recoverable

Carbon disulfide

Carbon tetrachloride

Chlordane

Chloride

4-Chloroaniline

Chlorobenzene

Chlorobenzilate

4-Chloro-m-cresol

Chloroethane

Chloroethene (Vinyl chloride)

2-Chloroethyl vinyl ether

Chloroform

Chloromethane (Methyl chloride)

2-Chloronaphthalene

2-Chlorophenol

4-Chlorophenyl phenyl ether

Chloroprene

Chromium, total recoverable

Chrysene

Cobatt, total recoverable

Copper, total recoverable

o-Cresol (2-Methviphenol)

$m, p$-Cresol

Cranide

$p, p^{\prime}-D D D$

$p, p^{\prime}-D D E$

p,p'-DDT

Diallate

Dibenzla,h]anthracene
1094

$\underline{2094}$

3094

4,320

$<20$

$<0.050$

$<50$

1,650

$<9.9$

$<9.9$

$<9.9$

$<2.0$

$<9.9$

$<2.0$

$<3.0$

$<1.0$

$<1.0$

$<1.0$

$<0.050$

$<0.050$

$<0.050$

$<9.9$

$<9.9$

$<9.9$

$<9.9$

$<9.9$

$<9.9$

$<3.0$

$<9.9$

$<9.9$

$<5.0$

$<9.9$

$<1.0$

$<1.0$

$<1.0$

$<1.0$

$<1.0$

$<1.0$

$<1.0$

$<2.0$

$<1.0$

$<1.0$

2,870

$<1.0$

$<1.0$

$<1.0$

$<1.0$

$<9.9$

$<9.9$

$<9.9$

$<2.0$

$<10$

$<1.0$

$<0.50$

$<0.50$

$<9.9$

$<1.0$

$<9.9$

$<9.9$

$<1.0$

$<1.0$

$<1.0$

$<1.0$

$<1.0$

$<1.0$

$<1.0$

$<1.0$

$<1.0$

$<1.0$

$<1.0$

$<1.0$

$<1.0$

$<9.9$

$<9.9$

$<9.9$

$<200$

$<4.0$

$<9.9$

$<4.0$

45

$<9.9$

$<9.9$

$<5.0$

$<0.10$

$<0.10$

$<0.10$

$<9.9$

$<9.9$
4094

DF Mod Unit Lab Flag

$<1.0 \quad 1 . \quad \mu$

GE 0

$\begin{array}{cc}\text { GE } & 0 \\ \text { GE } & 0 \\ \text { GE } & 0\end{array}$

$<1.0$
$<1.0$

$<1.0$

2,340

$<1.0$

$<1.0$

$<1.0$

$<1.0$

$<1.0$

$<1.0$

$<5.0$

1 $\mu g / L$

$\mu \mathrm{g} / \mathrm{L}$

$\mu \mathrm{gg} / \mathrm{L}$

$\mu g / L$

$\mu \mathrm{g} / \mathrm{L}$

$\mu \mathrm{g} / \mathrm{L}$

$\mu g / L$

$\mu g / L$

$\mu \mathrm{g} / \mathrm{L}$

$\mu \mathrm{g} / \mathrm{L}$

$\mu g / L$

$\mu \mathrm{g} / \mathrm{L}$

$\mu \mathrm{g} / \mathrm{L}$

$\mu \mathrm{g} / \mathrm{L}$

$\mu \mathrm{g} / \mathrm{L}$

$\mu \mathrm{g} / \mathrm{L}$

$\mu g / L$

$\mu g / L$

$\mu g / L$

$\mu g / L$

$\mu \mathrm{g} / \mathrm{L}$

$\mu \mathrm{g} / \mathrm{L}$

n

$\mu g / L$

$\mu g / L$

$\mu g / L$

$\mu g / L$

$\mu \mathrm{g} / \mathrm{L}$

$\mu g / L$

$\mu \mathrm{g} / \mathrm{L}$

Nh

$\mu g / L$

$\mu g / L$

$\mu g / L$

$\mu \mathrm{g} / \mathrm{L}$

$\mu g / L$

$\mu \mathrm{g} / \mathrm{L}$

$\mu g / L$

$\mu \mathrm{g} / \mathrm{L}$

$\mu g / L$.

$\mu \mathrm{g} / \mathrm{L}$

$\mu g / L$

$\mu \mathrm{g} / \mathrm{L}$

$\mu g / L$

$\mu g / L$

$\mu g / L$

$\mu g / L$

$\mu \mathrm{g} / \mathrm{L}$

$\mu g / L$

$\mu g / L$

$\mu g / L$

$\mu g / L$

$\mu g / L$

$\mu g / L$

$\mu g / L$

$\mu \mathrm{g} / \mathrm{L}$

$\mu g / L$

$\mu \mathrm{g} / \mathrm{L}$
GE 0

GE 0

GE 0

GE 0

GE 0

GE 0

GE 0

GE

0

Note: Flagging, dilution factors, modifiers, and laboratories are for fourth quarter 1994 data only. See Appendix B for flagging criteria. - = exceeded holding time for fourth quarter 1994.

- = exceeded screening level or final PDWS for fourth quarter 1994. 


$$
1094
$$

$\underline{2094}$

3094

Dibromochloromethane

1,2-Dibromo-3-chloropropane

1,2-Dibromoethane

Dibromomethane

Di-n-butyl phthalate

1,2-Dichlorobenzene

1.3-Dichlorobenzene

1,4-Dichlorobenzene

3,3'-Dichlorobenzidine

trans-1,4-Dichloro-2-butene

Dichlorodifluoromethane

1,1-Dichloroethane

1,2-Dichloroethane

1,1-Dichloroethylene

trans-1,2-Dichloroethylene

Dichloromethane

2,4-Dichlorophenol

2,6-Dichlorcphenol

2,4-Dichlorophenoxyacetic acid

1,2-Dichloropropane

cis-1,3-Dichloropropene

trans-1,3-Dichloropropene

Dieldrin

Diethyl phthalate

Dimethoate

2,4-Dimethyl phenol

Dimethyl phthalate

p-Dimethylaminoazobenzene

7,12-Dimethylbenz[a]anthracene

3,3'-Dimethylbenzidine

a,a-Dimethyiphenethylamine

1,3-Dinitrobenzene

2,4-Dinitrophenol

2,4-Dinitrotoluene

2,6-Dinitrotoluene

Di-n-octyl phthalate

1.4-Dioxane

Diphenylamine

Disulfoton

Endosulfan I

Endosulfan II

Endosulfan sulfate

Endrin

Endrin aldehyde

Ethyl methacrylate

Ethyl methanesulfonate

Ethylbenzene

Famphur

Fluoranthene

Fluorene

Fluoride

Gross alpha

Heptachlor

Heptachlor epoxide

$1,2,3,4,6,7,8-H P C D D$

Heptachlorodibenzo-p-dioxin isomers

$1,2,3,4,6,7,8$-HPCDF

Heptachlorodibenzo-p-furan isomers

Hexachlorobenzene

Hexachlorobutadiene

Hexachlorocyclopentadiene
$<1.0$

$<1.0$

$<1.0$

$<9.9$

$<1.0$

$<5.0$

$<20$

$<1.0$

$<9.9$

$<9.9$

$<9.9$

$<9.9$

$<9.9$

$<30$

$<1.0$

$<1.0$

$<1.0$

$<1.0$

$<1.0$

$<1.0$

$<9.9$

$<9.9$

$<0.0015$

$<1.0 \quad<1.0$

$<1.0$

$<1.0$

$<1.0$

$<1.0$

$<1.0$

$<1.0$

$<1.0$

$<0.025$

$<9.9$

$<9.9$

$<9.9$

$<9.9$

$<9.9$

$<9.9$

$<9.9$

$<9.9$

$<9.9$

$<45$

$<9.9$

$<9.9$

$<9.9$

$<9.9$

$<9.9$

$<9.9$

$<0.10$

$<0.10$

$<0.10$

$<50$

$<0.10$

$<9.9$

$<9.9$

$<1.0$

$<9.9$

$<9.9$

$<9.9$

4. $1 E+00$

$<100$

$1.4 E+\infty 0$

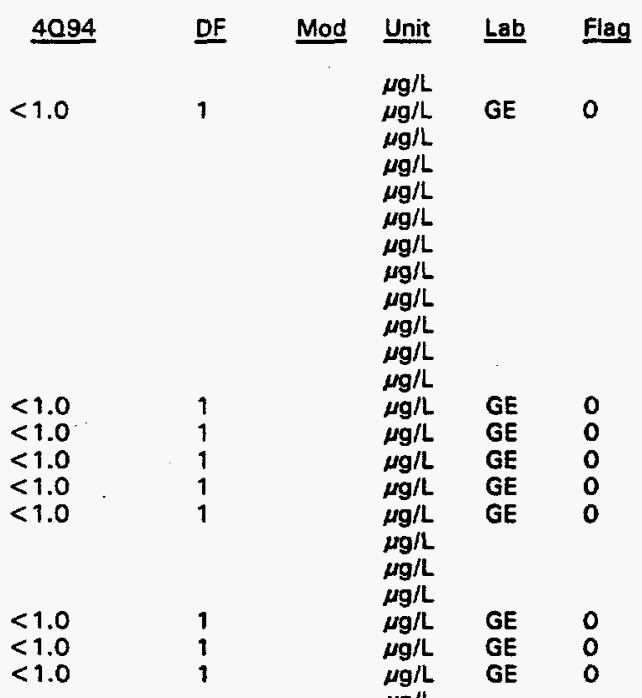

$<20$

$<1.0$

1

GE $O$

GE 0

Note: Flagging, dilution factors, modifiers, and laboratories are for fourth quarter 1994 data only. See Appendix B for flagging criteria. - = exceeded holding time for fourth quarter 1994.

- = exceeded screening level or final PDWS for fourth quarter 1994. 
ANALYTICAL DATA

\section{H ST Analyte}

$1,2,3,4,7,8-H \times C D D$

Hexachlorodibenzo-p-dioxin isomers

$1,2,3,4,7,8-\mathrm{HXCDF}$

Hexachlorodibenzo-p-furan isomers

Hexachloroethane

Hexachlorophene

Hexachioropropene

2-Hexanone

Indenol 1,2,3-c, d]pyrene

lodomethane (Methyl iodide)

Iron, total recoverable

Isobutyi alcohol

Isodrin

Isophorone

isosafrole

Kepone

Lead, total recoverable

Lindane

Manganese, total recoverable

Mercury, total recoverable

Methacrylonitrile

Methapyrilene

Methoxychlor

2-Methyl-4,6-dinitrophenol

Methyl ethyl ketone

Methyl isobutyl ketone

Methyl methacrylate

Methyl methanesulfonate

3-Methyicholanthrene

2-Methylnaphthalene

Naphthalene

1,4-Naphthoquinone

1-Naphthylamine

2-Naphthylamine

Nickel, total recoverable

Nitrate-nitrite as nitrogen

o-Nitroaniline

m-Nitroaniline

p-Nitroaniline

Nitrobenzene

2-Nitrophenol

4-Nitrophenol

4-Nitroquinoline-1-oxide

$\mathrm{N}$-Nitrosodi-n-butylamine

$\mathrm{N}$-Nitrosodiethylamine

N-Nitrosodimethylamine

$\mathrm{N}$-Nitrosodiphenylamine

$\mathrm{N}$-Nitrosodipropylamine

$\mathrm{N}$-Nitrosomethylethylamine

N-Nitrosomorpholine

$\mathrm{N}$-Nitrosopiperidine

$\mathrm{N}$-Nitrosopyrrolidine

5-Nitro-O-toluidine

Nonvolatile beta

$0,0,0$-Triethyl phosphorothioate

Octachlorodibenzo-p-dioxin

Octachlorodibenzo-p-furan

Parathion

Parathion methyl

PCB 1016

PCB 1221

PCB 1232
1094

$\underline{2094}$

3094

$<0.045$

$<0.045$

$<0.040$

$<0.040$

$<9.9$

$<9.9$

$<9.9$

$<10$

$<9.9$

$<15$

2,790

1,150

$<100$

$<9.9$

$<9.9$

$<9.9$

$<9.9$

57

10

6.7

$<0.20$

$<50$

$<9.9$

$<0.50$

$<9.9$

$<1.0$

$<10$

$<5.0$

$<9.9$

$<9.9$

$<9.9$

$<9.9$

$<9.9$

$<9.9$

$<9.9$

$<4.0$

240

$<4.0$

313

$<9.9$

$<9.9$

$<9.9$

$<9.9$

$<9.9$

$<9.9$

$<9.9$

$<9.9$

$<9.9$

$<9.9$

$<9.9$

$<9.9$

$<9.9$

$<9.9$

$<9.9$

$<9.9$

$<9.9$

$2.2 E+00$

$<9.9$

$<0.10$

$<0.10$

$<0.050$

$<0.050$

$<0.50$

$<0.50$

$<0.50$ $\mu g / L$

$\mu g / L$

$\mu g / L$

$\mu g / L$

$\mu g / L$

$\mu g / L$

$\mu g / L$

$\mu g / L$

$\mu g / L$

$\mu g / L$

$\mu g / L$

$\mu g / L$

$\mu g / L$

$\mu g / L$

$\mu g / L$

$\mu g / L$

$\mu g / L$

$\mu g / L$

$\mu g / L$

$\mu g / L$

$\mu \mathrm{g} / \mathrm{L}$

$\mu g / L$

$\mu g / L$

$\mu g / L$

$\mu g / L$

$\mu g / L$

igh

$\mu g / L$

$\mu g / L$

$\mu g / L$

4.9

236

$\mu g / L$

$\mu g / L$

$\mu g / L$

$\mu g / L$

pl

$\mu g / L$

$\mu \mathrm{g} / \mathrm{L}$

$\mu g / L$

$\mu g / L$

$\mu \mathrm{g} / \mathrm{L}$

$\mu g / L$

$\mu g / L$

$\mu g /$

$\mu g / L$

$\mu g / L$

$\mu \mathrm{g} / \mathrm{L}$

$\mu g / L$

$\mu g / L$

$\mu g / L$

$\mu g / L$

$\mu g / L$

$\mu g / L$

$\mu \mathrm{g} / \mathrm{L}$ $\underline{\text { Lab }} \quad$ Flag

GE $\quad 0$

Note: Flagging, dilution factors, modifiers, and laboratories are for fourth quarter 1994 data only. See Appendix B for flagging criteria.

- = exceeded holding time for fourth quarter 1994.

- = exceeded screening level or final PDWS for fourth quarter 1994. 
ANALYTICAL DATA

H ST Analyte

PCB 1242

PCB 1248

PCB 1254

PCB 1260

Pentachlorobenzene

$1,2,3,7,8-P C D D$

Pentachlorodibenzo-p-dioxin isomers

$1,2,3,7,8-P C D F$

Pentachloroethane

Pentachloronitrobenzene

Pentachlorophenol

Phenacetin

Phenanthrene

Phenol

p-Phenylenediamine

Phorate

2-Picoline

Pronamid

Propionitrile

Pyrene

Pyridine

Radium, total alpha-emitting

Safrole

Selenium, total recoverable

Silver, total recoverable

Styrene

Sulfide

Sulfotepp

1,2,4,5-Tetrachlorobenzene

$2,3,7,8-T C D D$

2,3,7,8-TCDF

Tetrachlorodibenzo-p-dioxin isomers

Tetrachlorodibenzo-p-furan isomers

1,1,1,2-Tetrachloroethane

1,1,2,2-Tetrachloroethane

Tetrachloroethylene

2,3,4,6-Tetrachlorophenol

Thallium, total recoverable

Thionazin

Tin, total recoverable

Toluene

o-Toluidine

Total organic carbon

Total organic halogens

Toxaphene

2,4,5-TP (Silvex)

1,2,4-Trichlorobenzene

1,1,1-Trichloroethane

1,1,2-Trichloroethane

- Trichloroethylene

Trichlorofluoromethane

2,4,5-Trichloropheno!

2,4,6-Trichlorophenol

$2,4,5-T$

1,2,3-Trichloropropane

1,3,5-Trinitrobenzene

Vanadium, total recoverable

Vinyl acetate

Xylenes

Zinc, total recoverable

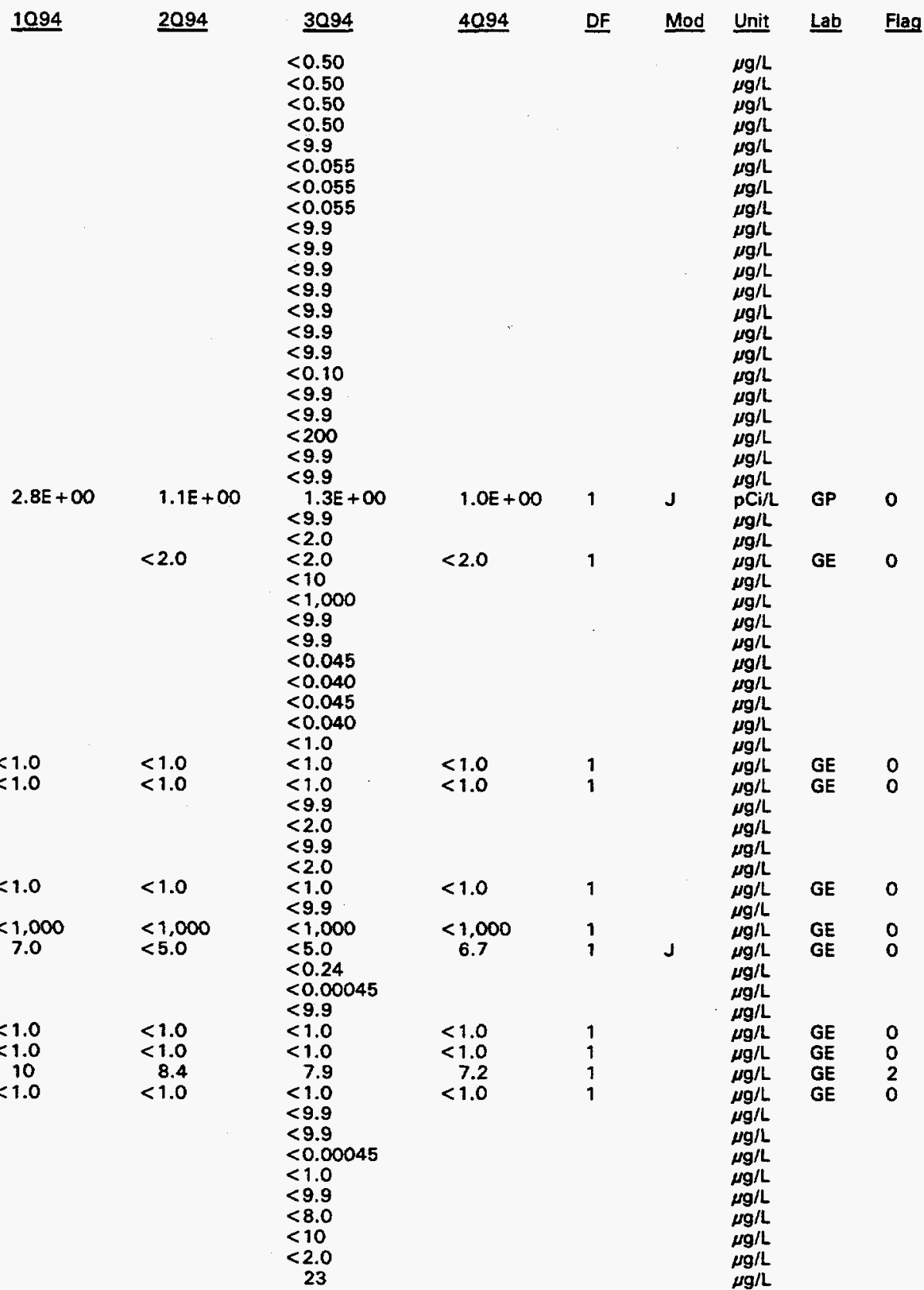

Note: Flagging, dilution factors, modifiers, and laboratories are for fourth quarter 1994 data only. See Appendix B for flagging criteria. - = exceeded holding time for fourth quarter 1994.

- = exceeded screening level or final PDWS for fourth quarter 1994. 
WELL AMB 7

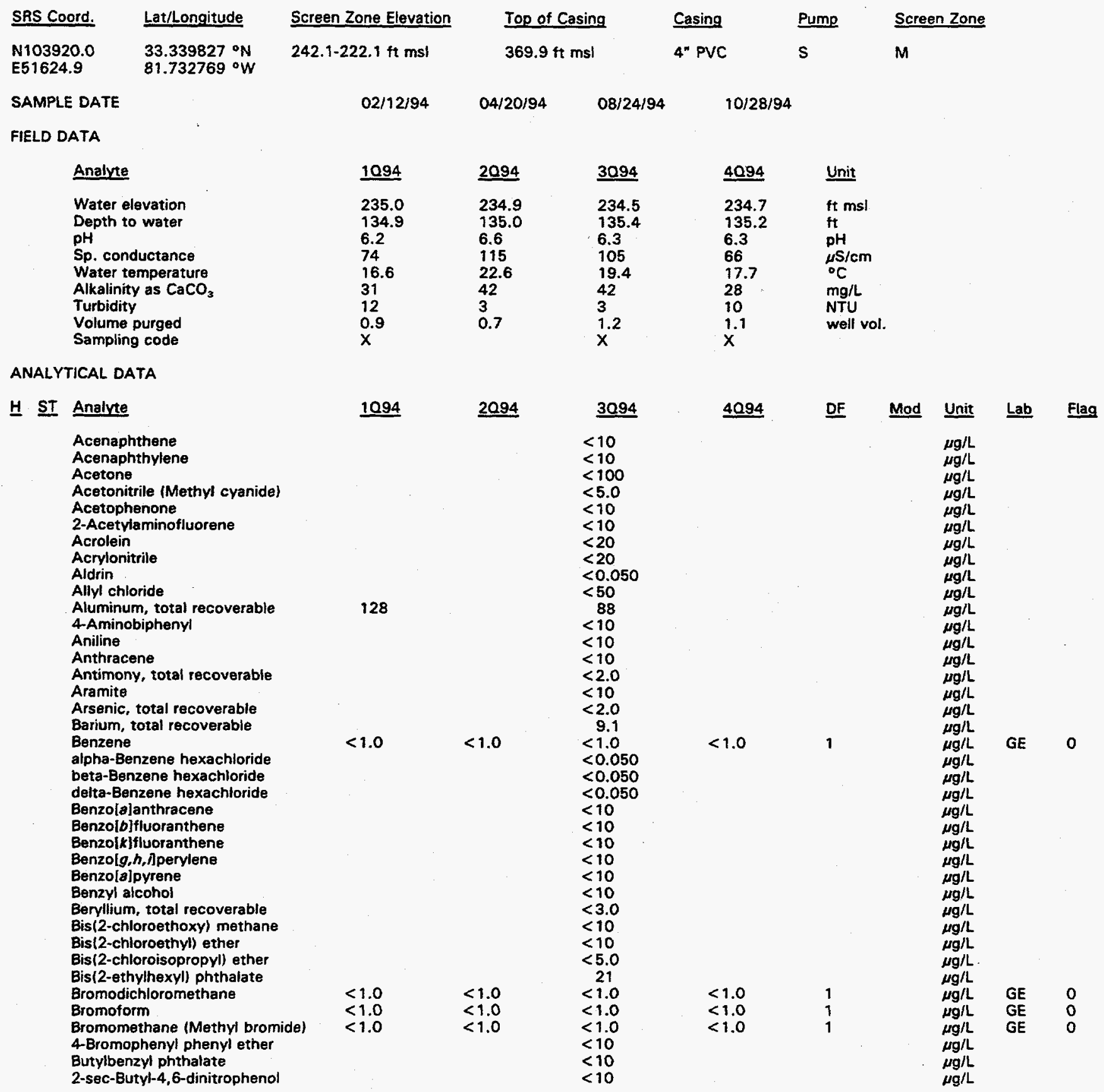

Note: Flagging, dilution factors, modifiers, and laboratories are for fourth quarter 1994 data only. See Appendix B for flagging criteria. - = exceeded holding time for fourth quarter 1994.

- = exceeded screening level or final PDWS for fourth quarter 1994. 


\section{H ST Analyte}

Cadmium, total recoverable

Carbon disulfide

Carbon tetrachloride

Chiordane

Chloride

4-Chloroaniline

Chlorobenzene

4-Chloro-m-cresol

Chloroethane

Chloroethene (Vinyl chloride)

2-Chloroethyl vinyl ether

Chioroform

Chloromethane (Methyl chloride)

2-Chloronaphthalene

2-Chlorophenol

4-Chlorophenyl phenyl ether

Chloroprene

Chromium, total recoverable

Chrysene

Cobalt, total recoverable

Copper, total recoverable

o-Cresol (2-Methylphenol)

m,p-Cresol

Cyanide

$p, p^{\prime}=D D D$

$p, p^{\prime}-D D E$

p,p'-DDT

Diallate

Dibenz $[a, h]$ anthracene

Dibenzofuran

Dibromochloromethane

1,2-Dibromo-3-chloropropane

1,2-Dibromoethane

Dibromomethane

Di-n-butyl phthalate

1,2-Dichlorobenzene

1,3-Dichlorobenzene

1,4-Dichlorobenzene

$3,3^{\prime}$-Dichlorobenzidine

trans-1,4-Dichloro-2-butene

Dichlorodifluoromethane

1,1-Dichloroethane

1,2-Dichloroethane

1,1-Dichloroethylene

trans-1,2-Dichloroethylene

Dichloromethane

2,4-Dichloropheno

2,6-Dichlorophenol

2,4-Dichlorophenoxyacetic acid

1.2-Dichloropropane

cis-1,3-Dichloropropene

trans-1,3-Dichloropropene

Dieldrin

Diethyl phthalate

Dimethoate

2,4-Dimethyl phenol

Dimethyl phthalate

p-Dimethylaminoazobenzene

7.12-Dimethylbenz[alanthracene

3, 3'-Dimethylbenzidine

a, a-Dimethylphenethylamine

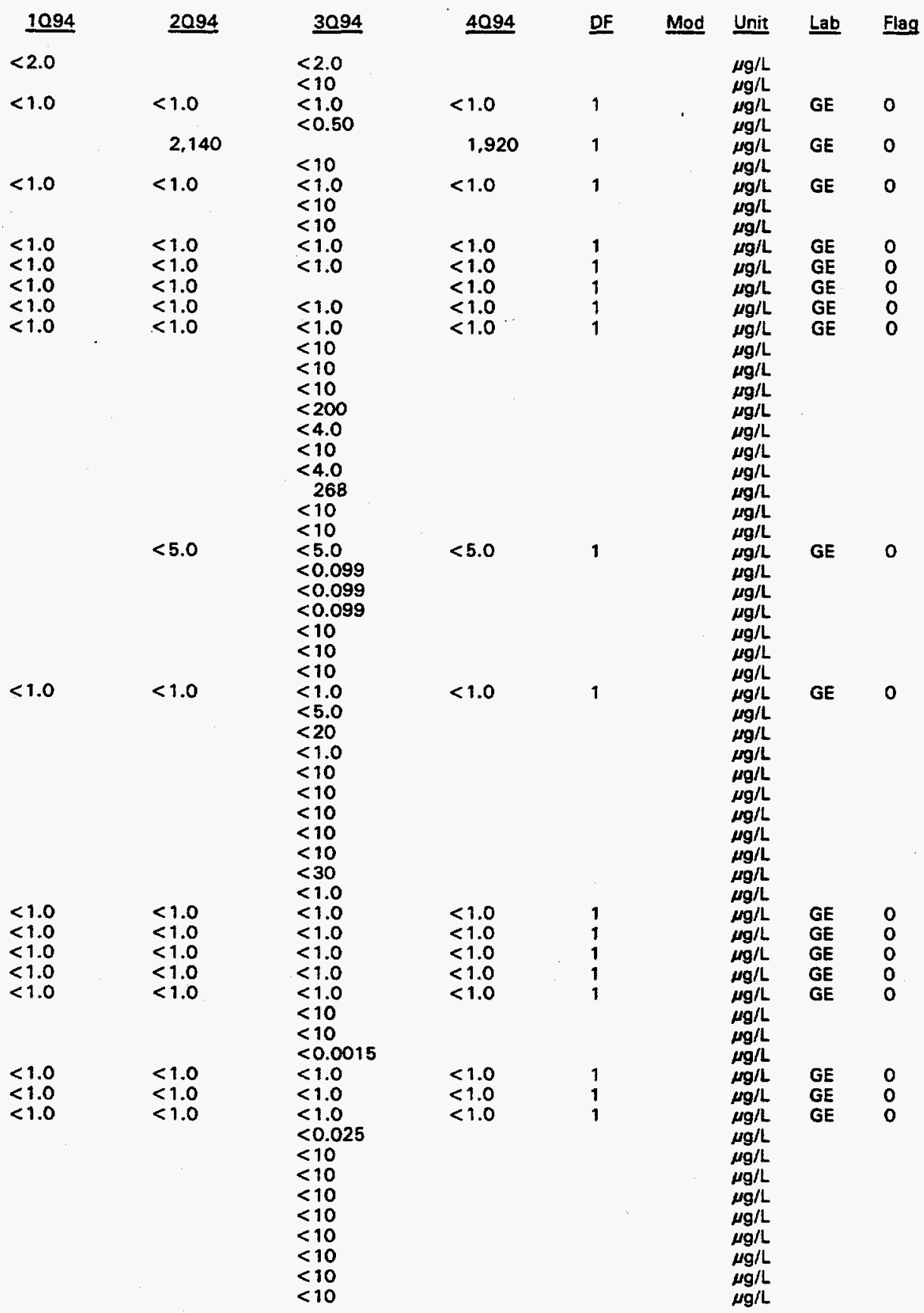

Note: Flagging, dilution factors, modifiers, and laboratories are for fourth quarter 1994 data only. See Appendix B for flagging criteria. - = exceeded holding time for fourth quarter 1994.

- =xceeded screening level or final PDWS for fourth quarter 1994. 
ANALYTICAL DATA

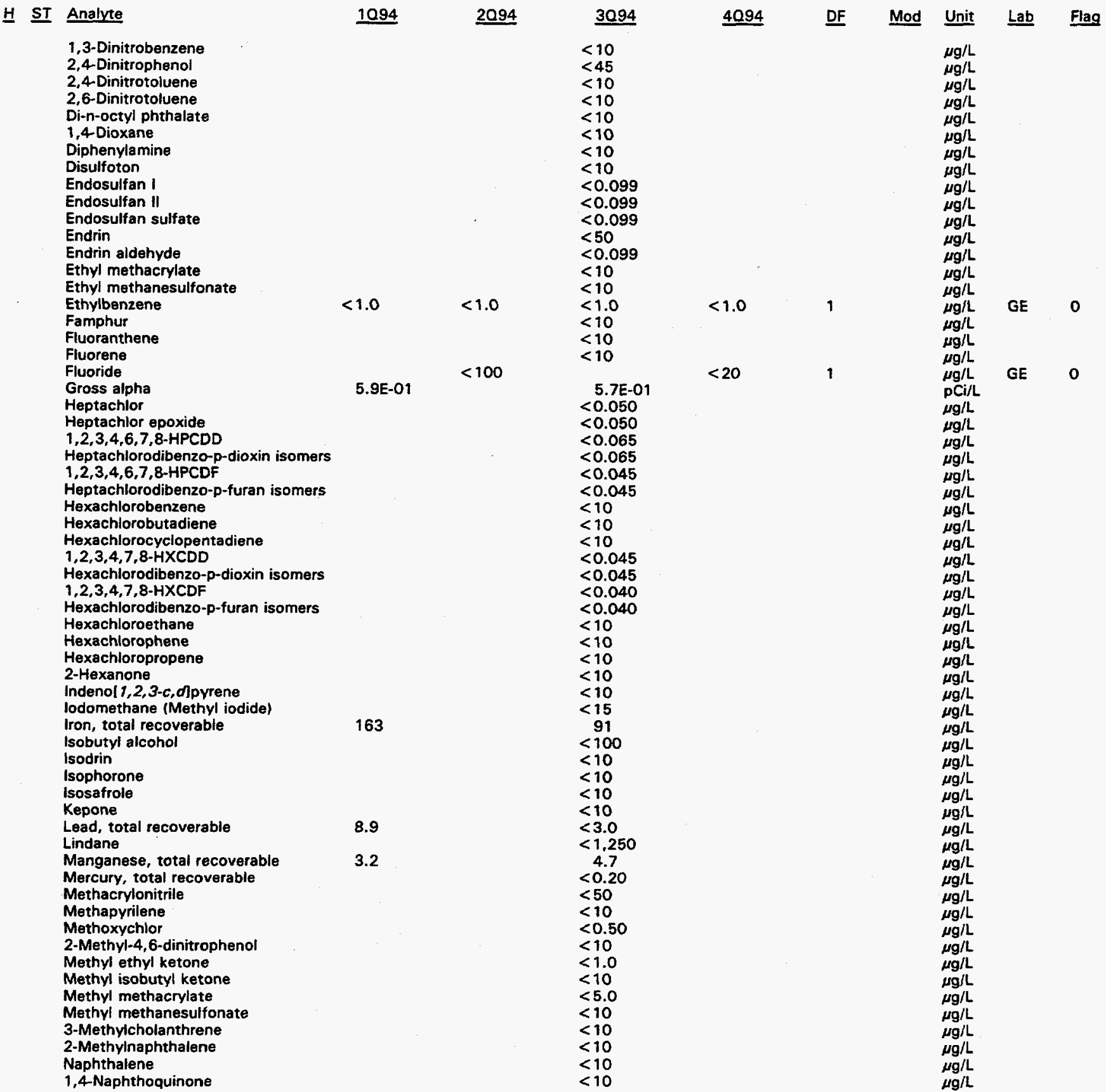

Note: Flagging, dilution factors, modifiers, and laboratories are for fourth quarter 1994 data only. See Appendix B for flagging criteria. - = exceeded holding time for fourth quarter 1994.

- = exceeded screening level or final PDWS for fourth quarter 1994. 
$\mu \mathrm{U} / \mathrm{U}$

1-Naphthylamine

2-Naphthylamine

Nickel, total recoverable

$<10$

Nitrate-nitrite as nitrogen

250

17

o-Nitroaniline

$m$-Nitroaniline

p-Nitroaniline

Nitrobenzene

2-Nitrophenol

4-Nitrophenol

4-Nitroquinoline-1-oxide

$\mathrm{N}$-Nitrosodi-n-butylamine

N-Nitrosodiethylamine

N-Nitrosodimethylamine

N-Nitrosodiphenylamine

$\mathrm{N}$-Nitrosodipropylamine

$\mathrm{N}$-Nitrosomethylethylamine

N-Nitrosomorpholine

N-Nitrosopiperidine

$\mathrm{N}$-Nitrosopyrrolidine

5-Nitro-o-toluidine

Nonvolatile beta

$0,0,0$-Triethyl phosphorothioate

Octachlorodibenzo-p-dioxin

Octachlorodibenzo-p-furan

Parathion

Parathion methyl

PCB 1016

PCB 1221

PCB 1232

PCB 1242

PCB 1248

PCB 1254

PCB 1260

Pentachlorobenzene

1,2,3,7,8-PCDD

Pentachlorodibenzo-p-dioxin isomers

$1,2,3,7,8-P C D F$

Pentachloroethane

Pentachloronitrobenzene

Pentachlorophenol

Phenacetin

Phenanthrene

Phenol

p-Phenylenediamine

Phorate

2-Picoline

Pronamid

Propionitrile

Pyrene

Pyridine

Radium, total alpha-emitting

Safrole

Selenium, total recoverable

Silver, total recoverable

Styrene

Sulfide

Sulfotepp

1,2,4,5-Tetrachlorobenzene

$2,3,7,8$-TCDD

$2,3,7,8-T C D F$

Tetrachlorodibenzo-p-dioxin isomers

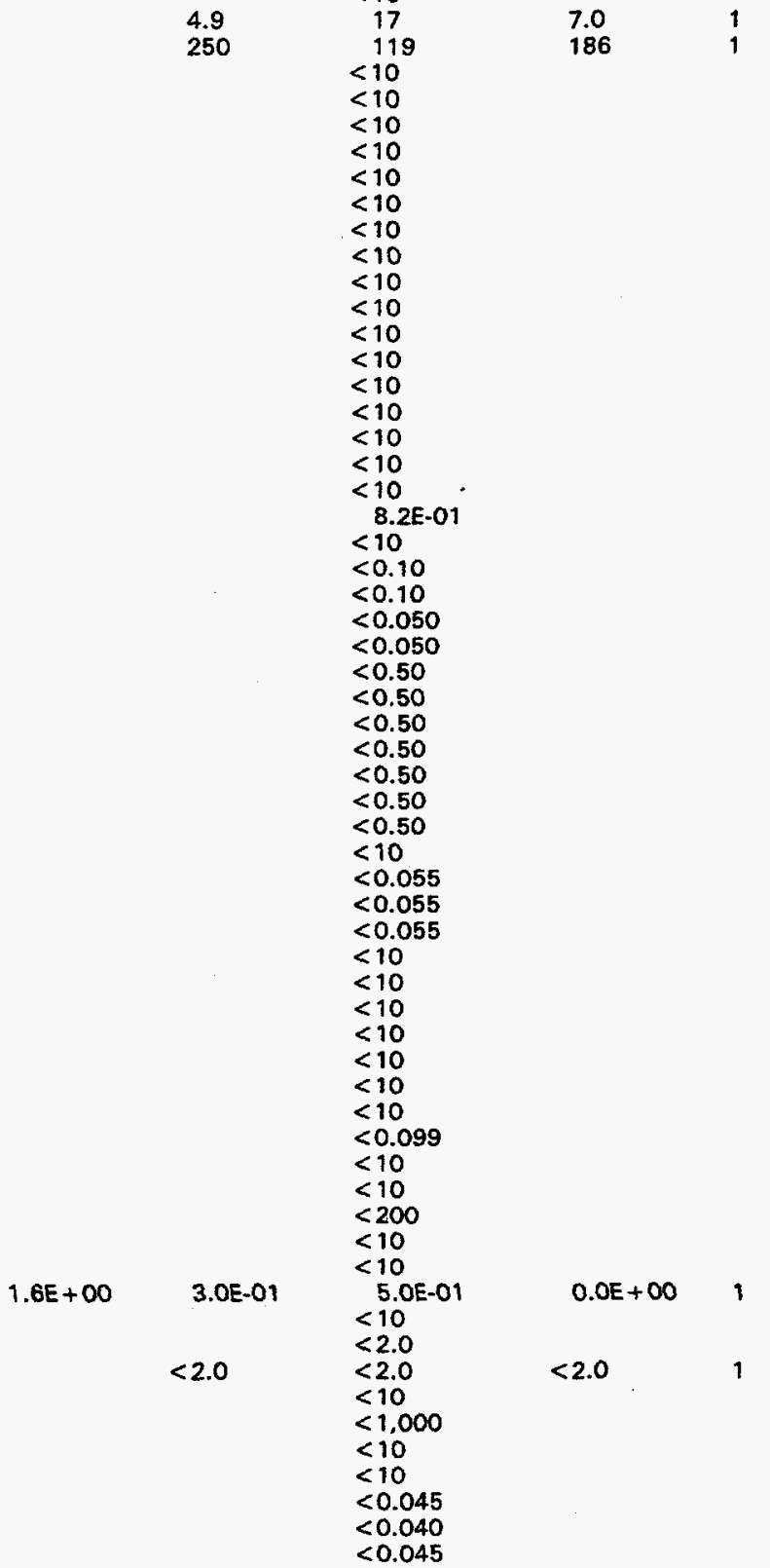

$\mu g /$

$\mu g / L$ GE $\quad 0$

$\mu g / L$

$\mu g / h$

$\mu g / L$

$\mu \mathrm{g} / \mathrm{L}$

$\mu \mathrm{g} / \mathrm{L}$

$\mu \mathrm{g} / \mathrm{L}$

$\mu g / L$

$\mu \mathrm{g} / \mathrm{L}$

$\mu \mathrm{g} / \mathrm{L}$

$\mu g / L$

$\mu g / L$

$\mu \mathrm{g} / \mathrm{L}$

$\mu \mathrm{g} / \mathrm{L}$

$\mu \mathrm{g} / \mathrm{L}$

$\mathrm{pCi} / \mathrm{L}$

$m g / L$

$\mu g / l$

$\mu \mathrm{g} / \mathrm{L}$

$\mu g / L$

$\mu g / L$

$\mu g / L$

$\mu \mathrm{g} / \mathrm{L}$

$\mu \mathrm{g} / \mathrm{h}$

$\mu \mathrm{g} / \mathrm{L}$

$\mu \mathrm{g} / \mathrm{L}$

$\mu g / L$

$\mu g / L$

$\mu g / L$

$\mu \mathrm{g} / \mathrm{L}$

$\mu \mathrm{g} / \mathrm{L}$

$\mu g / L$

$\mu \mathrm{\mu g} / \mathrm{L}$

$\mu g / L$

$\mu \mathrm{g} / \mathrm{L}$

$\mu g / L$

$\mu \mathrm{g} / \mathrm{L}$

$\mu \mathrm{g} / \mathrm{L}$

$\mu g / L$

$\mu \mathrm{g} / \mathrm{h}$

$\operatorname{gh}$

$\mu \mathrm{g} / \mathrm{L}$

$\mu \mathrm{g} / \mathrm{L}$

$\mathrm{pCi} / \mathrm{L}$

$\mu g / L$

$\mu g / L$

$\mu \mathrm{g} / \mathrm{L}$

$\mu g / L$

$\mu \mathrm{g} / \mathrm{L}$

$\mu g / L$

Lab $\quad$ Flag

GE $\quad 0$

GP $\quad 0$

GE $\quad 0$

Note: Flagging, dilution factors, modifiers, and laboratories are for fourth quarter 1994 data only. See Appendix B for flagging criteria. - = exceeded holding time for fourth quarter 1994.

- = exceeded screening level or final PDWS for fourth quarter 1994. 
Well AMB 7 continued

ANALYTICAL DATA

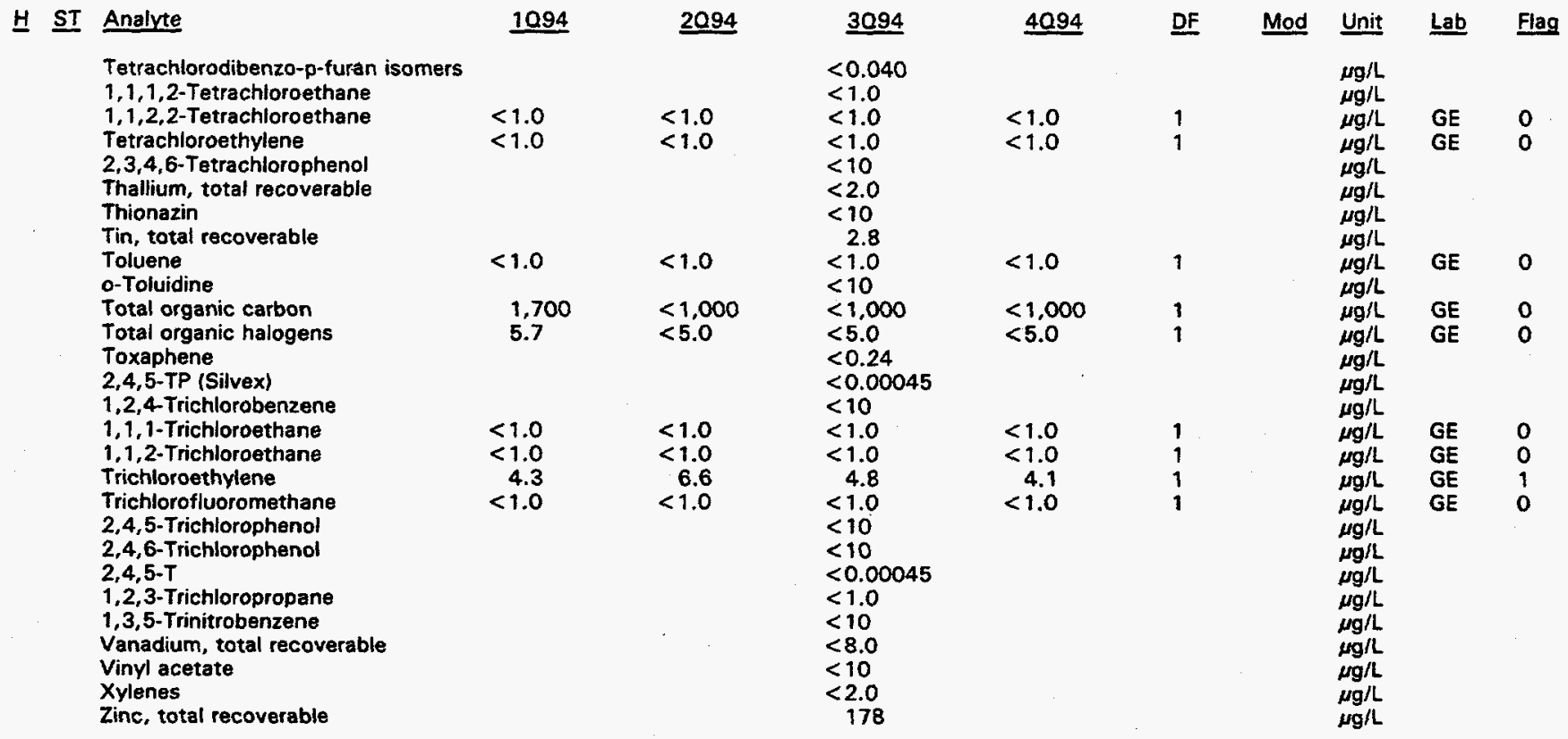

\section{WELL AMB 7A}

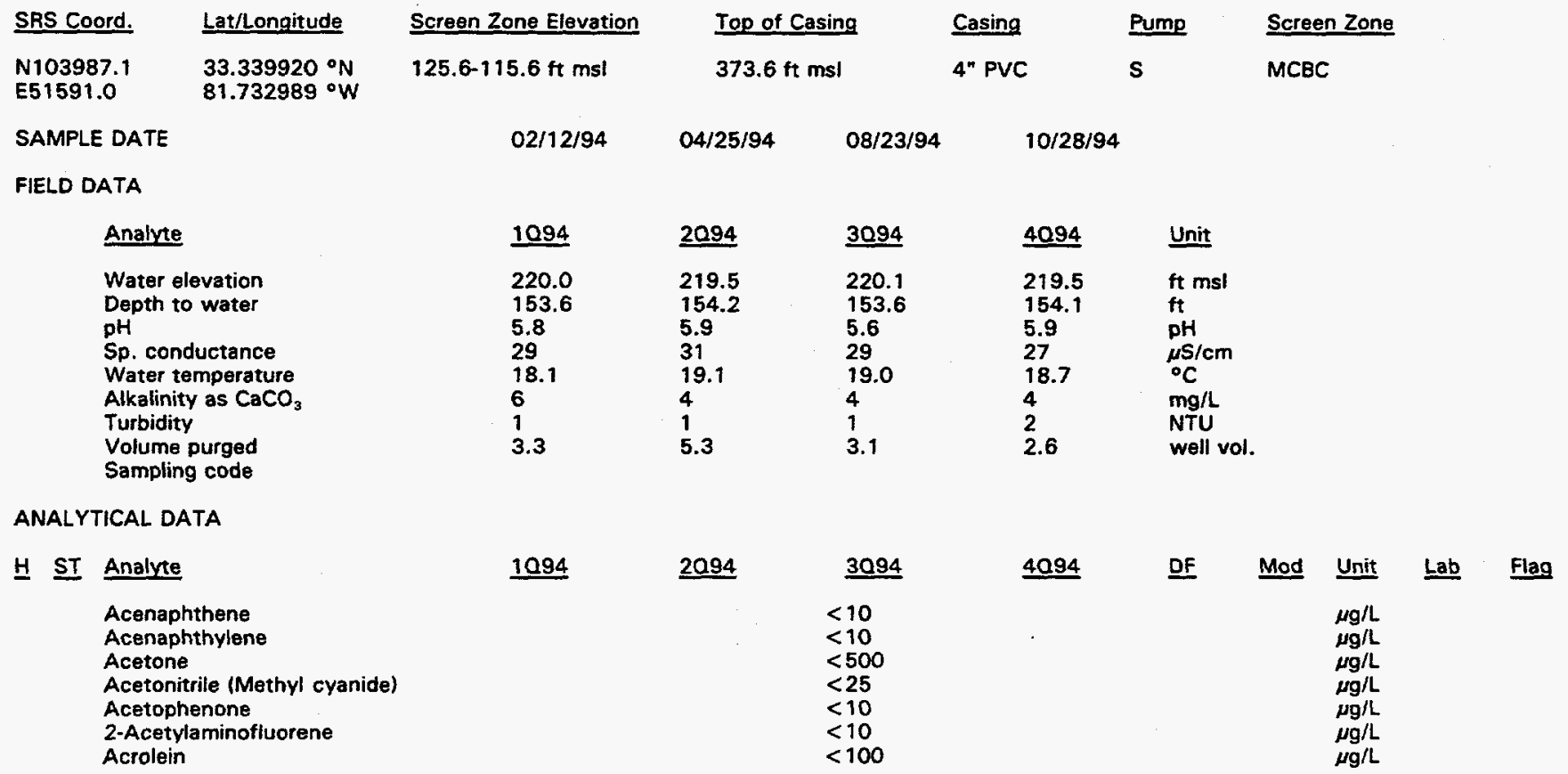

Note: Flagging, dilution factors, modifiers, and laboratories are for fourth quarter 1994 data only. See Appendix B for flagging criteria.

- = exceeded holding time for fourth quarter 1994.

- =xceeded screening level or final PDWS for fourth quarter 1994. 


\section{ANALYTICAL DATA}

\section{H ST Analvte}

Acrylonitrile

Aldrin

Allyl chloride

Aluminum, total recoverable

4-Aminobiphenyl

Aniline

Anthracene

Antimony, total recoverable

Aramite

Arsenic, total recoverable

Barium, total recoverable

Benzene

alpha-Benzene hexachloride

beta-Benzene hexachloride

delta-Benzene hexachloride

Benzo[a]anthracene

Benzolb]fluoranthene

Benzolk]fluoranthene

Benzolg, $h, i j p e r y l e n e$

Benzolalpyrene

Benzyl alcohol

Beryllium, total recoverable

Bis(2-chloroethoxy) methane

Bis(2-chloroethyl) ether

Bis(2-chloroisopropyl) ether

Bis(2-ethylhexyl) phthalate

Bromodichloromethane

Bromoform

Bromomethane (Methyl bromide)

4-Bromophenyl phenyl ether

Butylbenzyl phthalate

2-sec-Butyl-4,6-dinitrophenol

Cadmium, total recoverable

Carbon disulfide

Carbon tetrachloride

Chlordane

Chioride

4-Chloroaniline

Chlorobenzene

Chiorobenzilate

4-Chloro-m-cresol

Chloroethane

Chloroethene (Vinyl chloride)

2-Chloroethyl vinyl ether

Chloroform

Chloromethane (Methyl chloride)

2-Chloronaphthalene

2-Chlorophenol

4-Chlorophenyl phenyl ether

Chloroprene

Chromium, total recoverable

Chrysene

Cobalt, total recoverable

Copper, total recoverable

o-Cresol (2-Methylphenol)

m,p-Cresol

Cyanide

$p, p^{\prime}-D D D$

$p, p^{\prime}-D D E$

p, $p^{\prime}$-DDT

Diallate

Dibenz $[a, h]$ anthracene

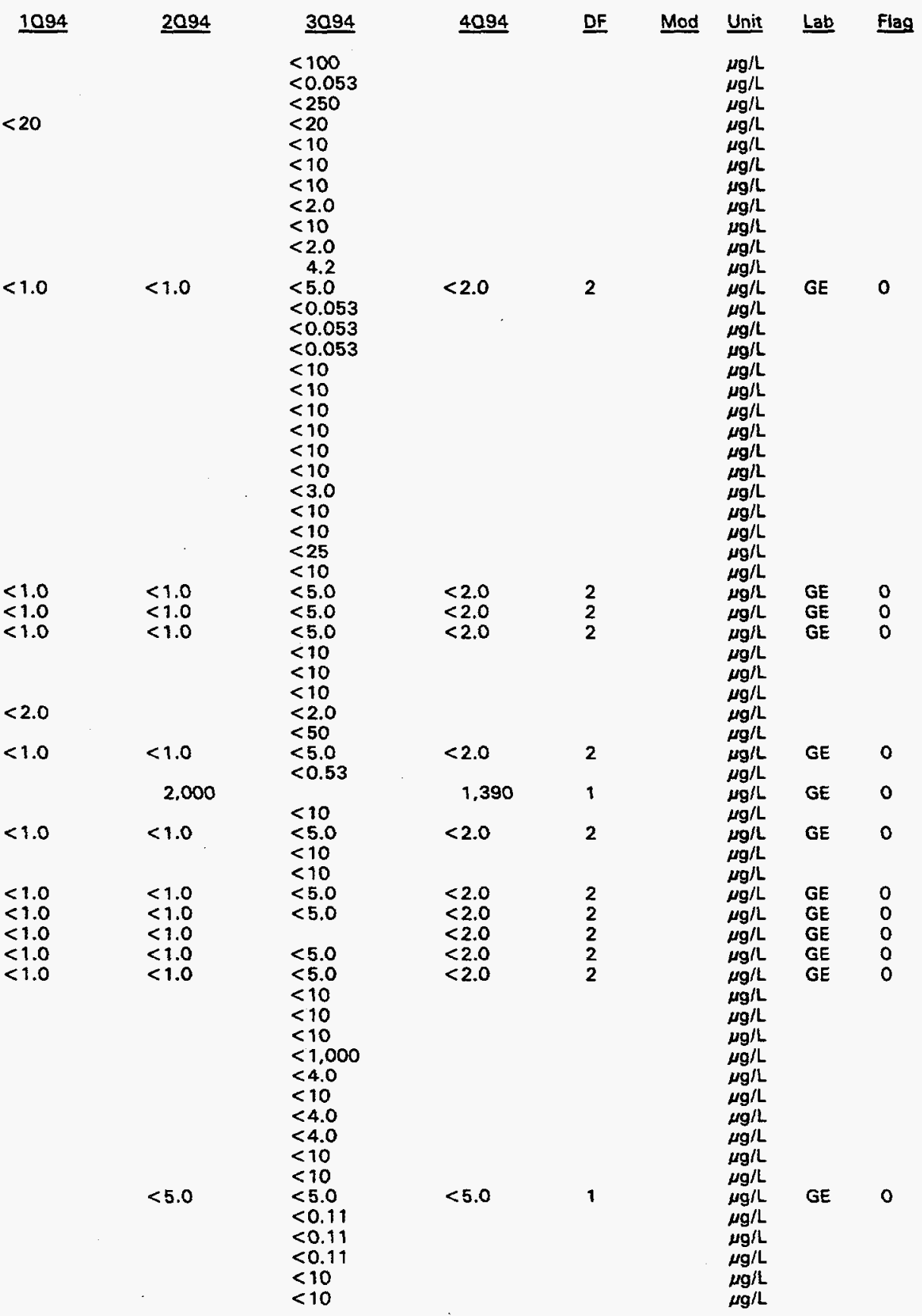

Note: Flagging, dilution factors, modifiers, and laboratories are for fourth quarter 1994 data only. See Appendix B for flagging criteria. - = exceeded holding time for fourth quarter 1994.

- = exceeded screening level or final PDWS for fourth quarter 1994. 
Well AMB 7A continued

ANALYTICAL DATA

H ST Analyte

Dibenzofuran

Dibromochloromethane

1,2-Dibromo-3-chloropropane

1,2-Dibromoethane

Dibromomethane

Di-n-butyl phthalate

1,2-Dichlorobenzene

1,3-Dichlorobenzene

1,4-Dichlorobenzene

3,3'-Dichlorobenzidine

trans-1,4-Dichloro-2-butene

Dichlorodifluoromethane

1,1-Dichloroethane

1,2-Dichloroethane

1,1-Dichloroethylene

trans-1,2-Dichloroethylene

Dichloromethane

2,4-Dichloropheno

2,6-Dichloriohenol

2,4-Dichlorophenoxyacetic acid

1,2-Dichloropropane

cis-1,3-Dichloropropene

trans-1,3-Dichloropropene

Dieldrin

Diethyl phthalate

Dimethoate

2,4-Dimethyl phenol

Dimethyl phthalate

p-Dimethyiaminoazobenzene

7.12-Dimethylbenzla]anthracene

3,3'-Dimethylbenzidine

a,a-Dimethylphenethylamine

1,3-Dinitrobenzene

2,4-Dinitrophenol

2,4-Dinitrotoluene

2,6-Dinitrotoluene

Di-n-octyl phthalate

1,4-Dioxane

Diphenylamine

Disulfoton

Endosulfan 1

Endosulfan II

Endosulfan sulfate

Endrin

Endrin aldehyde

Ethyl methacrylate

Ethyl methanesulfonate

Ethylbenzene

Famphur

Fluoranthene

Fluorene

Fluoride

Gross alpha

Heptachlor

Heptachlor epoxide

$1,2,3,4,6,7,8$-HPCDD

Heptachlorodibenzo-p-dioxin isomers

$1,2,3,4,6,7,8$-HPCDF

Heptachlorodibenzo-p-furan isomers

Hexachlorobenzene

Hexachlorobutadiene

Hexachlorocyciopentadiene

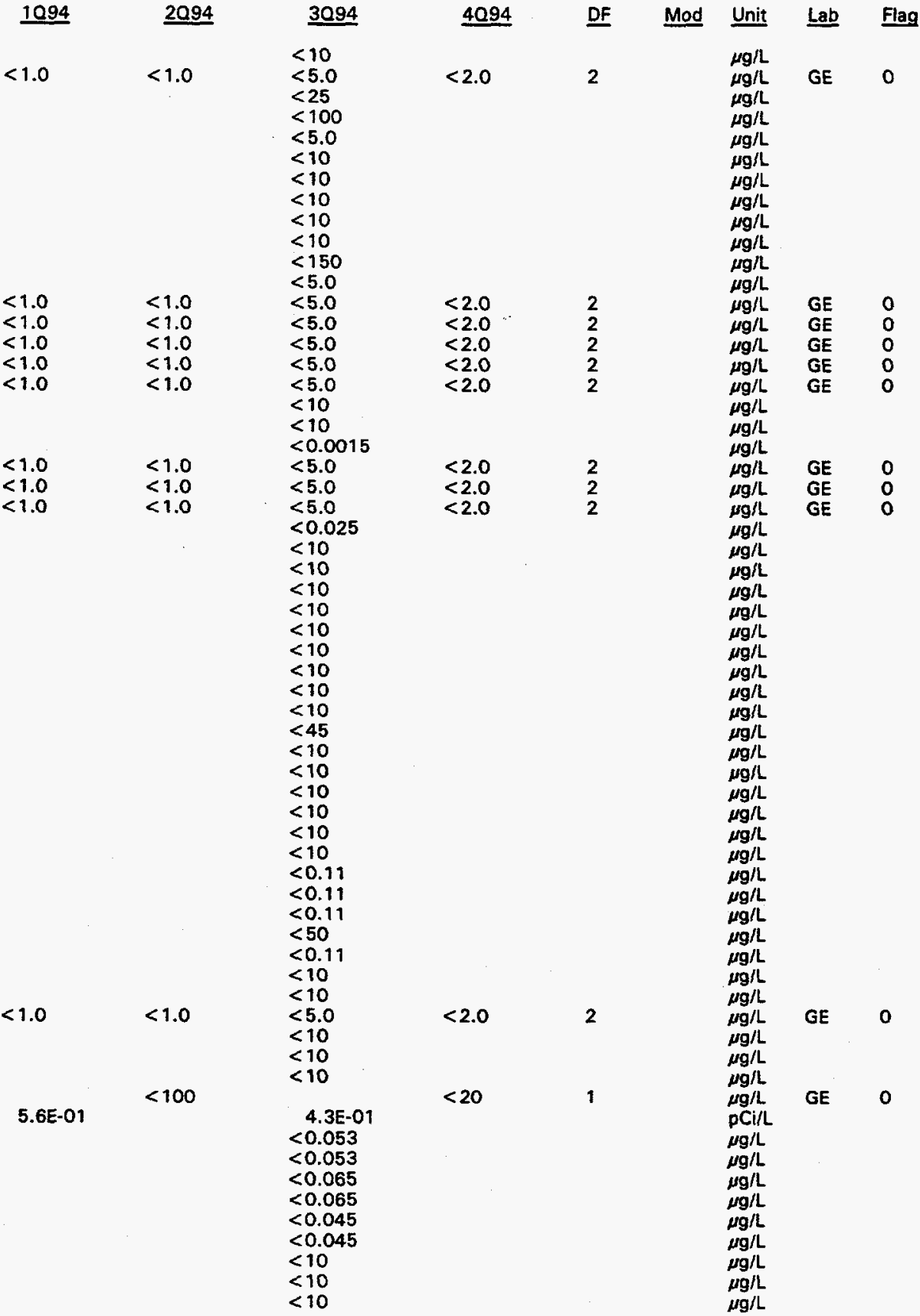

Note: Flagging, dilution factors, modifiers, and laboratories are for fourth quarter 1994 data only. See Appendix B for flagging criteria.

- exceeded holding time for fourth quarter 1994.

- = exceeded screening level or final PDWS for fourth quarter 1994. 


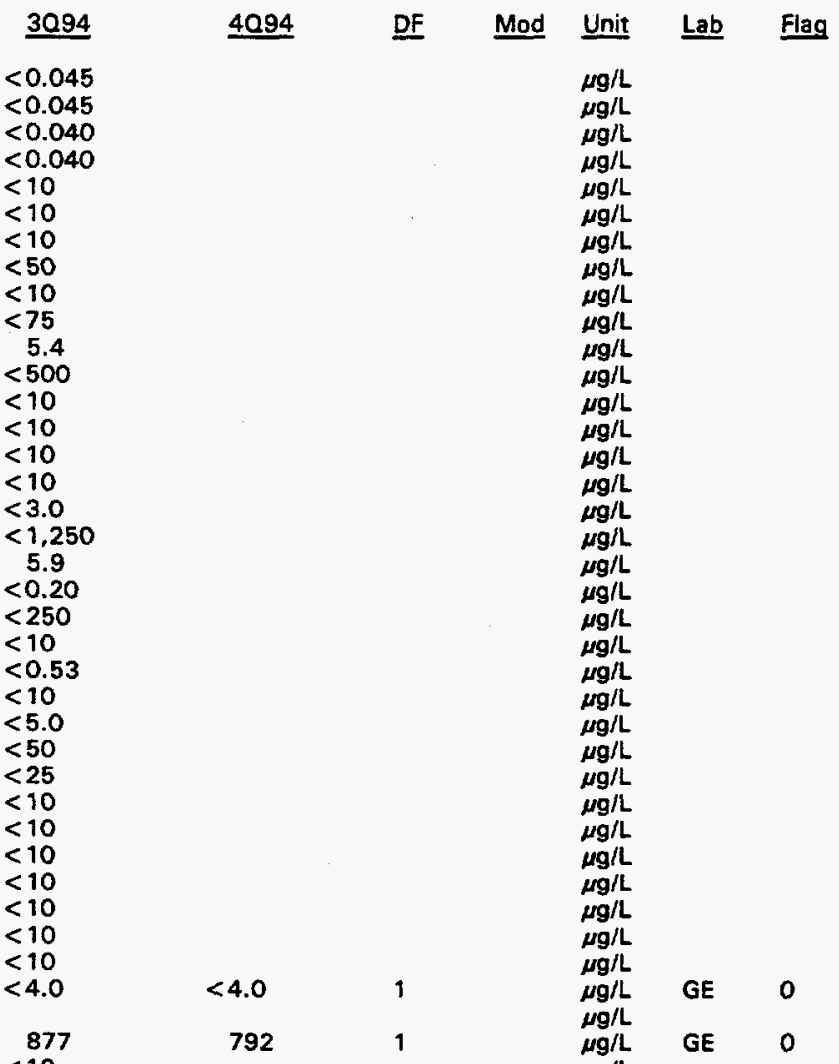

$1,2,3,4,7,8-\mathrm{HXCDD}$

Hexachlorodibenzo-p-dioxin isomers $1,2,3,4,7,8-H \times C D F$

Hexachlorodibenzo-p-furan isomers

Hexachloroethane

Hexachlorophene

Hexachloropropene

2-Hexanone

indenol $1,2,3-c, 0]$ pyrene

lodomethane (Methyl iodide)

Iron, total recoverable

Isobutyl alcohol

Isodrin

Isophorone

Isosafrole

Kepone

Lead, total recoverable

Lindane

Manganese, total recoverable

Mercury, total recoverable

Methacrylonitrile

Methapyrilene

Methoxychlor

2-Methyl-4,6-dinitrophenol

Methyl ethyl ketone

Methyl isobutyl ketone

Methyl methacrylate

Methyl methanesulfonate

3-Methylcholanthrene

2-Methylnaphthalene

Naphthalene

1.4-Naphthoquinone

1-Naphthylamine

2-Naphthylamine

Nickel, total recoverable

Nitrate as nitrogen

Nitrate-nitrite as nitrogen

o-Nitroaniline

m-Nitroaniline

p-Nitroaniline

Nitrobenzene

2-Nitropheno

4-Nitrophenol

4-Nitroquinoline-1-oxide

$\mathrm{N}$-Nitrosodi-n-butylamine

N-Nitrosodiethylamine

$\mathrm{N}$-Nitrosodimethylamine

N-Nitrosodiphenylamine

N-Nitrosodipropylamine

$\mathrm{N}$-Nitrosomethylethylamine

N-Nitrosomorpholine

$\mathrm{N}$-Nitrosopiperidine

$\mathrm{N}$-Nitrosopyrrolidine

5-Nitro-O-toluidine

Nonvolatile beta

0,0.0-Triethyl phosphorothioate

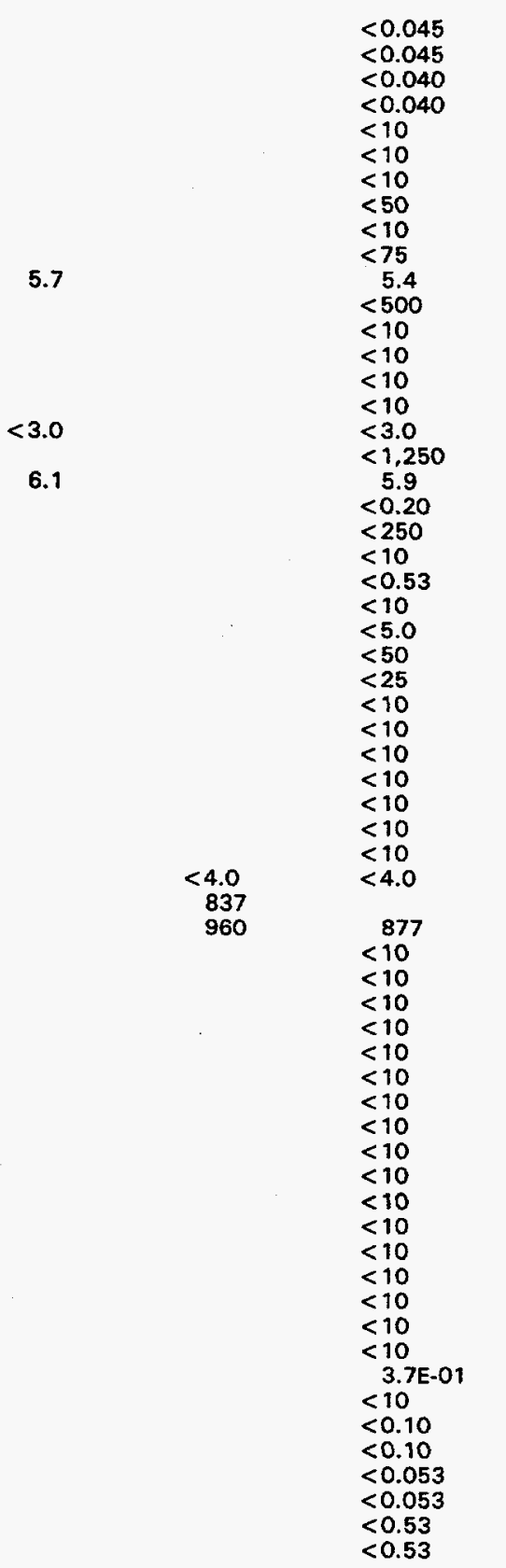

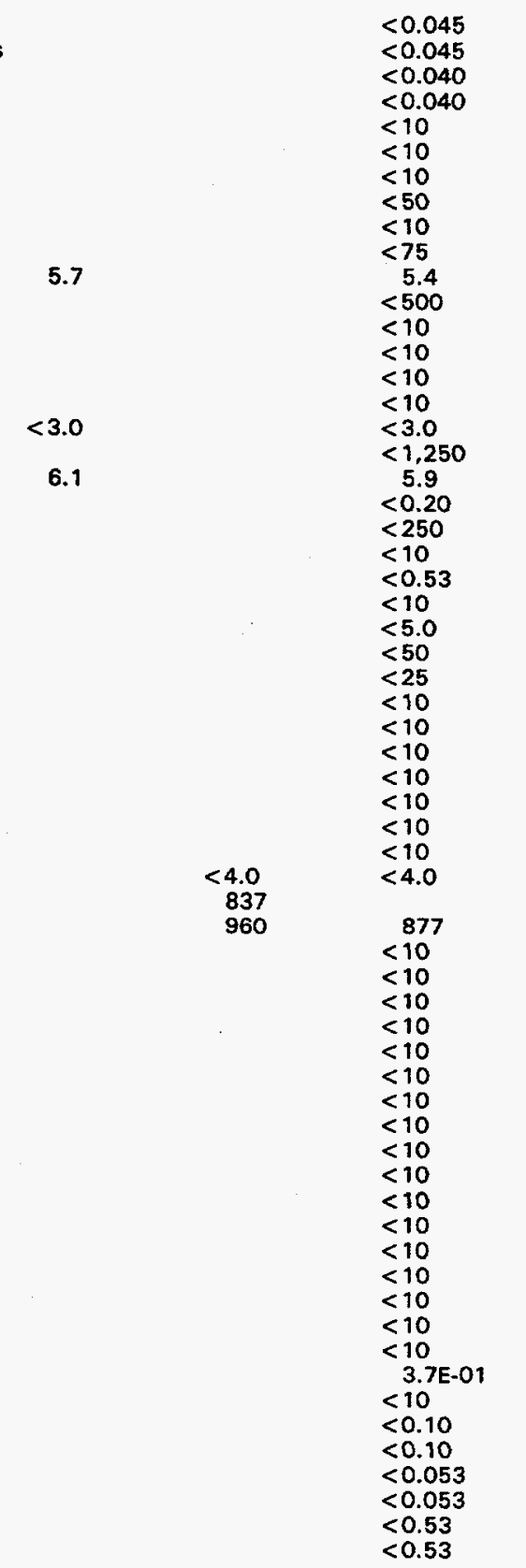

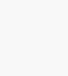

6.1

Octachlorodibenzo-p-dioxin

Octachlorodibenzo-p-furan

Parathion

Parathion methyl

PCB 1016

PCB 1221 
Well AMB 7A continued

\section{ANALYTICAL DATA}

H ST Analyte

PCB 1232

PCB 1242

PCB 1248

PCB 1254

PCB 1260

Pentachlorobenzene

1,2,3,7,8-PCDD

Pentachlorodibenzo-p-dioxin isomers

$1,2,3,7,8$-PCDF

Pentachloroethane

Pentachloronitrobenzene

Pentachlorophenol

Phenacetin

Phenanthrene

Phenol

p-Phenylenediamine

Phorate

2-Picoline

Pronamid

Propionitrile

Pyrene

Pyridine

Radium, total alpha-emitting

Radium-226

Radium-228

Safrole

Selenium, total recoverable

Silver, total recoverable

Styrene

Sulfide

Sulfotepp

1,2,4,5-Tetrachlorobenzene

\section{$2,3,7,8-T C D D$}

$2,3,7,8-T C D F$

Tetrachlorodibenzo-p-dioxin isomers Tetrachlorodibenzo-p-furan isomers

1,1,1,2-Tetrachloroethane

1,1,2,2-Tetrachloroethane

- Tetrachloroethylene

2,3,4,6-Tetrachlorophenol

Thallium, total recoverable

Thionazin

Tin, total recoverable

Toluene

o-Toluidine

Total organic carbon

Total organic halogens

Toxaphene

2,4,5-TP (Silvex)

1,2,4-Trichlorabenzene

1,1,1-Trichloroethane

1,1,2-Trichloroethane

- Trichloroethylene

Trichlorofluoromethane

2,4,5-Trichlorophenol

$2,4,6$-Trichlorophenol

2,4,5-T

1,2,3-Trichloropropane

1,3,5-Trinitrobenzene

Vanadium, total recoverable

Vinyl acetate
1094

$\underline{2094}$

$<0.53$

$<0.53$

$<0.53$

$<0.53$

$<0.53$

$<10$

$<0.055$

$<0.055$

$<0.055$

$<10$

$<10$

$<10$

$<10$

$<10$

$<10$

$<10$

$<0.11$

$<10$

$<10$

$<1,000$

$<10$
$<10$

$1.2 E+\infty<<0.0 E+\infty$

$1.9 \mathrm{E}-01$

$<2.0 \mathrm{E}-01$

$1.2 E+\infty 0$

$<10$

$<2.0$

$<2.0$

$<50$

$<1,000$

$<10$

$<0.045$

$<0.045$

$<0.045$

$<0.040$

$<5.0$

$<5.0$
17

$<1.0$
15

$<1.0$
12

$<1.0$

$<1.0$

$<1,000$

91

$<10$

$<2.0$

$<10$

$<2.0$

$<5.0$

$<10$

$<1,000$

114
$<0.25$

$<0.00045$

$<10$

$<5.0$

$<5.0$

331

$<5.0$

$<10$

$<10$

$<0.00045$

$<5.0$

$<10$

$<8.0$

$<50$
2.OE-O

4094 DF $\underline{\text { Mod Unit }} \underline{\text { Lab }}$ Flag

$<2.0$

$\mu g / h$

$\mu g h$

$\mu g / L$

$\mu \mathrm{g} / \mathrm{L}$

$\mu g / 1$

$\mu g / L$

$\mu g / L$

$\mu g / L$

$\mu g / L$

$\mu \mathrm{\mu g} / \mathrm{L}$

$\mu g / L$

$\mu g / L$

$\mu \mathrm{g} / \mathrm{L}$

$\mu \mathrm{g} / \mathrm{L}$

$\mu g / L$

$\mu g /$

$\mu g / L$

$\mu g / L$

$\mu \mathrm{g} / \mathrm{L}$

pCi/L

pCin

$\mu \mathrm{g} / \mathrm{L}$

ugll

$\mu \mathrm{g} / \mathrm{L}$

$\mu g / L$

$\mu g / L$

$\mu g / L$

$\mu g / L$

$\mu g / L$

$\mu g / L$

glL

$\mu \mathrm{g} / \mathrm{L}$

$\mu g / h$

$\mu g / L$

$\mu g / L$

$\mu g / L$

$\mu g / L$
$\mu g / L$
$\mu g / L$

$\mu g / L$

$\mu g / \mathrm{L}$

$\mu g / L$

$\mu g / L$

$\mu \mathrm{g} / \mathrm{L}$

$\mu \mathrm{g} / \mathrm{L}$

u

$\mu \mathrm{g} / \mathrm{L}$

$\mu \mathrm{g} / \mathrm{L}$

$\mu g / L$

$\mu g / L$

$\mu \mathrm{g} / \mathrm{L}$

$\mu g / L$
GP $\quad 0$

GE 0

$\begin{array}{ll}\text { GE } & 0 \\ \text { GE } & 2\end{array}$

GE $\quad 0$

$\begin{array}{ll}\mathrm{GE} & 0 \\ \mathrm{GE} & 2\end{array}$

$\mathrm{GE} \quad 2$

Note: Flagging, dilution factors, modifiers, and laboratories are for fourth quarter 1994 data only. See Appendix B for flagging criteria.

- = exceeded holding time for fourth quarter 1994.

- = exceeded screening level or final PDWS for fourth quarter 1994. 
WSRC-TR-94-0615

Unclassified

Well AMB 7A continued

ANALYTICAL DATA

H ST Analyte

Xylenes

Zinc, total recoverable
1094

$2094 \quad \begin{array}{r}3094 \\ <10 \\ 21\end{array}$ $\underline{4094}$

DF

Mod

$\mu \mathrm{g} /$

$\mu \mathrm{g} / \mathrm{L}$

Lab

Flag

\section{WELL AMB 7B}

\begin{tabular}{|c|c|}
\hline SRS Coord. & Lat/Longitude \\
\hline $\begin{array}{l}\text { N103972.0 } \\
\text { E51590.3 }\end{array}$ & $\begin{array}{l}33.339885^{\circ} \mathrm{N} \\
81.732961^{\circ} \mathrm{W}\end{array}$ \\
\hline
\end{tabular}

SAMPLE DATE

$\frac{\text { Screen Zone Elevation }}{162.9-152.9 \mathrm{ft} \text { msl }}$

\section{Top of Casing}

$373 \mathrm{ft} \mathrm{msl}$

Casing

4" PVC

Pump

s

Screen Zone

$02 / 12 / 94$

$05 / 02 / 94$

$08 / 23 / 94$

$10 / 28 / 94$

FIELD DATA

Analyte

Water elevation

Depth to water

$\mathrm{pH}$

Sp. conductance

Water temperature

Alkalinity as $\mathrm{CaCO}_{3}$

Turbidity

Volume purged

Sampling code

ANALYTICAL DATA

H ST Analyte

Acenaphthene

Acenaphthylene

Acetone

Acetonitrile (Methyl cyanide)

Acetophenone

2-Acetylaminofluorene

Acrolein

Acrylonitrile

Aldrin

Allyl chloride

Aluminum, total recoverable

4-Aminobiphenyl

Aniline

Anthracene

Antimony, total recoverable

Aramite

Arsenic, total recoverable

Barium, total recoverable

Benzene

alpha-Benzene hexachloride

beta-Benzene hexachloride

delta-Benzene hexachloride

Benzolalanthracene

Benzolb]fluoranthene

Benzolk]fluoranthene

Benzo[g, $h$, Aperylene

Benzola]pyrene

Benzyl alcohol

Beryllium, total recoverable

Bis (2-chloroethoxy) methane

Bis(2-chloroethyl) ether

Bis (2-chloroisopropyl) ether

Bis \{2-ethylhexyl) phthalate

Bromodichloromethane

1094
225.5
147.6
5.1
27
18.0
1
0
3.7

2094
225.5
147.6
5.2
29
18.4
1
1
4.0

3094
225.7
147.3
5.0
28
19.0
1
1
3.0

1094

2094

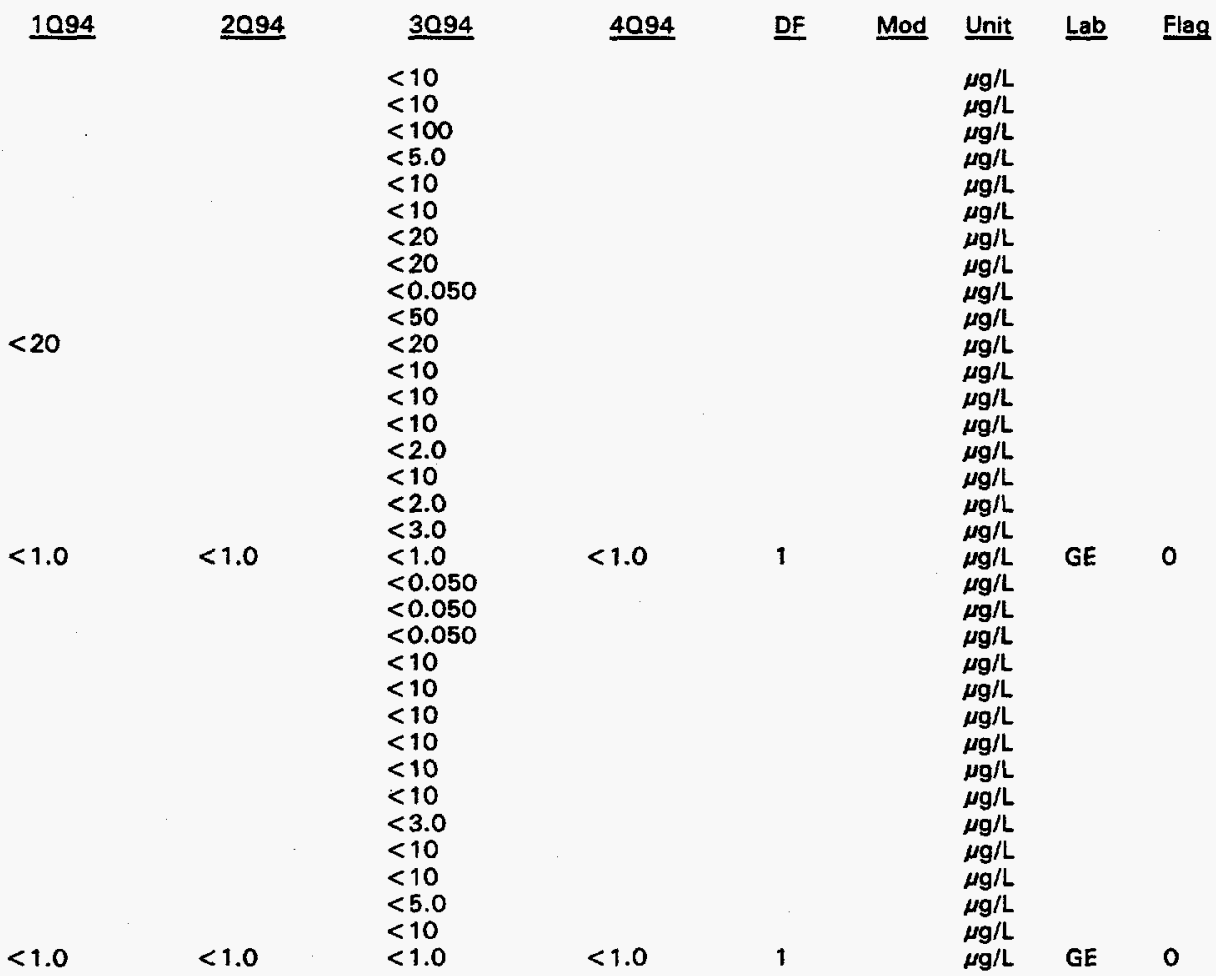

4094
225.2
147.8
5.1
26
18.6
1
1
3.2

Unit

ft msl

$\mathrm{ft}$

pH

$\mu \mathrm{S} / \mathrm{cm}$

$\mathrm{mg} / \mathrm{L}$

NTU

well vol.
LL

Note: Flagging, dilution factors, modifiers, and laboratories are for fourth quarter 1994 data only. See Appendix B for flagging criteria. - = exceeded holding time for fourth quarter 1994.

- = exceeded screening level or final PDWS for fourth quarter 1994. 
H ST Analvte

Bromoform

Bromomethane (Methyl bromide)

4-Bromophenyl phenyl ether

Butylbenzyl phthalate

2-sec-Butyl-4, 6-dinitrophenol

Cadmium, total recoverable

Carbon disulfide

Carbon tetrachloride

Chlordane

Chloride

4-Chioroaniline

Chlorobenzene

Chlorobenzilate

4-Chloro-m-cresol

Chloroethane

Chloroethene (Vinyl chloride)

2-Chloroethyl vinyl ether

Chloroform

Chloromethane (Methyl chloride)

2-Chloronaphthalene

2-Chiorophenol

4-Chlorophenyl phenyl ether

Chloroprene

Chromium, total recoverable

Chrysene

Cobalt, total recoverable

Copper, total recoverable

o-Cresol (2-Methylphenol)

m,p-Cresol

Cyanide

P.P'-DDD

p.p'-DDE

p.p'-DDT

Diallate

Dibenz $[a, h]$ anthracene

Dibenzofuran

Dibromochloromethane

1.2-Dibromo-3-chloropropane

1,2-Dibromoethane

Dibromomethane

Di-n-butyl phthalate

1,2-Dichlorobenzene

1,3-Dichlorobenzene

1,4-Dichlorobenzene

3,3'-Dichlorobenzidine

trans-1,4-Dichloro-2-butene

Dichlorodifluoromethan $\theta$

1.1-Dichloroethane

1,2-Dichloroethane

1,1-Dichloroethylene

trans-1,2-Dichloroethylene

Dichloromethane

2,4-Dichlorophenol

2,6-Dichlorophenol

2,4-Dichlorophenoxyacetic acid

1,2-Dichloropropane

cis-1,3-Dichloropropene

trans-1,3-Dichloropropene

Dieldrin

Diethyl phthalate

Dimethoate

2,4-Dimethyl phenol

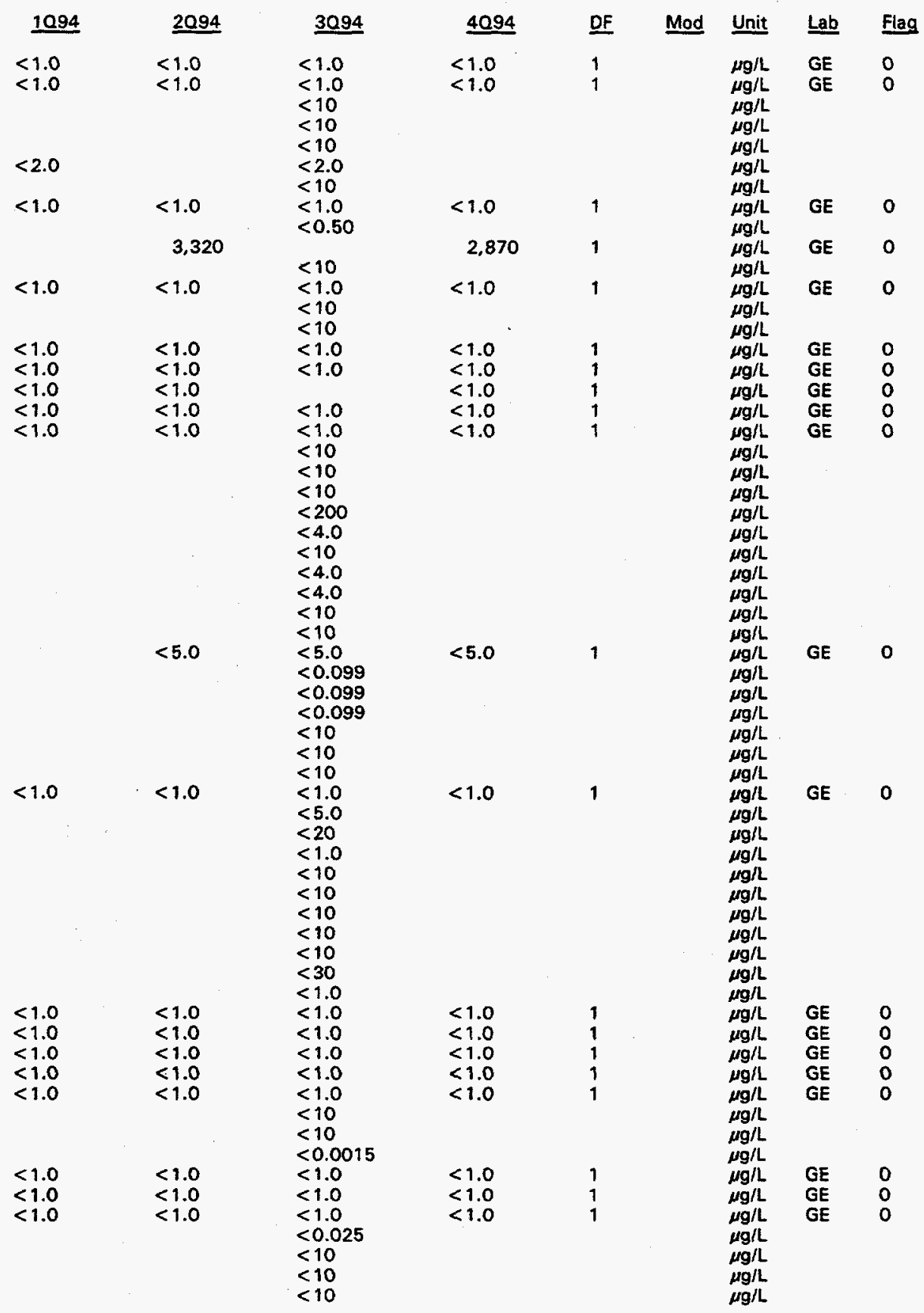

Note: Flagging, dilution factors, modifiers, and laboratories are for fourth quarter 1994 data only. See Appendix B for flagging criteria. - = exceeded holding time for fourth quarter 1994.

- = exceeded screening level or final PDWS for fourth quarter 1994. 
ANALYTICAL DATA

Dimethyl phthalate

p-Dimethylaminoazobenzene

7,12-Dimethylbenz[a]anthracene

3,3'-Dimethylbenzidine

a,a-Dimethylphenethylamine

1,3-Dinitrobenzene

2,4-Dinitrophenol

2,4-Dinitrotoluene

2,6-Dinitrotoluene

Di-n-octyl phthalate

1,4-Dioxane

Diphenylamine

Disulfoton

Endosulfan I

Endosulfan II

Endosulfan sulfate

Endrin

Endrin aldehyde

Ethyl methacrylate

Ethyl methanesulfonate

Ethylbenzene

$<1.0$

Famphur

Fluoranthene

Fluorene

Gross alpha

$1.1 \mathrm{E}-01$
$<0.050$

Heptachlor

Heptachlor epoxide

1,2,3,4,6,7,8-HPCDD

Heptachlorodibenzo-p-dioxin isomers

$1,2,3,4,6,7,8-\mathrm{HPCDF}$

Heptachlorodibenzo-p-furan isomers

Hexachlorobenzene

Hexachlorobutadiene

Hexachlorocyclopentadiene

$1,2,3,4,7,8-H \times C D D$

Hexachlorodibenzo-p-dioxin isomers

$1,2,3,4,7,8-H X C D F$

Hexachlorodibenzo-p-furan isomers

Hexachloroethane

Hexachlorophene

Hexachloropropene

2-Hexanone

Indenol 1,2,3-c, d)pyrene

lodomethane (Methyl iodide)

Iron, total recoverable

Isobutyl alcohol

Isodrin

Isophorone

Isosafrole

Kepone

Lead, total recoverable

Lindane

Manganese, total recoverable

Mercury, total recoverable

Methacrylonitrile

Methapyrilene

Methoxychlor

2-Methyl-4,6-dinitrophenol

Methyl ethyl ketone

Methyl isobutyl ketone

Methyl methacrylate

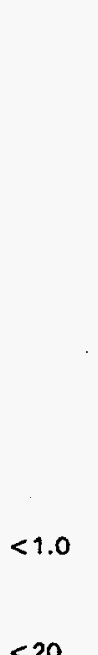

$\mu g /$

$\mu g / L$

$\mu \mathrm{g} / \mathrm{L}$

$\mu \mathrm{g} / \mathrm{L}$

$\mu \mathrm{g} / \mathrm{L}$

$\mu \mathrm{g} / \mathrm{L}$

$\mu \mathrm{g} / \mathrm{L}$

$\mu g / L$

$\mu g / L$

$\mu g / L$

$\mu g / L$

$\mu g / L$

$\mu g / L$

$\mu g / L$

$\mu g / L$

$\mu \mathrm{g} / \mathrm{h}$

pg

$\mu g / L$

GE $\quad 0$

$<20$

$\mu \mathrm{g} / \mathrm{L}$

$\mu g / L$

$<0.050$

$<0.065$

$<0.065$

$<0.045$

$<0.045$

$<10$

$<10$

$<10$

$<0.045$

$<0.045$

$<0.040$

$<0.040$

$<10$

$<10$

$<10$

$<10$

$<10$

$<15$

5.6

$<100$

$<10$

$<10$

$<10$

$<10$

$<3.0$

$<1.250$

$<2.0$

$<0.20$

$<50$

$<10$

$<0.50$

$<10$

$<1.0$

$<10$

$<5.0$

$\mathrm{pCi} / \mathrm{L}$

$\mu g / L$

$\mu g / L$

$\mu g / L$

$\mu g / L$

$\mu g / L$

$\mu \mathrm{g} / \mathrm{L}$

$\mu g / L$

$\mu \mathrm{g} / L$

$\mu g / L$

$\mu g / L$

$\mu g / L$

$\mu g / L$

$\mu g / L$

ug

$\mu g / L$

$\mu g / L$

$\mu g / L$

$\mu \mathrm{g} / \mathrm{L}$

$\mu g /$

$\mu g / L$

$\mu g / L$

$\mu g / L$

$\mu g / L$

$\mu g /$

$\mu g / L$

ag/L

$\mu g / L$

$\mu \mathrm{g} / \mathrm{L}$

$\mu \mathrm{g} / \mathrm{L}$

$\mu g / L$

$\mu g / L$

$\mu g / L$

GE 0

Note: Flagging, dilution factors, modifiers, and laboratories are for fourth quarter 1994 data only. See Appendix B for flagging criteria. - = exceeded holding time for fourth quarter 1994.

- = exceeded screening level or final PDWS for fourth quarter 1994. 


\section{H ST Analyte}

Methyl methanesulfonate

3-Methylcholanthrene

2-Methylnaphthalene

Naphthalene

1,4-Naphthoquinone

1-Naphthylamine

2-Naphthylamine

Nickel, total recoverable

Nitrate-nitrite as nitrogen

o-Nitroaniline

m-Nitroaniline

p-Nitroaniline

Nitrobenzene

2-Nitropheno

4-Nitrophenol

4-Nitroquinoline-1-oxide

$N$-Nitrosodi-n-butylamine

$\mathrm{N}$-Nitrosodiethylamine

$\mathrm{N}$-Nitrosodimethylamine

$N$-Nitrosodiphenylamine

N-Nitrosodipropylamine

$\mathrm{N}$-Nitrosomethylethylamine

$\mathrm{N}$-Nitrosomorpholine

$\mathrm{N}$-Nitrosopiperidine

N-Nitrosopyrrolidine

5-Nitro-0-toluidine

Nonvolatile beta

$0,0,0$-Triethyl phosphorothioate

Octachlorodibenzo-p-dioxin

Octachlorodibenzo-p-furan

Parathion

Parathion methyl

PCB 1016

PCB 1221

PCB 1232

PCB 1242

PCB 1248

PCB 1254

PCB 1260

Pentachlorobenzene

$1,2,3,7,8-P C D D$

Pentachlorodibenzo-p-dioxin isomers

$1,2,3,7,8-P C D F$

Pentachloroethane

Pentachloronitrobenzene

Pentachlorophenol

Phenacetin

Phenanthrene

Phenol

p-Phenylenediamine

Phorate

2-Picoline

Pronamid

Propionitrile

Pyrene

Pyridine

Radium, total alpha-emitting

Safrole

Selenium, total recoverable

Silver, total recoverable

Styrene

Sulfide

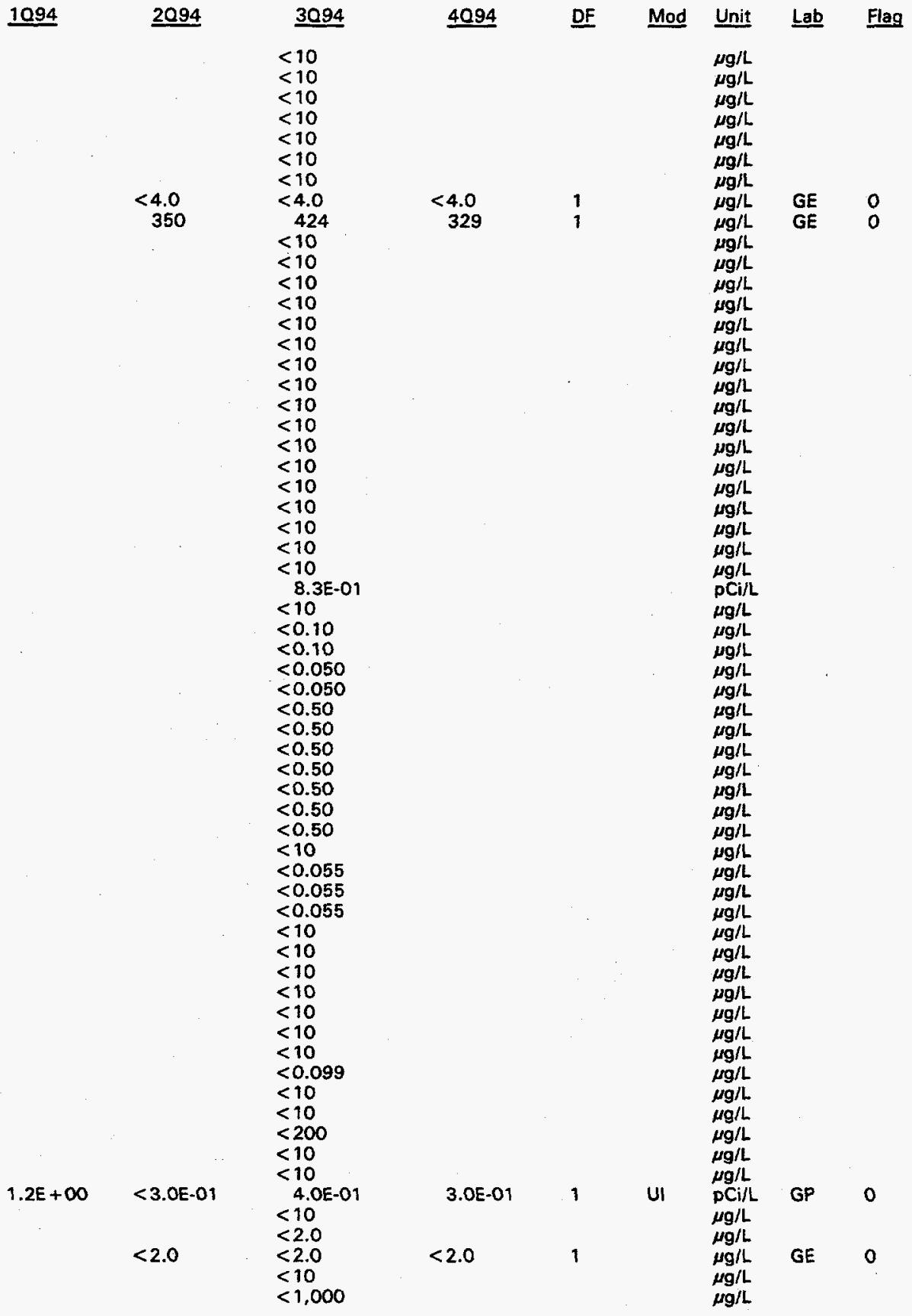

Note: Flagging, dilution factors, modifiers, and laboratories are for fourth quarter 1994 data only. See Appendix B for flagging criteria.

- = exceeded holding time for fourth quarter 1994.

- =xceeded screening level or final PDWS for fourth quarter 1994. 
ANALYTICAL DATA

HE ST Analyte

Sulfotepp

1,2,4,5-Tetrachlorobenzene

2,3,7,8-TCDD

2,3,7,8-TCDF

Tetrachlorodibenzo-p-dioxin isomers

Tetrachlorodibenzo-p-furan isomers

1,1,1,2-Tetrachloroethane

1,1,2,2-Tetrachloroethane

Tetrachloroethylene

2,3,4,6-Tetrachlorophenol

Thallium, total recoverable

Thionazin

Tin, total recoverable

Toluene

o.Toluidine

Total organic carbon

Total organic halogens

Toxaphene

2,4,5-TP (Silvex)

1,2,4-Trichlorobenzene

1,1,1-Trichloroethane

1,1,2-Trichloroethane

Trichloroethylene

Trichlorofluoromethane

2,4,5-Trichlorophenol

2,4,6-Trichlorophenol

2,4,5-T

1,2,3-Trichloropropane

1,3,5-Trinitrobenzene

Vanadium, total recoverable

Vinyl acetate

Xylenes

Zinc, total recoverable
1094

$\underline{2094}$

3094

$\underline{4094}$

DF

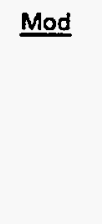

$<2.0$

$<10$

$<1.0<1.0 \quad<2.0$

$<1,000$

$<5.0$

$<10$

$<1,000$

15

$<1,000$

8.6

$<1.0$

$<0.24$

$<0.00046$

$<1.0$

$<10$

$<1.0$

$<1.0$

$<1.0$

$<1.0$

$<1.0$
3.0

$<1.0$

$<1.0$

3.8

$<1.0$

$<10$

$<10$

$<0.00046$

$<1.0$

$<10$

$<8.0$

$<10$

$<10$
$<2.0$

18$$
\begin{aligned}
& \text { Unit } \\
& \mu g / L \\
& \mu g / L \\
& \mu g / L \\
& \mu g / L \\
& \mu g \\
& \mu g / \\
& \mu g / \\
& \mu g / L \\
& \mu g / L \\
& \mu g
\end{aligned}
$$

$\mu g$
$\mu g$
$\mu g$
$\mu g$
$\mu g$
$\mu g$
$\mu g$
$\mu g$
$\mu g$
$\mu g$

Lab Flag

g/L

$\mu \mathrm{g} / \mathrm{L}$

$\mu \mathrm{g} / \mathrm{L}$

$\mu g / L$

$\mu g / L$

$\mu g / L$

$\mu \mathrm{g} / \mathrm{L}$

$\mu g / L$

g/L

$\mu g / L$

$\mu g / L$

$\mu g / L$

$\mu g / L$

$\mu g / L$

$\mu g / L$

$\underset{\mu g / L}{\mu g / L}$

$\mu \mathrm{g} / \mathrm{L}$

$\mu g / L$

$\mu g / L$

$\mu g / L$

$\mu g / L$

$\mu g / L$

$\mu \mathrm{g} / \mathrm{L}$

$\mu \mathrm{g} /$

$\begin{array}{cc}\text { GE } & 0 \\ \text { GE } & 0 \\ & \\ & \\ & \\ \text { GE } & 0 \\ \text { GE } & 0 \\ \text { GE } & 0 \\ & \\ & \\ \text { GE } & 0 \\ \text { GE } & 0 \\ \text { GE } & 1 \\ \text { GE } & 0\end{array}$

Note: Flagging, dilution factors, modifiers, and laboratories are for fourth quarter 1994 data only. See Appendix B for flagging criteria. - = exceeded holding time for fourth quarter 1994.

- exceeded screening level or final PDWS for fourth quarter 1994. 
WELL AMB 8D

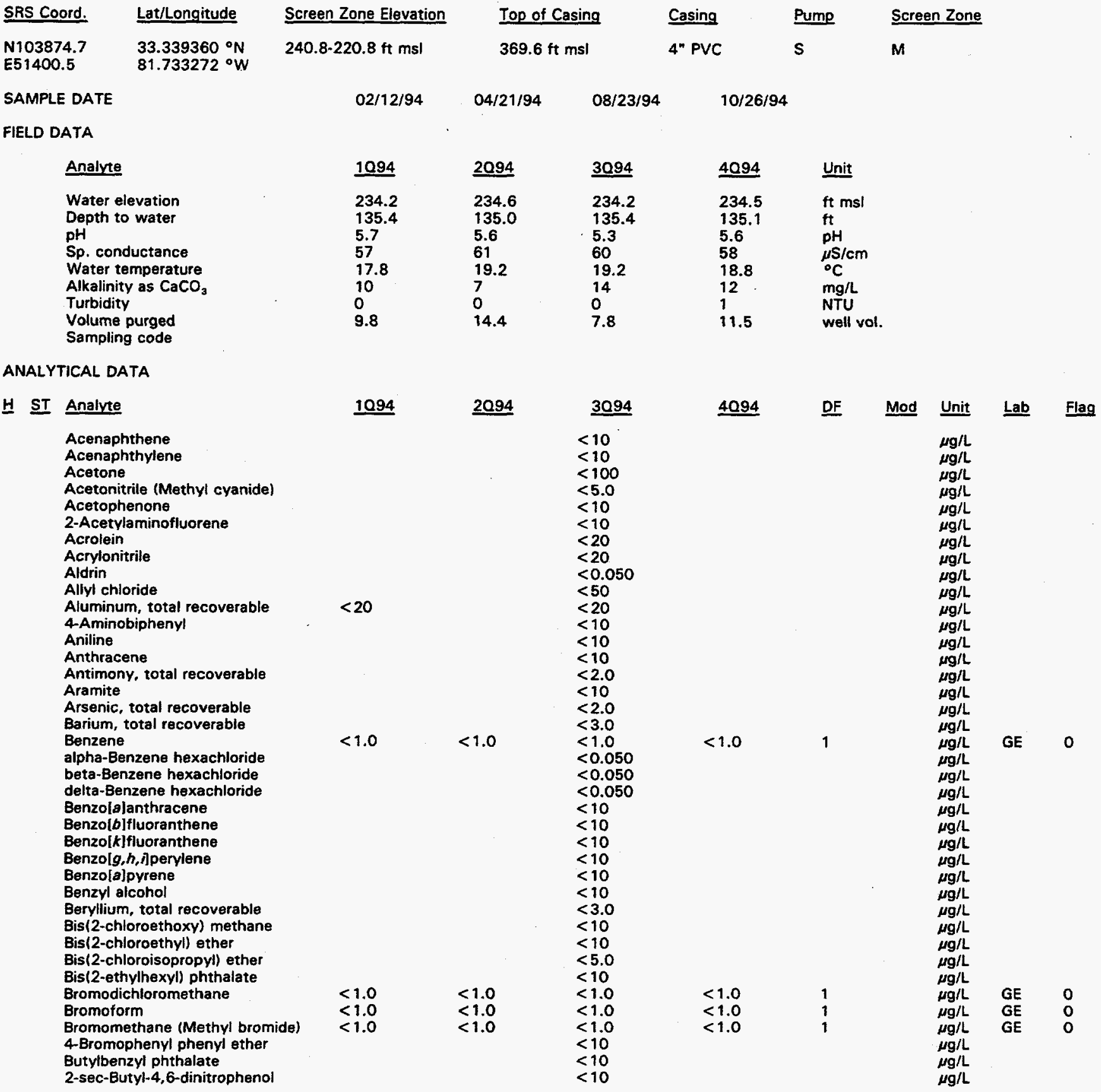

Note: Flagging, dilution factors, modifiers, and laboratories are for fourth quarter 1994 data only. See Appendix B for flagging criteria. - = exceeded holding time for fourth quarter 1994.

- = exceeded screening level or final PDWS for fourth quarter 1994. 


\section{ANALYTICAL DATA}

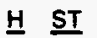

Analyte

Cadmium, total recoverable

Carbon disulfide

Carbon tetrachloride

Chlordane

Chloride

4-Chloroaniline

Chlorobenzene

Chlorobenzilate

4-Chloro-m-cresol

Chloroethane

Chloroethene (Vinyl chloride)

2-Chloroethyl vinyl ether

Chloroform

Chioromethane (Methyl chloride)

2-Chloronaphthalene

2-Chiorophenol

4-Chlorophenyl phenyl ether

Chloroprene

Chromium, total recoverable

Chrysene

Cobalt, total recoverable

Copper, total recoverable

o-Cresol (2-Methylphenol)

m,p-Cresol

Cyanide

$p, p^{\prime}-D D D$

$p, p^{\prime}-D D E$

D.p'-DDT

Diallate

Dibenzla,hlanthracene

Dibenzofuran

Dibromochloromethane

1,2-Dibromo-3-chloropropane

1,2-Dibromoethane

Dibromomethane

Di-n-butyl phthalate

1,2-Dichlorobenzene

1,3-Dichlorobenzene

1,4-Dichlorobenzene

3,3'-Dichlorobenzidine

trans-1,4-Dichloro-2-butene

Dichlorodifluoromethane

1,1-Dichloroethane

1,2-Dichloroethane

1,1-Dichloroethylene

trans-1,2-Dichloroethylene

Dichloromethane

2,4-Dichloropheno

2,6-Dichlorophenol

2,4-Dichlorophenoxyacetic acid

1,2-Dichloropropane

cis-1,3-Dichloropropene

trans-1,3-Dichloropropene

Dieldrin

Diethyl phthalate

Dimethoate

2,4-Dimethyl phenol

Dimethyl phthalate

p-Dimethylaminoazobenzene

7,12-Dimethylbenz[a]anthracene

3,3'-Dimethylbenzidine

a,a-Dimethylphenethylamine

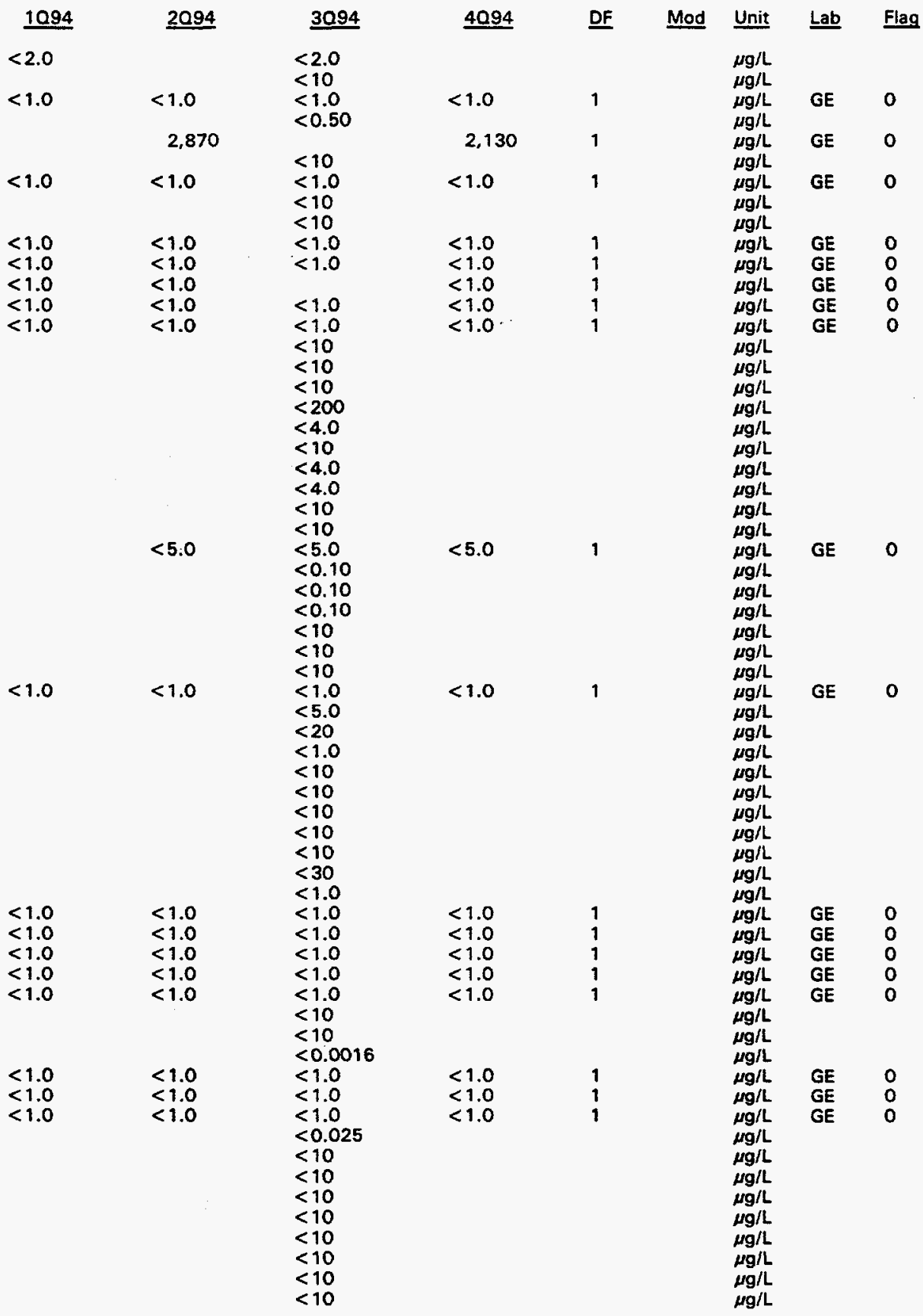

Note: Flagging, dilution factors, modifiers, and laboratories are for fourth quarter 1994 data only. See Appendix B for flagging criteria.

- = exceeded holding time for fourth quarter 1994.

- = exceeded screening level or final PDWS for fourth quarter 1994. 
WSRC-TR-94-0615

Unclassified

Well AMB 8D continued

ANALYTICAL DATA

H ST Analyte

1,3-Dinitrobenzene

2,4-Dinitrophenol

2,4-Dinitrotoluene

2,6-Dinitrotoluene

Di-n-octyl phthalate

1,4-Dioxane

Diphenylamine

Disulfoton

Endosulfan I

Endosulfan II

Endosulfan sulfate

Endrin

Endrin aldehyde

Ethyl methacrylate

Ethyl methanesulfonate

Ethylbenzene

Famphur

Fluoranthene

Fluorene

Fluoride

Gross alpha

Heptachlor

Heptachlor epoxide

$1,2,3,4,6,7,8$-HPCDD

Heptachlorodibenzo-p-dioxin isomers

$1,2,3,4,6,7,8-H P C D F$

Heptachlorodibenzo-p-furan isomers

Hexachlorobenzene

Hexachlorobutadiene

Hexachlorocyclopentadiene

$1,2,3,4,7,8-\mathrm{HXCDD}$

Hexachlorodibenzo-p-dioxin isomers

$1,2,3,4,7,8-\mathrm{HXCDF}$

Hexachlorodibenzo-p-furan isomers

Hexachloroethane

Hexachlorophene

Hexachloropropene

2-Hexanone

Indenol 1,2,3-c, d]pyrene

lodomethane (Methyl iodide)

Iron, total recoverable

Isobutyl alcohol

Isodrin

Isophorone

isosafrole

Kepone

Lead, total recoverable

Lindane

Manganese, total recoverable

Mercury, total recoverable

Methacrylonitrile

Methapyrilene

Methoxychlor

2-Methyl-4,6-dinitrophenol

Methyl ethyl ketone

Methyl isobutyl ketone

Methyl methacrylate

Methyl methanesulfonate

3-Methylcholanthrene

2-Methylnaphthalene

Naphthalene

1,4-Naphthoquinone
1094

$\underline{2094}$

$\begin{aligned} & 3094 \\ & 309 \\ &<<10 \\ &<46 \\ &<10 \\ &<10 \\ &<10 \\ &<10 \\ &<10 \\ &<10 \\ &<10 \\ &<0.10 \\ &<0.10 \\ &<0.10 \\ &<50 \\ &<0.10 \\ &<10 \\ &<10 \\ &<1.0 \\ &<10 \\ &<10 \\ &<10\end{aligned}$

$1.2 E+00$

$<100$

$<10$

$\begin{aligned} & 1.6 E+\infty 0 \\ < & 0.050 \\ < & 0.050 \\ < & 0.065\end{aligned}$

$<1.0$

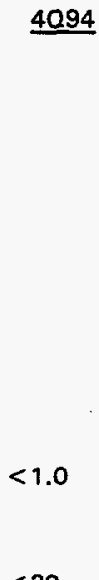

DF

Mod

$<20$

$<0.065$

$<0.045$

$<0.045$

$<10$

$<10$

$<10$

$<0.045$

$<0.045$

$<0.040$

$<0.040$

$<10$

$<10$

$<10$

$<10$

$<10$

$<10$

$<15$

7.4

18

$<100$

$<10$

$<10$

$<10$

$<10$

$<3.0$

$<1,250$

$<2.0$

$<0.20$

$<50$

$<10$

$<0.50$

$<10$

$<1.0$

$<10$

$<5.0$

$<10$
$<10$

$<10$

$<10$

$<10$ $\underline{\text { Lab }}$ Flag

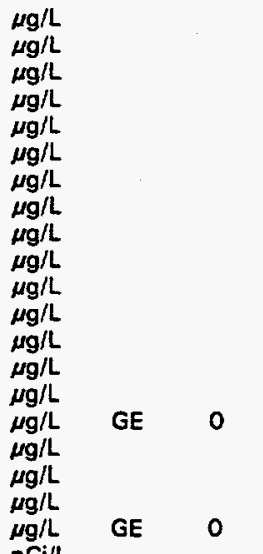

pCill

$\mu \mathrm{g} / \mathrm{L}$

$\mu g / L$

$\mu g / L$

$\mu \mathrm{g} / \mathrm{L}$

$\mu g / L$

$\mu g / L$

$\mu g / L$

$\mu \mathrm{g} / \mathrm{L}$

$\mu g / L$

$\mu \mathrm{g} / \mathrm{L}$

$\mu g / L$

$\mu \mathrm{g} / \mathrm{L}$

$\mu g / L$

$\mu \mathrm{g} / \mathrm{L}$

$\mu g / L$

$\mu \mathrm{g} / \mathrm{L}$

$\mu g / L$

$\mu \mathrm{g} / \mathrm{L}$

$\mu g / L$

$\mu \mathrm{g} / \mathrm{L}$

$\mu g / L$

$\mu g / L$

$\mu g / L$

$\mu \mathrm{g} / \mathrm{L}$

$\mu \mathrm{g} / \mathrm{L}$

$\mu \mathrm{g} / \mathrm{L}$

$\mu g / L$

$\mu \mathrm{g} / \mathrm{L}$

$\mu g / L$

$\mu \mathrm{g} / \mathrm{L}$

$\mu \mathrm{g} / \mathrm{L}$

$\mu g / L$

$\mu g / L$

$\mu \mathrm{g} / \mathrm{L}$

$\mu g / L$

Note: Flagging, dilution factors, modifiers, and laboratories are for fourth quarter 1994 data only. See Appendix B for flagging criteria. - = exceeded holding time for fourth quarter 1994.

- = exceeded screening level or final PDWS for fourth quarter 1994 
ANALYTICAL DATA

H ST Analyte

1-Naphthylamine

2-Naphthylamine

Nickel, total recoverable

Nitrate-nitrite as nitrogen

o-Nitroaniline

m-Nitroaniline

p-Nitroaniline

Nitrobenzene

2-Nitrophenol

4-Nitrophenol

4-Nitroquinoline-1-oxide

N-Nitrosodi-n-butylamine

N-Nitrosodiethylamine

$\mathrm{N}$-Nitrosodimethylamine

N-Nitrosodiphenylamine

N-Nitrosodipropylamine

N-Nitrosomethylethylamine

$\mathrm{N}$-Nitrosomorpholine

N-Nitrosopiperidine

$\mathrm{N}$-Nitrosopyrrolidine

5-Nitro-o-toluidine

Nonvolatile beta

$0,0,0$-Triethyl phosphorothioate

Octachlorodibenzo-p-dioxin

Octachlorodibenzo-p-furan

Parathion

Parathion methyl

PCB 1016

PCB 1221

PCB 1232

PCB 1242

PCB 1248

PCB 1254

PCB 1260

Pentachlorobenzene

1,2,3,7,8-PCDD

Pentachlorodibenzo-p-dioxin isomers

$1,2,3,7,8$-PCDF

Pentachloroethane

Pentachloronitrobenzene

Pentachlorophenol

Phenacetin

Phenanthrene

Phenol

p-Phenylenediamine

Phorate

2-Picoline

Pronamid

Propionitrile

Pyrene

Pyridine

Radium, total alpha-emitting

Safrole

Selenium, total recoverable

Silver, total recoverable

Styrene

Sulfide

Sulfotepp

$1,2,4,5$-Tetrachlorobenzene

2,3,7,8-TCDD

$2,3,7,8-$ TCDF

Tetrachlorodibenzo-p-dioxin isomers

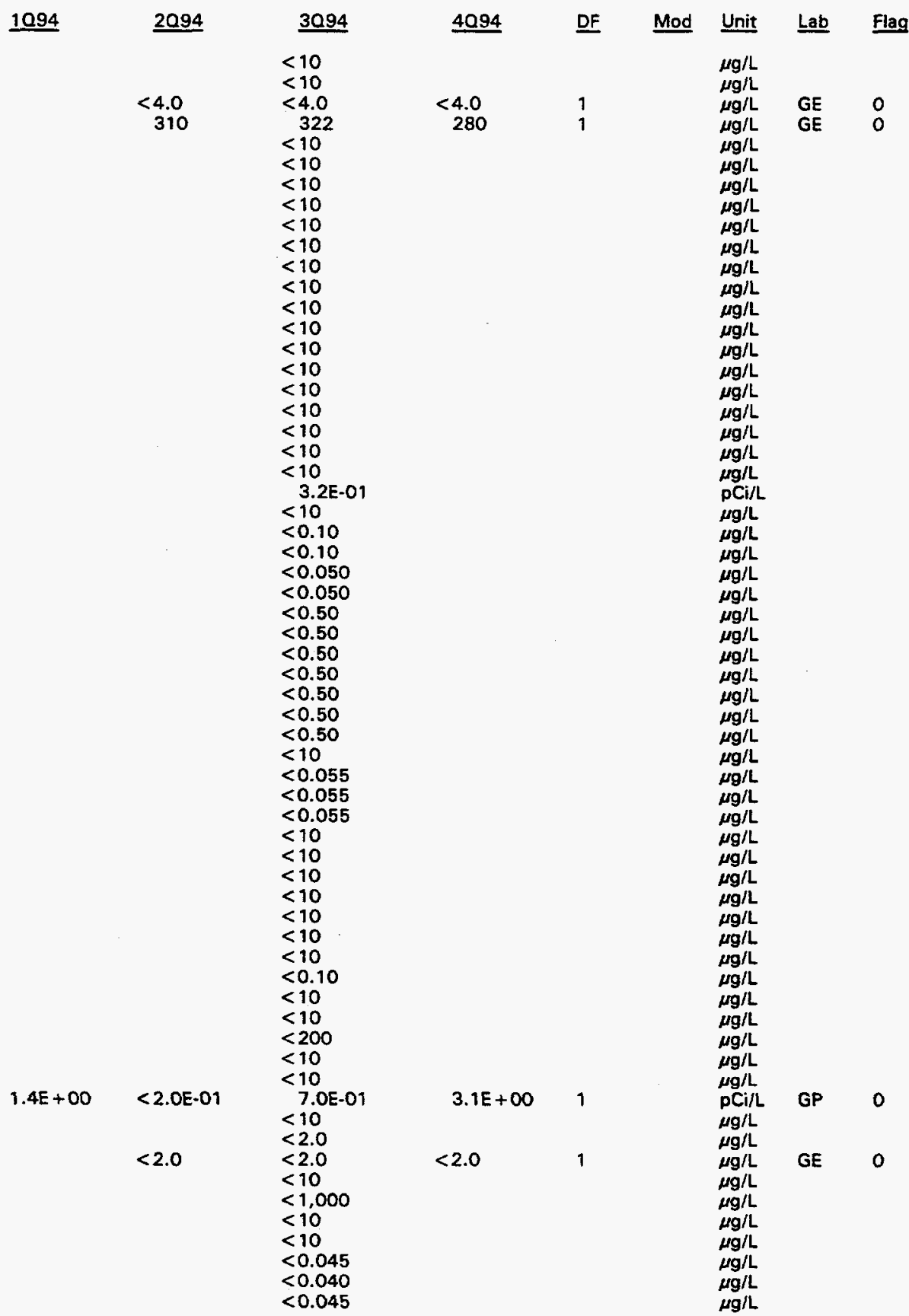

Note: Flagging, dilution factors, modifiers, and laboratories are for fourth quarter 1994 data only. See Appendix B for flagging criteria. - = exceeded holding time for fourth quarter 1994.

- = exceeded screening level or final PDWS for fourth quarter 1994. 
Well AMB 8D continued

ANALYTICAL DATA

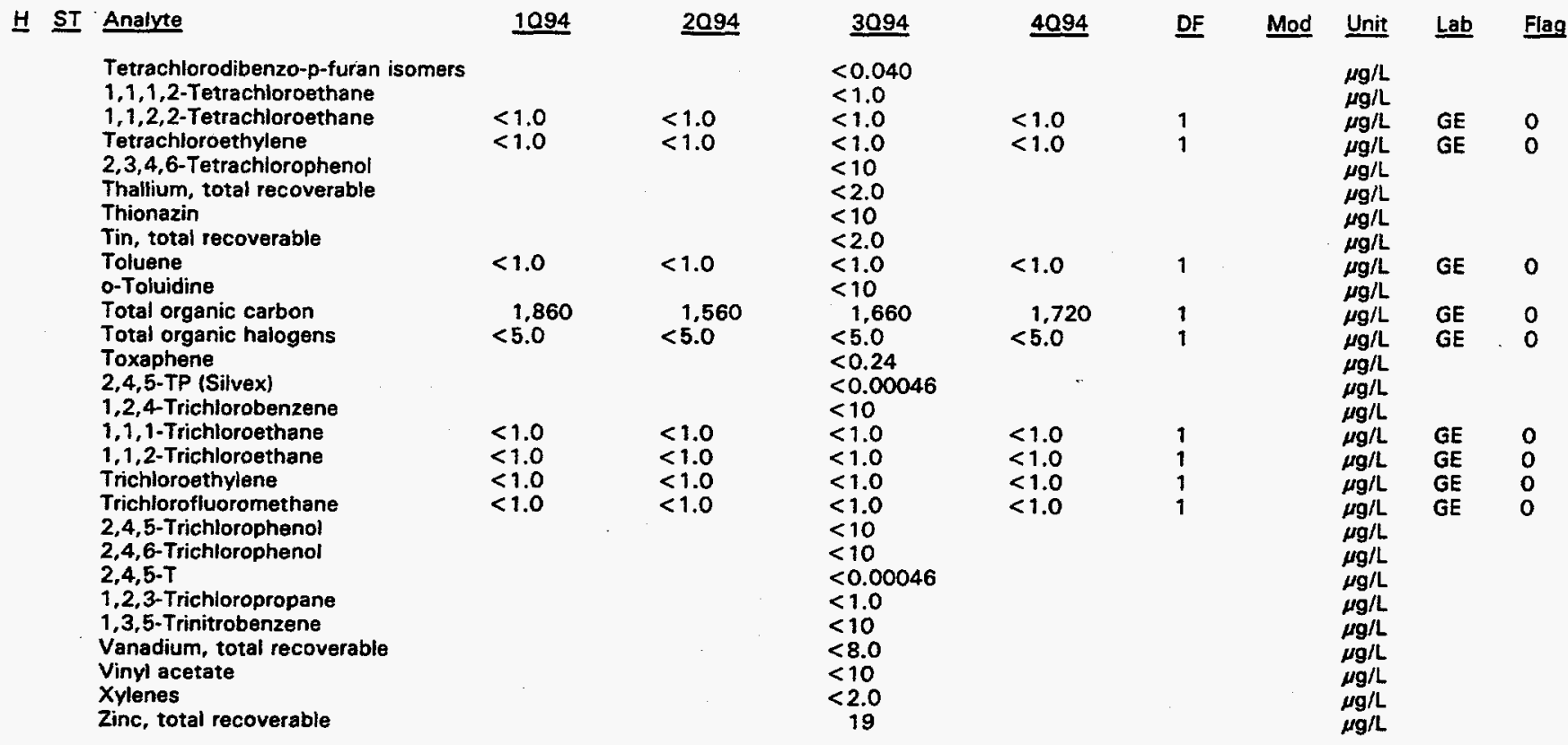

\section{WELL AMB 9D}

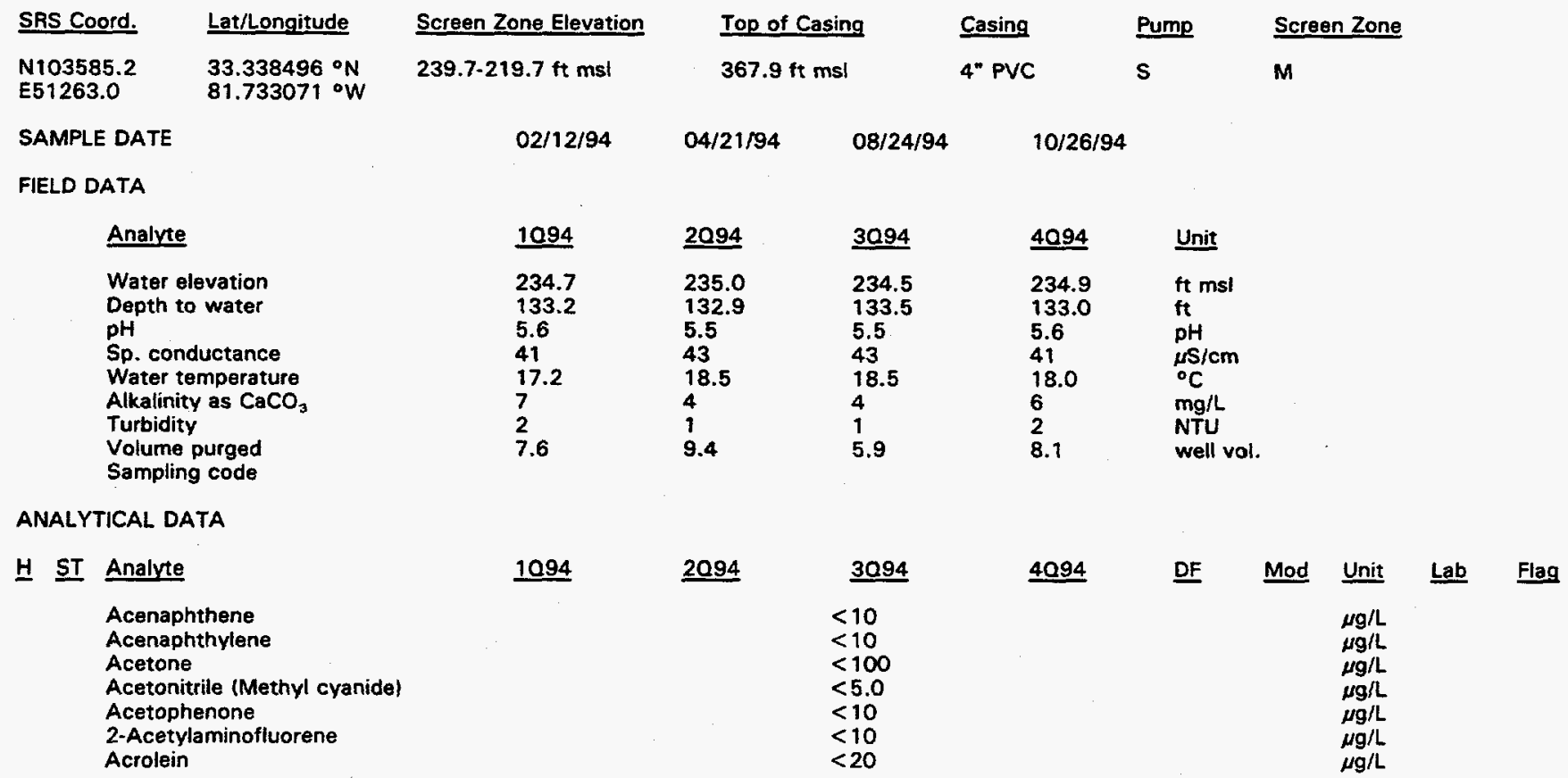

Note: Flagging, dilution factors, modifiers, and laboratories are for fourth quarter 1994 data only. See Appendix B for flagging criteria. - = exceeded holding time for fourth quarter 1994.

- = exceeded screening level or final PDWS for fourth quarter 1994. 
ANALYTICAL DATA

H ST Analyte

Acrylonitrile

Aldrin

Allyl chloride

Aluminum, total recoverable

4-Aminobipheny!

Aniline

Anthracene

Antimony, total recoverable

Aramite

Arsenic, total recoverable

Barium, total recoverable

Benzene

alpha-Benzene hexachloride

beta-Benzene hexachloride

detta-Benzene hexachloride

Benzolajanthracene

Benzolblfluoranthene

Benzolk)fluoranthene

Benzolg, $h, i]$ perylene

Benzolajpyrene

Benzyl alcohol

Beryllium, total recoverable

Bis(2-chloroethoxy) methane

Bis(2-chloroethyl) ether

Bis(2-chloroisopropyl) ether

Bis(2-ethylhexyl) phthalate

Bromodichloromethane

Bromoform

Bromomethane (Methyl bromide)

4-Bromophenyl phenyl ether

Butylbenzyl phthalate

2-sec-Butyl-4, 6-dinitrophenol

Cadmium, total recoverable

Carbon disulfide

Carbon tetrachloride

Chlordane

Chloride

4-Chloroaniline

Chlorobenzene

Chlorobenzilate

4-Chloro-m-cresol

Chloroethane

Chloroethene (Vinyl chloride)

2-Chloroethyl vinyl ether

Chloroform

Chloromethane (Methyl chloride)

2-Chloronaphthalene

2-Chlorophenol

4-Chlorophenyl phenyl ether

Chloroprene

Chromium, total recoverable

Chrysene

Cobalt, total recoverable

Copper, total recoverable

o-Cresol (2-Methylphenol)

m,p-Cresol

Cyanide

$P, P^{\prime}-D D D$

p.p'-DDE

$p, p^{\prime}-D D T$

Diallat

Dibenzla, hlanthracene

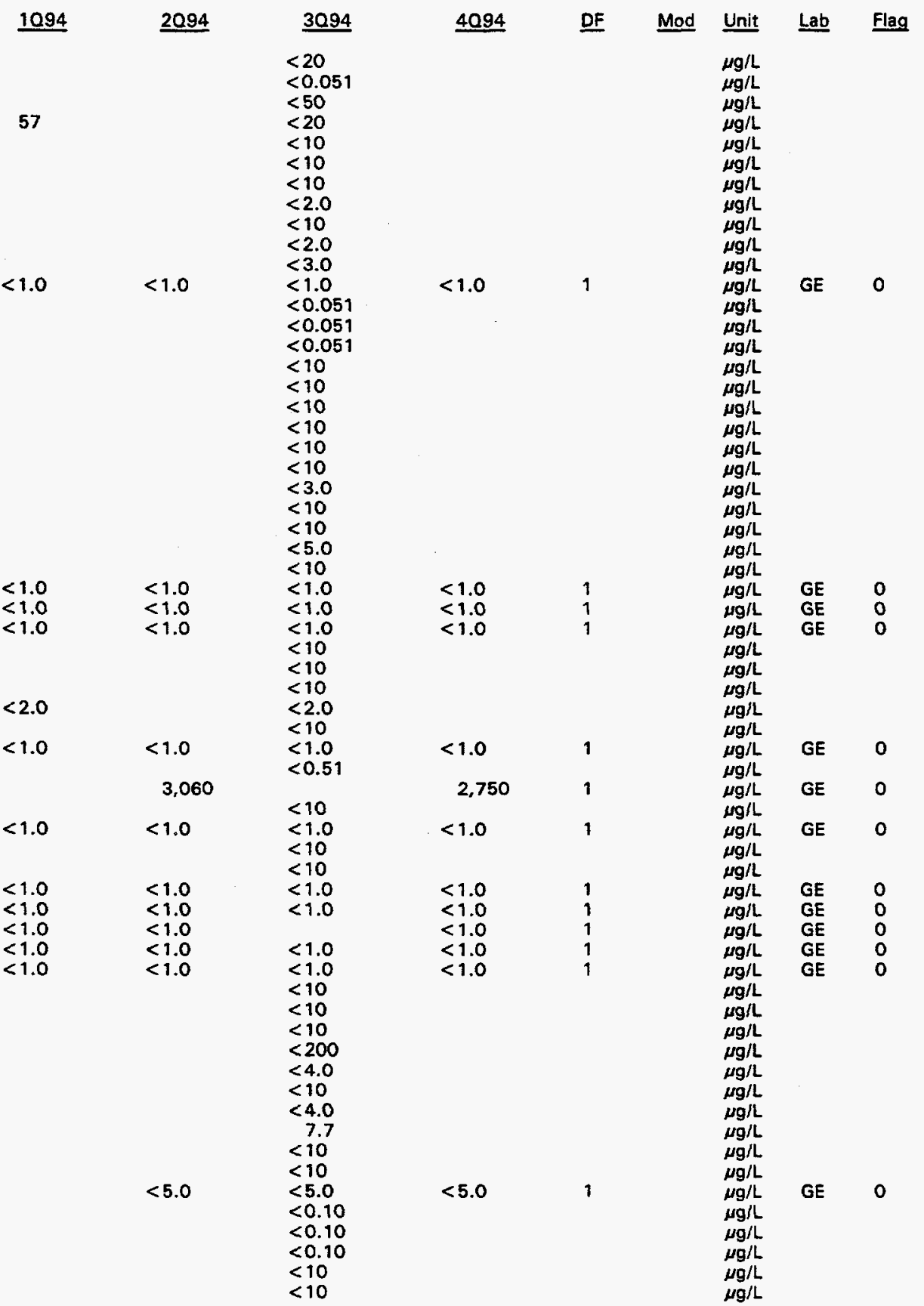

Note: Flagging, dilution factors, modifiers, and laboratories are for fourth quarter 1994 data only. See Appendix B for flagging criteria. - = exceeded holding time for fourth quarter 1994.

- = exceeded screening level or final PDWS for fourth quarter 1994. 


\section{H ST Analyte}

Dibenzofuran

Dibromochloromethane

1,2-Dibromo-3-chloropropane

1,2-Dibromoethane

Dibromomethane

Di-n-butyl phthaiate

1.2-Dichlorobenzene

1,3-Dichlorobenzene

1,4-Dichlorobenzene

3,3'-Dichlorobenzidine

trans-1,4-Dichloro-2-butene

Dichlorodifluoromethane

1.1-Dichloroethane

1,2-Dichloroethane

1,1-Dichlorosthylene

trans-1,2-Dichloroethylene

Dichloromethane

2,4-Dichlorophenol

2,6-Dichlorophenol

2,4-Dichlorophenoxyacetic acid

1,2-Dichloroprodane

cis-1,3-Dichloropropene

trans-1,3-Dichloropropene

Dieldrin

Diethyl phthalate

Dimethoate

2,4-Dimethyl pheno

Dimethyl phthalate

p-Dimethylaminoazobenzene

7,12-Dimethylbenz [a]anthracene

3,3'-Dimethylbenzidine

a, a-Dimethylphenethylamine

1.3-Dinitrobenzene

2,4-Dinitrophenol

2,4-Dinitrotoluene

2,6-Dinitrotoluene

Di-n-octyl phthalate

1.4-Dioxane

Diphenylamine

Disulfoton

Endosulfan

Endosulfan II

Endosulfan sulfate

Endrin

Endrin aldehyde

Ethyl methacrylate

Ethyl methanesulfonate

Ethylbenzene

Famphur

Fluoranthene

Fluorene

Fluoride

Gross aipha

Heptachlor

Heptachlor epoxide

$1,2,3,4,6,7,8$-HPCDD

Heptachlorodibenzo-p-dioxin isomers

$1,2,3,4,6,7,8-H P C D F$

Heptachlorodibenzo-p-furan isomers

Hexachiorobenzene

Hexachlorobutadiene

Hexachlorocyclopentadiene

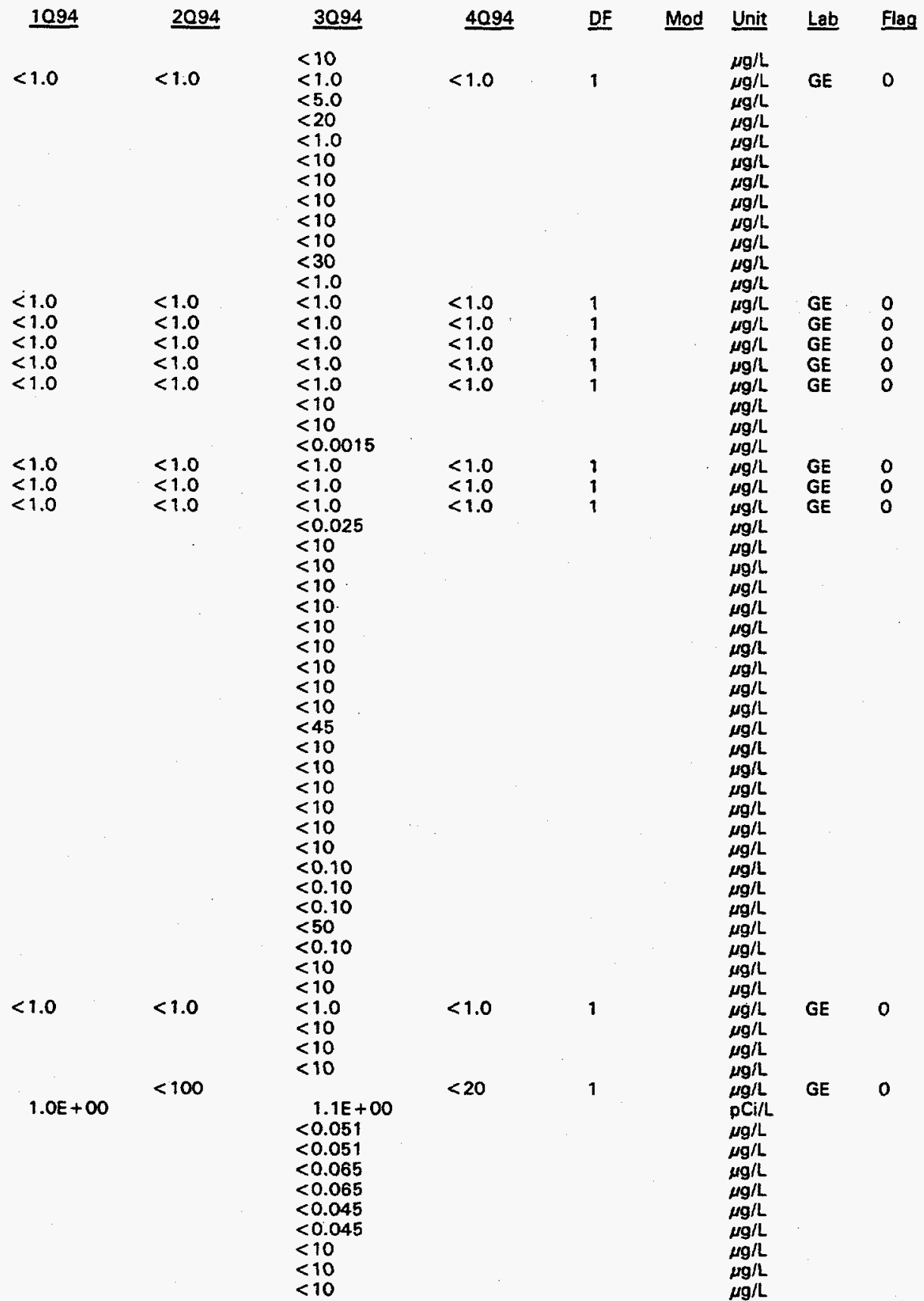

Note: Flagging, dilution factors, modifiers, and laboratories are for fourth quarter 1994 data only. See Appendix B for flagging criteria. - = exceeded holding time for fourth quarter 1994.

- exceeded screening level or final PDWS for fourth quarter 1994. 


\section{ANALYTICAL DATA}

H ST Analyte

$1,2,3,4,7,8-H \times C D D$

Hexachlorodibenzo-p-dioxin isomers

$1,2,3,4,7,8 \cdot H \times C D F$

Hexachlorodibenzo-p-furan isomers

Hexachloroethane

Hexachlorophene

Hexachloropropene

2-Hexanone

Indeno[1,2,3-c, d]pyrene

lodomethane (Methyl iodide)

Iron, total recoverable

Isobutyl alcohol

Isophorone

Isosafrole

Kepone

Lead, total recoverable

Lindane

Manganese, total recoverable

Mercury, total recoverable

Methacrylonitrile

Methapyrilene

Methoxychlor

2-Methyl-4,6-dinitrophenol

Methyl ethyl ketone

Methyl isobutyl ketone

Methyl methacrylate

Methyl methanesulfonate

3-Methylcholanthrene

2-Methylnaphthalene

Naphthalene

1,4-Naphthoquinone

1-Naphthylamine

2-Naphthylamine

Nickel, total recoverable

Nitrate-nitrite as nitrogen

o-Nitroaniline

m-Nitroaniline

p-Nitroaniline

Nitrobenzene

2-Nitrophenol

4-Nitrophenol

4-Nitroquinoline-1-oxide

$\mathrm{N}$-Nitrosodi-n-butylamine

$\mathrm{N}$-Nitrosodiethylamine

N-Nitrosodimethylamine

N-Nitrosodiphenylamine

N-Nitrosodipropylamine

$\mathrm{N}$-Nitrosomethylethylamine

$\mathrm{N}$-Nitrosomorpholine

$\mathrm{N}$-Nitrosopiperidine

$\mathrm{N}$-Nitrosopyrrolidine

5-Nitro-o-toluidine

Nonvolatile beta

$0,0,0$-Triethyl phosphorothioate

Octachlorodibenzo-p-dioxin

Octachiorodibenzo-p-furan

Parathion

Parathion methy

PCB 1016

PCB 1221

PCB 1232
86

1094

2094

3094

$<0.045$

$<0.045$

$<0.040$

$<0.040$

$<10$

$<10$

$<10$

$<10$

$<10$

$<15$

14

$<100$

$<10$

$<10$

$<10$

$<10$

$<3.0$

$<1,250$

2.3

$<0.20$

$<50$

$<10$

$<0.51$

$<10$

$<1.0$

$<10$

$<5.0$

$<10$

$<10$

$<10$

$<10$

$<10$

$<10$

$<10$

$<4.0$

$<4.0$

118

$<10$

$<10$

$<10$

$<10$

$<10$

$<10$

$<10$

$<10$

$<10$

$<10$

$<10$

$<10$

$<10$

$<10$

$<10$

$<10$

$<10$

$<10$

$<10$

4.2E-02

$<10$

$<0.10$

$<0.10$

$<0.051$

$<0.051$

$<0.51$

$<0.51$

$<0.51$

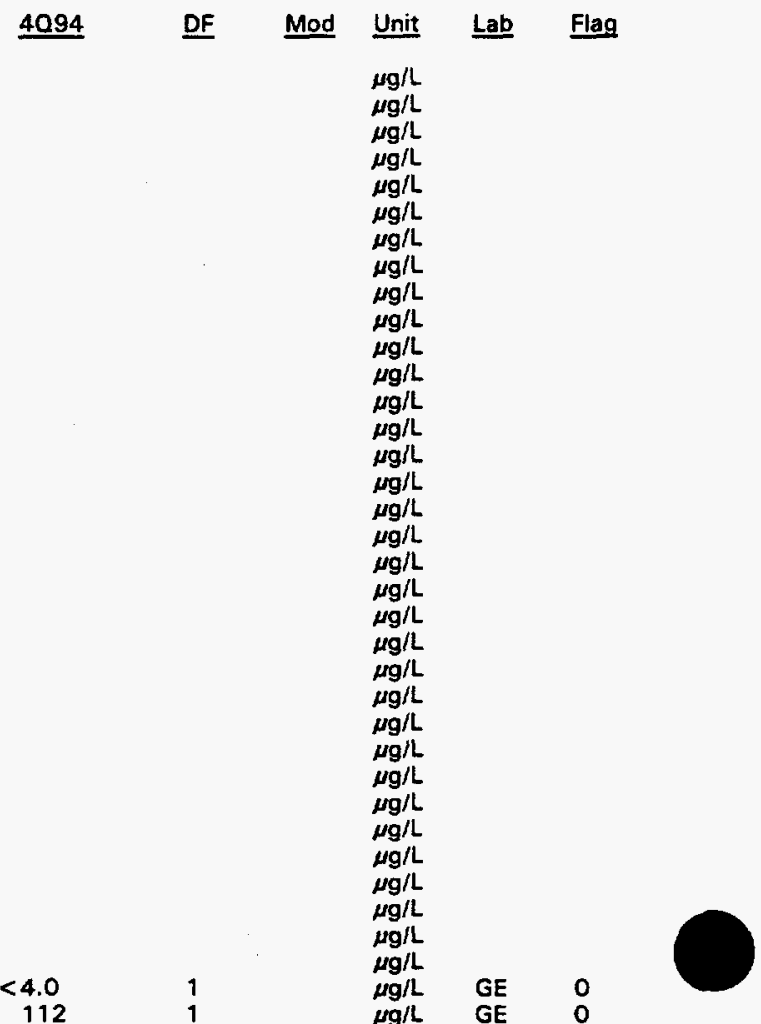

$\mu g / L$
$\mu g / L$
$\mu g / L$

$\mu g / L$

$\mu \mathrm{g} / \mathrm{h}$

$\mu g / L$

$\mu \mathrm{g} / \mathrm{L}$

$\mu g / 2$

$\mu \mathrm{g} / \mathrm{h}$

$\mu \mathrm{g} / \mathrm{L}$

$\mu g / L$

$\mu g / L$

$\mu \mathrm{g} / \mathrm{h}$

$\mu g / L$

$\mu \mathrm{g} / \mathrm{L}$

$\mu g / L$

$\mu g / L$

$\mu g / L$

pCi/L

$\mu \mathrm{g} / \mathrm{L}$

$\mu \mathrm{g} / \mathrm{L}$

$\mu g / L$

$\mu g / L$

$\mu g / L$

$\mu g / L$

$\mu \mathrm{g} / \mathrm{h}$

$\mu \mathrm{g} / \mathrm{L}$

GE $\quad 0$


Well AMB 9D continued

ANALYTICAL DATA

H ST Analyte

PCB 1242

PCB 1248

PCB 1254

PCB 1260

Pentachlorabenzene

1,2,3,7,8-PCDD

Pentachlorodibenzo-p-dioxin isomers

1,2,3,7,8-PCDF

Pentachloroethane

Pentachloronitrobenzene

Pentachlorophenol

Phenacetin

Phenanthrene

Phenol

p.Phenylenediamine

Phorate

2-Picoline

Pronamid

Propionitrile

Pyrene

Pyridine

Radium, total alpha-emitting

Safrole

Selenium, total recoverable

Silver, total recoverable

Styrene

Sulfide

Sulfotepp

1,2,4,5-Tetrachlorobenzene

$2,3,7,8-T C D D$

$2,3,7,8$-TCD

Tetrachlorodibenzo-p-dioxin isomers

Tetrachlorodibenzo-p-furan isomers

1,1,1,2-Tetrachloroethane

1, 1,2,2-Tetrachloroethane

Tetrachloroethylene

2,3,4,6-Tetrachloropheno

Thallium, total recoverable

Thionazin

Tin, total recoverable

Toluene

o-Toluidine

Total organic carbon

Total organic halogens

Toxaphene

2,4,5-TP (Silvex)

1,2,4-Trichlorobenzene

1,1,1-Trichloroethane

1,1,2-Trichloroethane

Trichloroethylene

Trichlorofluoromethane

2,4,5-Trichlorophenol

2,4,6-Trichlorophenol

2,4,5-T

1,2,3-Trichloropropane

1,3,5-Trinitrobenzene

Vanadium, total recoverable

Vinyl acetate

Xylenes

Zinc, total recoverable
1094

$\underline{2094}$

$<0.51$

$<0.51$

$<0.51$

$<0.51$

$<10$

$<0.055$

$<0.055$

$<0.055$

$<10$

$<10$

$<10$

$<10$

$<10$

$<10$

$<10$

$<0.10$

$<10$

$<200$

$<10$

$<10$

$2.2 E+\infty 0 \quad 8.0 E-01 \quad 9.0 E-01$

$<10$

$<2.0$

$<2.0$

$<2.0$

$<10$

$<1,000$

$<10$

$<10$

$<0.045$

$<0.045$

$<0.040$

$<1.0$

$<1.0$

$<1.0$

$<10$

$<2.0$

$<10$

3.0

$<1.0$

$<10$

$<1,000$

$<5.0$

$<0.00046$

$<0.000$

$<1.0$

$<1.0$

$<1.0$

$<1.0$

$<10$

$<10$

$<0.00046$

$<1.0$

$<10$

$<8.0$

$<10$

$<2.0$

$\begin{array}{cc}7.0 E-01 & 1\end{array}$

GP 0

GE $O$

$<1.0$

$<1.0$

$<1.0$

1,120

$<5.0$

$<1.0$

$<1.0$

$<1.0$

$<1.0$

$\mathrm{pCi} /$

$\mu g / L$

$\mu g / L$

$\mu \mathrm{g} / \mathrm{L}$

$\mu g / L$

$\mu \mathrm{g} / \mathrm{L}$

$\mu g / L$

$\mu g / L$

$\mu \mathrm{g} / \mathrm{L}$

$\mu g / L$

$\mu g / L$
$\mu g / L$

$\mu g / L$

$\mu \mathrm{g} / \mathrm{L}$

$\mu g / L$

$\mu g / L$

$\mu g / L$

$\mu g / L$

$\mu g / L$

$\mu g / L$

GE 0

GE $\quad 0$

$\mu g / L$

$\mu g / L$

$\mu g / L$

$\mu g / L$

$\mu g / L$

$\nu g /$

$\mu \mathrm{g} / \mathrm{L}$

$\mu \mathrm{g} / \mathrm{L}$

$\mu g$

$\mu g / L$

$\mu g / L$

$\mu g / L$

$\mu g / L$

$\mu g / L$

$\mu g / L$

$\mu \mathrm{g} / \mathrm{L}$

$\mu g / L$

$\mu g / h$

$\mu g / L$

$\mu \mathrm{g} / \mathrm{L}$

$\mu \mathrm{g} / \mathrm{L}$

$\mu g / L$

$\mu g / L$

$\mu g / L$

$\mu g / L$

$\mu \mathrm{g} / \mathrm{L}$

$\mu g / L$

$\mu g / L$

$\mu \mathrm{g} / \mathrm{L}$

Note: Flagging, dilution factors, modifiers, and laboratories are for fourth quarter 1994 data only. See Appendix B for flagging criteria.

- = exceeded holding time for fourth quarter 1994.

- = exceeded screening level or final PDWS for fourth quarter 1994. 
WELL AMB 10A

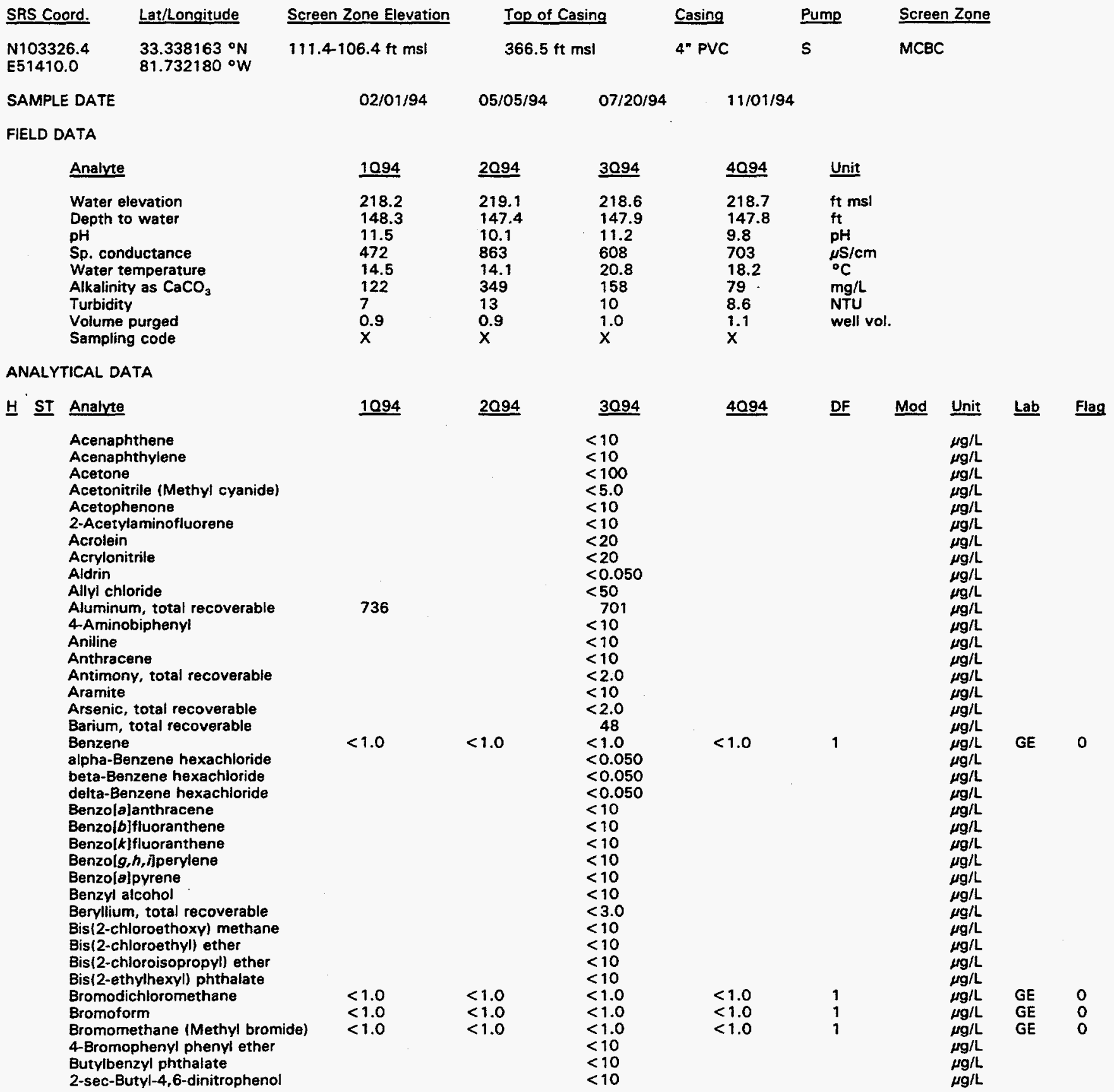

Note: Flagging, dilution factors, modifiers, and laboratories are for fourth quarter 1994 data only. See Appendix B for flagging criteria. - = exceeded holding time for fourth quarter 1994.

- = exceeded screening level or final PDWS for fourth quarter 1994. 
Well AMB 10A continued

ANALYTICAL DATA

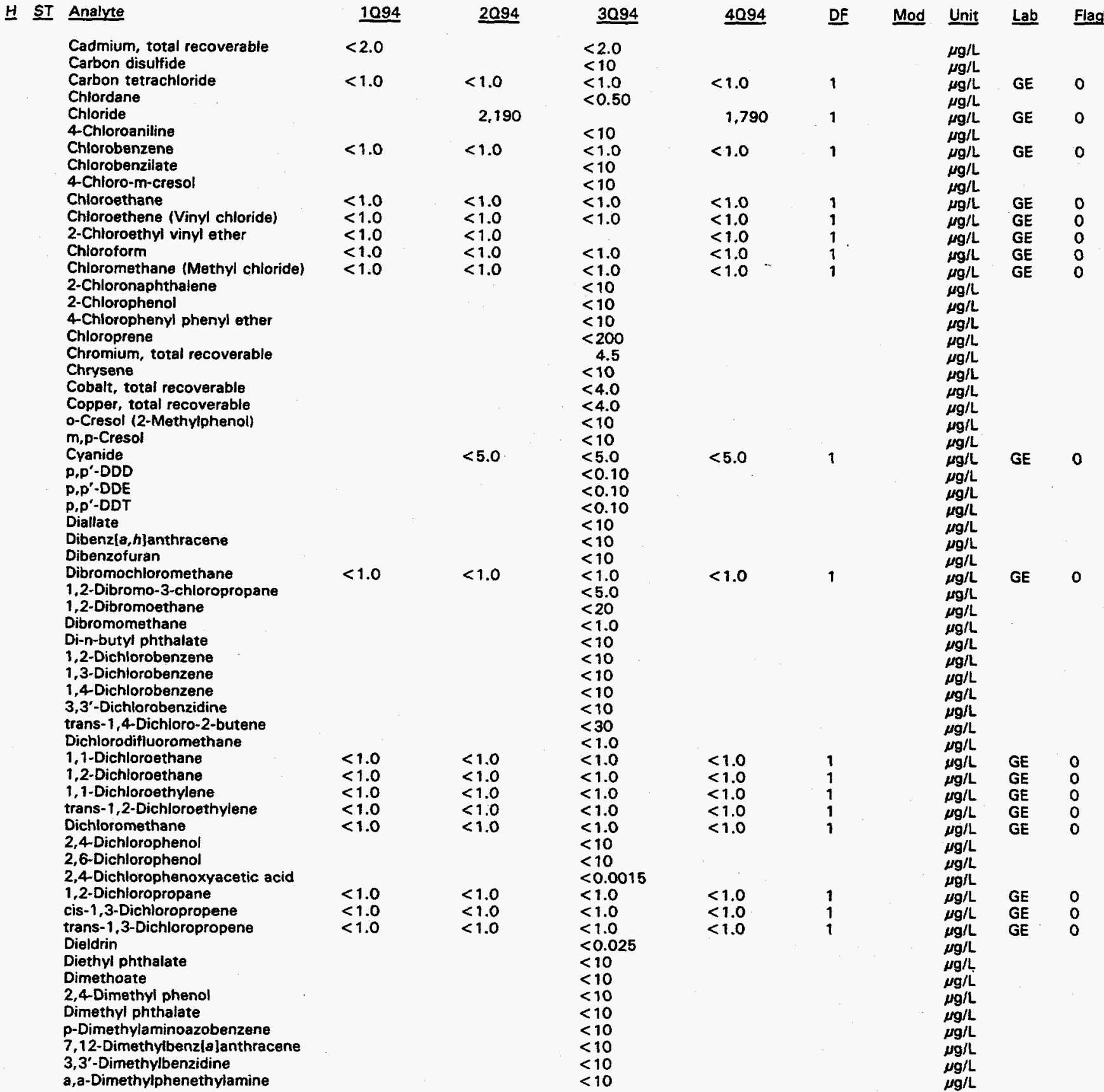

Note: Flagging, dilution factors, modifiers, and laboratories are for fourth quarter 1994 data only. See Appendix B for flagging criteria. - exceeded holding time for fourth quarter 1994 .

- = exceeded screening level or final PDWS for fourth quarter 1994. 
H ST Analyte

1,3-Dinitrobenzene

2,4-Dinitrophenol

2,4-Dinitrotoluene

2,6-Dinitrotoluene

Di-n-octyl phthalate

1,4-Dioxane

Diphenylamine

Disulfoton

Endosulfan I

Endosulfan II

Endosulfan sulfate

Endrin

Endrin aldehyde

Ethyl methacrylate

Ethyl methanesulfonate

Ethylbenzene

Famphur

Fluoranthene

Fluorene

Fluoride

Gross alpha

Heptachlor

Heptachlor epoxide

$1,2,3,4,6,7,8$-HPCDD

Heptachlorodibenzo-p-dioxin isomers

$1,2,3,4,6,7,8-H P C D F$

Heptachlorodibenzo-p-furan isomers

Hexachlorobenzene

Hexachlorobutadiene

Hexachlorocyclopentadiene

$1,2,3,4,7,8-H \times C D D$

Hexachlorodibenzo-p-dioxin isomers

$1,2,3,4,7,8-H X C D F$

Hexachlorodibenzo-p-furan isomers

Hexachloroethane

Hexachlorophene

Hexachloropropene

2-Hexanone

Indenol 1,2,3-c, d]pyrene

lodomethane (Methyl iodide)

Iron, total recoverable

Isobutyl alcohol

Isodrin

Isophorone

Isosafrole

Kepone

Lead, total recoverable

Lindane

Manganese, total recoverable

Mercury, total recoverable

Methacrylonitrile

Methapyrilene

Methoxychior

2-Methyl-4,6-dinitrophenol

Methyl ethyl ketone

Methyl isobutyl ketone

Methyl methacrylate

Methyl methanesulfonate

3-Methylcholanthrene

2-Methvinaphthalene

Naphthalene

1,4-Naphthoquinone
1094

$\underline{2094}$

$\begin{aligned} & 3094 \\ &<<10 \\ &<45 \\ &<10 \\ &<10 \\ &<10 \\ &<10 \\ &<10 \\ &<10 \\ &<10 \\ &<0.10 \\ &<0.10 \\ &<0.10 \\ &<50 \\ &<0.10 \\ &<10 \\ &<10 \\ &<1.0 \\ &<10 \\ &<10 \\ &<10 \\ &<10\end{aligned}$

$<5.0 E-01$

$<100$

9.6E-02

$<0.050$

$<0.050$

$<0.065$

$<0.065$

$<0.045$

$<0.045$

$<10$

$<10$

$<10$

$<0.045$

$<0.045$

$<0.040$

$<0.040$

$<10$

$<10$

$<10$

$<10$

$<10$

$<15$

16

21

$<100$

$<10$

$<10$

$<10$

$<10$

$<3.0$

$<1,250$

2.1

$<0.20$

$<50$

$<10$

$<0.50$

$<10$

$<1.0$

$<10$

$<5.0$

$<10$

$<10$

$<10$

$<10$

$<10$

Note: Flagging, dilution factors, modifiers, and laboratories are for fourth quarter 1994 data only. See Appendix B for flagging criteria. - = exceeded holding time for fourth quarter 1994.

- = exceeded screening level or final PDWS for fourth quarter 1994.

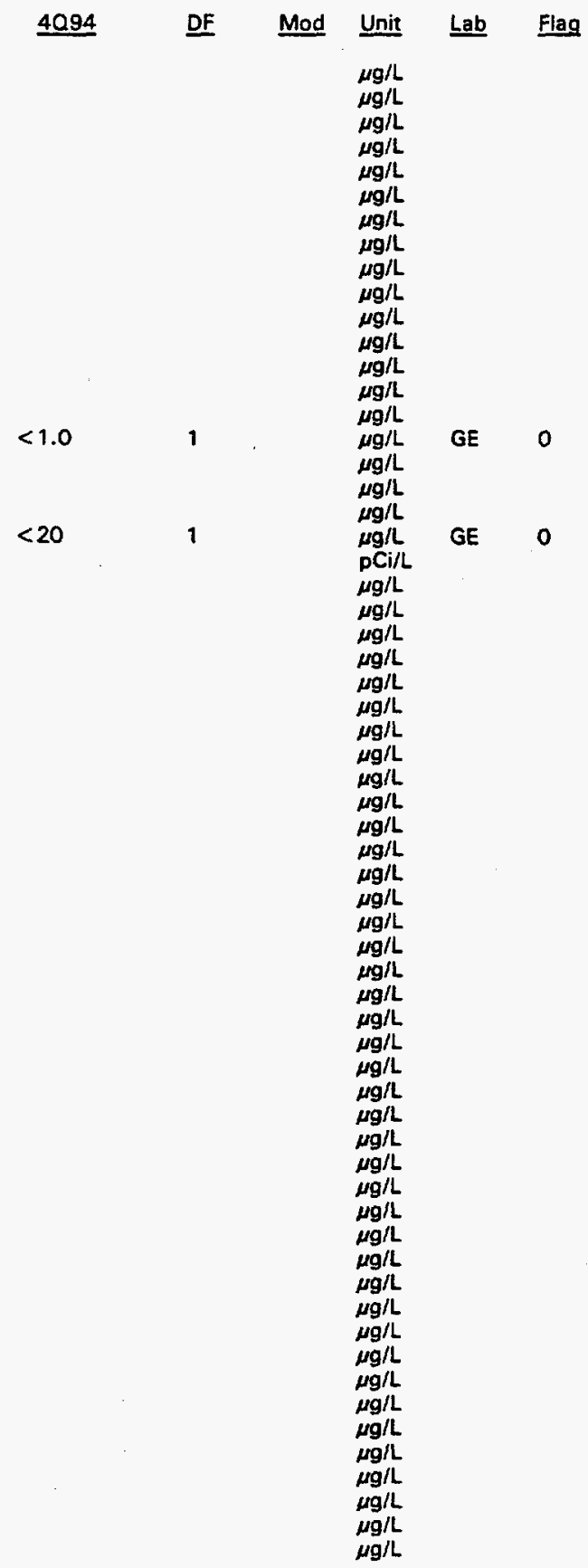


ANALYTICAL DATA

\section{H ST Analyte}

1-Naphthylamine

2-Naphthylamine

Nickel, total recoverable

Nitrate-nitrite as nitrogen

o-Nitroaniline

m-Nitroaniline

p-Nitroaniline

2-Nitrophenol

4-Nitrophenol

4-Nitroquinoline-1-oxide

N-Nitrosodi-n-butylamine

N-Nitrosodiethylamine

$\mathrm{N}$-Nitrosodimethylamine

$\mathrm{N}$-Nitrosodiphenviamine

N-Nitrosodipropylamine

N-Nitrosomethylethylamine

N-Nitrosomorpholine

N-Nitrosopiperidine

N-Nitrosopyrrolidine

5-Nitro-0-toluidine

Nonvolatile beta

$0,0,0$-Triethyl phosphorothioate

Octachlorodibenzo-p-dioxin

Octachlorodibenzo-p-furan

Parathion

Parathion methyl

PCB 1016

PCB 1221

PCB 1232

PCB 1242

PCB 1248

PCB 1254

PCB 1260

Pentachlorobenzene

1,2,3,7,8-PCDD

Pentachlorodibenzo-p-dioxin isomers

1,2,3, 7, 8-PCDF

Pentachloroethane

Pentachloronitrobenzene

Pentachlorophenol

Phenacetin

Phenanthrene

Phenol

p-Phenylenediamine

Phorate

2-Picoline

Pronamid

Propionitrile

Prrene

Pyridine

Radium, total alpha-emitting

Safrole

Selenium, total recoverable

Silver, total recoverable

Styrene

Sulfide

Sulfotepp

1,2,4,5-Tetrachlorobenzene

2,3,7,8-TCDD

$2,3,7,8-T C D F$

Tetrachlorodibenzo-p-dioxin isomers

Tetrachlorodibenzo-p-furan isomers

\begin{tabular}{|c|c|c|c|c|c|c|c|c|}
\hline 1094 & 2094 & 3094 & 4094 & DF & Mod & Unit & Lab & Fle \\
\hline & $\begin{array}{r}<8.0 \\
130\end{array}$ & $\begin{array}{l}<10 \\
<10 \\
<4.0 \\
152 \\
<10 \\
<10 \\
<10 \\
<10 \\
<10 \\
<10 \\
<10 \\
<10 \\
<10 \\
<10 \\
<10 \\
<10 \\
<10 \\
<10 \\
<10 \\
<10 \\
1.0 E+01 \\
<10 \\
<0.10 \\
<0.10 \\
<0.050 \\
<0.050 \\
<0.50 \\
<0.50 \\
<0.50 \\
<0.50 \\
<0.50 \\
<0.50 \\
<0.50 \\
<10 \\
<0.055 \\
<0.055 \\
<0.055 \\
<10 \\
<10 \\
<10 \\
<10 \\
<10 \\
<10 \\
<10 \\
<0.10 \\
<10 \\
<10 \\
<200 \\
<10 \\
<10 \\
< \\
\end{array}$ & $\begin{array}{r}<4.0 \\
231\end{array}$ & $\begin{array}{l}1 \\
1\end{array}$ & & $\begin{array}{l}\mu g / L \\
\mu g / L \\
\mu g / L \\
\mu g / L \\
\mu g / L \\
\mu g / L \\
\mu g / L \\
\mu g / L \\
\mu g / L \\
\mu g / L \\
\mu g / L \\
\mu g / L \\
\mu g / L \\
\mu g / L \\
\mu g / L \\
\mu g / L \\
\mu g / L \\
\mu g / L \\
\mu g / L \\
\mu g / L \\
p C i / L \\
\mu g / L \\
\mu g / L \\
\mu g / L \\
\mu g / L \\
\mu g / L \\
\mu g / L \\
\mu g / L \\
\mu g / L \\
\mu g / L \\
\mu g / L \\
\mu g / L \\
\mu g / L \\
\mu g / L \\
\mu g / L \\
\mu g / L \\
\mu g / L \\
\mu g / L \\
\mu g / L \\
\mu g / L \\
\mu g / L \\
\mu g / L \\
\mu g / L \\
\mu g / L \\
\mu g / L \\
\mu g / L \\
\mu g / L \\
\mu g / L \\
\mu g / L \\
\mu g / L\end{array}$ & $\begin{array}{l}\mathrm{GE} \\
\mathrm{GE}\end{array}$ & $\begin{array}{l}0 \\
0\end{array}$ \\
\hline $9.0 E-01$ & $<4.0 \mathrm{E}-01$ & $\begin{array}{l}<3.0 \mathrm{E}-01 \\
<10 \\
<2.0\end{array}$ & $2.0 E-01$ & 1 & UI & $\begin{array}{l}\mathrm{PCi} / \mathrm{L} \\
\mu \mathrm{g} / \mathrm{L} \\
\mu \mathrm{g} / \mathrm{L}\end{array}$ & GP & 0 \\
\hline & $<4.0$ & $\begin{array}{l}<2.0 \\
<10 \\
<1,000 \\
<10 \\
<10 \\
<0.045 \\
<0.040 \\
<0.045 \\
<0.040\end{array}$ & $<2.0$ & 1 & & $\begin{array}{l}\mu g / L \\
\mu g / L \\
\mu g / L \\
\mu g / L \\
\mu g / L \\
\mu g / L \\
\mu g / L \\
\mu g / L \\
\mu g / L\end{array}$ & GE & 0 \\
\hline
\end{tabular}

Note: Flagging, dilution factors, modifiers, and laboratories are for fourth quarter 1994 data only. See Appendix B for flagging criteria. - = exceeded holding time for fourth quarter 1994.

- =xceeded screening level or final PDWS for fourth quarter 1994. 
브 ST Analyte

1,1,1,2-Tetrachioroethane

1,1,2,2-Tetrachloroethane

Tetrachloroethylene

2,3,4,6-Tetrachlorophenol

Thallium, total recoverable

Thionazin

Tin, total recoverable

Toluene

o-Toluidine

Total organic carbon

Total organic halogens

Toxaphene

2,4,5-TP (Silvex)

1,2,4-Trichiorobenzene

1,1,1-Trichloroethane

1,1,2-Trichloroethane

Trichloroethylene

Trichlorofluoromethane

2,4,5-Trichlorophenol

2,4,6-Trichlorophenol

2,4,5-T

1,2,3-Trichloropropane

1,3,5-Trinitrobenzene

Vanadium, total recoverable

Vinyl acetate

Xylenes

Zinc, total recoverable

\begin{tabular}{|c|c|}
\hline 10.94 & $\underline{2094}$ \\
\hline $\begin{array}{l}<1.0 \\
<1.0\end{array}$ & $\begin{array}{l}<1.0 \\
<1.0\end{array}$ \\
\hline$<1.0$ & $<1.0$ \\
\hline $\begin{array}{l}1,040 \\
8.6\end{array}$ & $\begin{aligned} & 2,130 \\
&<5.0\end{aligned}$ \\
\hline $\begin{array}{l}<1.0 \\
<1.0 \\
<1.0 \\
<1.0\end{array}$ & $\begin{array}{l}<1.0 \\
<1.0 \\
<1.0 \\
<1.0\end{array}$ \\
\hline
\end{tabular}

\begin{tabular}{|c|c|}
\hline 3094 & 3094 \\
\hline & \\
\hline$<1.0$ & $<1.0$ \\
\hline$<1.0$ & $<1.0$ \\
\hline$<10$ & \\
\hline$<2.0$ & \\
\hline$<10$ & \\
\hline 30 & \\
\hline$<1.0$ & $<1.0$ \\
\hline$<10$ & \\
\hline 1,230 & 1,630 \\
\hline $\begin{array}{c}18 \\
<0.24\end{array}$ & \\
\hline$<0.00044$ & \\
\hline$<10$ & \\
\hline$<1.0$ & $<1.0$ \\
\hline$<1.0$ & $<1.0$ \\
\hline$<1.0$ & $<1.0$ \\
\hline$<1.0$ & $<1.0$ \\
\hline$<10$ & \\
\hline$<10$ & \\
\hline$<0.00044$ & \\
\hline $\begin{array}{l}<1.0 \\
<10\end{array}$ & \\
\hline$<8.0$ & \\
\hline$<10$ & \\
\hline 21 & \\
\hline
\end{tabular}

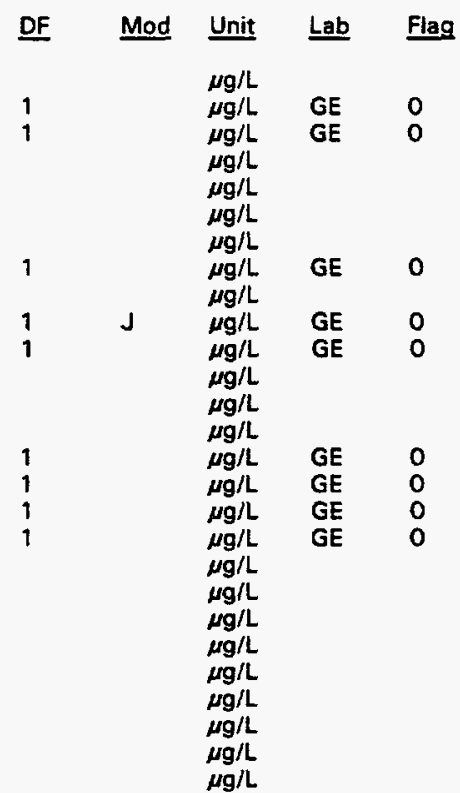

WELL AMB 10B

\begin{tabular}{|c|c|c|c|c|c|c|c|c|c|c|c|}
\hline SRS Co & ord. & Lat/Longitude & Screen Zone Elevation & Top of & ing & Casing & Pump & Scre & n Zone & & \\
\hline $\begin{array}{l}\text { N1033 } \\
\text { E51418 }\end{array}$ & 1.3 & $\begin{array}{l}33.338201^{\circ} \mathrm{N} \\
81.732180^{\circ} \mathrm{W}\end{array}$ & $154.3-149.3 \mathrm{ft} \mathrm{msl}$ & $366.4 f$ & & 4" PVC & s & LL & & & \\
\hline SAMPL & $E D^{-}$ & & $02 / 12 / 94$ & $04 / 25 / 94$ & $08 / 23 / 94$ & $10 / 31 / 94$ & & & & & \\
\hline FIELD D & ATA & & & & & & & & & & \\
\hline & Ana & & 1094 & $\underline{2094}$ & $\underline{3094}$ & $\underline{4094}$ & Unit & & & & \\
\hline & $\begin{array}{l}\text { Wat } \\
\text { Dep } \\
\text { pH } \\
\text { Sp. } \\
\text { Wat } \\
\text { Alka } \\
\text { Turb } \\
\text { Volu } \\
\text { Sam }\end{array}$ & $\begin{array}{l}\text { evation } \\
\text { water } \\
\text { luctance } \\
\text { mperature } \\
\text { as } \mathrm{CaCO}_{3} \\
\text { purged } \\
\text { code }\end{array}$ & $\begin{array}{l}223.9 \\
142.5 \\
6.1 \\
59 \\
17.3 \\
19 \\
1 \\
3.6\end{array}$ & $\begin{array}{l}223.5 \\
142.9 \\
6.2 \\
63 \\
19.0 \\
18 \\
1 \\
5.5\end{array}$ & $\begin{array}{l}224.0 \\
142.4 \\
5.8 \\
61 \\
18.9 \\
10 \\
1 \\
3.3\end{array}$ & $\begin{array}{l}223.7 \\
142.8 \\
5.9 \\
51 \\
18.0 \\
4 \\
1 \\
5.2\end{array}$ & 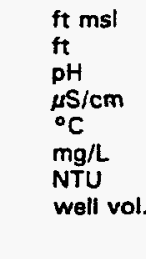 & & & & \\
\hline ANALY & IICAl & TA & & & & & & & & & \\
\hline$\underline{H} \underline{S T}$ & Ana & & 1094 & $\underline{2094}$ & 3094 & 4094 & DF & Mod & $\underline{\text { Unit }}$ & $\underline{\text { Lab }}$ & Flag \\
\hline & $\begin{array}{l}\text { Ace } \\
\text { Ace } \\
\text { Ace } \\
\text { Ace } \\
\text { Ace } \\
2-A C \\
\text { Acr } \\
\text { Acr }\end{array}$ & $\begin{array}{l}\text { thene } \\
\text { thylene } \\
\text { rile (Methyl cyanide) } \\
\text { enone } \\
\text { aminofluorene } \\
\text { trile }\end{array}$ & & & $\begin{array}{l}<10 \\
<10 \\
<100 \\
<5.0 \\
<10 \\
<10 \\
<20 \\
<20\end{array}$ & - & & & $\begin{array}{l}\mu g / L \\
\mu g / L \\
\mu g / L \\
\mu g / L \\
\mu g / L \\
\mu g / L \\
\mu g / L \\
\mu g / L\end{array}$ & & \\
\hline
\end{tabular}

Note: Flagging, dilution factors, modifiers, and laboratories are for fourth quarter 1994 data only. See Appendix B for flagging criteria. - = exceeded holding time for fourth quarter 1994.

- = exceeded screening level or final PDWS for fourth quarter 1994. 
Well AMB $10 B$ continued

ANALYTICAL DATA

\section{H ST Analyte}

Aldrin

Allyl chloride

Aluminum, total recoverable

4-Aminobiphenyl

Aniline

Anthracene

Antimony, total recoverable

Aramite

Arsenic, total recoverable

Barium, total recoverable

Benzene

alpha-Benzene hexachloride

beta-Benzene hexachloride

delta-Benzene hexachloride

Benzolajanthracene

Benzolblfluoranthene

Benzol $k]$ fluoranthene

Benzo[g,h,i]perylene

Benzola]pyrene

Benzyl alcohol

Berylium, total recoverable

Bis(2-chloroethoxy) methane

Bis(2-chloroethyl) ether

Bis(2-chloroisopropyl) ether

Bis(2-ethylhexyl) phthalate

Bromodichloromethane

Bromoform

Bromomethane (Methyl bromide)

4-Bromophenyl phenyl ether

Butylbenzyl phthalate

2-sec-Butyl-4,6-dinitrophenol

Cadmium, total recoverable

Carbon disulfide

Carbon tetrachloride

Chlordane

Chloride

4-Chloroaniline

Chlorobenzene

Chlorobenzilate

4-Chloro-m-cresol

Chloroethane

Chloroethene (Vinyl chloride)

2-Chloraethyl vinyl ether

Chloroform

Chloromethane (Methyl chloride)

2-Chloronaphthalene

2-Chlorophenol

4-Chlorophenyl phenyl ether

Chloroprene

Chromium, total recoverable

Chrysene

Cobalt, total recoverable

Copper, total recoverable

o-Cresol (2-Methylphenol)

$\mathrm{m}, \mathrm{p}-$ Cresol

Cyanide

p. $p^{\prime}-D D D$

$P, p^{\prime}-D D E$

p, $p^{\prime}$-DDT

Diallate

Dibenzla,hlanthracene

Dibenzofuran

\begin{tabular}{|c|c|c|c|c|c|c|c|c|}
\hline 1094 & 2094 & 3094 & 4094 & DF & Mod & Unit & $\underline{\text { Lab }}$ & Flag \\
\hline 23 & & $\begin{array}{l}<0.052 \\
<50 \\
<20 \\
<10 \\
<10 \\
<10 \\
<2.0 \\
<10 \\
<2.0 \\
\quad 16\end{array}$ & & & & $\begin{array}{l}\mu g / L \\
\mu g / L \\
\mu g / L \\
\mu g / L \\
\mu g / L \\
\mu g / L \\
\mu g / L \\
\mu g / L \\
\mu g / L \\
\mu g / L\end{array}$ & & \\
\hline$<1.0$ & $<1.0$ & $\begin{array}{l}<1.0 \\
<0.052 \\
<0.052 \\
<0.052 \\
<10 \\
<10 \\
<10 \\
<10 \\
<10 \\
<10 \\
<3.0 \\
<10 \\
<10 \\
<5.0 \\
<10\end{array}$ & $<1.0$ & 1 & & $\begin{array}{l}\mu g / L \\
\mu g / L \\
\mu g / L \\
\mu g / L \\
\mu g / L \\
\mu g / L \\
\mu g / L \\
\mu g / L \\
\mu g / L \\
\mu g / L \\
\mu g / L \\
\mu g / L \\
\mu g / L \\
\mu g / L \\
\mu g / L\end{array}$ & .. & 0 \\
\hline $\begin{array}{l}<1.0 \\
<1.0\end{array}$ & $\begin{array}{l}<1.0 \\
<1.0\end{array}$ & $\begin{array}{l}<1.0 \\
<1.0\end{array}$ & $\begin{array}{l}<1.0 \\
<10\end{array}$ & 1 & & $\mu \mathrm{g} / \mathrm{L}$ & GE & 0 \\
\hline $\begin{array}{l}<1.0 \\
<1.0\end{array}$ & $\begin{array}{l}<1.0 \\
<1.0\end{array}$ & $\begin{array}{l}<1.0 \\
<1.0 \\
<10 \\
<10 \\
<10\end{array}$ & $\begin{array}{l}<1.0 \\
<1.0\end{array}$ & $\begin{array}{l}1 \\
1\end{array}$ & & $\begin{array}{l}\mu g / L \\
\mu g / L \\
\mu g / L \\
\mu g / L \\
\mu g / L\end{array}$ & $\begin{array}{l}\text { GE } \\
\text { GE }\end{array}$ & $\begin{array}{l}0 \\
0\end{array}$ \\
\hline$<2.0$ & & $\begin{array}{l}<2.0 \\
<10\end{array}$ & & & & $\underset{\mu g / L}{\mu g / L}$ & & \\
\hline$<1.0$ & $<1.0$ & $\begin{array}{l}<1.0 \\
<0.52\end{array}$ & $<1.0$ & 1 & & $\mu \mathrm{g} / \mathrm{L}$ & GE & 0 \\
\hline & 3,430 & $<10$ & 3,940 & 1 & & $\stackrel{\mu g / L}{\mu g / L}$ & WA & 0 \\
\hline$<1.0$ & $<1.0$ & $\begin{array}{l}<1.0 \\
<10 \\
<10\end{array}$ & $<1.0$ & 1 & & $\begin{array}{l}\mu g / L \\
\mu g / L \\
\mu g / L\end{array}$ & GE & 0 \\
\hline$<1.0$ & $<1.0$ & $<1.0$ & $<1.0$ & 1 & & $\mu \mathrm{g} / \mathrm{L}$ & GE & 0 \\
\hline $\begin{array}{l}<1.0 \\
<1.0\end{array}$ & $\begin{array}{l}<1.0 \\
<1.0\end{array}$ & $<1.0$ & $\begin{array}{l}<1.0 \\
<1.0\end{array}$ & $\begin{array}{l}1 \\
1\end{array}$ & & $\mu \mathrm{g} / \mathrm{L}$ & $\begin{array}{l}\text { GE } \\
\text { GE }\end{array}$ & $\begin{array}{l}0 \\
0\end{array}$ \\
\hline$<1.0$ & $<1.0$ & $<1.0$ & $<1.0$ & 1 & & $\mu \mathrm{g} / \mathrm{L}$ & $\mathrm{GE}$ & 0 \\
\hline$<1.0$ & $<1.0$ & $\begin{array}{l}<1.0 \\
<10 \\
<10 \\
<10 \\
<200 \\
<4.0 \\
<10 \\
<4.0 \\
<4.0 \\
<10 \\
<10\end{array}$ & $<1.0$ & 1 & & $\begin{array}{l}\mu g / L \\
\mu g / L \\
\mu g / L \\
\mu g / L \\
\mu g / L \\
\mu g / L \\
\mu g / L \\
\mu g / L \\
\mu g / L \\
\mu g / L \\
\mu g / L\end{array}$ & GE & 0 \\
\hline & $<5.0$ & $\begin{array}{l}<5.0 \\
<0.10 \\
<0.10 \\
<0.10 \\
<10 \\
<10 \\
<10\end{array}$ & $<5.0$ & 1 & & $\begin{array}{c}\mu \mathrm{g} / \mathrm{L} \\
\mu \mathrm{g} / \mathrm{L} \\
\mu \mathrm{g} / \mathrm{L} \\
\mu \mathrm{g} / \mathrm{L} \\
\mu \mathrm{g} / \mathrm{L} \\
\mu \mathrm{g} / \mathrm{L} \\
\mu \mathrm{g} / \mathrm{L}\end{array}$ & GE & 0 \\
\hline
\end{tabular}

Note: Flagging, dilution factors, modifiers, and laboratories are for fourth quarter 1994 data only. See Appendix B for flagging criteria.

- = exceeded holding time for fourth quarter 1994.

- exceeded screening level or final PDWS for fourth quarter 1994. 
H ST Analyte

Dibromochloromethane

1,2-Dibromo-3-chloropropane

1,2-Dibromoethane

Dibromomethane

Di-n-butyl phthalate

1,2-Dichlorobenzene

1.3-Dichlorobenzene

1,4-Dichlorobenzene

3, 3'-Dichlorobenzidine

trans-1,4-Dichloro-2-butene

Dichlorodifluoromethane

1,1-Dichloroethane

1,2-Dichloroethane

1,1-Dichloroethylene

trans-1,2-Dichloroethylene

Dichloromethane

2,4-Dichiorophenol

2,6-Dichlorophenol

2,4-Dichlorophenoxyacetic acid

1,2-Dichloropropane

cis-1,3-Dichloropropene

trans-1,3-Dichloropropene

Dieldrin

Diethyl phthaiate

Dimethoate

2,4-Dimethyl pheno

Dimethyl phthalate

p-Dimethylaminoazobenzene

7,1 2-Dimethylbenz [a]anthracene

$3,3^{r}$-Dimethylbenzidine

a,a-Dimethylphenethylamine

1,3-Dinitrobenzene

2,4-Dinitrophenol

2,4-Dinitrotoluene

2,6-Dinitrotoluene

Di-n-octyl phthalate

1.4-Dioxane

Diphenylamin

Disulfoton

Endosulfan I

Endosulfan II

Endosulfan suifate

Endrin

Endrin aldehyde

Ethyl methacrylate

Ethyl methanesulfonate

Ethylbenzene

Famphur

Fluoranthene

Fluorene

Fluoride

Gross alpha

Heptachlor

Heptachlor epoxide

1, 2, 3, 4,6,7,8-HPCDD

Heptachlorodibenzo-p-dioxin isomers

$1,2,3,4,6,7,8$-HPCDF

Heptachlorodibenzo-p-furan isomers

Hexachlorobenzene

Hexachlorobutadiene

Hexachlorocyclopentadiene

$1,2,3,4,7,8-\mathrm{HXCDD}$

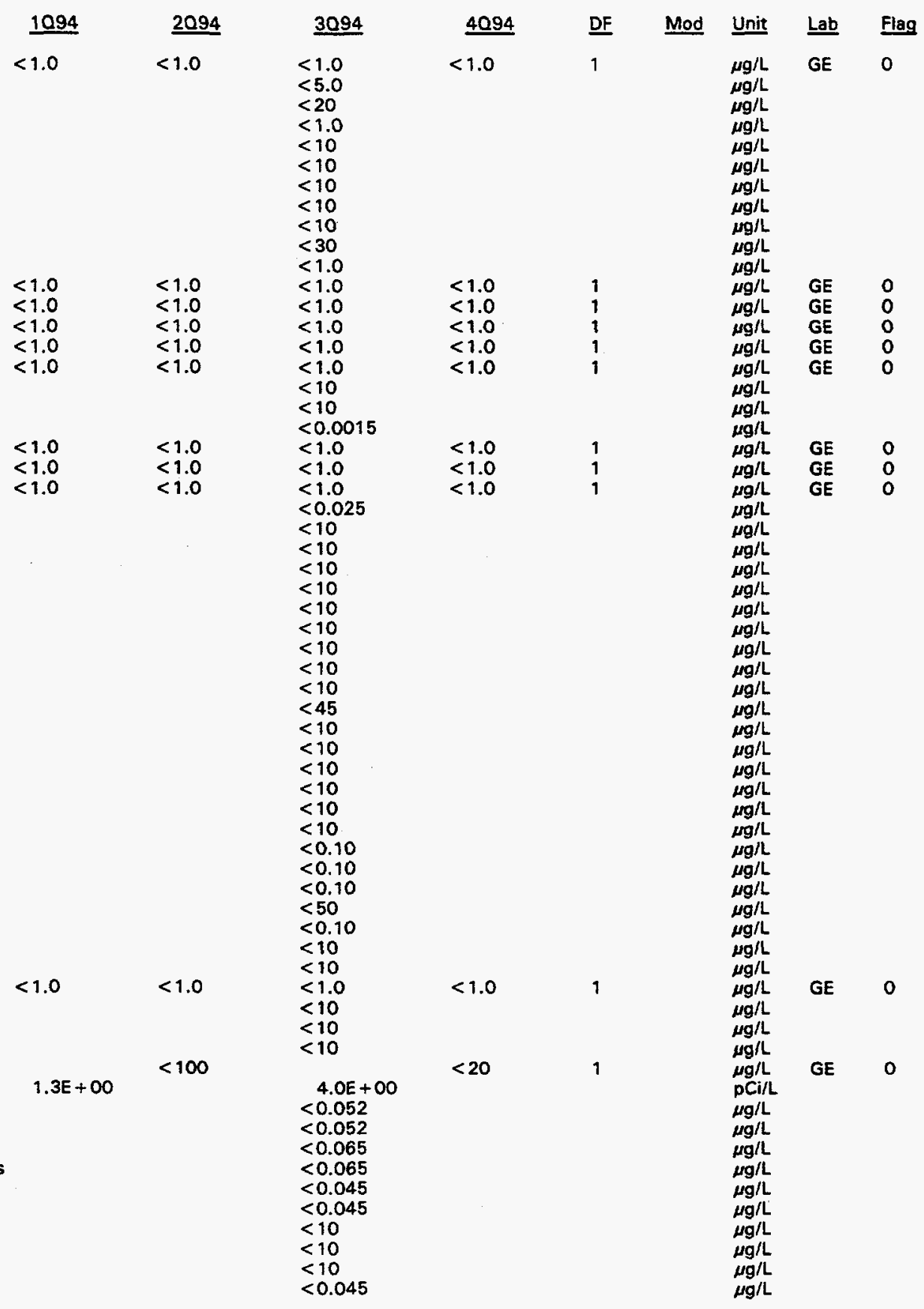

Note: Flagging, dilution factors, modifiers, and laboratories are for fourth quarter 1994 data only. See Appendix B for flagging criteria. $-=$ exceeded holding time for fourth quarter 1994

- = exceeded screening level or final PDWS for fourth quarter 1994. 


\section{H ST Analyte}

Hexachlorodibenzo-p-dioxin isomers $1,2,3,4,7,8-\mathrm{HXCDF}$

Hexachlorodibenzo-p-furan isomers

Hexachloroethane

Hexachlorophene

Hexachloropropene

2-Hexanone

Indenol $1,2,3-c$, olpyrene

lodomethane (Methyl iodide)

Iron, total recoverable

isobutyl alcohol

Isodrin

isophorone

Isosafrole

Kepone

Lead, total recoverable

Lindane

Manganese, total recoverable

Mercury, total recoverable

Methacrylonitrile

Methapyrilene

Methoxychlor

2-Methyl-4,6-dinitrophenol

Methyl ethyl ketone

Methyl isobutyl ketone

Methyl methacrylate

Methyl methanesulfonate

3-Methylcholanthrene

2-Methyinaphthalene

Naphthalene

1.4-Naphthoquinone

1-Naphthylamine

2-Naphthylamine

Nickel, total recoverable

Nitrate as nitrogen

Nitrate-nitrite as nitrogen

o-Nitroaniline

m-Nitroaniline

p-Nitroaniline

Nitrobenzene

2-Nitrophenol

4-Nitrophenol

4-Nitroquinoline-1-oxide

$\mathrm{N}$-Nitrosodi-n-butylamine

N-Nitrosodiethylamine

N-Nitrosodimethylamine

$\mathrm{N}$-Nitrosodiphenylamine

$\mathrm{N}$-Nitrosodipropylamine

N-Nitrosomethylethylamine

N-Nitrosomorpholine

$\mathrm{N}$-Nitrosopiperidine

$\mathrm{N}$-Nitrosopyrrolidine

5-Nitro-o-toluidine

Nonvolatile beta

$0,0,0$-Triethyl phosphorothioate

Octachlorodibenzo-p-dioxin

Octachlorodibenzo-p-furan

Parathion

Parathion methyl

PCB 1016

PCB 1221

PC8 1232

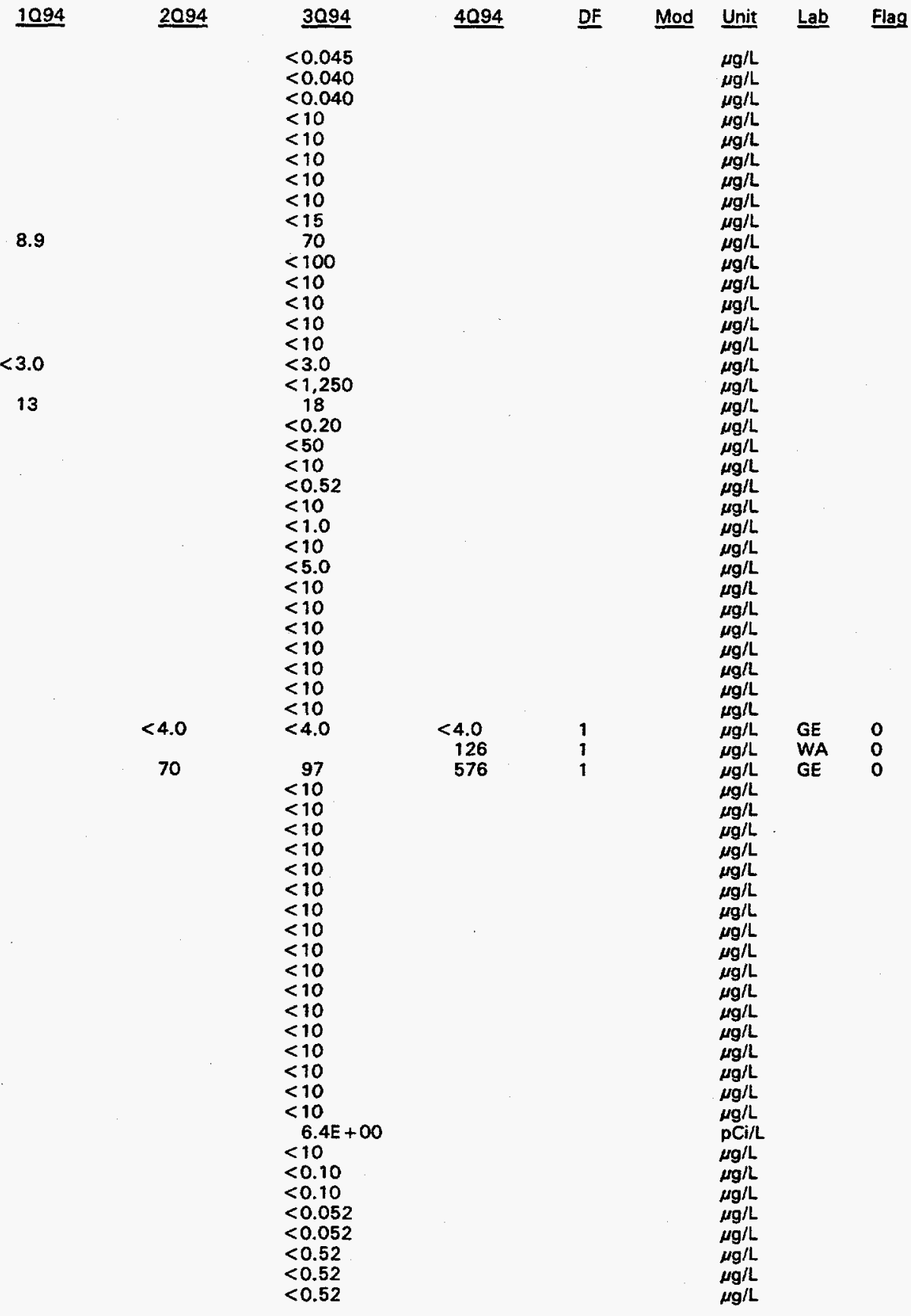

Note: Flagging, dilution factors, modifiers, and laboratories are for fourth quarter 1994 data only. See Appendix B for flagging criteria. - = exceeded holding time for fourth quarter 1994.

- = exceeded screening level or final PDWS for fourth quarter 1994. 
ANALYTICAL DATA

H ST Analvate

PCB 1242

РCB 1248

PCB 1254

PCB 1260

Pentachlorobenzene

$1,2,3,7,8-P C D D$

Pentachlorodibenzo-p-dioxin isomers

$1,2,3,7,8 \cdot P C D F$

Pentachloroethane

Pentachloronitrobenzene

Pentachlorophenol

Phenacetin

Phenanthrene

Phenol

p-Phenylenediamine

Phorate

2-Picoline

Pronamid

Propionitrile

Pyrene

Pyridine

Radium, total alpha-emitting

Radium-226

Radium-228

Safrole

Selenium, total recoverable

Silver, total recoverable

Styrene

Sulfide

Sulfotepp

1,2,4,5-Tetrachlorobenzene

2,3,7,8-TCDD

$2,3,7,8-\mathrm{TCDF}$

Tetrachlorodibenzo-p-dioxin isomers

Tetrachlorodibenzo-p-furan isomers

1,1,1,2-Tetrachloroethane

1,1,2,2-Tetrachloroethane

Tetrachioroethyiene

2,3,4,6-Tetrachlorophenol

Thallium, total recoverable

Thionazin

Tin, total recoverable

Toluene

o-Toluidine

Total organic carbon

Total organic halogens

Toxaphene

2,4,5-TP (Silvex)

1,2,4-Trichlorobenzene

$1,1,1-$ Trichloroethane

1,1,2-Trichloroethane

Trichloroethylene

Trichlorofluoromethane

2,4,5-Trichlorophenol

2,4,6-Trichlorophenol

2,4,5-T

1,2,3-Trichloropropane

1,3,5-Trinitrobenzene

Vanadium, total recoverable

Vinyl acetate

Xylenes

Zinc, total recoverable
1094

$\underline{2094}$

3094

$<0.52$

$<0.52$

$<0.52$

$<0.52$

$<10$

$<0.055$

$<0.055$

$<0.055$

$<10$

$<10$

$<10$

$<10$

$<10$

$<10$

$<10$

$<0.10$

$<10$
$<10$

$<200$

$<10$

$<10$

$1.3 E+00<1.0 E-01$

5.0E-01

$<10$

$<2.0$

$<2.0$

$<2.0$

$<1,000$

$<10$

$<10$

$<0.045$

$<0.040$

$<0.045$

$<0.040$

$<1.0$

$<1.0$

$<1.0$

$<10$

$<2.0$

$<10$

$<2.0$

$<1.0$

$<10$

$<1,000$

$<5.0$

$<0.25$

$<0.00046$

$<10$

$<1.0$

$<1.0$

$<1.0$

$<1.0$

$<10$

$<10$

$<0.00046$

$<1.0$

$<10$

$<8.0$

$<10$

$<2.0$

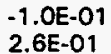

2.6E-01

$\mu \mathrm{g} /$

$\mu g / L$

$\mu \mathrm{g} / \mathrm{L}$

$\mu \mathrm{g} / \mathrm{L}$

$\mu \mathrm{g} / \mathrm{L}$

$\mu g / L$

$\mu g / L$

$\mu g h$

$\mu g / L$

$\mu \mathrm{rg} / \mathrm{L}$

$\mu g / L$

$\mu g / L$

$\mu g / L$

$\mu g / L$

$\mu g / L$

$\mu g / h$

$\mu g / L$

$\mu g / L$

DCi/L GP 0

$\begin{array}{lll}\mathrm{PC} \text { Ci/L } & T M & 0 \\ \mathrm{PCI} / \mathrm{L} & \mathrm{TM} & 0\end{array}$

$\mu g / L$

ugll

$\mu g / L$

$<2.0 \quad 1$

GE 0

$<1.0$

$<1.0$

1

\section{$<1.0$}

1,200

19

$\mu g / L$

$\mu g / h$

$\mu g /$

$\mu g / L$

$\mu g / L$

$\mu g / L$

$\mu g / L$

$\mu g / L$

$\mu g / L$

$\mu \mathrm{g} /$

$\mu g / L$

$\mu \mathrm{g} / \mathrm{L}$

$\mu g / L$

$\mu \mathrm{g} / \mathrm{L}$

$\mu g / L$ GE 0

$\mu g / L$

$\mu g / L$

$\mu g / L$

$\mu \mathrm{g} / \mathrm{L}$

$\mu g / L$

$\mu g / L$

$\mu g / L$

$\mu g / L$

$\mu g / L$

$\mu g / \mathrm{L}$

$\mu g / L$

$\mu g / L$

$\mu g / L$

$\mu g / L$

$\mu g / L$

$\mu g / L$

$\mu g / L$

$\mu g / L$

Note: Flagging, dilution factors, modifiers, and laboratories are for fourth quarter 1994 data only. See Appendix B for flagging criteria. - = exceeded holding time for fourth quarter 1994.

- = exceeded screening level or final PDWS for fourth quarter 1994. 
WELL AMB 10D

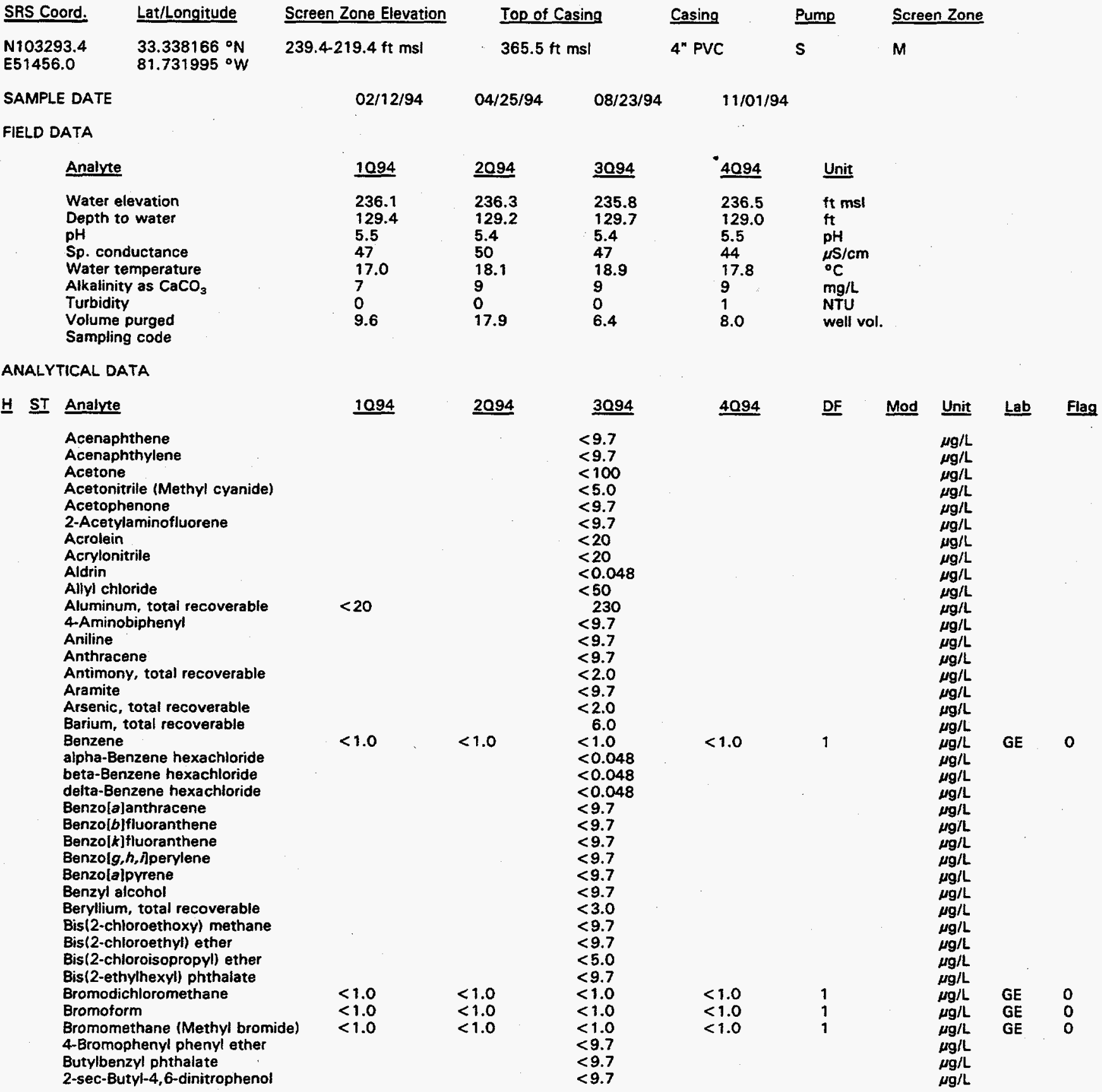

Note: Flagging, dilution factors, modifiers, and laboratories are for fourth quarter 1994 data only. See Appendix B for flagging criteria. - = exceeded holding time for fourth quarter 1994.

- = exceeded screening level or final PDWS for fourth quarter 1994. 


\section{H ST Analyte}

Cadmium, total recoverable

Carbon disulfide

Carbon tetrachloride

Chlordane

Chloride

4-Chloroaniline

Chlorobenzene

Chlorobenzilate

4-Chloro-m-cresol

Chloroethane

Chloroethene Rinyl chloride)

2-Chloroethyl vinyl ether

Chloroform

Chloromethane (Methyl chloride)

2-Chloronaphthalene

2-Chlorophenol

4-Chlorophenyl phenyl ether

Chloroprene

Chromium, total recoverable

Chrysene

Cobalt, total recoverable

Copper, total recoverable

o-Cresol (2-Methylphenol)

$m, p$-Cresol

Cyanide

$p, p^{\prime}-D D D$

$p, p^{\prime}-D D E$

$p, p^{\prime}-D D T$

Diallate

Dibenz $[a, h]$ anthracene

Dibenzofuran

Dibromochloromethane

1.2-Dibromo-3-chloropropane

1,2-Dibromoethane

Dibromomethane

Di-n-butyl phthalate

1,2-Dichlorobenzene

1,3-Dichlorobenzene

1,4-Dichlorobenzene

$3,3^{\prime}$-Dichlorobenzidine

trans-1,4-Dichloro-2-butene

Dichlorodifluoromethane

1,1-Dichloroethane

1.2-Dichloroethane

1,1-Dichloroethylene

trans-1,2-Dichloroethylene

Dichloromethane

2,4-Dichlorophenol

2,6-Dichlorophenol

2,4-Dichlorophenoxyacetic acid

1,2-Dichloropropane

cis-1.3-Dichloropropene

trans-1,3-Dichloropropene

Dieldrin

Diethyl phthalate

Dimethoate

2.4-Dimethyl phenol

Dimethyl phthalate

p-Dimethylaminoazobenzene

7,12-Dimethylbenzlalanthracene

3,3'-Dimethylbenzidine

a,a-Dimethylphenethylamine

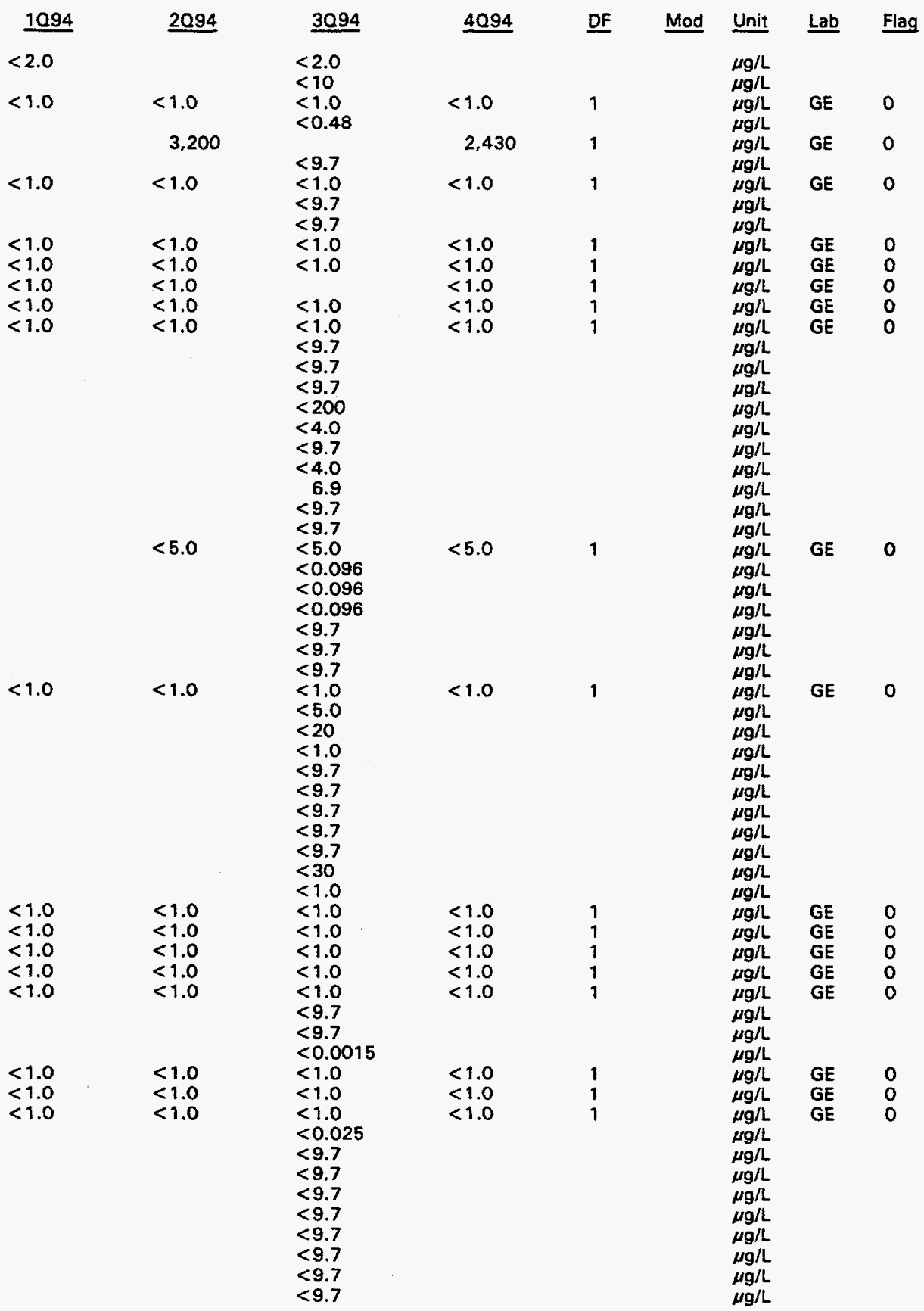

Note: Flagging, dilution factors, modifiers, and laboratories are for fourth quarter 1994 data only. See Appendix B for flagging criteria. - = exceeded holding time for fourth quarter 1994.

a = exceeded screening level or final PDWS for fourth quarter 1994. 
Well AMB 100 continued

ANALYTICAL DATA

H ST Analyte

1,3-Dinitrobenzene

2,4-Dinitrophenol

2,4-Dinitrotoluene

2,6-Dinitrotoluene

Di-n-octyl phthalate

1,4-Dioxane

Diphenylamine

Disulfoton

Endosulfan

Endosulfan II

Endosulfan sulfate

Endrin

Endrin aldehyde

Ethyl methacrylate

Ethyl methanesulfonate

Ethyibenzene

Famphur

Fluoranthene

Fluorene

Fluoride

Gross alpha

Heptachlor

Heptachlor epoxide

$1,2,3,4,6,7,8$-HPCDD

Heptachlorodibenzo-p-dioxin isomers

$1,2,3,4,6,7,8-\mathrm{HPCDF}$

Heptachlorodibenzo-p-furan isomers

Hexachlorobenzene

Hexachlorobutadiene

Hexachlorocyclopentadiene

$1,2,3,4,7,8-\mathrm{HXCDD}$

Hexachlorodibenzo-p-dioxin isomers

$1,2,3,4,7,8-H \times C D F$

Hexachlorodibenzo-p-furan isomers

Hexachloroethane

Hexachlorophene

Hexachloropropene

2-Hexanone

Indenol 1,2,3-c, of pyrene

lodomethane (Methyl iodide)

Iron, total recoverable

Isobutyl alcohol

Isodrin

Isophorone

Isosafrole

Kepone

Lead, total recoverable

Lindane

Manganese, total recoverable

Mercury, total recoverable

Methacrylonitrile

Methapyrilene

Methoxychlor

2-Methyl-4,6-dinitrophenol

Methyl ethyl ketone

Methyl isobutyl ketone

Methyl methacrylate

Methy! methanesulfonate

3-Methylcholanthrene

2-Methyinaphthalene

Naphthalene

1,4-Naphthoquinone $\underline{2094}$

3094

$<44$

$<9.7$

$<9.7$

$<9.7$

$<9.7$

$<9.7$

$<9.7$

$<0.096$

$<0.096$

$<0.096$

$<0.09$

$<9.7$

$<9.7$

$<1.0$

$<9.7$

$<9.7$

$<9.7$

9.4E-01

$<100$

$1.2 E+\infty$

$<0.048$

$<0.048$

$<0.065$

$<0.065$

$<0.045$

$<0.045$

$<9.7$

$<9.7$

$<9.7$

$<0.045$

$<0.045$

$<0.040$

$<0.040$

$<9.7$

$<9.7$

$<9.7$

$<10$

$<9.7$

$<9.7$
$<15$
25

12

$<100$

$<9.7$

$<9.7$

$<9.7$

$<9.7$

$<3.0$

$<1,250$

5.0

$<0.20$

$<50$

$<9.7$

$<0.48$

$<9.7$

$<1.0$

$<10$

$<5.0$

$<9.7$

$<9.7$

$<9.7$

$<9.7$

$<9.7$ $\underline{4094}$

DF

Mod

Un
$\mu g / L$
$\mu g / L$
$\mu g / L$
$\mu g / L$
$\mu g / L$
$\mu g /$
$\mu g / L$
$\mu g / L$
$\mu g / L$
$\mu g / L$
$\mu g$
$\mu g / L$

$<1.0$

Lab Flag

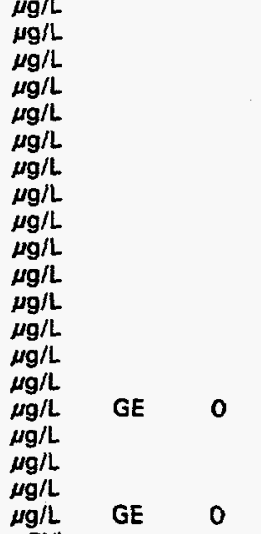

$<20$

pCi/L

pg/L

$\mu g / L$

$\mu g / L$

$\mu g / L$

$\mu \mathrm{g} / \mathrm{L}$

$\mu \mathrm{g} / \mathrm{h}$

$\mu g / L$

$\mu \mathrm{g} / \mathrm{L}$

$\mu g / L$

$\mu g / L$

$\mu g / L$

$\mu \mathrm{g} / \mathrm{L}$

$\mu \mathrm{g} / \mathrm{L}$

$\mu g /$

$\mu \mathrm{g} / \mathrm{L}$

$\mu \mathrm{g} / \mathrm{L}$

$\mu g / L$

$\mu \mathrm{g} / \mathrm{L}$

$\mu g / L$

$\mu g / L$

$\mu g / L$

$\mu g /$

$\mu g / L$

$\mu g / L$

$\mu \mathrm{g} / \mathrm{L}$

ug/l

$\mu g / L$

$\mu g / L$

$\mu g / L$

$\mu g / L$

$\mu \mathrm{gg} / \mathrm{L}$

$\mu g / L$

$\mu g / L$

$\mu g / L$

$\mu g / L$

$\mu g / L$

$\mu g / L$

ugil

Note: Flagging, dilution factors, modifiers, and laboratories are for fourth quarter 1994 data only. See Appendix B for flagging criteria. - = exceeded holding time for fourth quarter 1994.

- = exceeded screening level or final PDWS for fourth quarter 1994. 
1-Naphthylamine

2-Naphthylamine

Nickel, total recoverable

Nitrate-nitrite as nitrogen

o-Nitroaniline

m-Nitroaniline

p-Nitroaniline

Nitrobenzene

2-Nitrophenol

4-Nitrophenol

4-Nitroquinoline-1-oxide

N-Nitrosodi-n-butylamine

$\mathrm{N}$-Nitrosodiethylamine

$\mathrm{N}$-Nitrosodimethylamine

$\mathrm{N}$-Nitrosodiphenylamine

$\mathrm{N}$-Nitrosodipropylamine

$\mathrm{N}$-Nitrosomethylethylamine

$\mathrm{N}$-Nitrosomorpholine

N-Nitrosopiperidine

$\mathrm{N}$-Nitrosopytrolidine

5-Nitro-o-toluidine

Nonvolatile beta

0.0.0-Triethyl phosphorothioate

Octachlorodibenzo-p-dioxin

Octachlorodibenzo-p-furan

Parathion

Parathion methyl

PCB 1016

PCB 1221

PCB 1232

PCB 1242

PCB 1248

PCB 1254

PCB 1260

Pentachlorobenzene

1,2,3,7.8-PCDD

Pentachlorodibenzo-p-dioxin isomers

$1,2,3,7,8$-PCDF

Pentachloroethane

Pentachloronitrobenzene

Pentachlorophenol

Phenacetin

Phenanthrene

Phenol

p-Phenylenediamine

Phorate

2-Picoline

Pronamid

Propionitrile

Pyrene

Pyridine
Radium, total alpha-emitting

Safrole

Selenium, total recoverable

Silver, total recoverable

Styrene

Sulfide

Sulfotepp

1,2,4,5-Tetrachlorobenzene

2,3,7,8-TCDD

$2,3,7,8$-TCDF

Tetrachlorodibenzo-p-dioxin isomers
1094

$\underline{2094}$

$<9.7$

$<9.7$

$<4.0$

100

$<4.0$

103

$<9.7$

$<9.7$

$<9.7$

$<9.7$

$<9.7$

$<9.7$

$<9.7$

$<9.7$

$<9.7$

$<9.7$

$<9.7$

$<9.7$

$<9.7$

$<9.7$

$<9.7$

$<9.7$

$<9.7$

$1.2 E+00$

$<9.7$

$<0.10$

$<0.10$

$<0.048$

$<0.048$

$<0.48$

$<0.48$

$<0.48$

$<0.48$

$<0.48$

$<0.48$

$<0.48$

$<9.7$

$<0.055$

$<0.055$

$<0.055$

$<9.7$

$<9.7$

$<9.7$

$<9.7$

$<9.7$

$<9.7$

$<9.7$

$<0.096$

$<9.7$

$<9.7$

$<200$

$<9.7$

$<9.7$

$1.3 E+00$

1. $0 E+00$

1.7E + 00

$<9.7$

$<2.0$

$<2.0$

$<10$

$<1,000$

$<9.7$

$<9.7$

$<0.045$

$<0.040$

$<0.045$

\begin{tabular}{|c|c|c|c|c|}
\hline 4094 & DF & Mod & Unit & Lab \\
\hline & & & $\begin{array}{l}\mu g / L \\
\mu g / L\end{array}$ & \\
\hline $\begin{array}{c}<4.0 \\
82\end{array}$ & 1 & $J$ & $\mu g / L$ & $\begin{array}{l}\mathrm{GE} \\
\mathrm{GE}\end{array}$ \\
\hline
\end{tabular}

$\mu g / L$ GE

$\mu \mathrm{g} / \mathrm{L}$

$\mu g / L$

$\mu g / L$

$\mu g / L$

$\mu \mathrm{g} / \mathrm{L}$

$\mu \mathrm{g} / \mathrm{L}$

$\mu g / L$

$\mu \mathrm{g} / \mathrm{L}$

$\mu g / L$

$\mu g / L$

$\mu g / L$

$\mu \mathrm{g} / \mathrm{L}$

$\mu \mathrm{g} / \mathrm{L}$

$\mu g / L$

$\mu g$ 닌

$\mu \mathrm{g} / \mathrm{L}$

$\mu \mathrm{g} / \mathrm{L}$

$\mu \mathrm{g} / \mathrm{L}$

$\mu g / L$

$\mu \mathrm{g} / \mathrm{L}$

$\mu g / L$

$\mu \mathrm{g} / \mathrm{L}$

$\mu g / \mathrm{L}$

$\mu g / L$

$\mu g / L$

$\mu \mathrm{g} / \mathrm{L}$

$\mu g / L$

$\mu \mathrm{g} / \mathrm{L}$

$\mu \mathrm{g} / \mathrm{L}$

$\mu g /$

$\mu \mathrm{g} / \mathrm{L}$

$\mu \mathrm{g} / \mathrm{L}$

$\mu \mathrm{g} / \mathrm{L}$

$\mu / 2$

$\mu \mathrm{g} / \mathrm{L}$

$\mu \mathrm{g} / \mathrm{L}$

$\mu g / h$

$\mu \mathrm{g} / \mathrm{L}$

$\mu g / L$

$\mu g / L$

$\mu g / L$

$\mathrm{pCi} / \mathrm{L}$
$\mu \mathrm{g} / \mathrm{L}$

$\mu g / L$

$\mu g / L$

$\mu \mathrm{g} / \mathrm{L}$

$\mu \mathrm{g} / \mathrm{L}$

$\mu g / L$

$\mu g / L$

$\mu \mathrm{g} / \mathrm{L}$

$\mu g / L$

$\mu g / L$

Note: Flagging, dilution factors, modifiers, and laboratories are for fourth quarter 1994 data only. See Appendix B for flagging criteria. - = exceeded holding time for fourth quarter 1994.

- = exceeded screening level or final PDWS for fourth quarter 1994. 
Well AMB 100 continued

ANALYTICAL DATA

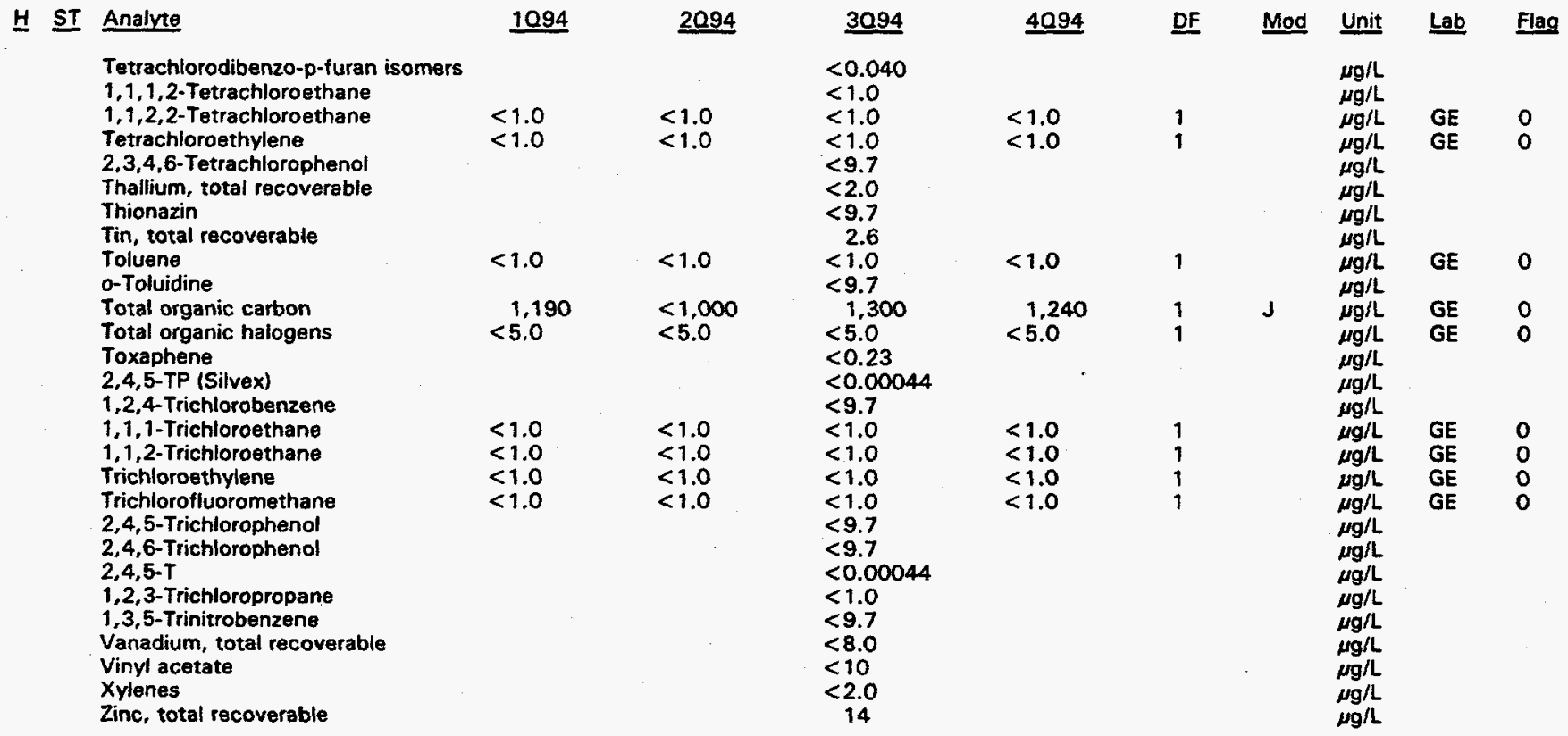

\section{WELL AMB 10DD}

$\begin{array}{lll}\text { SRS Coord. } & \text { Lat/Longitude } & \text { Screen Zone Elevation } \\ \text { N103278.7 } & \begin{array}{ll}33.338133^{\circ} \mathrm{N} & 358.6-338.6 \mathrm{ft} \mathrm{msl} \\ \text { E51456.0 } & 81.731966^{\circ} \mathrm{W}\end{array}\end{array}$

$\begin{array}{llll}\text { Top of Casing } & \text { Casing } & \text { Pump } & \text { Screen Zone } \\ 365.4 \mathrm{ft} \mathrm{msl} & 4^{n \text { PVC }} & \mathrm{s} & \mathrm{M}\end{array}$

SAMPLE DATE

$02 / 12 / 94$

$04 / 25 / 94$

$08 / 23 / 94$

$11 / 01 / 94$

FIELD DATA

Analyte
Water elevation
Depth to water
pH
Sp. conductance
Water temperature
Alkalinity as $\mathrm{CaCO}_{3}$
Turbidity
Volume purged
Sampling code

ANALYTICAL DATA

H ST Analyte

Aluminum, total recoverable Barium, total recoverable

Benzene

Bromodichloromethane

Bromoform

Bromomethane (Methyl bromide)

Cadmium, total recoverable

1094
359.2
6.2
6.4
136
16.0
65
1
11.3

1094

$<20$

$<1.0$

$<1.0$

$<1.0$

$<1.0$

$<2.0$

2094
359.1
6.3
6.2
126
17.2
54
1
13.9

3094
359.3
6.1
6.2
134
20.7
41
1
8.4

\section{4}

359.3

6.1
6.4

139

20.0

65

1.6

7.6 NTU

13.9

$\begin{aligned} & 2094 \\ & \\ & <1.0 \\ < & 1.0 \\ < & 1.0 \\ < & 1.0\end{aligned}$

$\begin{aligned} & 4094 \\ &<1.0 \\ &< 1.0 \\ &<1.0 \\ &<1.0\end{aligned}$

Unit
ft msl
$\mathrm{ft}$
pH
pS/cm
${ }^{\circ} \mathrm{C}$
$\mathrm{mg} / \mathrm{L}$
$\mathrm{NTU}$
well vol.

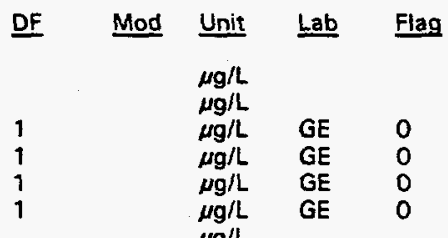

Note: Flagging, dilution factors, modifiers, and laboratories are for fourth quarter 1994 data only. See Appendix B for flagging criteria.

- exceeded holding time for fourth quarter 1994.

a = exceeded screening level or final PDWS for fourth quarter 1994. 


\section{H ST Analyte}

Carbon tetrachloride

Chloride

Chlorobenzene

Chloroethane

Chloroethene (Vinyl chloride)

2-Chloroethyl vinyl ether

Chloroform

Chloromethane (Methyl chloride)

Cobalt, total recoverable

Copper, total recoverable

Cyanide

Dibromochloromethane

1,1-Dichloroethane

1,2-Dichloroethane

1,1-Dichloroethylene

trans-1,2-Dichloroethylene

Dichloromethane

1,2-Dichloropropane

cis-1,3-Dichloropropene

trans-1,3-Dichloropropene

Ethylbenzene

Fluoride

Gross alpha

Iron, total recoverable

Lead, total recoverable

Manganese, total recoverable

Nickel, total recoverable

Nitrate as nitrogen

Nitrate-nitrite as nitrogen

Nonvolatile beta

Radium, total alpha-emitting

Radium-226

Radium-228

Selenium, total recoverable

Silver, total recoverable

1,1,2,2-Tetrachloroethane

Tetrachloroethylene

Toluene

Total organic carbon

Total organic halogens

1,1,1-Trichloroethane

1,1,2-Trichloroethane

Trichloroethylene

Trichlorofluoromethane

Zinc, total recoverable

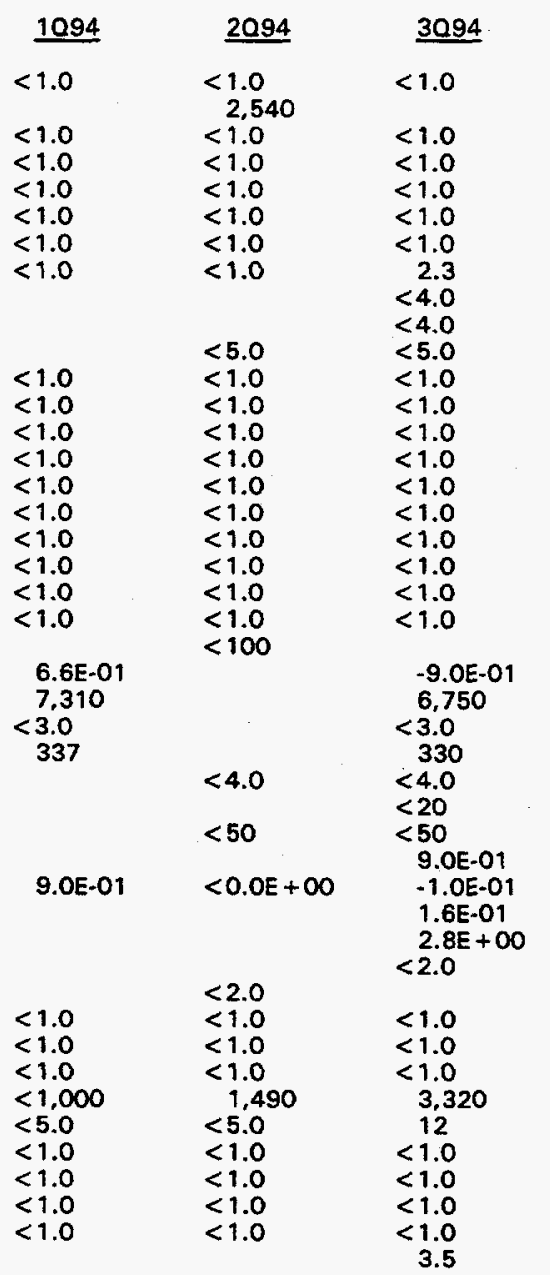

Mod

$<1.0$

1,410

$<1.0$

$<1.0$

$<1.0$

$<1.0$

$<1.0$

$<5.0$

$<1.0$

$<1.0$

$<1.0$

$<1.0$

$<1.0$

$<1.0$

$<1.0$

$<1.0$

$<1.0$

$<1.0$

$<20$

$<4.0$

$<50$

1.OE-O1

1.OE-O

$<2.0$

$<1.0$

$<1.0$

$<1.0$

2,880

$<5.0$

$<1.0$

$<1.0$

$<1.0$

$<1.0$

DF Mod
1
1
1
1
1
1
1
1

1
1
1
1
1
1
1
1
1
1
1

\begin{tabular}{lll} 
Unit & Lab & Flag \\
\cline { 1 - 1 }$\mu \mathrm{g} / \mathrm{L}$ & GE & 0 \\
$\mu \mathrm{g} / \mathrm{L}$ & $\mathrm{GE}$ & 0 \\
$\mu \mathrm{g} / \mathrm{L}$ & $\mathrm{GE}$ & 0 \\
$\mu \mathrm{g} / \mathrm{L}$ & $\mathrm{GE}$ & 0 \\
$\mu \mathrm{g} / \mathrm{L}$ & $\mathrm{GE}$ & 0 \\
$\mu \mathrm{g} / \mathrm{L}$ & $\mathrm{GE}$ & 0 \\
$\mu \mathrm{g} / \mathrm{L}$ & $\mathrm{GE}$ & 0 \\
$\mu \mathrm{g} / \mathrm{L}$ & $\mathrm{GE}$ & 0 \\
$\mu \mathrm{g} / \mathrm{L}$ & &
\end{tabular}

$\mu \mathrm{rg} / \mathrm{L}$

$\mu g / L \quad G E$

$\mu \mathrm{g} / \mathrm{L} \quad \mathrm{GE}$

$\mu \mathrm{g} / \mathrm{L} \quad \mathrm{GE}$

$\mu \mathrm{g} / \mathrm{L} \quad \mathrm{GE}$

$\mu g / L \quad G E$

$\mu g / L \quad G E$

$\mu \mathrm{g} / \mathrm{L}$ GE

$\mu g /$ GE

$\mu \mathrm{g} / \mathrm{L} \quad \mathrm{GE}$

g/L GE

$\mu \mathrm{g} / \mathrm{L} \quad \mathrm{GE}$

$\mathrm{pCi} / \mathrm{L}$

$\mu \mathrm{gg} / \mathrm{L}$

$\mu g / L$

$\mu \mathrm{g} / \mathrm{L}$

$\mu \mathrm{g} / \mathrm{L}$

$\mu g / L$

pCill

$\mathrm{pCi} / \mathrm{L}$
$\mathrm{pCi} / \mathrm{L}$

pCi/L

$\mu \mathrm{g} / \mathrm{L}$

$\mu g / L$

$\mu g / L$

$\mu \mathrm{gg} / \mathrm{L}$

$\mu g / L$

$\mu g / L$

$\mu \mathrm{g} / \mathrm{L}$

$\mu g / L$

$\mu g / L$

$\mu \mathrm{gg} / \mathrm{L}$

$\mu \mathrm{g} / \mathrm{L}$

\section{GE 0}

GE 0

GP 0

GE 0

GE 0

0 
WELL AMB 11B

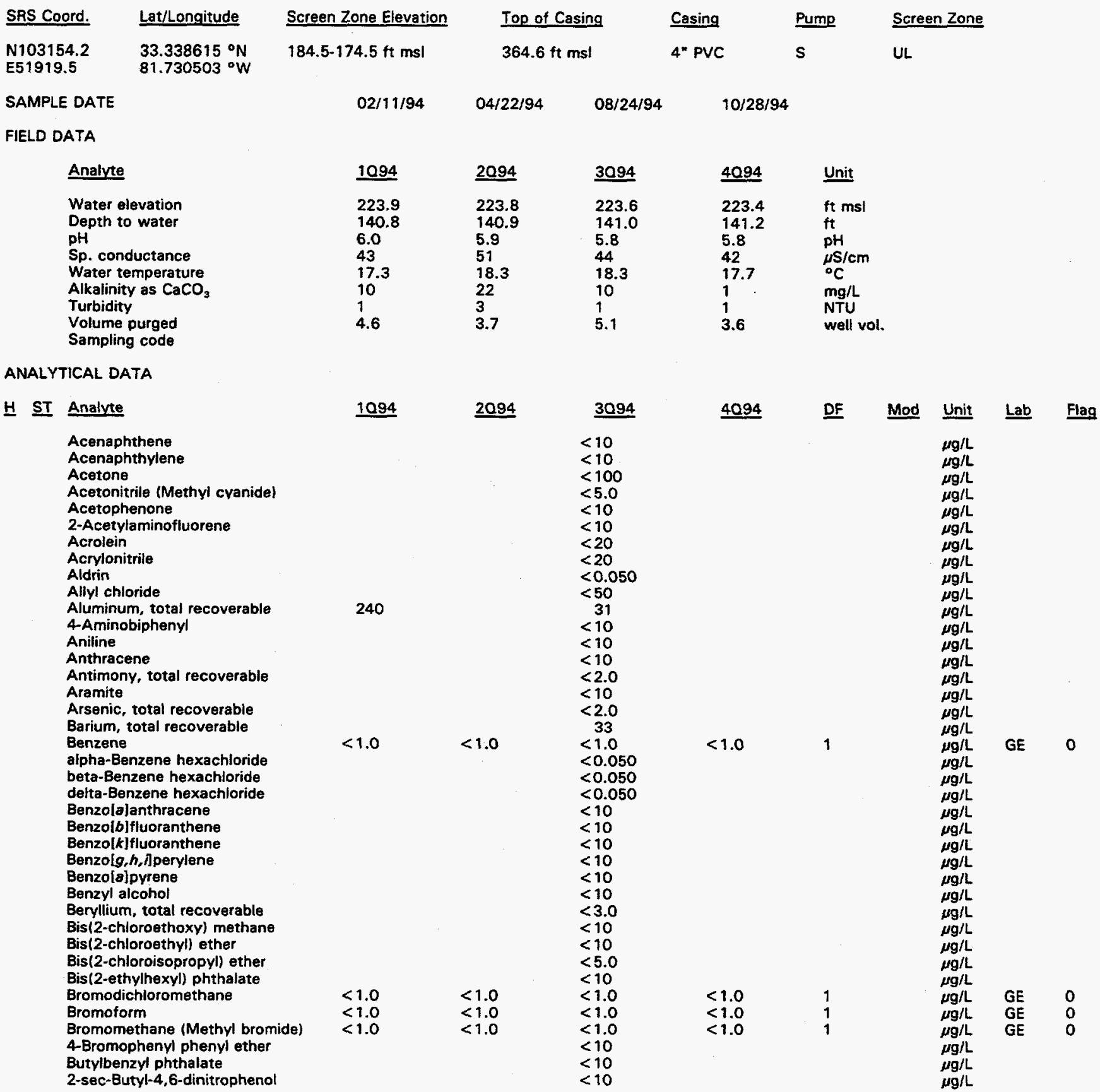

Note: Flagging, dilution factors, modifiers, and laboratories are for fourth quarter 1994 data only. See Appendix B for flagging criteria. - = exceeded holding time for fourth quarter 1994.

- = exceeded screening level or final PDWS for fourth quarter 1994. 


\section{H ST Analyte}

Cadmium, total recoverable

Carbon disulfide

Carbon tetrachloride

Chlordane

Chloride

4Chloroaniline

Chlorobenzene

Chlorobenzilate

4-Chloro-m-cresol

Chloroethane

Chloroethene (Vinyl chloride)

2-Chloroethyl vinyl ether

Chloroform

Chloromethane (Methyl chloride)

2-Chloronaphthalene

2-Chlorophenol

4-Chlorophenyl phenyl ether

Chloroprene

Chromium, total recoverable

Chrysene

Cobalt, total recoverable

Copper, total recoverable

o-Cresol (2-Methylphenol)

$\mathrm{m}, \mathrm{p}$-Cresol

Cyanide

$p, p^{\prime}-D D D$

$p, p^{\prime}-D D E$

$p, p^{\prime}-D D T$

Diallate

Dibenzla,hlanthracene

Dibenzofuran

Dibromochtoromethane

1,2-Dibromo-3-chloropropane

1,2-Dibromoethane

Dibromomethane

Di-n-butyl phthalate

1,2-Dichlorobenzene

1.3-Dichlorobenzene

1,4-Dichlorobenzene

3,3'-Dichlorobenzidine

trans-1,4-Dichloro-2-butene

Dichlorodifluoromethane

1,1-Dichloroethane

1,2-Dichloroethane

1,1-Dichloroethylene

trans-1,2-Dichloroethylene

Dichloromethane

2,4-Dichlorophenol

2,6-Dichlorophenol

2,4-Dichlorophenoxyacetic acid

1,2-Dichloropropane

cis-1,3-Dichloropropene

trans-1,3-Dichloropropene

Dieldrin

Diethyl phthalate

Dimethoate

2,4-Dimethyl phena

Dimethyl phthalate

p-Dimethylaminoazobenzene

7,12-Dimethylbenzialanthracene

3,3'-Dimethylbenzidine

a,a-Dimethylphenethylamine

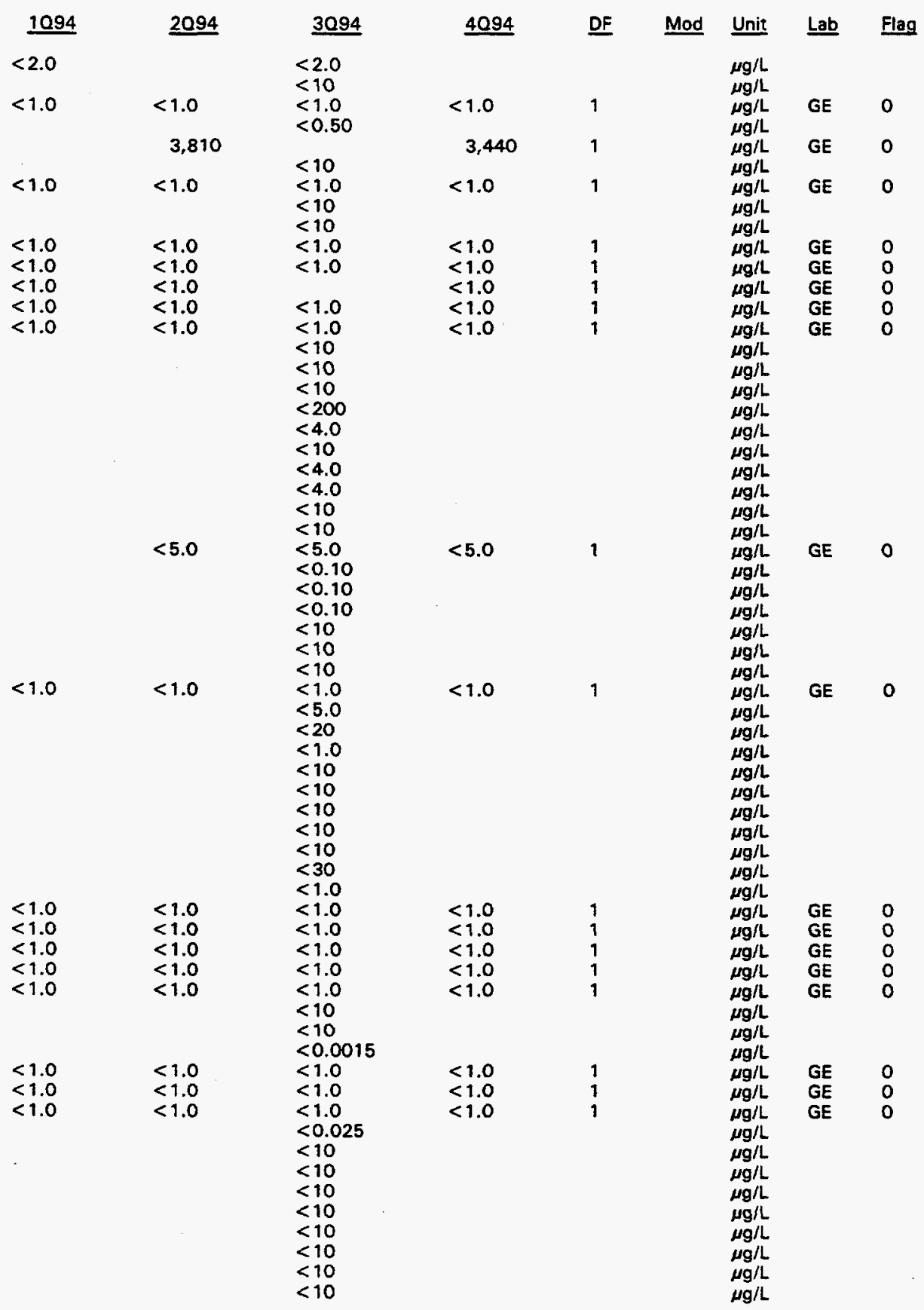

Note: Flagging, dilution factors, modifiers, and laboratories are for fourth quarter 1994 data only. See Appendix B for flagging criteria. - = exceeded holding time for fourth quarter 1994 .

- = exceeded screening level or final PDWS for fourth quarter 1994. 
Well AMB 118 continued

ANALYTICAL DATA

\section{Analyte}

1,3-Dinitrobenzene

2,4-Dinitrophenal

2,4-Dinitrotoluene

2,6-Dinitrotoluene

Di-n-octyl phthalate

1,4-Dioxane

Diphenylamine

Disulfoton

Endosulfan 1

Endosulfan II

Endosulfan sulfate

Endrin

Endrin aldehyde

Ethyl methacrylate

Ethyl methanesulfonate

Ethylbenzene

Famphur

Fluoranthene

Fluorene

Fluoride

Gross alpha

Heptachlor

Heptachlor epoxide

$1,2,3,4,6,7,8$-HPCDD

Heptachlorodibenzo-p-dioxin isomers

$1,2,3,4,6,7,8-H P C D F$

Heptachlorodibenzo-p-furan isomers

Hexachlorobenzene

Hexachlorobutadiene

Hexachlorocyclopentadiene

$1,2,3,4,7,8-H X C D D$

Hexachlorodibenzo-p-dioxin isomers

$1,2,3,4,7,8-H X C D F$

Hexachlorodibenzo-p-furan isomers

Hexachloroethane

Hexachlorophene

Hexachloropropene

2-Hexanone

Indeno[ $7,2,3-c, 0]$ pyrene

lodomethane (Methyl iodide)

Iron, total recoverable

Isobutyl aicohol

Isodrin

Isophorone

Isosafrole

Kepone

Lead, total recoverable

Lindane

Manganese, total recoverable

Mercury, total recoverable

Methacryionitrile

Methapyrilene

Methoxychlor

2-Methyl-4, 6-dinitrophenol

Methyl ethyl ketone

Methyl isobutyl ketone

Methyl methacrylate

Methyl methanesulfonate

3-Methylcholanthrene

2-Methylnaphthalene

Naphthalene

1,4-Naphthoquinone

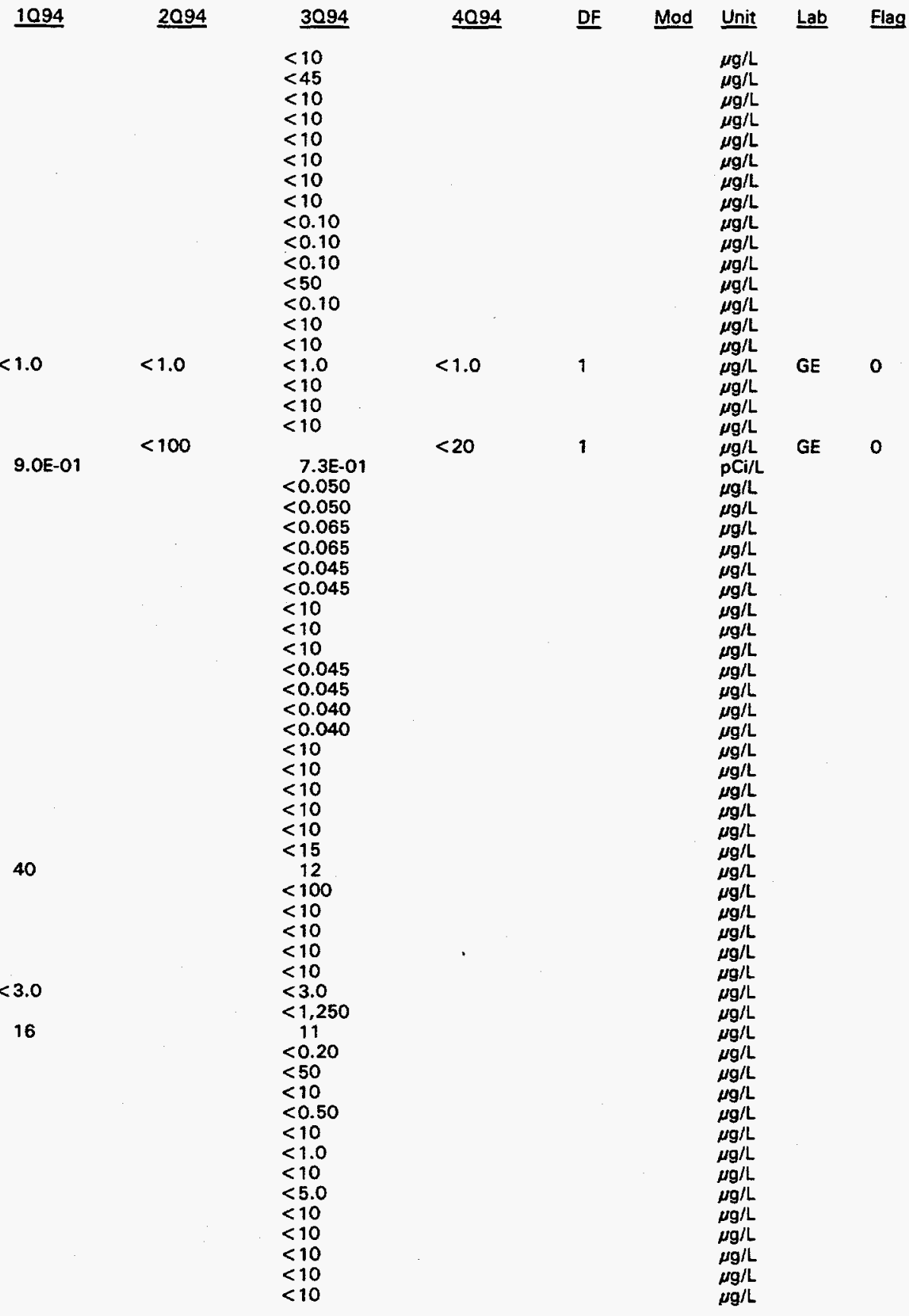

Note: Flagging, dilution factors, modifiers, and laboratories are for fourth quarter 1994 data only. See Appendix B for flagging criteria. - = exceeded holding time for fourth quarter 1994.

- = exceeded screening level or final PDWS for fourth quarter 1994. 


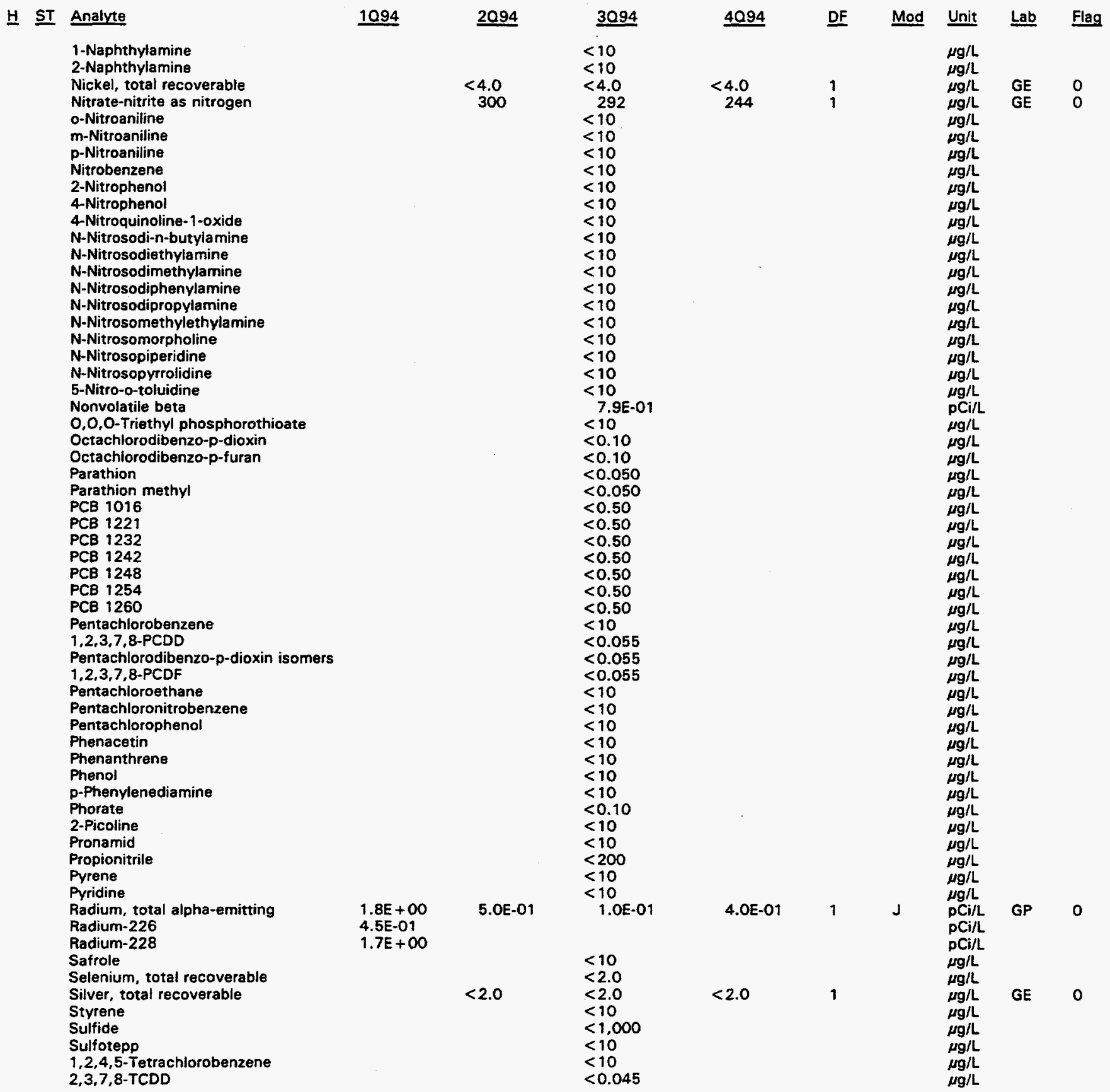

Note: Flagging, dilution factors, modifiers, and laboratories are for fourth quarter 1994 data only. See Appendix B for flagging criteria. - = exceeded holding time for fourth quarter 1994.

- = exceeded screening level or final PDWS for fourth quarter 1994. 
Well AMB 118 continued

ANALYTICAL DATA

H ST Analyte

2,3,7,8-TCDF

Tetrachlorodibenzo-p-dioxin isomers

Tetrachlorodibenzo-p-furan isomers

1,1,1,2-Tetrachloroethane

1,1,2,2-Tetrachloroethane

Tetrachloroethylene

2,3,4,6-Tetrachlorophenol

Thallium, total recoverable

Thionazin

Tin, total recoverable

Toluene

o-Toluidine

Total organic carbon

Total organic halogens

Toxaphene

2,4,5-TP (Silvex)

1,2,4-Trichlorobenzene

1,1,1-Trichloroethane

1,1,2-Trichloroethane

Trichloroethylene

Trichlorofluoromethane

2,4,5-Trichlorophenol

2,4,6-Trichlorophenol

2,4,5-T

1,2,3-Trichloropropane

1,3,5-Trinitrobenzene

Vanadium, total recoverable

Vinyl acetate

Xylenes

Zinc, total recoverable

\begin{tabular}{|c|c|c|c|c|c|c|c|c|}
\hline 1094 & 2094 & 3094 & 4094 & DF & Mod & Unit & Lab & Fiag \\
\hline & & $\begin{array}{l}<0.040 \\
<0.045 \\
<0.040 \\
<1.0\end{array}$ & & & & $\begin{array}{l}\mu g / L \\
\mu g / L \\
\mu g / L \\
\mu g / L\end{array}$ & & \\
\hline $\begin{array}{l}<1.0 \\
<1.0\end{array}$ & $\begin{array}{l}<1.0 \\
<1.0\end{array}$ & $\begin{array}{c}<1.0 \\
<1.0 \\
<10 \\
<2.0 \\
<10 \\
3.3\end{array}$ & $\begin{array}{l}<1.0 \\
<1.0\end{array}$ & $\begin{array}{l}1 \\
1\end{array}$ & & $\begin{array}{l}\mu g / L \\
\mu g / L \\
\mu g / L \\
\mu g / L \\
\mu g / L \\
\mu g / L\end{array}$ & $\begin{array}{l}\text { GE } \\
\text { GE }\end{array}$ & $\begin{array}{l}0 \\
0\end{array}$ \\
\hline$<1.0$ & $<1.0$ & $\begin{array}{l}<1.0 \\
<10\end{array}$ & $<1.0$ & 1 & & $\begin{array}{l}\mu \mathrm{g} / \mathrm{L} \\
\mu \mathrm{g} / \mathrm{L}\end{array}$ & GE & 0 \\
\hline $\begin{array}{c}<1,000 \\
12\end{array}$ & $\begin{array}{l}<1,000 \\
<5.0\end{array}$ & $\begin{array}{l}<1,000 \\
<5.0 \\
<0.24 \\
<0.00044 \\
<10\end{array}$ & $\begin{array}{l}1,120 \\
6.1\end{array}$ & $\begin{array}{l}1 \\
1\end{array}$ & $J$ & $\begin{array}{l}\mu g / L \\
\mu g / L \\
\mu g / L \\
\mu g / L \\
\mu g / L\end{array}$ & $\begin{array}{l}G E \\
G E\end{array}$ & $\begin{array}{l}0 \\
0\end{array}$ \\
\hline $\begin{array}{l}<1.0 \\
<1.0 \\
<1.0 \\
<1.0\end{array}$ & $\begin{array}{l}<1.0 \\
<1.0 \\
<1.0 \\
<1.0\end{array}$ & $\begin{aligned}<1.0 \\
<1.0 \\
<1.0 \\
<1.0 \\
<10 \\
<10 \\
<10 \\
<0.00044 \\
<1.0 \\
<10 \\
<8.0 \\
<10 \\
<2.0 \\
<1.5\end{aligned}$ & $\begin{array}{l}<1.0 \\
<1.0 \\
<1.0 \\
<1.0\end{array}$ & $\begin{array}{l}1 \\
1 \\
1 \\
1\end{array}$ & & $\begin{array}{l}\mu g / L \\
\mu g / L \\
\mu g / L \\
\mu g / L \\
\mu g / L \\
\mu g / L \\
\mu g / L \\
\mu g / L \\
\mu g / L \\
\mu g / L \\
\mu g / L \\
\mu g / L \\
\mu g / L\end{array}$ & $\begin{array}{l}\text { GE } \\
\text { GE } \\
\text { GE } \\
\text { GE }\end{array}$ & $\begin{array}{l}0 \\
0 \\
0 \\
0\end{array}$ \\
\hline
\end{tabular}

\section{WELL AMB 11D}

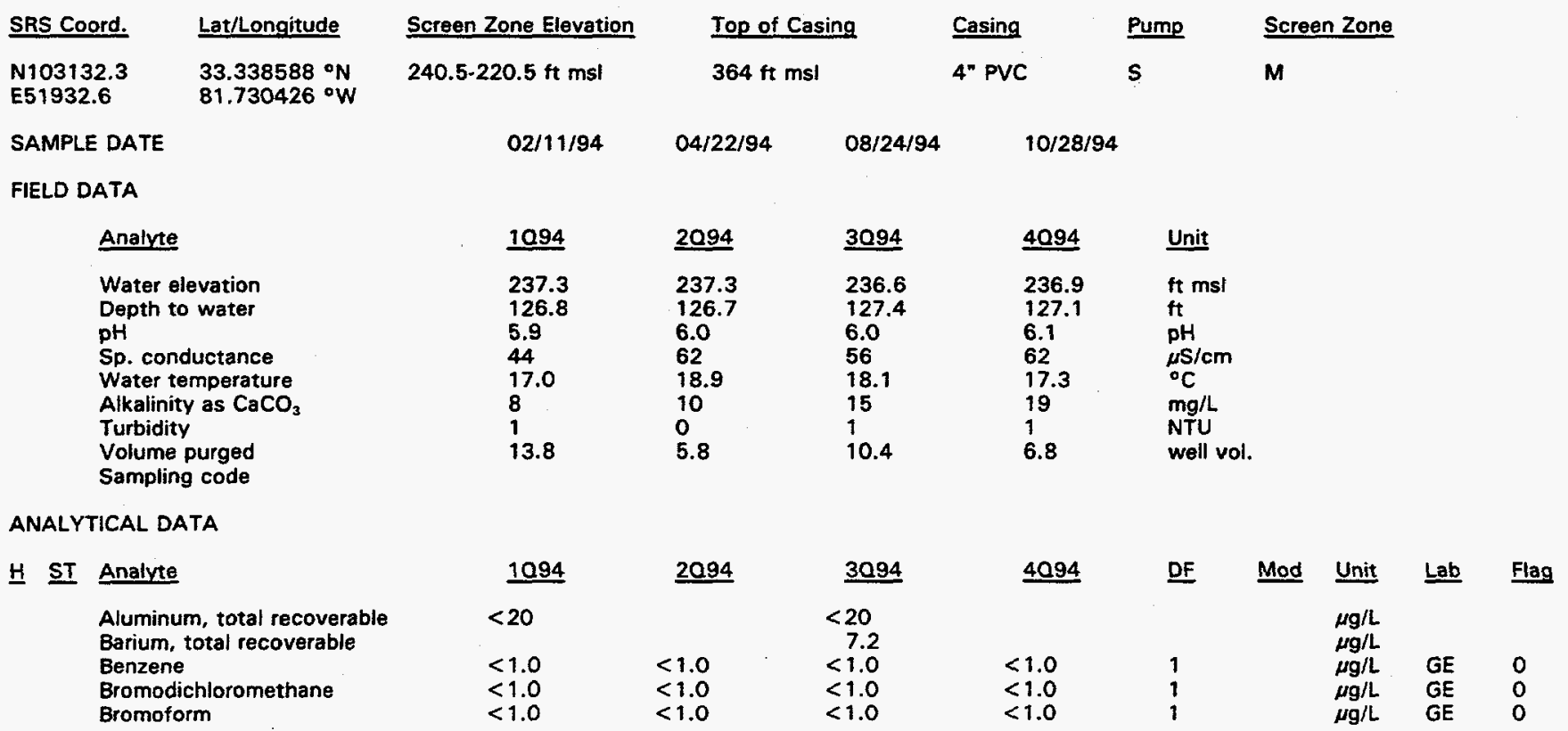

Note: Flagging, dilution factors, modifiers, and laboratories are for fourth quarter 1994 data only. See Appendix B for flagging criteria. - = exceeded holding time for fourth quarter 1994.

- = exceeded screening level or final PDWS for fourth quarter 1994. 


\section{브 ST Analyte}

Bromomethane (Methyl bromide) Cadmium, total recoverable Carbon tetrachloride

Chloride

Chlorobenzene

Chloroethane

Chloroethene (Vinyl chloride)

2-Chloroethyl vinyl ether

Chloroform

Chloromethane (Methyl chloride)

Cobalt, total recoverable

Copper, total recoverable

Cyanide

Dibromochloromethane

1,1-Dichloroethane

1.2-Dichloroethane

1,1-Dichloroethyiene

trans-1,2-Dichloroethylene

Dichloromethane

1,2-Dichloropropane

cis-1,3-Dichloropropene

trans-1, 3-Dichloropropene

Ethylbenzene

Fiuoride

Gross alpha

Iron, total recoverable

Lead, total recoverable

Manganese, total recoverable

Nickel, total recoverable

Nitrate-nitrite as nitrogen

Nonvolatile beta

Radium, total alpha-emitting

Selenium, total recoverable

Silver, total recoverable

$1,1,2,2$-Tetrachloroethane

Tetrachloroethylene

Toluene

Total organic carbon

Total organic halogens

1,1,1-Trichloroethane

1, 1,2-Trichloroethane

Trichloroethylene

Trichlorofluoromethane

Zinc, total recoverable

$\begin{aligned} & 1094 \\ &< 1.0 \\ &<2.0 \\ &<1.0 \\ & \\ & 1.8 \\ &<1.0 \\ &<1.0 \\ &<1.0 \\ &<1.0 \\ &<1.0 \\ & \\ & \\ &<1.0 \\ &<1.0 \\ &<1.0 \\ &<1.0 \\ &<1.0 \\ &<1.0 \\ &<1.0 \\ &<1.0 \\ &<1.0 \\ &<1.0 \\ & \\ & 1.5 E+00 \\ &<3.4 \\ &<3.0 \\ &<2.0 \\ &\end{aligned}$

$\begin{aligned} & 2094 \\ &<1.0 \\ &<1.0 \\ & 2.590 \\ &<1.0 \\ &<1.0 \\ &<1.0 \\ &<1.0 \\ &<1.0 \\ &<1.0\end{aligned}$

3094

$<1.0$

$<1.0$

$<1.0$

$<1.0$

$<1.0$

$<1.0$

$<1.0$

$<1.0$

$<4.0$

$<4.0$

$<5.0$

$<1.0$

$<1.0$

$<1.0$

$<1.0$

$<1.0$

$<1.0$

$<1.0$

$<1.0$

$<1.0$

$<1.0$

$<100$

$<5.0$

$<1.0$

$<1.0$

$<1.0$

$<1.0$

$<1.0$

$<1.0$

$<1.0$

$<1.0$

4.4E-01

4.3

$<3.0$

$<2.0$
$<4.0$

$<4.0$

170

8.0E-01

$<-1.0 E-01$

67

5.2E-O1
$2.0 E-01$

$<2.0$

$<2.0$
$<1.0$

$<1.0$

$<1.0$

$<1,000$

$<5.0$

$<1.0$

$<1.0$

$<1.0$

$<1.0$
$<1.0$

$<1.0$

$<1.0$

$<1,000$

$<5.0$

$<1.0$

$<1.0$

$<1.0$

$<1.0$

15

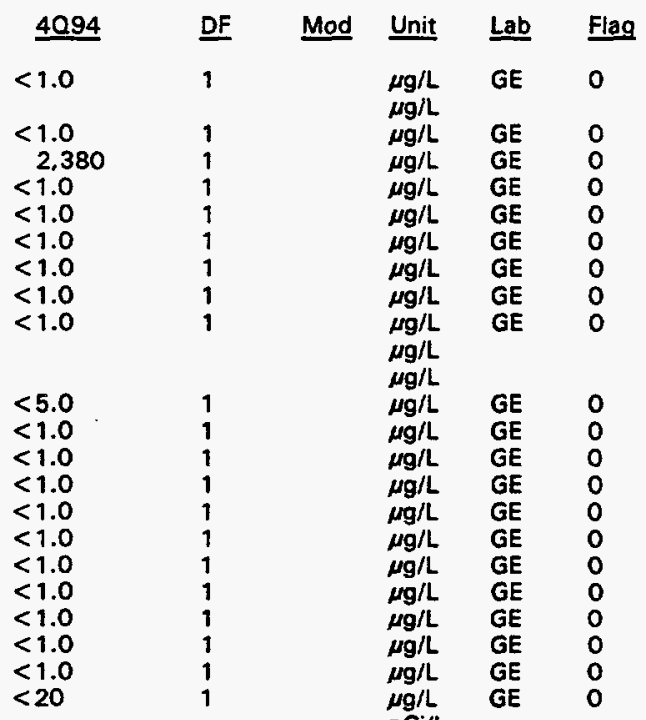

$<4.0$

56
$2.0 E-01$

$<2.0$

$<1.0$

$<1.0$

$<1.0$

1,120

$<5.0$

$<1.0$

$<1.0$

$<1.0$

$<1.0$

$\mathrm{pCi} / \mathrm{L}$

$\mu g / L$

$\mu g / L$

$\mu /$

$\mu g / L$

$\mu \mathrm{pCi} / \mathrm{L}$

pCi/L

$\mu g / L$

$\mu g / L$

$\mu \mathrm{g} / \mathrm{L}$

$\mu g / L$

$\mu g / L$

$\mu g / L$

$\mu \mathrm{g} / \mathrm{L}$

$\mu \mathrm{g} / \mathrm{L}$

$\mu g / L$

$\mu \mathrm{g} / \mathrm{L}$

$\mu g / L$

$\begin{array}{cc}\text { GE } & 0 \\ \text { GE } & 0 \\ \text { GP } & 0 \\ & \\ \text { GE } & 0 \\ \text { GE } & 0 \\ \text { GE } & 0 \\ \text { GE } & 0 \\ \text { GE } & 0 \\ \text { GE } & 0 \\ \text { GE } & 0 \\ \text { GE } & 0 \\ \text { GE } & 0 \\ \text { GE } & 0\end{array}$

Note: Flagging, dilution factors, modifiers, and laboratories are for fourth quarter 1994 data only. See Appendix B for flagging criteria. - = exceeded holding time for fourth quarter 1994.

- = exceeded screening level or final PDWS for fourth quarter 1994. 
WELL AMB 12D

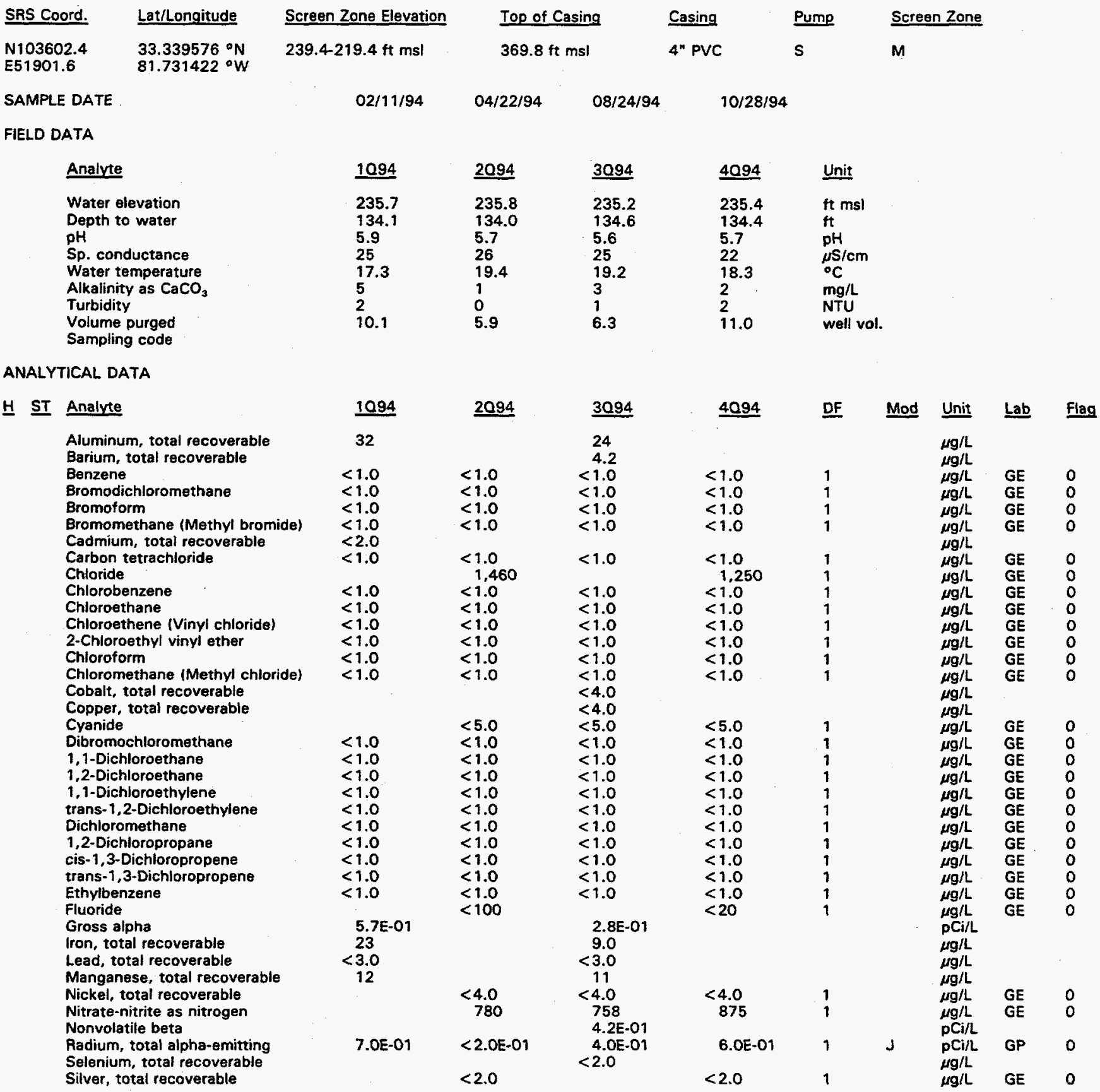

Note: Flagging, dilution factors, modifiers, and laboratories are for fourth quarter 1994 data only. See Appendix B for flagging criteria. - = exceeded holding time for fourth quarter 1994.

- = exceeded screening level or final PDWS for fourth quarter 1994. 


\begin{tabular}{|c|c|c|}
\hline 1094 & 2094 & 3094 \\
\hline $\begin{array}{l}<1.0 \\
<1.0 \\
<1.0 \\
<1.000 \\
<5.0 \\
<1.0 \\
<1.0 \\
<1.0 \\
<1.0\end{array}$ & $\begin{array}{l}<1.0 \\
<1.0 \\
<1.0 \\
<1.000 \\
<5.0 \\
<1.0 \\
<1.0 \\
<1.0 \\
<1.0\end{array}$ & $\begin{aligned}<1.0 \\
<1.0 \\
<1.0 \\
<1.000 \\
33 \\
<1.0 \\
<1.0 \\
<1.0 \\
<1.0 \\
2.1\end{aligned}$ \\
\hline
\end{tabular}

$\begin{aligned} & 4094 \\ &<1.0 \\ &<1.0 \\ &<1.0 \\ &<1.000 \\ &<5.0 \\ &<1.0 \\ &<1.0 \\ &<1.0 \\ &<1.0\end{aligned}$

DF
1
1
1
1
1
1
1
1
1

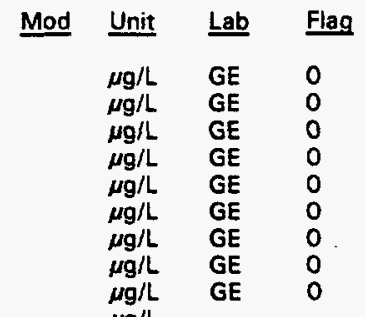

\section{WELL AMB 13AR}

\begin{tabular}{|c|c|c|c|c|c|c|c|c|c|c|}
\hline \multicolumn{2}{|c|}{ SRS Coord. } & Screen Zone Elevation & \multicolumn{2}{|c|}{ Top of Casing } & Casing & Pump & \multicolumn{2}{|c|}{ Screen Zone } & & \\
\hline $\begin{array}{l}\text { N103082.0 } \\
\text { E51396.0 }\end{array}$ & $\begin{array}{l}33.337600^{\circ} \mathrm{N} \\
81.731742^{\circ} \mathrm{W}\end{array}$ & $110.9-100.9 \mathrm{ft} \mathrm{msl}$ & \multicolumn{2}{|c|}{$365.1 \mathrm{ft} \mathrm{msl}$} & 4" PVC & $\mathbf{s}$ & \multirow{2}{*}{\multicolumn{2}{|c|}{ MCBC }} & & \\
\hline SAMPLE & E DATE & $02 / 13 / 94$ & $04 / 26 / 94$ & $08 / 24 / 94$ & $11 / 01 / 94$ & & & & & \\
\hline \multicolumn{10}{|c|}{ FIELD DATA } & \\
\hline & Analyte & 1094 & $\underline{2094}$ & $\underline{3094}$ & 4099 & Unit & & & & \\
\hline & $\begin{array}{l}\text { Water elevation } \\
\text { Depth to water } \\
\text { pH } \\
\text { Sp. conductance } \\
\text { Water temperature } \\
\text { Alkalinity as } \mathrm{CaCO}_{3} \\
\text { Turbidity } \\
\text { Volume purged } \\
\text { Sampling code }\end{array}$ & $\begin{array}{l}219.6 \\
145.5 \\
10.9 \\
234 \\
16.6 \\
63 \\
2 \\
0.8 \\
x\end{array}$ & $\begin{array}{l}219.3 \\
145.8 \\
11.1 \\
274 \\
18.3 \\
2 \\
3 \\
0.8 \\
x\end{array}$ & $\begin{array}{l}219.7 \\
145.4 \\
10.7 \\
139 \\
19.0 \\
41 \\
21 \\
0.7 \\
\times\end{array}$ & $\begin{array}{l}219.7 \\
145.4 \\
10.2 \\
190 \\
19.3 \\
100 \\
7 \\
0.9 \\
x\end{array}$ & $\begin{array}{l}\text { ft msl } \\
\text { ft } \\
\mathrm{pH} \\
\mu \mathrm{S} / \mathrm{cm} \\
{ }^{\circ} \mathrm{C} \\
\mathrm{mg} / \mathrm{L} \\
\mathrm{NTU} \\
\text { well vol. }\end{array}$ & & & & \\
\hline \multicolumn{11}{|c|}{ ANALYTICAL DATA } \\
\hline \multirow[t]{2}{*}{ H $\underline{\text { ST }}$} & Analyte & 1094 & $\underline{2094}$ & $\underline{3094}$ & 4099 & DF & Mod & Unit & Lab & Flag \\
\hline & $\begin{array}{l}\text { Acenaphthene } \\
\text { Acenaphthylene } \\
\text { Acetone } \\
\text { Acetonitrile (Methyl cyanide) } \\
\text { Acetophenone } \\
\text { 2-Acetylaminofluorene } \\
\text { Acrolein } \\
\text { Acrylonitrile } \\
\text { Aldrin } \\
\text { Allyl chloride } \\
\text { Aluminum, total recoverable } \\
\text { 4-Aminobiphenyl } \\
\text { Aniline } \\
\text { Anthracene } \\
\text { Antimony, total recoverable } \\
\text { Aramite } \\
\text { Arsenic, total recoverable } \\
\text { Barium, total recoverable } \\
\text { Benzene } \\
\text { alpha-Benzene hexachloride } \\
\text { beta-Benzene hexachloride } \\
\text { delta-Benzene hexachloride } \\
\text { Benzolalanthracene } \\
\text { Benzolblfluoranthene } \\
\text { Benzolklfluoranthene }\end{array}$ & $<1.0$ & $<1.0$ & $\begin{array}{l}<10 \\
<10 \\
<100 \\
<5.0 \\
<10 \\
<10 \\
<20 \\
<20 \\
<0.050 \\
<50 \\
128 \\
<10 \\
<10 \\
<10 \\
<2.0 \\
<10 \\
<2.0 \\
442 \\
<1.0 \\
<0.050 \\
<0.050 \\
<0.050 \\
<10 \\
<10 \\
<10\end{array}$ & $<1.0$ & 1 & & $\begin{array}{l}\mu g / L \\
\mu g / L \\
\mu g / L \\
\mu g / L \\
\mu g / L \\
\mu g / L \\
\mu g / L \\
\mu g / L \\
\mu g / L \\
\mu g / L \\
\mu g / L \\
\mu g / L \\
\mu g / L \\
\mu g / L \\
\mu g / L \\
\mu g / L \\
\mu g / L \\
\mu g / L \\
\mu g / L \\
\mu g / L \\
\mu g / L \\
\mu g / L \\
\mu g / L \\
\mu g / L \\
\mu g / L\end{array}$ & GE & 0 \\
\hline
\end{tabular}

Note: Flagging, dilution factors, modifiers, and laboratories are for fourth quarter 1994 data only. See Appendix B for flagging criteria. - = exceeded holding time for fourth quarter 1994.

- = exceeded screening level or final PDWS for fourth quarter 1994. 


\section{H $\underline{\text { ST Analyte }}$}

Benzolg, $h$, iperylene

Benzo[a]pyrene

Benzyl alcohol

Beryllium, total recoverable

Bis(2-chloroethoxy) methane

Bis(2-chloroethyl) ether

Bis (2-chloroisopropyl) ether

Bis(2-ethylhexyl) phthalate

Bromodichloromethane

Bromoform

Bromomethane (Methyl bromide)

4-Bromophenyl phenyl ether

Butylbenzyl phthalate

2-sec-Butyl-4,6-dinitrophenol

Cadmium, total recoverable

Carbon disulfide

Carbon tetrachloride

Chlordane

Chloride

4-Chloroaniline

Chlorobenzene

Chlorobenzilate

4-Chloro-m-cresol

Chloroethane

Chloroethene (Vinyl chloride)

2-Chloroethyl vinyl ether

Chioroform

Chloromethane (Methyl chloride)

2-Chloronaphthalene

2-Chlorophenol

4-Chlorophenyl phenyl ether

Chloroprene

Chromium, total recoverable

Chrysene

Cobalt, total recoverable

Copper, total recoverable

o-Cresol (2-Methylphenol)

m,p-Creso

Cyanide

p, $p^{\prime}-D D D$

$D, p^{\prime}-D D E$

D. P'-DDT

Diallate

Dibenzla, hlanthracene

Dibenzofuran

Dibromochloromathane

1,2-Dibromo-3-chloropropane

1,2-Dibromoethane

Dibromomethane

Di-n-butyl phthalate

1,2-Dichlorobenzene

1,3-Dichlorobenzene

1,4-Dichlorobenzene

3,3'-Dichlorobenzidine

trans-1,4-Dichloro-2-buten

Dichlorodifluoromethane

1,1-Dichloroethane

1,2-Dichloroethane

1,1-Dichloroethylene

trans-1,2-Dichloroethylene

Dichloromethane

2,4-Dichlorophenol

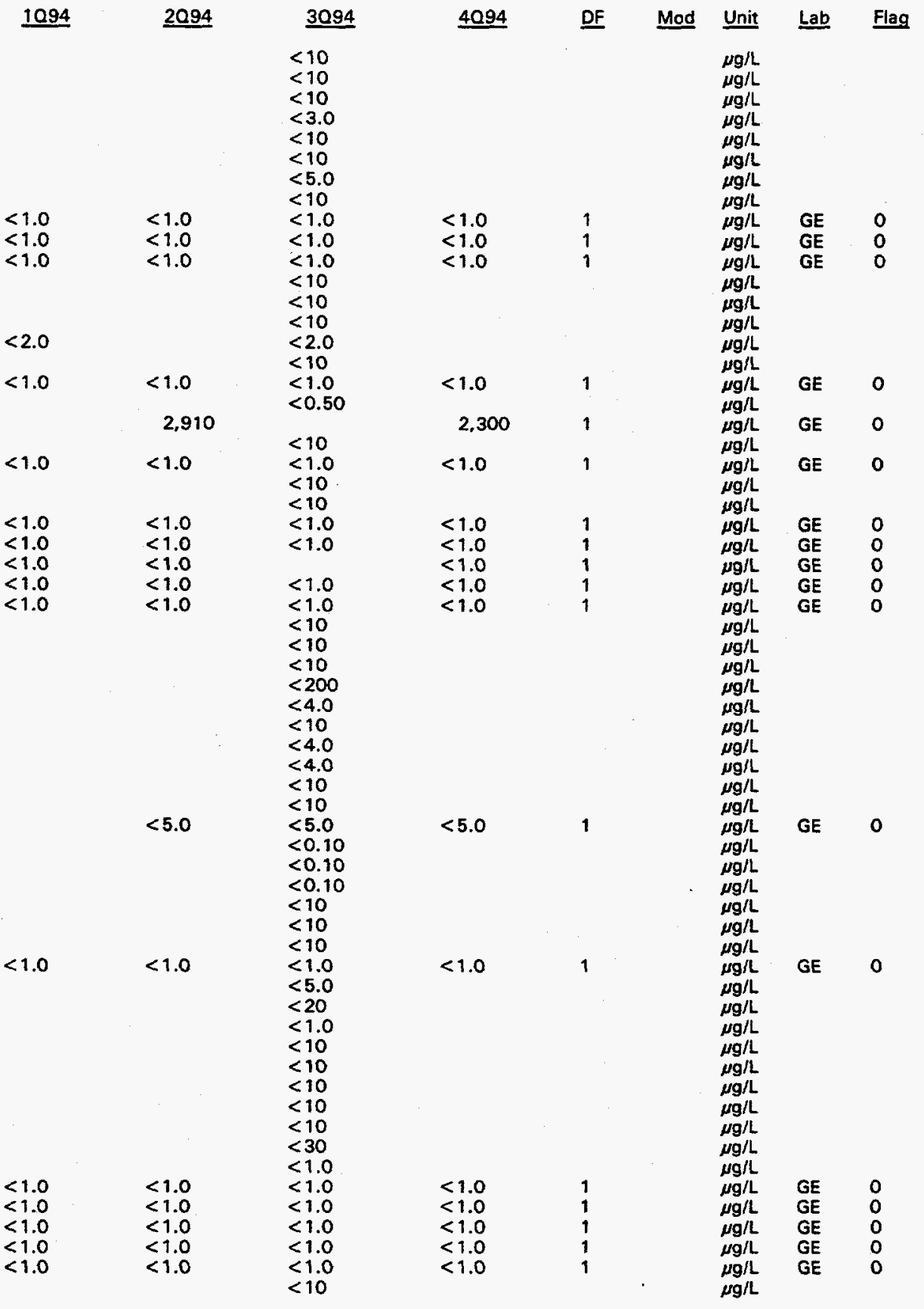

Note: Flagging, dilution factors, modifiers, and laboratories are for fourth quarter 1994 data only. See Appendix B for flagging criteria. - = exceeded holding time for fourth quarter 1994.

- = exceeded screening level or final PDWS for fourth quarter 1994. 


\section{H ST Analyte}

2,6-Dichlorophenol

2,4-Dichlorophenoxyacetic acid

1,2-Dichloropropane

cis-1,3-Dichloropropene

trans-1,3-Dichloropropene

Dieldrin

Diethyl phthalate

Dimethoate

2,4-Dimethyl phenol

Dimethyl phthalate

p-Dimethylaminoazobenzene

7, 12-Dimethylbenz[a]anthracene

3,3'-Dimethylbenzidine

a,a-Dimethylphenethylamine

1,3-Dinitrobenzene

2,4-Dinitrophenol

2,4-Dinitrotoluene

2,6-Dinitrotoluene

Di-n-octyl phthalate

1,4-Dioxane

Diphenylamine

Disulfoton

Endosulfan I

Endosulfan II

Endosulfan sulfate

Endrin

Endrin aldehyde

Ethyl methacrylate

Ethyl methanesulfonate

Ethylbenzene

Famphur

Fiuoranthene

Fluorene

Fluoride

Gross alpha

Heptachlor

Heptachlor epoxide

$1,2,3,4,6,7,8-H P C D D$

Heptachlorodibenzo-p-dioxin isomers

$1,2,3,4,6,7,8-\mathrm{HPCDF}$

Heptachlorodibenzo-p-furan isomers

Hexachlorobenzene

Hexachlorobutadiene

Hexachlorocyclopentadiene

$1,2,3,4,7,8-H \times C D D$

Hexachlorodibenzo-p-dioxin isomers

$1,2,3,4,7,8-H X C D F$

Hexachlorodibenzo-p-furan isomers

Hexachloroethane

Hexachlorophene

Hexachloropropene

2-Hexanone

Indeno $[1,2,3-c, d]$ pyrene

lodomethane (Methyl iodide)

Iron, total recoverable

Isobutyl alcohol

Isodrin

Isophorone

Isosafrole

Kepone

Lead, total recoverable

Lindane

24

10.94
$\begin{aligned} & <1.0 \\ & <1.0\end{aligned} \quad<1.0$
$<1.0$
$<3.0$
24
$<4.8 E-01$
$<1.0$

$<4.8 \mathrm{E}-01$

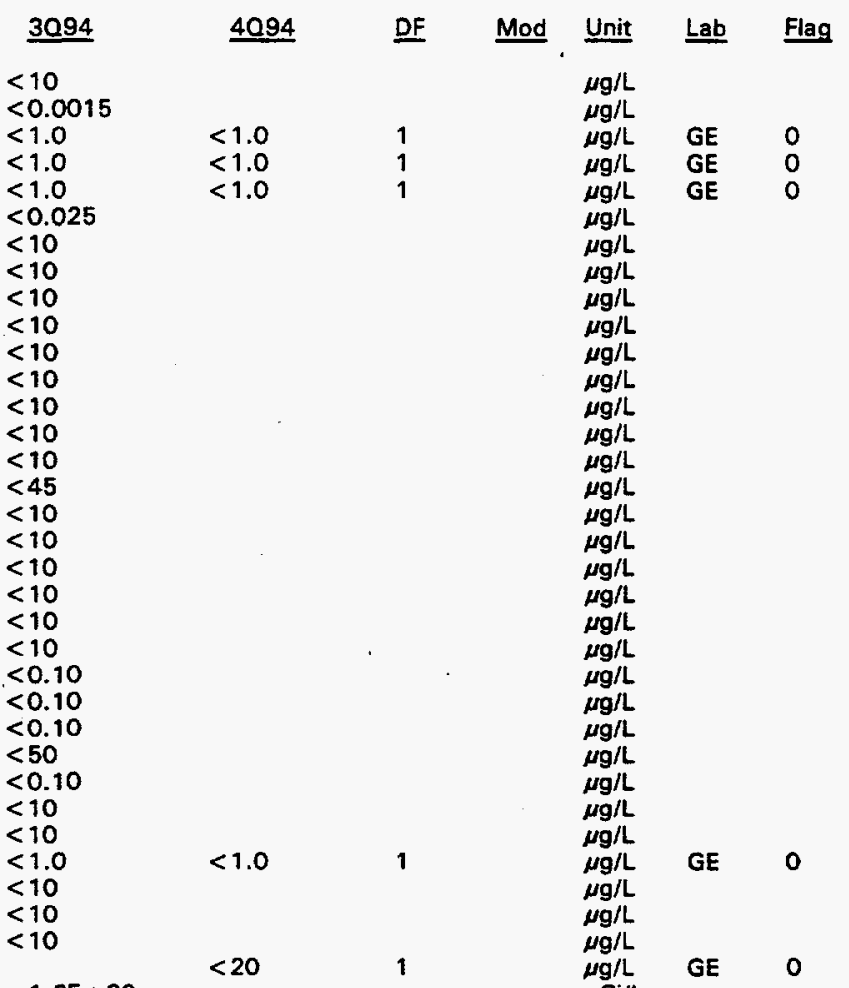

$1.6 \mathrm{E}+00$

$<0.050$

$<0.050$

$<0.065$

$<0.065$

$<0.045$

$<0.045$

$<10$

$<10$

$<10$

$<0.045$

$<0.045$

$<0.040$

$<0.040$

$<10$

$<10$

$<10$

$<10$

$<10$

$<15$
78

$<100$
$<10$

$<10$
$<10$

$<10$

$<10$

$<3.0$

$<1,250$

Note: Flagging, dilution factors, modifiers, and laboratories are for fourth quarter 1994 data only. See Appendix B for flagging criteria. - = exceeded holding time for fourth quarter 1994.

- = exceeded screening level or final PDWS for fourth quarter 1994. 
Well AMB 13AR continued

ANALYTICAL DATA

\section{H ST Analyte}

Manganese, total recoverable

Mercury, total recoverable

Methacrylonitrile

Methapyrilene

Methoxychlor

2-Methyi-4,6-dinitrophenol

Methyl ethyl ketone

Methyl isobutyl ketone

Methyl methacrylate

Methyl methanesulfonate

3-Methylcholanthrene

2-Methyinaphthalene

Naphthalene

1,4-Naphthoquinone

1-Naphthylamine

2-Naphthylamine

Nickel, total recoverable

Nitrate-nitrite as nitrogen

o-Nitroaniline

m-Nitroanilirie

p-Nitroaniline

Nitrobenzene

2-Nitrophenol

4-Nitrophenol

4-Nitroquinoline-1-oxide

N-Nitrosodi-n-butylamine

N-Nitrosodiethylamine

N-Nitrosodimethylamine

N-Nitrosodiphenylamine

$\mathrm{N}$-Nitrosodipropylamine

$\mathrm{N}$-Nitrosomethylethylamine

N-Nitrosomorpholine

$\mathrm{N}$-Nitrosopiperidine

N-Nitrosopyrrolidine

5-Nitro-o-toluidine

Nonvolatile beta

$0,0,0$-Triethyl phosphorothioate

Octachlorodibenzo-p-dioxin

Octachlorodibenzo-p-furan

Parathion

Parathion methyl

PCB 1016

PCB 1221

PCB 1232

PCB 1242

PCB 1248

PCB 1254

PCB 1260

Pentachlorobenzene

1,2,3,7,8-PCDD

Pentachlorodibenzo-p-dioxin isomers

1,2,3,7,8-PCDF

Pentachloroethane

Pentachloronitrobenzene

Pentachlorophenol

Phenacetin

Phenanthrene

Phenol

p.Phenylenediamine

Phorate

2-Picoline

Pronamid

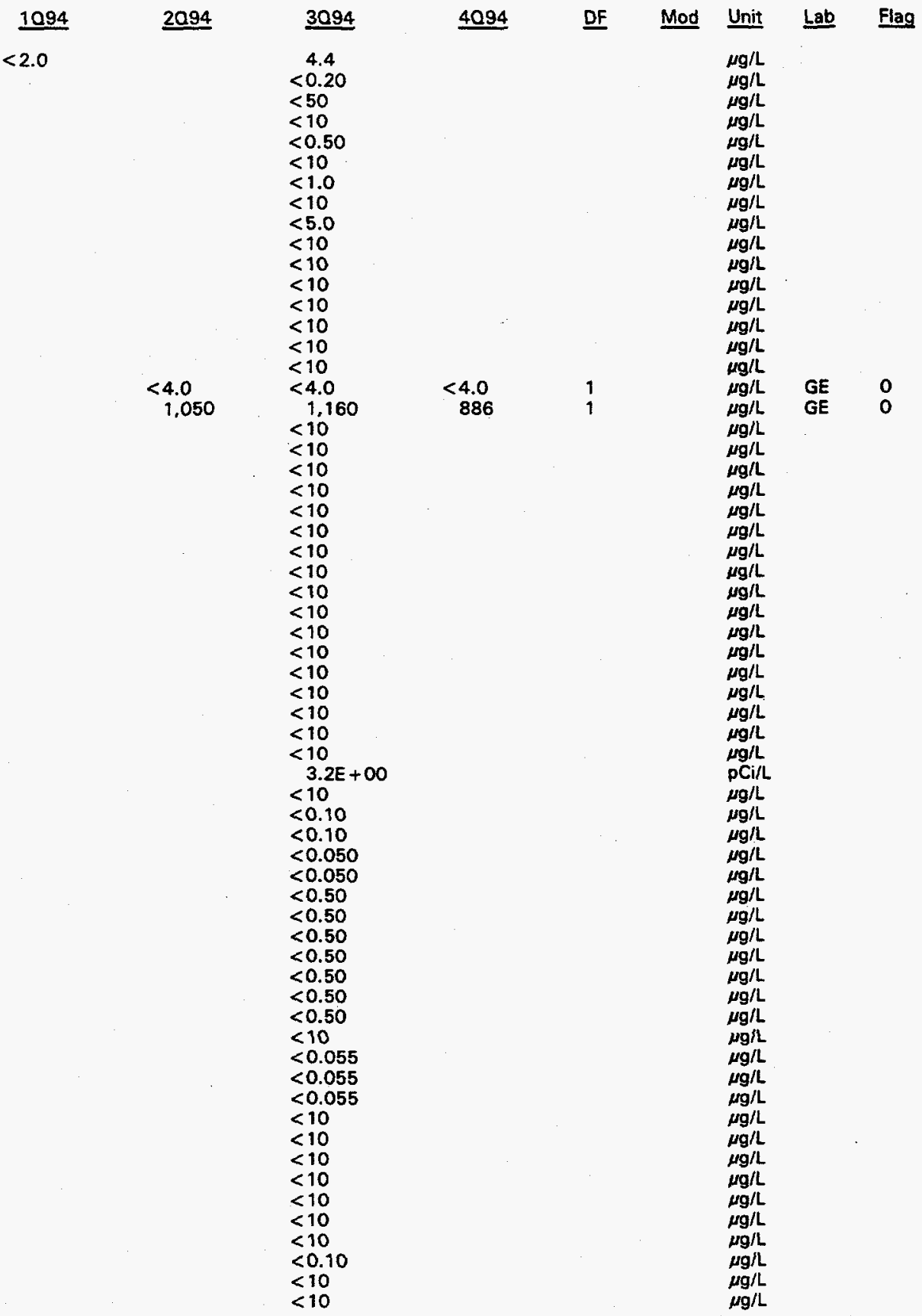

Note: Flagging, dilution factors, modifiers, and laboratories are for fourth quarter 1994 data only. See Appendix B for flagging criteria. - = exceeded holding time for fourth quarter 1994.

- exceeded screening level or final PDWS for fourth quarter 1994. 
ANALYTICAL DATA

H ST Analyte

Propionitrile

Pyrene

Pyridine

Radium, total alpha-emitting

Safrole

Selenium, total recoverable

Silver, total recoverable

Styrene

Sulfide

Sulfotepp

1,2,4,5-Tetrachlorobenzene

2,3,7,8-TCDD

$2,3,7,8-T C D F$

Tetrachlorodibenzo-p-dioxin isomers

Tetrachlorodibenzo-p-furan isomers

1,1,1,2-Tetrachloroethane

1,1,2,2-Tetrachloroethane

Tetrachloroethylene

2,3,4,6-Tetrachlorophenol

Thallium, total recoverable

Thionazin

Tin, total recoverable

Toluene

o-Toluidine

Total organic carbon

Total organic halogens

Toxaphene

2,4,5-TP (Silvex)

1,2,4-Trichlorobenzene

1,1,1-Trichloroethane

1,1,2-Trichioroethane

Trichloroethylene

Trichlorofluoromethane

2,4,5-Trichlorophenol

2,4,6-Trichlorophenol

2,4,5-T

1,2,3-Trichloropropane

1.3.5-Trinitrobenzene

Vanadium, total recoverable

Vinyl acetate

Xylenes

Zinc, total recoverable

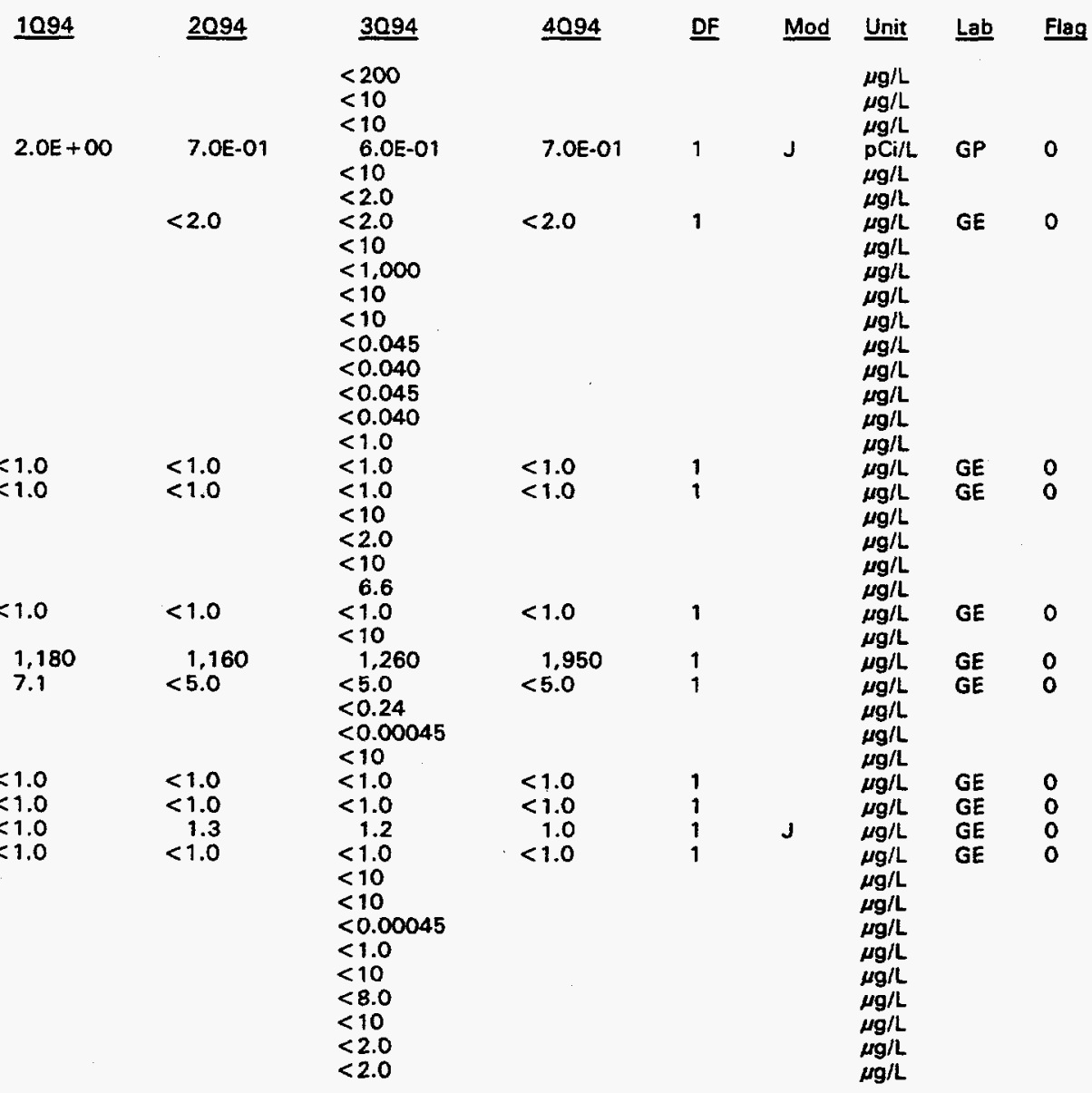

Note: Flagging, dilution factors, modifiers, and laboratories are for fourth quarter 1994 data only. See Appendix B for flagging criteria.

- = exceeded holding time for fourth quarter 1994.

- exceeded screening level or final PDWS for fourth quarter 1994. 


\section{Appendix E}

\section{Data Quality/Usability Assessment}


WSRC-TR-94-0615

Unclassified

THIS PAGE LEFT BLANK INTENTIONALLY. 


\section{Data Quality/Usability Assessment}

Quality assurance/quality control (QA/QC) procedures relating to accuracy and precision of analyses performed on groundwater samples are followed in the field and laboratory and are reviewed prior to publication of results. The review by the Environmental Protection Department/Environmental Monitoring Section (EPD/EMS) of the volume of analytical data acquired each quarter and presented in various reports is an ongoing process; its review of the QAVQ data cannot be completed in time to meet the deadlines for the reports required by the Resource Conservation and Recovery Act and associated regulations. Other site and regulatory personnel can obtain further information on the data quality and usability in a variety of ways, including those described below.

\section{Data Qualification}

The contract laboratories continually assess their own accuracy and precision according to U.S. Environmental Protection Agency (EPA) guidelines. They submit sample- or batch-specific QA QC information either at the same time as analytical results or in quarterly summaries. Properly defined and used result modifiers (also referred to as qualifiers) can be a key component in assessing data usability. Result modifiers designed by EPD/EMS and used by the primary laboratories are presented in Appendix D.

\section{Assessment of Accuracy of the Data}

Accuracy, or the nearness of the reported result to the true concentration of a constituent in a sample, can be assessed in several ways.

A laboratory's general accuracy can be judged by analysis of results obtained from known samples. The non-radionuclide contract laboratories analyze commercial reference samples every quarter at EPD/EMS' request. The results of these analyses are presented in the EPD/EMS groundwater monitoring quarterly reports. The primary laboratories also seek or maintain state certification by participating periodically in performance studies; reference samples and analysis of results are provided by EPA. Results of these studies also are published in the EPD/EMS quarterly reports.

Analysis of blanks provides a tool for assessing the accuracy of both sampling and laboratory analysis. Results for all field blanks for the quarter can be found in the EPD/EMS quarterly reports. Any field or laboratory blanks that exceed established minimums are identified in the same reports, in tables associating them with groundwater samples analyzed in the same batches.

Surrogates, organic compounds similar in chemical behavior to the compounds of interest but not normally found in environmental samples, are used to monitor the effect of the matrix on the accuracy of analyses for organic parameters. For example, for analyses of volatile organics by EPA Method 8240 , three surrogate compounds are added to all samples and blanks in each analytical batch. In analyses of semivolatile organics, three acid compounds and three base/ neutral compounds are used. Two surrogates are used in organochlorine pesticides analyses. Percent recoveries for surrogate analyses are calculated by laboratory personnel, reported to EPD/EMS, reviewed, and entered into the database, but they are not published. If recoveries are not within specified limits, the laboratory is expected to reanalyze the samples or attach qualifiers to the data identifying the anomalous results. 
Sample-specific accuracy for both organic and inorganic parameters can be assessed by examination of matrix spike/matrix spike duplicate results. A sample is analyzed unspiked to determine a baseline set of values. A second portion of the sample is spiked with known concentrations of compounds appropriate to the analyses being performed, typically five volatile organic compounds for volatile organics analyses, eleven semivolatile compounds for semivolatiles, six pesticide compounds for pesticides, all metals for metals analyses by SW-846 methods (EPA, 1986), and a known quantity of cyanide for cyanide analysis. The percentage of the spike compound that is recovered (i.e., measured in excess of the value obtained for the unspiked sample) is a direct measure of analytical accuracy. EPA requires matrix spike/matrix spike duplicates to be run at least once per 20 samples of similar matrix.

Matrix spike/matrix spike duplicate results are reported to EPD/EMS but are not published. For organic compounds, according to EPA guidelines, no action is taken on the basis of matrix spike/ matrix spike duplicate data alone (i.e., no result modifiers are assigned solely on the basis of matrix spike results); however, the results can indicate if a laboratory is having a systematic problem in the analysis of one or more analytes.

In the case of inorganic compounds, such as metals, the matrix spike sample analysis provides information about the effect of each sample matrix on the digestion and measurement methodology. Data qualifiers assigned by the laboratories on the basis of the percentage of spike recovery are reported in the published results tables.

\section{Assessment of Precision}

Precision of the analyses, or agreement of a set of replicate results among themselves, is assessed through the use of duplicates initiated by the laboratory and blind replicates provided by EPD/EMS. The results of duplicate and replicate analyses are presented in those results tables of the quarterly reports which report only one quarter of data, usually during first, second, and third quarters. Duplicate and replicate results are not presented in results tables that report more than one quarter of data, generally provided in fourth quarter reports. In this case, the results tables instead present only the highest result for each analyte for each quarter of the year.

The laboratories assess precision by calculating the relative percent difference (RPD) for each pair of laboratory-initiated duplicate results. One of the contract laboratories uses a data qualifier (J3) to modify metals analyses when the RPD for laboratory duplicates is greater than 20 percent.

Additional statistical comparisons of laboratory duplicate and blind replicate results, both intraand interlaboratory, are presented in the EPD/EMS quarterly reports. The calculation used for these reports is the mean relative difference (MRD) which is similar to EPA's RPD except that the MRD is the average of all the RPD values from one laboratory for each compound (intralaboratory MRD) or all the RPD values from all laboratories for each compound (interlaboratory $M R D$ ), during one quarter. Because detection limits may vary among samples, the MRD requires calculation of a reference detection limit, which is the detection limit at the 90th percentile of the array of limits in the population of all duplicate and replicate analyses for a given analyte during a particular quarter. The MRD is not method-specific.

\section{Method-Specific Accuracy and Precision}

The contract laboratories' EPA-approved laboratory procedures include QAVC requirements as an integral part of the methods. Thus, knowledge of the method used in obtaining data is an important component of determining data usability. EPA has conducted extensive research and 
development on the methods approved for the analysis of water and waste water; information on the accuracy and precision of a method is available from EPA publications, as is full information on required $Q A V Q C$ procedures. A listing of the methods used by the primary laboratories during fourth quarter 1993 is given below along with the source for the method description. Many, if not all, of these sources include presentations of representative accuracy and precision results.

\section{Methods Used by the Contract Laboratories}

\begin{tabular}{|c|c|c|}
\hline Method & Used to Analyze & Source \\
\hline EPA120.1 & Specific conductance & EPA EMSL, 1983 \\
\hline EPA150.1 & $\mathrm{pH}$ & EPA EMSL, 1983 \\
\hline EPA160.1 & Total dissolved solids & EPA EMSL, 1983 \\
\hline EPA160.2 & Total dissolved solids, total suspended solids & EPA EMSL, 1983 \\
\hline EPA180.1 & Turbidity & EPA EMSL, 1983 \\
\hline EPA200.7 & Metals & EPA EMSL, 1983 \\
\hline EPA204.2 & Antimony & EPA EMSL, 1983 \\
\hline EPA206.2 & Arsenic & EPA EMSL, 1983 \\
\hline EPA239.2 & Lead & EPA EMSL, 1983 \\
\hline EPA245.1 & Mercury & EPA EMSL, 1983 \\
\hline EPA270.2 & Selenium & EPA EMSL, 1983 \\
\hline EPA279.2 & Thallium & EPA EMSL, 1983 \\
\hline EPA300.0 & Chloride, nitrite, sulfate & EPA EMSL, 1991 \\
\hline EPA310.1 & Alkalinity & EPA EMSL, 1983 \\
\hline EPA325.2 & Chloride & EPA EMSL, 1983 \\
\hline EPA335.3 & Cyanide & EPA EMSL, 1983 \\
\hline EPA340.2 & Fluoride & EPA EMSL, 1983 \\
\hline EPA353.1 & Nitrogen, nitrate-nitrite & EPA EMSL, 1983 \\
\hline EPA353.2 & Nitrogen, nitrate, nitrite, or combined & EPA EMSL, 1983 \\
\hline EPA365.1 & Phosphorus, all forms (reported as total phosphates) & EPA EMSL, 1983 \\
\hline EPA365.2 & Phosphorus, all forms (reported as total phosphates) & EPA EMSL, 1983 \\
\hline EPA376.2 & Sulfide & EPA EMSL, 1983 \\
\hline EPA413.1 & Oil \& grease & EPA EMSL, 1983 \\
\hline EPA415.1 & $\begin{array}{l}\text { Dissolved organic carbon, total inorganic carbon, total organic } \\
\text { carbon }\end{array}$ & EPA EMSL, 1983 \\
\hline EPA418.1 & Total petroleum hydrocarbons & EPA EMSL, 1983 \\
\hline EPA420.2 & Phenols & EPA EMSL, 1983 \\
\hline EPA900.0 & Gross alpha, nonvolatile beta & EPA EMSL, 1980 \\
\hline EPA900.1 & Total alpha-emitting radium & EPA EMSL, 1980 \\
\hline EPA906.0 & Tritium & EPA EMSL, 1980 \\
\hline EPA6010 & Metals & EPA, 1986 \\
\hline EPA7041 & Antimony & EPA, 1986 \\
\hline EPA7060 & Arsenic & EPA, 1986 \\
\hline EPA7421 & Lead & EPA, 1986 \\
\hline EPA7470 & Mercury & EPA, 1986 \\
\hline EPA7740 & Selenium & EPA, 1986 \\
\hline EPA7841 & Thallium & EPA, 1986 \\
\hline EPA8010 & Chlorinated volatile organics & EPA, 1986 \\
\hline EPA8080 & Organochlorine pesticides and PCBs & EPA, 1986 \\
\hline EPA8150 & Chlorinated herbicides & EPA, 1986 \\
\hline EPA8240 & GCMS volatiles & EPA, 1986 \\
\hline EPA8270 & GCMS semivolatiles & EPA, 1986 \\
\hline EPA8280 & Dioxins and furans & EPA, 1986 \\
\hline EPA9012 & Cyanide & EPA, 1986 \\
\hline EPA9020 & Total organic halogens & EPA, 1986 \\
\hline
\end{tabular}


Method

EPA9020A

EPA9030

EPA9060
Used to Analyze

Total organic halogens

Sulfide

Dissolved organic carbon, total inorganic carbon, total organic carbon
Source

EPA, 1986

EPA, 1986

EPA, 1986

An example of available method-specific QAVC information is that for the analysis of metals by EPA Method 6010/200.7 (EPA, 1986/EPA EMSL, 1983). The primary laboratories, General Engineering Laboratories (GE) and Roy F. Weston, Inc. (Weston), use this inductively coupled plasma (ICP) atomic emission spectrometric method.

The following precision and accuracy data are based on the experience of seven laboratories that applied the ICP technique to acid-distilled water matrices that had been spiked with various metal concentrates. (Note: Not all seven laboratories analyzed all 14 elements.) The references give results for samples having three concentration ranges; the results here are for samples having the lowest values, similar to actual groundwater results for SRS.

\section{ICP PRECISION AND ACCuRACY DATA}

\begin{tabular}{llll} 
Element & True value $(\mu \mathrm{g} / \mathrm{L})$ & $\begin{array}{l}\text { Mean reported } \\
\text { value }(\mu \mathrm{g} / \mathrm{L})\end{array}$ & $\begin{array}{l}\text { Mean } \\
\text { RSS }\end{array}$ \\
\cline { 2 - 3 } & & & 33 \\
Aluminic & 60 & 62 & 23 \\
Beryllium & 22 & 19 & 9.8 \\
Cadmium & 20 & 20 & 16 \\
Chromium & 2.5 & 2.9 & 18 \\
Cobalt & 10 & 10 & 4.1 \\
Copper & 20 & 20 & 40 \\
Iron & 11 & 11 & 15 \\
Lead & 20 & 19 & 32 \\
Manganese & 24 & 30 & 6.7 \\
Nickel & 15 & 15 & 11 \\
Selenium & 30 & 28 & 42 \\
Vanadium & 6 & 8.5 & 2.9 \\
Zinc & 70 & 69 & 45
\end{tabular}

a Relative standard deviation. In EPA (1986), the column heading is Mean Standard Deviation (\%).

As another example, EPA Method 601/8010 (EPA, 1991/EPA, 1986) is used by both GE and Weston for analyses of halogenated volatile organics. In the presentation of the method in both references, the following table gives method-specific accuracy and precision as functions of concentration. Contract laboratories are expected to achieve or at least approach these limits.

\section{Accuracy and Precision as functions of Concentration for EPA Method 601/8010}

\section{Parameter}

Bromodichloromethane

Bromoform

Bromomethane
Accuracy as recover $X^{\prime a}(\mu g / L)$

$1.12 \mathrm{C}-1.02^{\mathrm{d}}$

$0.96 C-2.05$

$0.76 C-1.27$
Single analyst

${\text { precision }(\mu \mathrm{g} / \mathrm{L})^{b}}^{\text {b }}$

$0.11 \bar{X}+0.04^{e}$

$0.12 \bar{X}+0.58$

$0.28 \bar{X}+0.27$
Overall precision $(\mu \mathrm{g} / \mathrm{L})^{\mathrm{C}}$

$0.20 \bar{X}+1.00$

$0.21 \bar{X}+2.41$

$0.36 \bar{X}+0.94$ 
Parameter

Carbon tetrachloride

Chlorobenzene

Chloroethane

2-Chloroethyl vinyl ether ${ }^{f}$

Chloroform

Chloromethane

Dibromochloromethane

1,2-Dichlorobenzene

1,3-Dichlorobenzene

1,4-Dichlorobenzene

1,1-Dichloroethane

1,2-Dichloroethane

1,1-Dichloroethene

trans-1,2-Dichloroethene

Dichloromethane

(Methylene chloride)

1,2-Dichloropropane $f$ cis-1,3-Dichloropropene $f$ trans-1,3-Dichloropropene ${ }^{f}$

1,1,2,2-Tetrachloroethane

Tetrachloroethylene

1,1,1-Trichloroethane

1,1,2-Trichloroethane

Trichloroethylene

Trichlorofluoromethane

Vinyl chloride

$\begin{array}{ll}\text { Accuracy as } & \text { Single analyst } \\ \text { recovery, } X^{\prime}(\mu \mathrm{g} / \mathrm{L}) & \text { precision }(\mu \mathrm{g} / \mathrm{L})\end{array}$

$0.98 \mathrm{C}-1.04$

$1.00 C-1.23$

$0.99 C-1.53$

$1.00 \mathrm{C}$

$0.93 C-0.39$

$0.77 \mathrm{C}+0.18$

$0.94 C+2.72$

$0.93 C+1.70$

$0.95 C+0.43$

$0.93 C-0.09$

$0.95 C-1.08$

$1.04 C-1.06$

$0.98 C-0.87$

$0.97 C-0.16$

$0.91 C-0.93$

$1.00 \mathrm{C}$

$1.00 \mathrm{C}$

$1.00 \mathrm{C}$

$0.95 C+0.19$

$0.94 C+0.06$

$0.90 C-0.16$

$0.86 C+0.30$

$0.87 C+0.48$

$0.89 C-0.07$

$0.97 C-0.36$
$0.15 \bar{X}+0.38$

$0.15 \bar{X}-0.02$

$0.14 \bar{X}-0.13$

$0.20 \bar{X}$

$0.13 \bar{X}+0.15$

$0.28 \bar{X}-0.31$

$0.11 \bar{X}+1.10$

$0.20 \bar{X}+0.97$

$0.14 \bar{X}+2.33$

$0.15 \bar{X}+0.29$

$0.09 \bar{X}+0.17$

$0.11 \bar{X}+0.70$

$0.21 \bar{X}-0.23$

$0.11 \bar{X}+1.46$

$0.11 \bar{X}+0.33$

$0.13 \bar{X}$

$0.18 \bar{X}$

$0.18 \bar{X}$

$0.14 \bar{X}+2.41$

$0.14 \bar{X}+0.38$

$0.15 \bar{X}+0.04$

$0.13 \bar{X}-0.14$

$0.13 \bar{X}-0.03$

$0.15 \bar{X}+0.67$

$0.13 \bar{X}+0.65$
Overall

precision $(\mu \mathrm{g} / \mathrm{L}$ )

$0.20 \bar{X}+0.39$

$0.18 \bar{X}+1.21$

$0.17 \bar{X}+0.63$

$0.35 \bar{X}$

$0.19 \bar{X}-0.02$

$0.52 \bar{X}+1.31$

$0.24 \bar{X}+1.68$

$0.13 \bar{X}+6.13$

$0.26 \bar{X}+2.34$

$0.20 \bar{X}+0.41$

$0.14 \bar{X}+0.94$

$0.15 \bar{X}+0.94$

$0.29 \bar{X}-0.40$

$0.17 \overline{\mathrm{X}}+1.46$

$0.21 \bar{X}+1.43$

$0.23 \bar{X}$

$0.32 \bar{X}$

$0.32 \bar{X}$

$0.23 \bar{X}+2.79$

$0.18 \bar{X}+2.21$

$0.20 \bar{X}+0.37$

$0.19 \bar{X}+0.67$

$0.23 \bar{X}+0.30$

$0.26 \bar{X}+0.91$

$0.27 \bar{X}+0.40$

a $X^{\prime}=$ expected recovery for one or more measurements of a sample containing a concentration of $C$, in $\mu \mathrm{g} / \mathrm{L}$.

b Expected single analyst standard deviation of measurements.

c Expected interlaboratory standard deviation of measurements.

d $C=$ true value for the concentration, in $\mu \mathrm{g} / \mathrm{L}$.

e $\bar{X}=$ average recovery found for measurements of samples containing a concentration of $C$, in $\mu g / L$.

$f$ Estimates based on performance of a single laboratory.

\section{References Cited}

EPA (U.S. Environmental Protection Agency), 1986. Test Methods for Evaluating Solid Waste (SW-846), Volumes IA-IC. Washington, DC.

EPA (U.S. Environmental Protection Agency), 1991. Guidelines Establishing Test Procedures for the Analysis of Pollutants, Code of Federal Regulations, Title 40, Part 136, Appendix A. Washington, DC.

EPA EMSL (U.S. Environmental Protection Agency, Environmental Monitoring and Systems Laboratory), 1980. Prescribed Procedures for Measurement of Radioactivity in Drinking Water, EPA-600/4-80-032. Cincinnati, OH.

EPA EMSL (U.S. Environmental Protection Agency, Environmental Monitoring and Systems Laboratory); 1983. Methods for Chemical Analysis of Water and Wastes. Cincinnati, $\mathrm{OH}$. 
EPA EMSL (U.S. Environmental Protection Agency, Environmental Monitoring and Systems Laboratory), 1991. Test Method, The Determination of Inorganic Anions in Water by lon Chromatography-Method 300.0. Cincinnati, $\mathrm{OH}$. 
WSRC-TR-94-0615

Unclassified

\section{Appendix F}

Time Series Plots 
THIS PAGE LEFT BLANK INTENTIONALLY. 


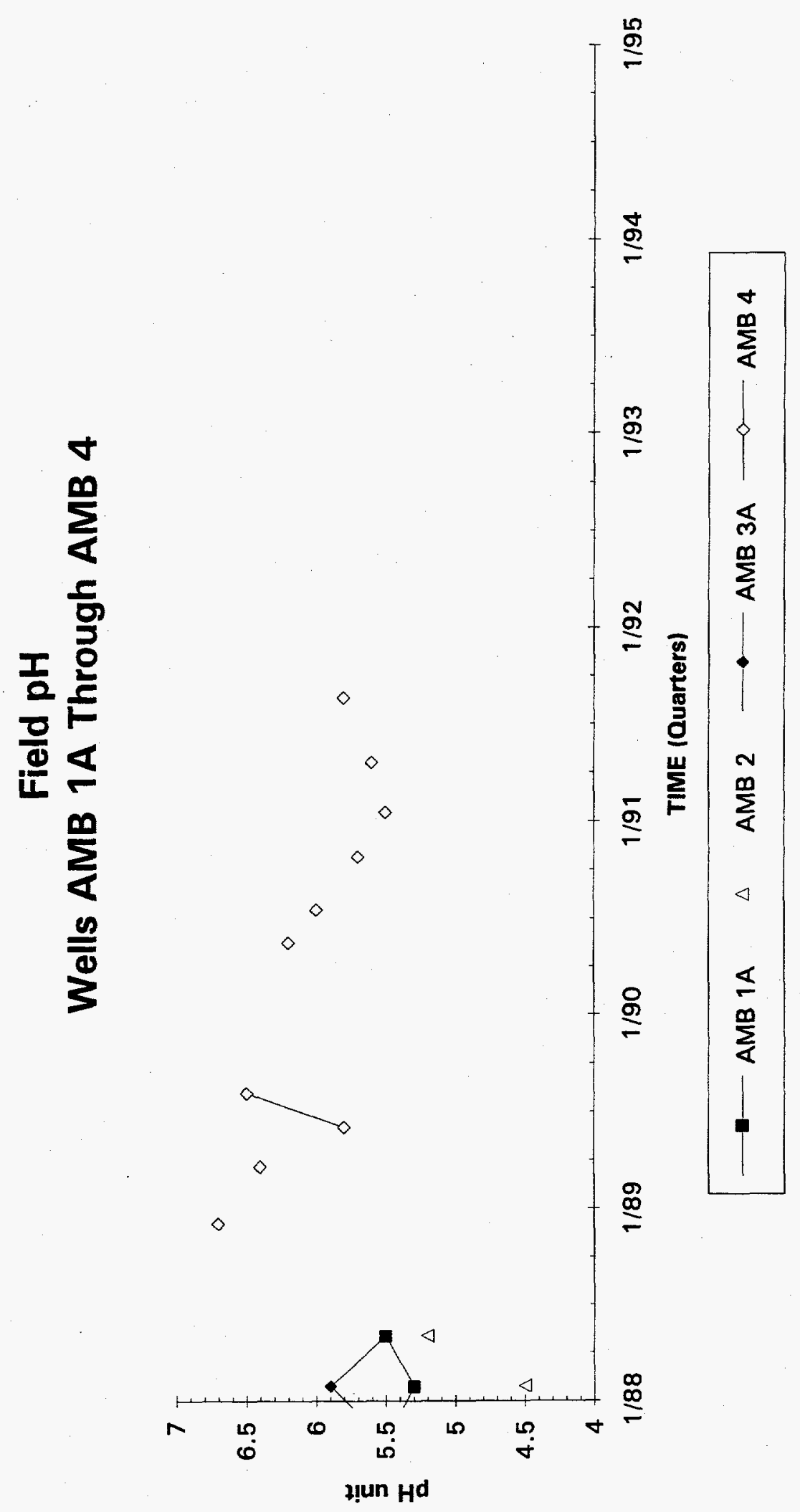

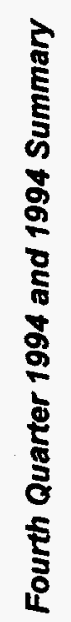



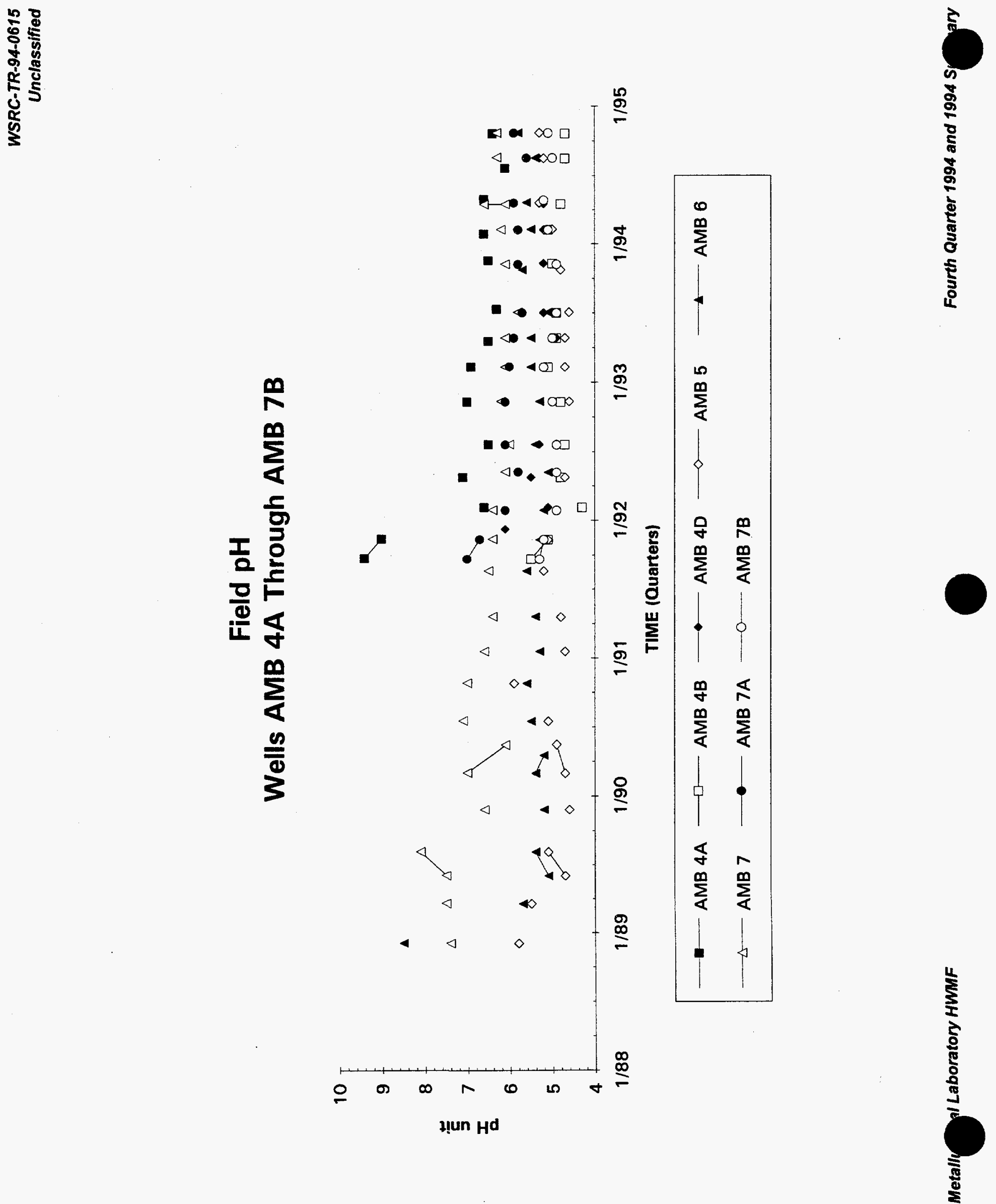


\section{Field $\mathrm{pH}$}

Wells AMB 8D Through AMB 13AR

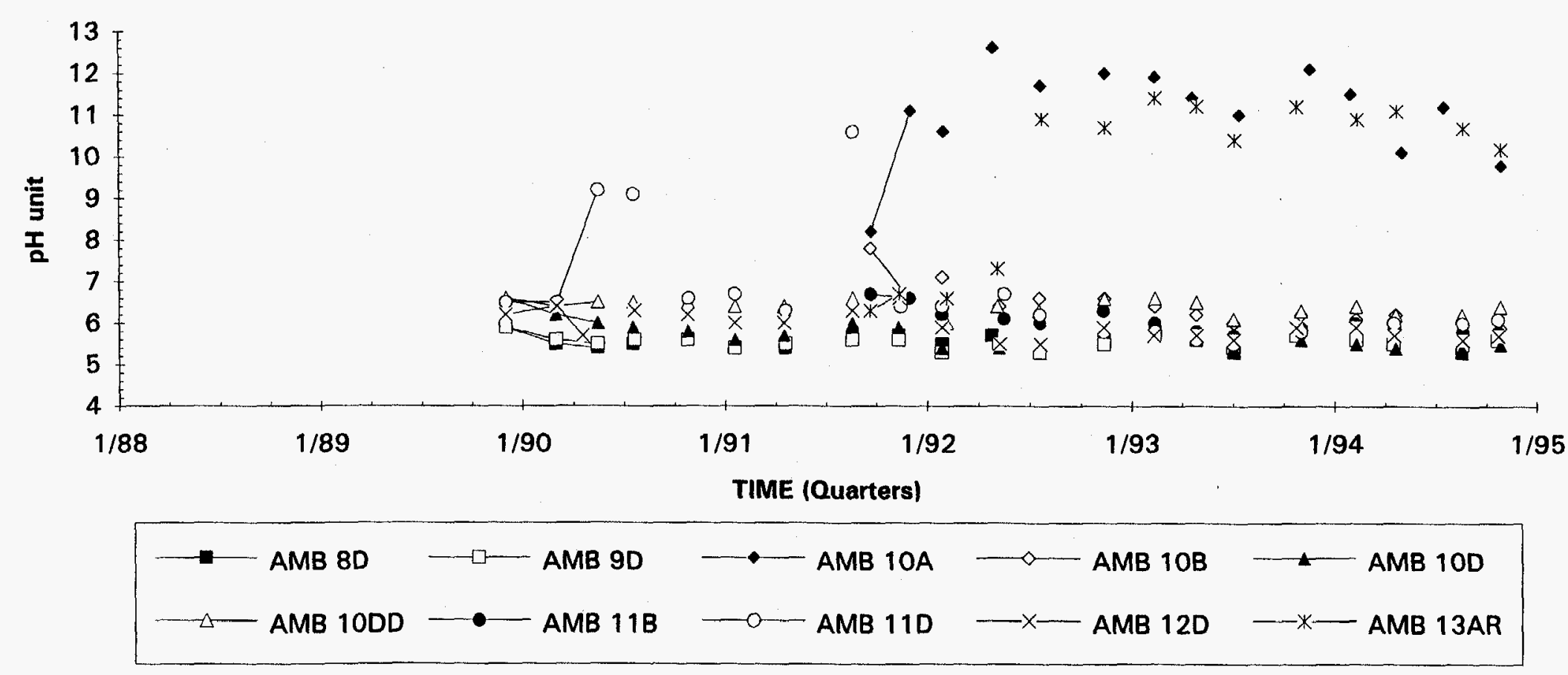




\section{Field Specific Conductance}

\section{Wells AMB 1A Through AMB 4}

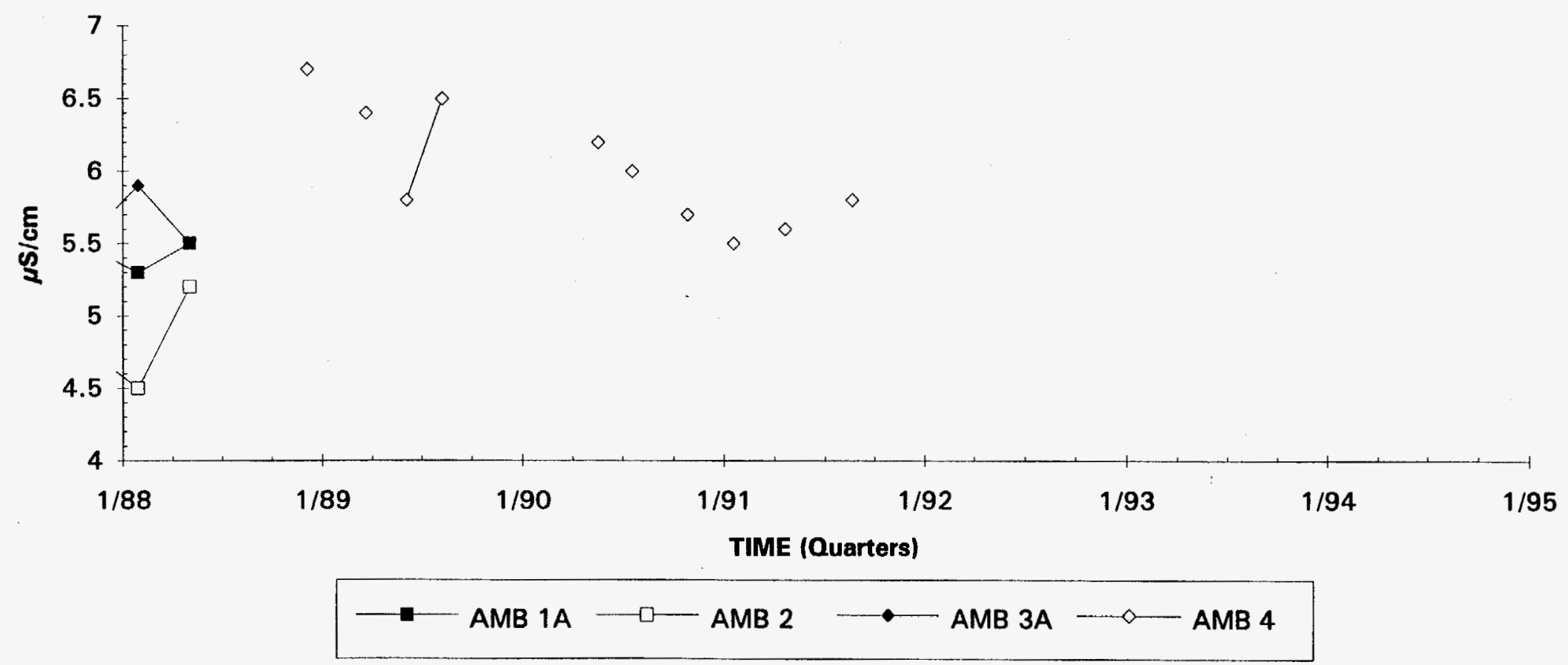


Field Specific Conductance

Wells AMB 4A Through AMB 7B

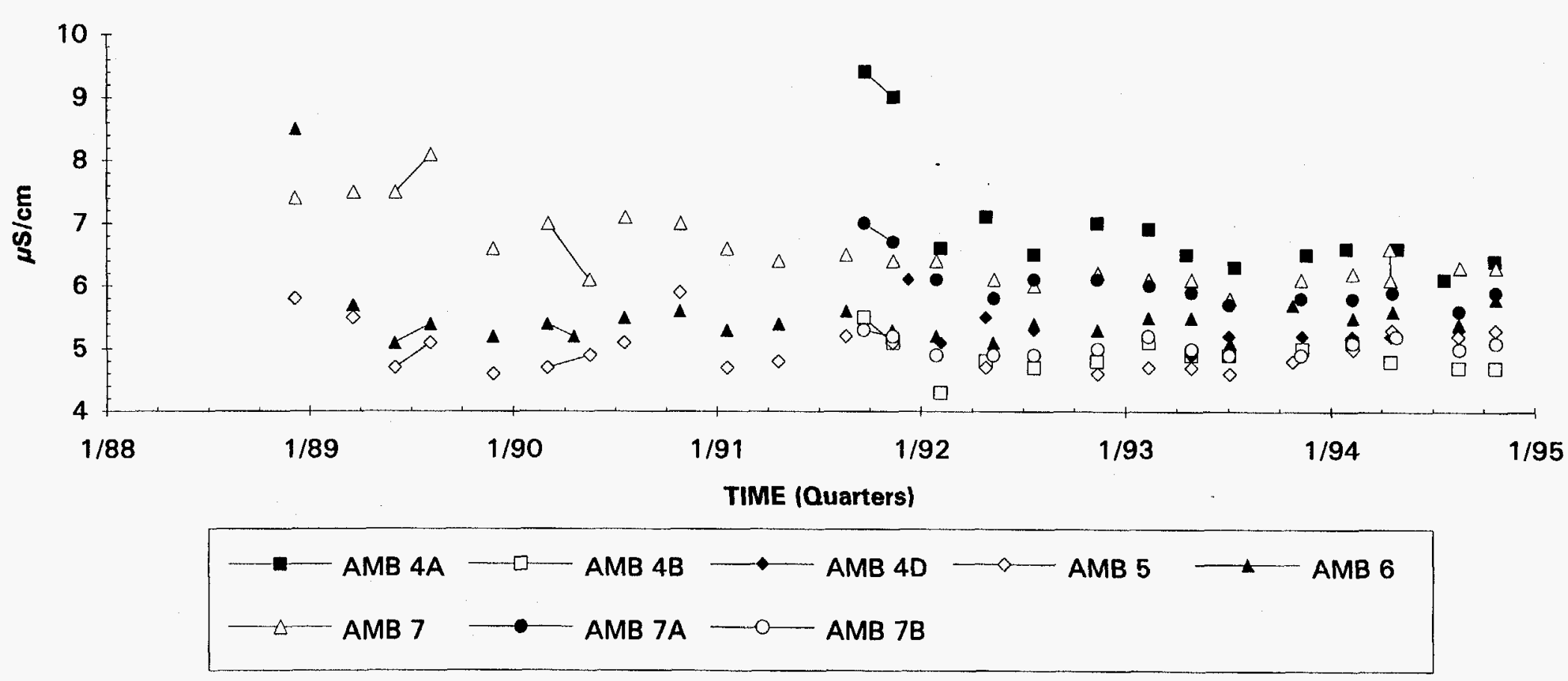



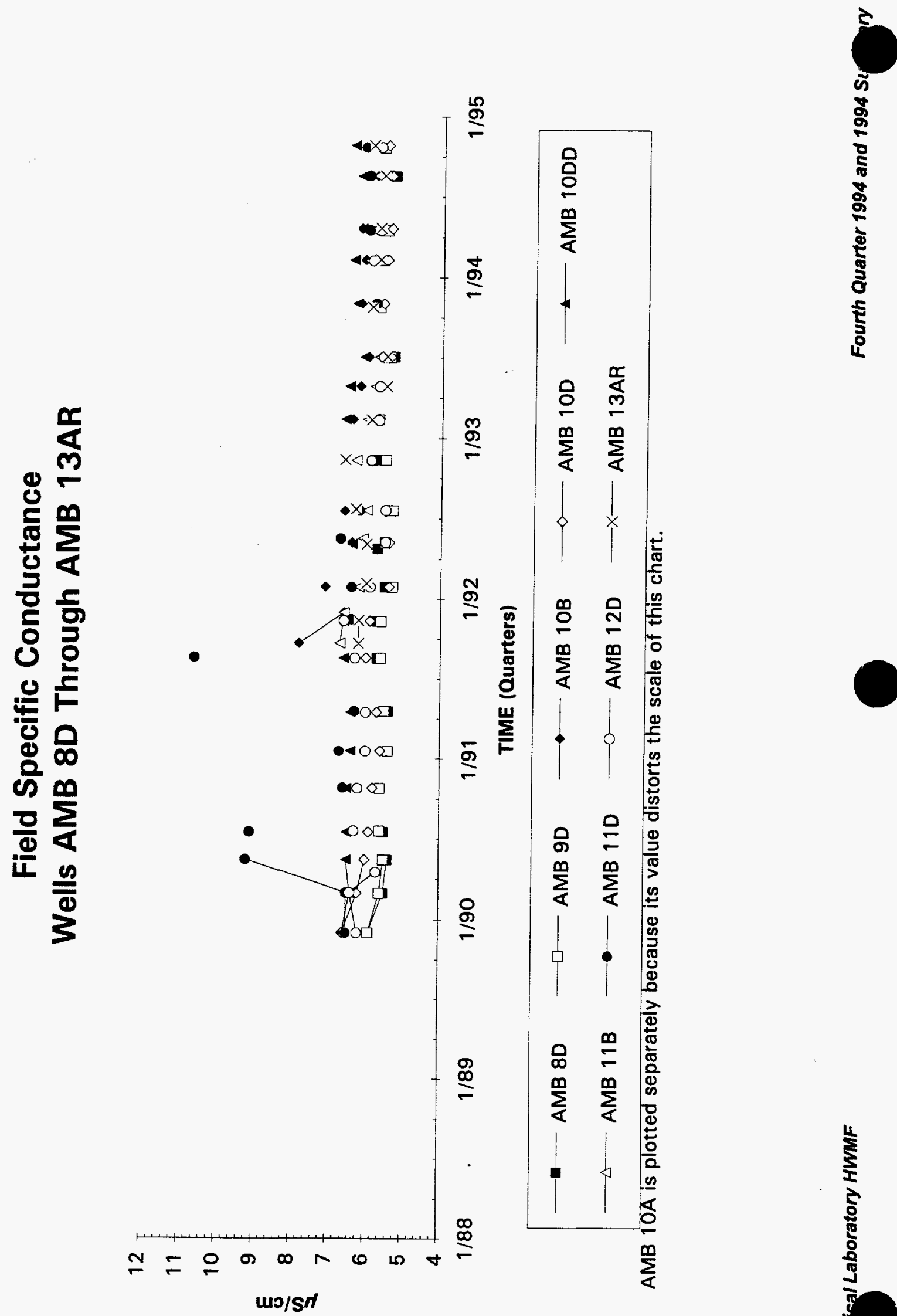

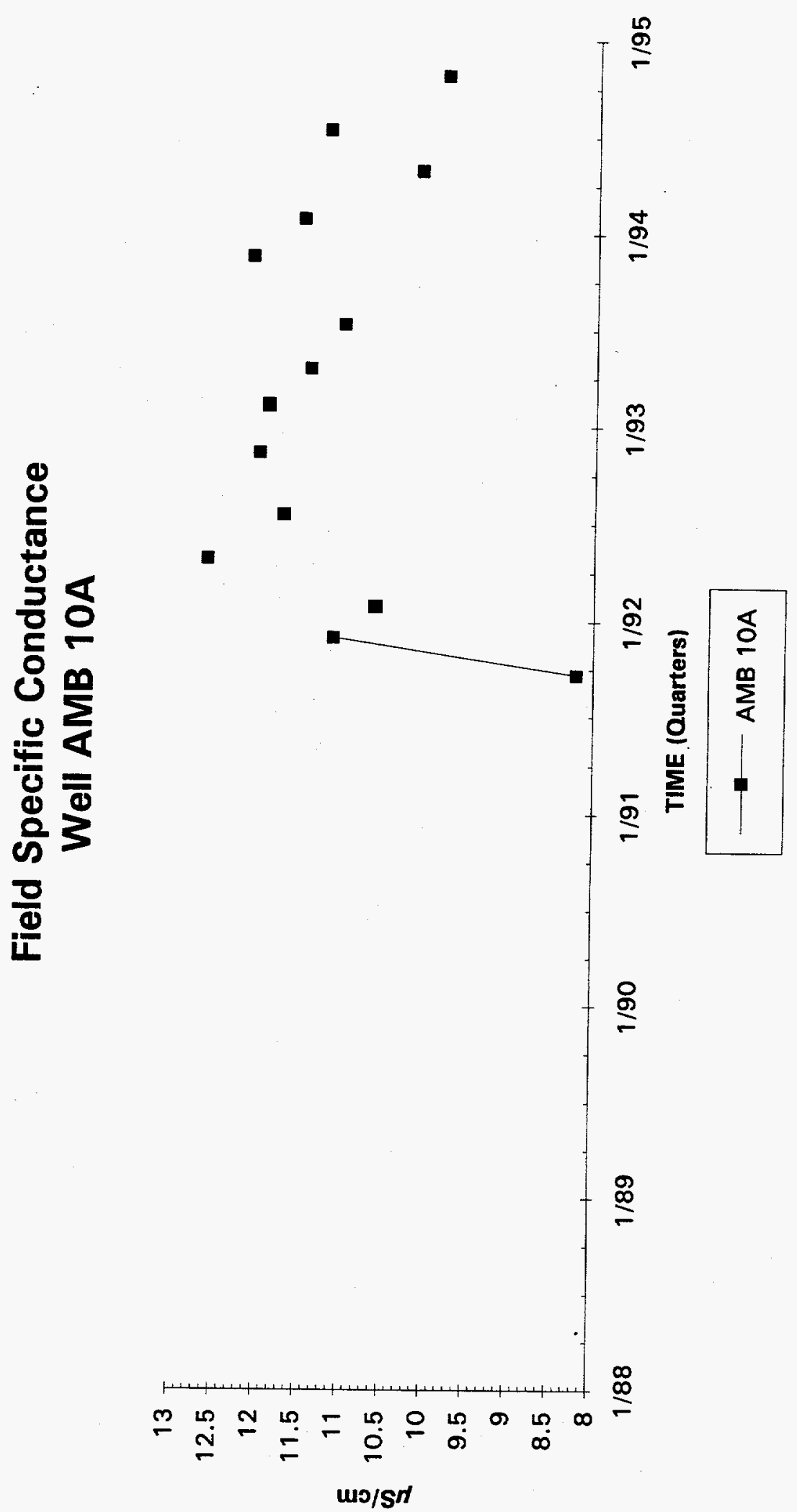


\section{Tetrachloroethylene Concentrations}

Well AMB 1A

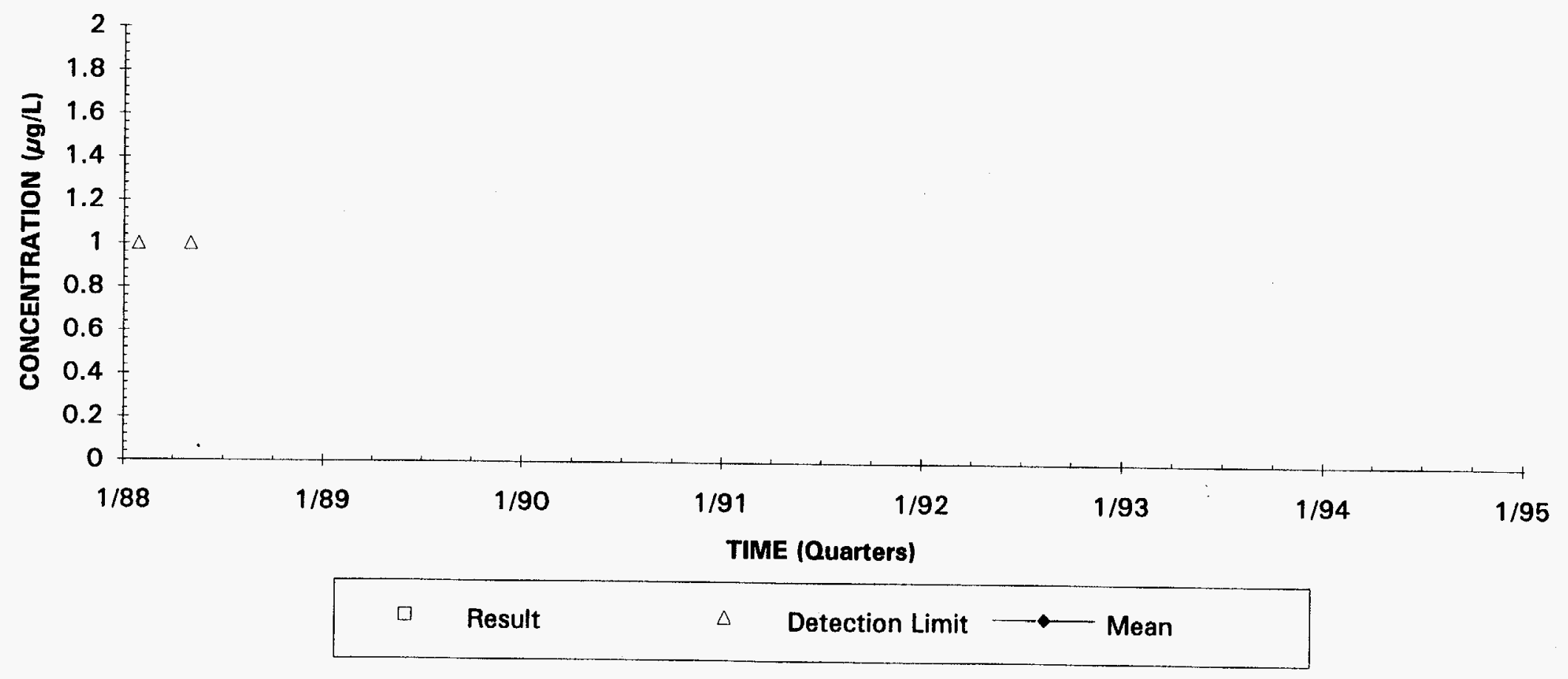



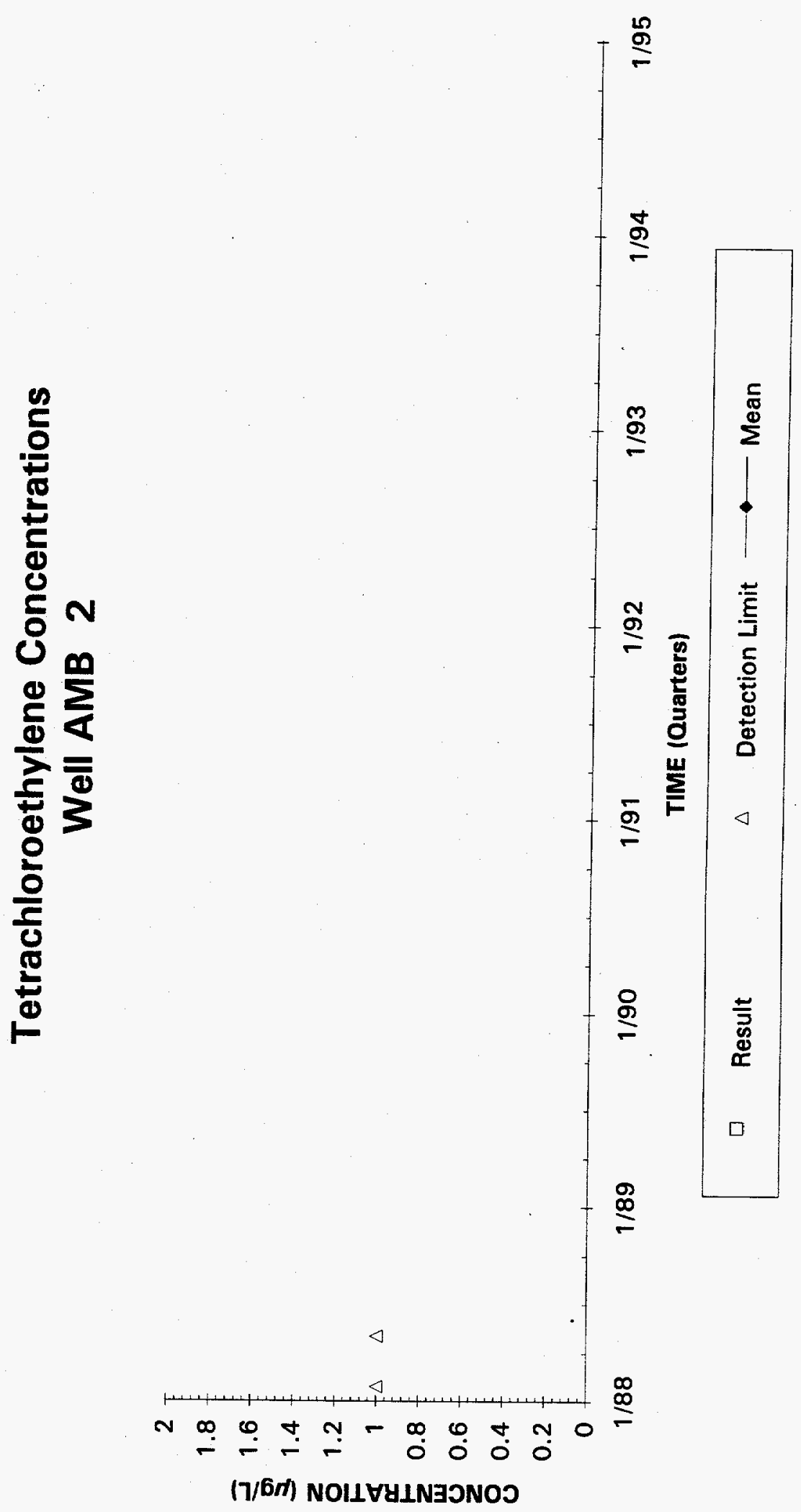


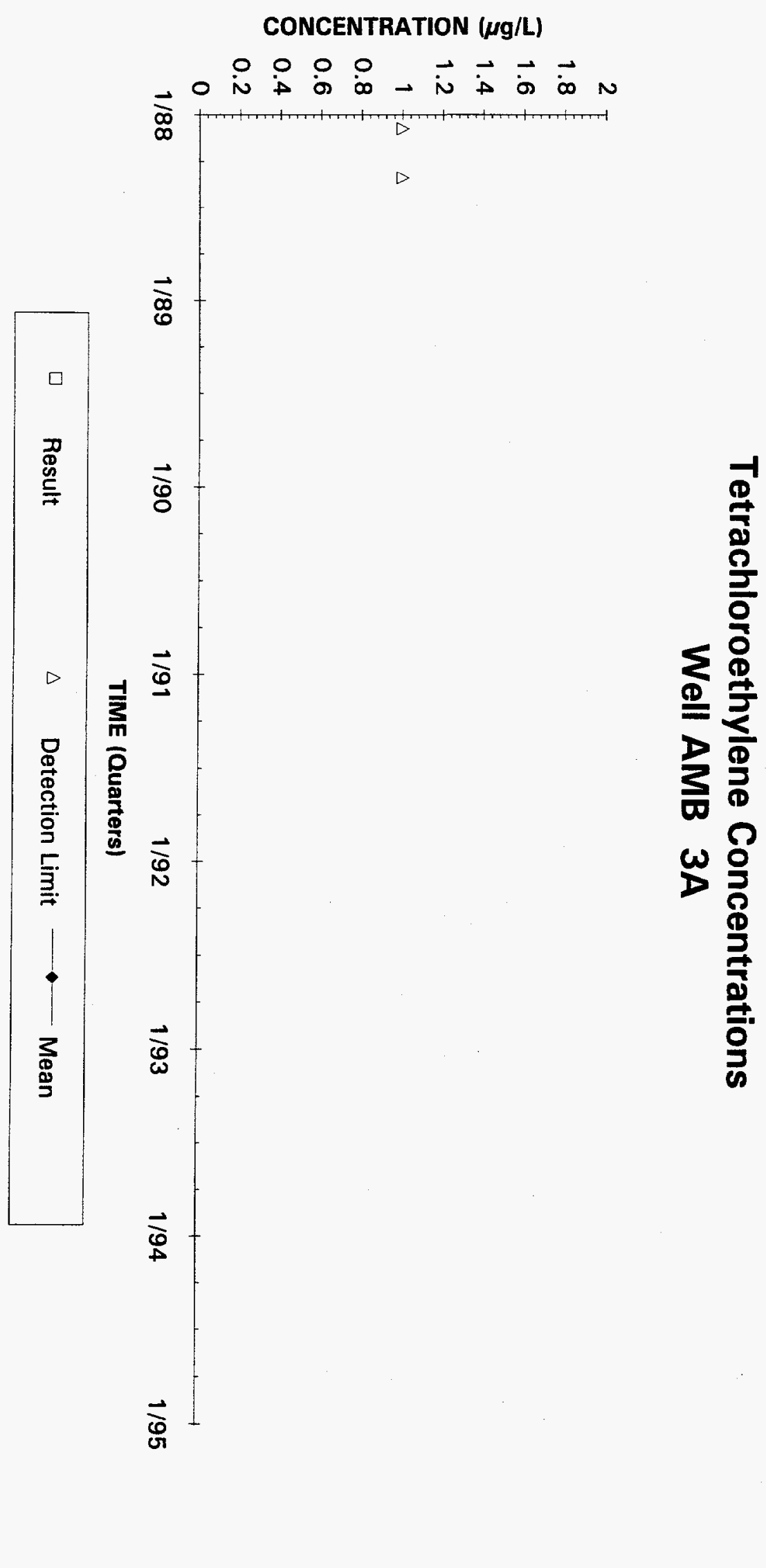

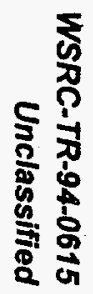


产
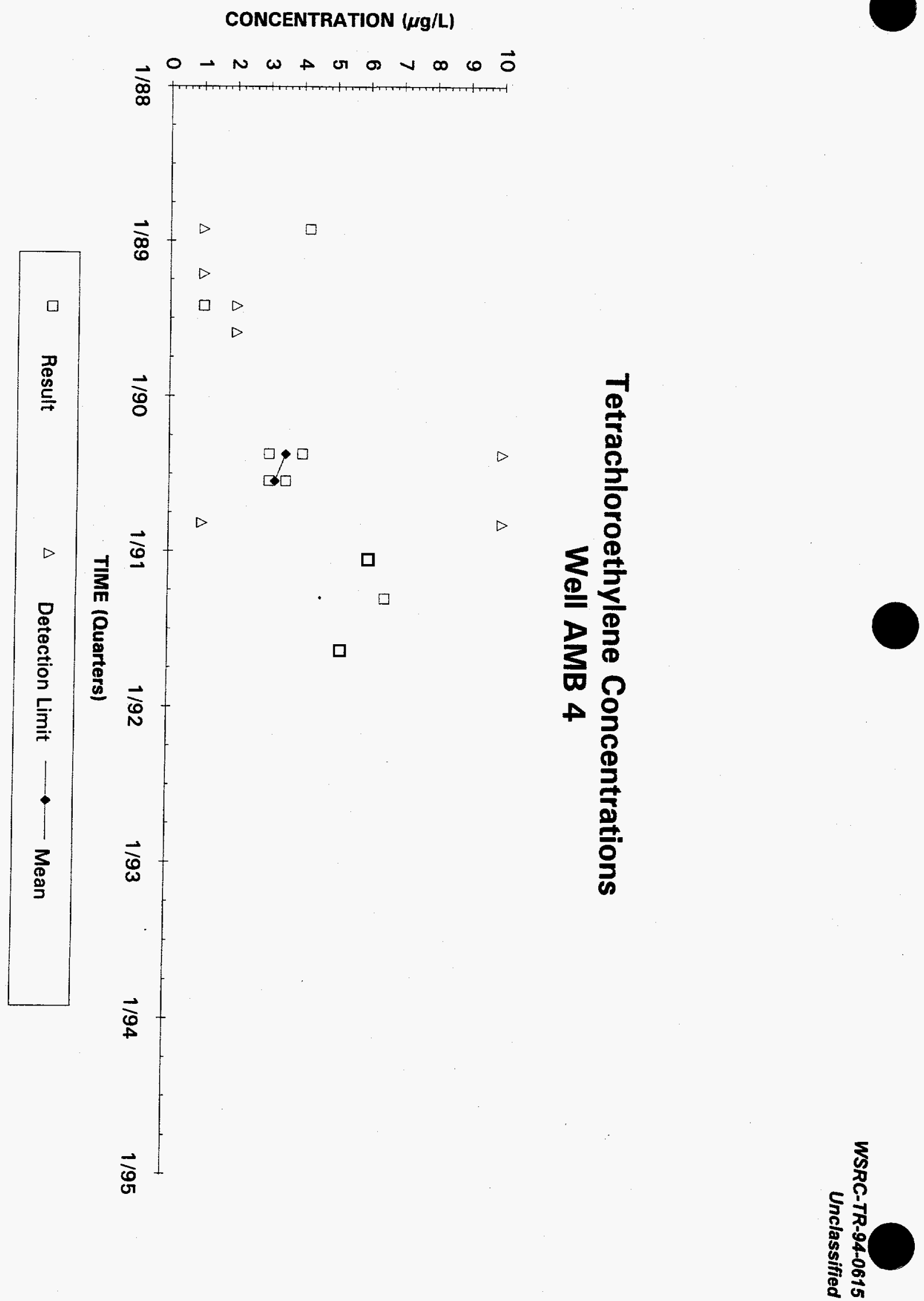


\section{Tetrachloroethylene Concentrations}

Well AMB 4A

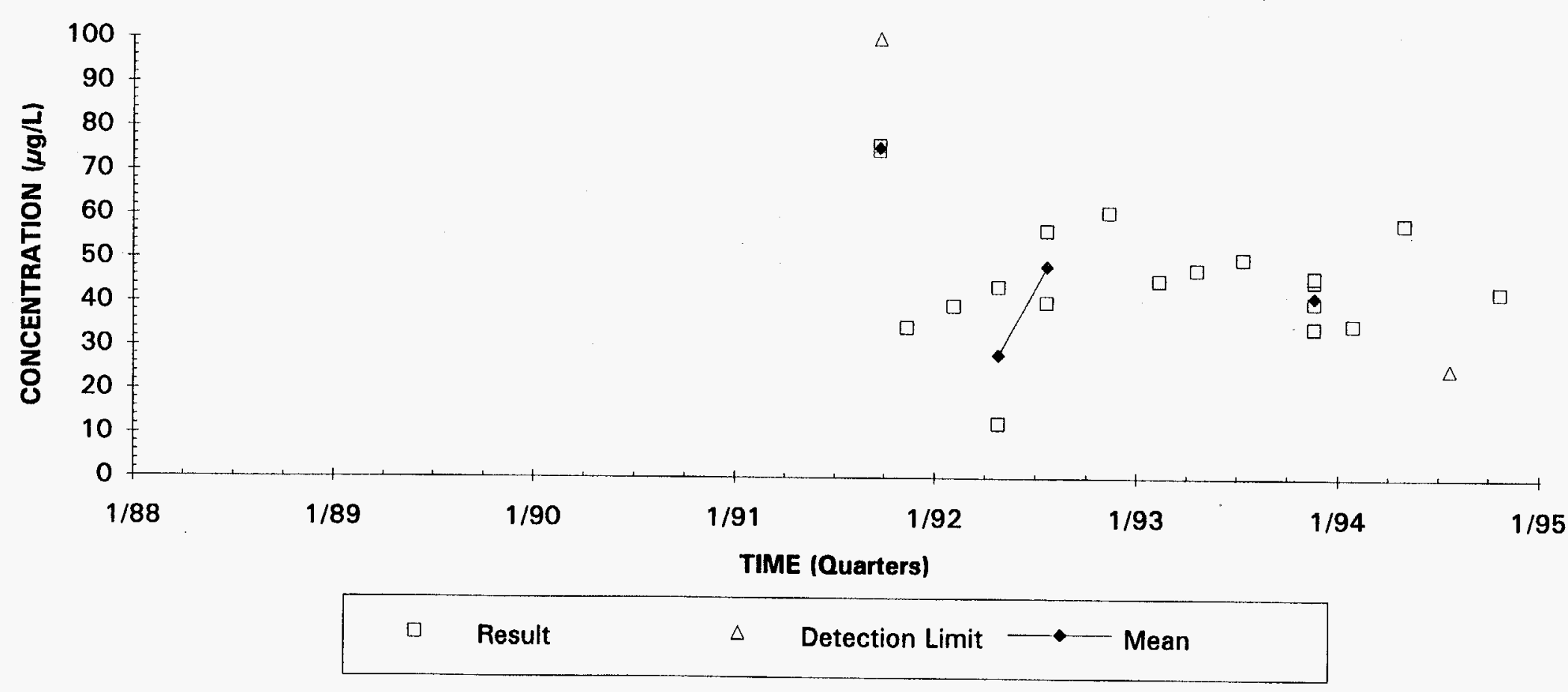



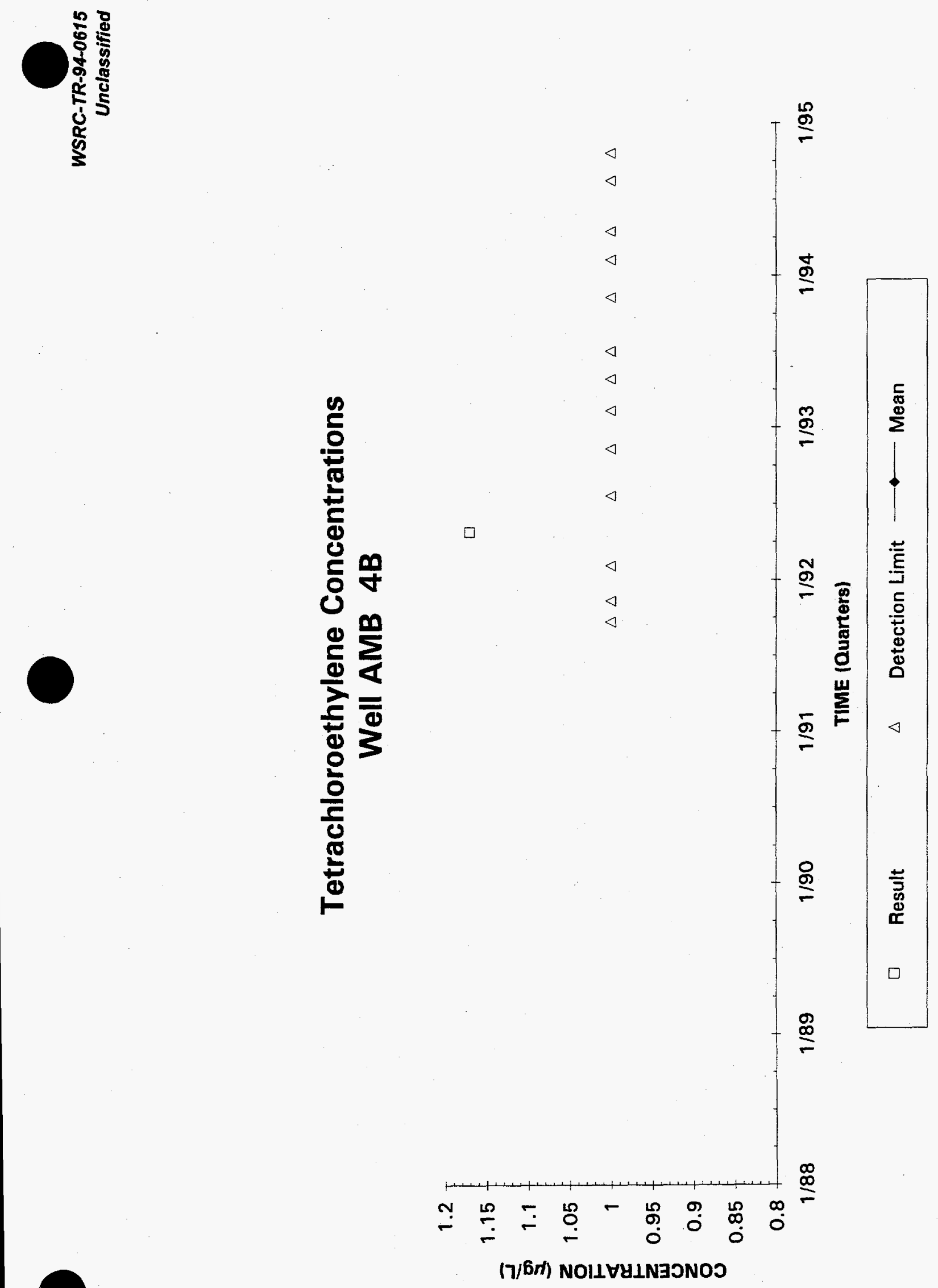

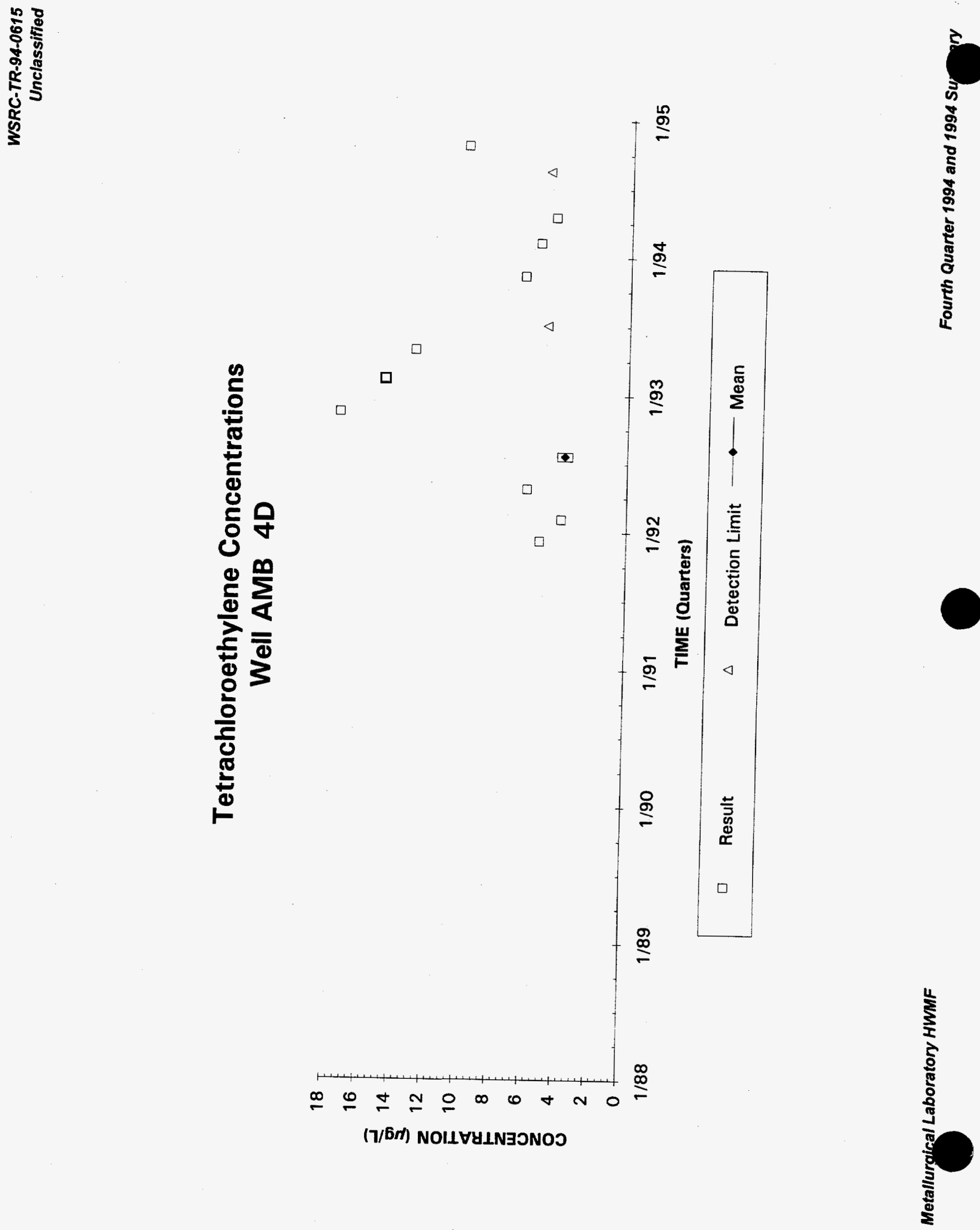


\section{Tetrachloroethylene Concentrations}

Well AMB 5

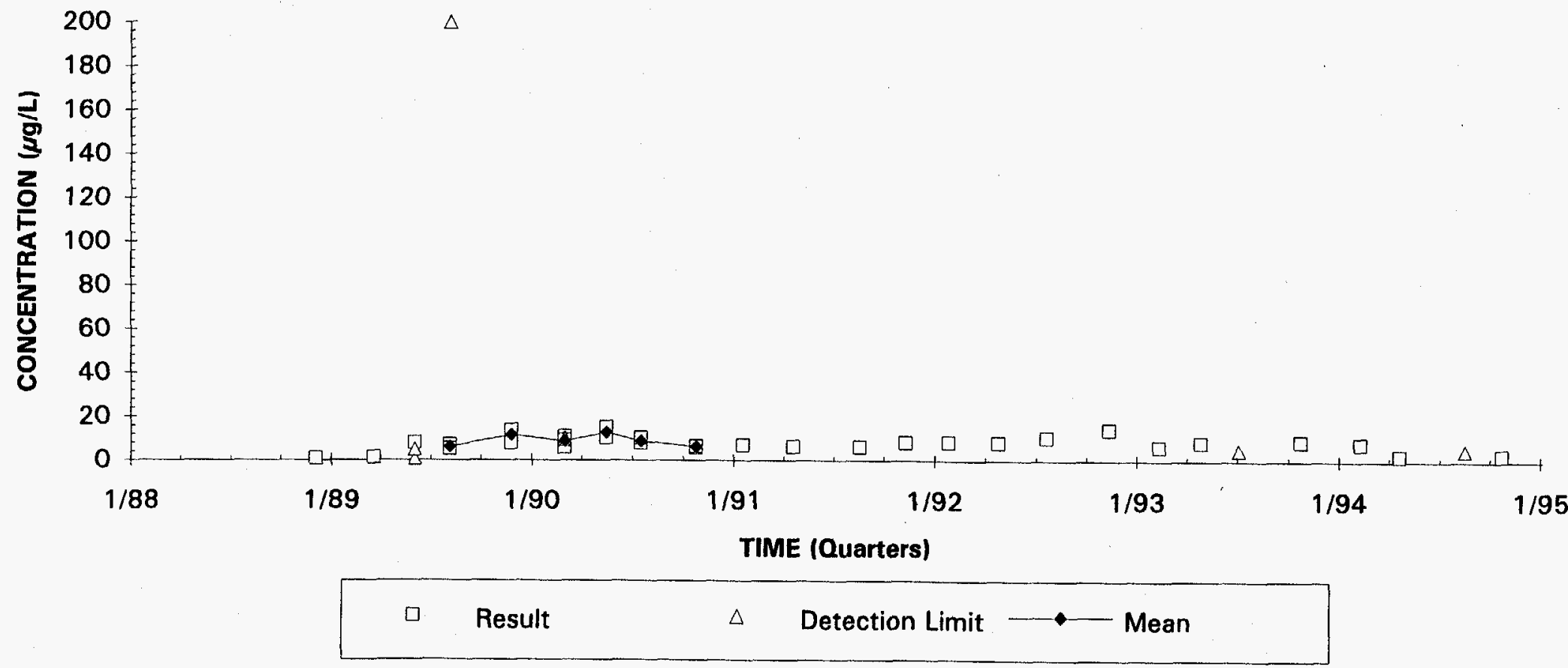




\section{Tetrachloroethylene Concentrations}

Well AMB 6

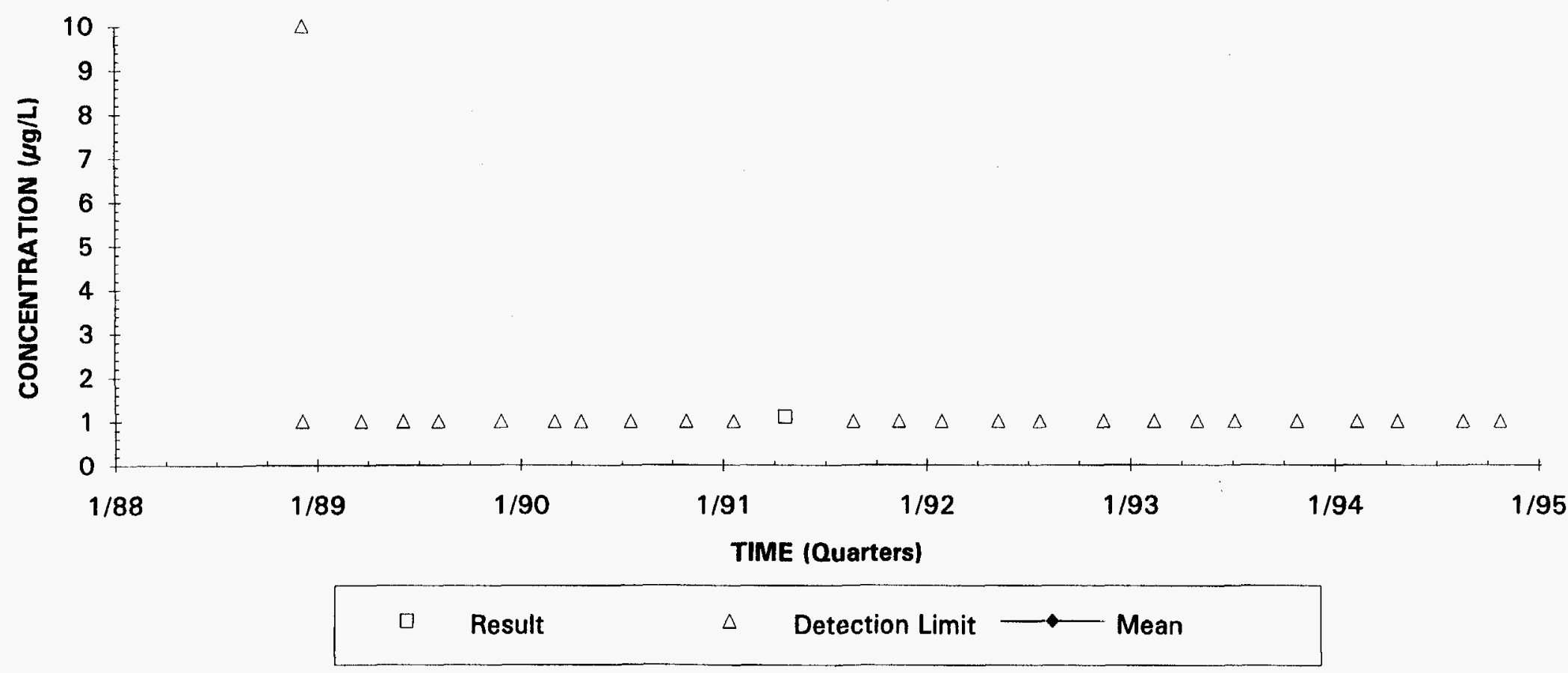




\section{Tetrachloroethylene Concentrations}

Well AMB 7

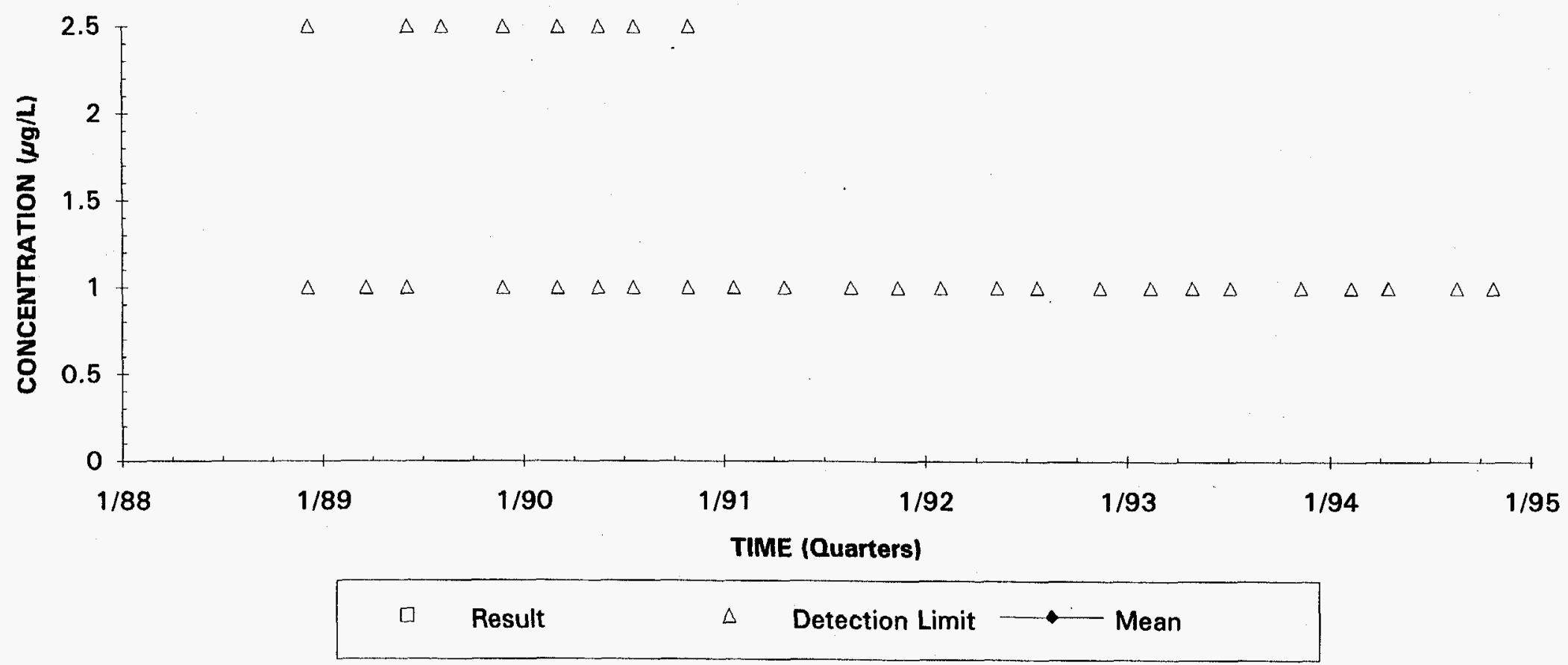




\section{Tetrachloroethylene Concentrations}

\section{Well AMB 7A}

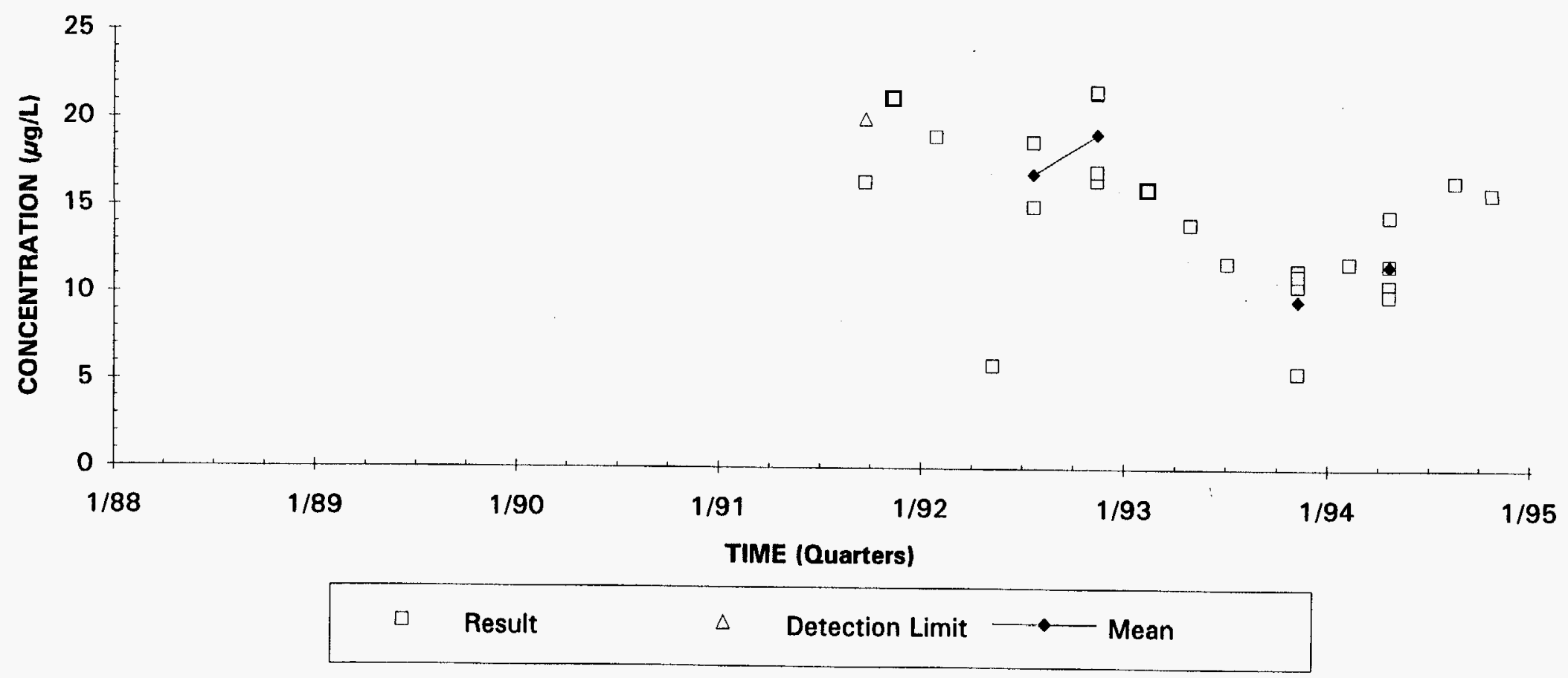





\section{Tetrachloroethylene Concentrations}

Well AMB 8D

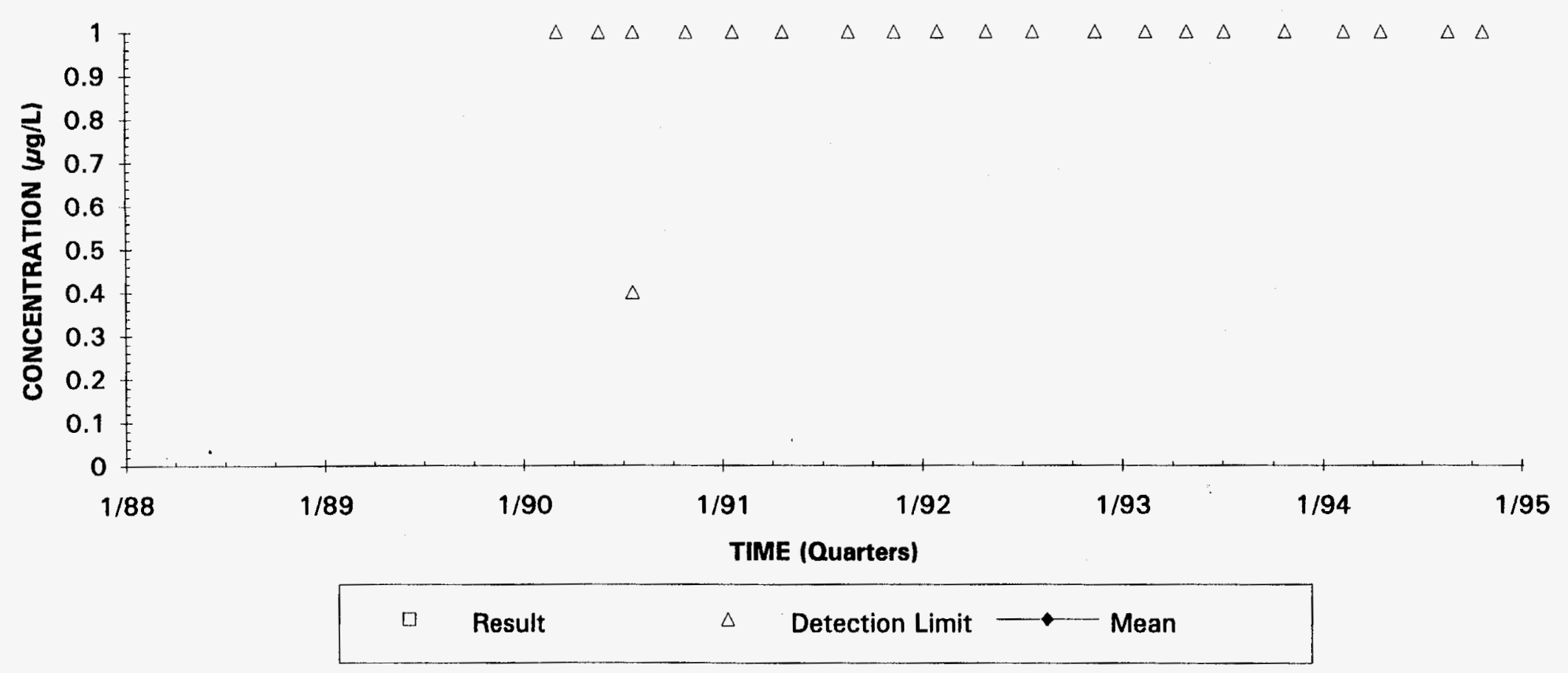




\section{Tetrachloroethylene Concentrations}

\section{Well AMB 9D}

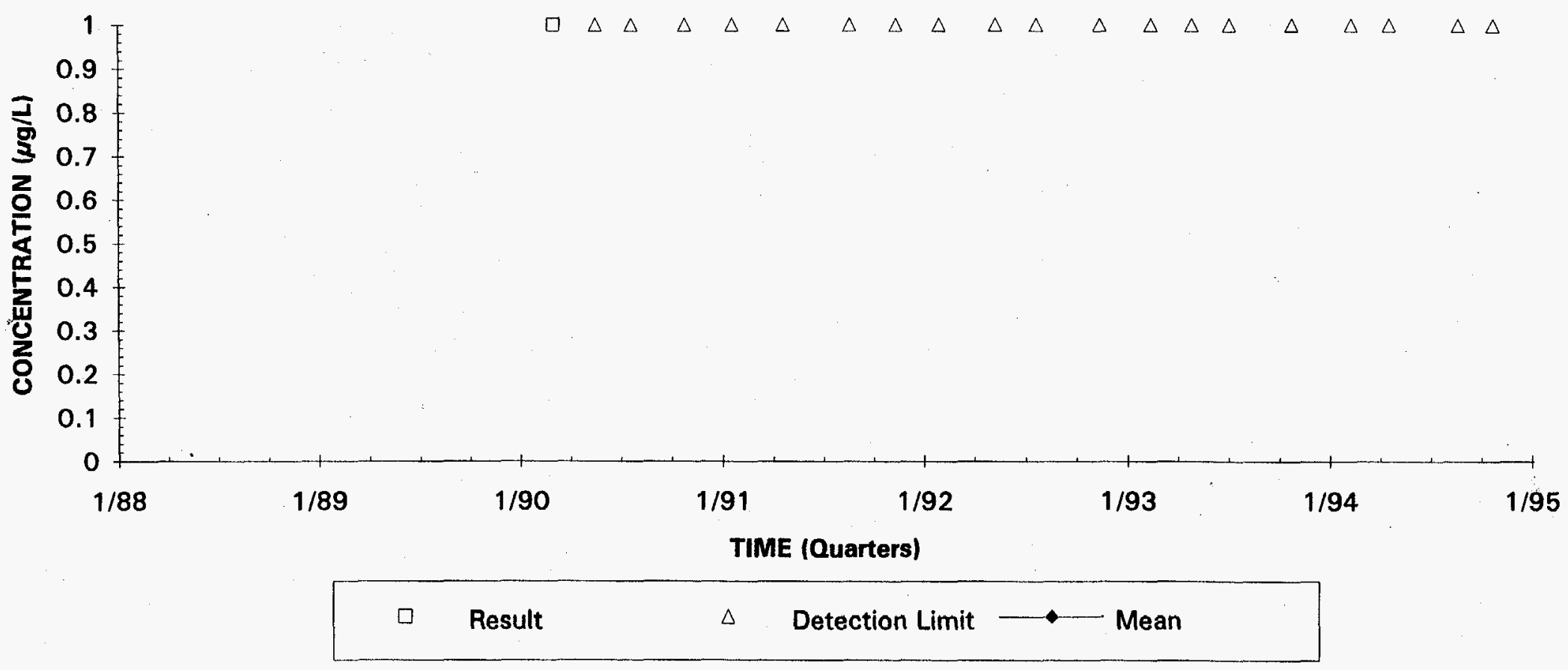




\section{Tetrachloroethylene Concentrations}

Well AMB 10A

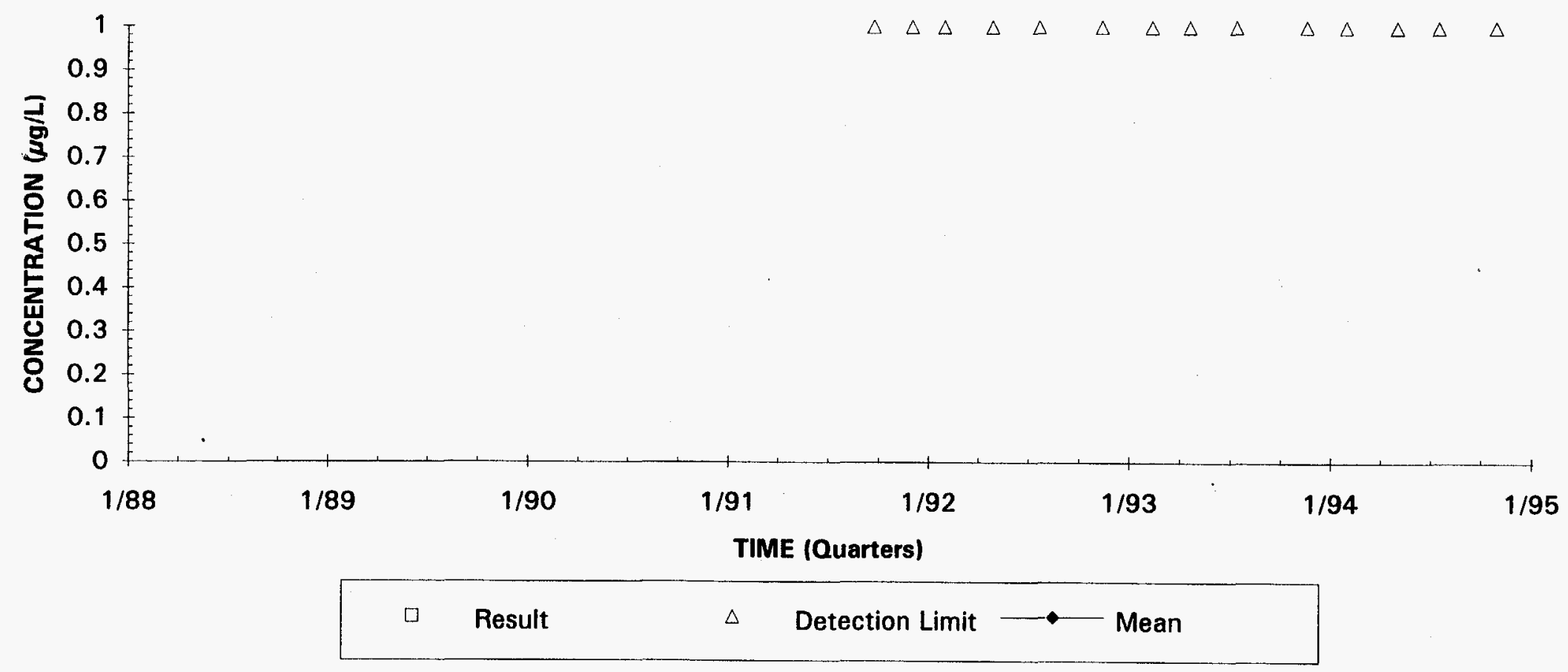




\section{Tetrachloroethylene Concentrations}

Well AMB 10B

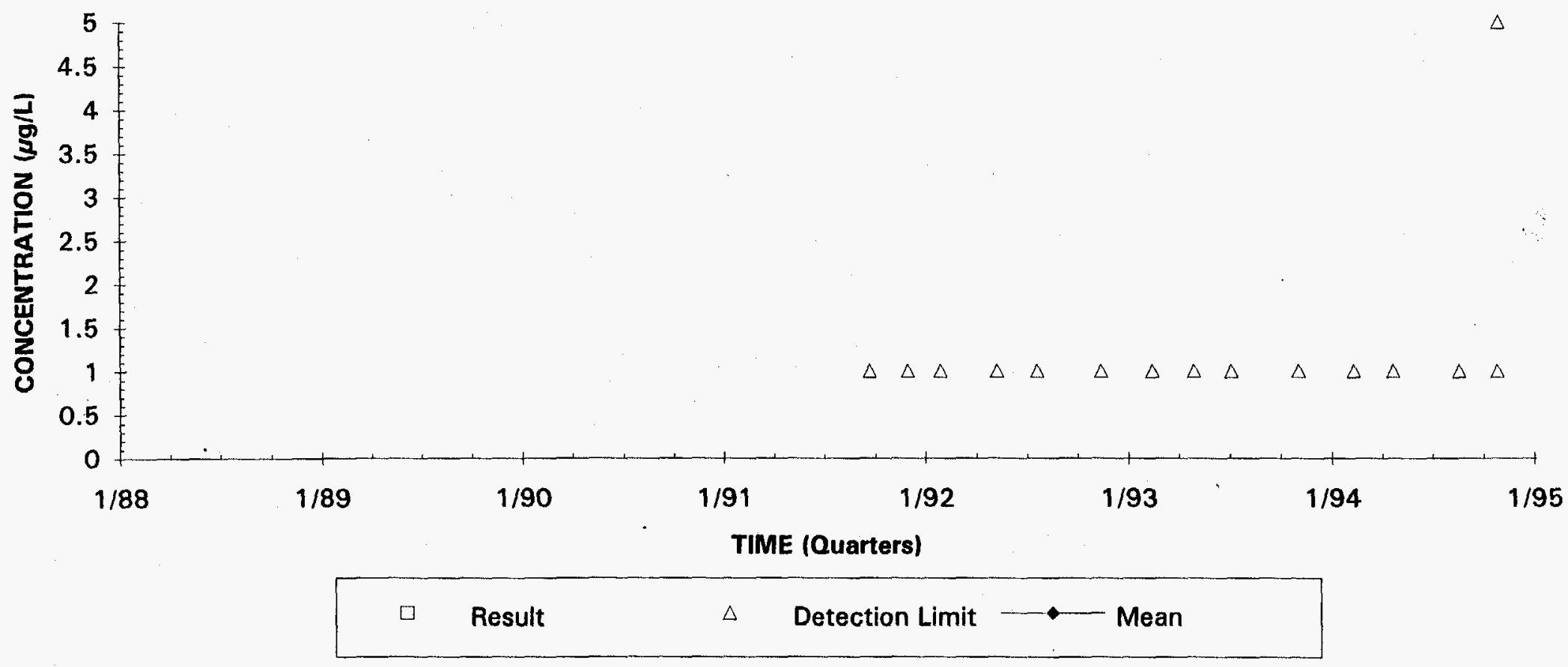




\section{Tetrachloroethylene Concentrations}

Well AMB 10D

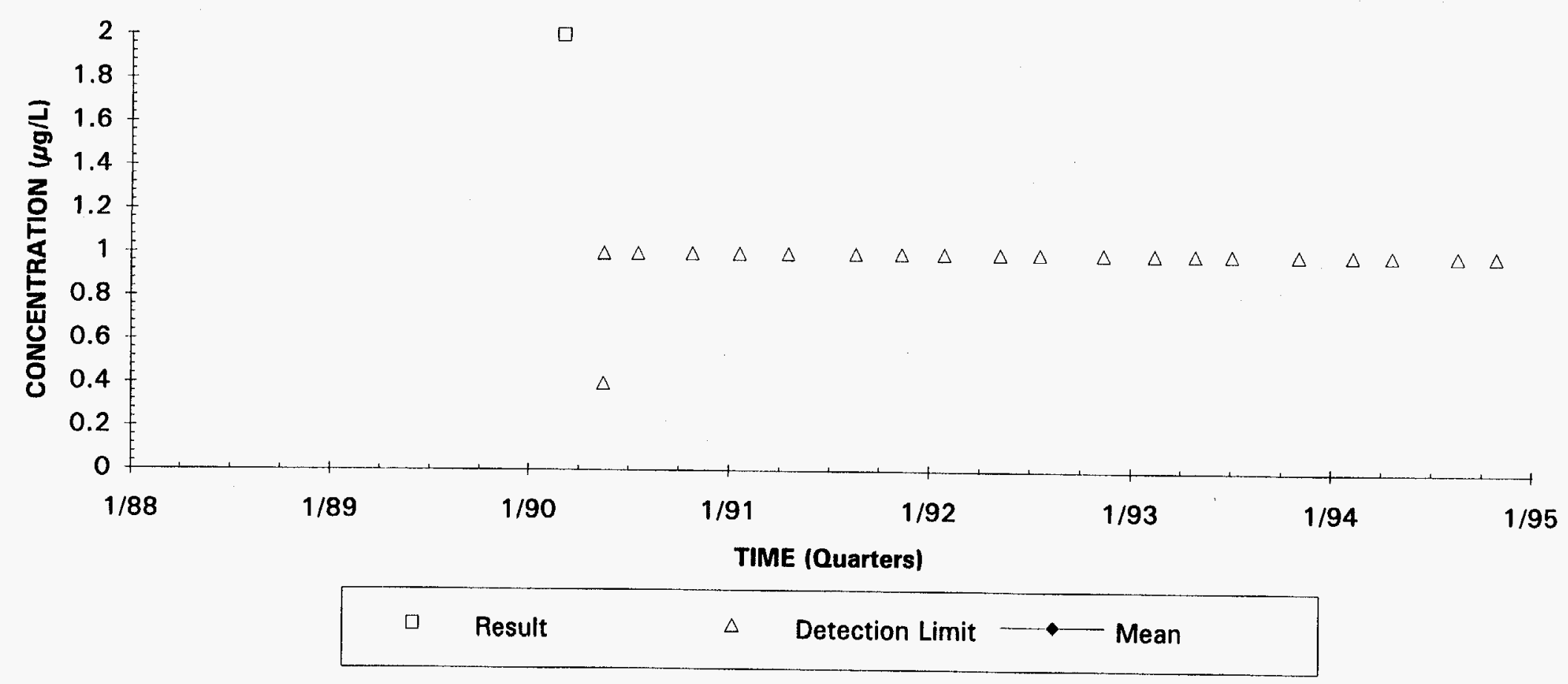




\section{Tetrachloroethylene Concentrations}

\section{Well AMB 10DD}

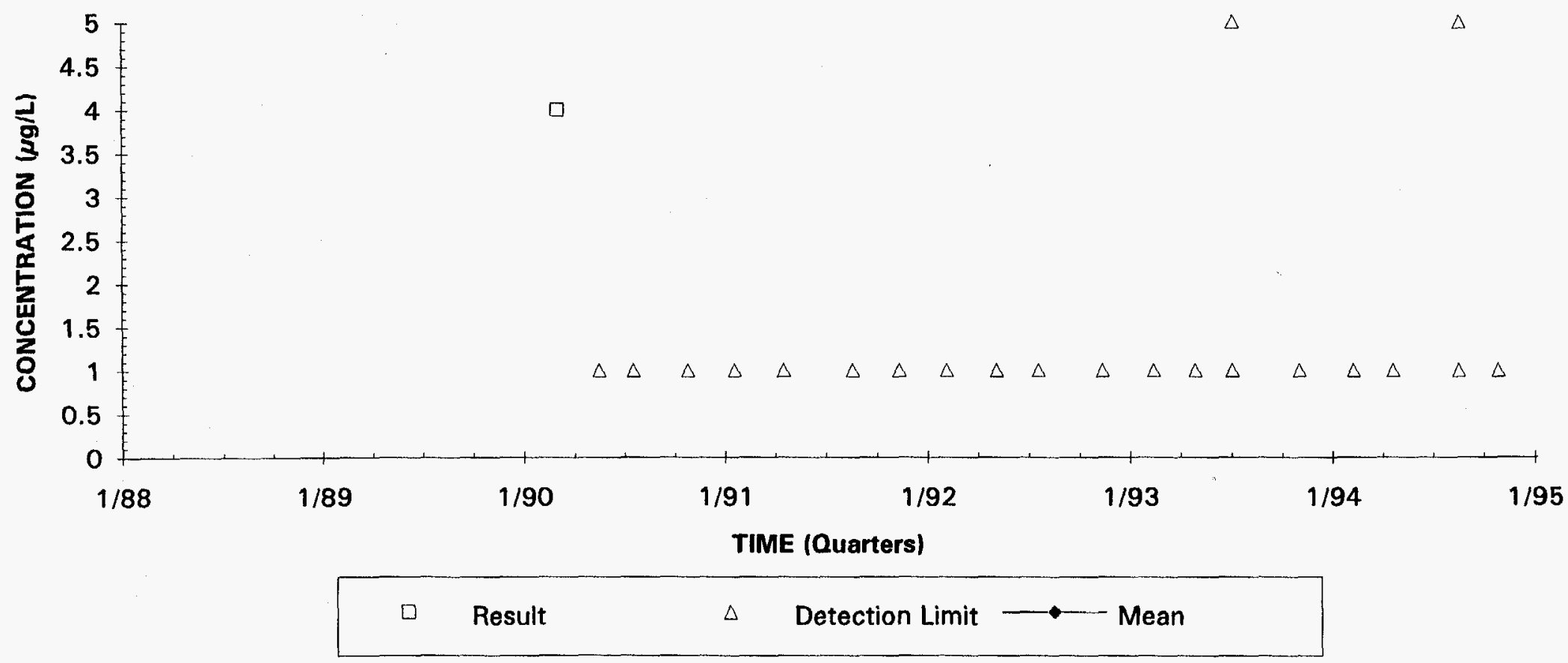


Tetrachloroethylene Concentrations

Well AMB 11B

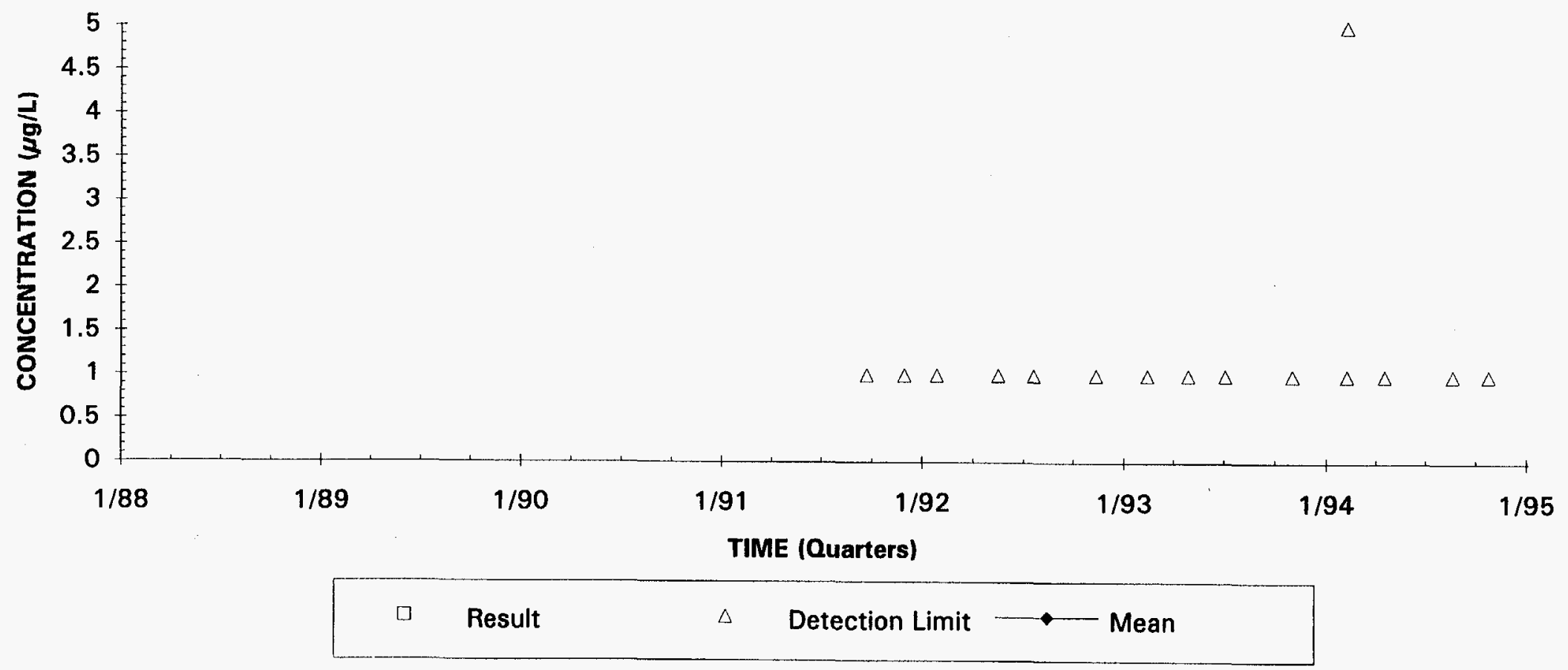




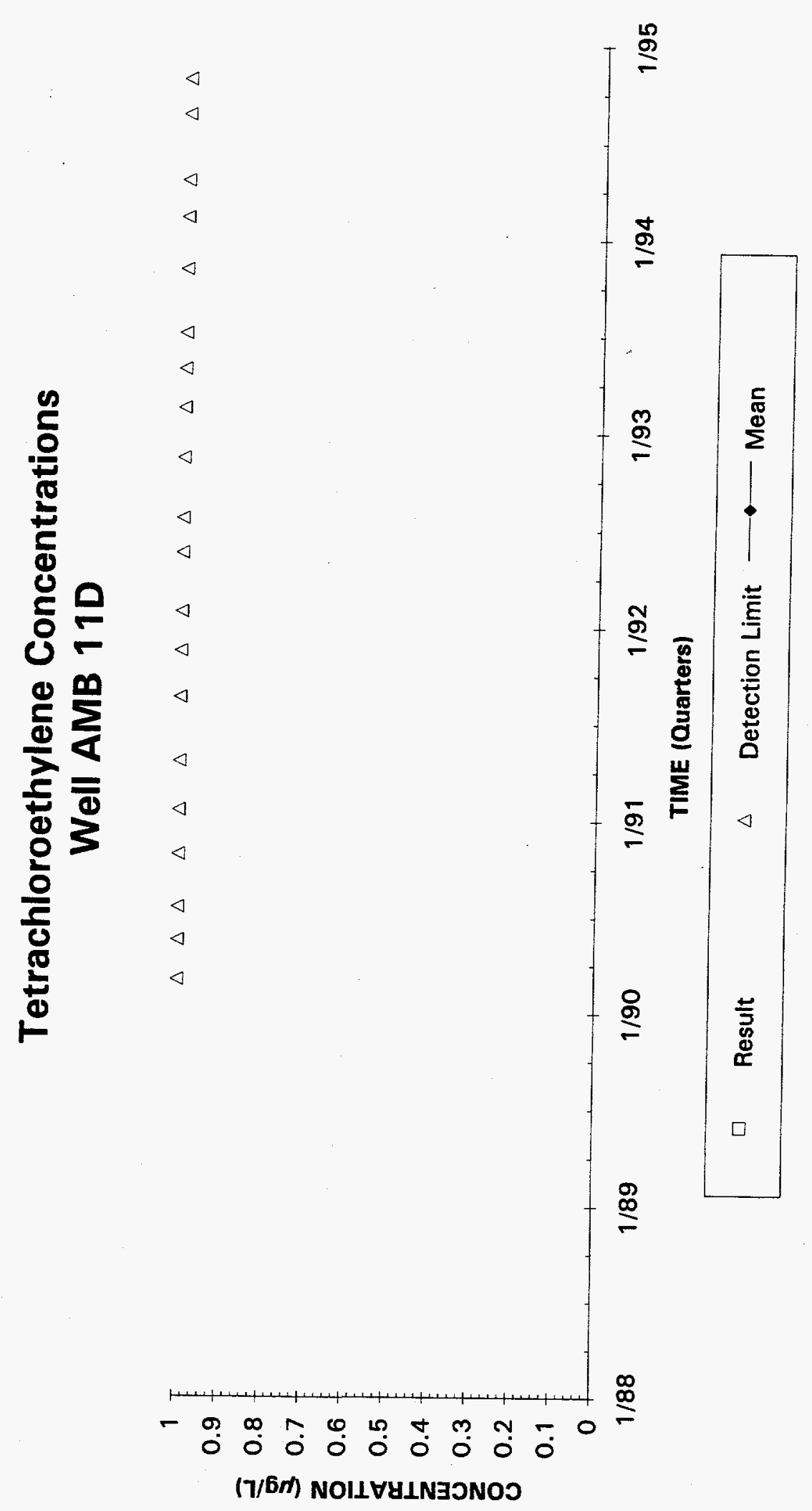

ह 


\section{Tetrachloroethylene Concentrations}

Well AMB 12D

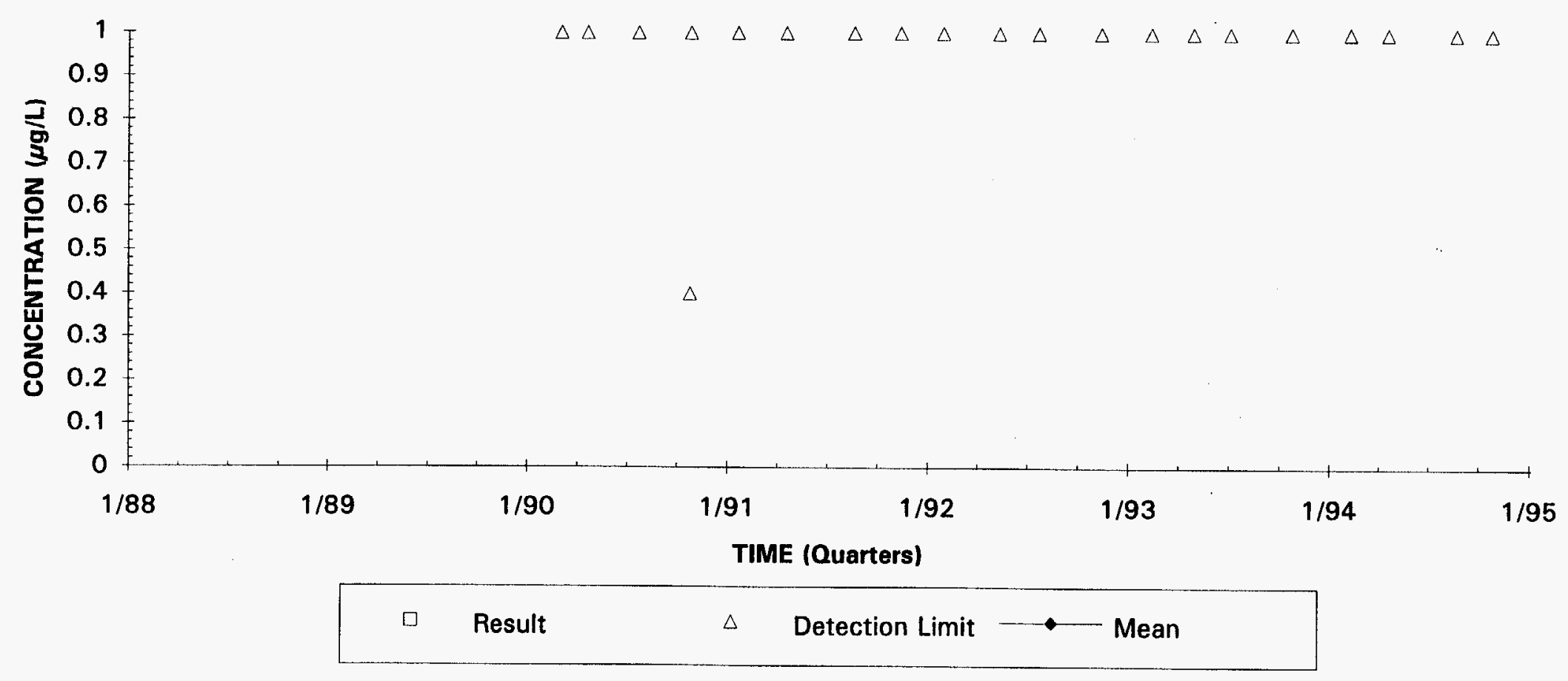




\section{Tetrachloroethylene Concentrations}

Well AMB 13AR

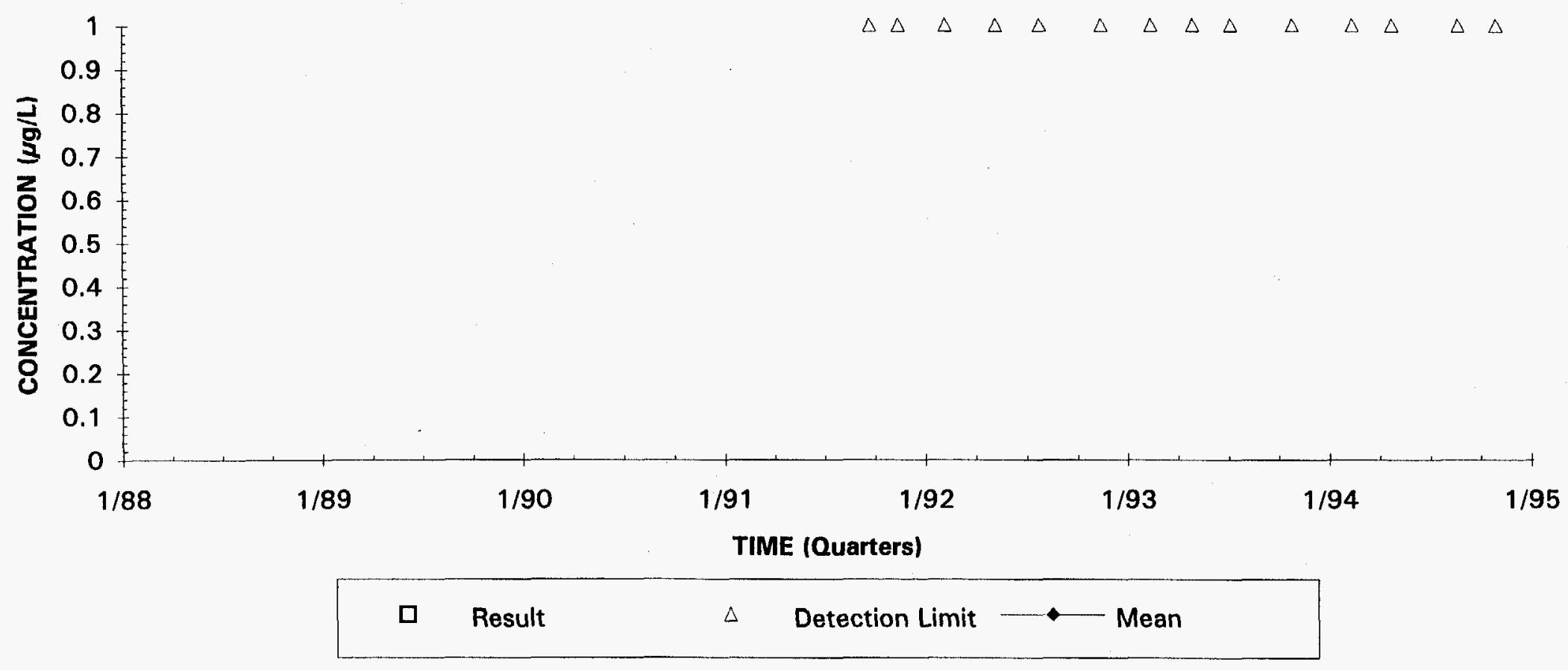



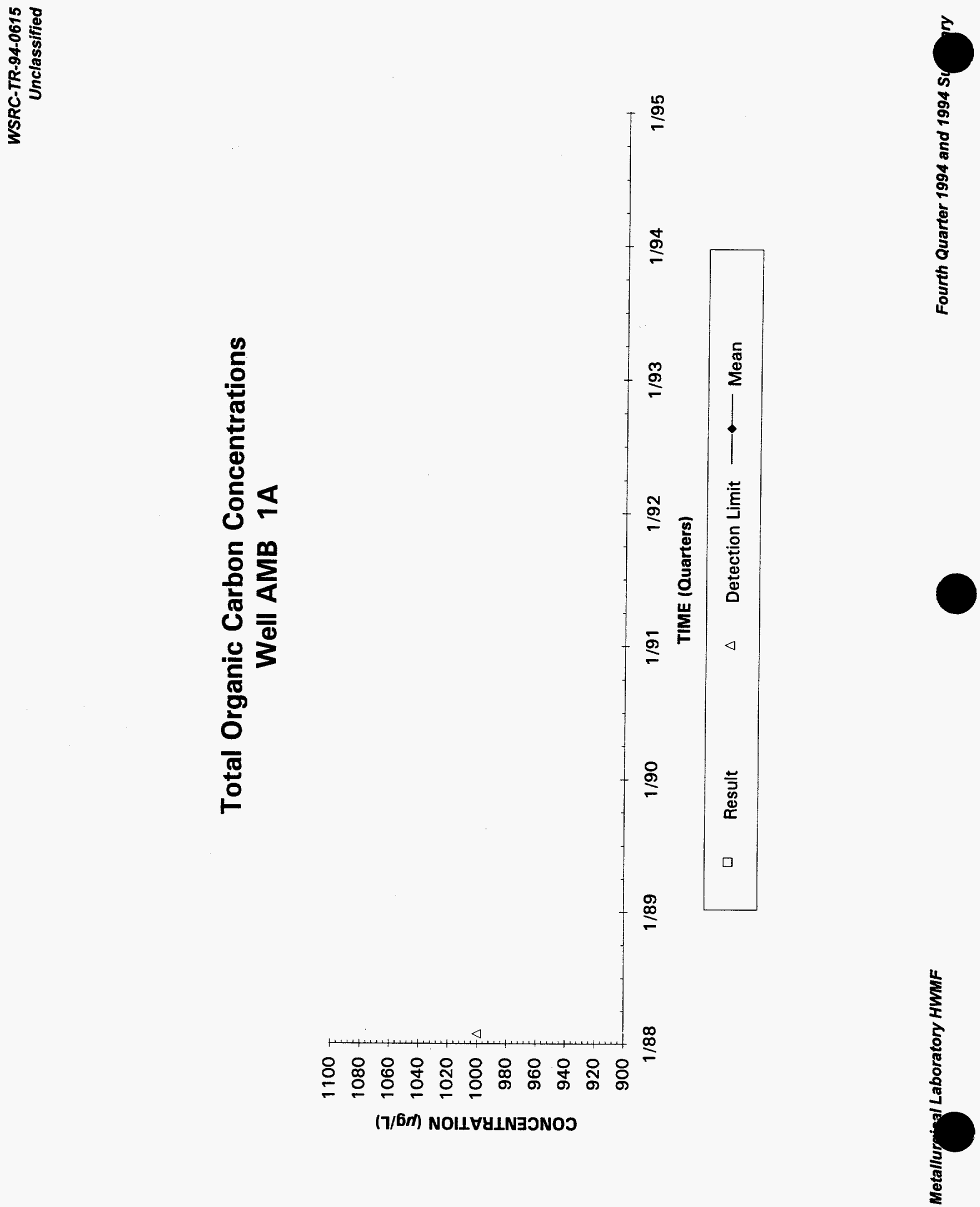


\section{Total Organic Carbon Concentrations}

Well AMB 2

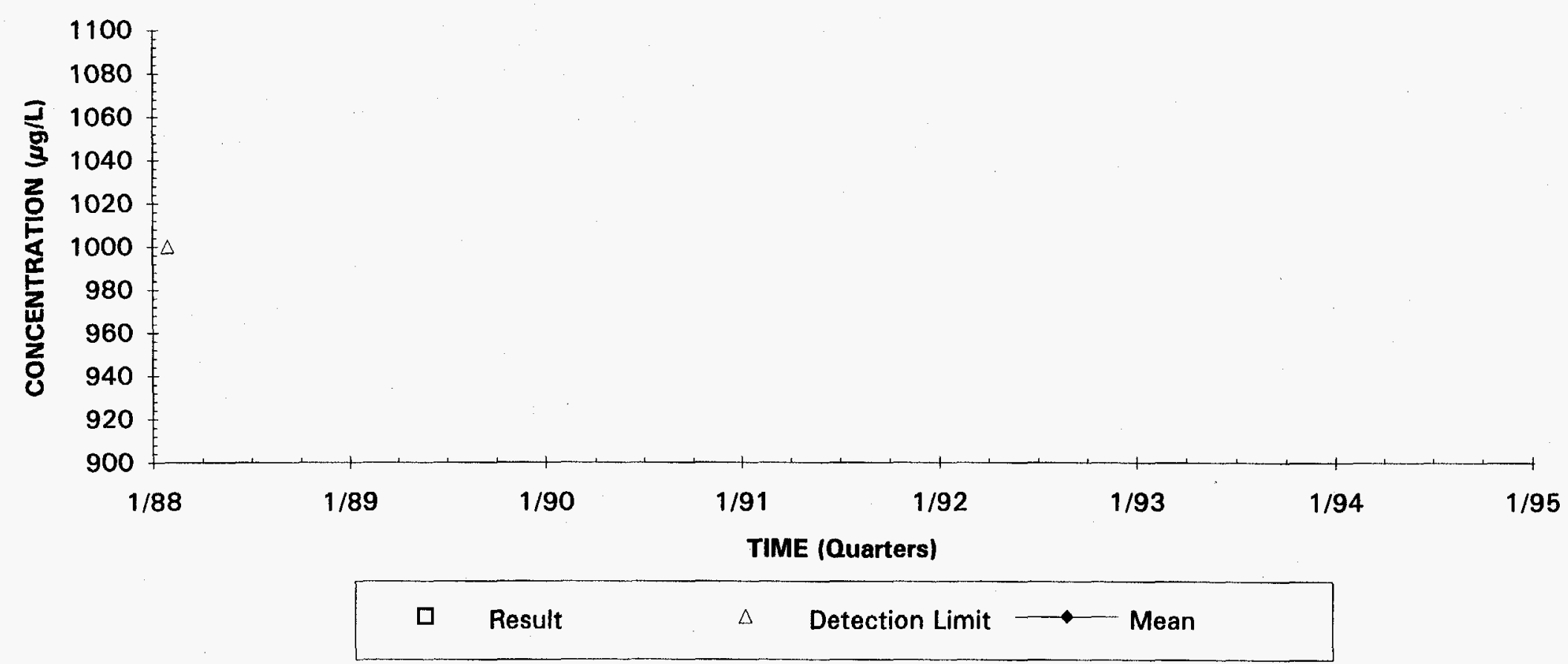




\section{Total Organic Carbon Concentrations}

Well AMB 3A

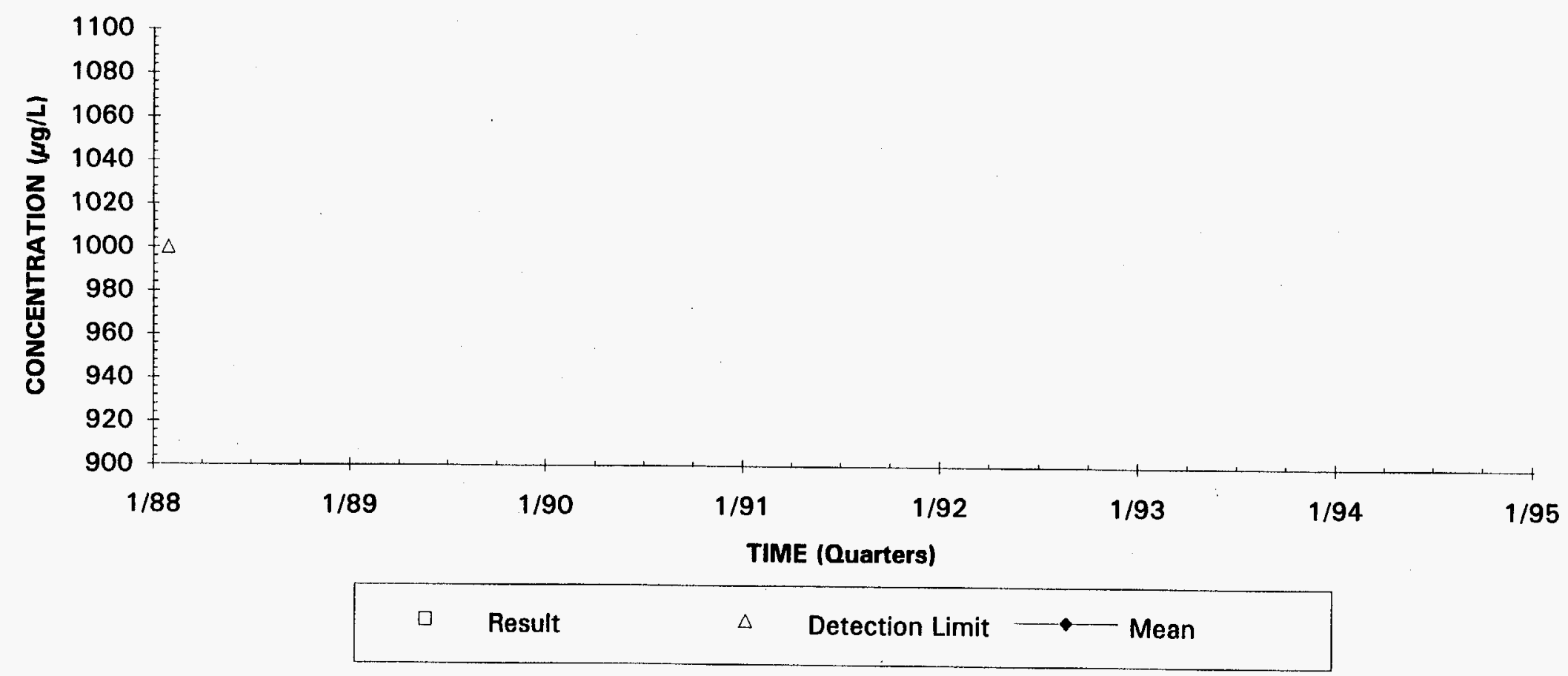


ํํำ
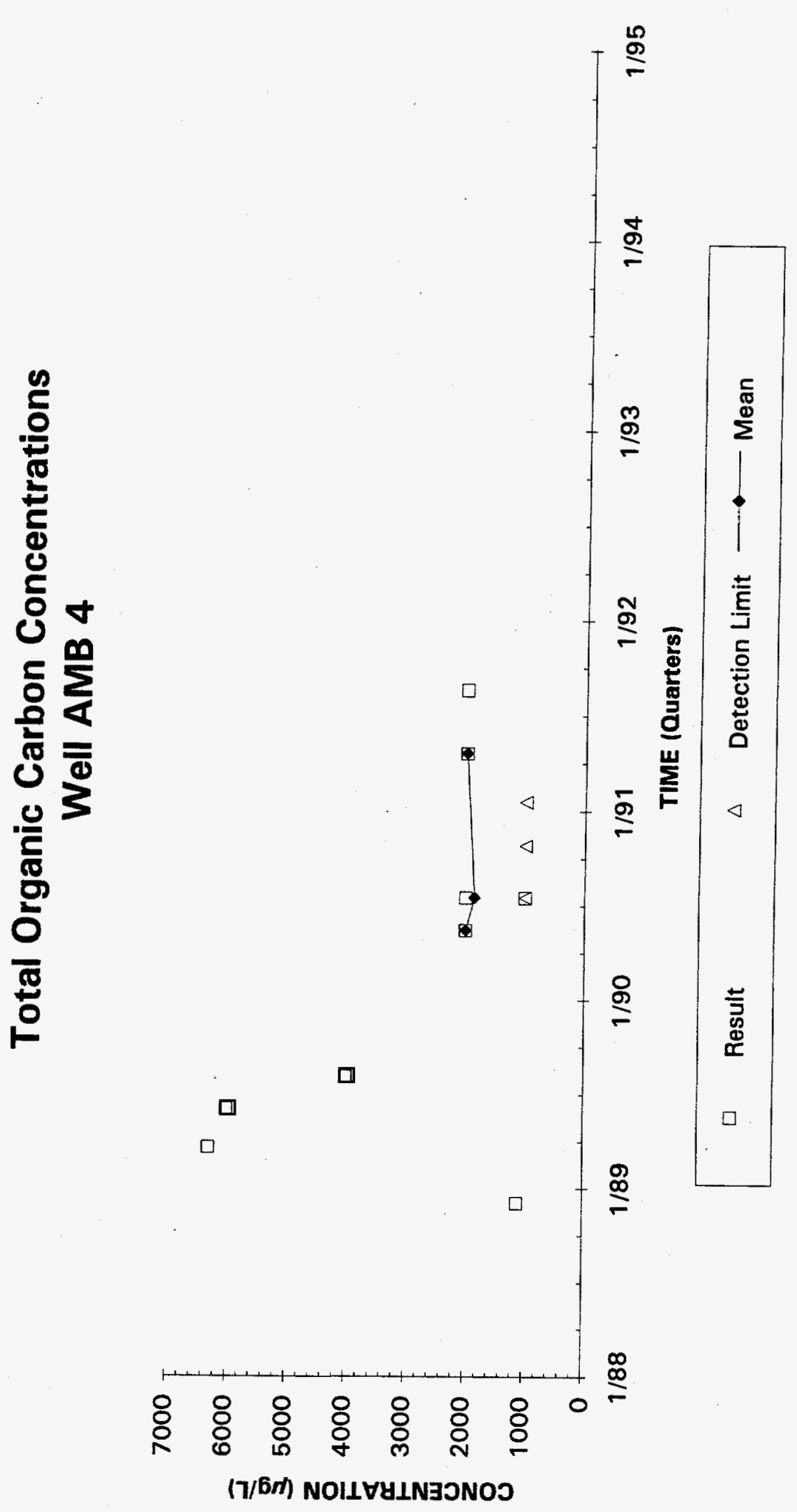


\section{Total Organic Carbon Concentrations \\ Well AMB 4A}

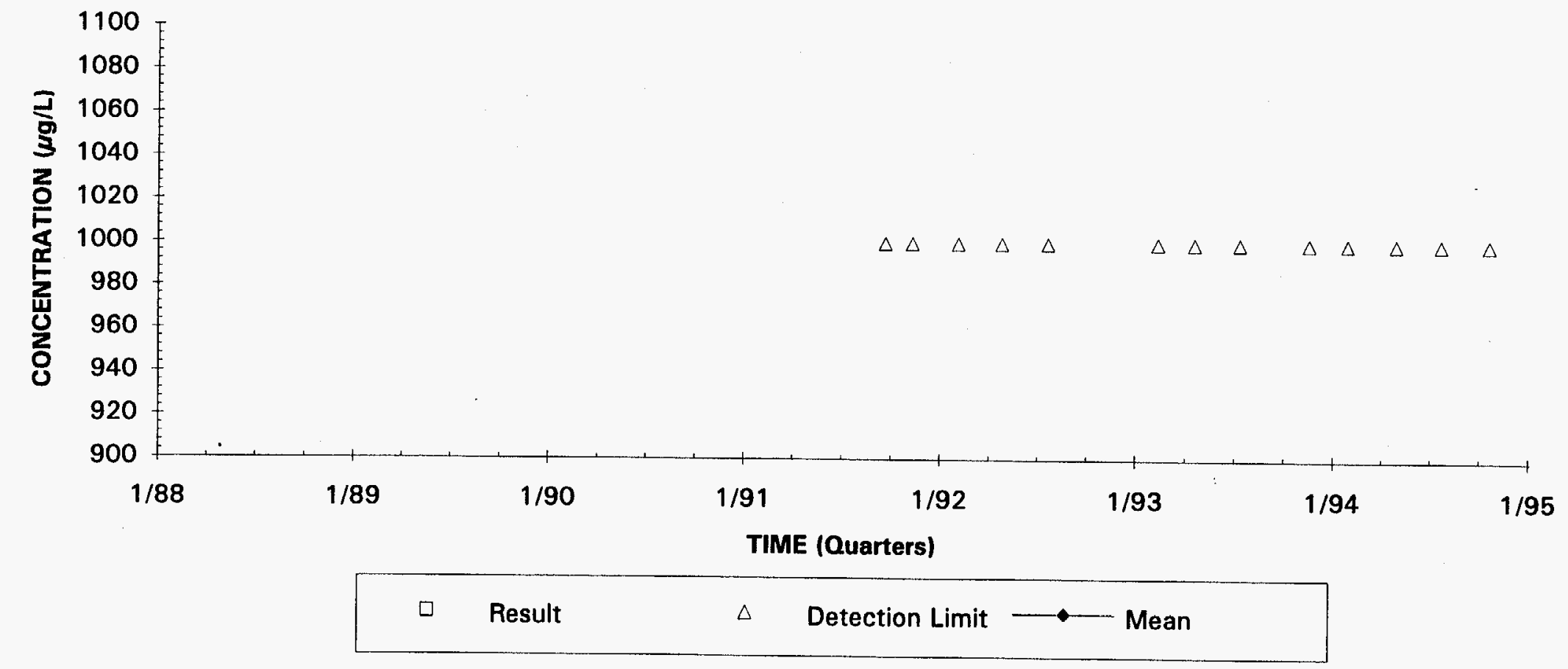



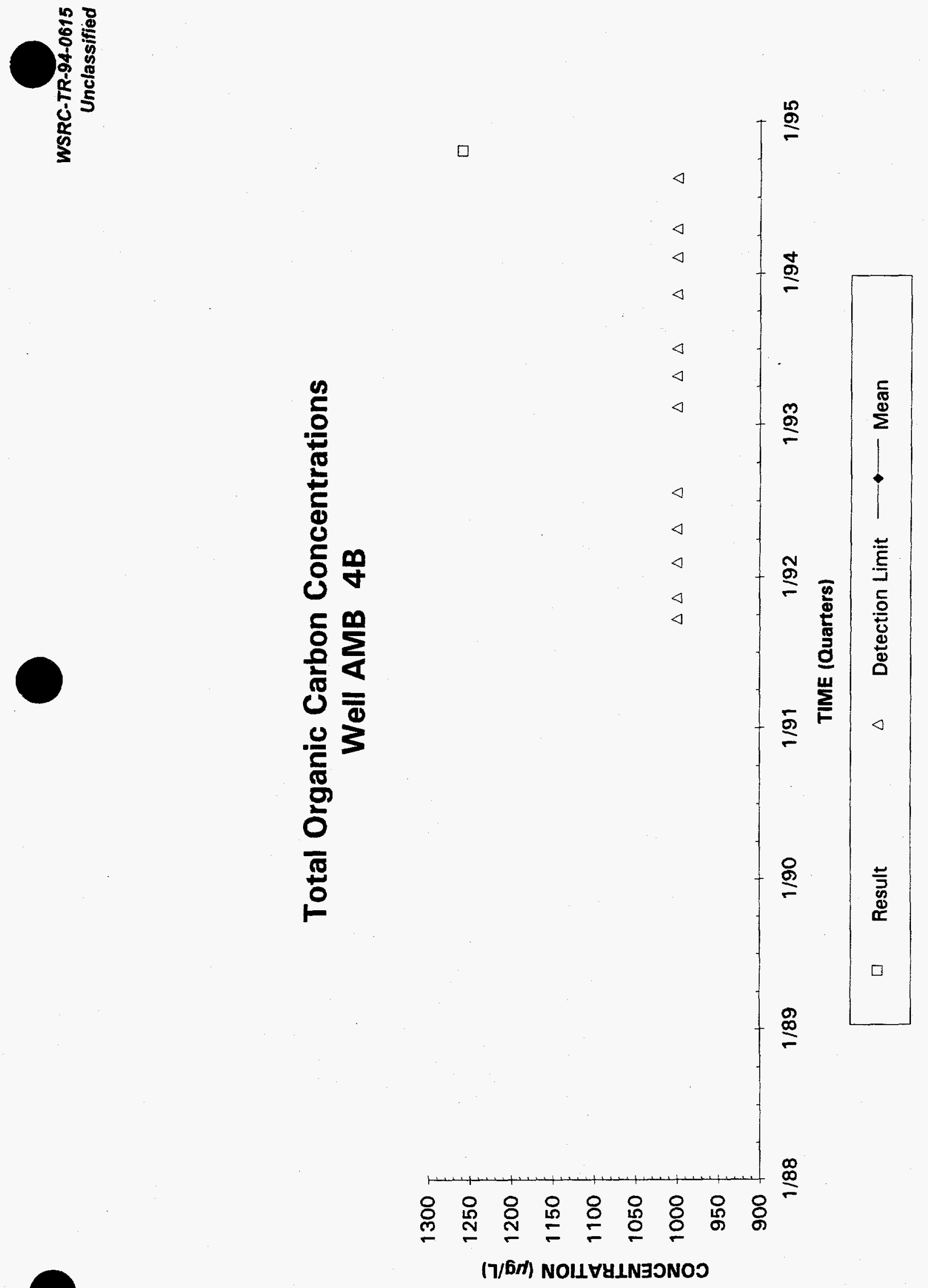


\section{Total Organic Carbon Concentrations}

Well AMB 4D

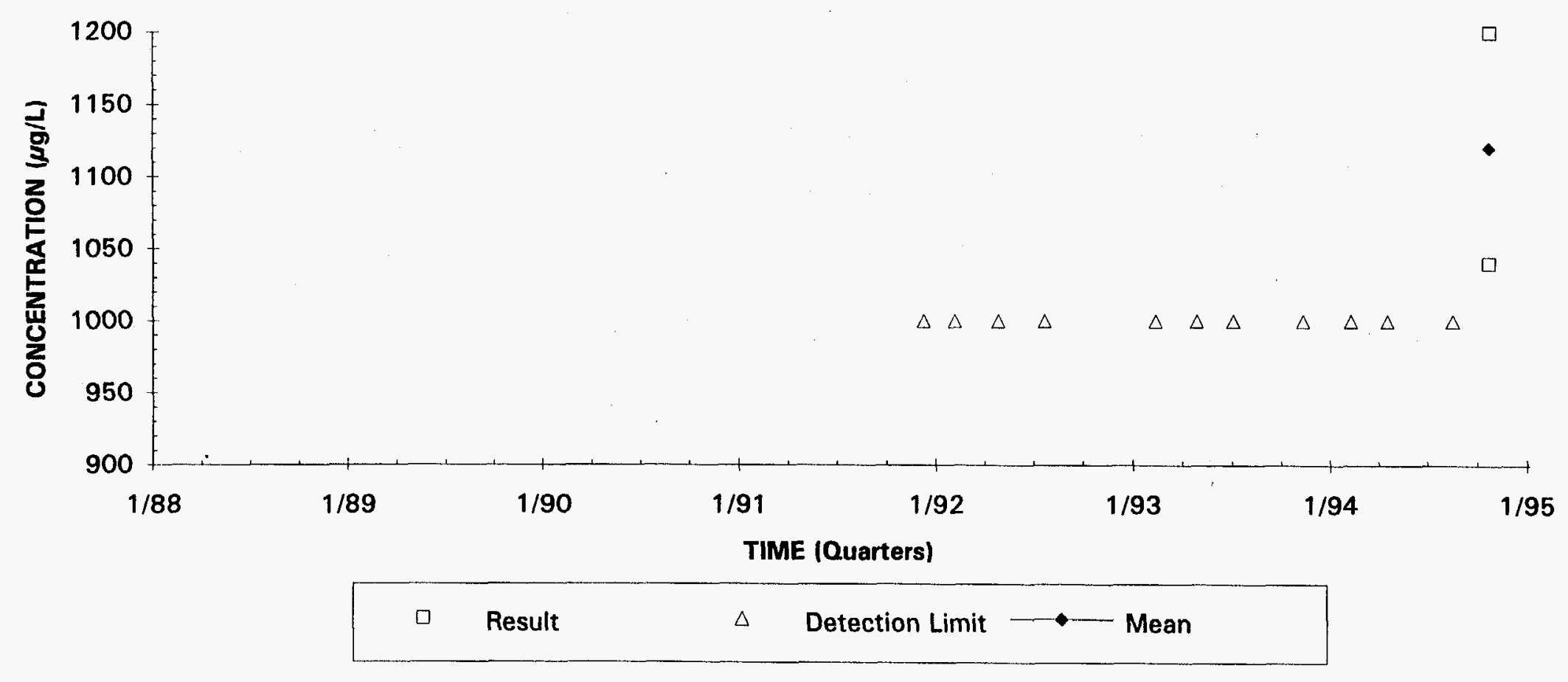




\section{Total Organic Carbon Concentrations}

\section{Well AMB 5}

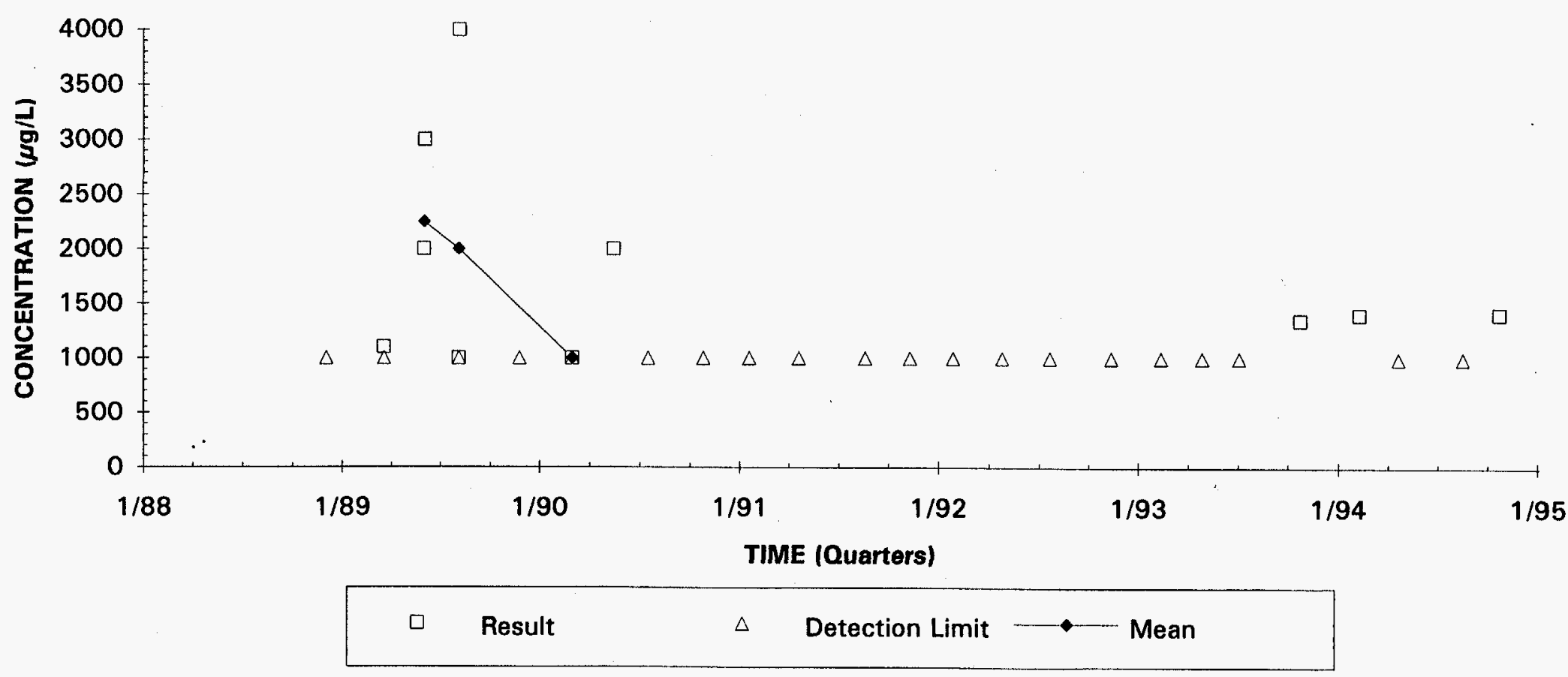




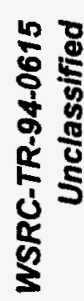
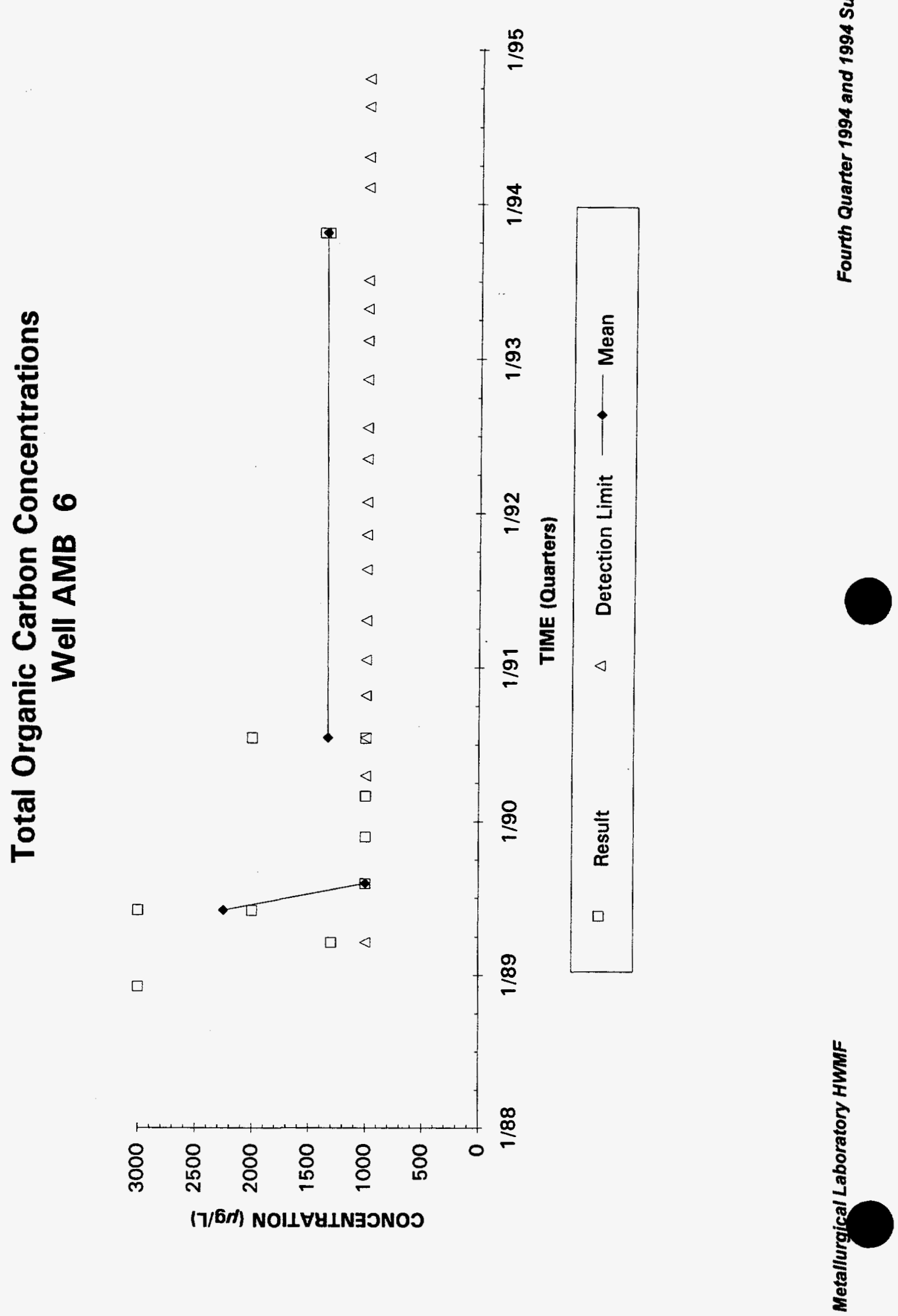


\section{Total Organic Carbon Concentrations}

Well AMB 7

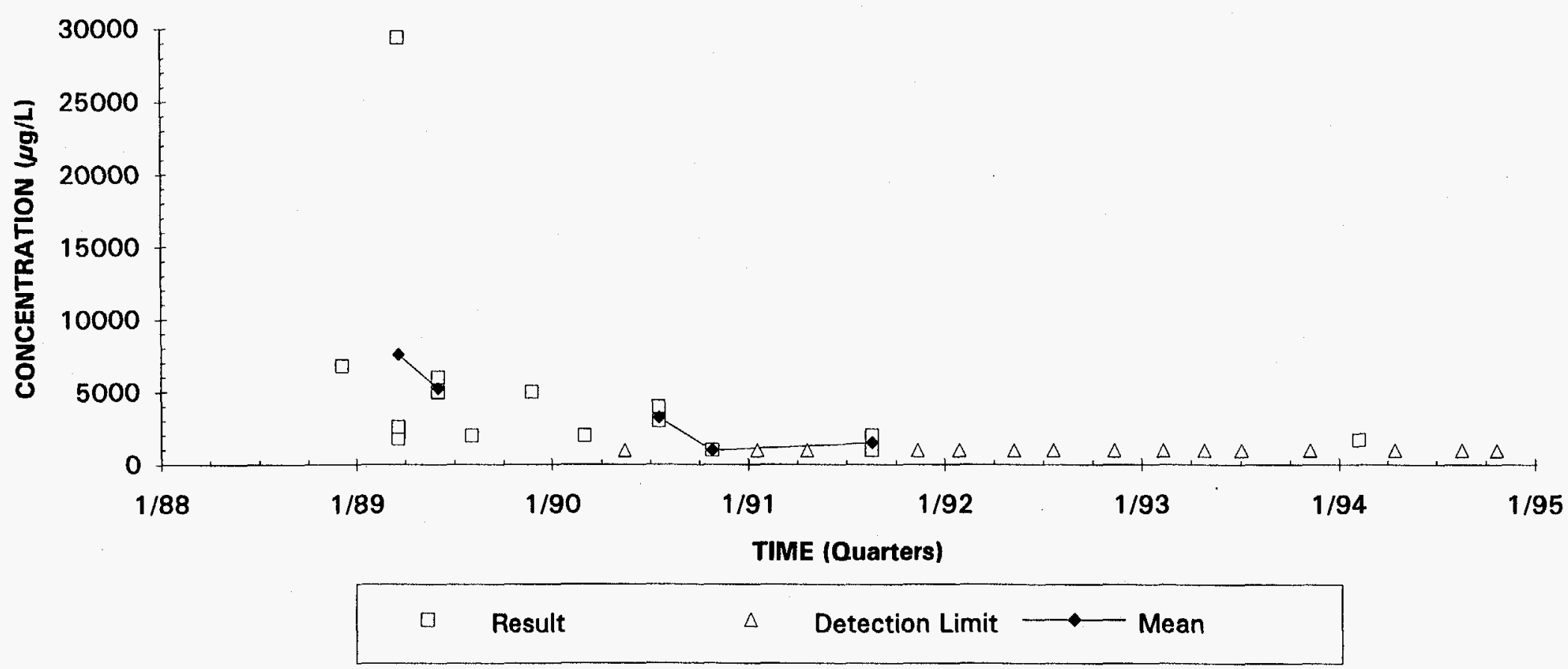



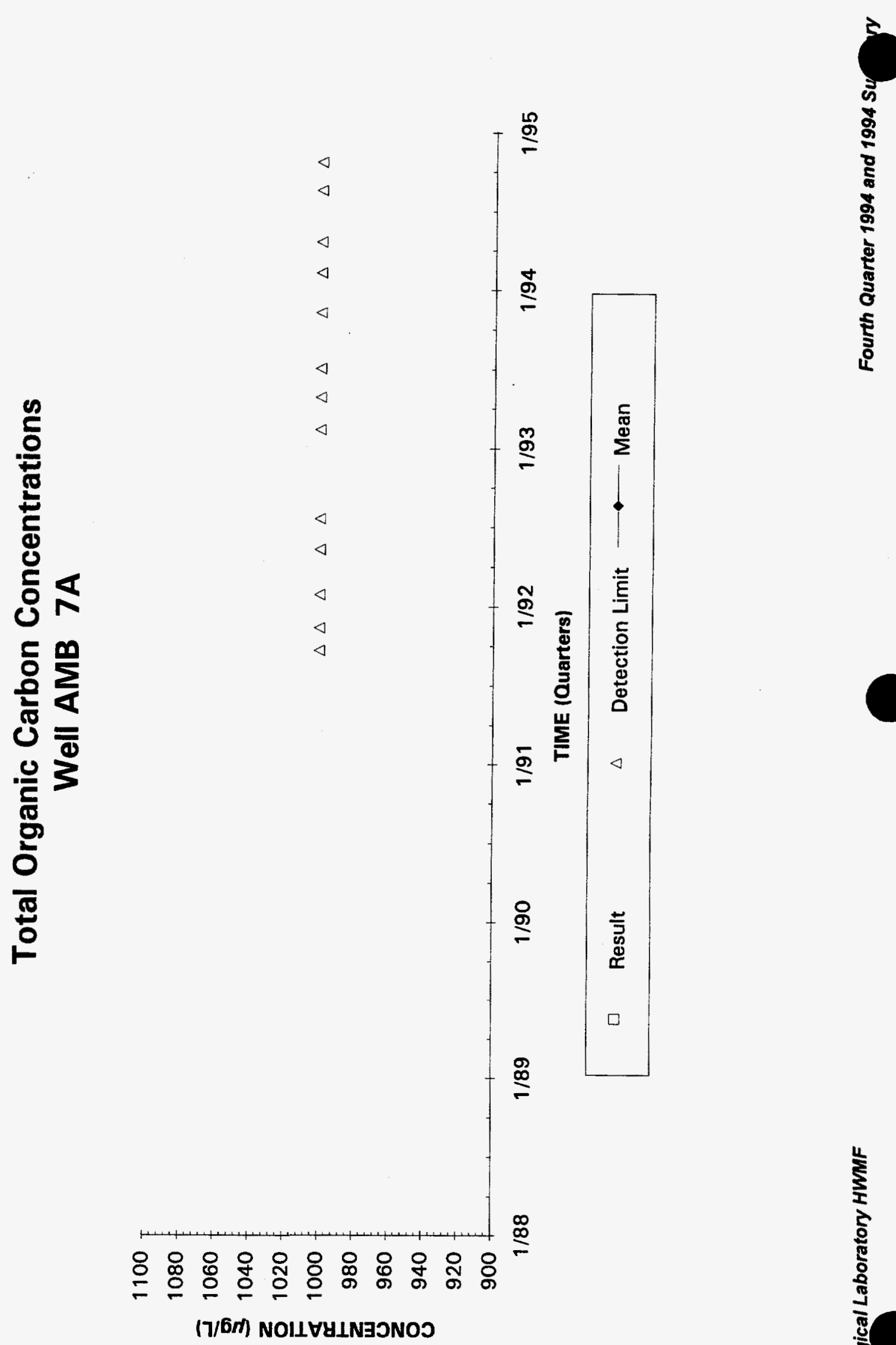


\section{Total Organic Carbon Concentrations}

Well AMB 7B

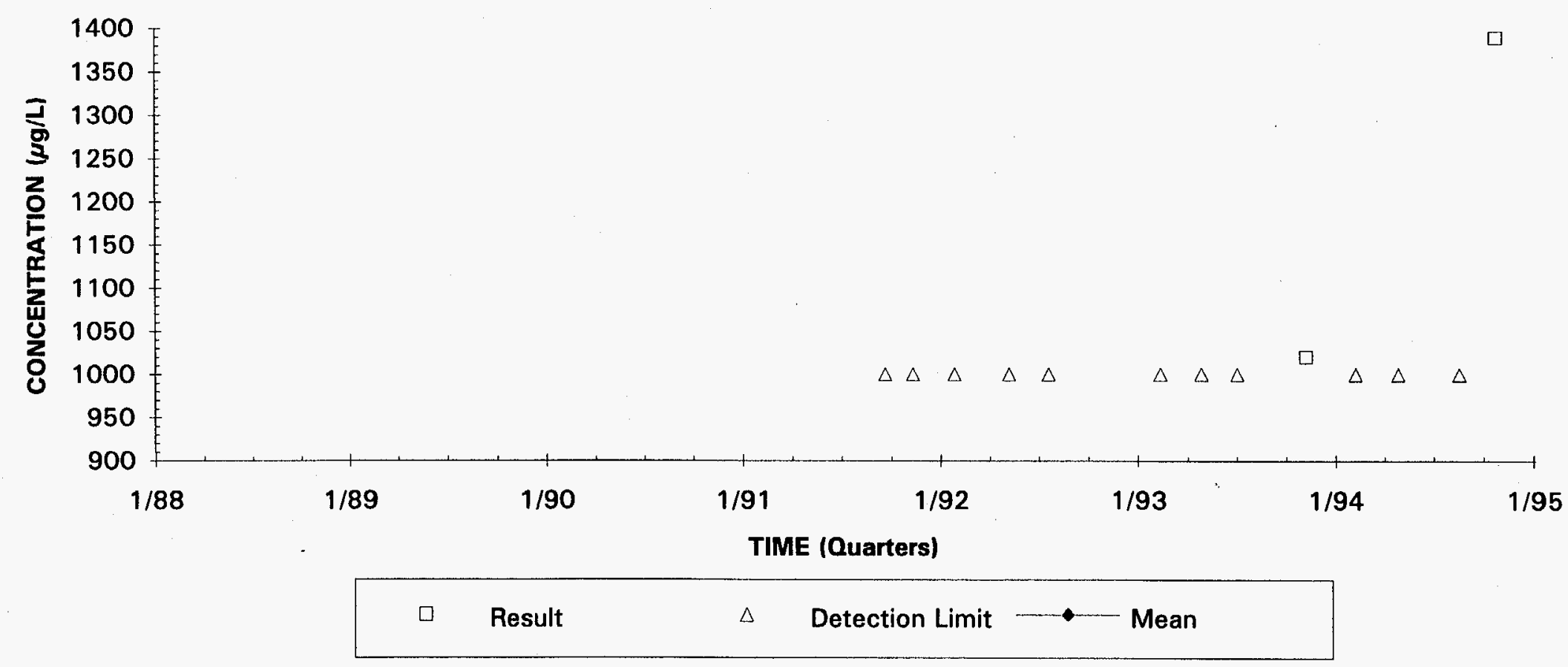




\section{Total Organic Carbon Concentrations}

\section{Well AMB 8D}

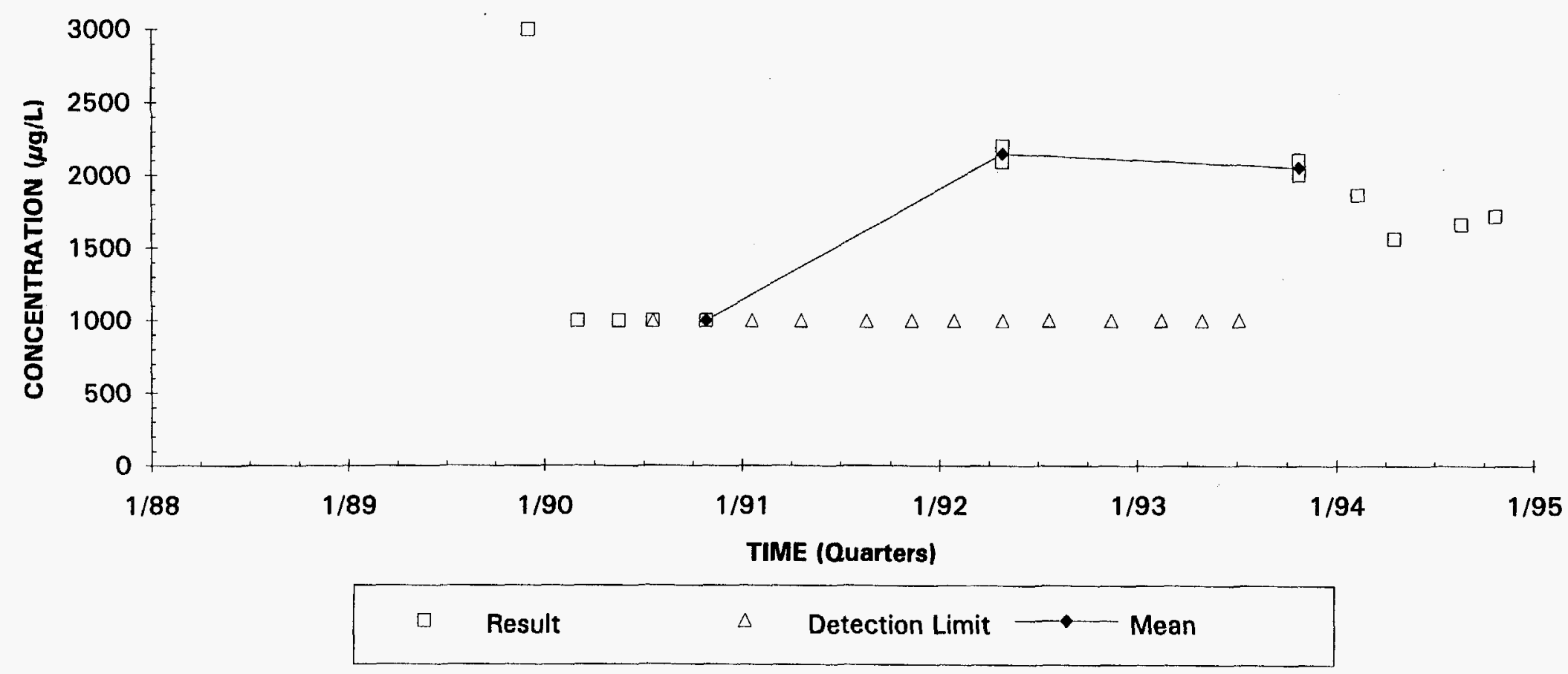



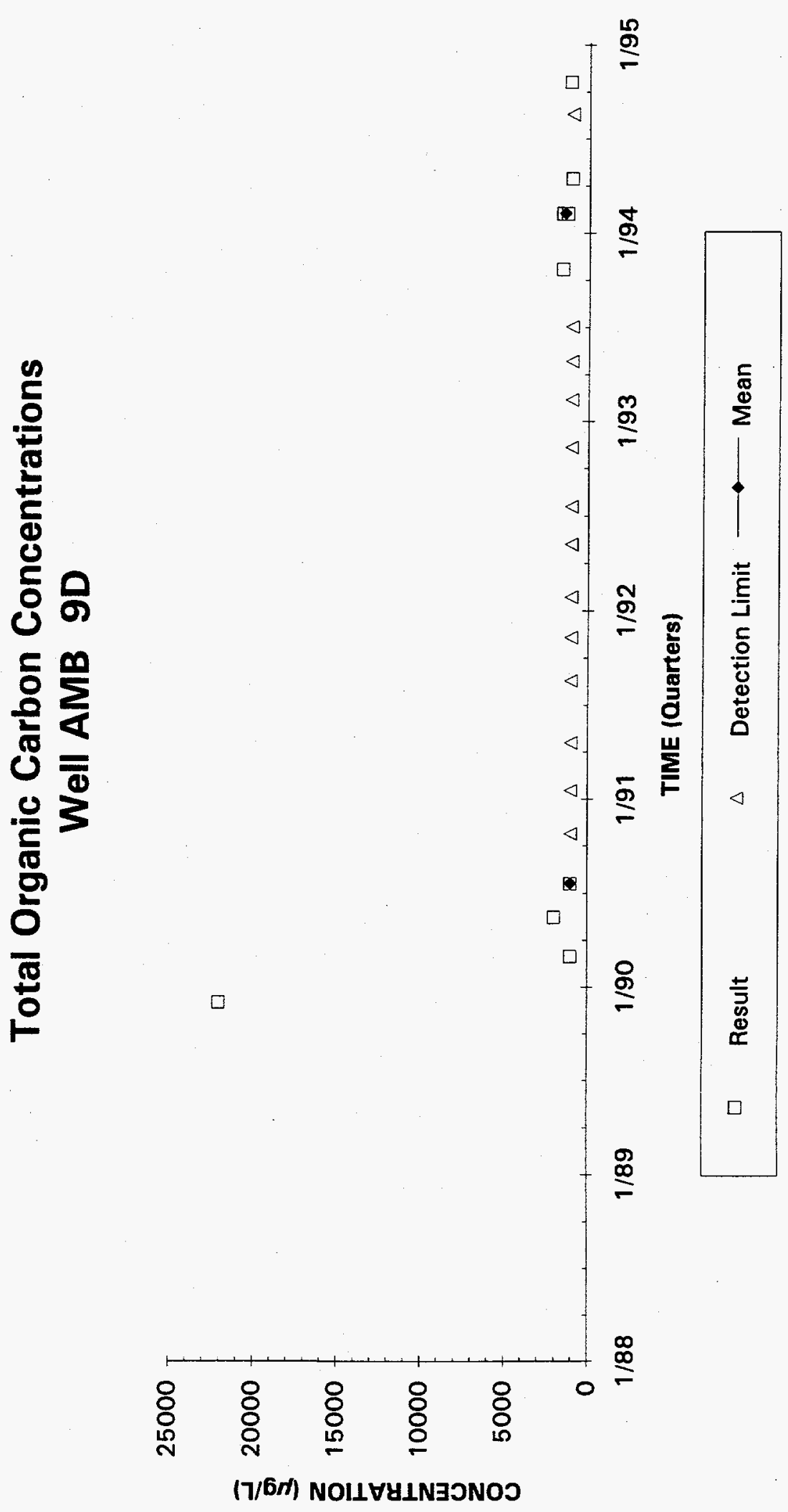


\section{Total Organic Carbon Concentrations}

Well AMB 10A

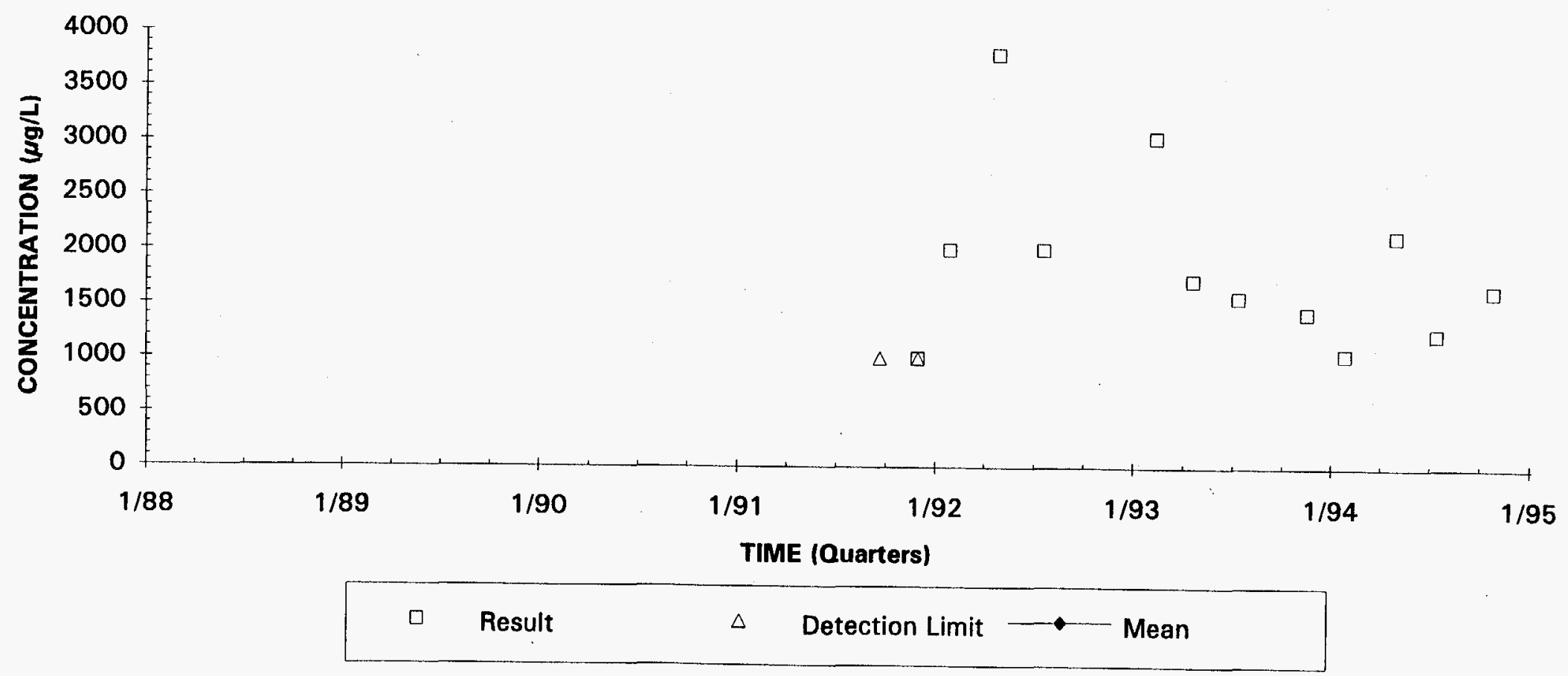




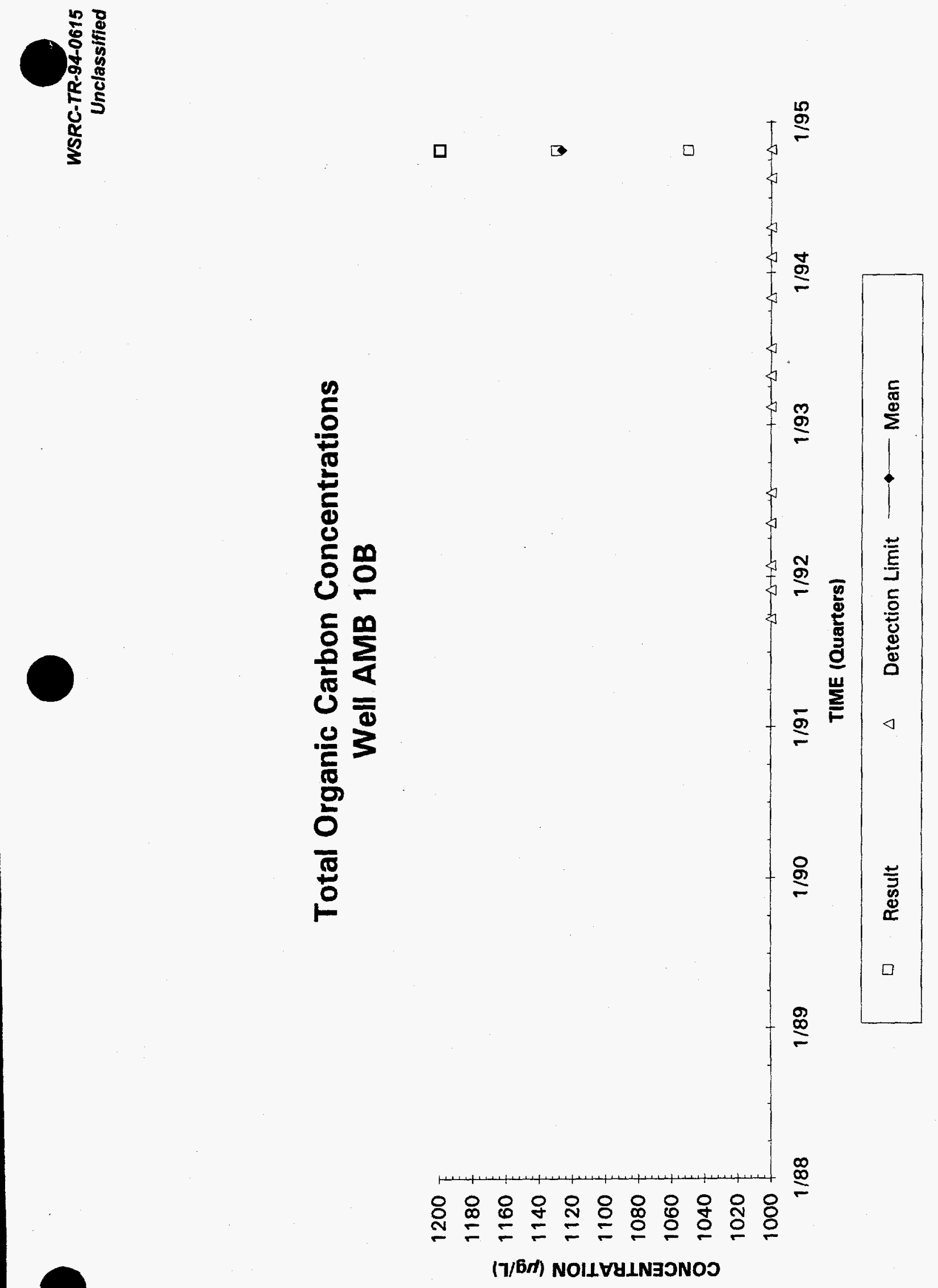




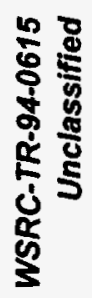
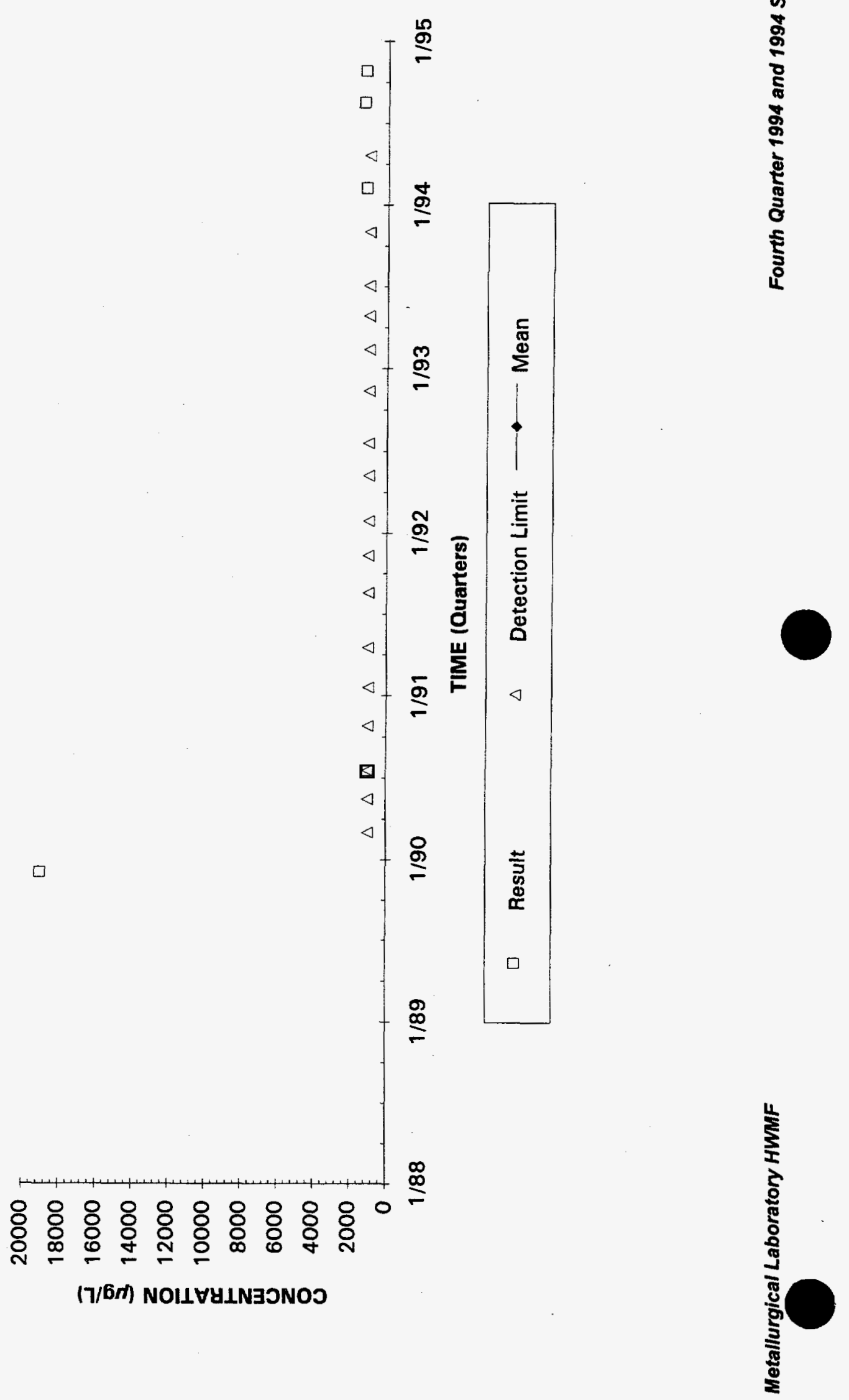

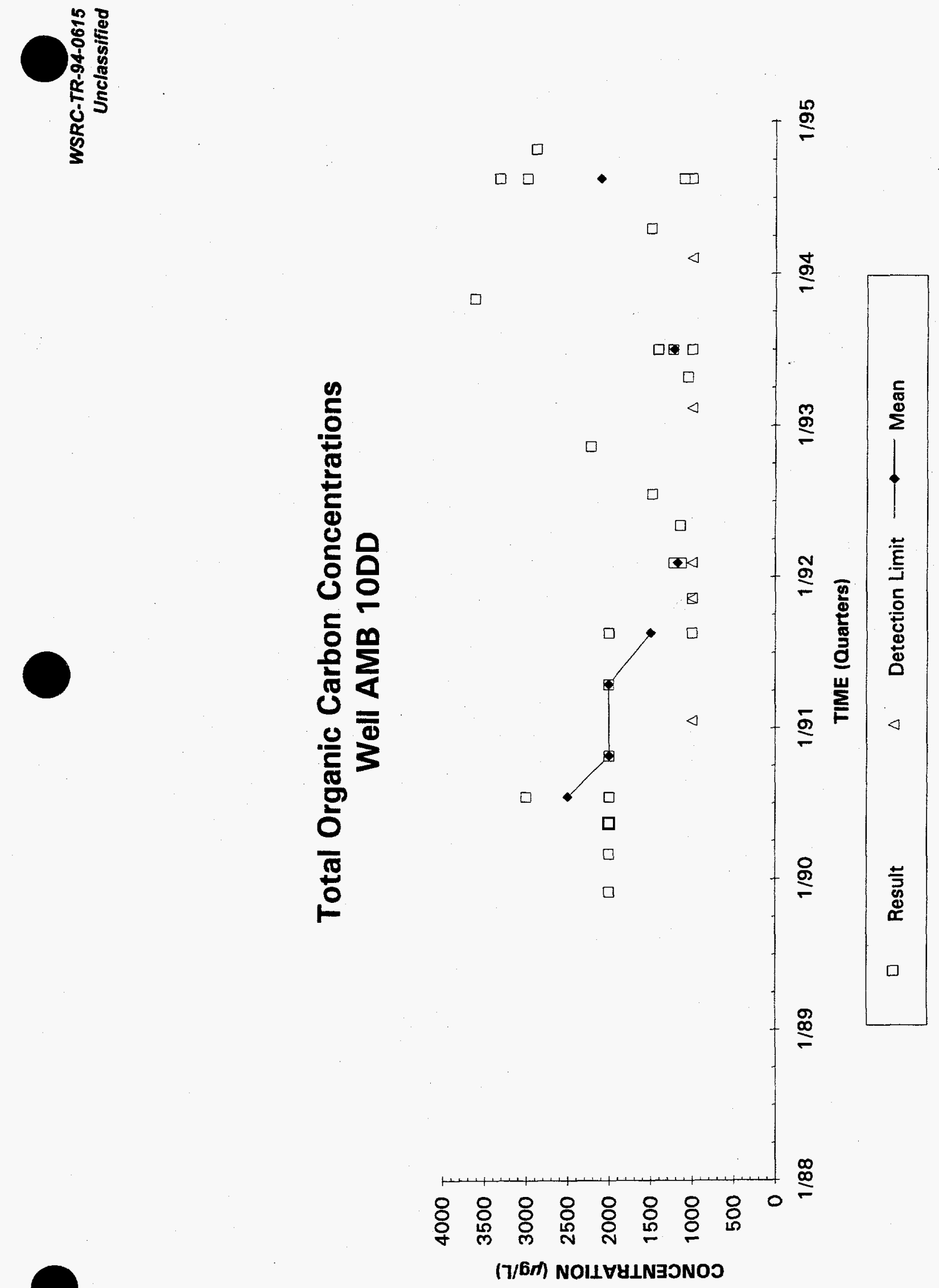

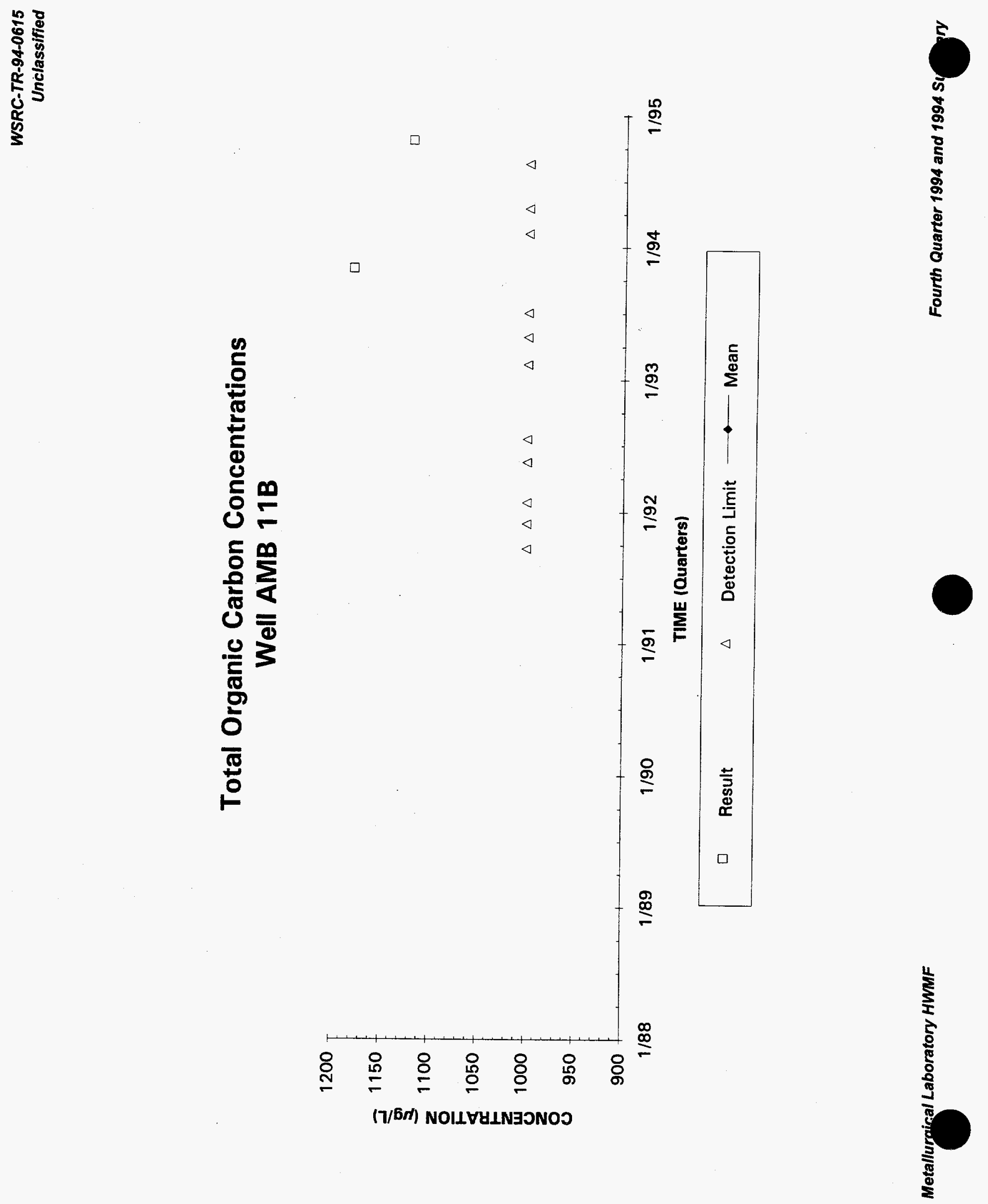


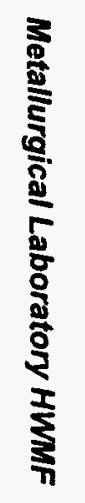

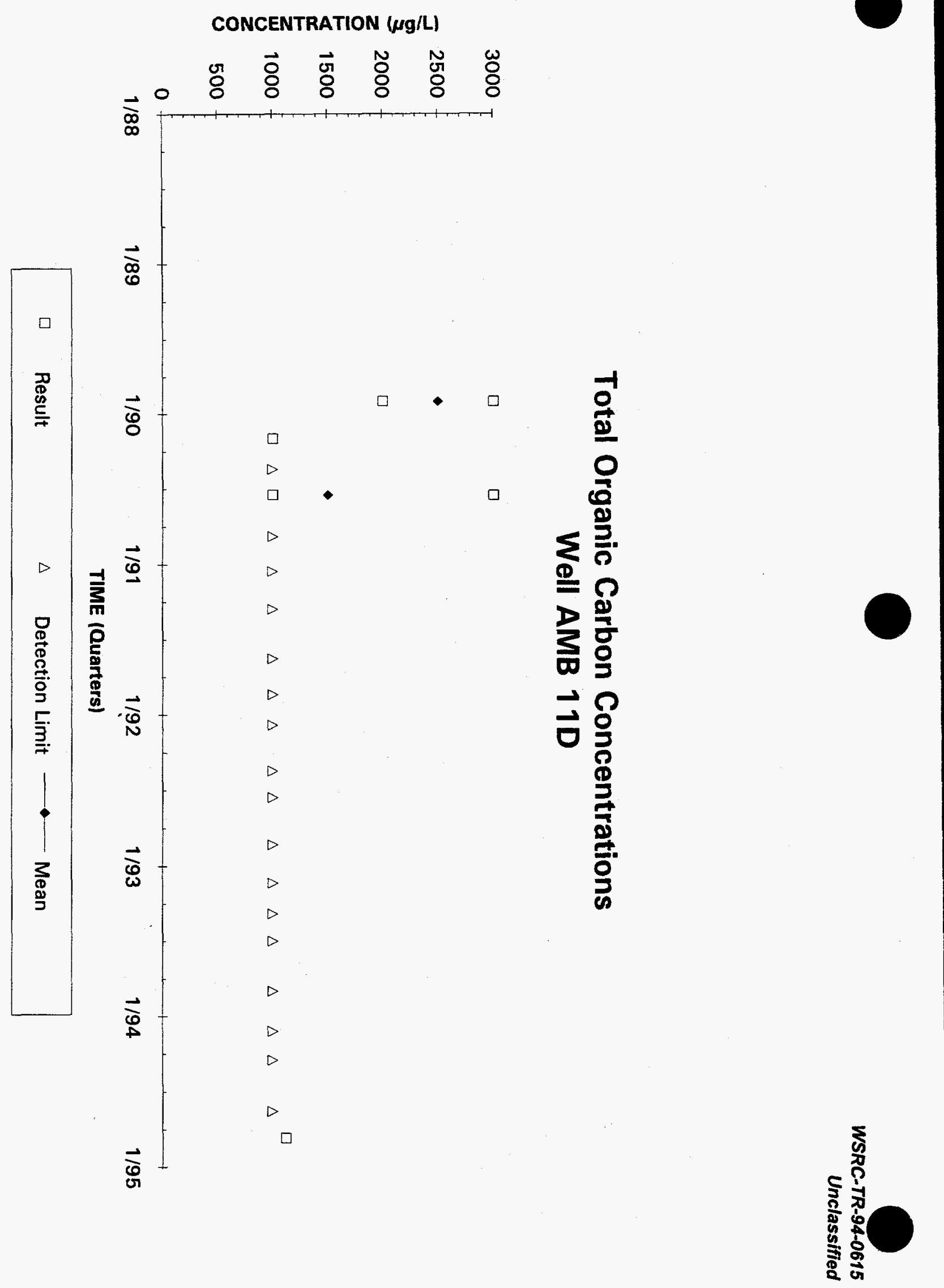




\section{Total Organic Carbon Concentrations}

Well AMB 12D

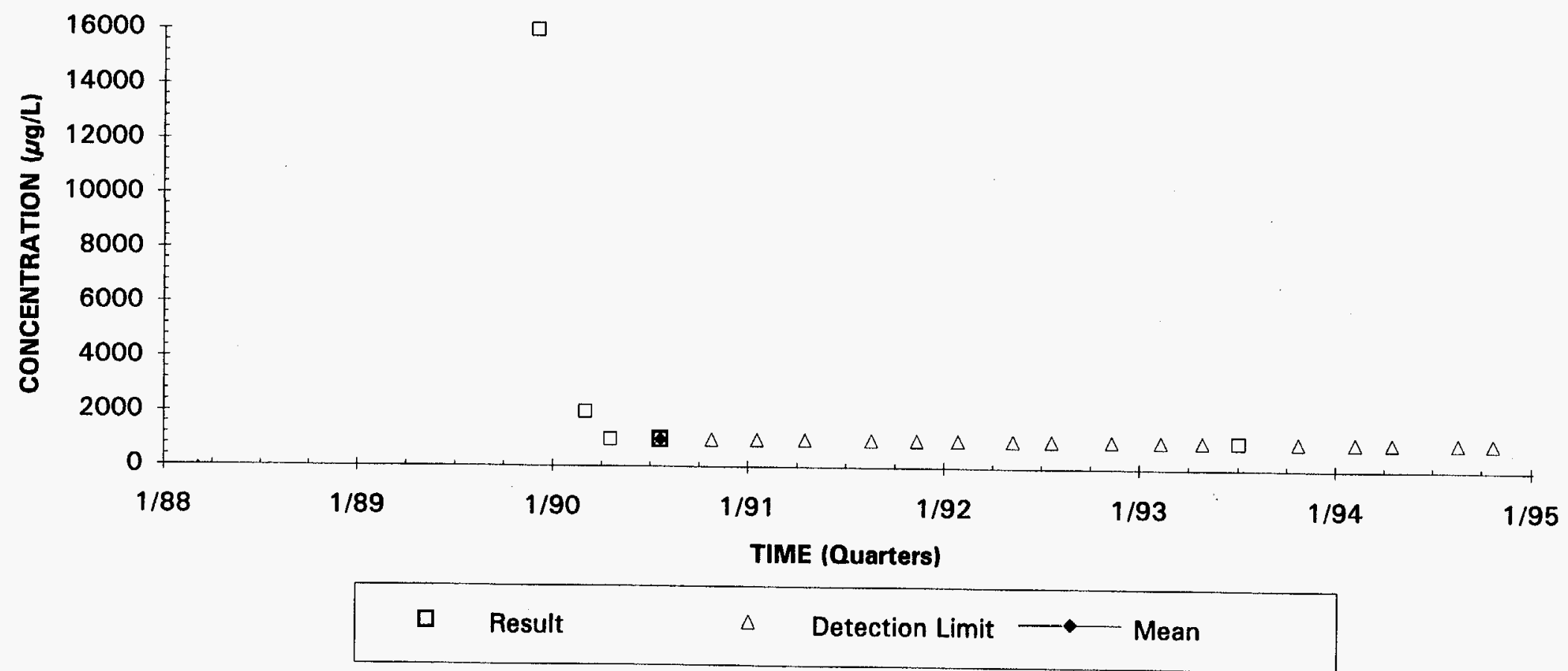




\section{Total Organic Carbon Concentrations \\ Well AMB 13AR}

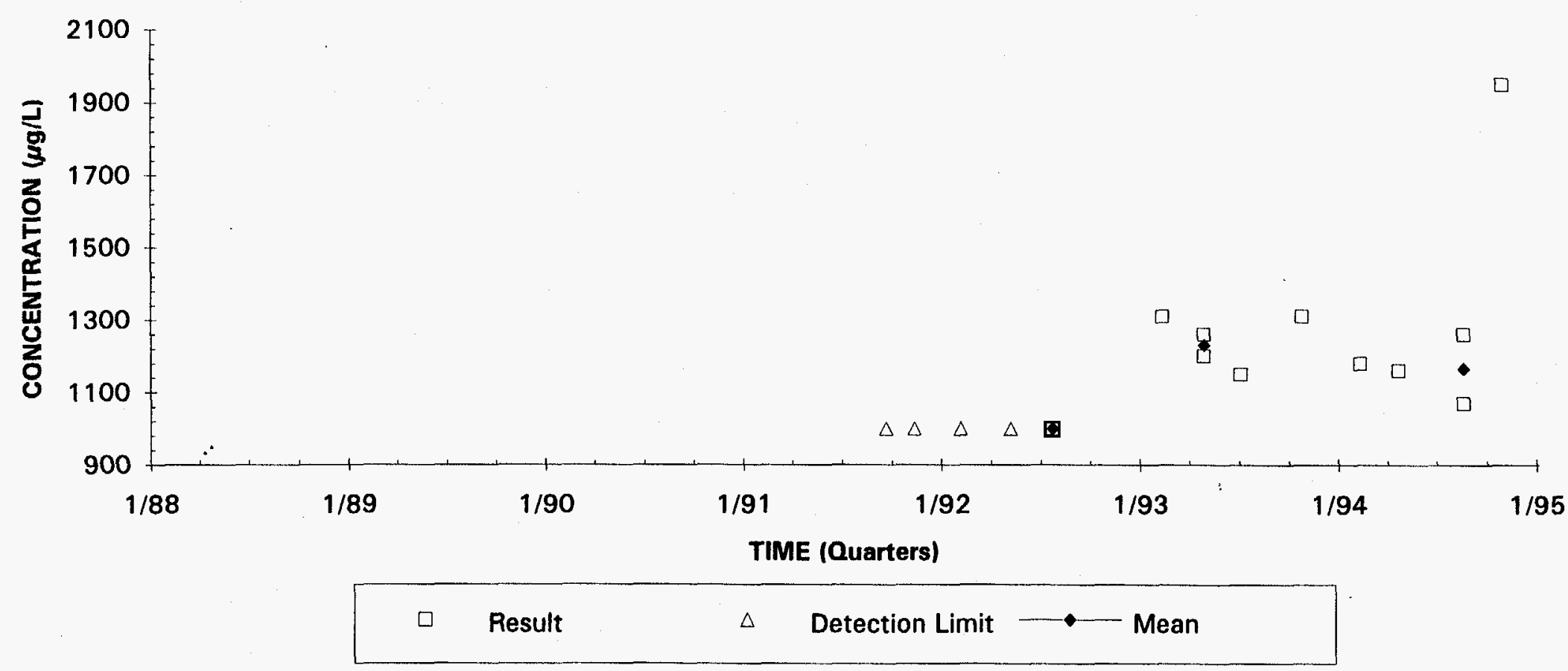




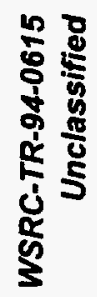
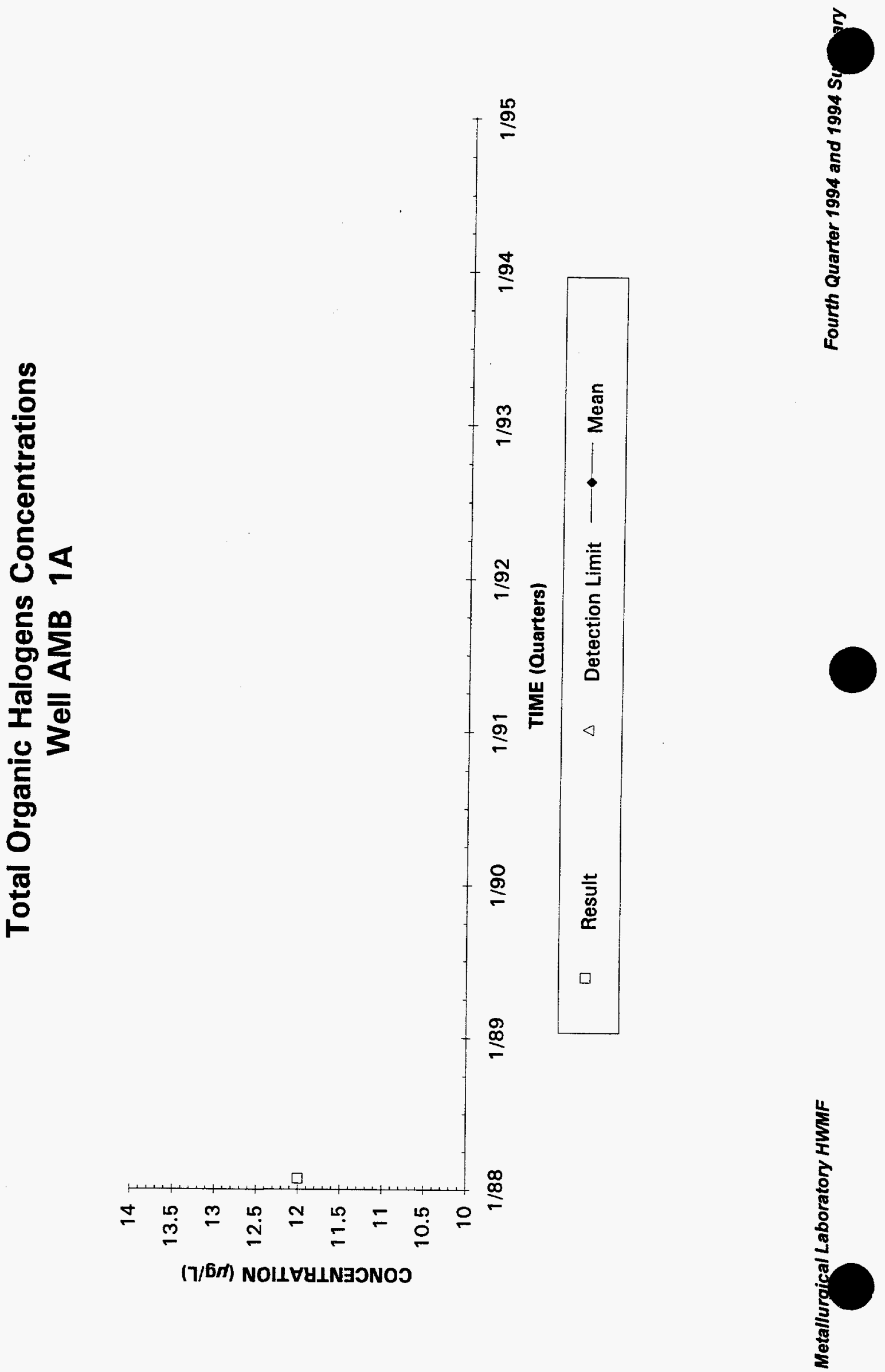


\section{Total Organic Halogens Concentrations}

Well AMB 2

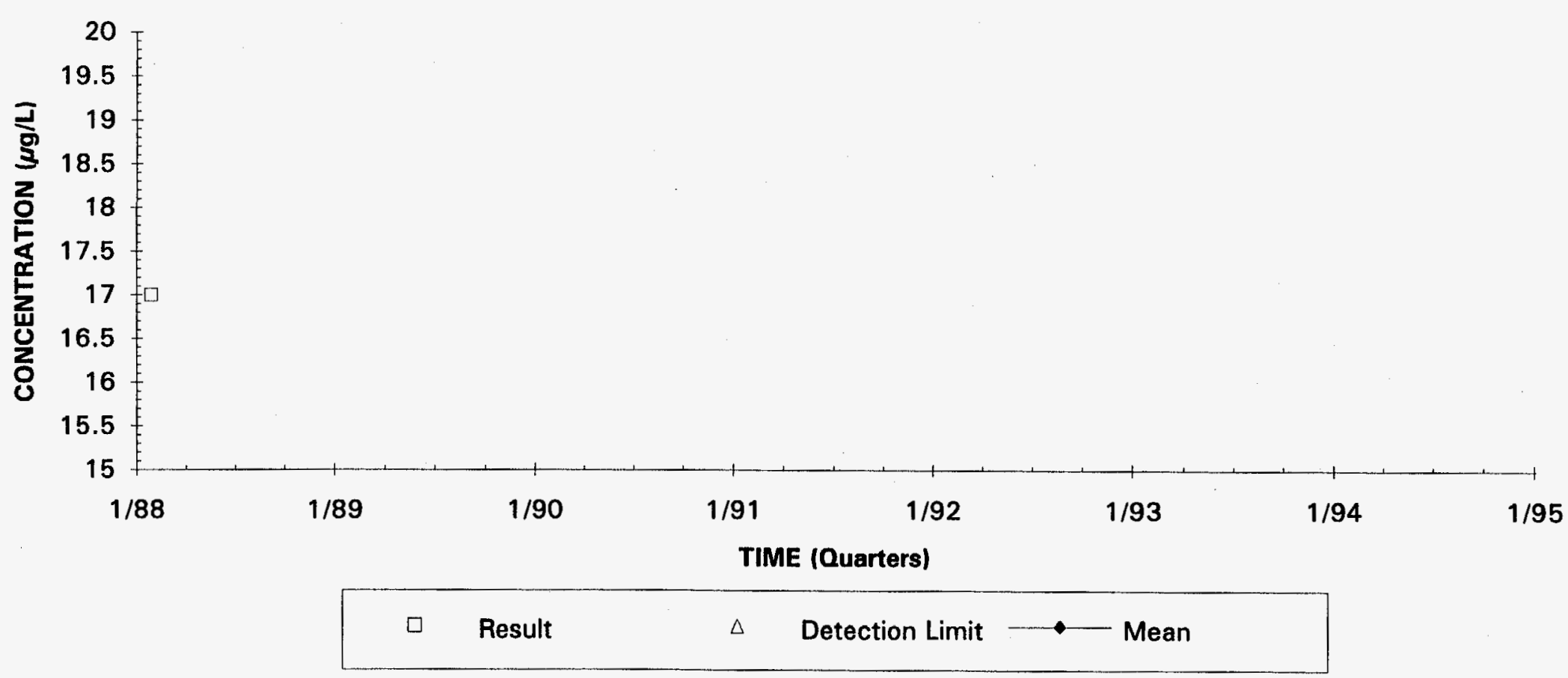




\section{Total Organic Halogens Concentrations}

Well AMB 3A

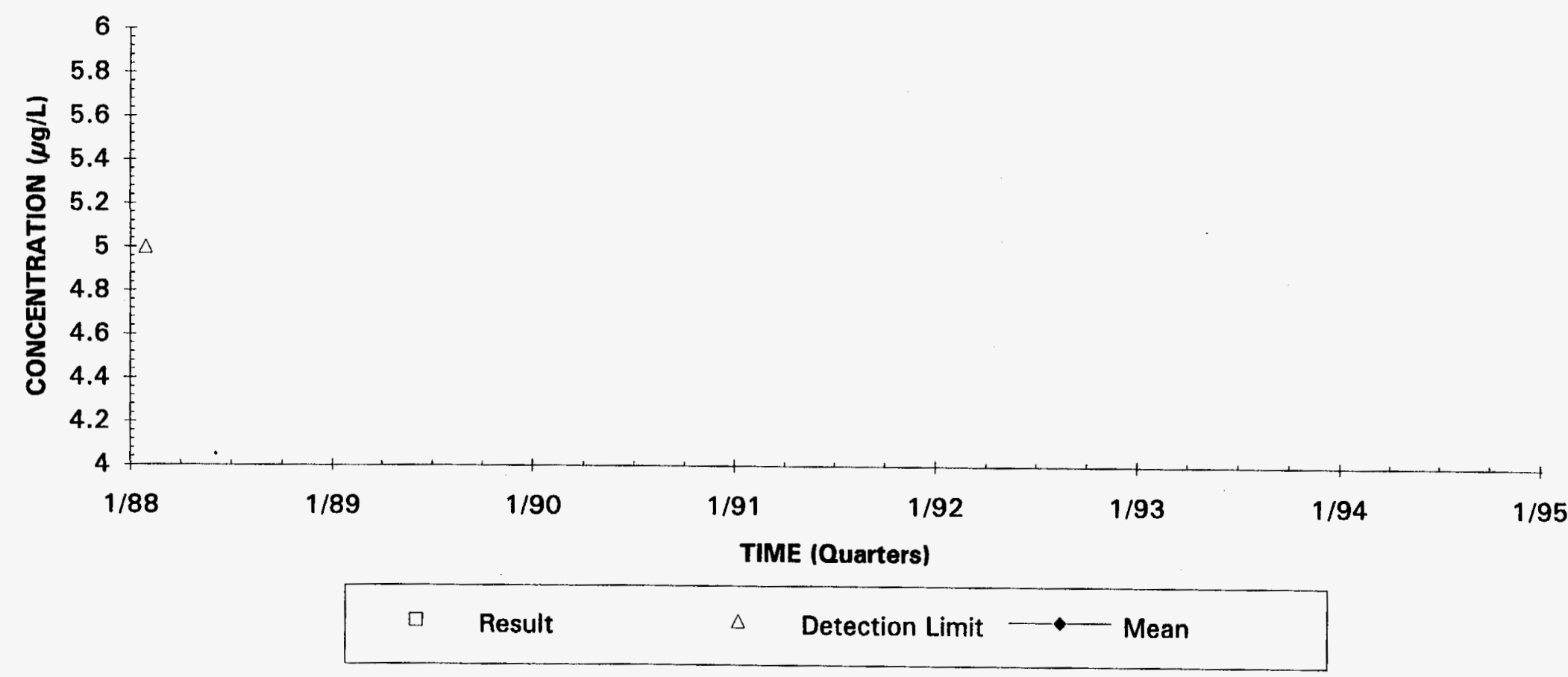



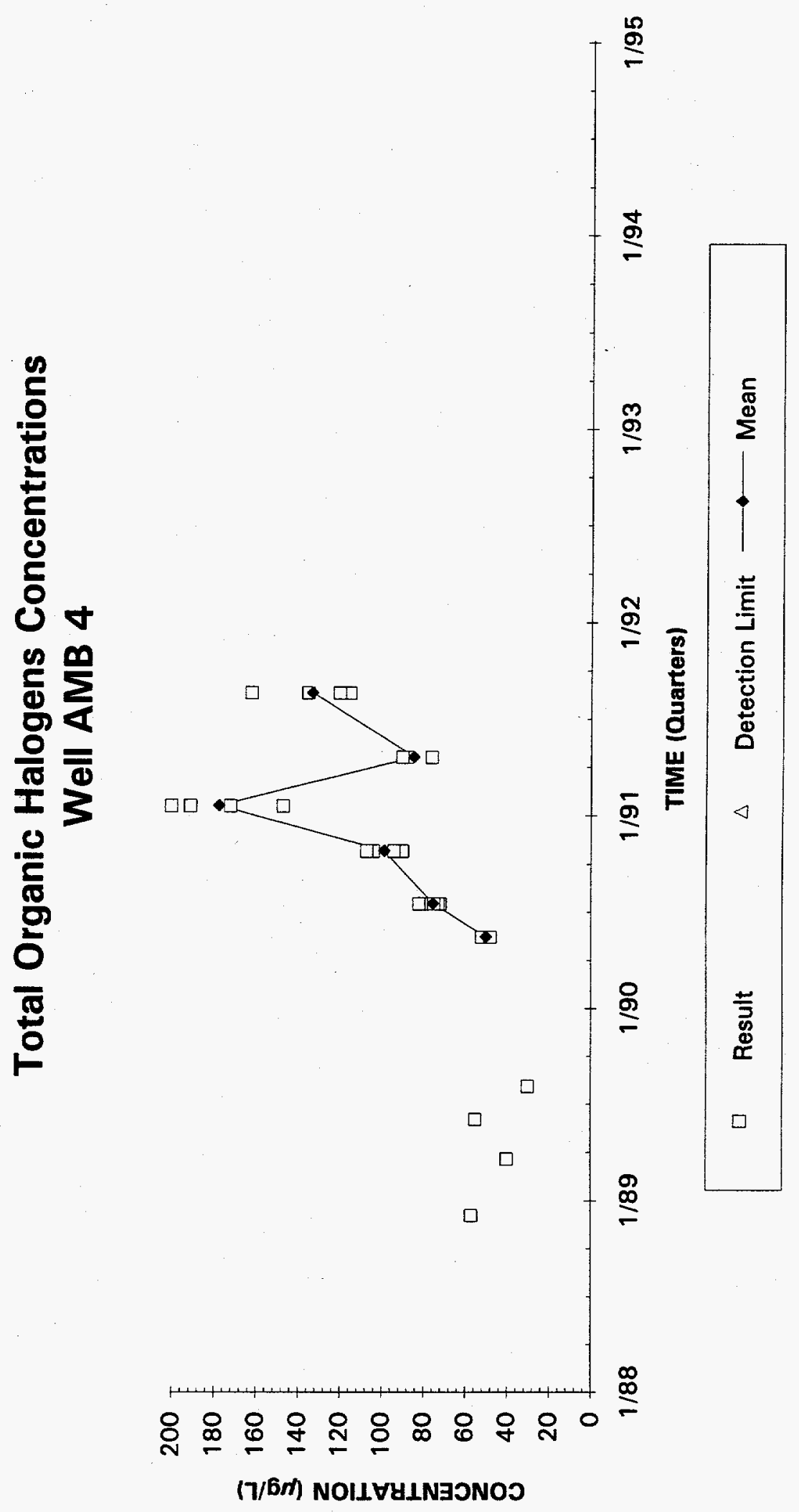


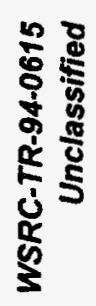
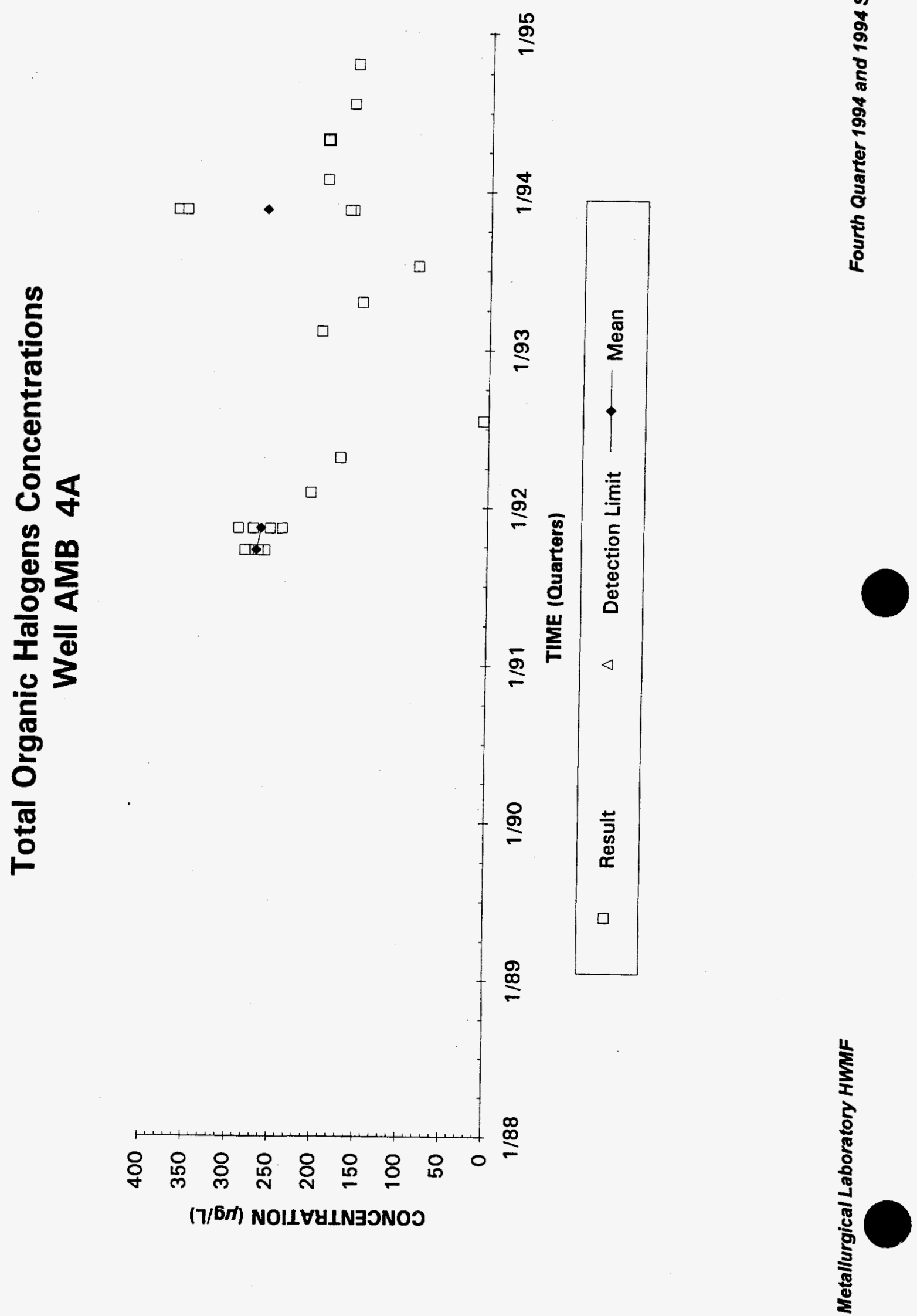

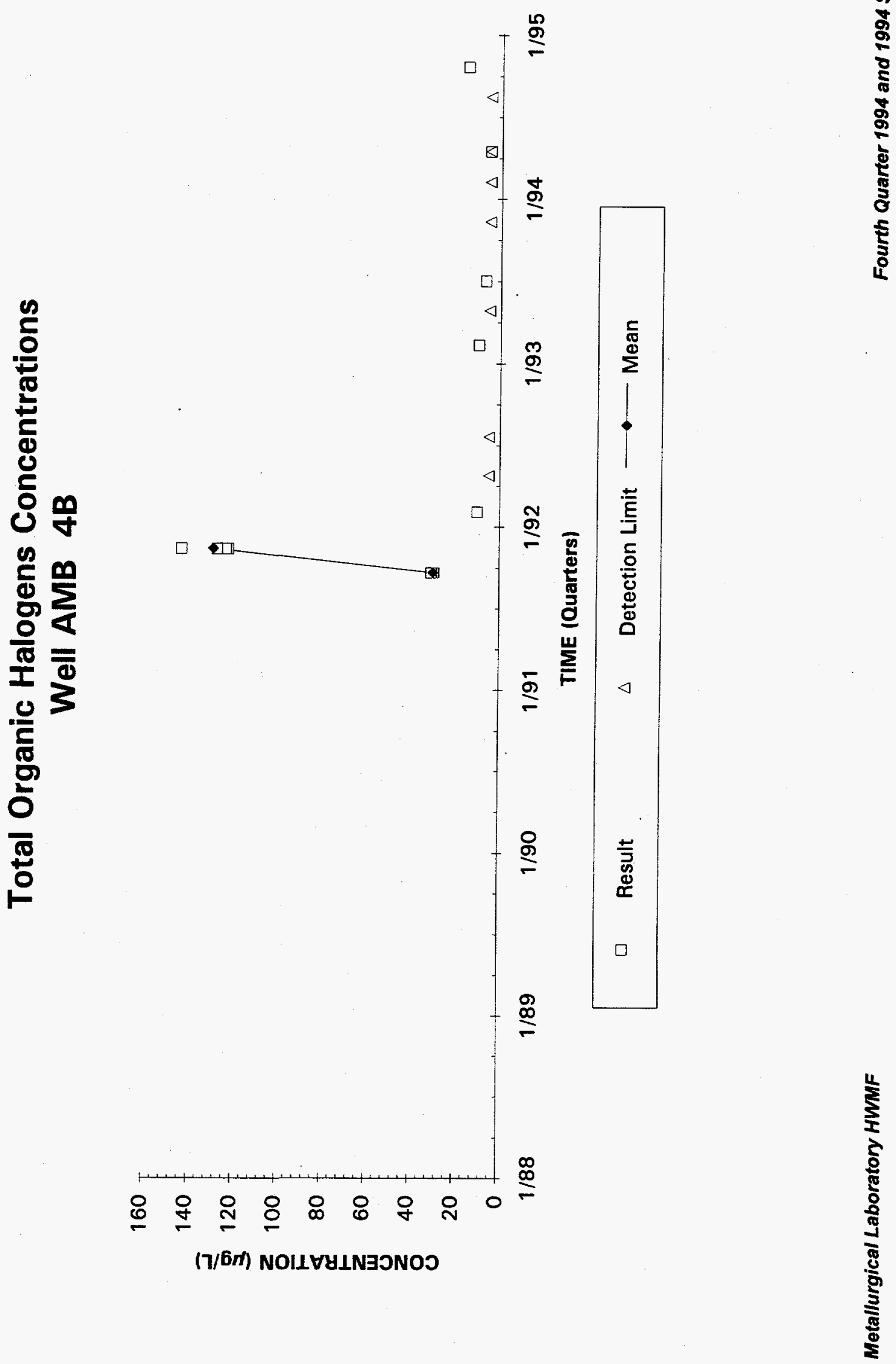


\section{Total Organic Halogens Concentrations}

Well AMB 4D

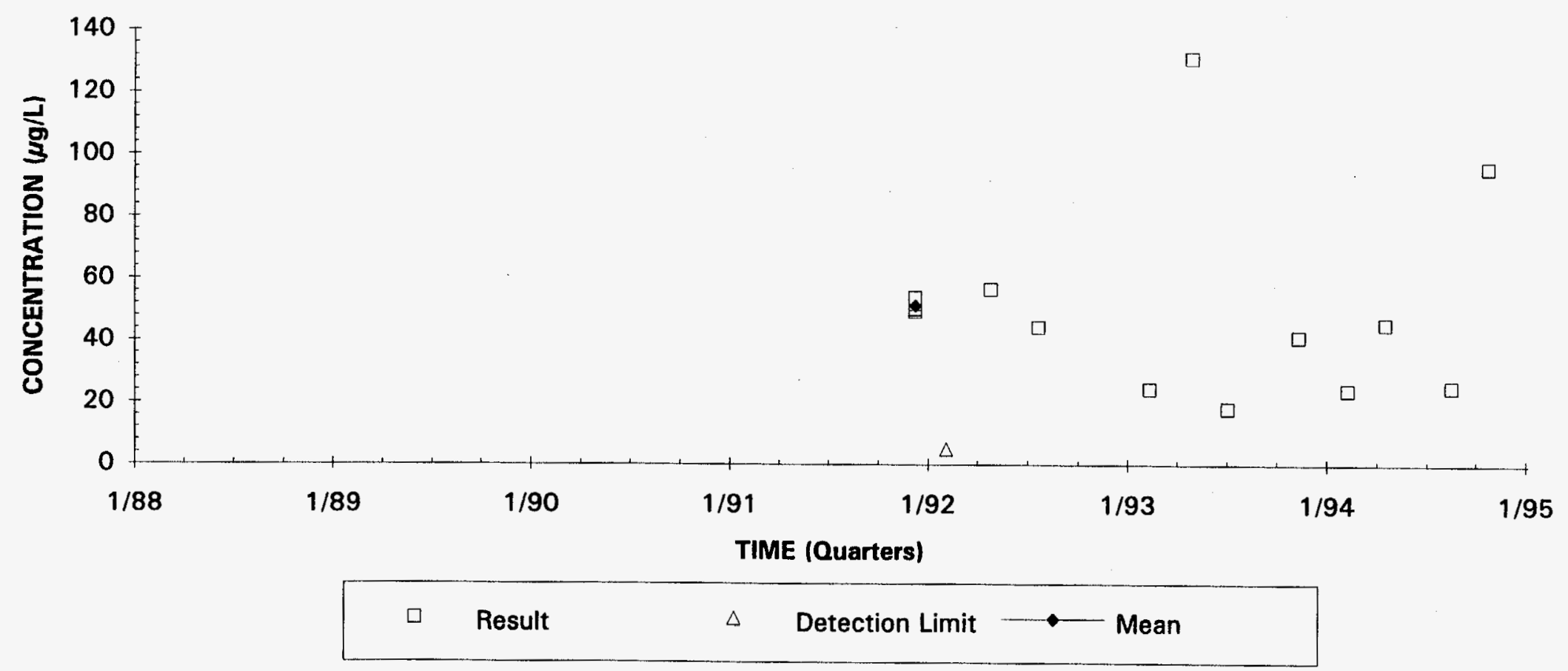



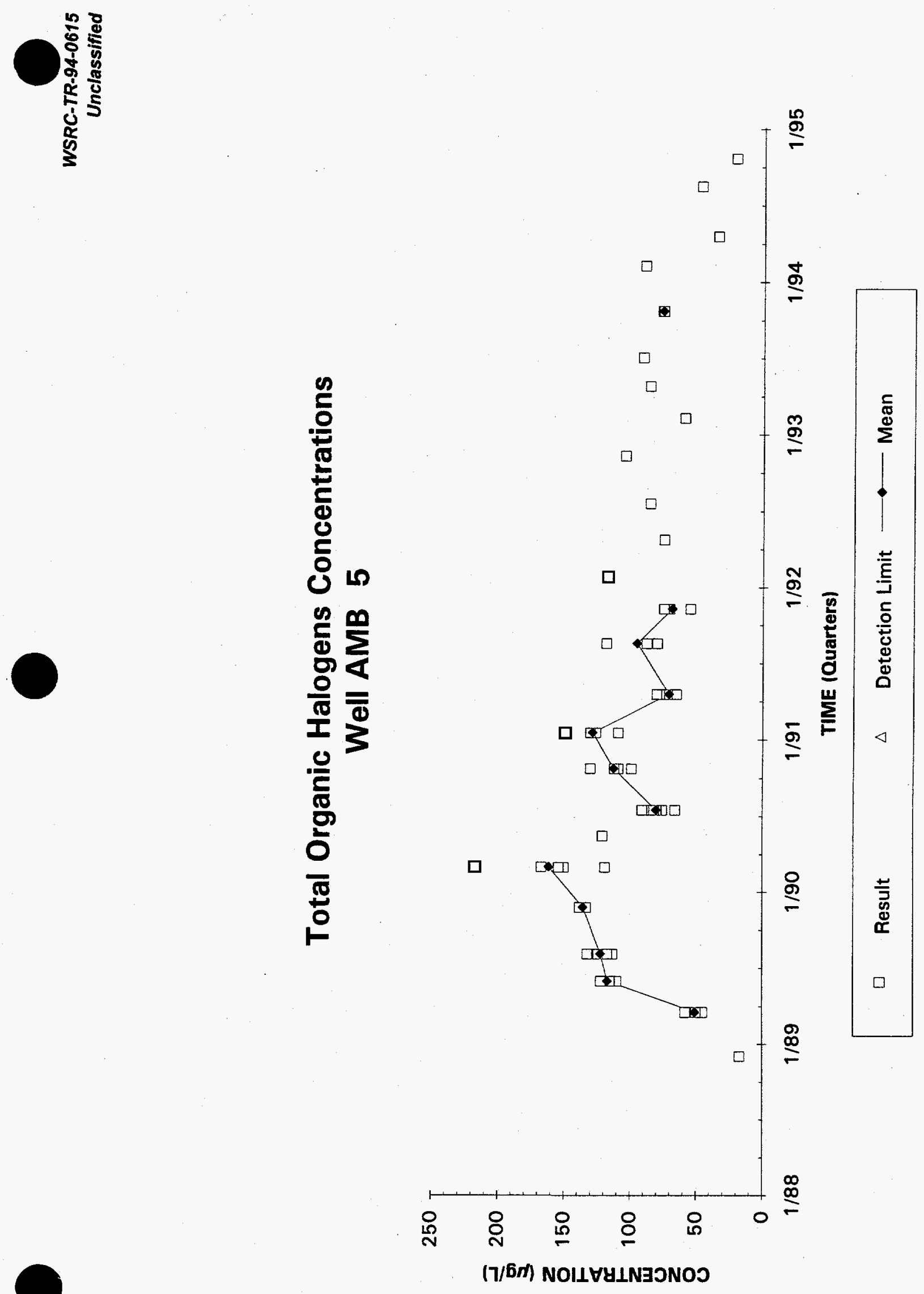

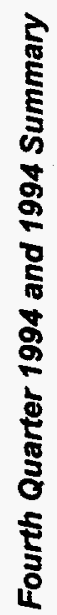

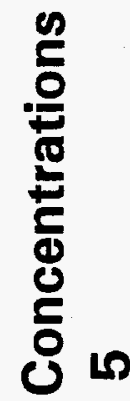

0
$\frac{0}{0} \geq$
0
$\frac{0}{10}=$

을

응

可

1 


\section{Total Organic Halogens Concentrations}

Well AMB 6

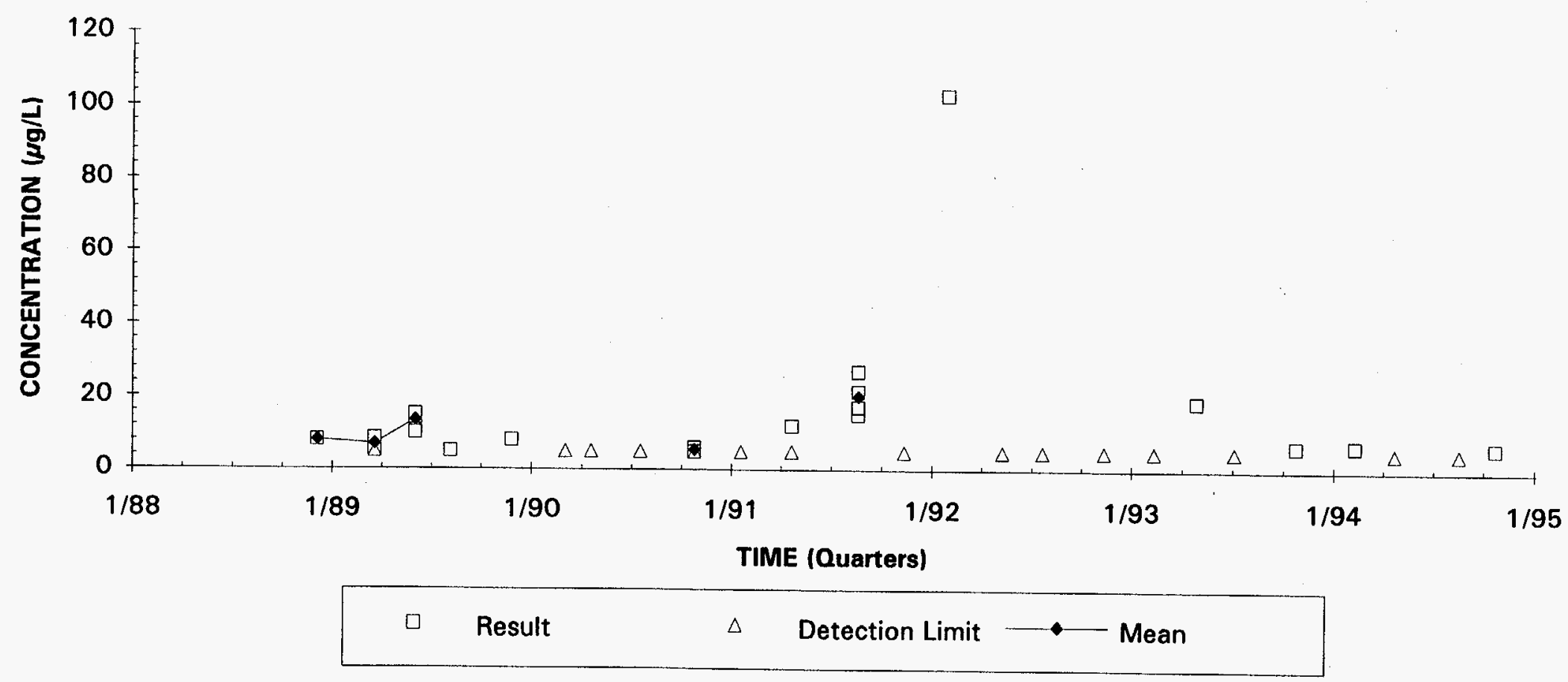



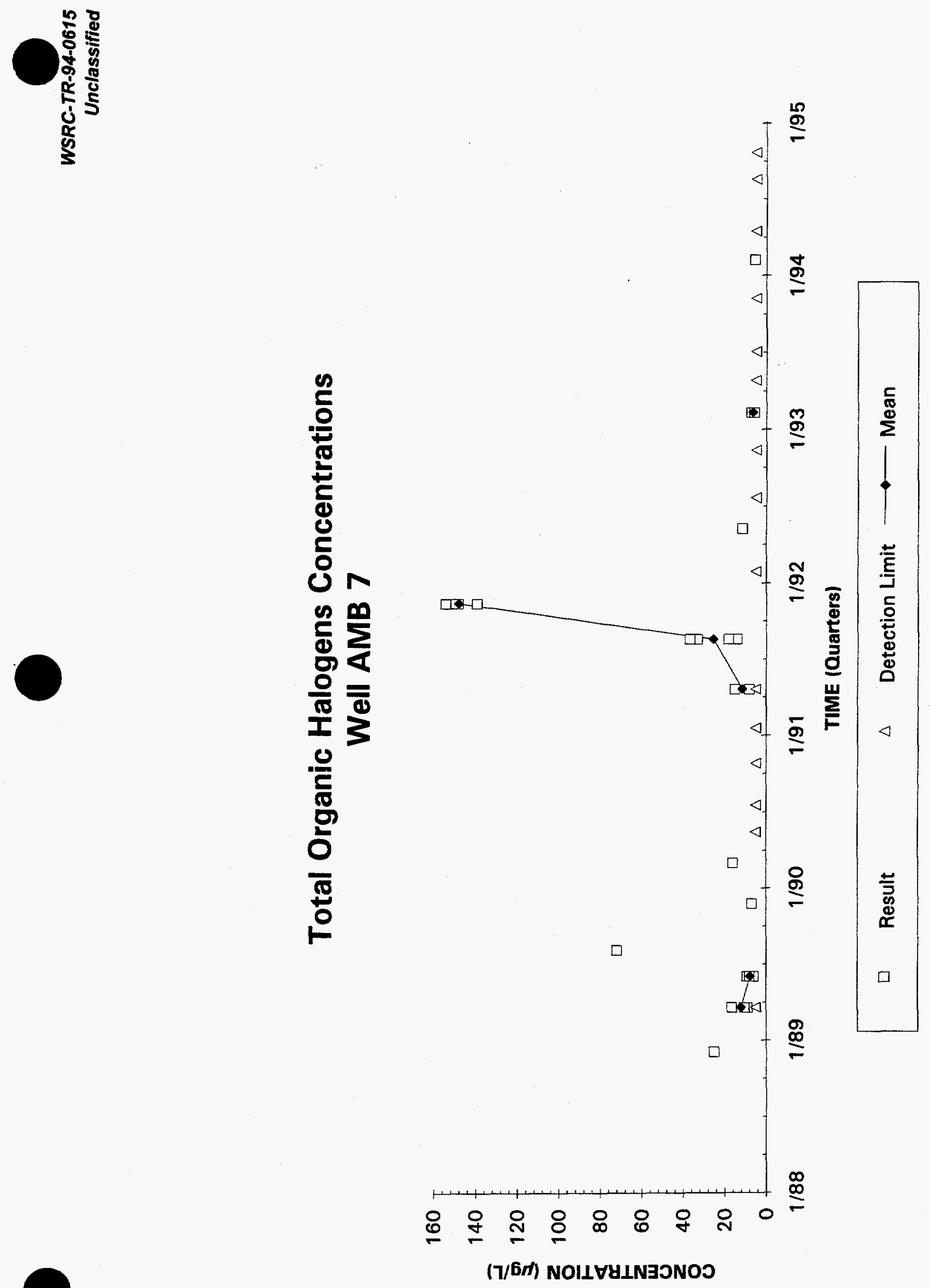

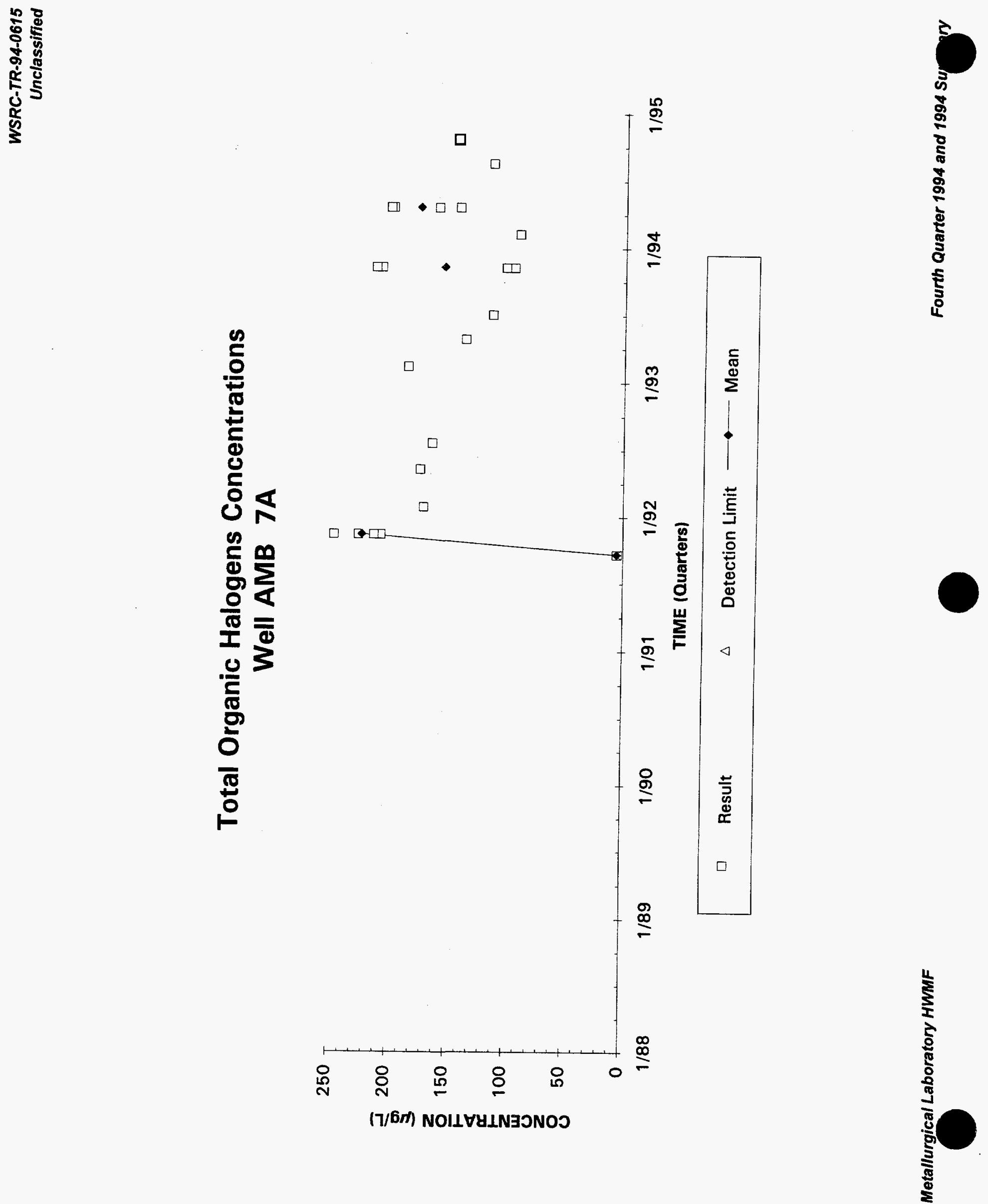

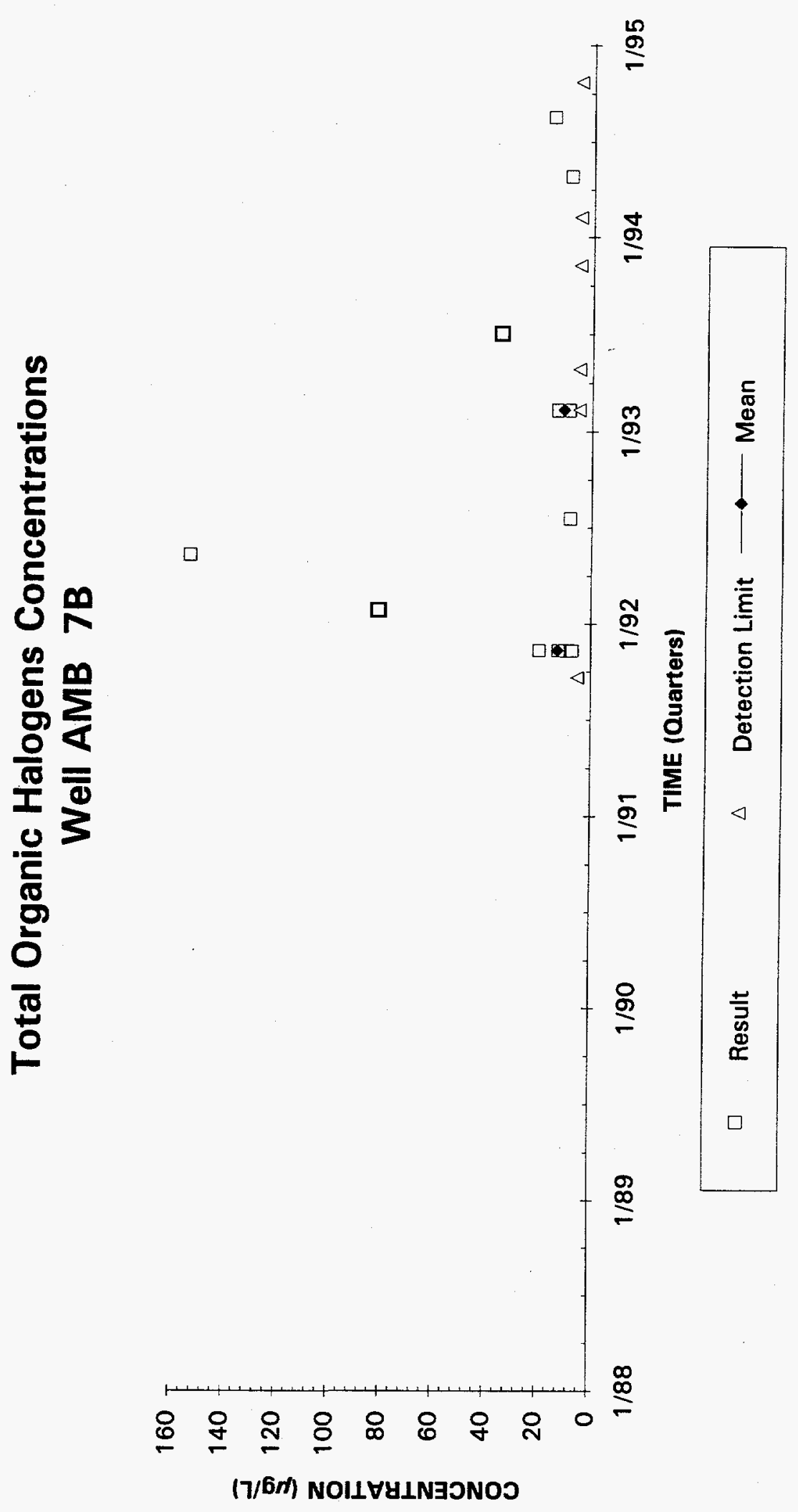


\section{Total Organic Halogens Concentrations}

\section{Well AMB 8D}

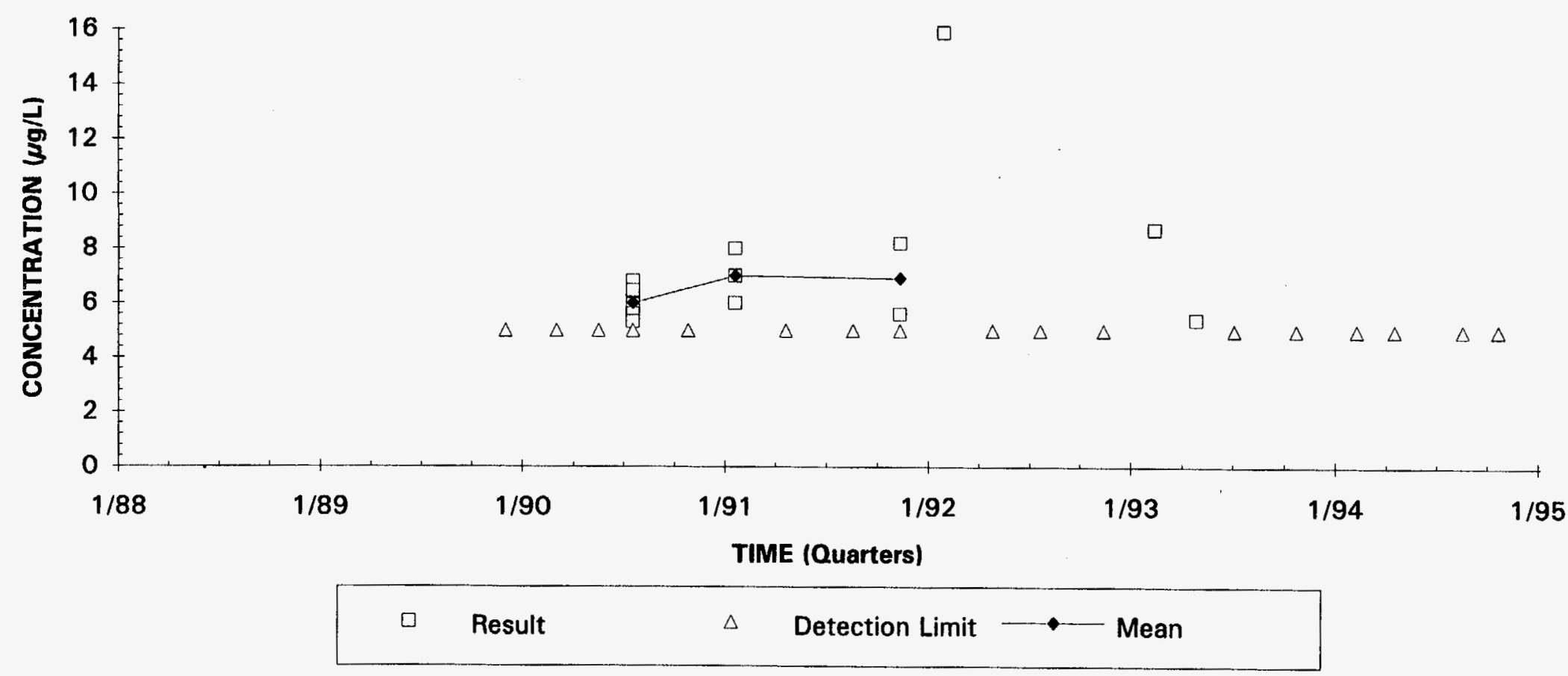



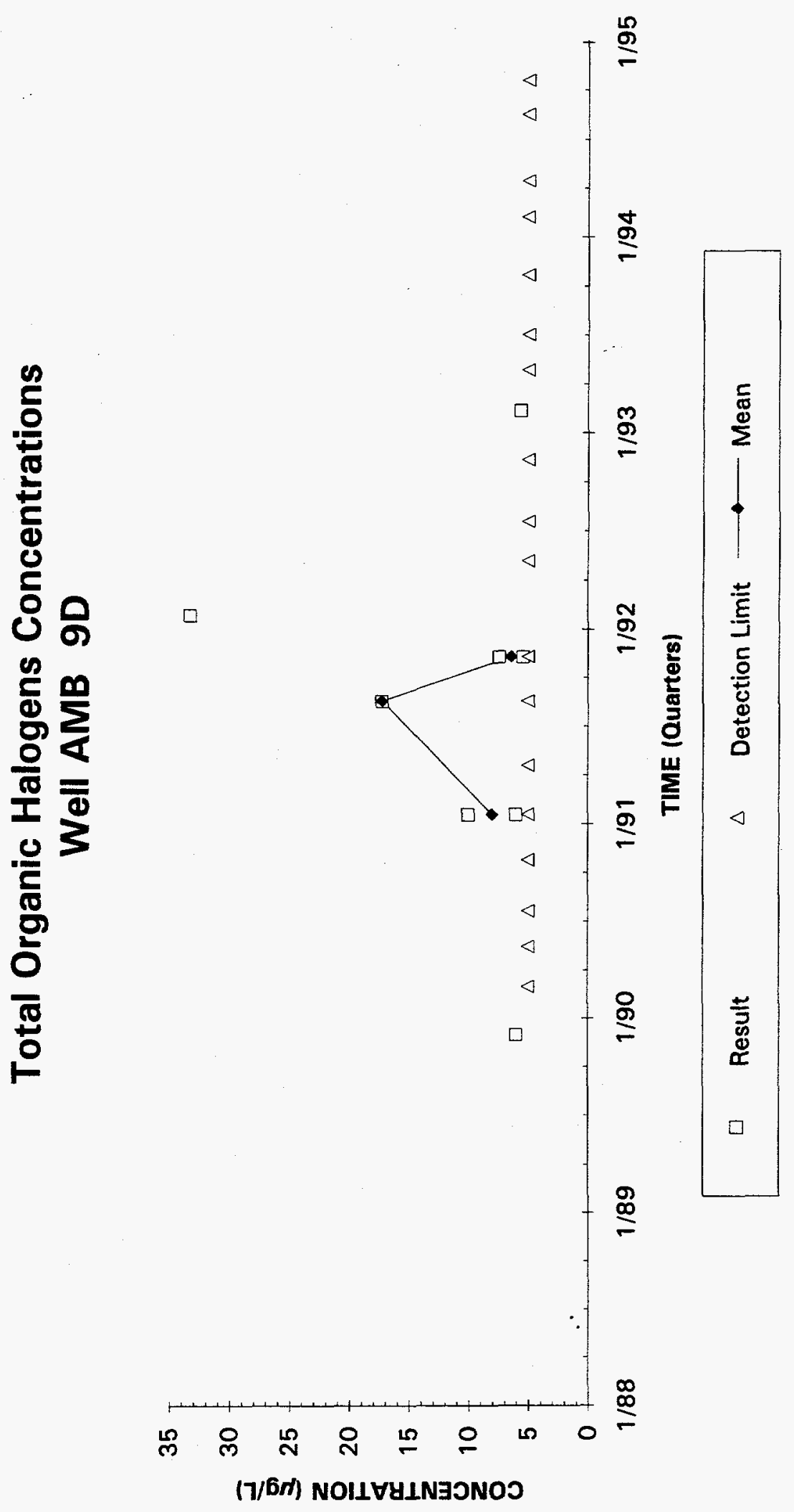


\section{Total Organic Halogens Concentrations}

Well AMB 10A

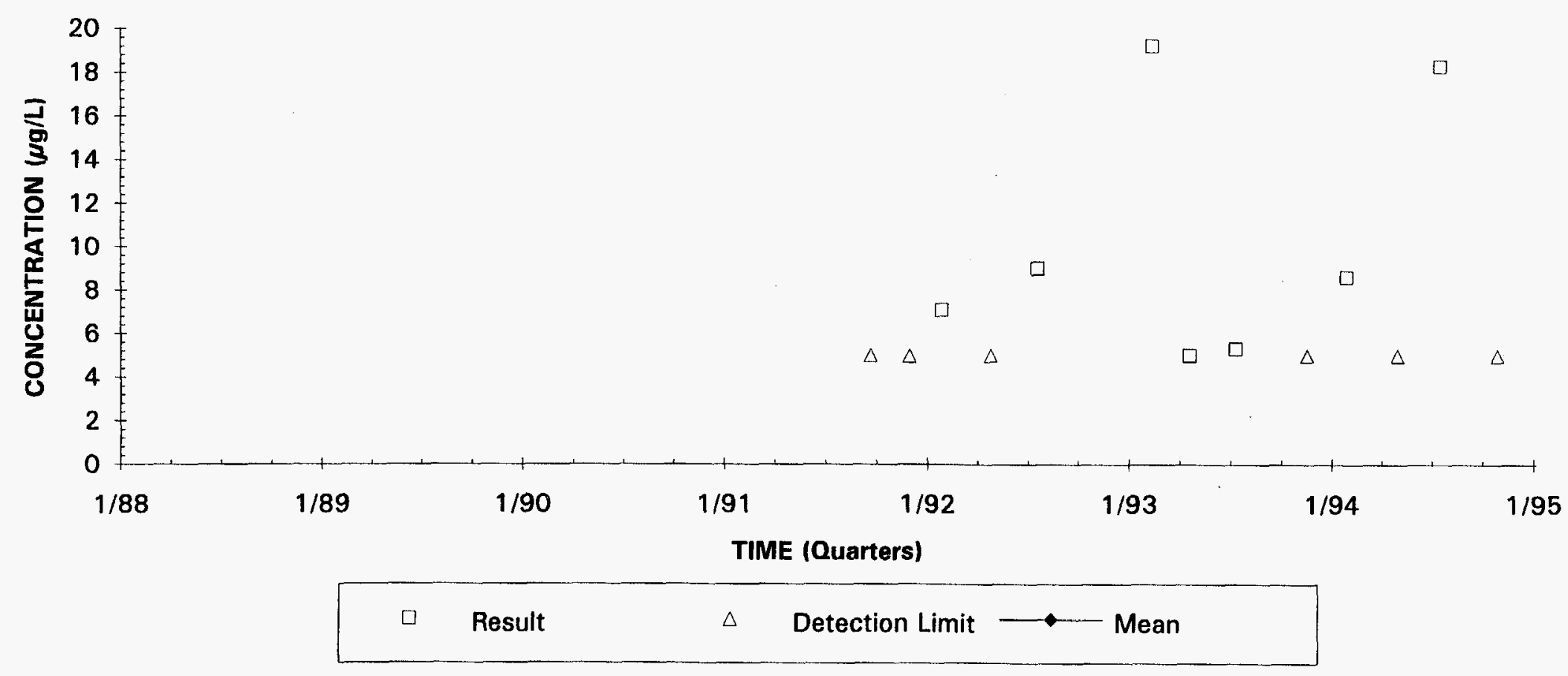



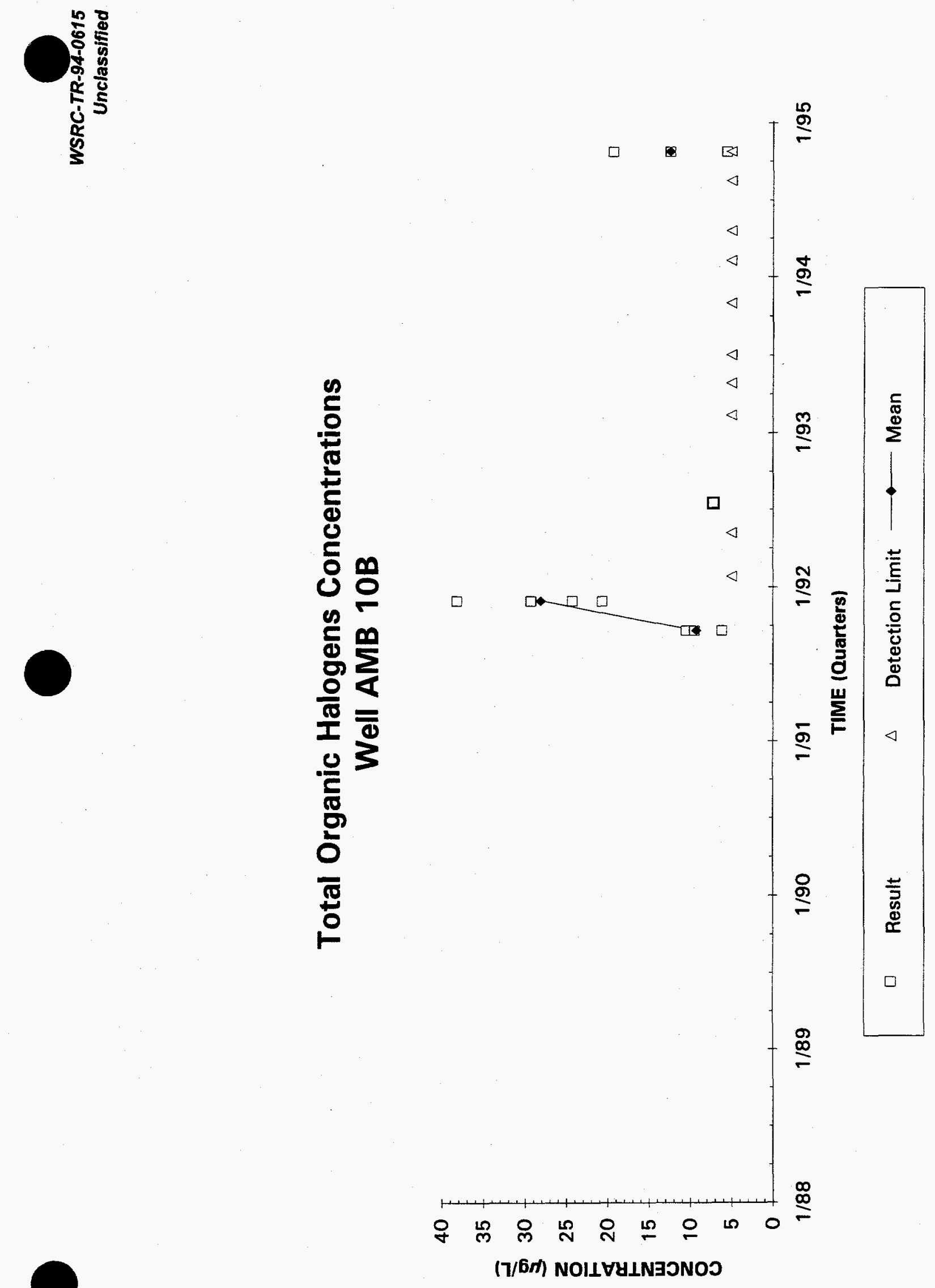

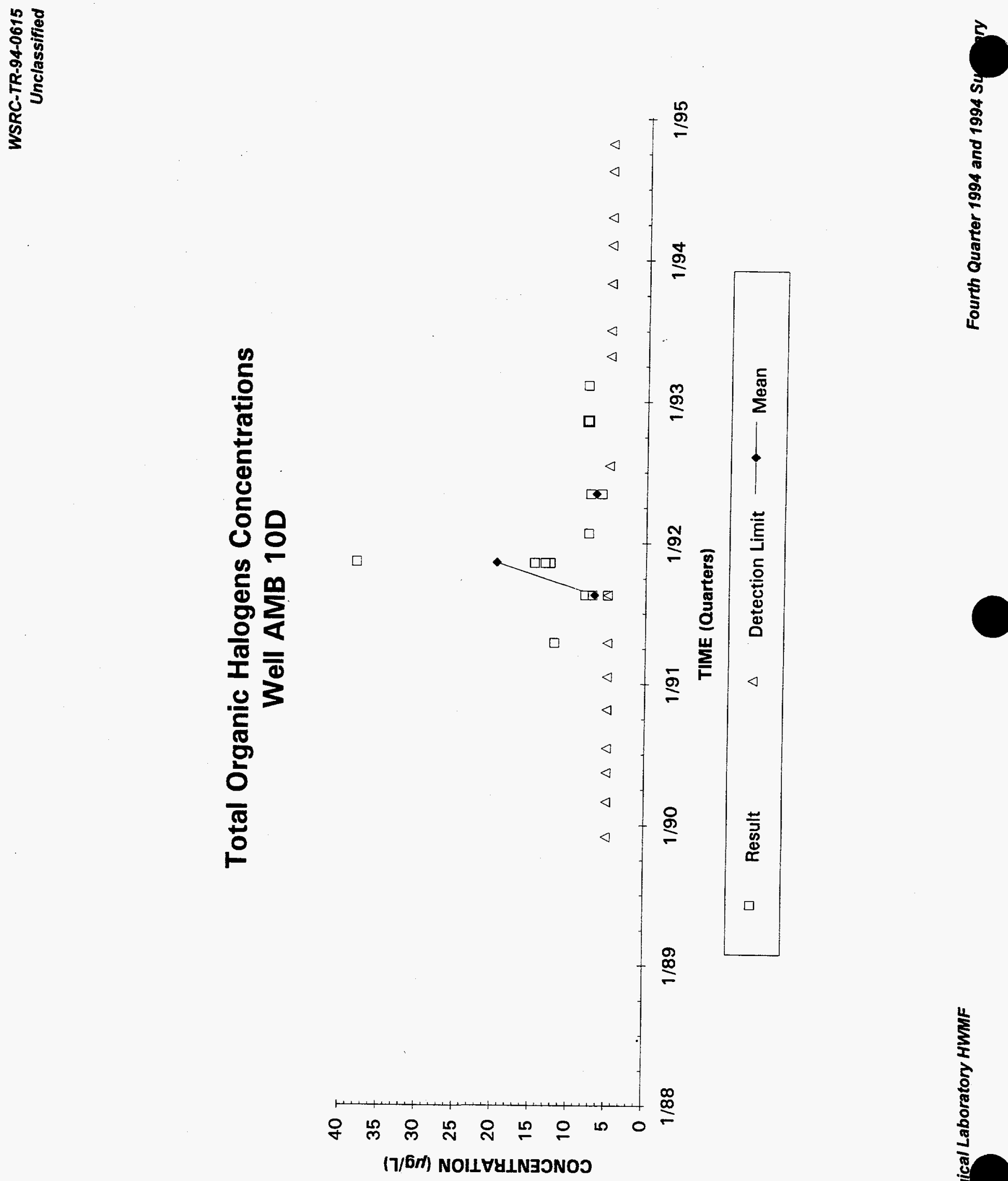

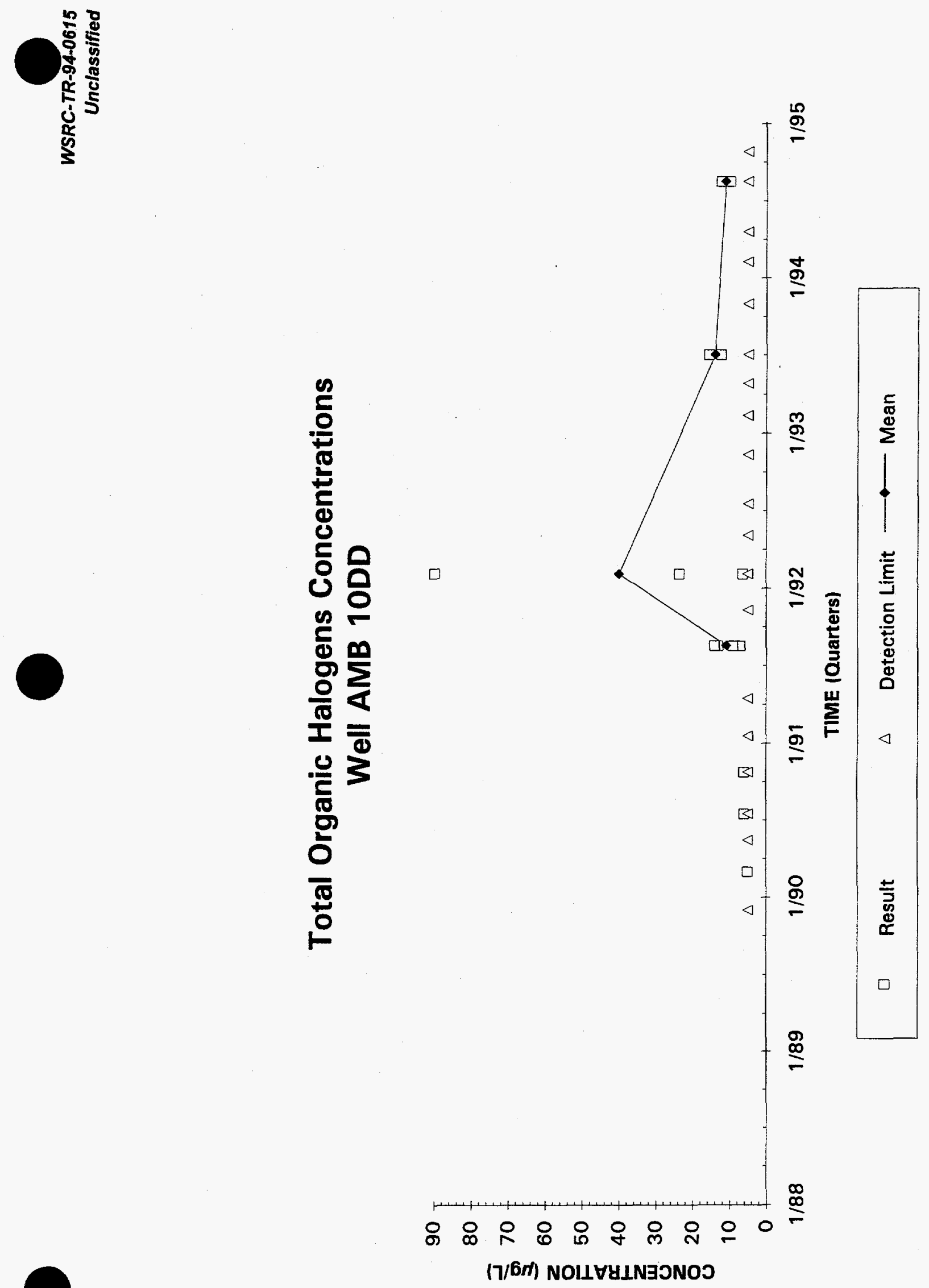


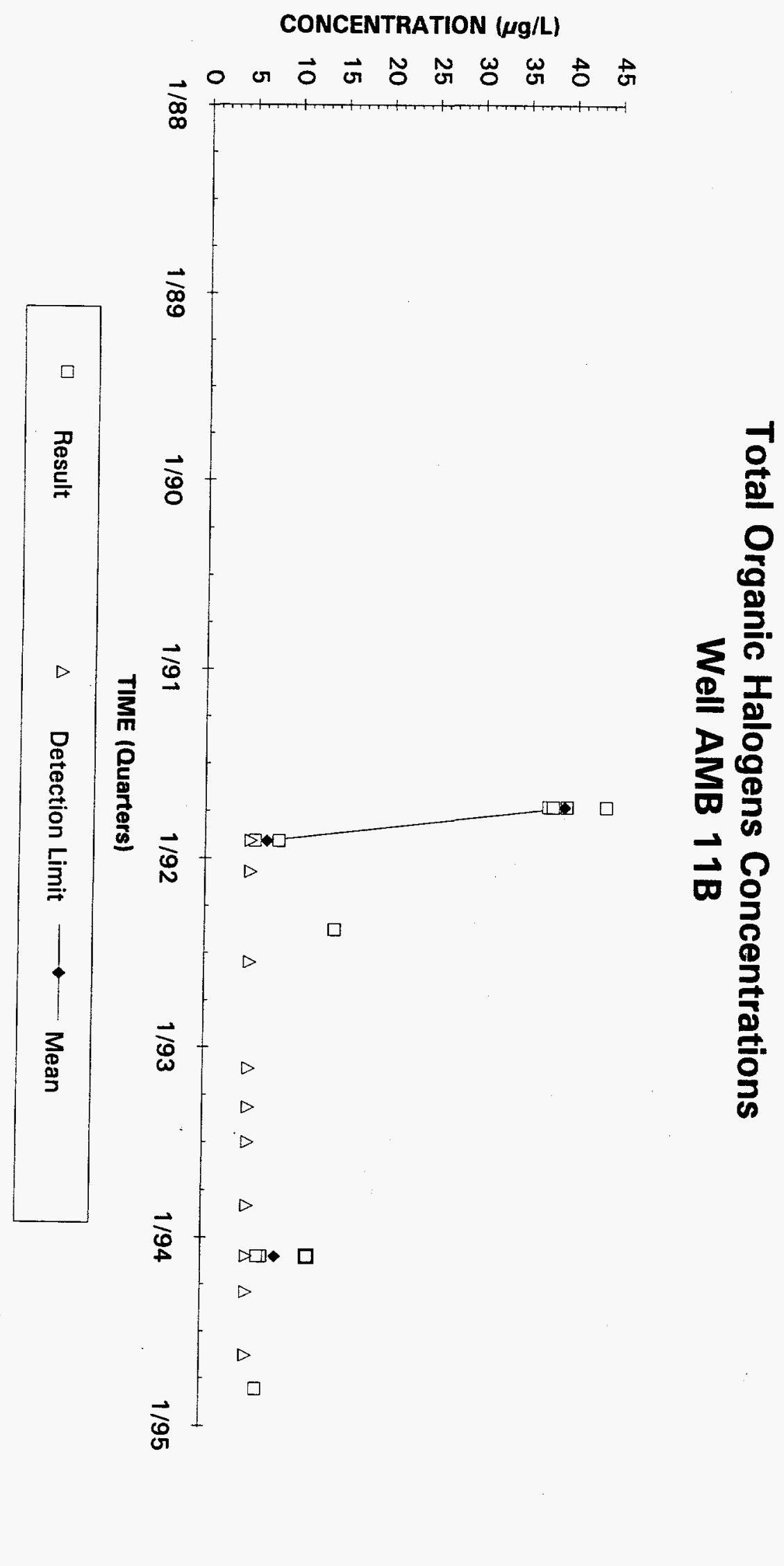

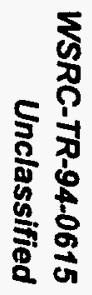


בิ๊

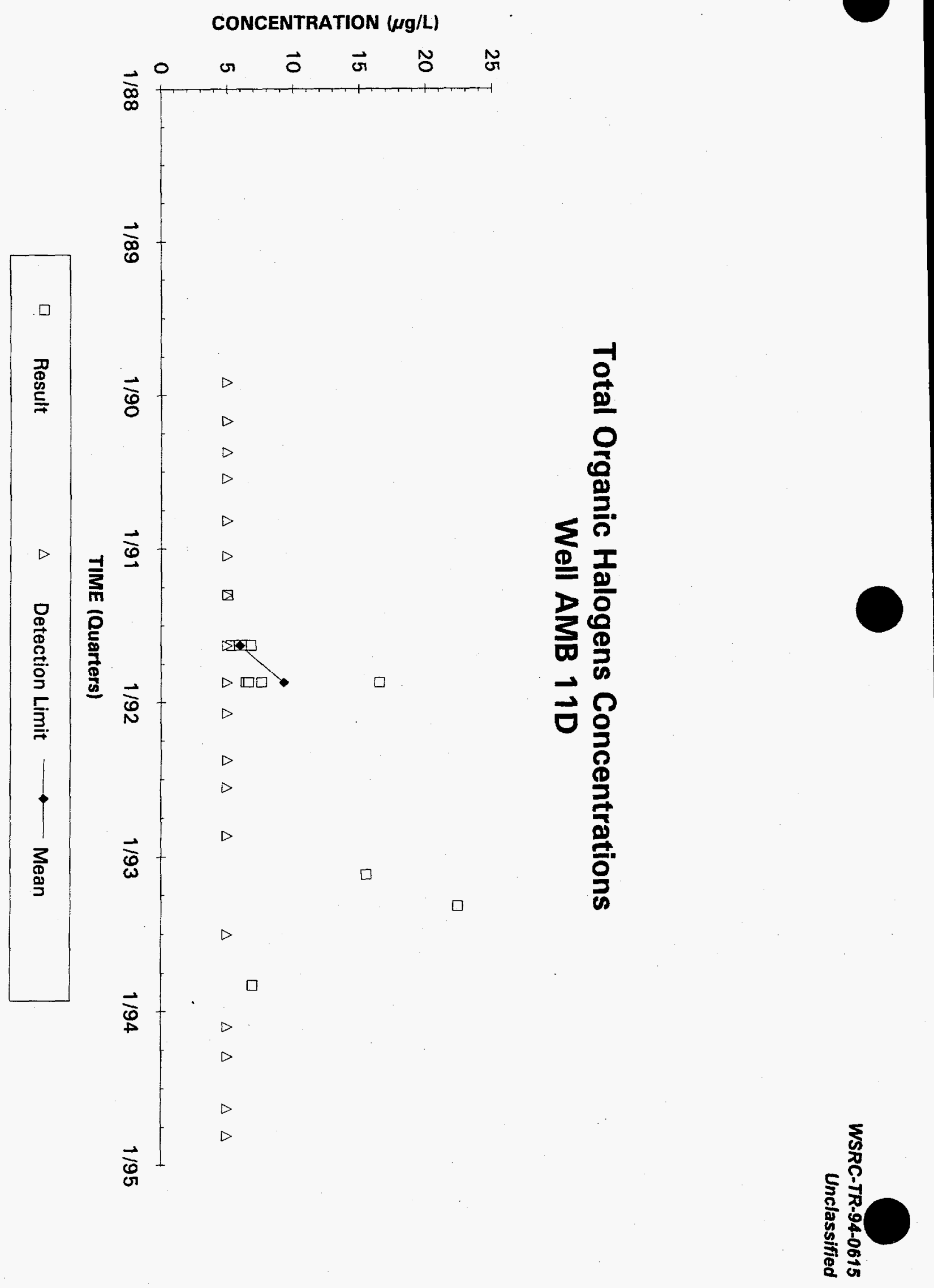




\section{Total Organic Halogens Concentrations}

Well AMB 12D

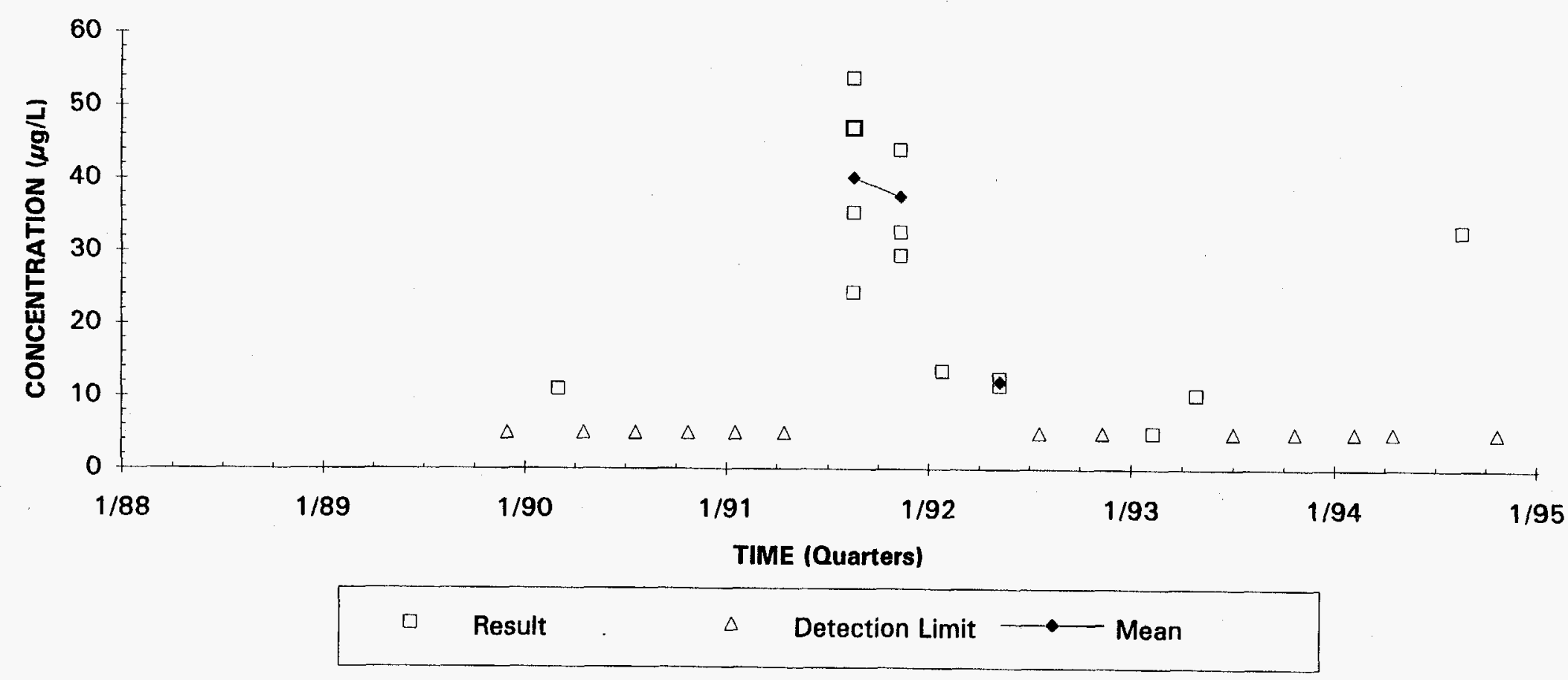




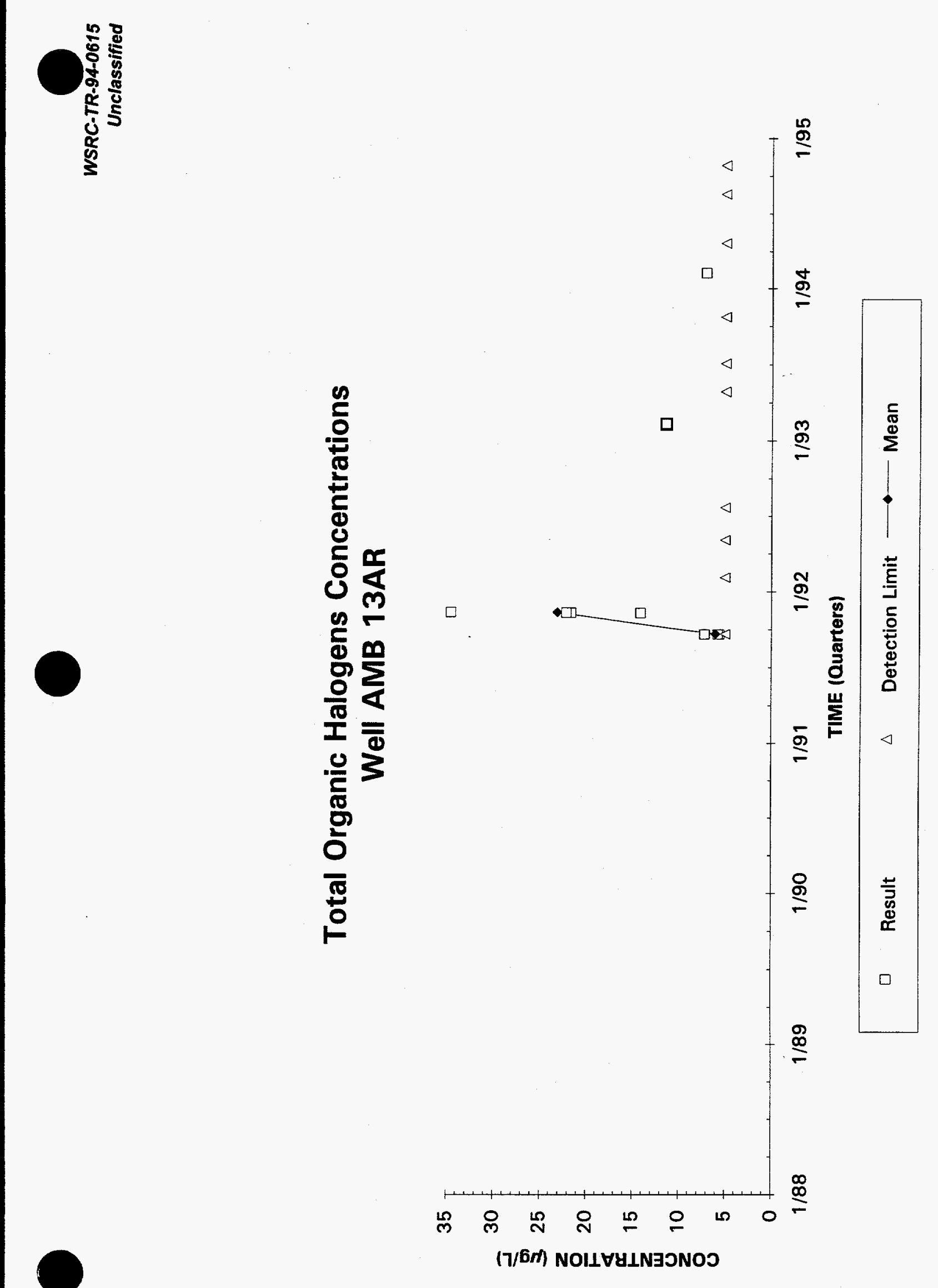

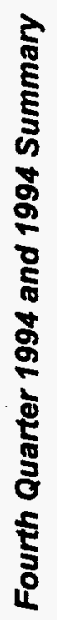



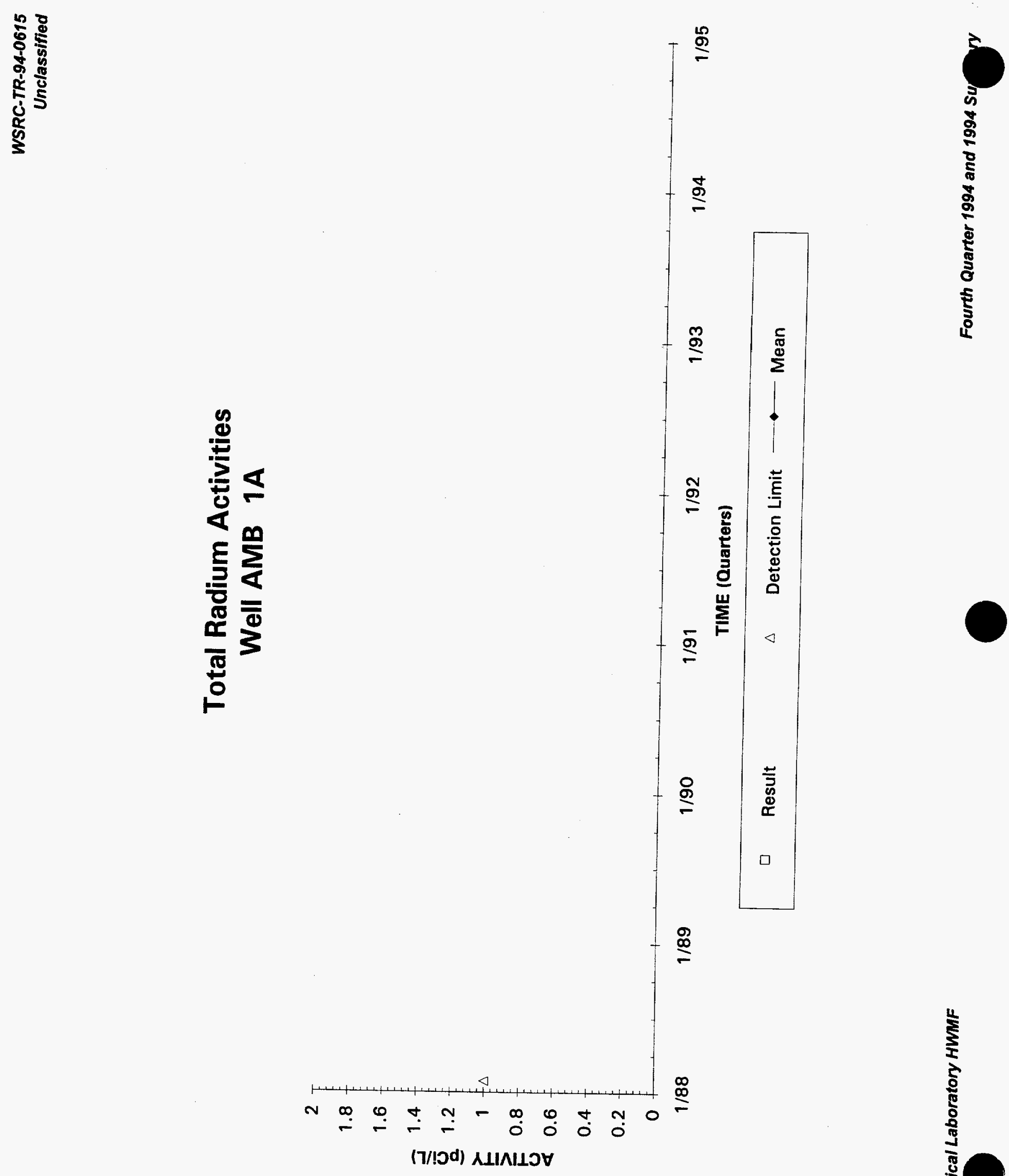

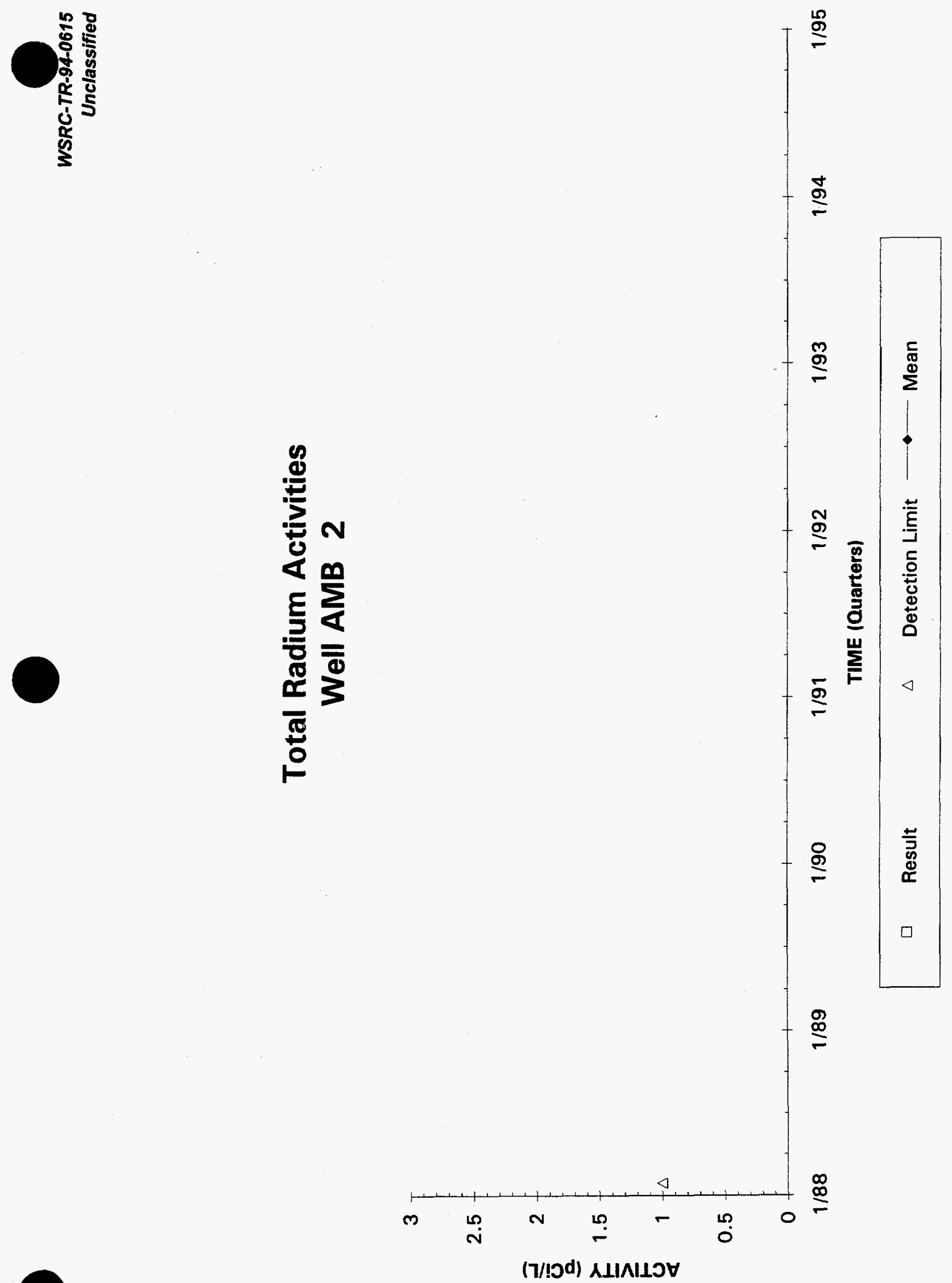

हे

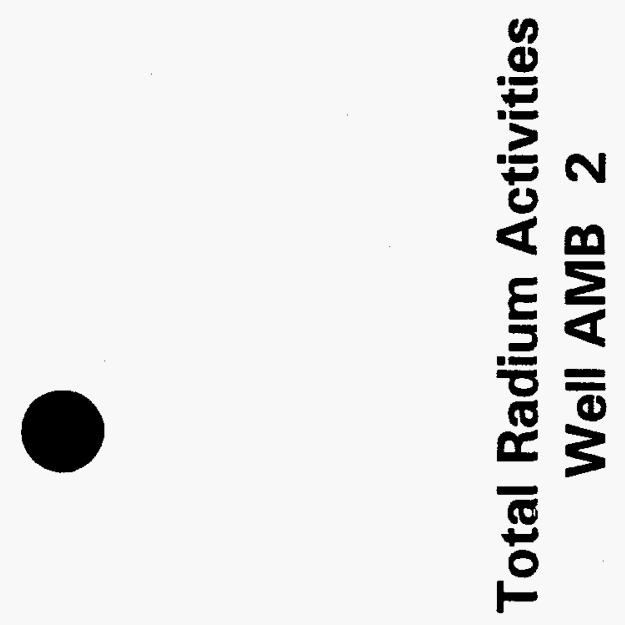

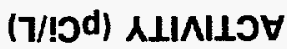




\section{Total Radium Activities \\ Well AMB 3A}

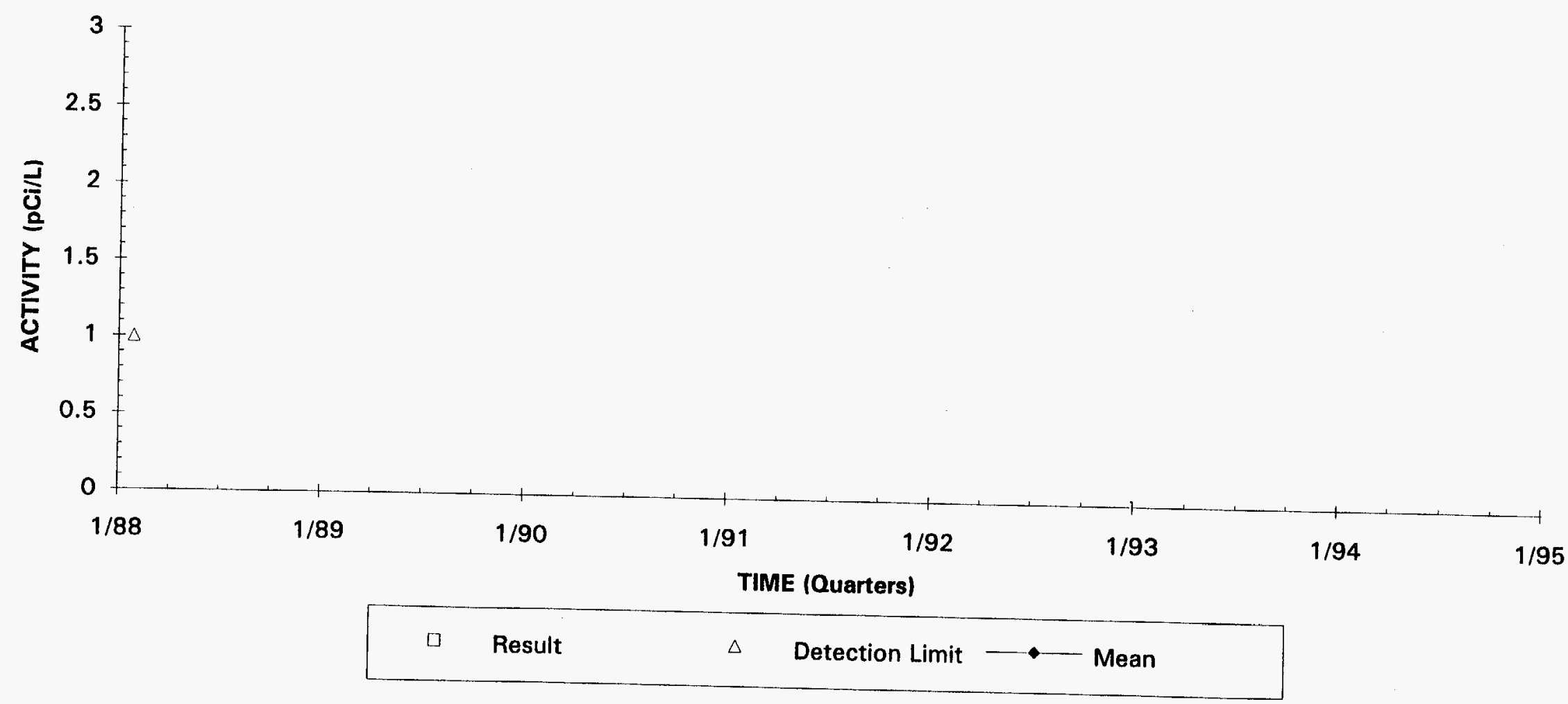



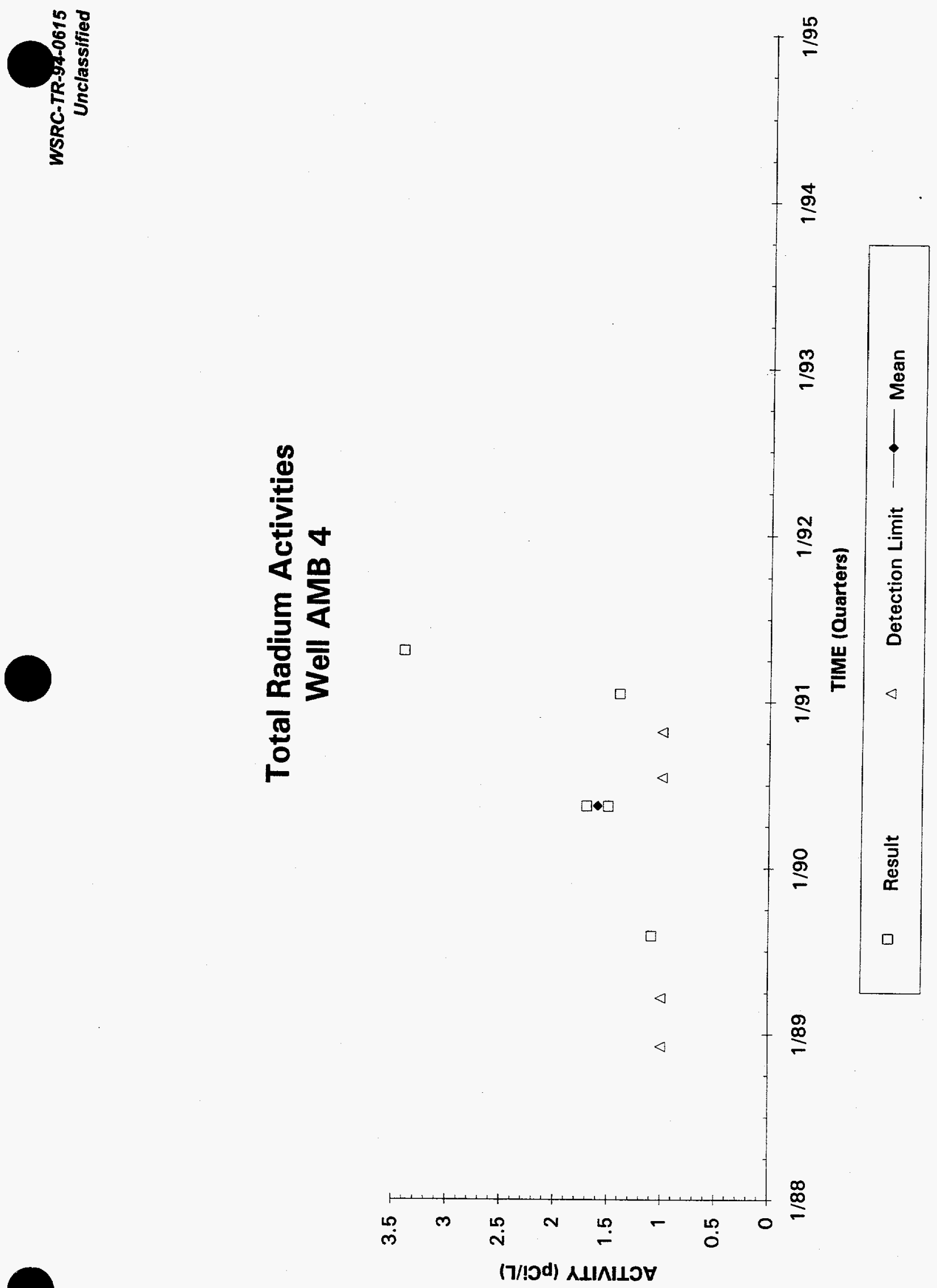

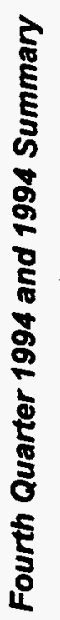

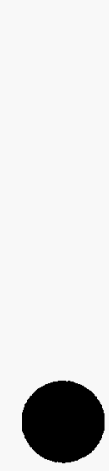



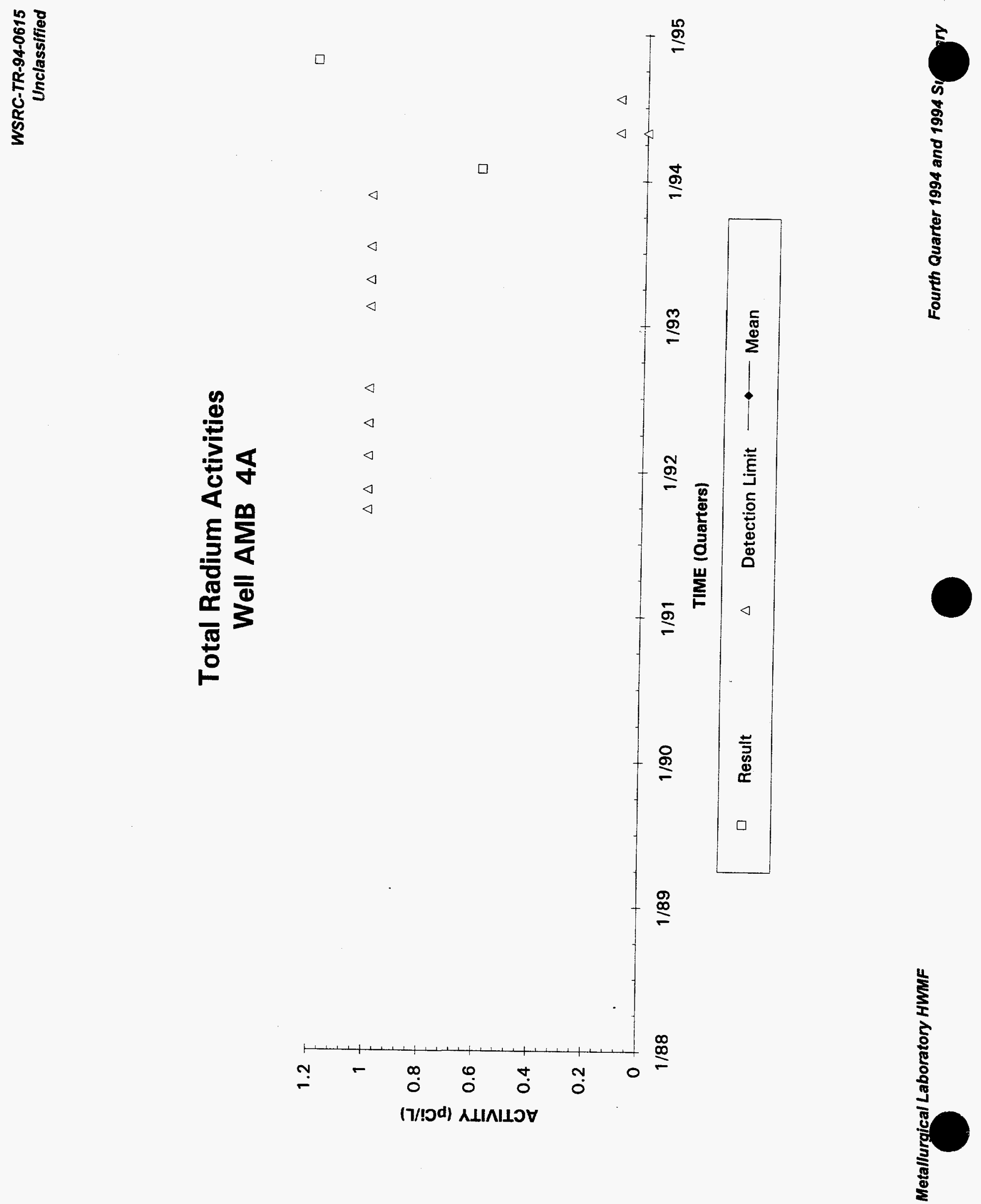

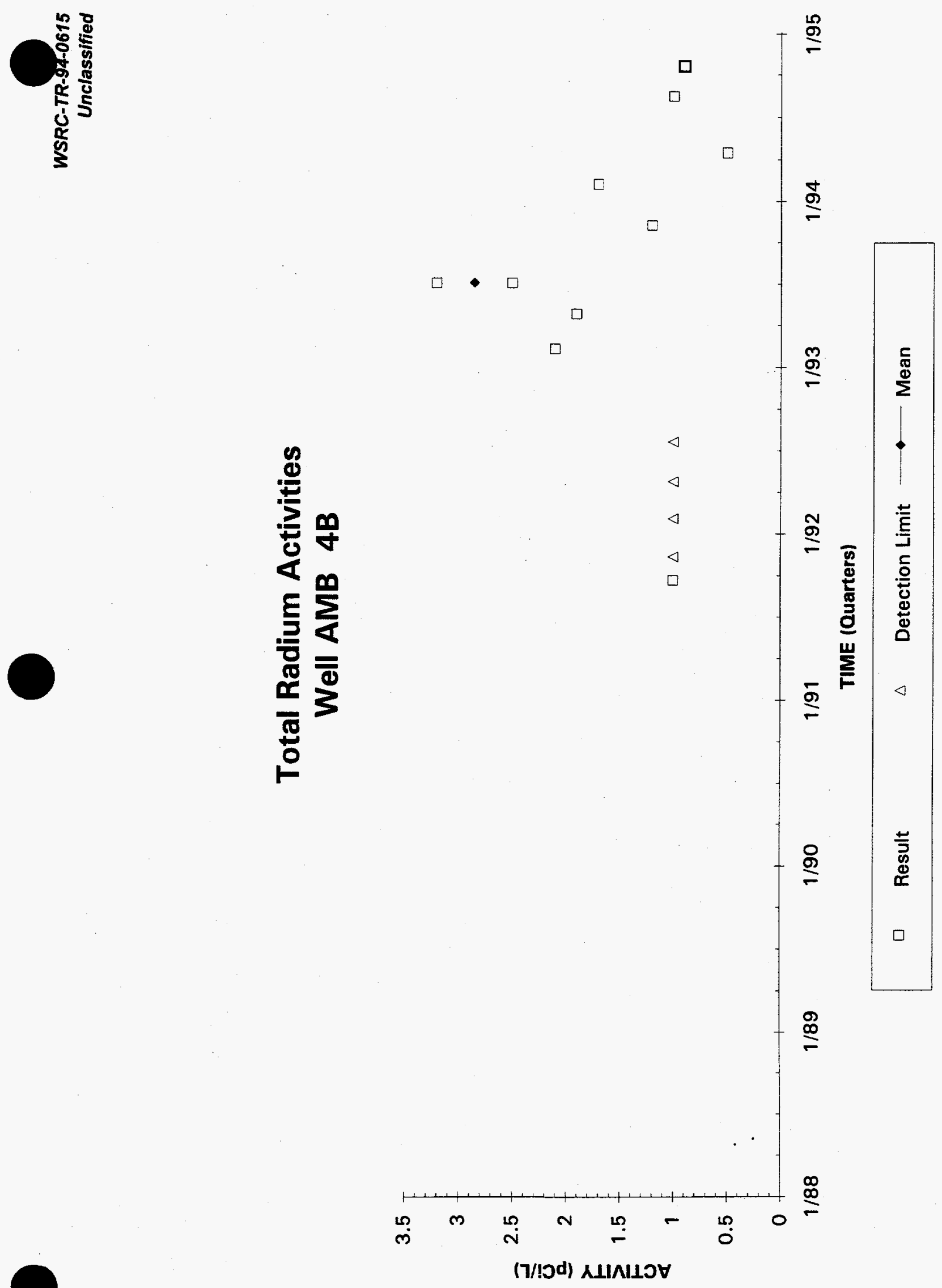

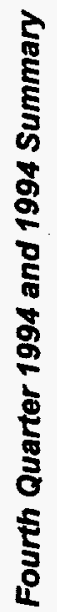

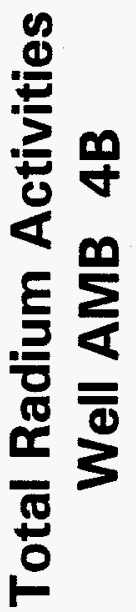



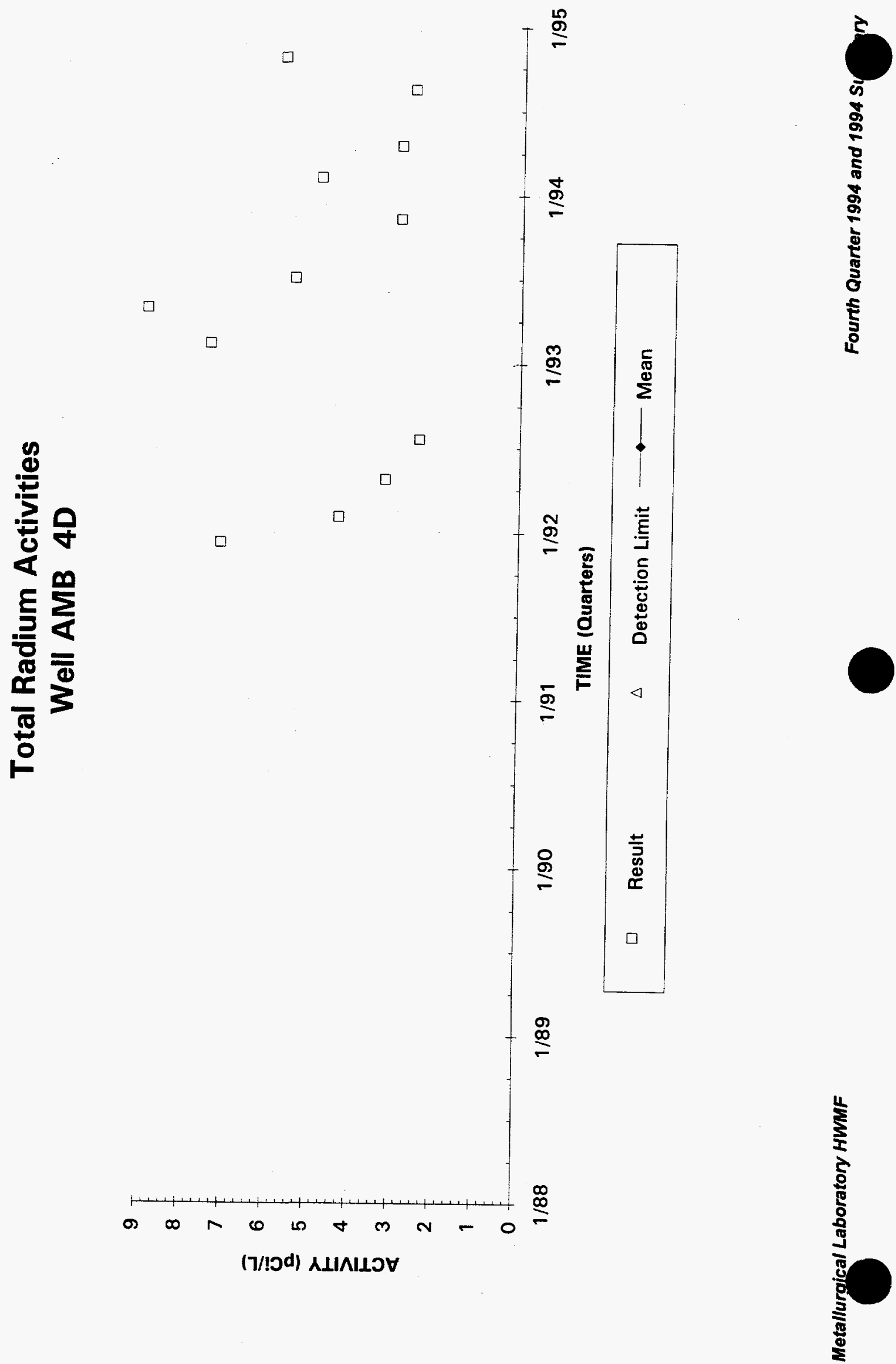

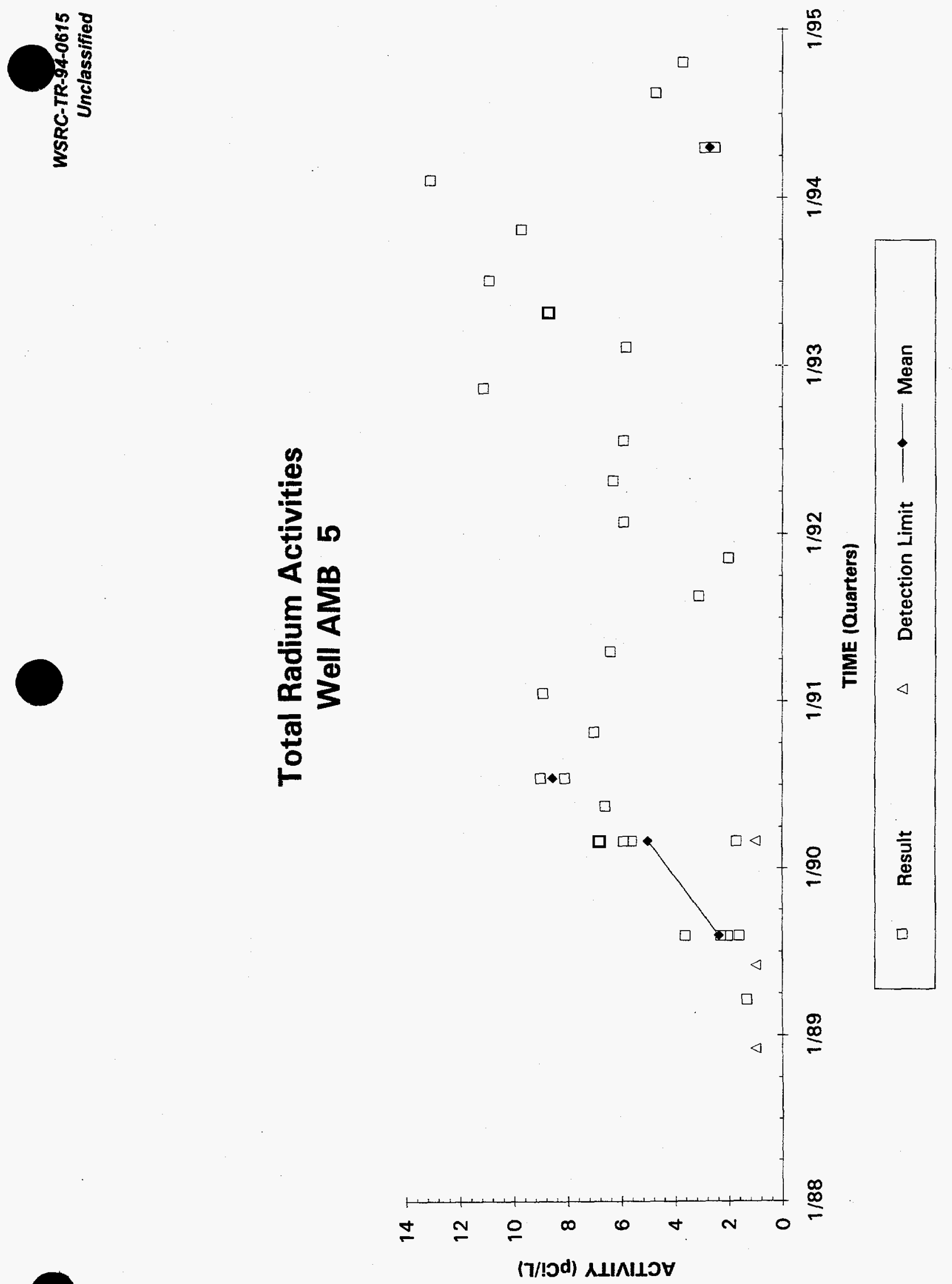

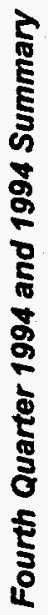

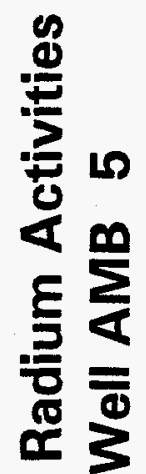

$\underset{10}{0}$

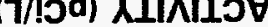




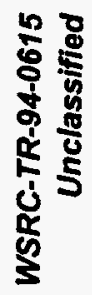
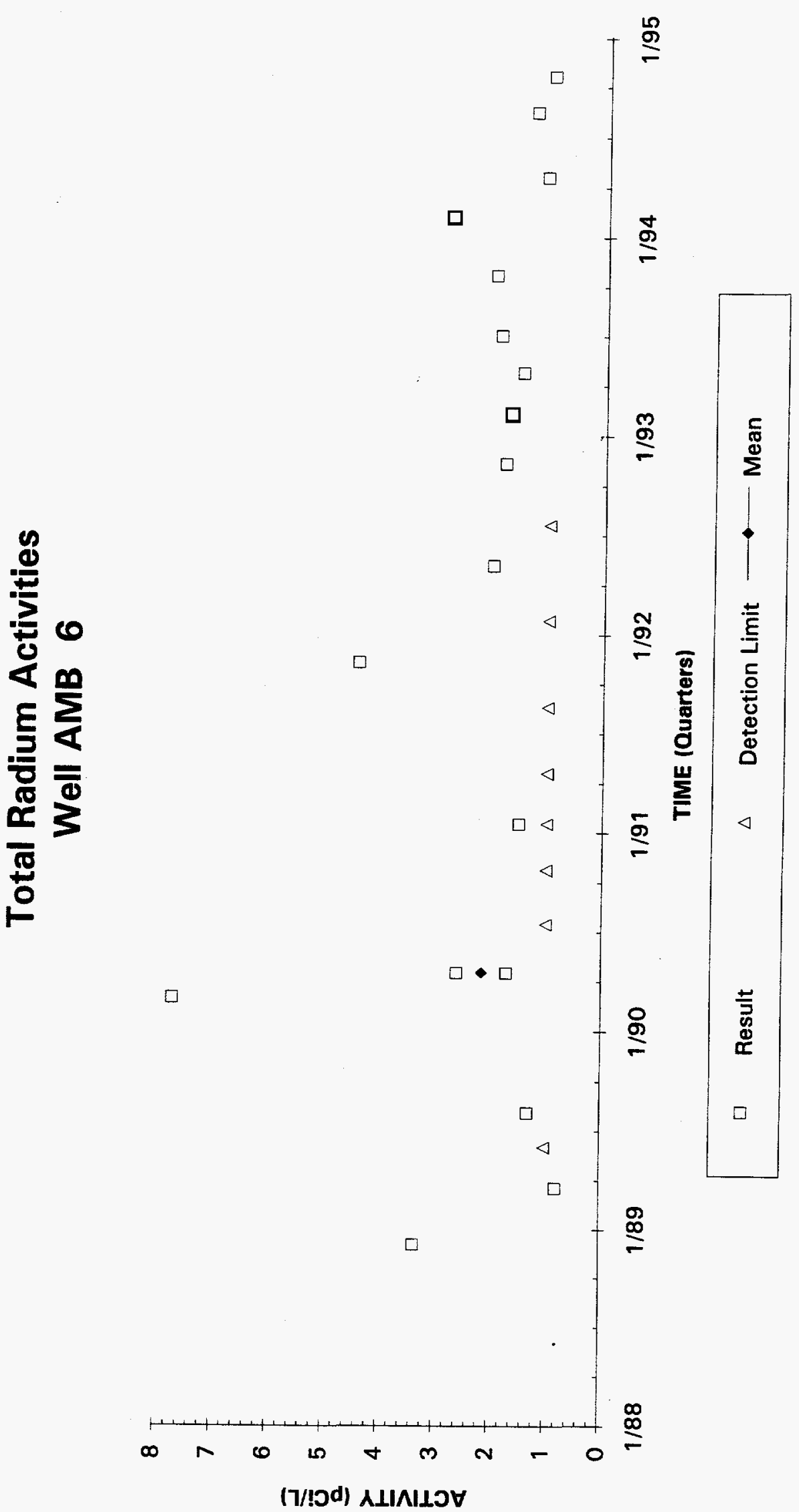

ह
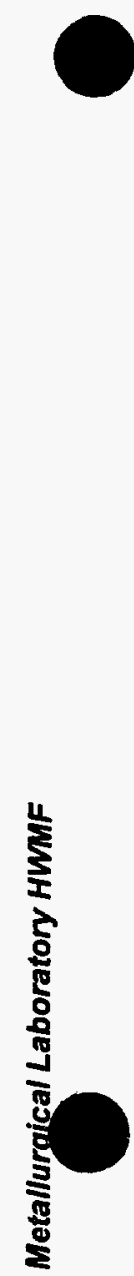


\section{Total Radium Activities}

Well AMB 7

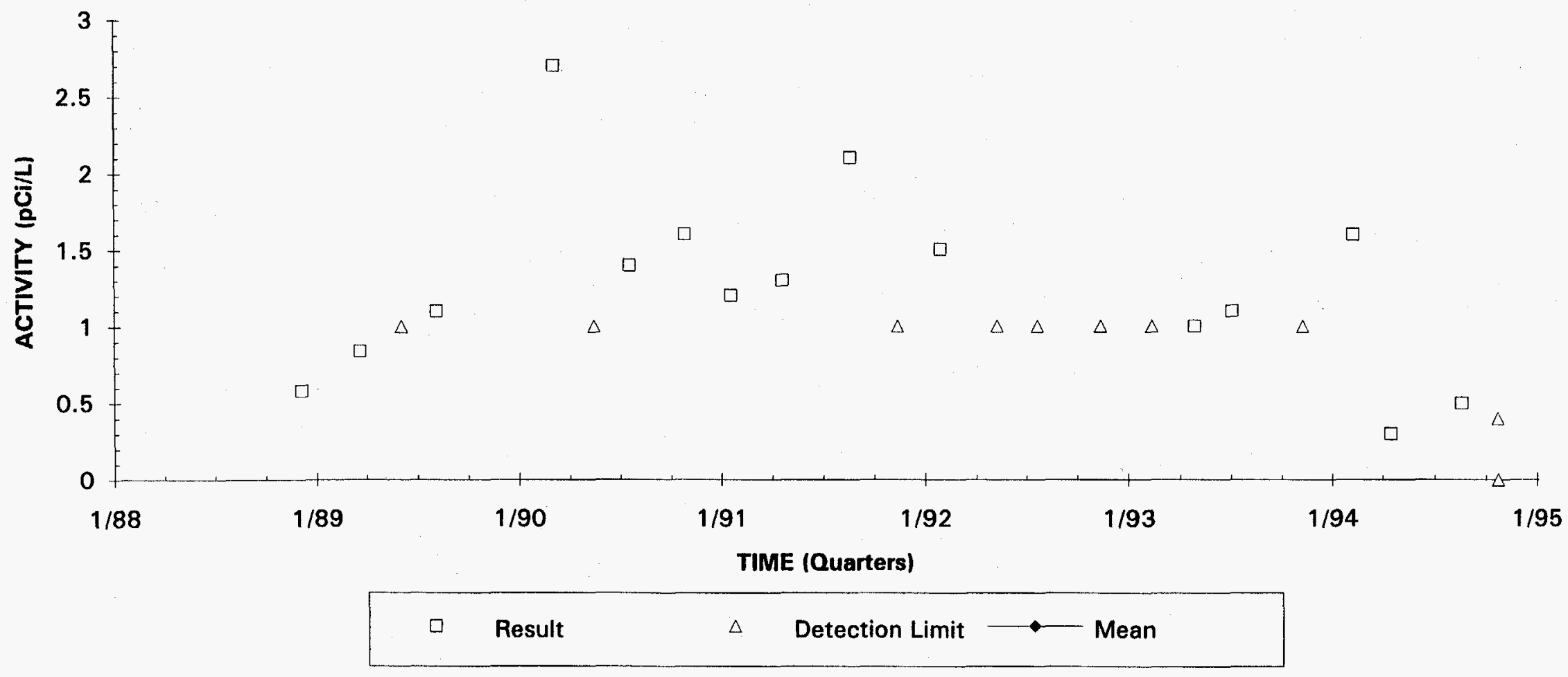




\section{Total Radium Activities}

Well AMB 7A

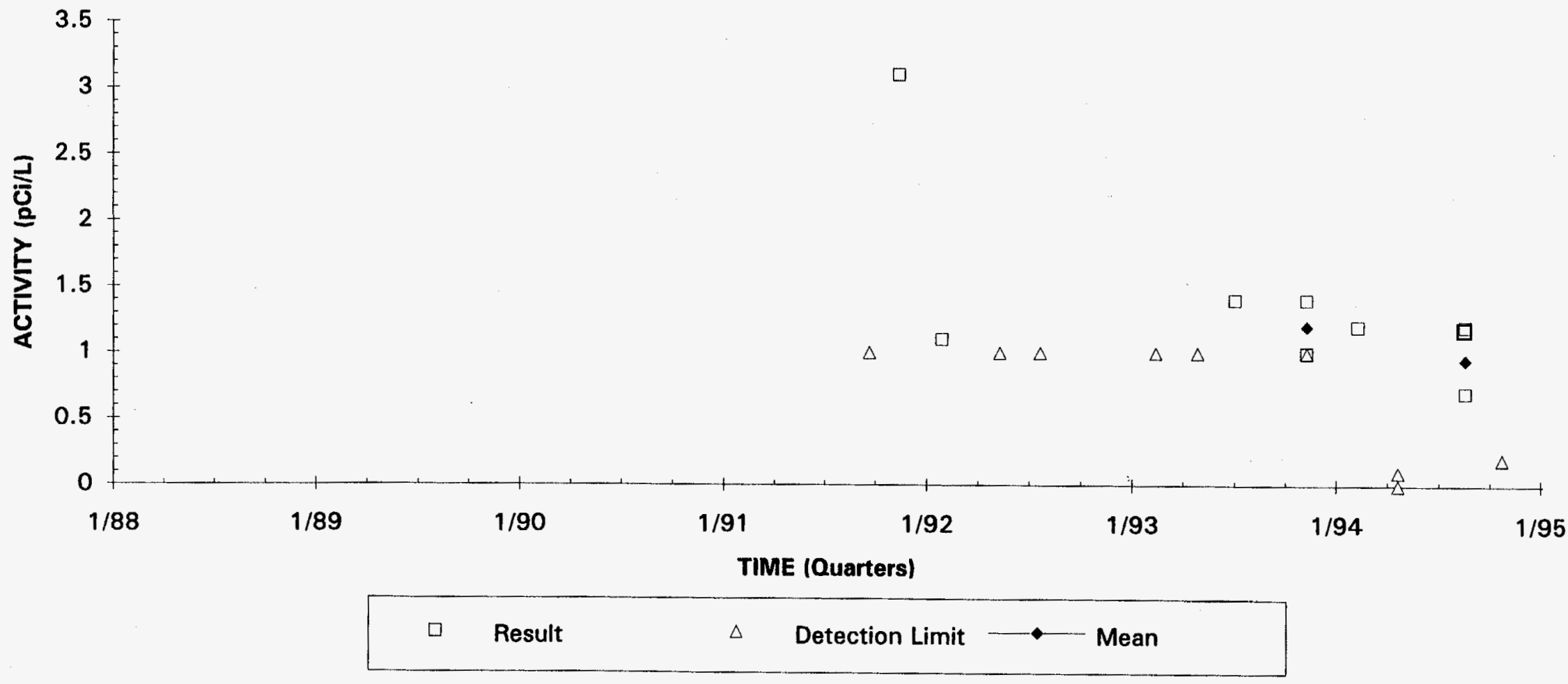




\section{Total Radium Activities}

Well AMB 7B

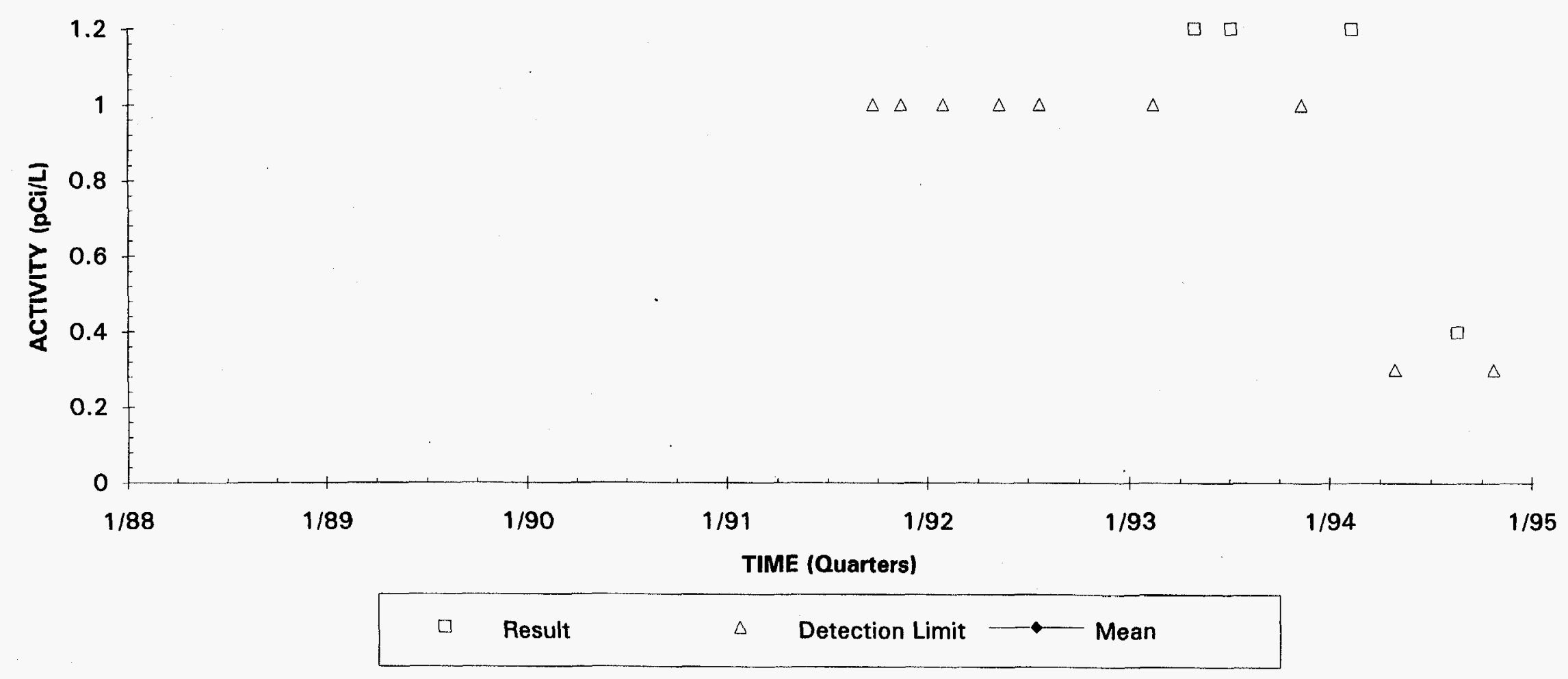




\section{Total Radium Activities}

Well AMB 8D

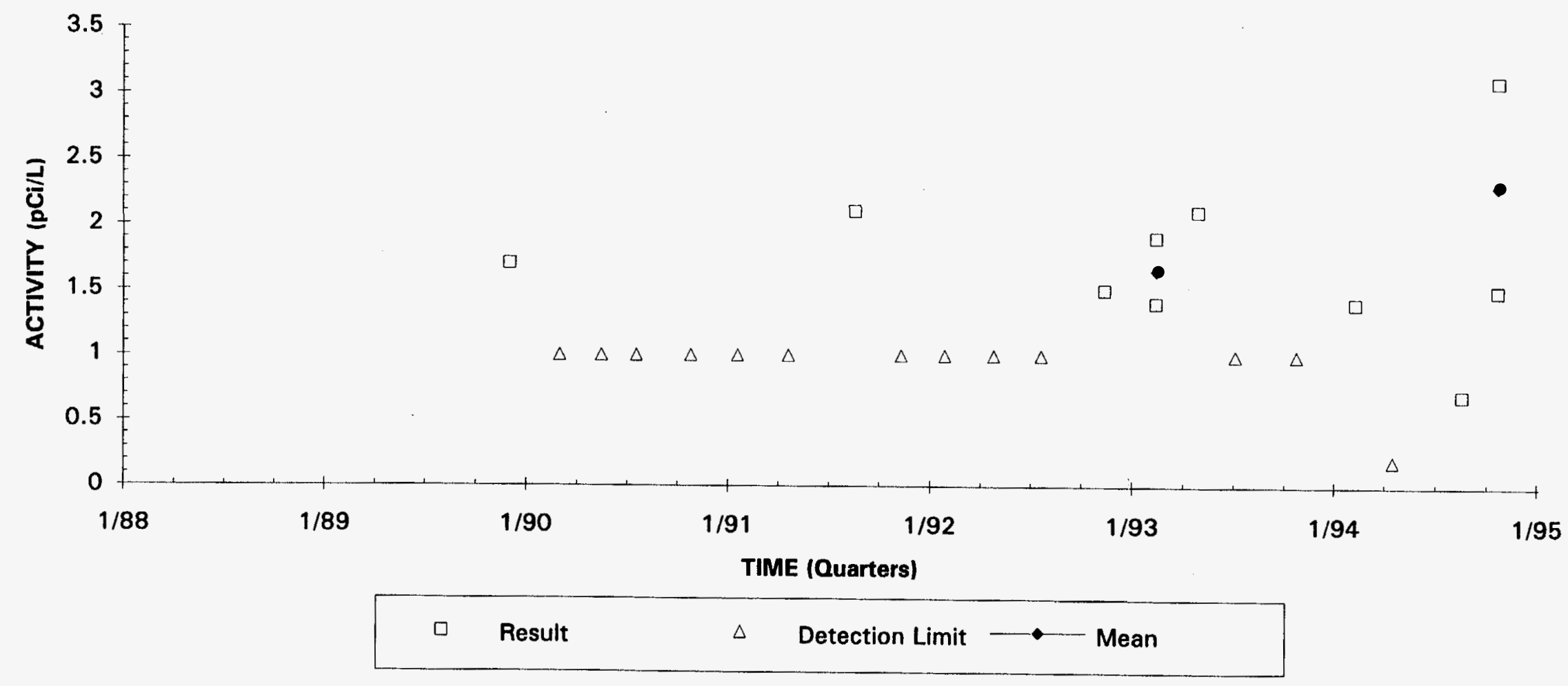



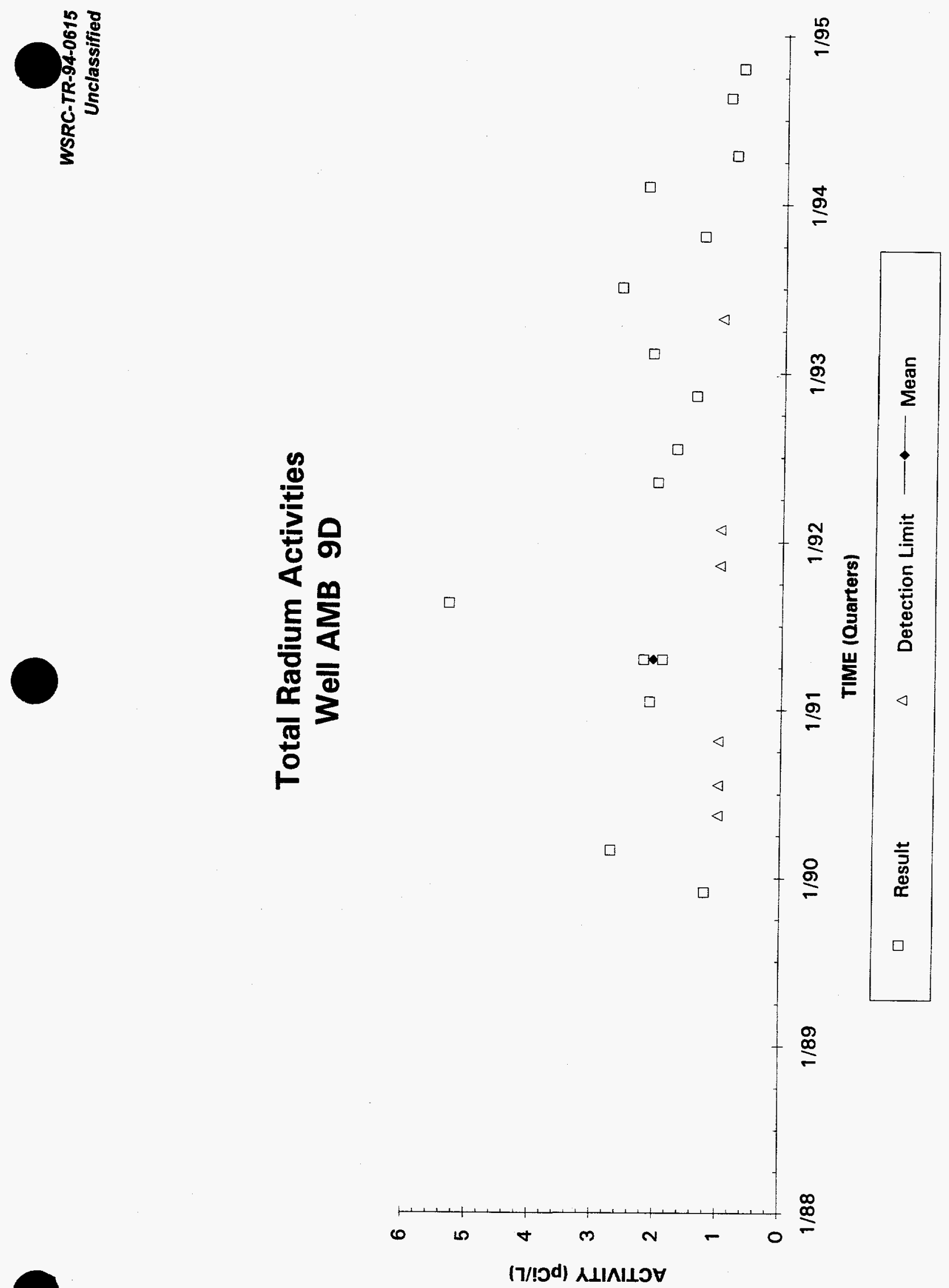

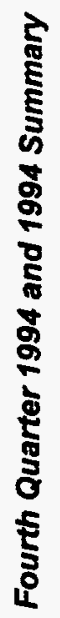

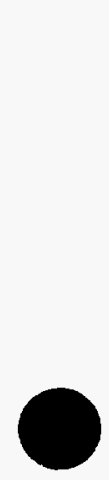

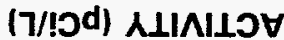




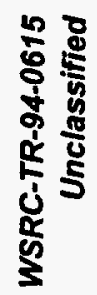
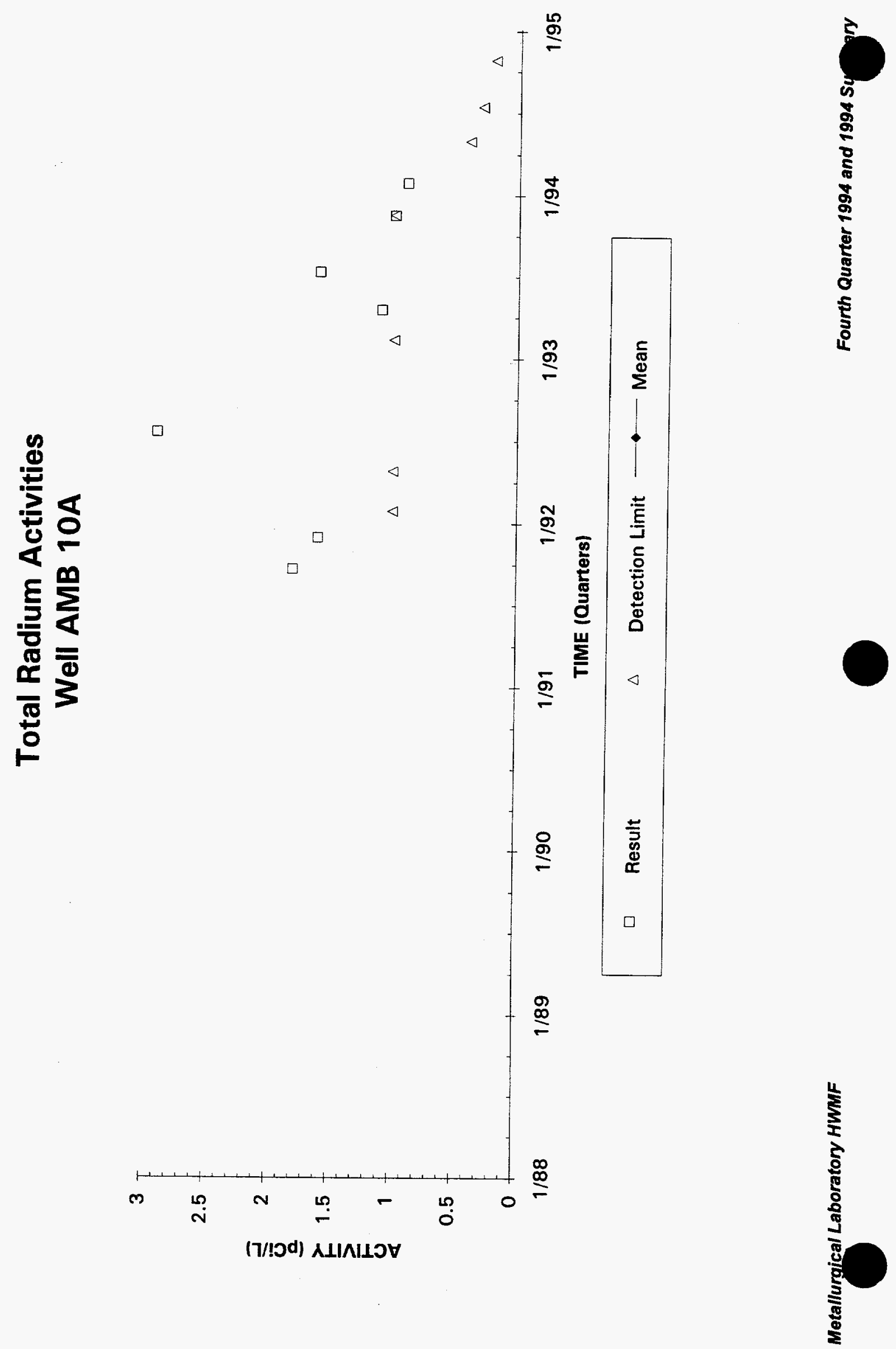


\section{Total Radium Activities}

Well AMB 10B

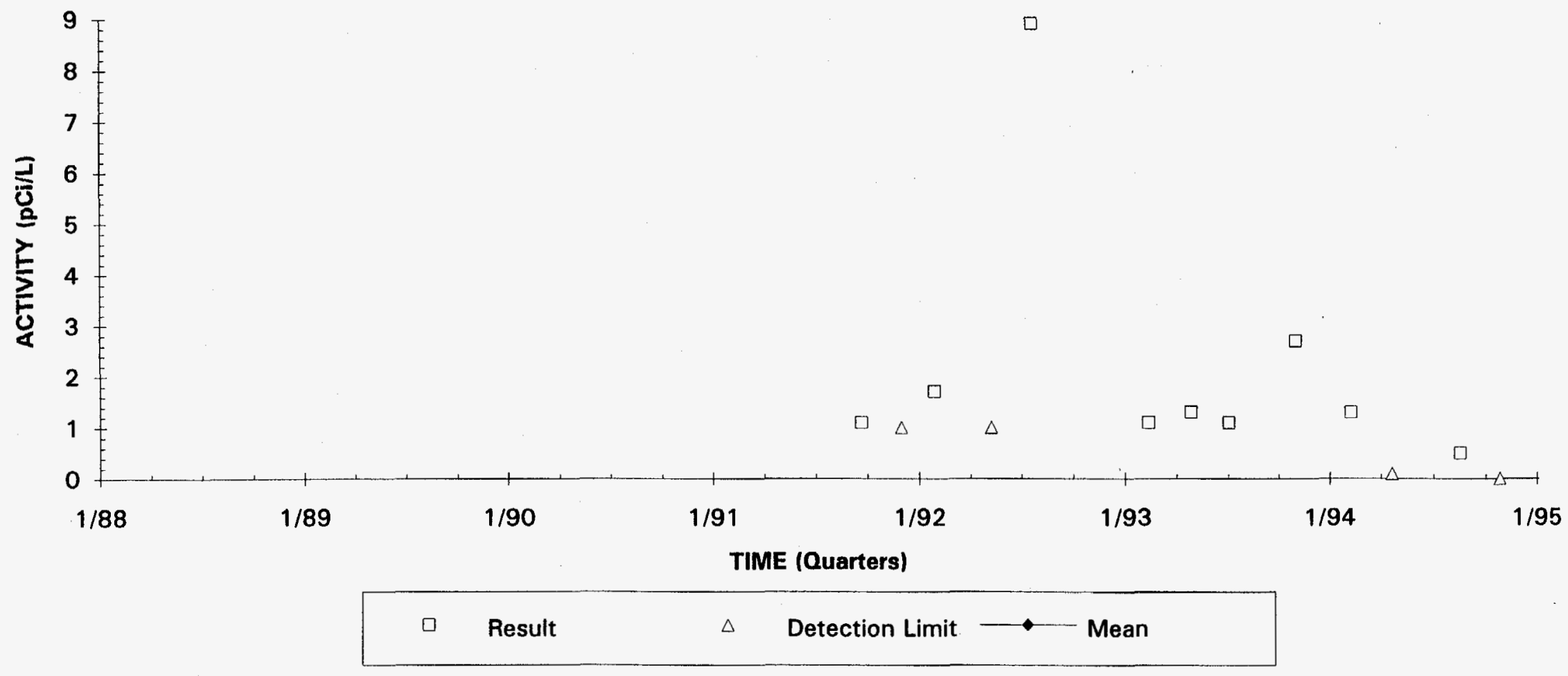




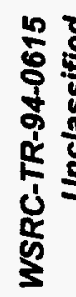
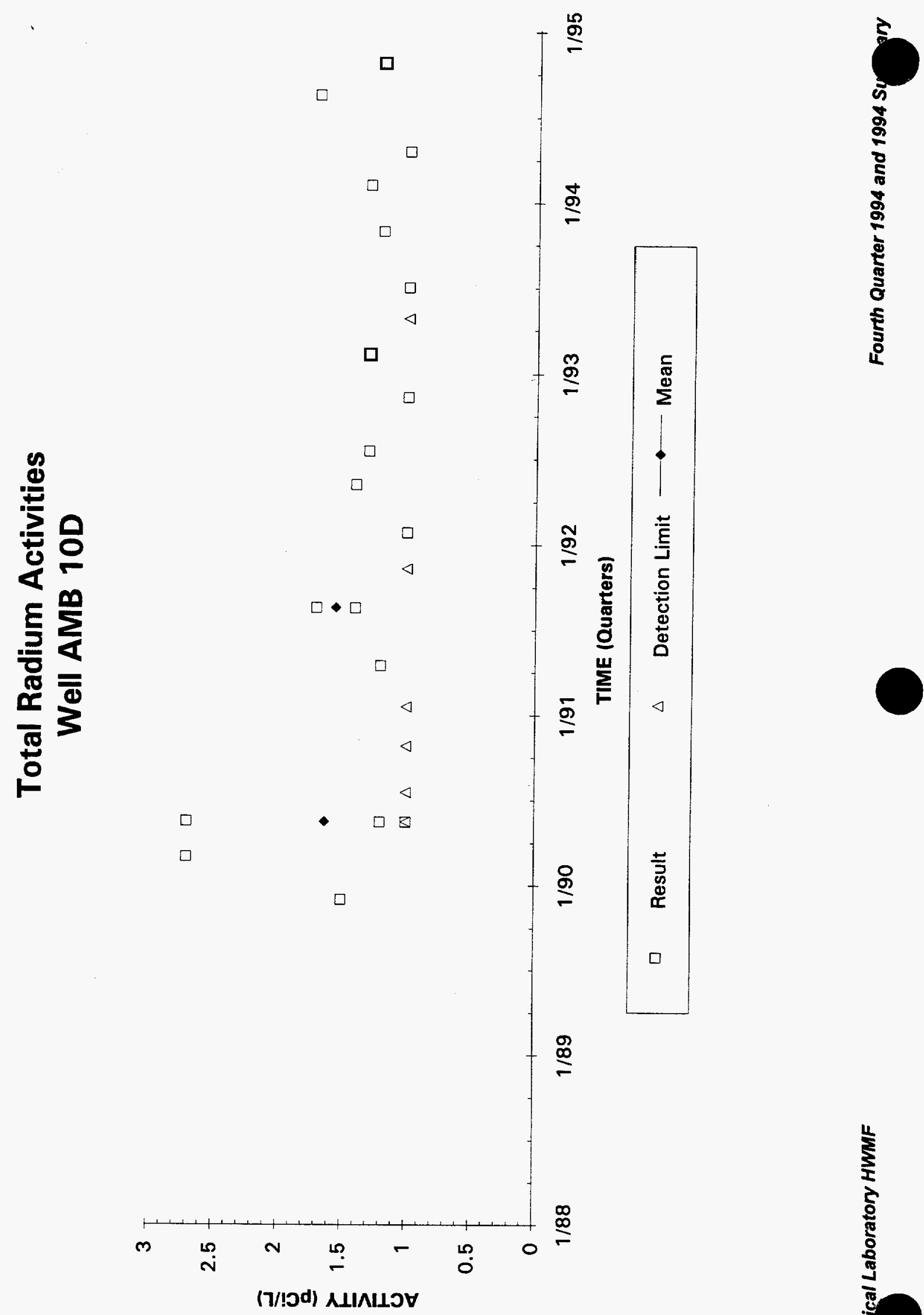

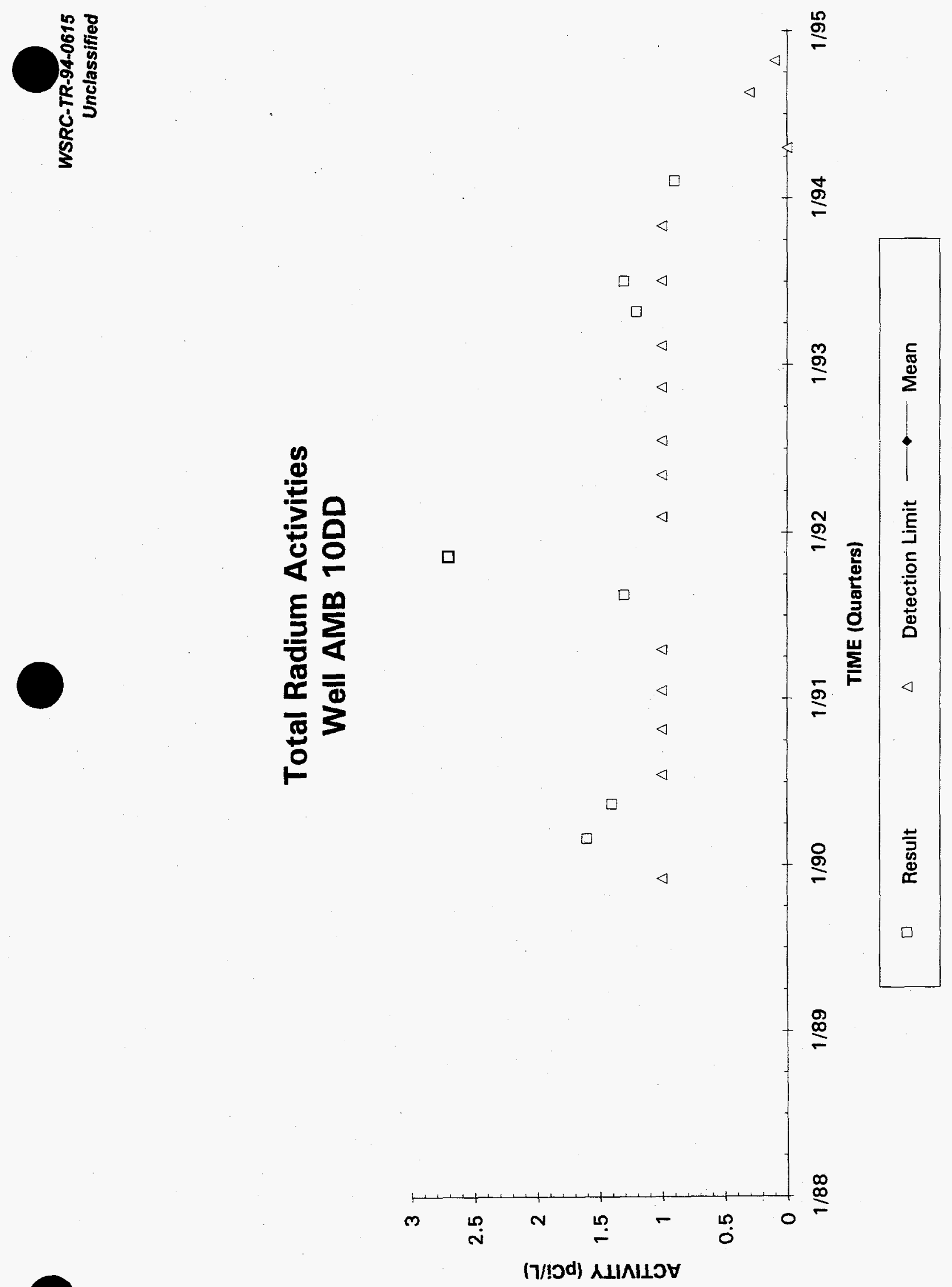

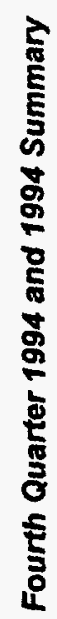

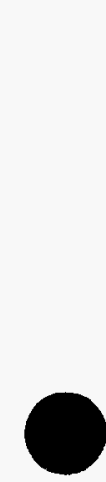




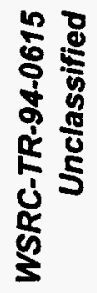
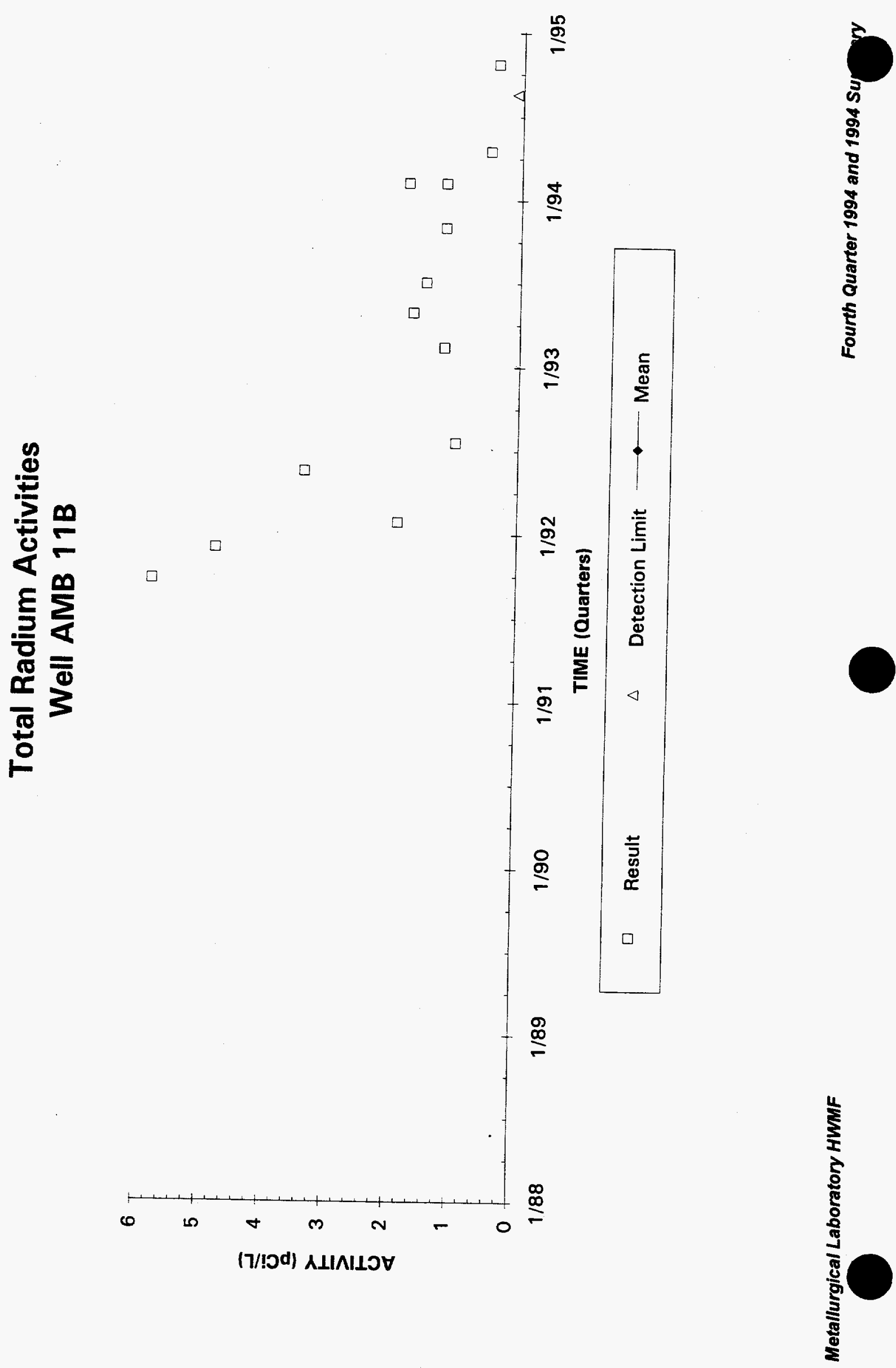

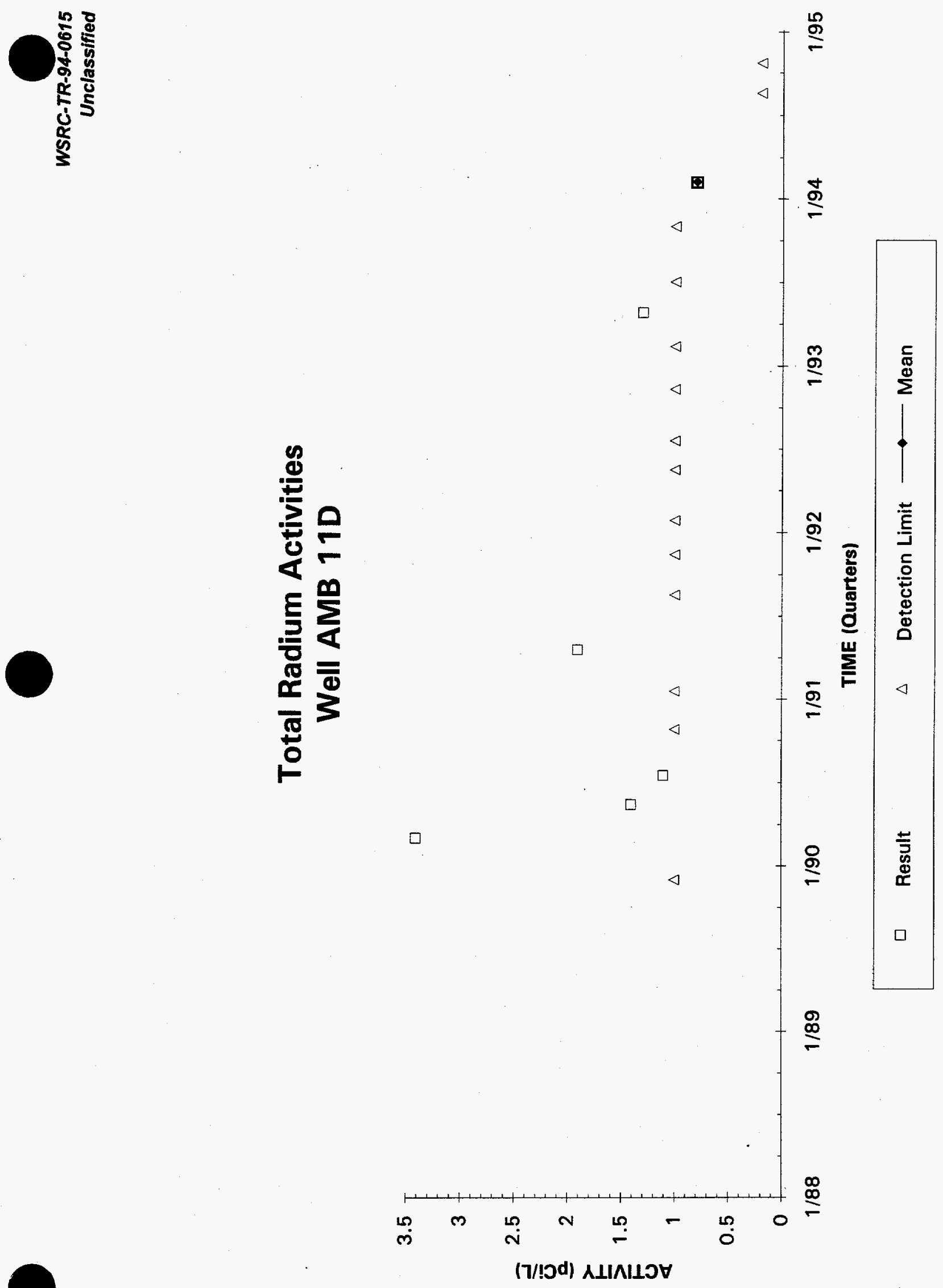

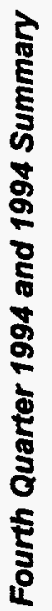
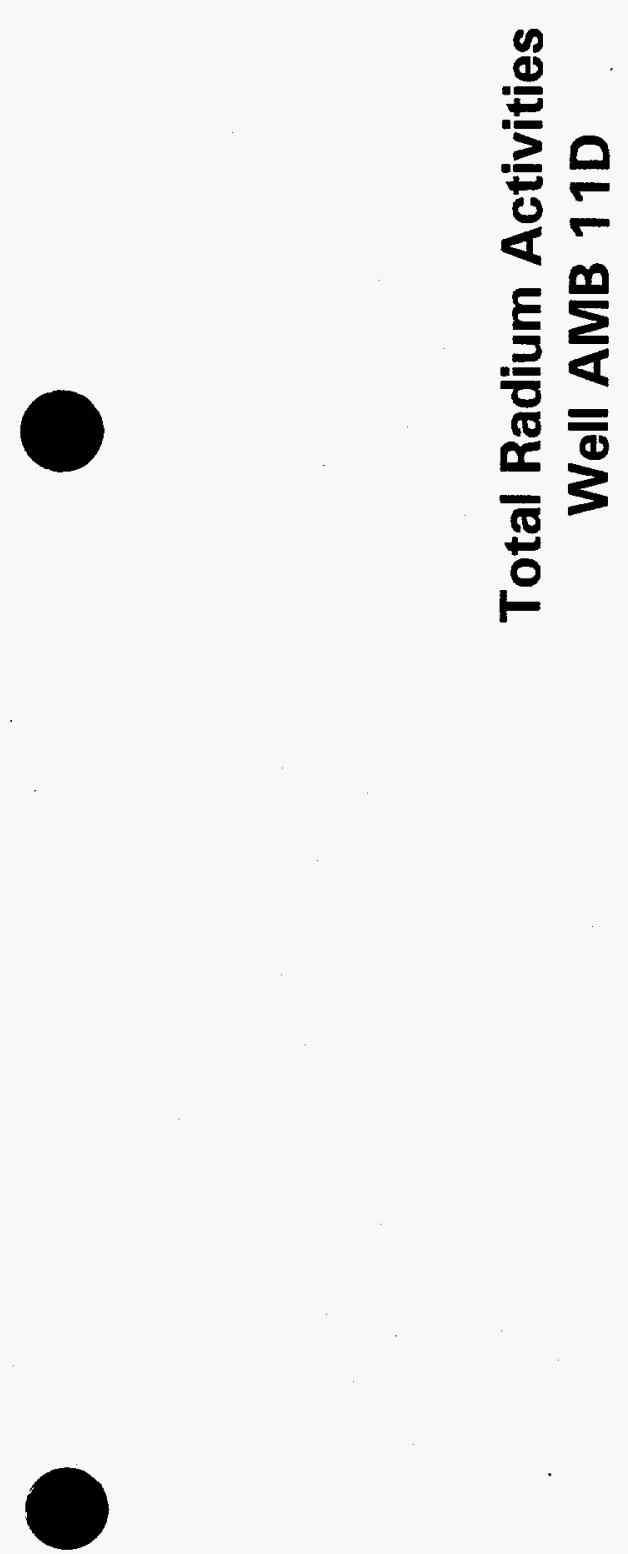

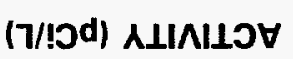




\section{Total Radium Activities}

Well AMB 12D

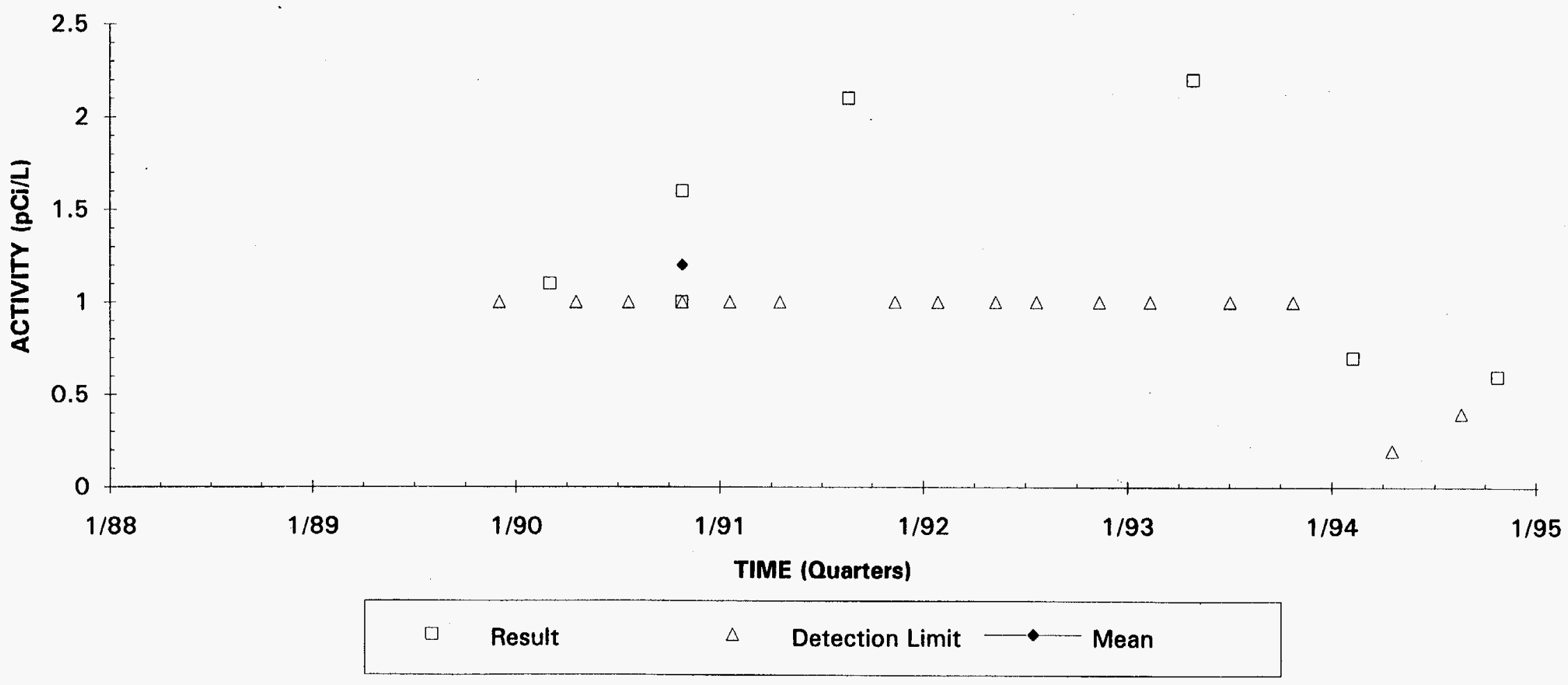



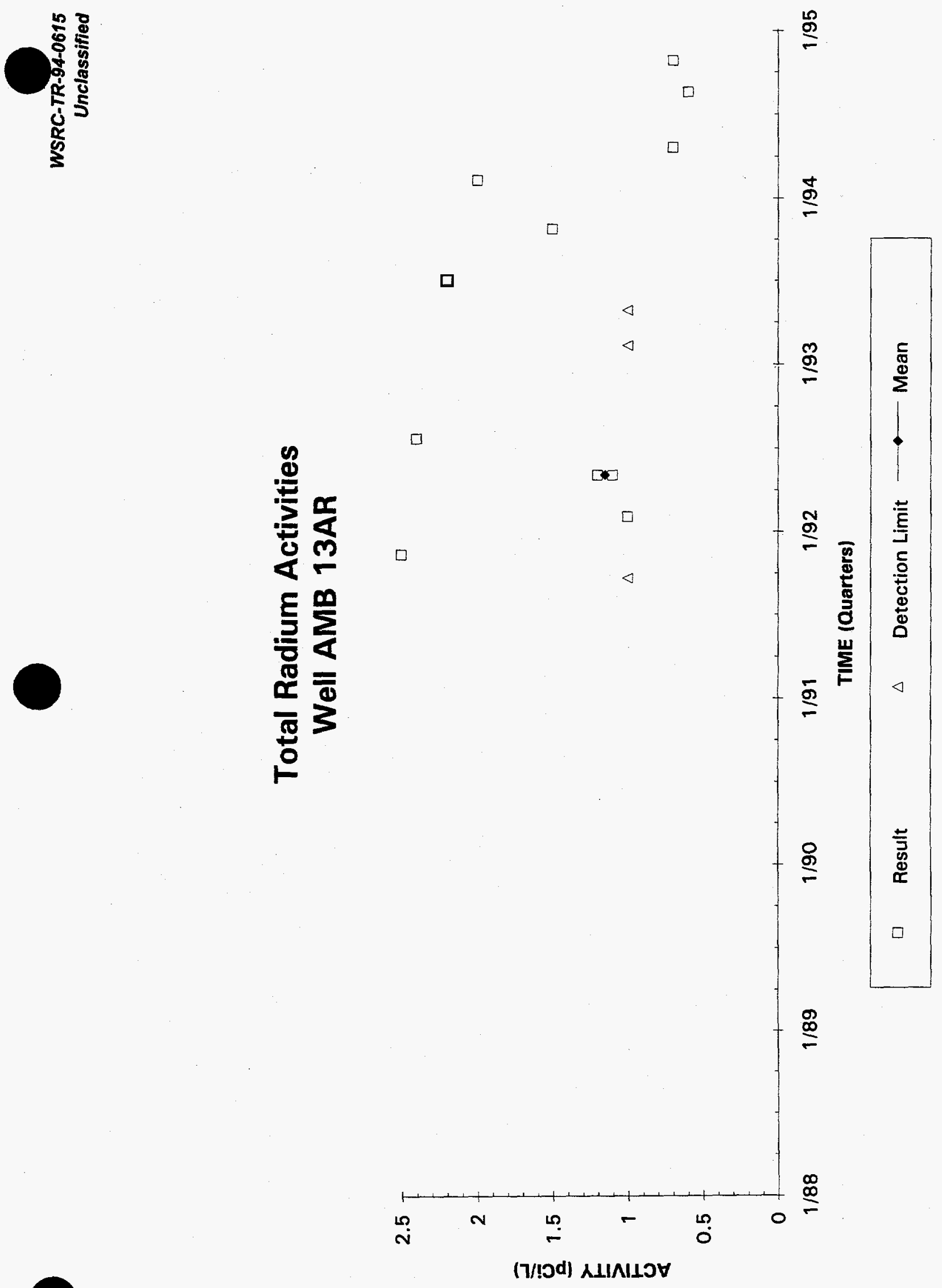

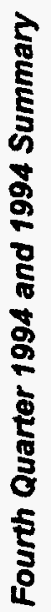

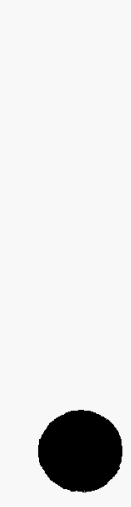




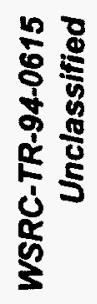

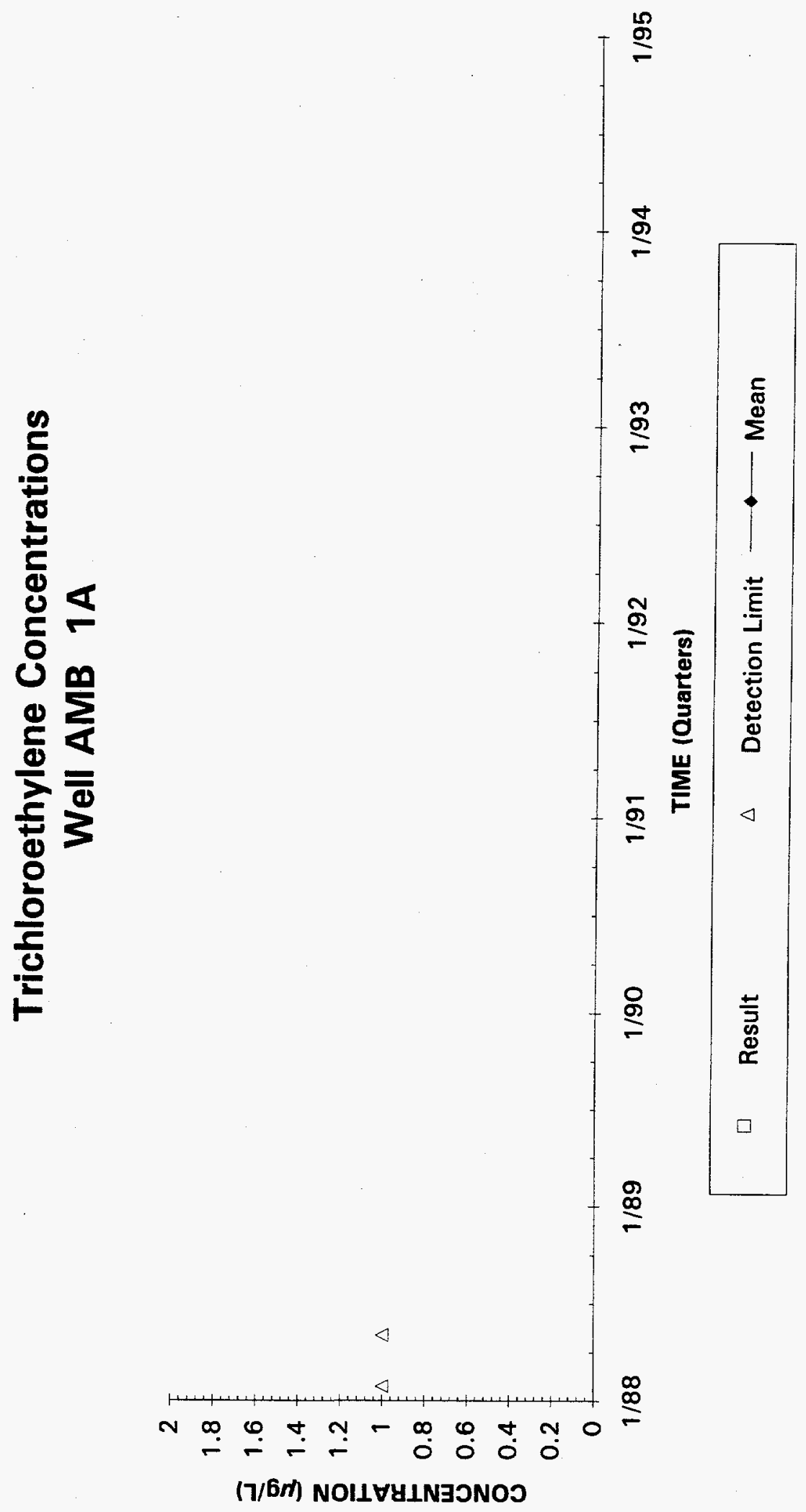

है

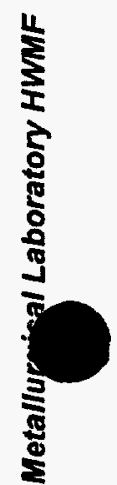


Trichloroethylene Concentrations

Well AMB 2

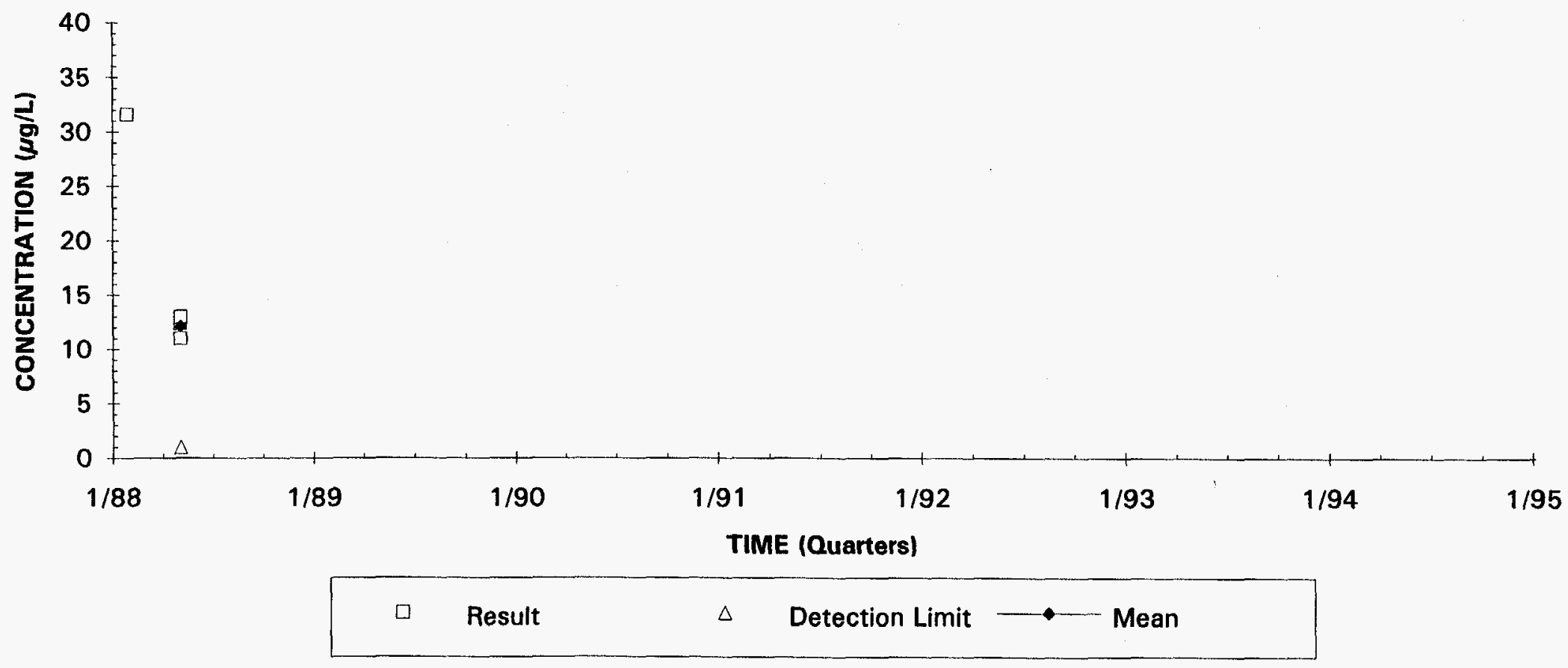



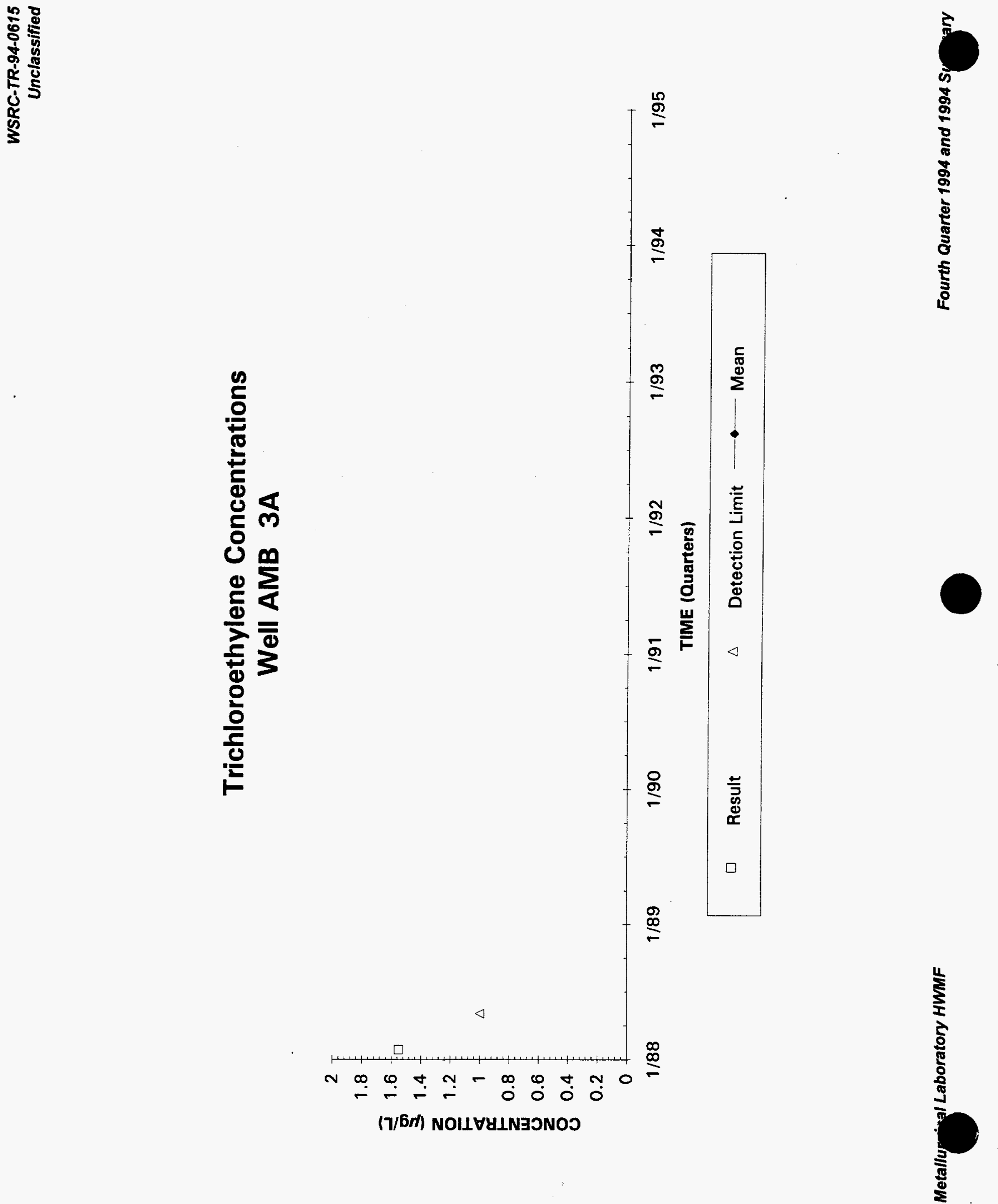


\section{Trichloroethylene Concentrations}

Well AMB 4

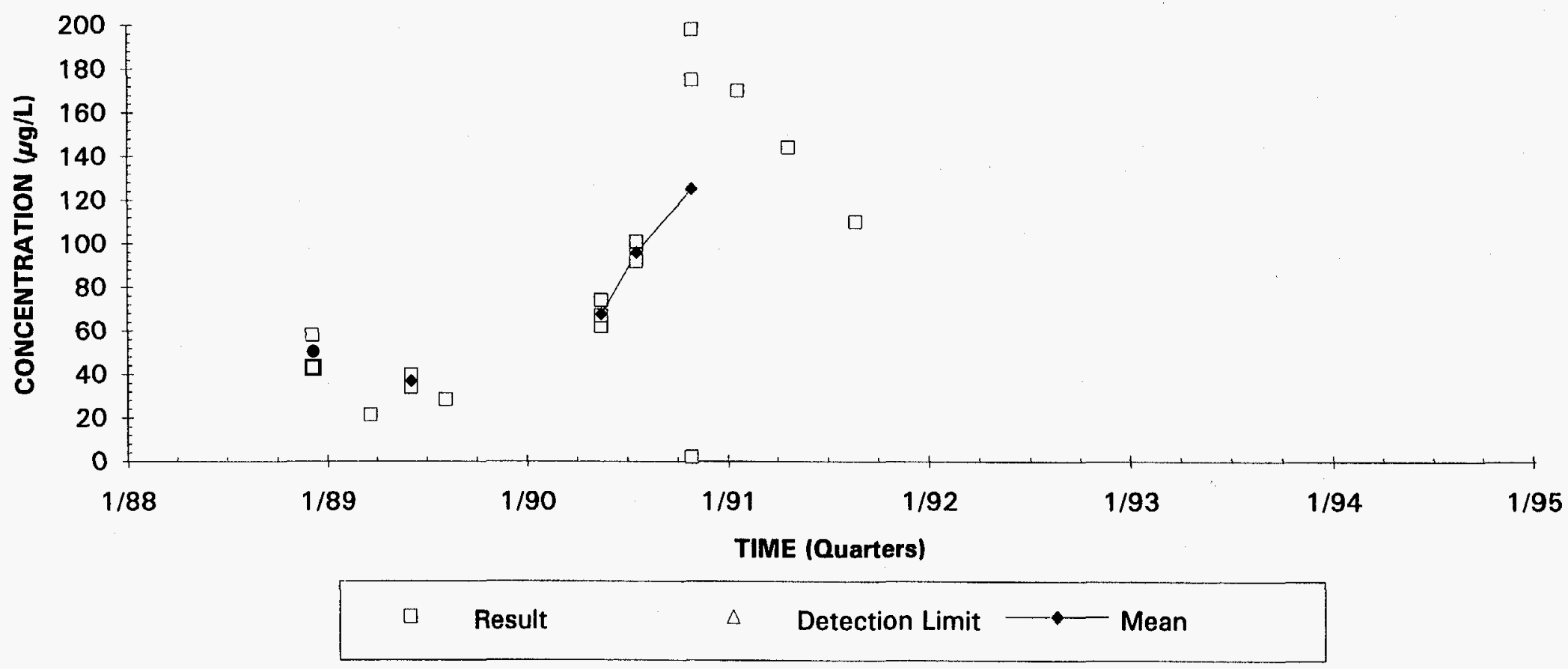




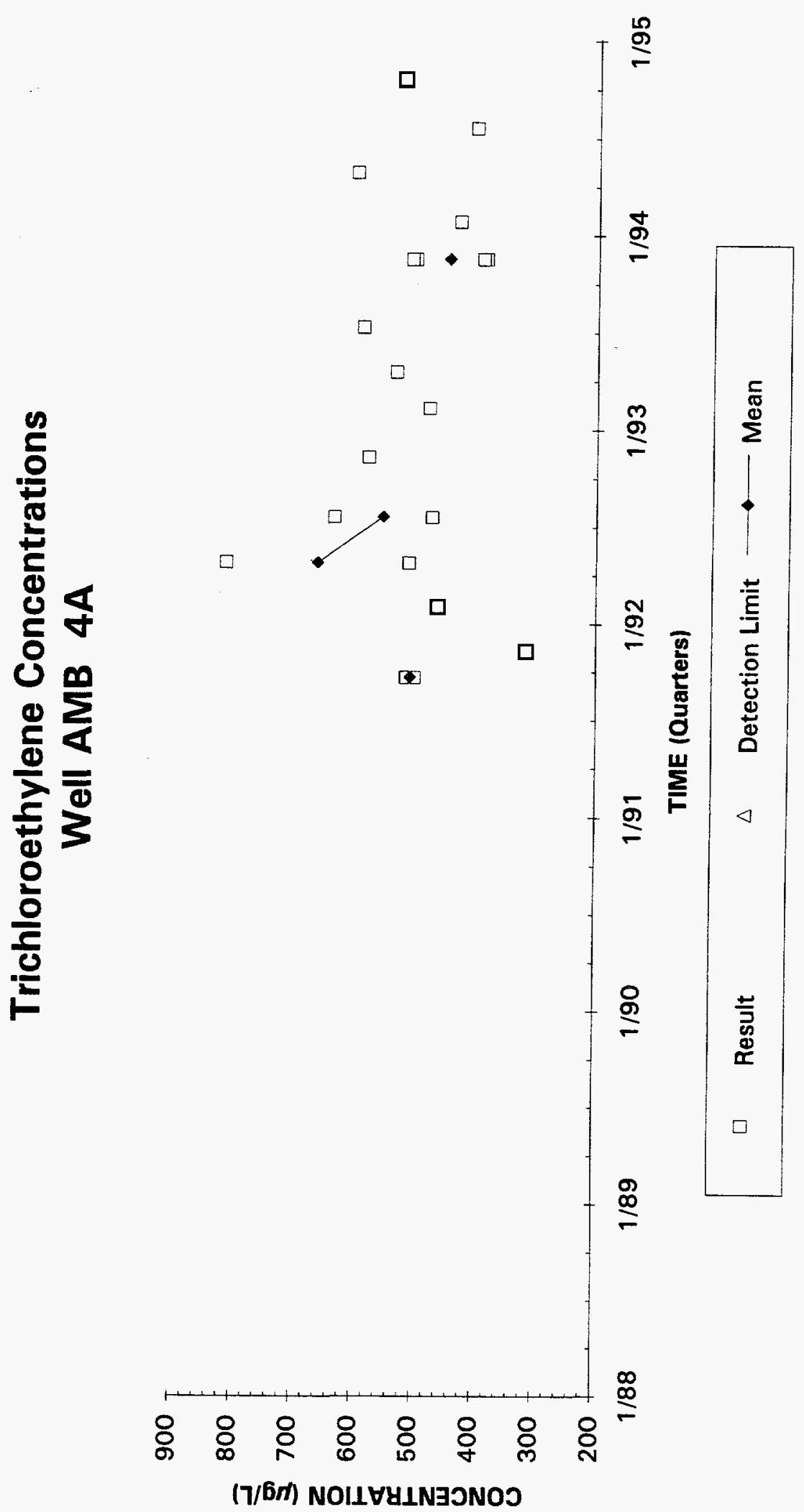

के 


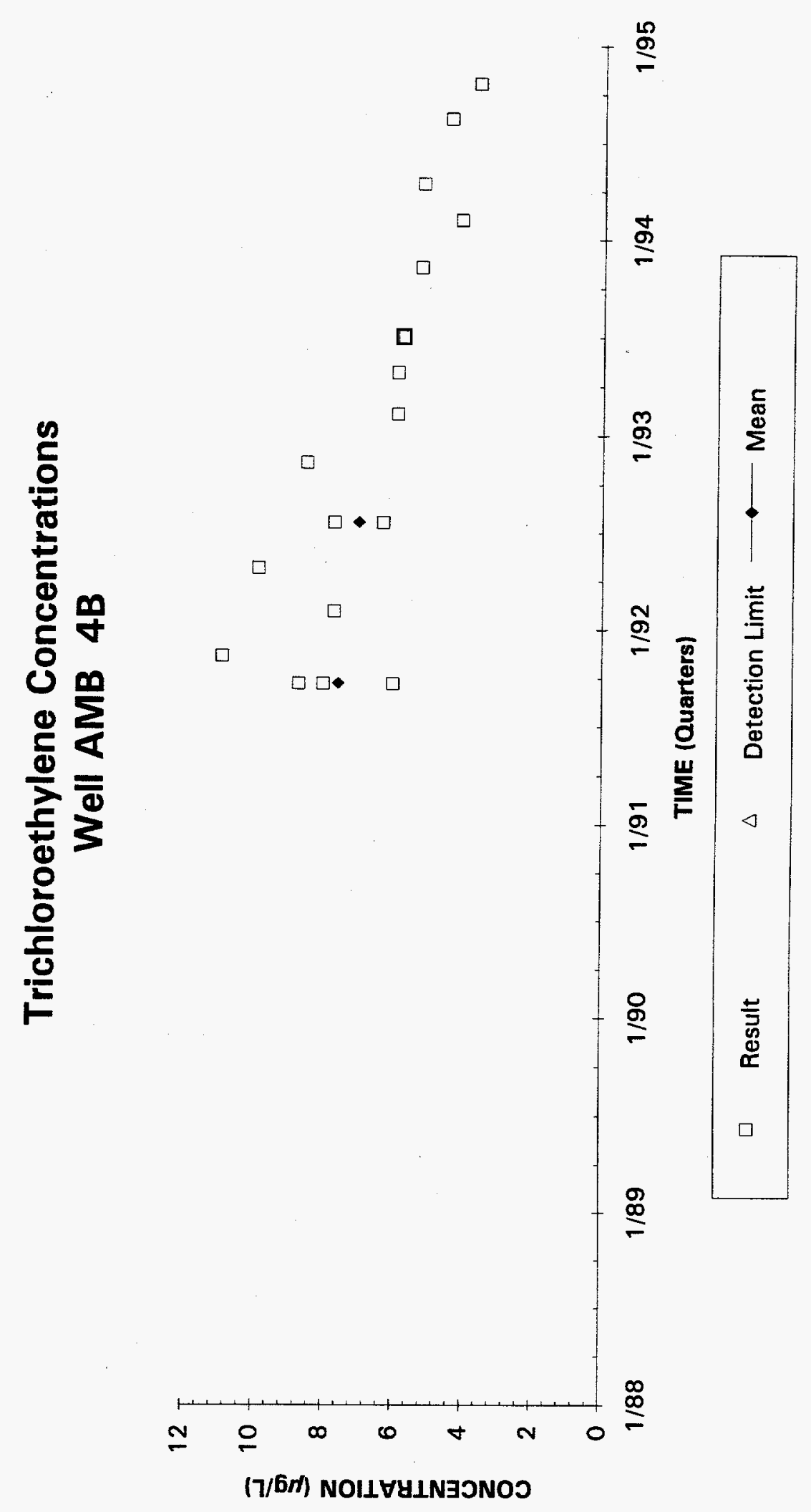




\section{Trichloroethylene Concentrations}

Well AMB 4D

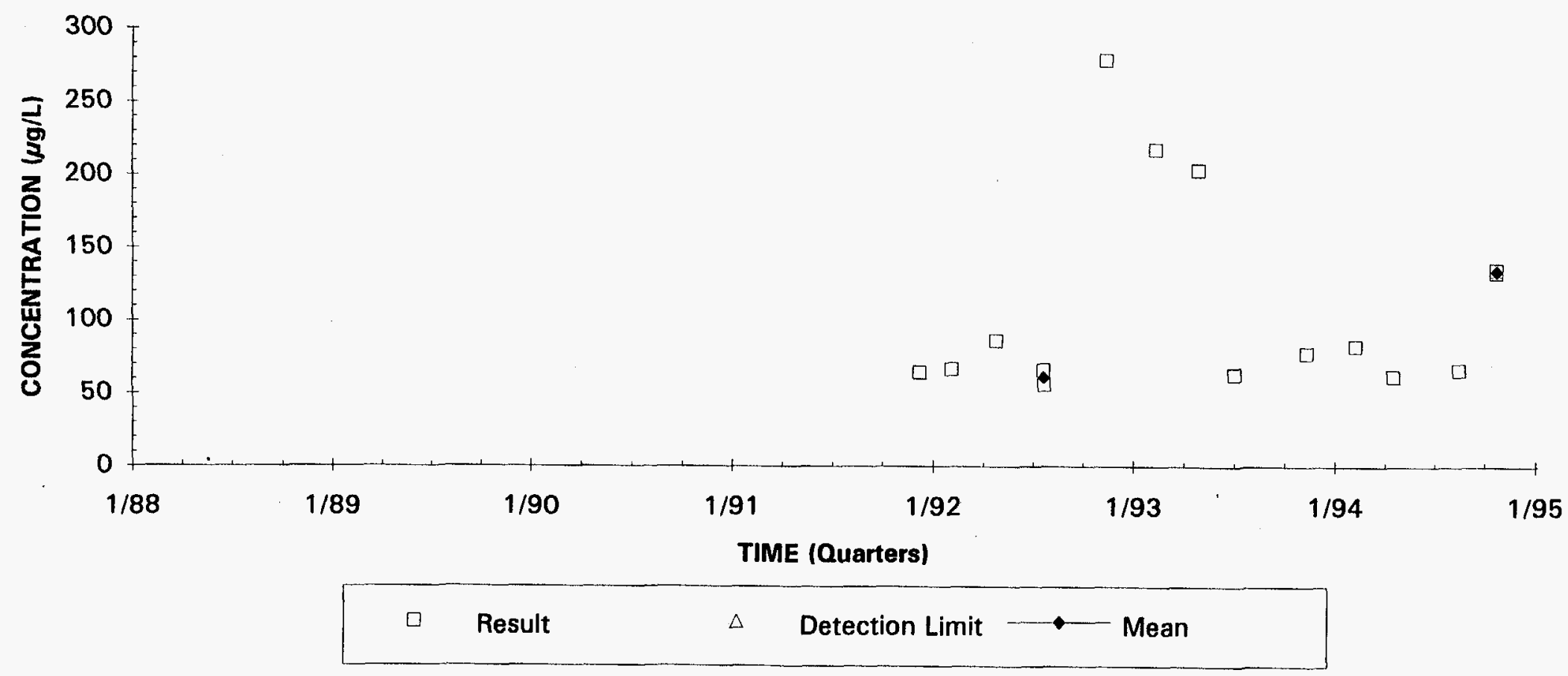




\section{Trichloroethylene Concentrations}

\section{Well AMB 5}

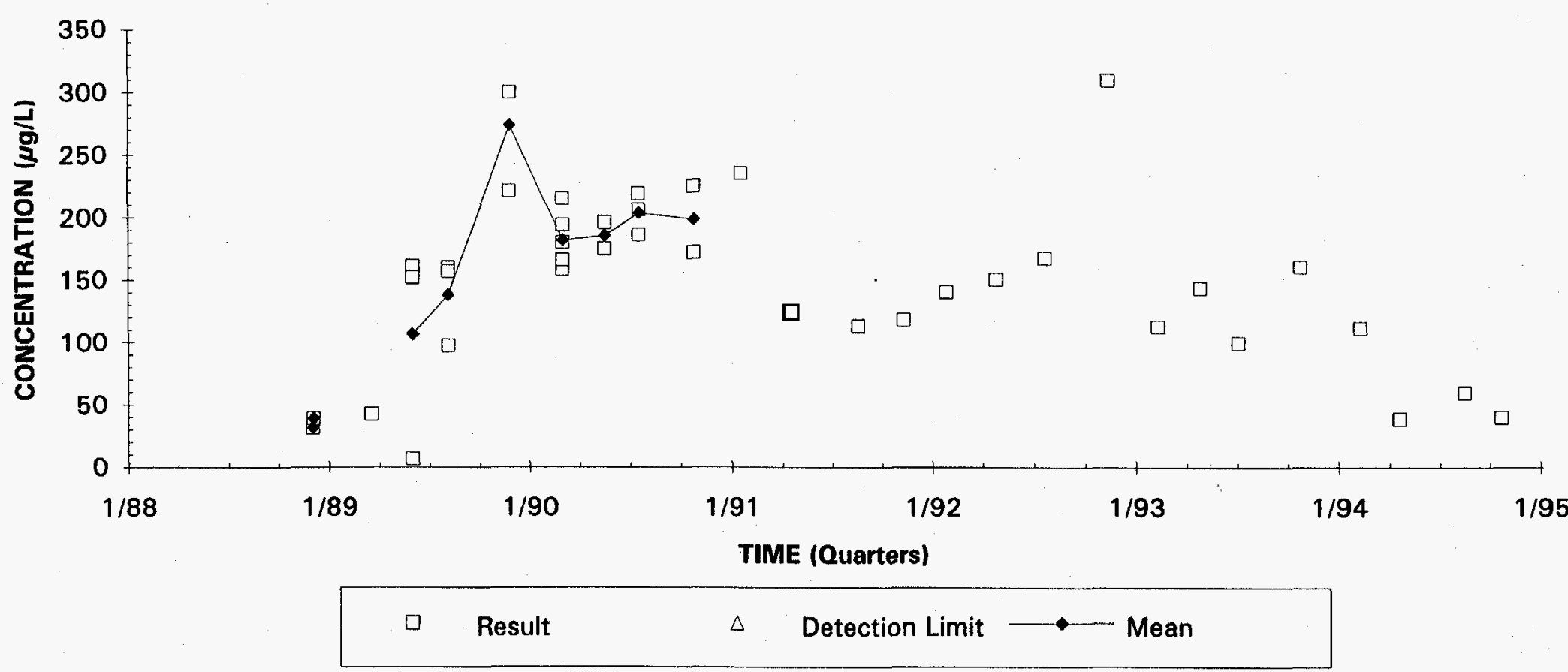



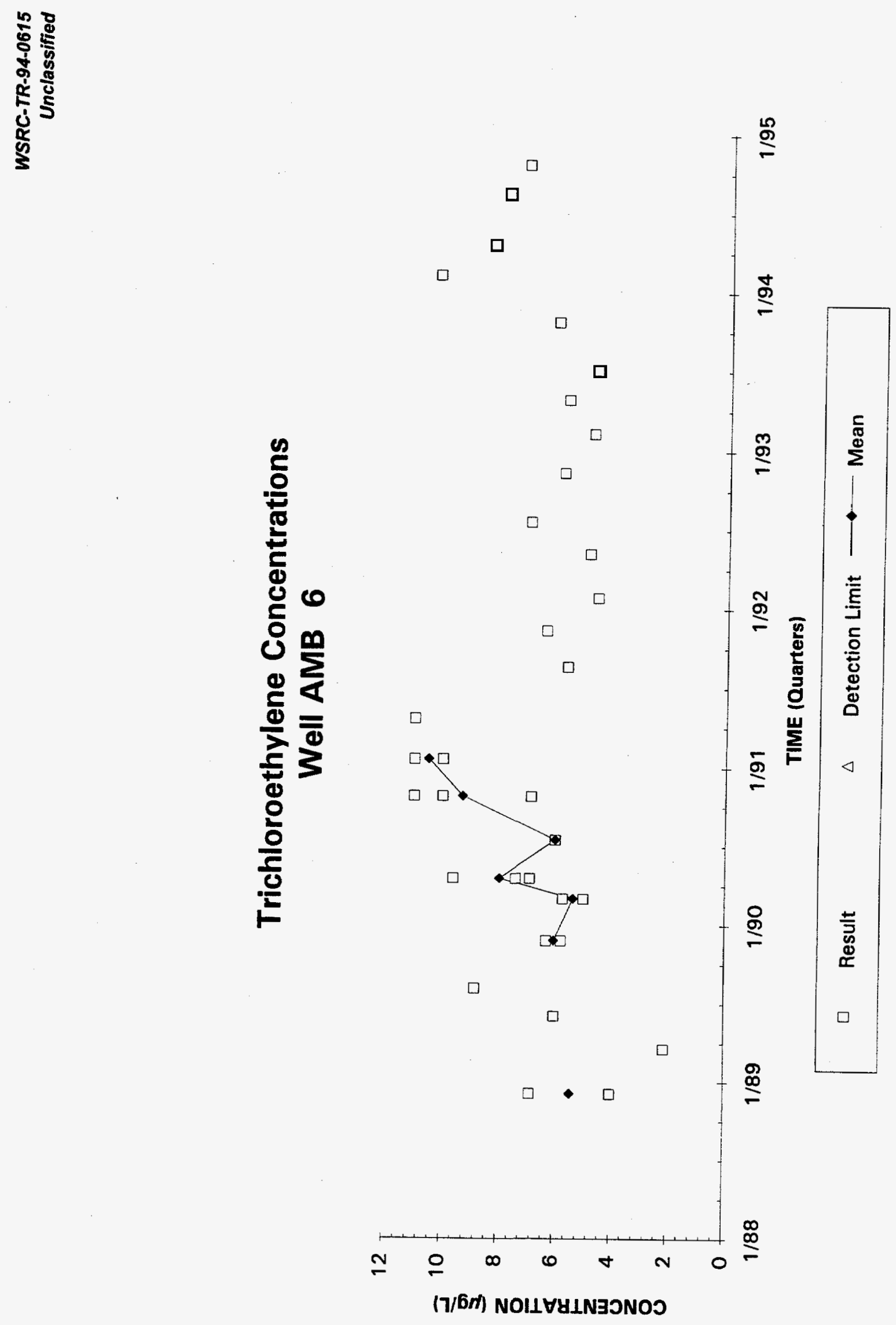


\section{Trichloroethylene Concentrations}

Well AMB 7

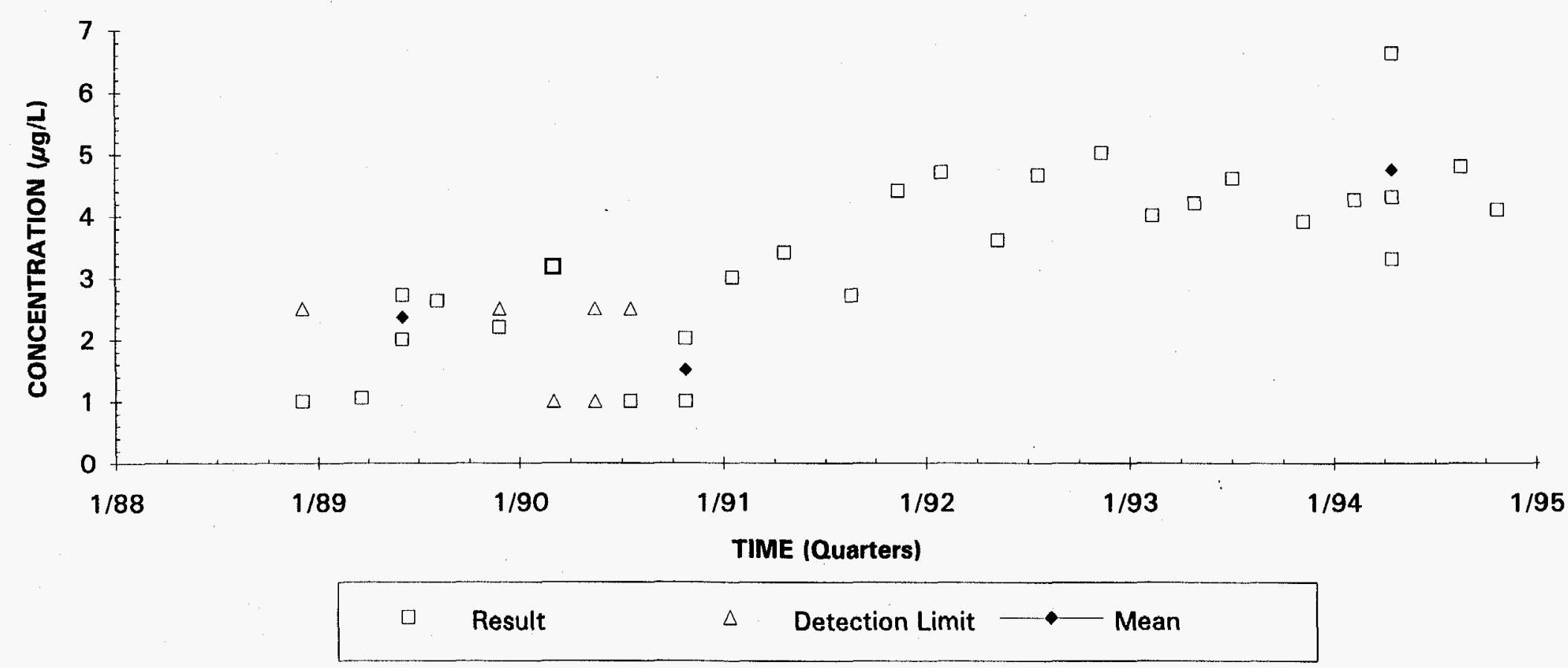




\section{Trichloroethylene Concentrations}

Well AMB 7A

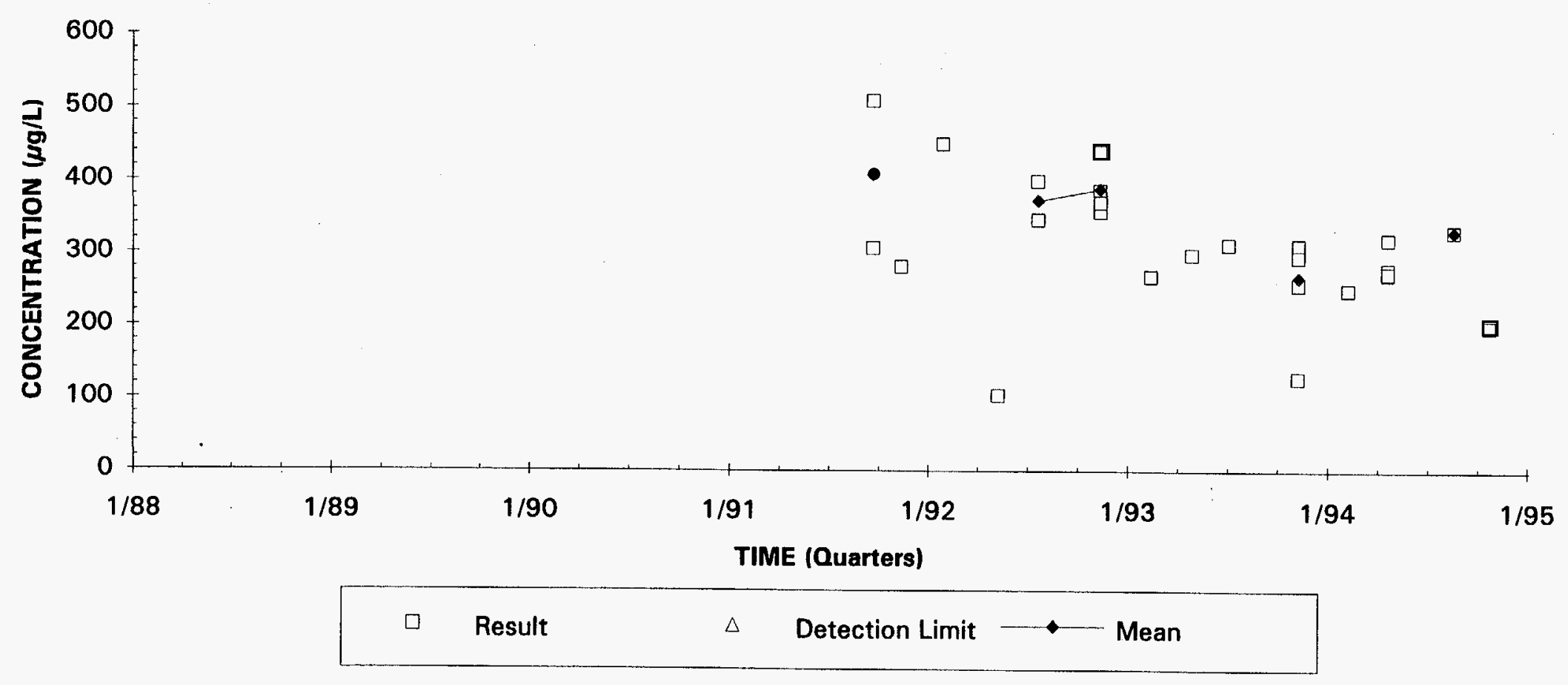




\section{Trichloroethylene Concentrations}

Well AMB 7B

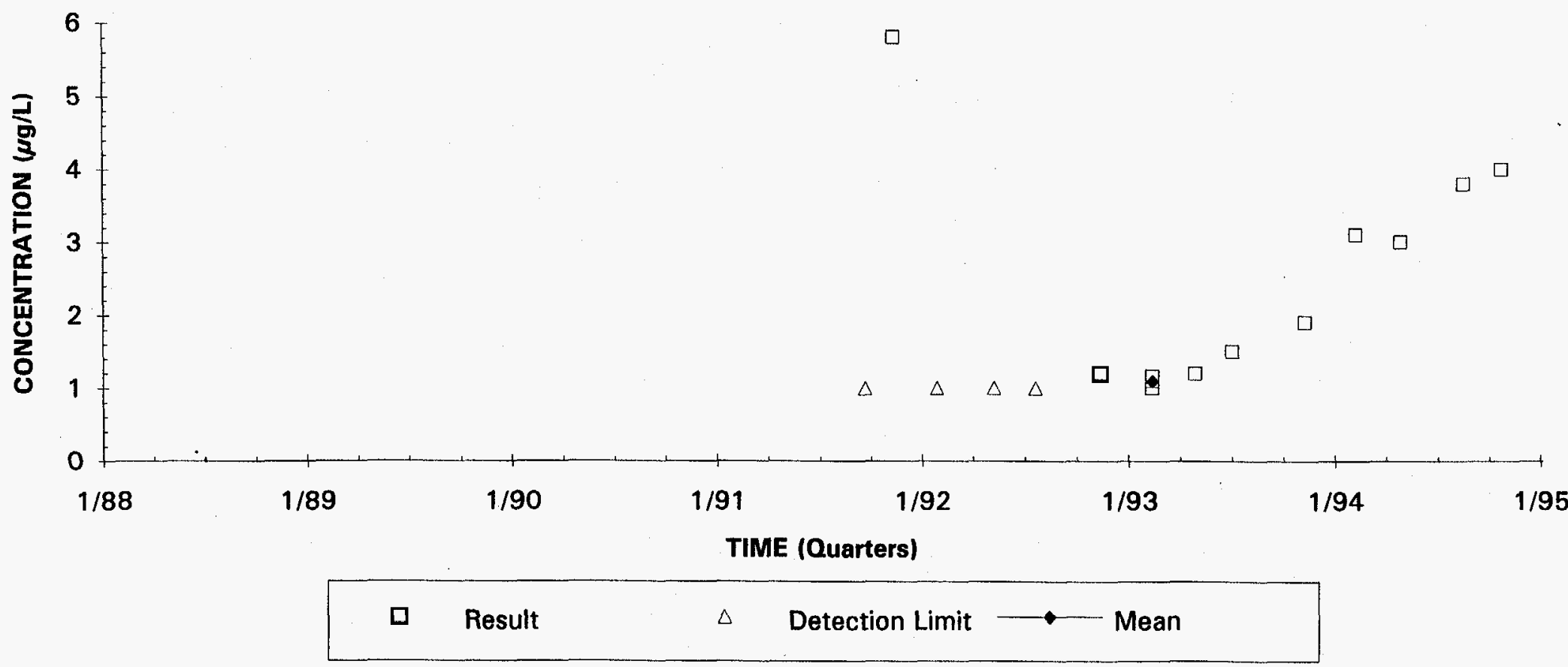



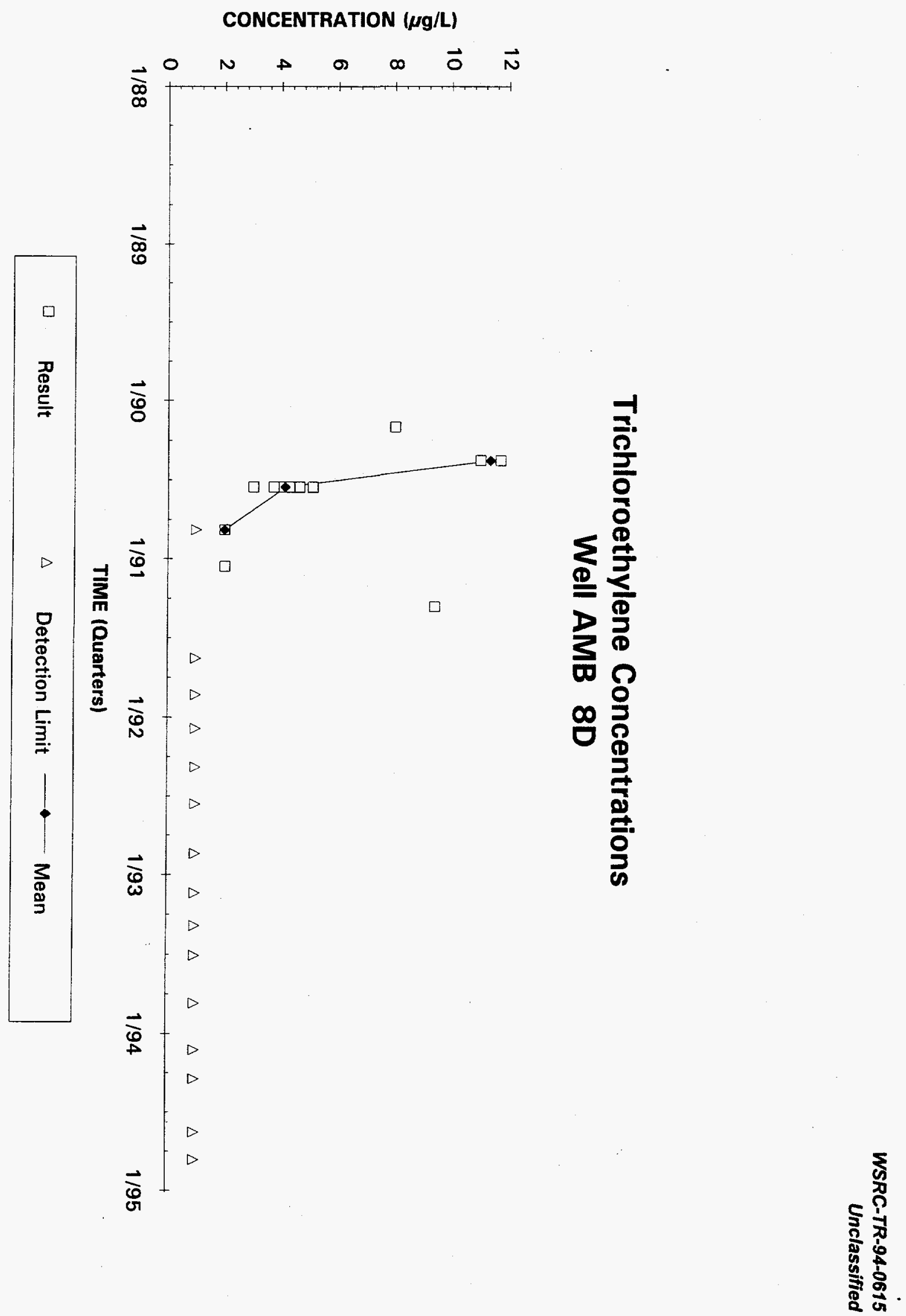

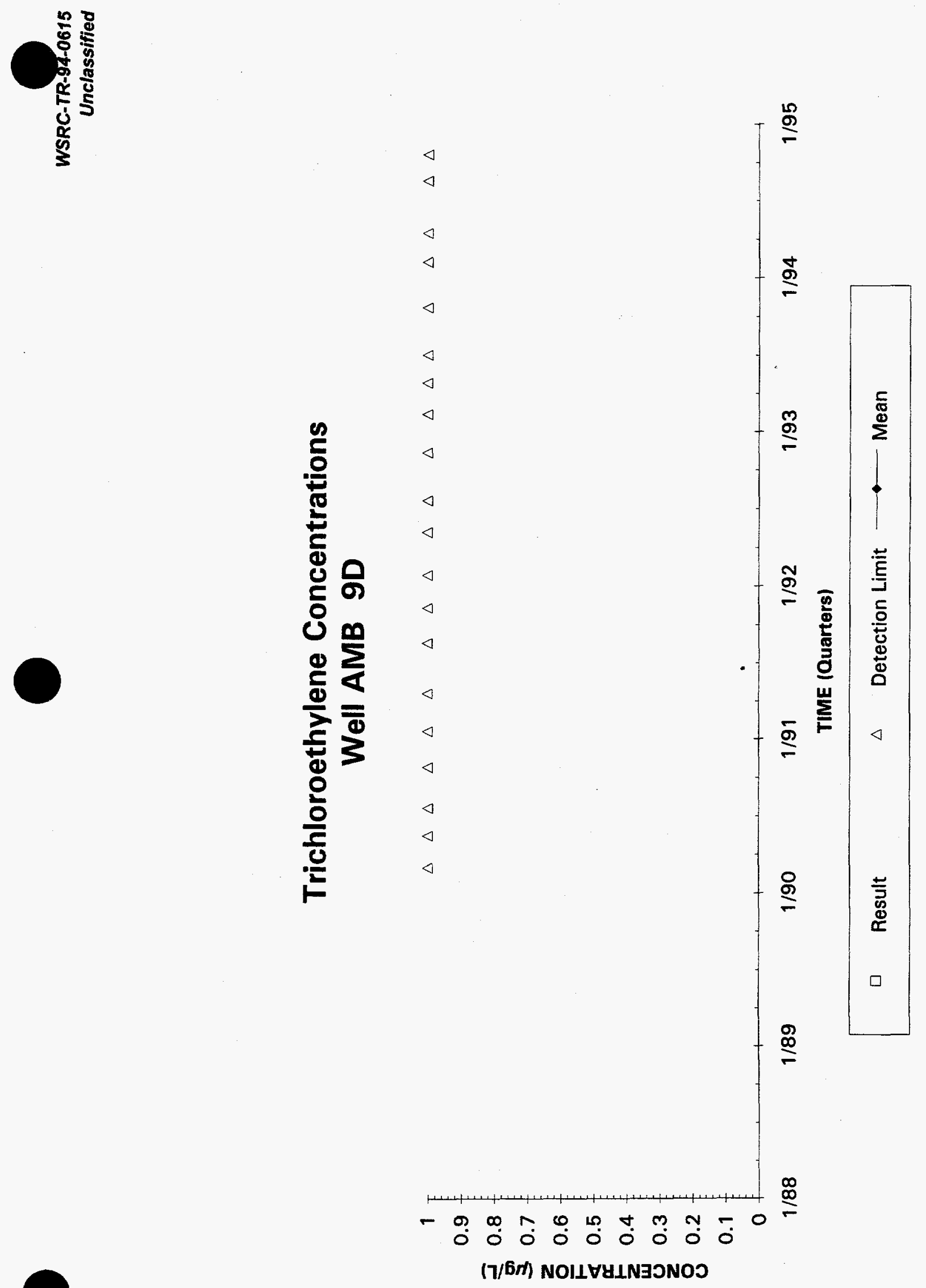


\section{Trichloroethylene Concentrations}

\section{Well AMB 10A}

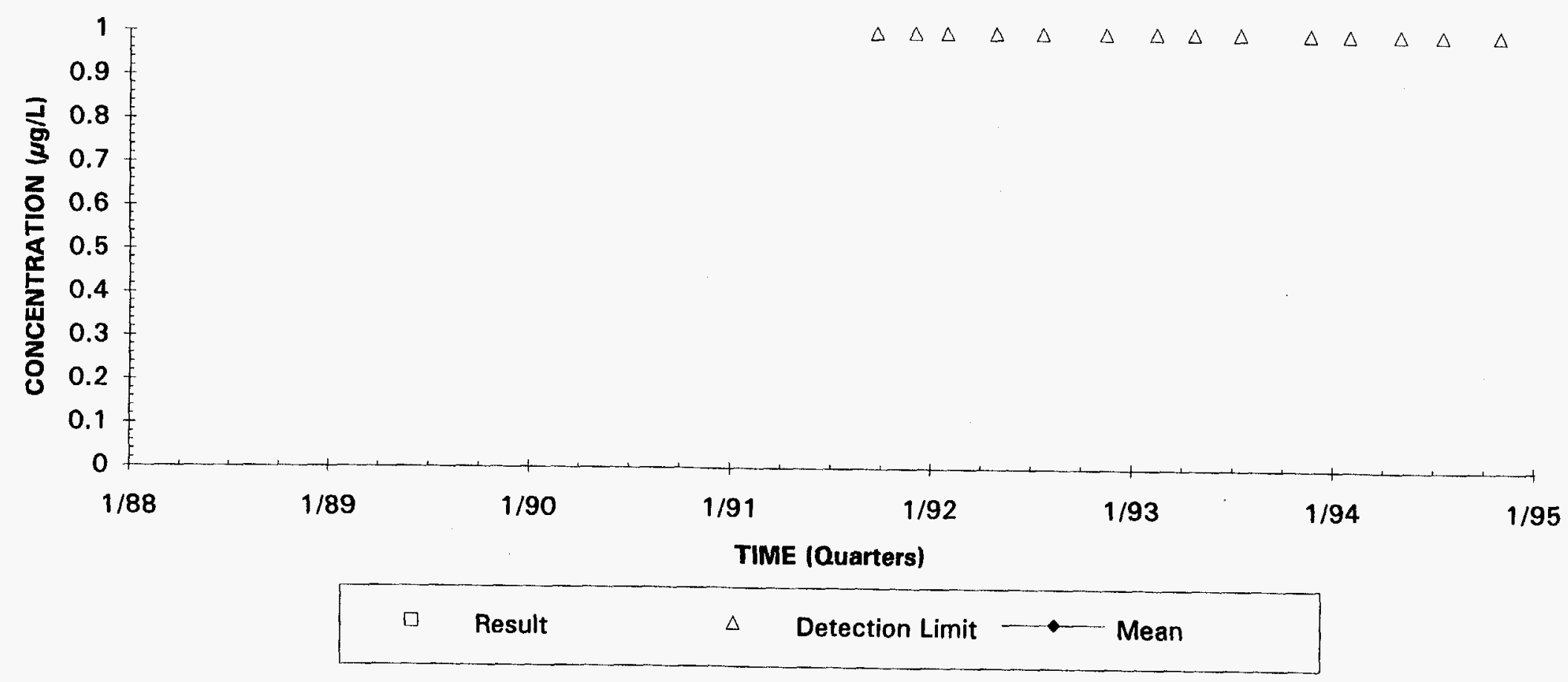



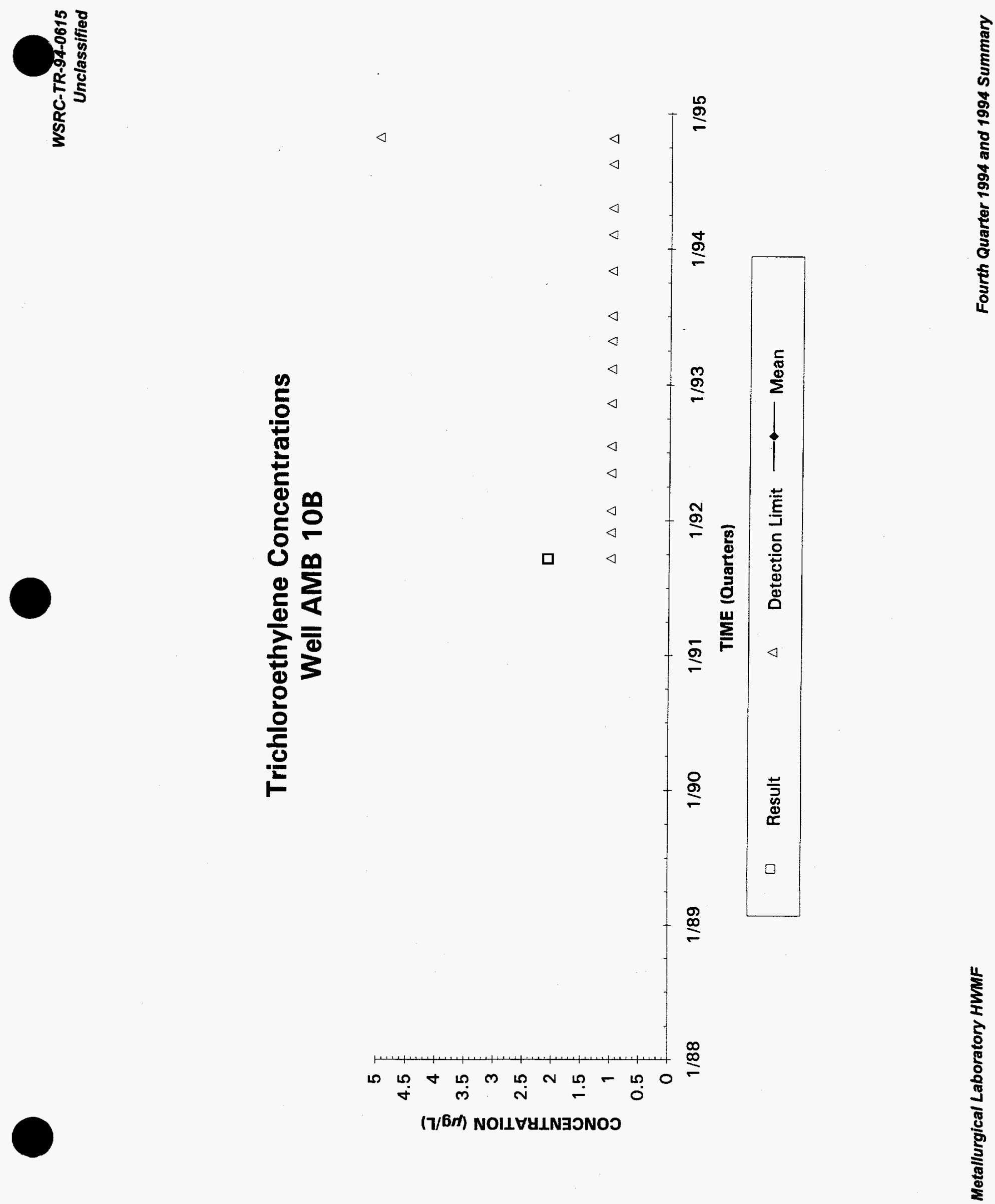


\section{Trichloroethylene Concentrations}

\section{Well AMB 10D}

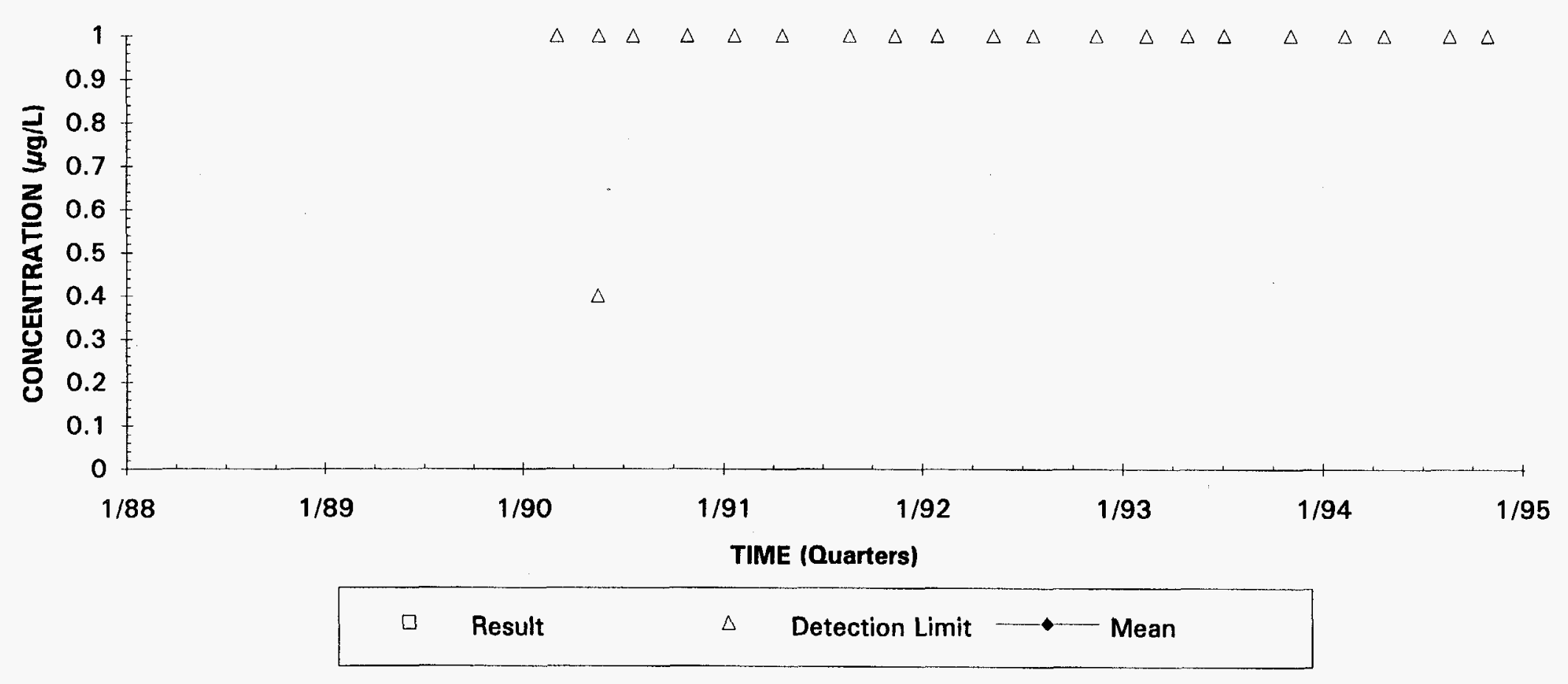



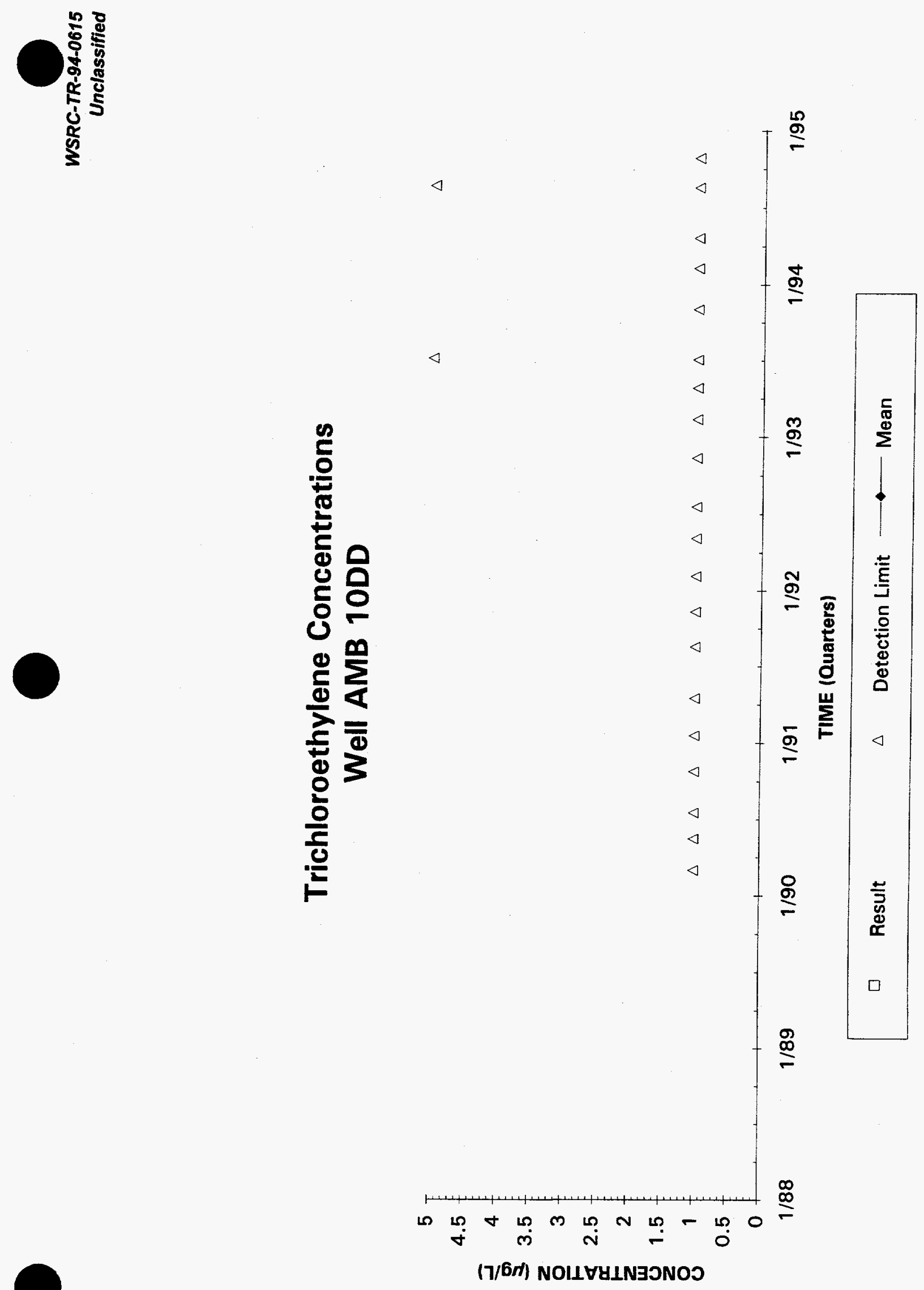


\section{Trichloroethylene Concentrations}

\section{Well AMB 11B}

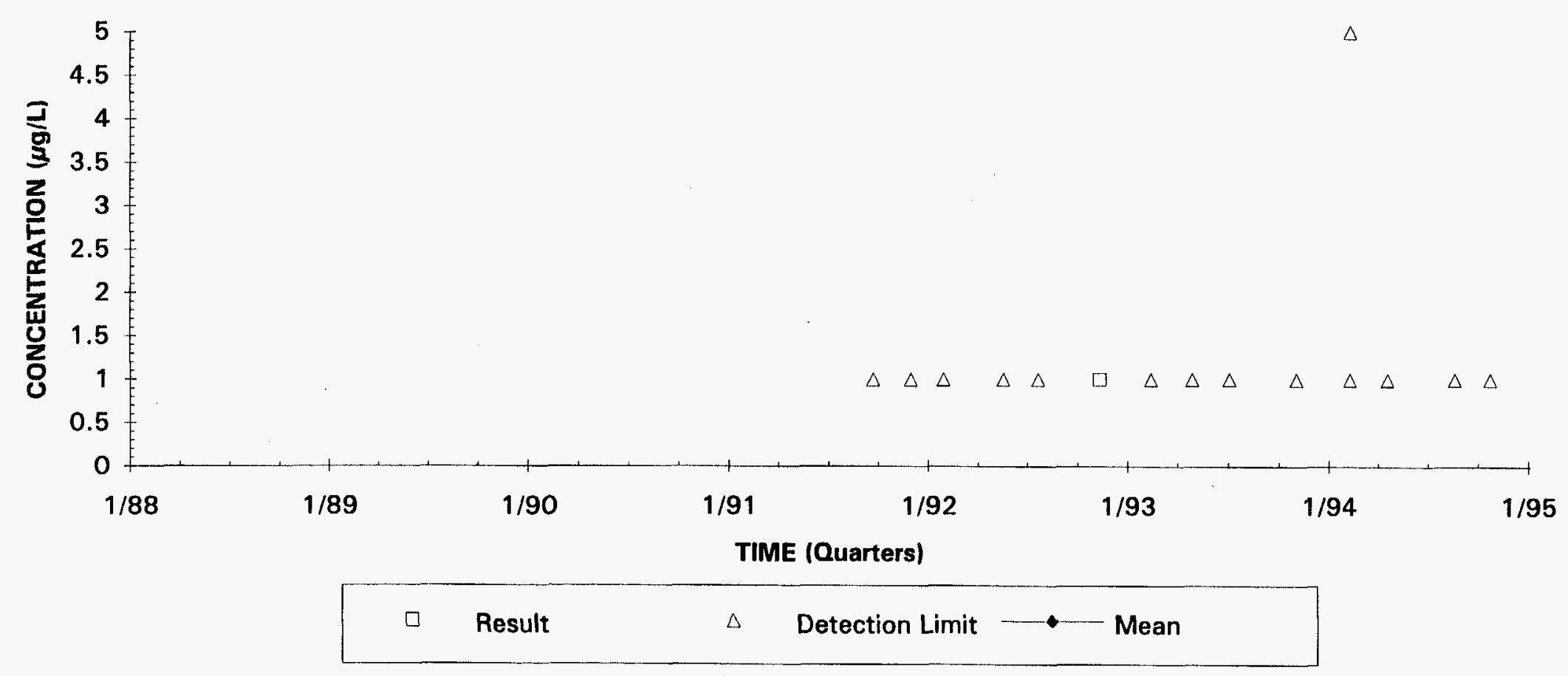


בั.
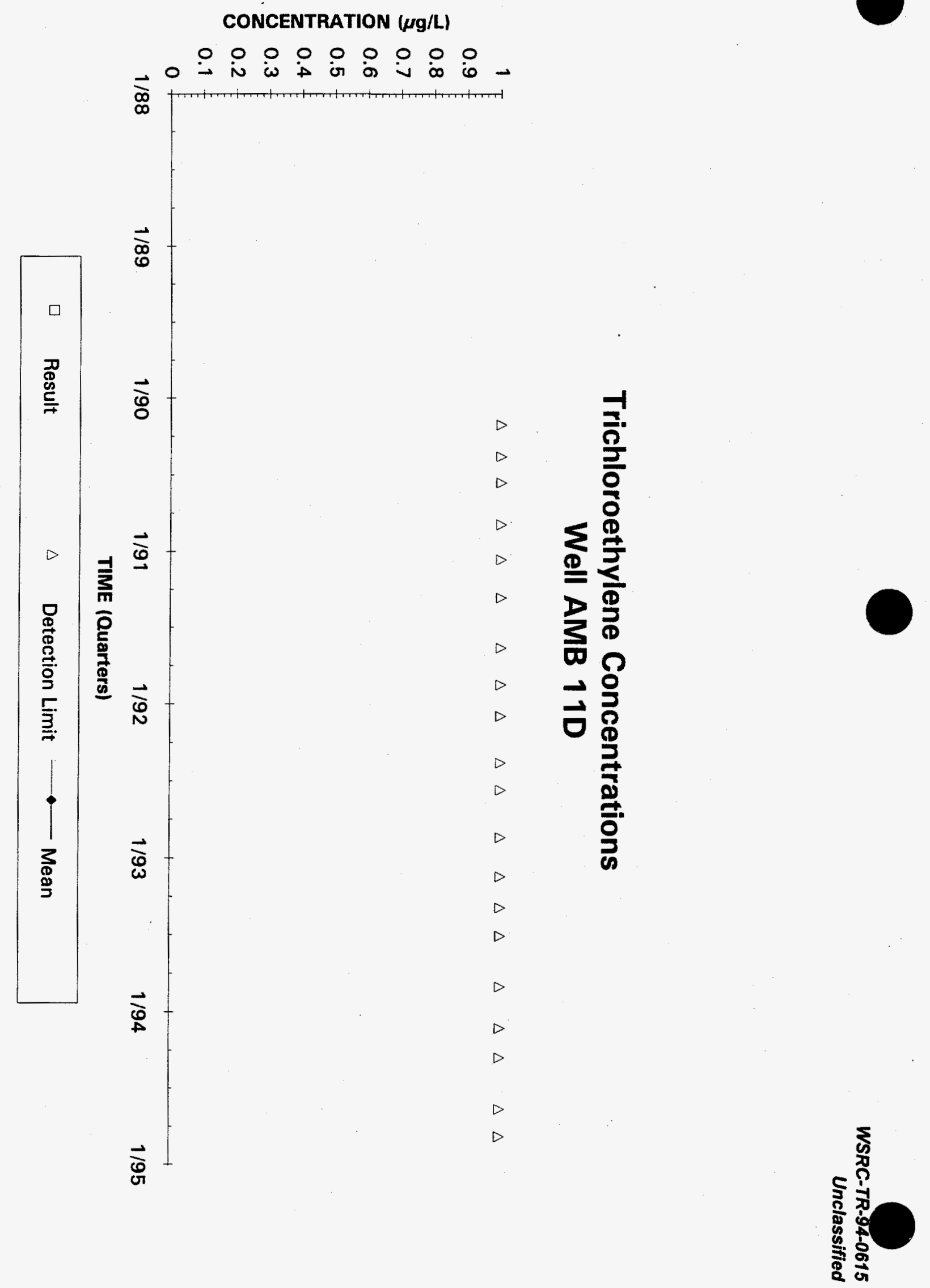


\section{Trichloroethylene Concentrations \\ Well AMB 12D}

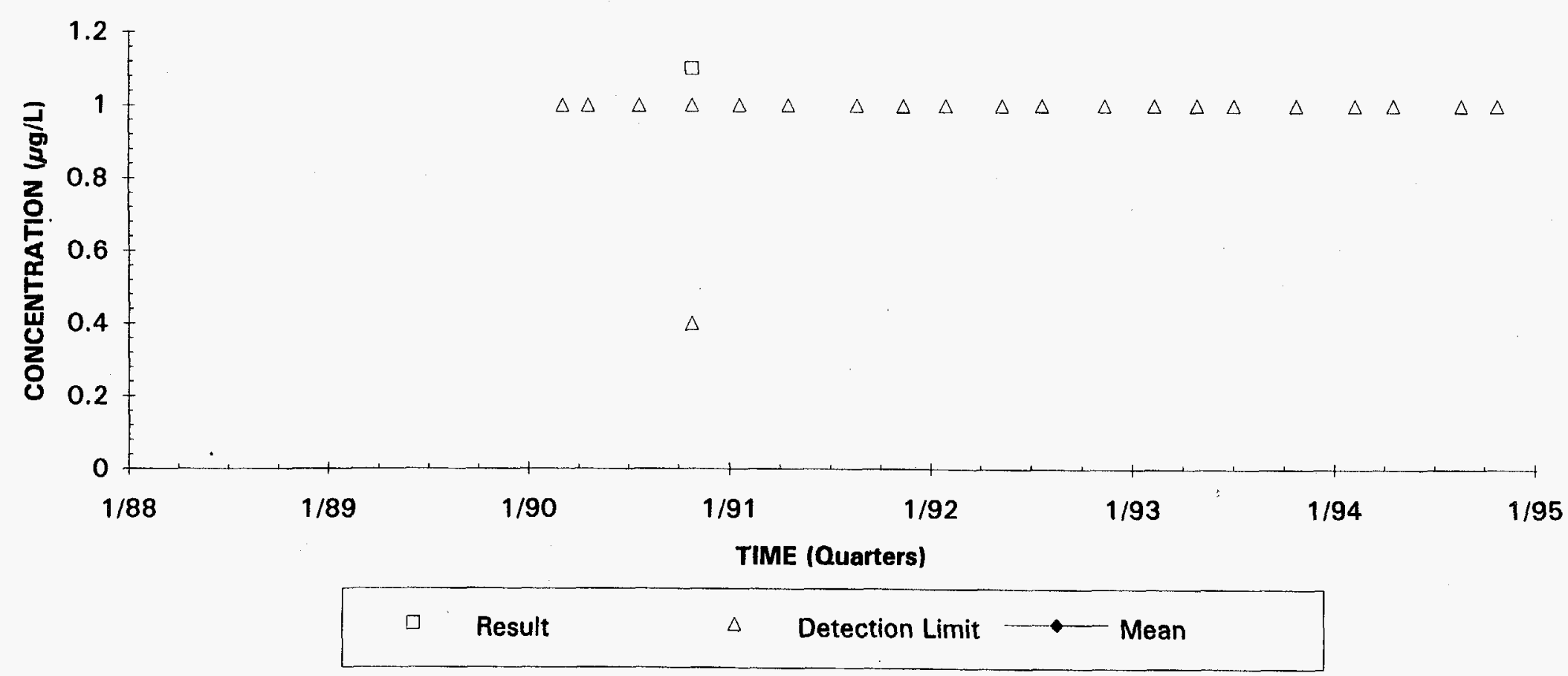



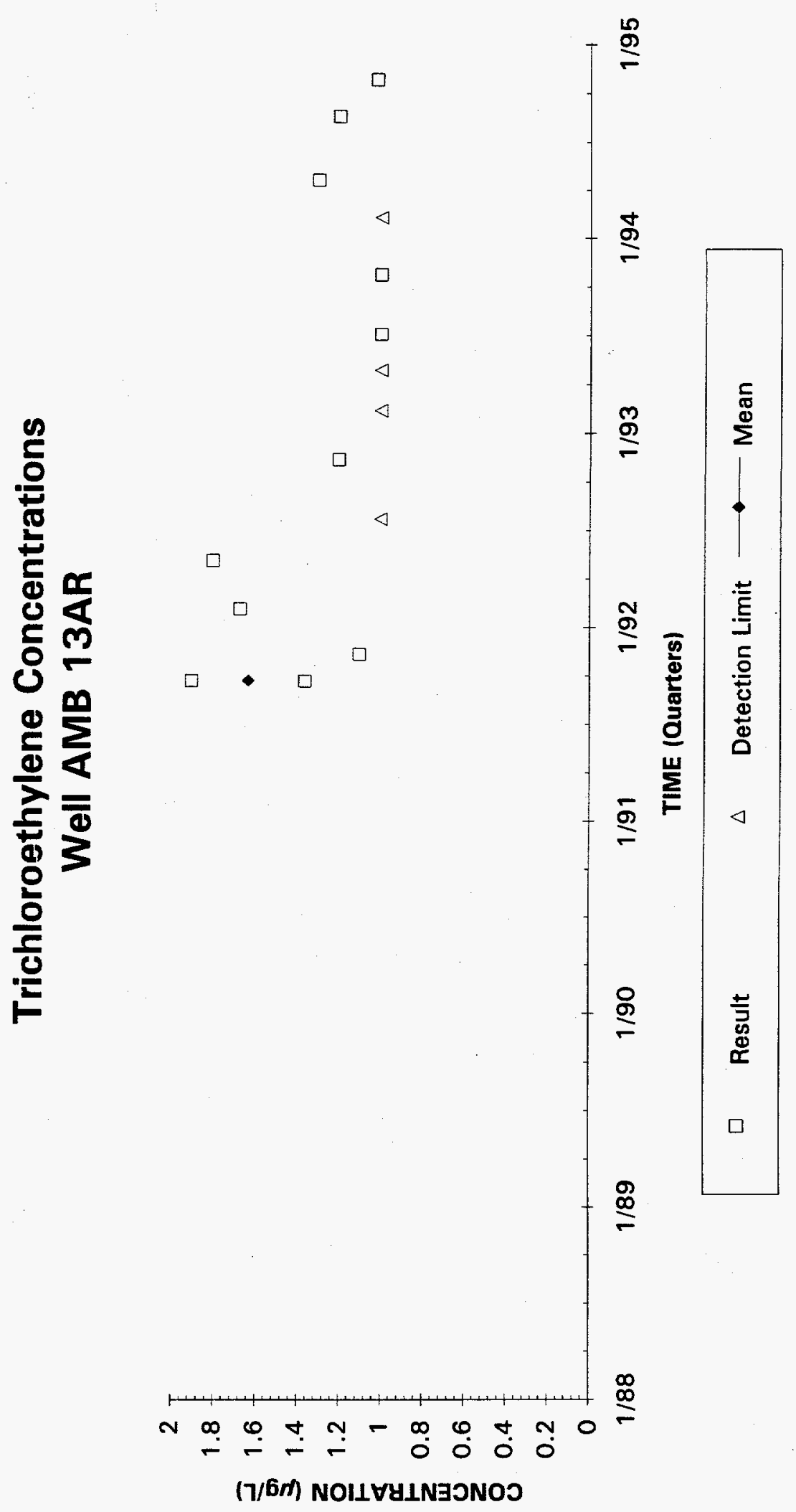
WSRC-TR-94-0615 Unclassified

THIS PAGE LEFT BLANK INTENTIONALLY. 
WSRC-TR-94-0615 Unclassified

\section{Appendix G}

\section{Hydrographs}


THIS PAGE LEFT BLANK INTENTIONALLY. 


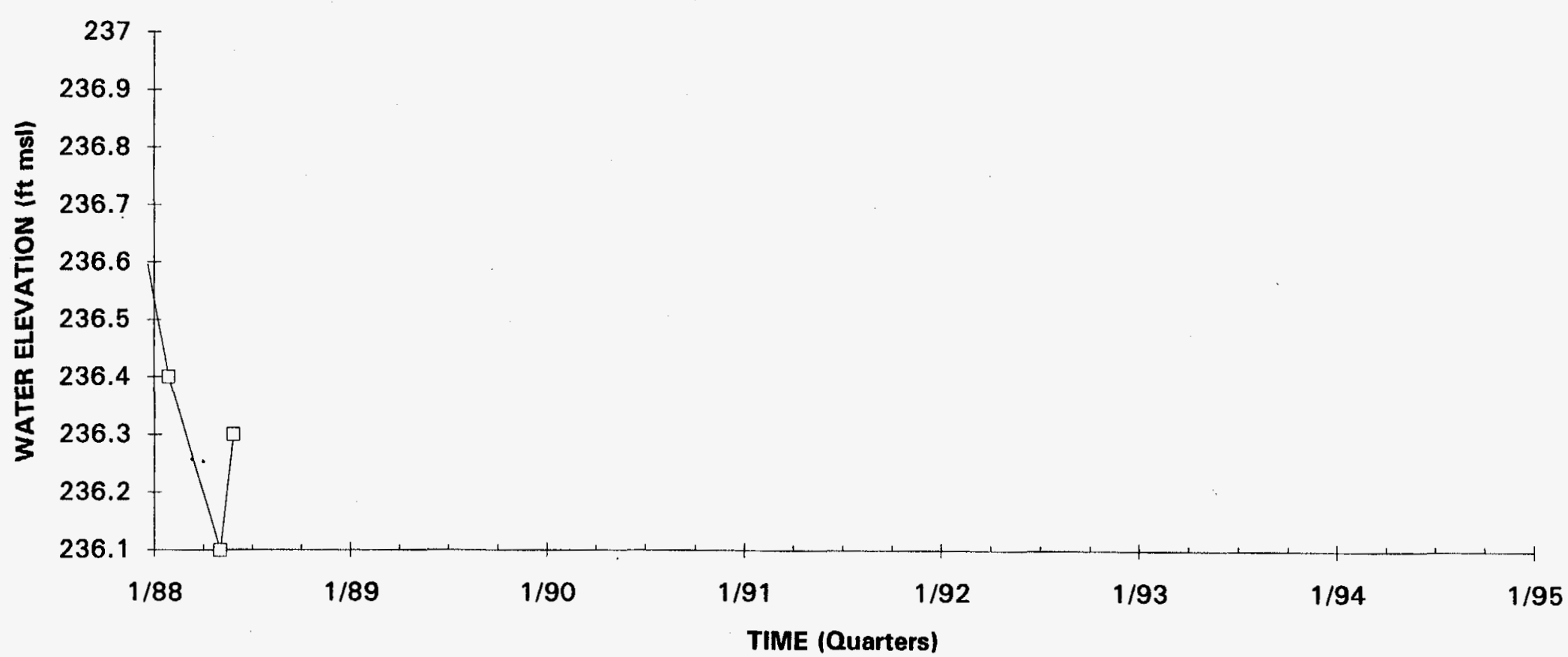



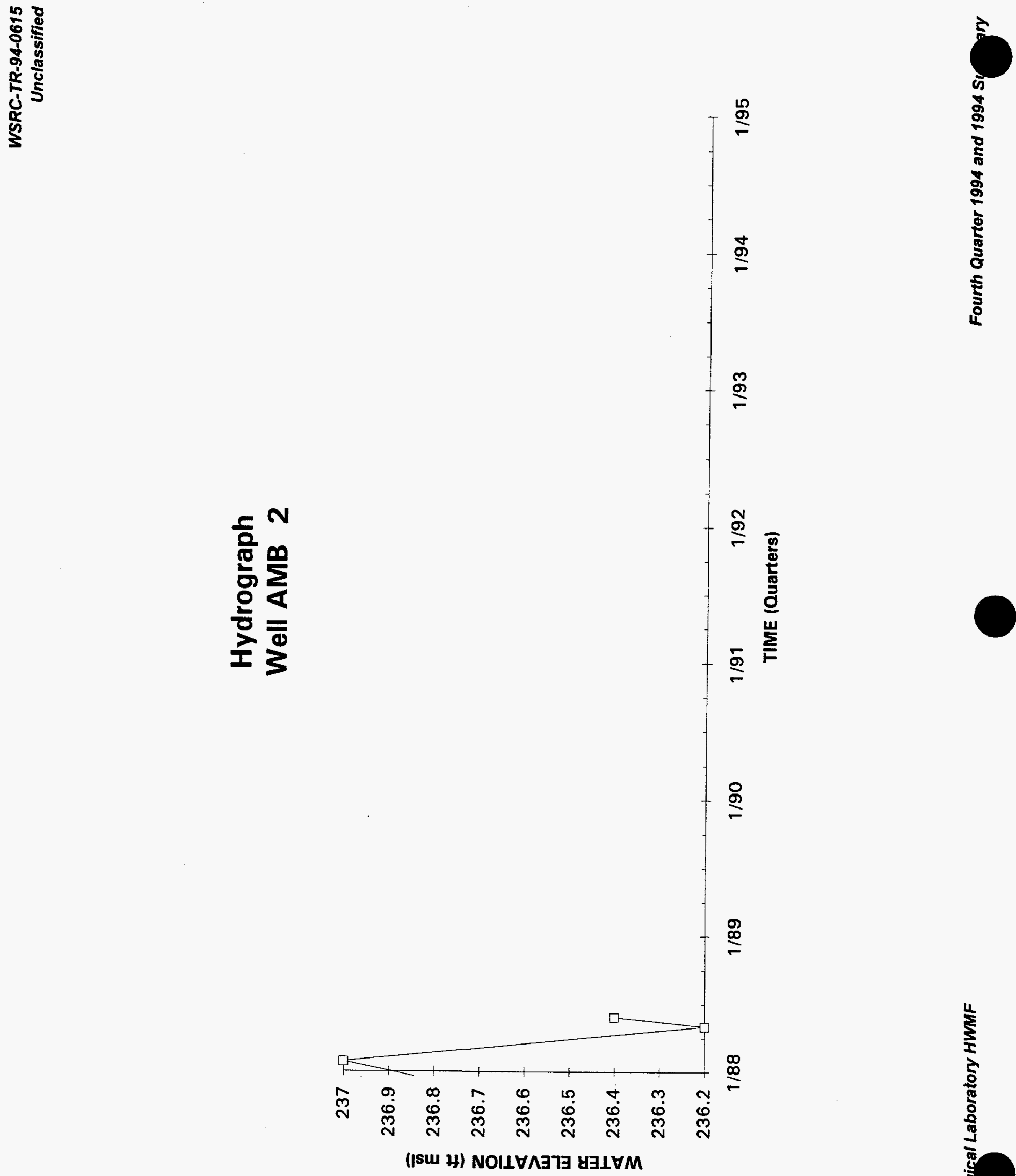

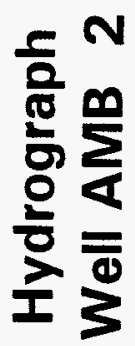
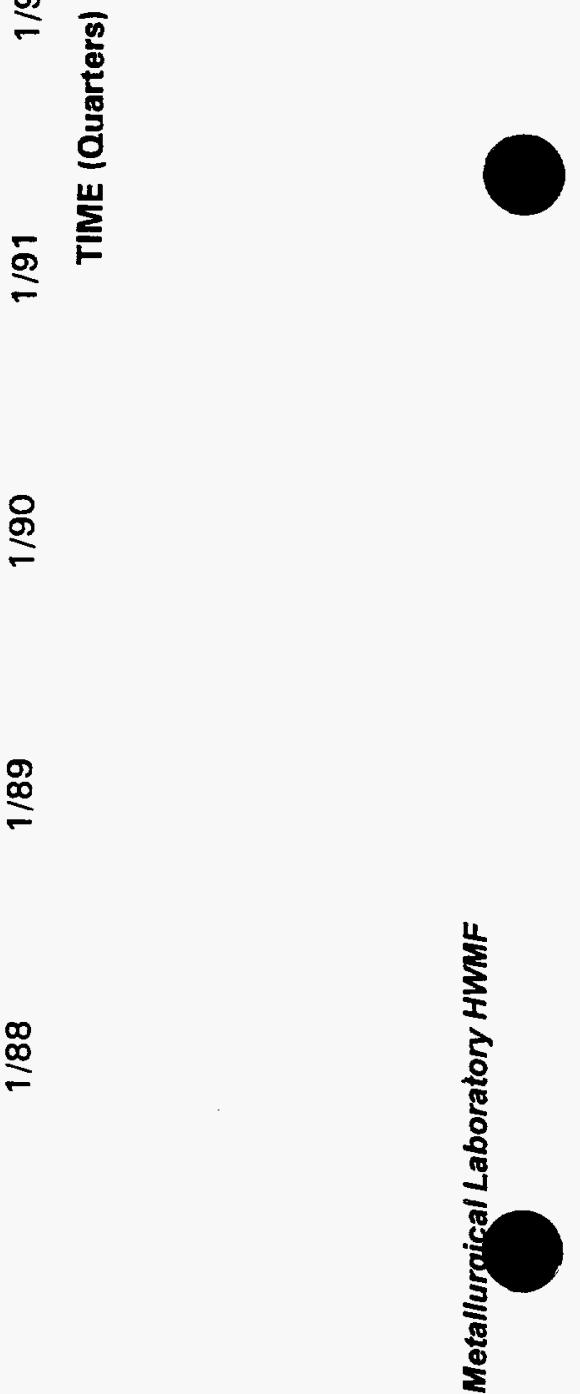


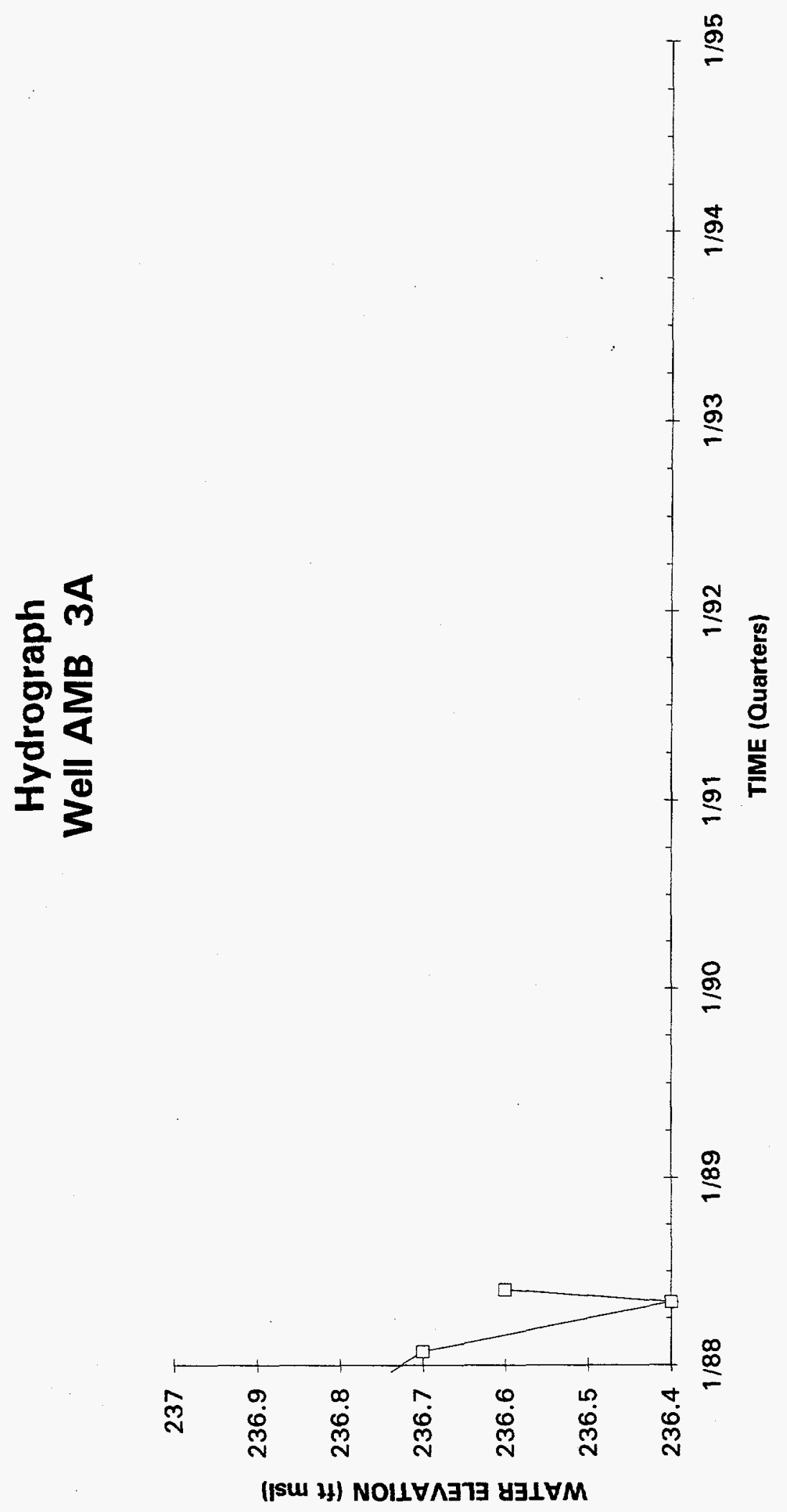




\section{Hydrograph}

Well AMB 4

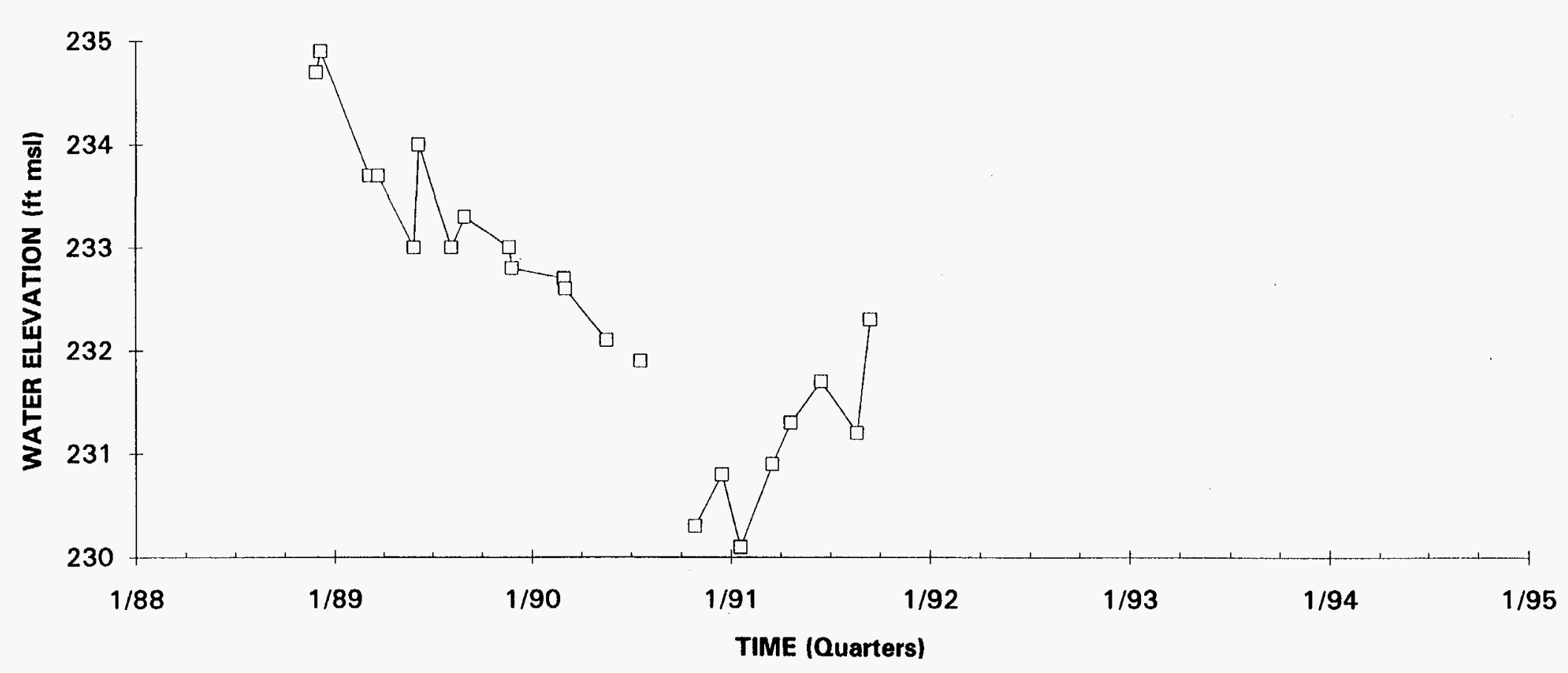




\section{Hydrograph}

Well AMB 4A

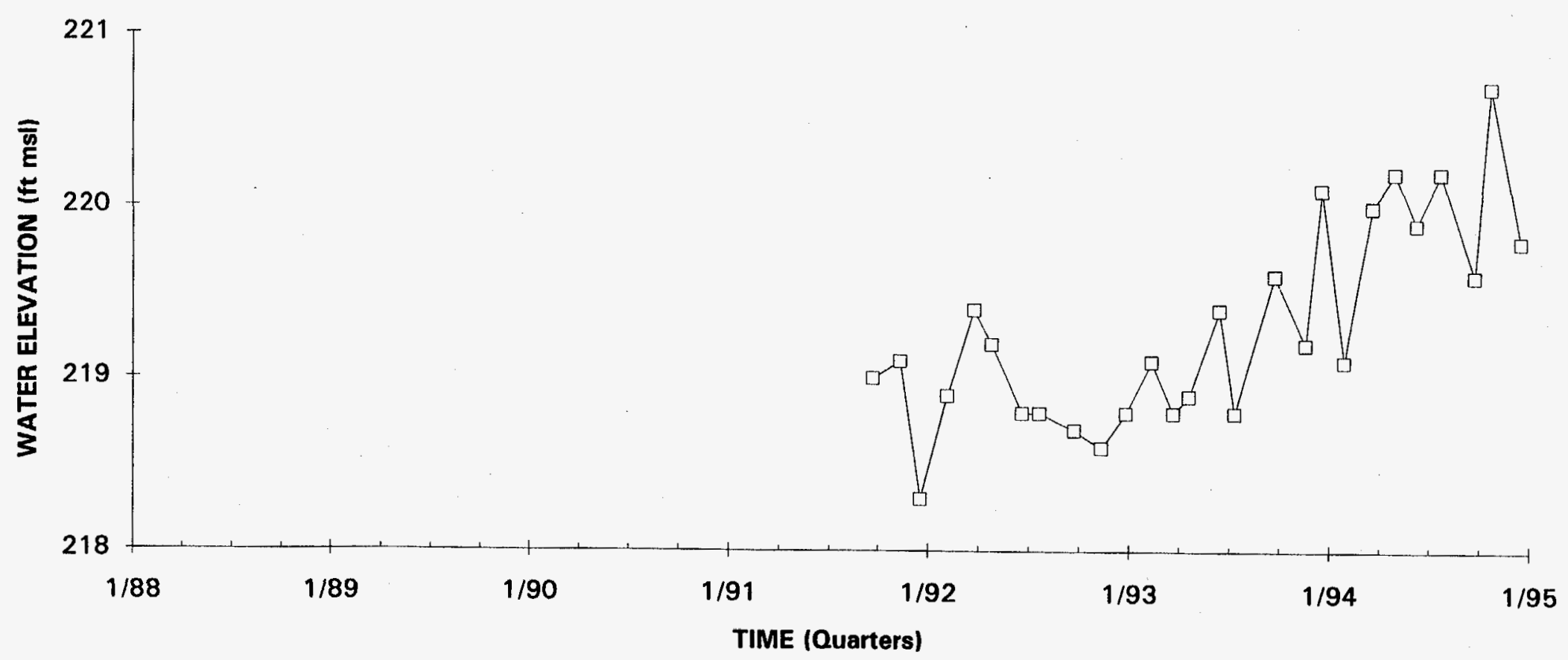




\section{Hydrograph}

Well AMB 4B

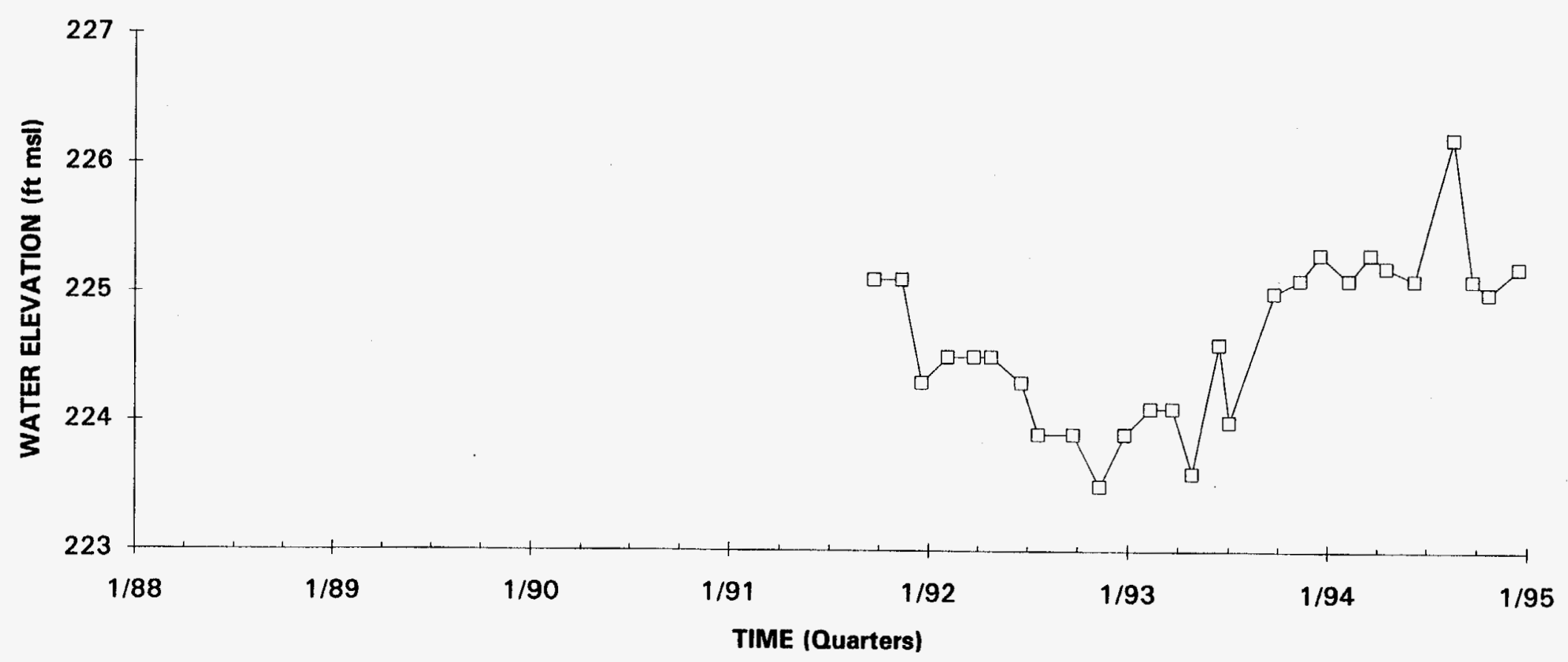




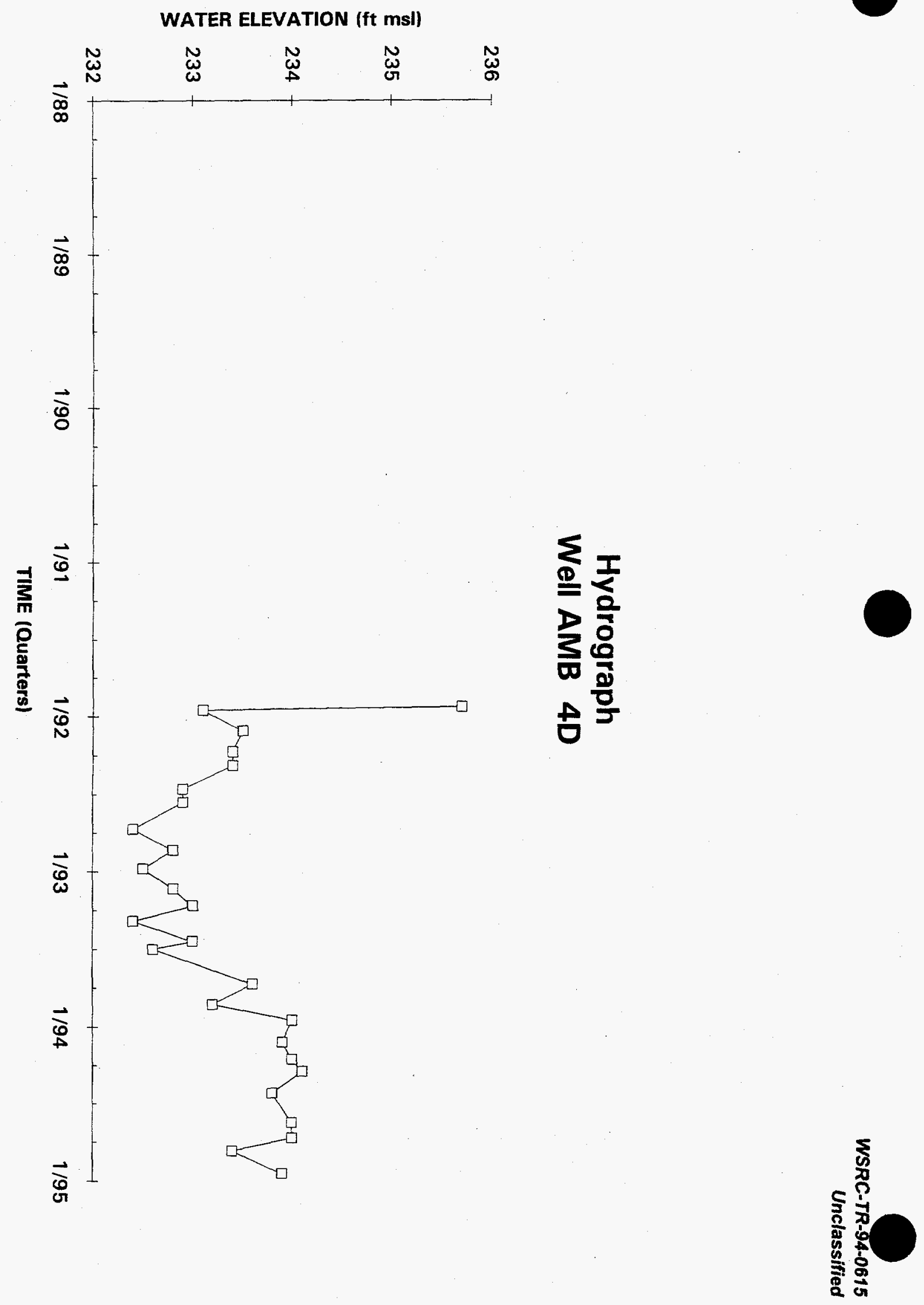



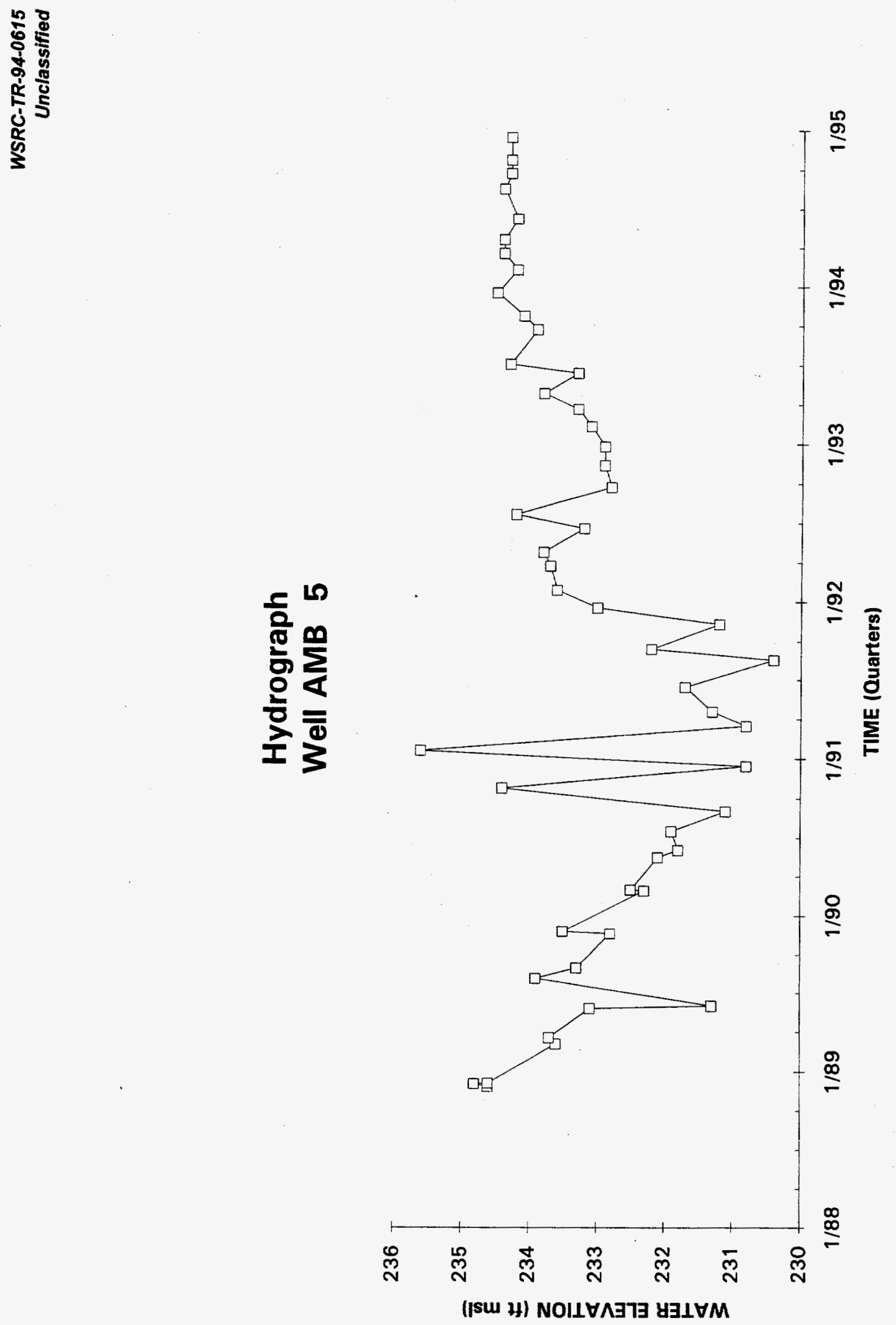

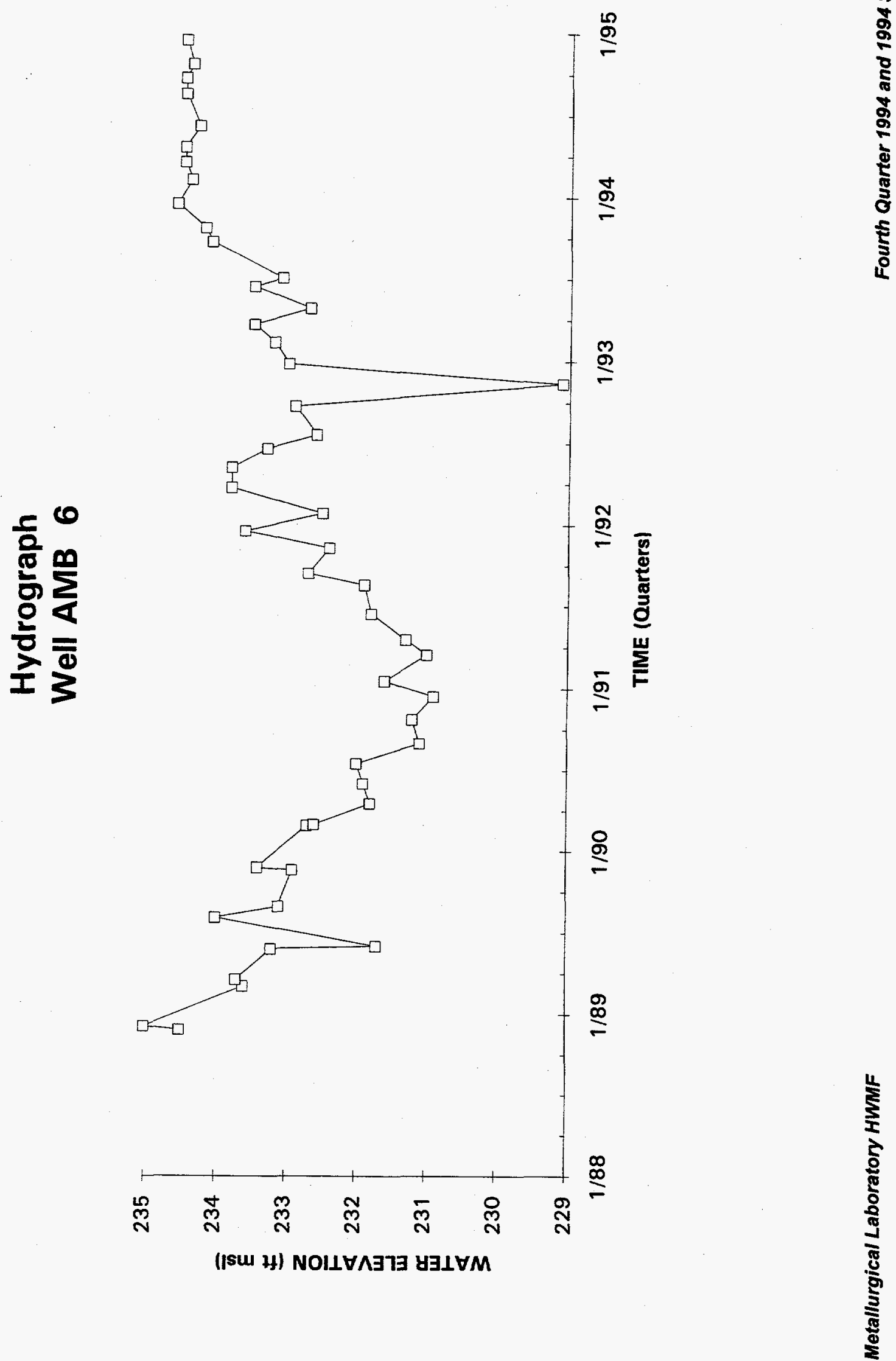


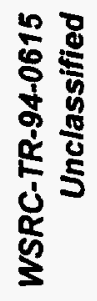
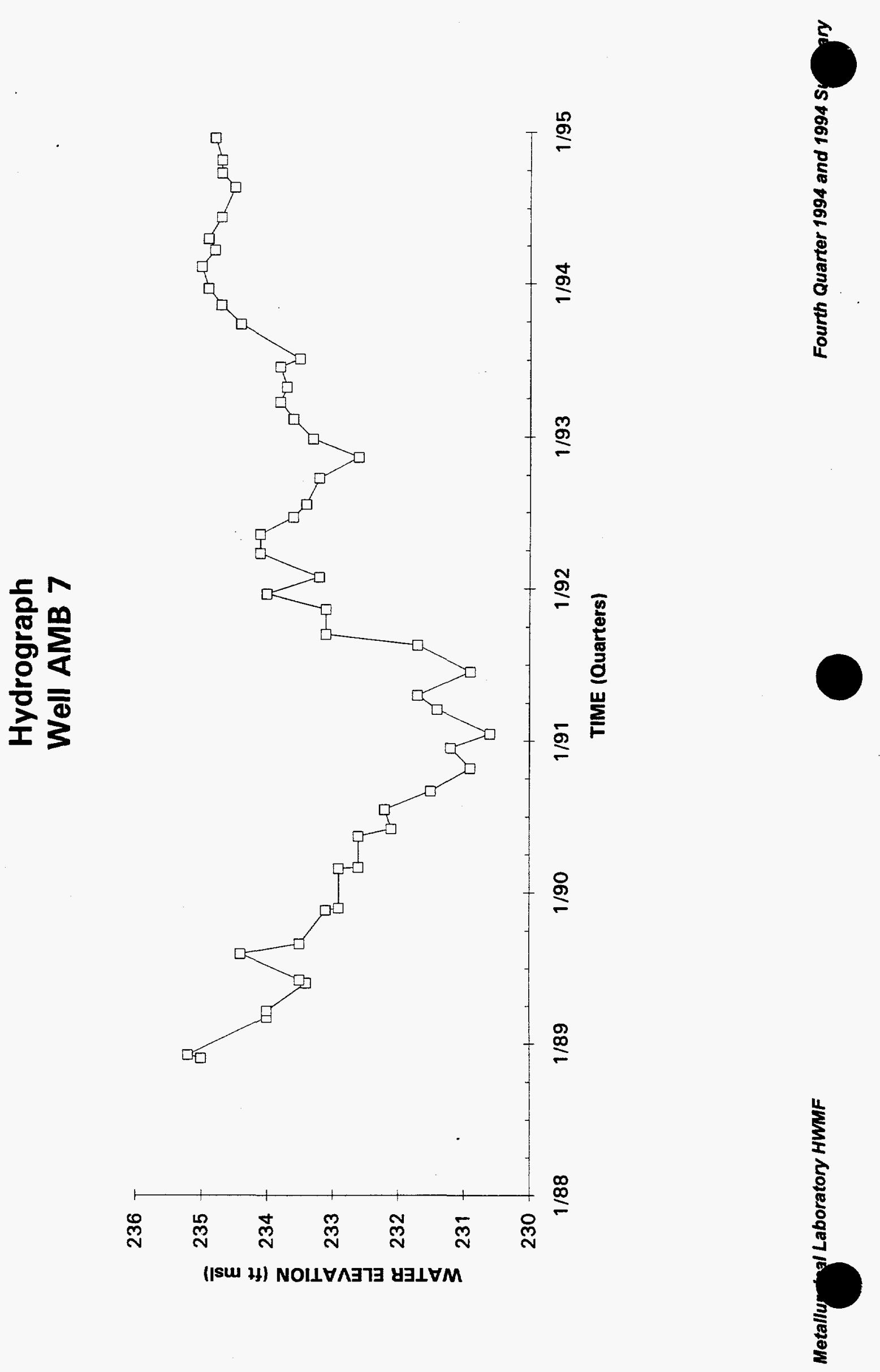


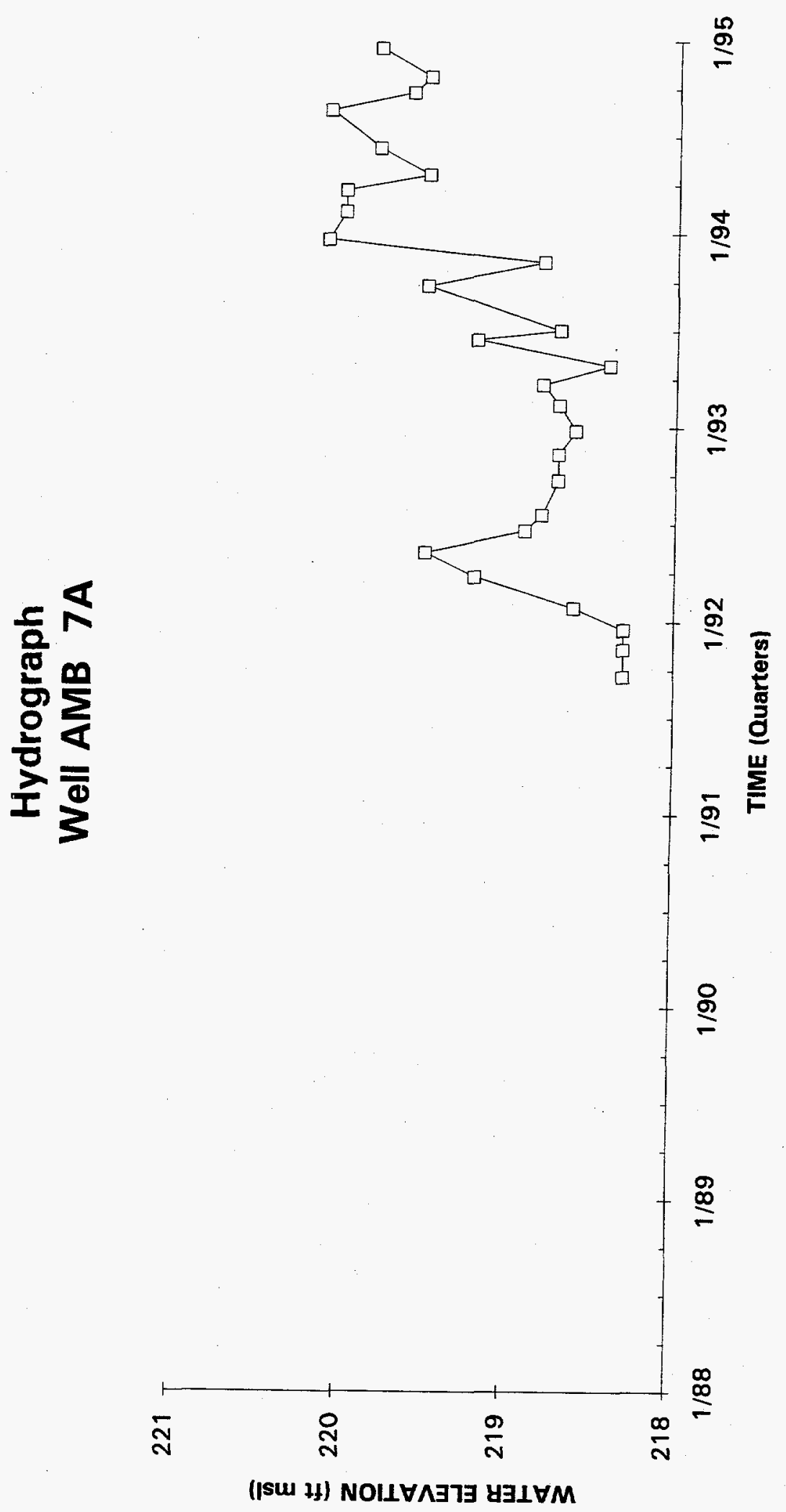




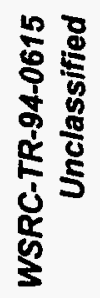

定是
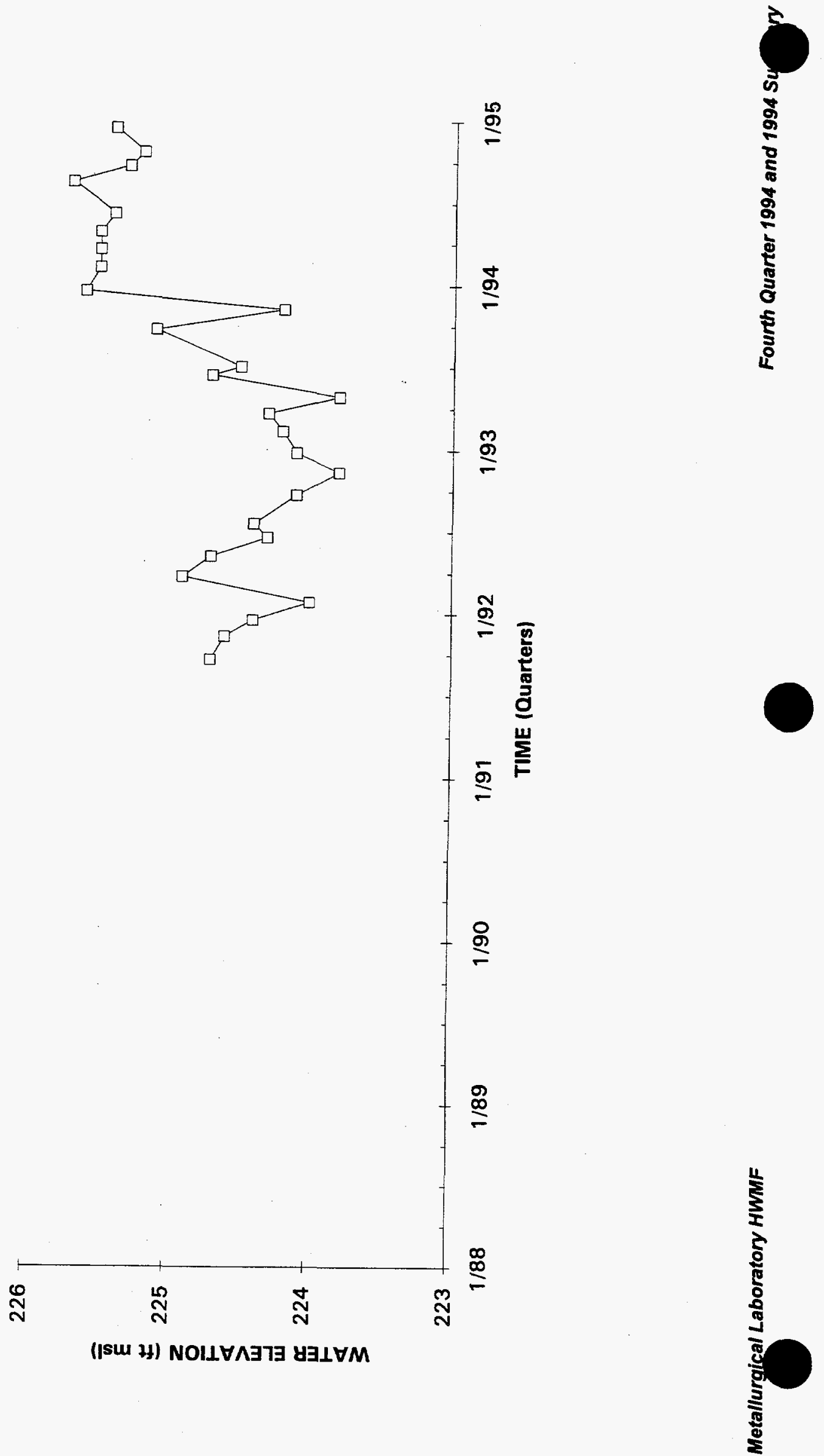


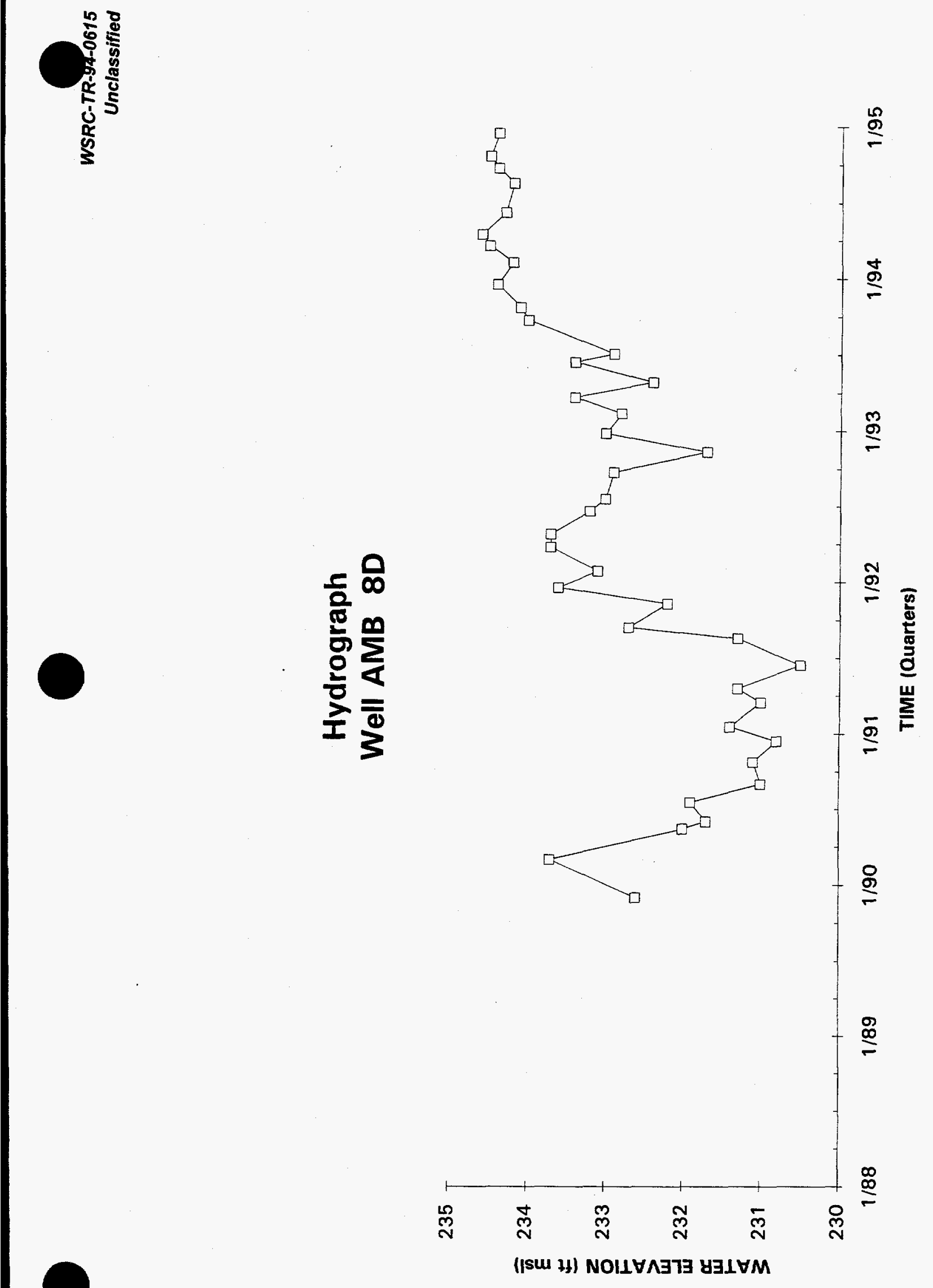




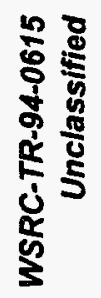
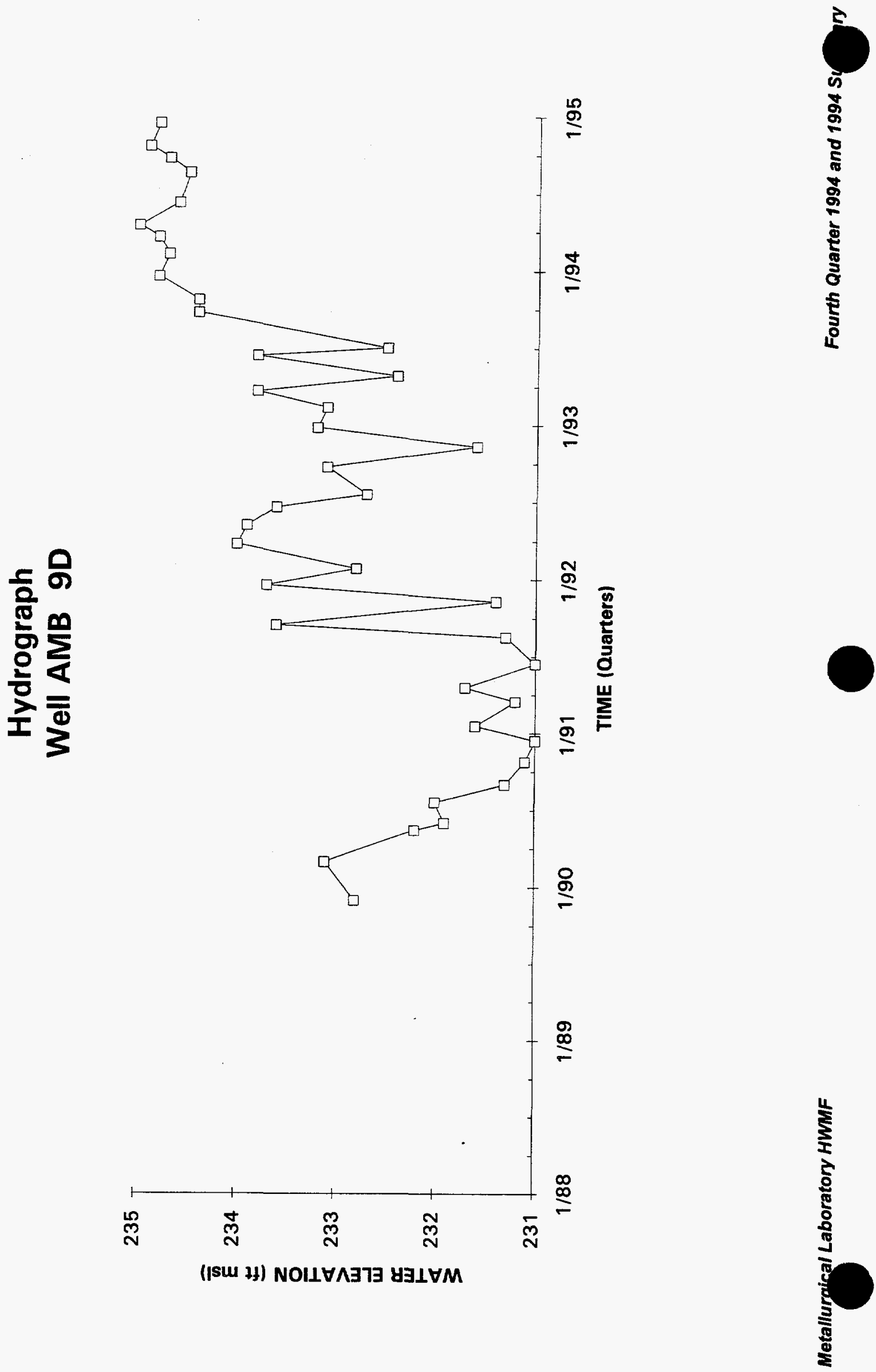

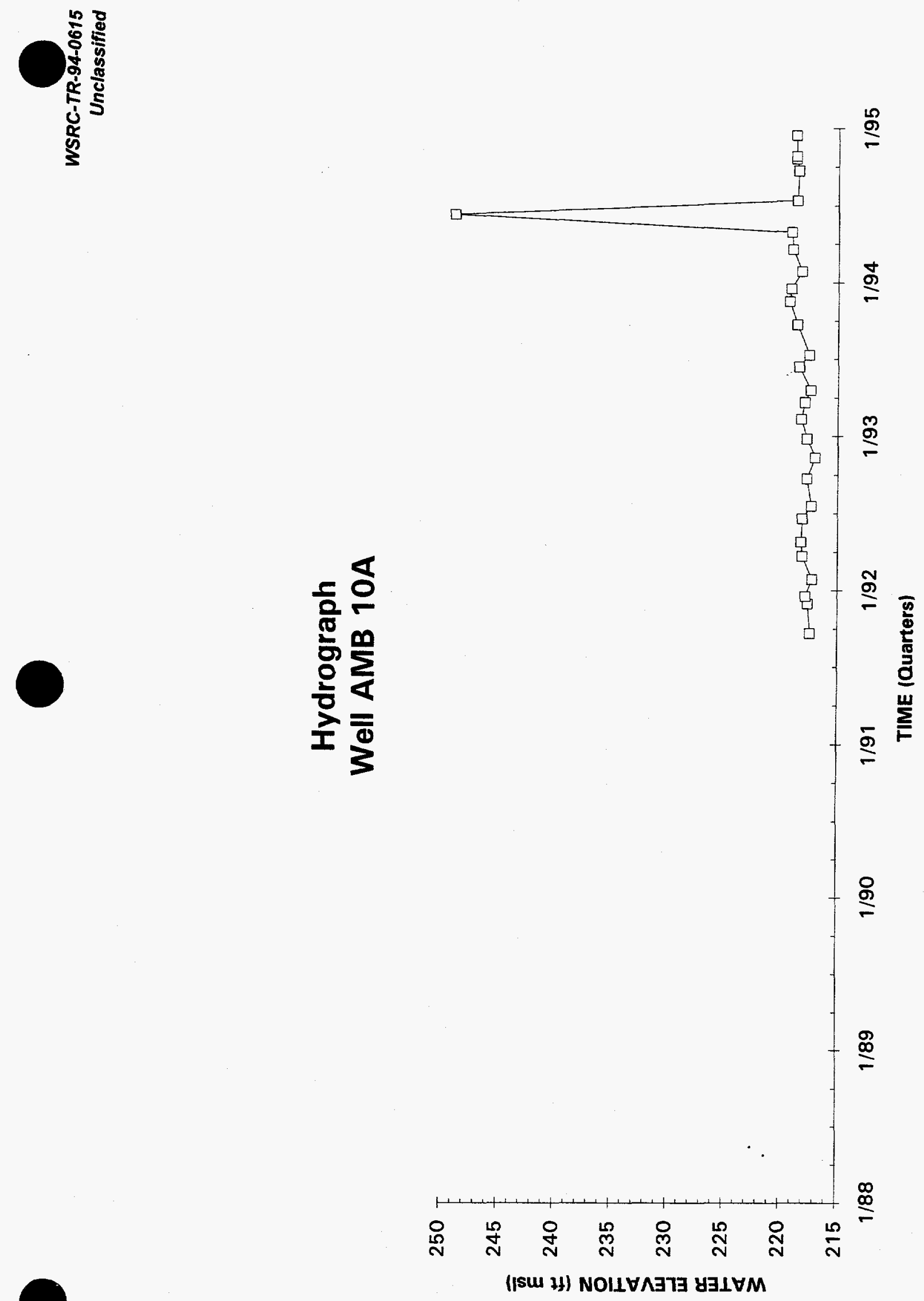


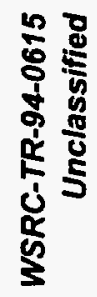
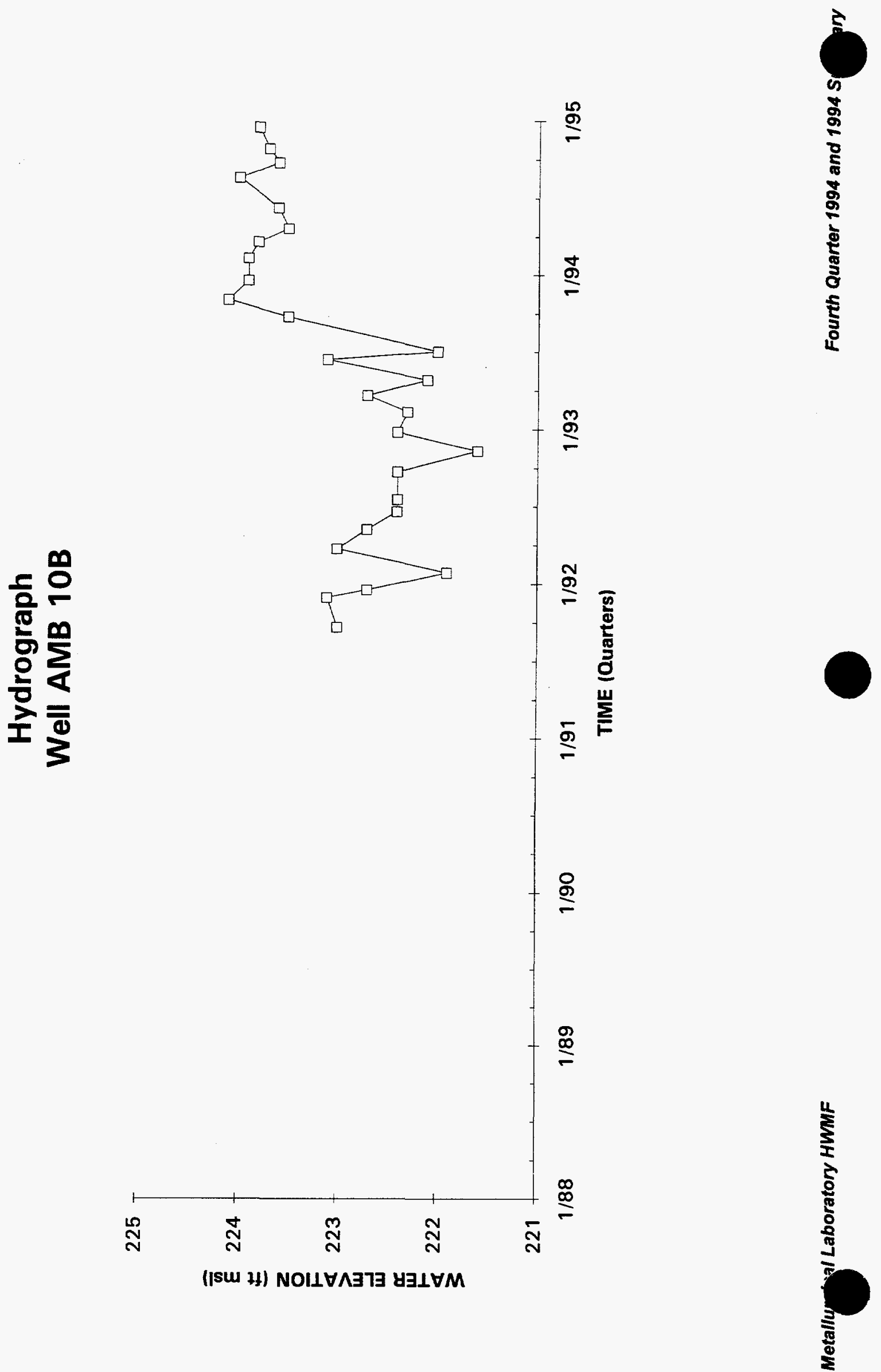

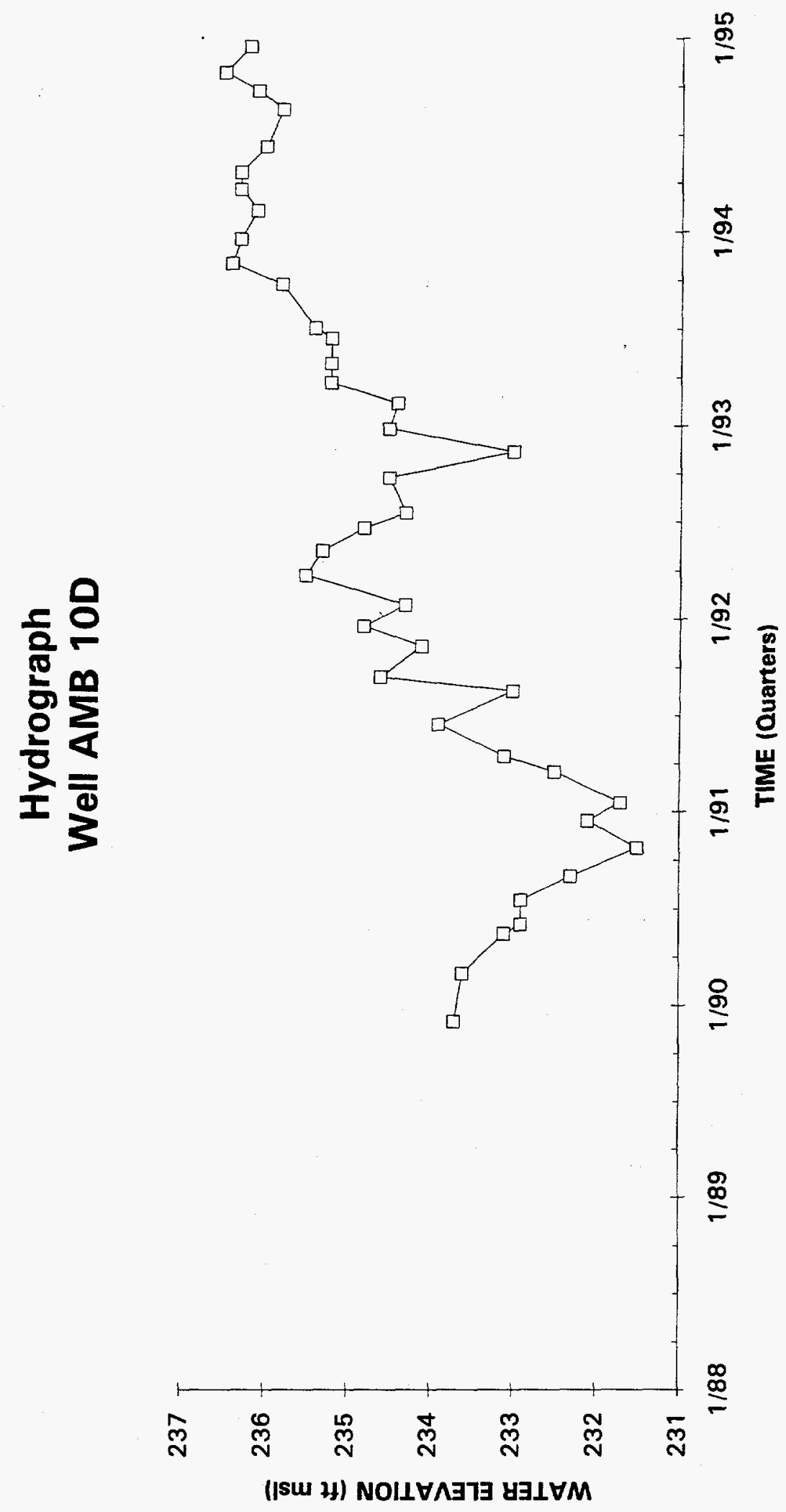

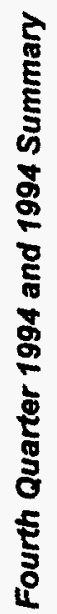




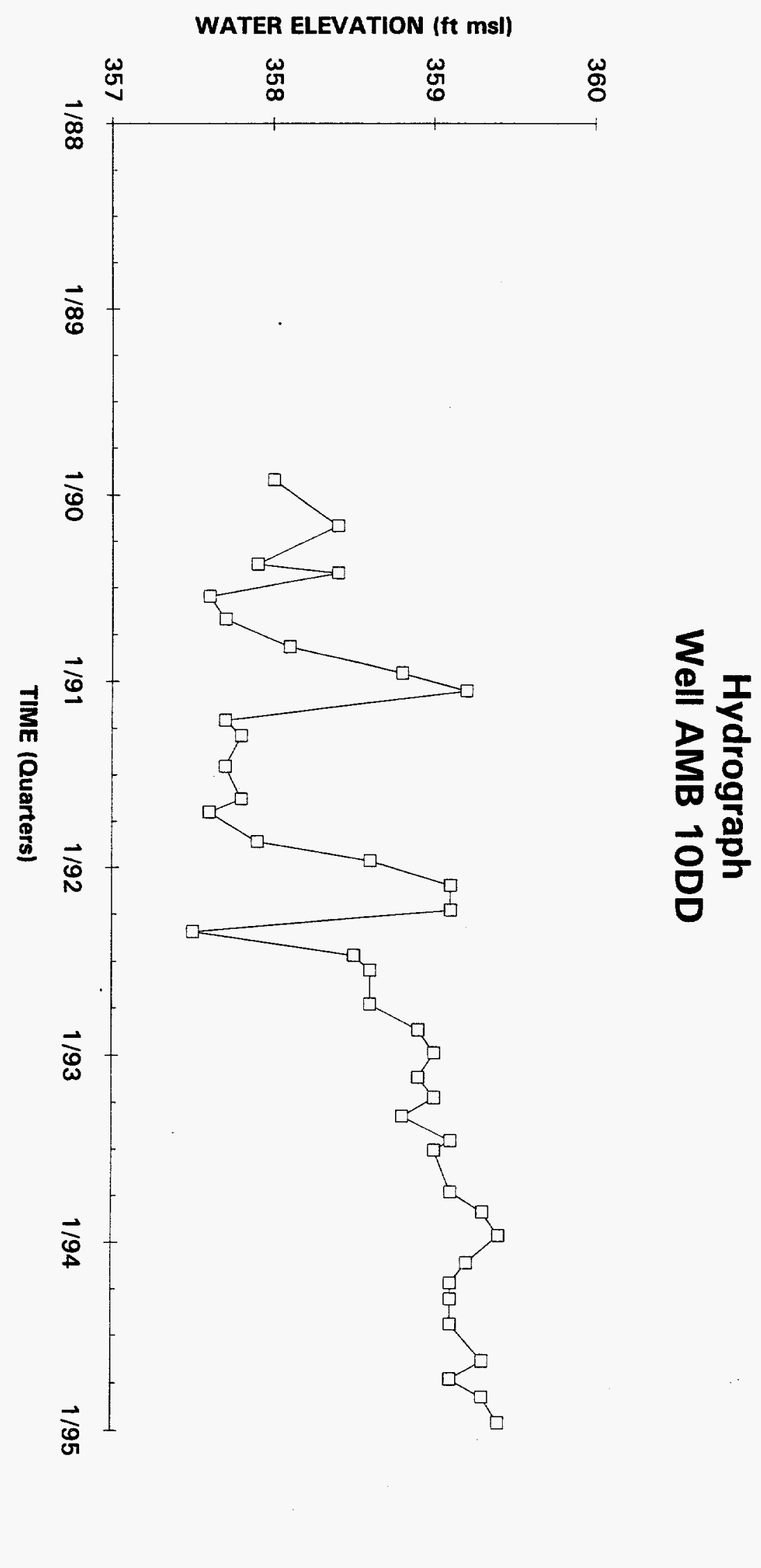

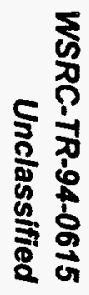



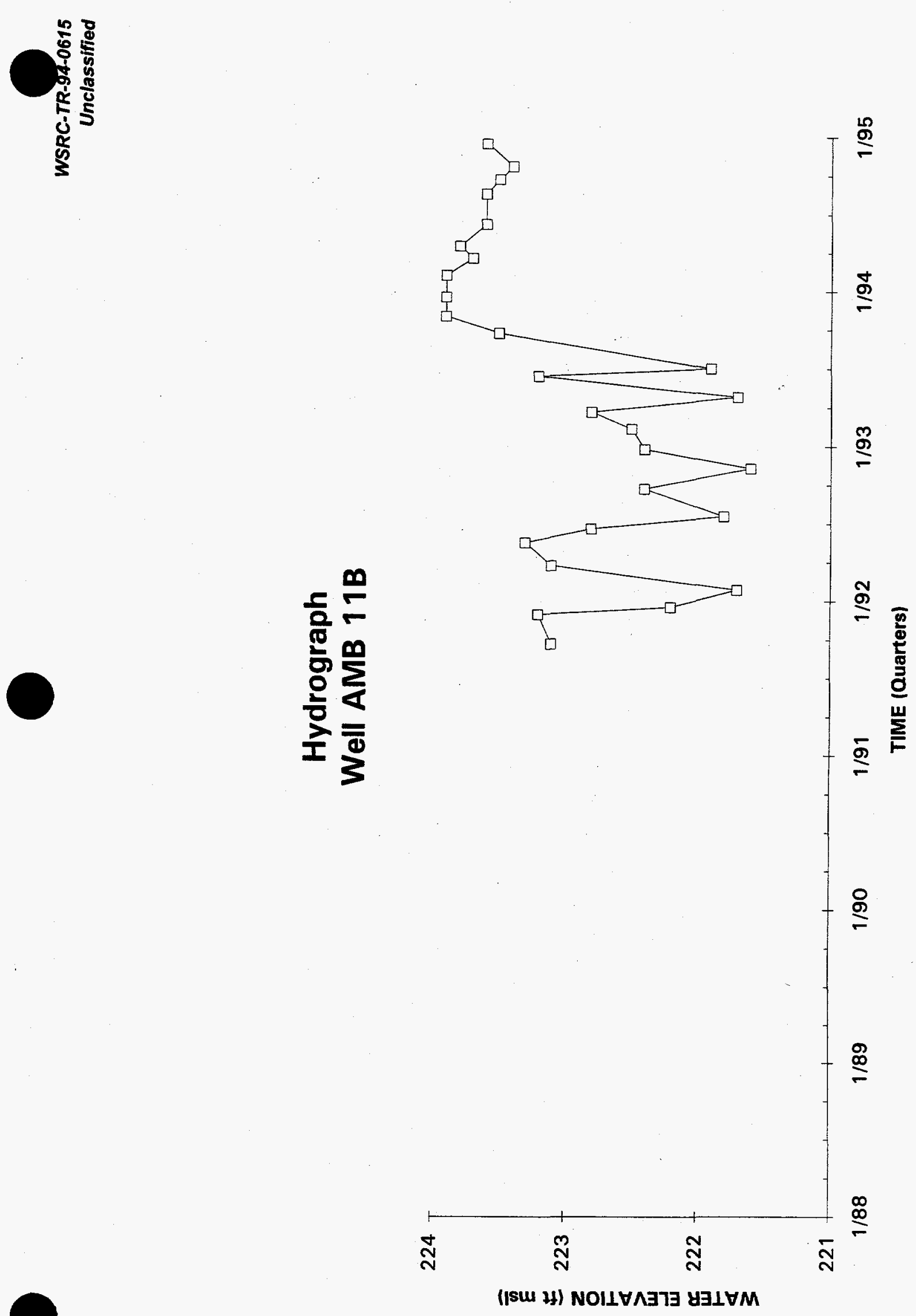


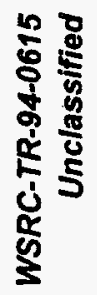
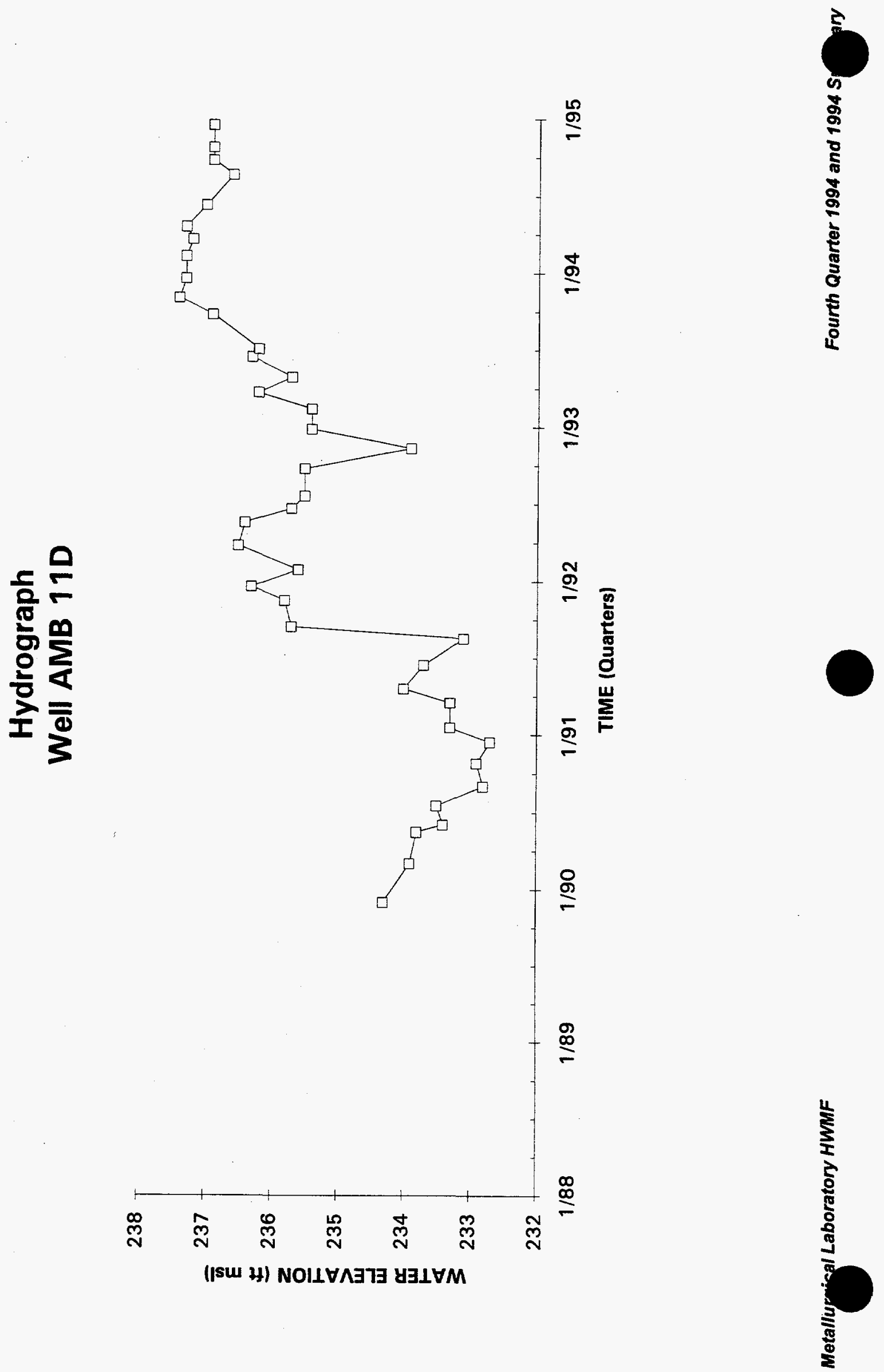

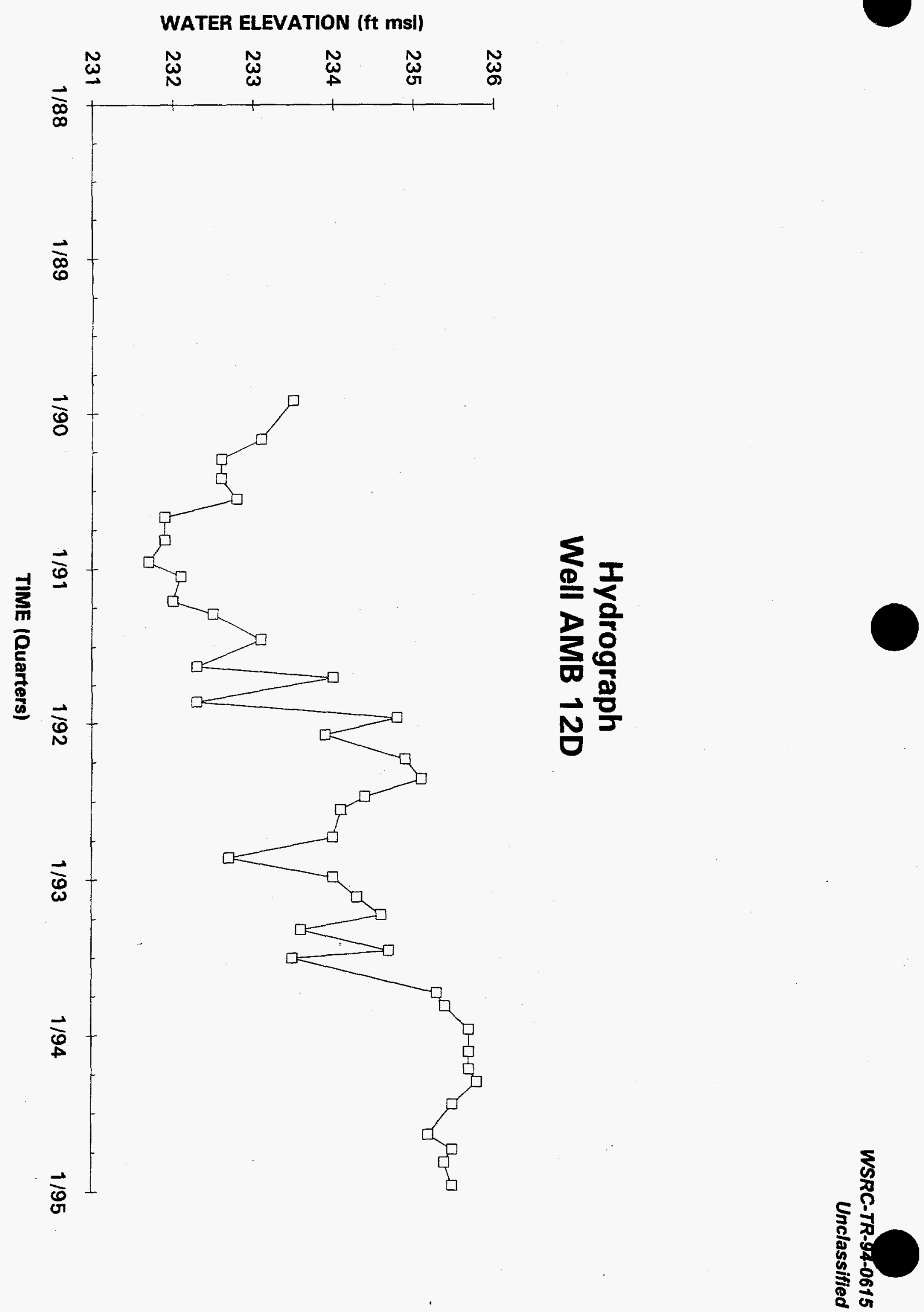


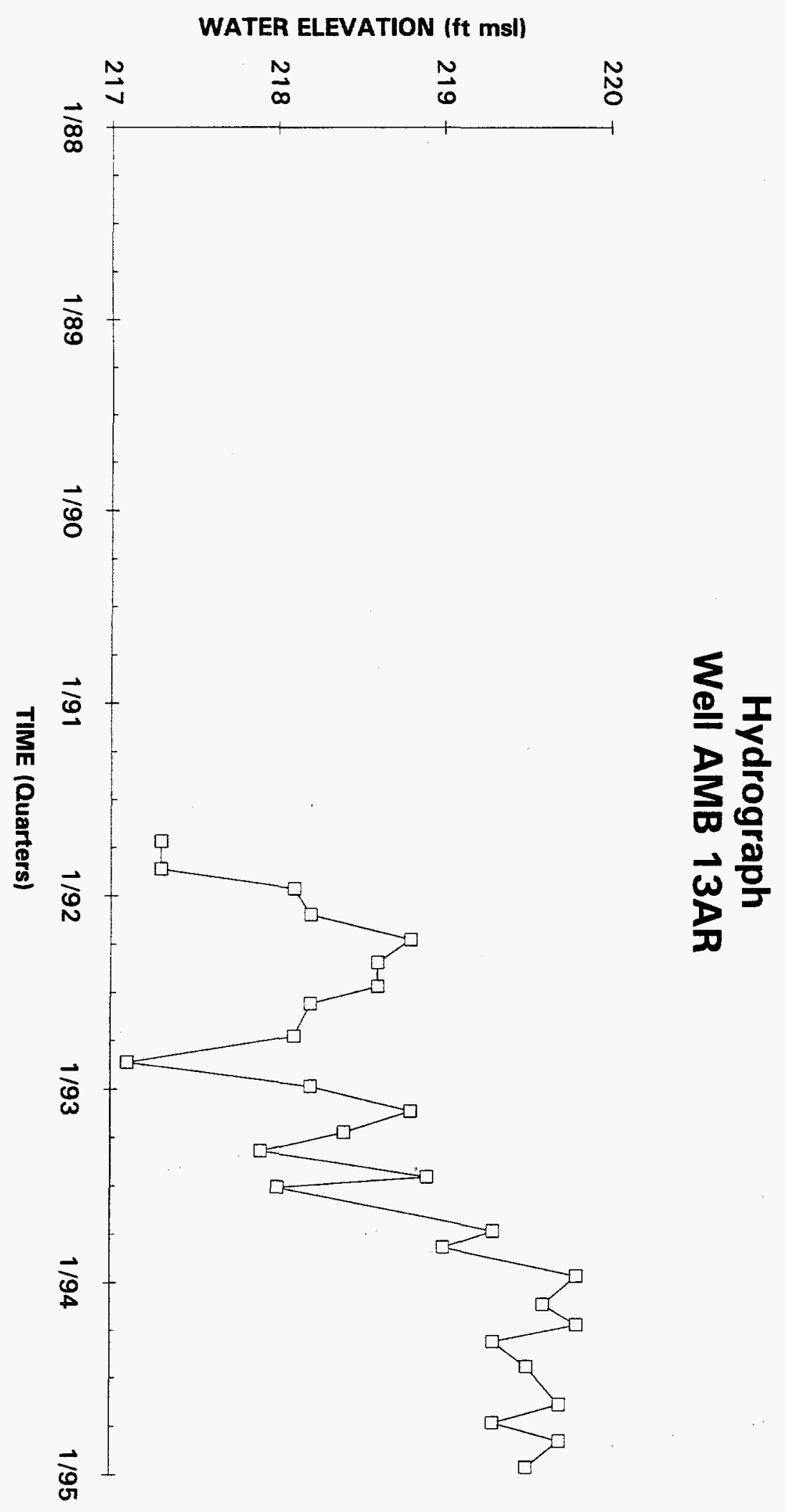




\section{Appendix $\mathrm{H}$}

\section{M-Area Water-Elevation Maps}


THIS PAGE LEFT BLANK INTENTIONALLY. 


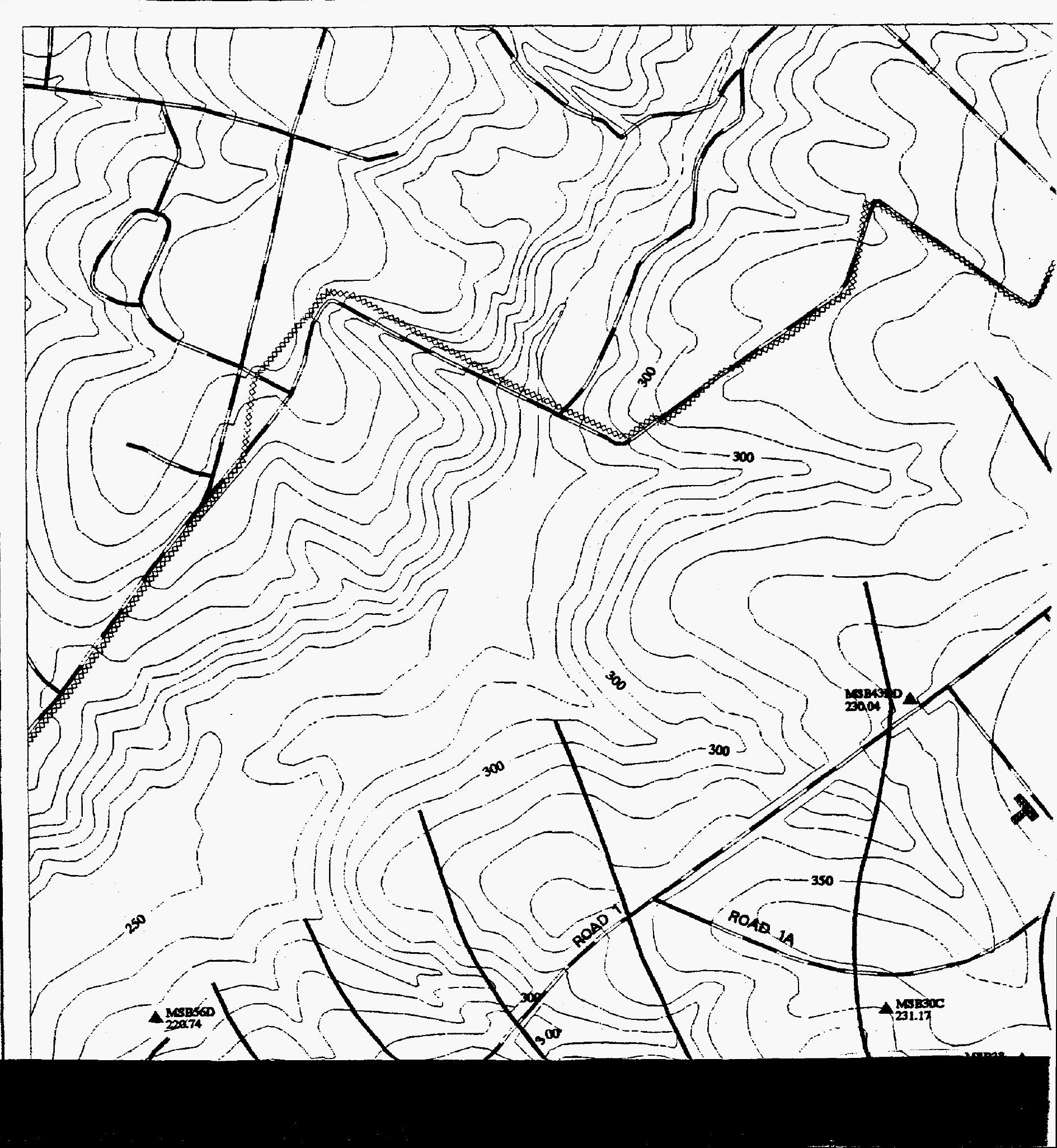




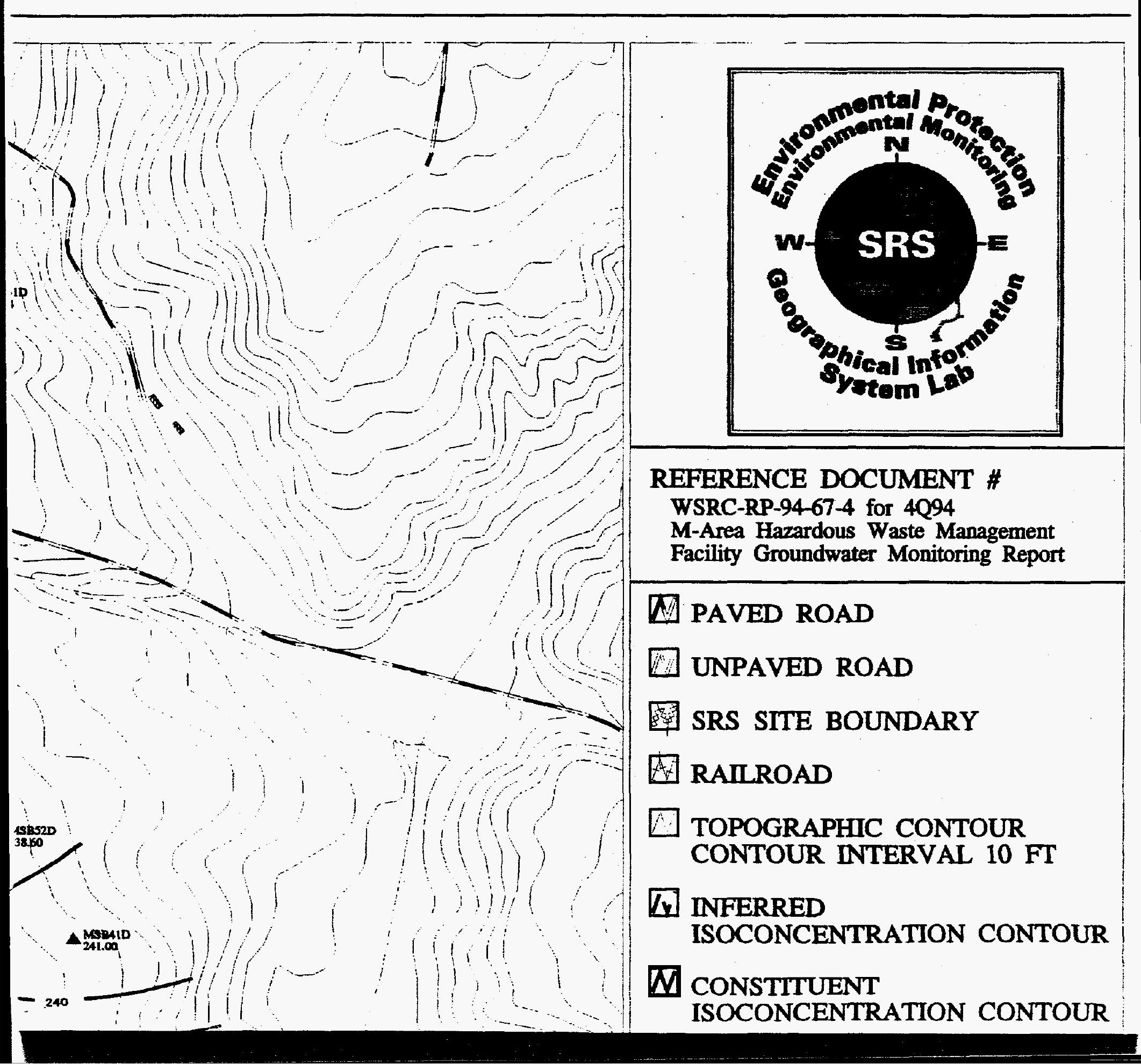




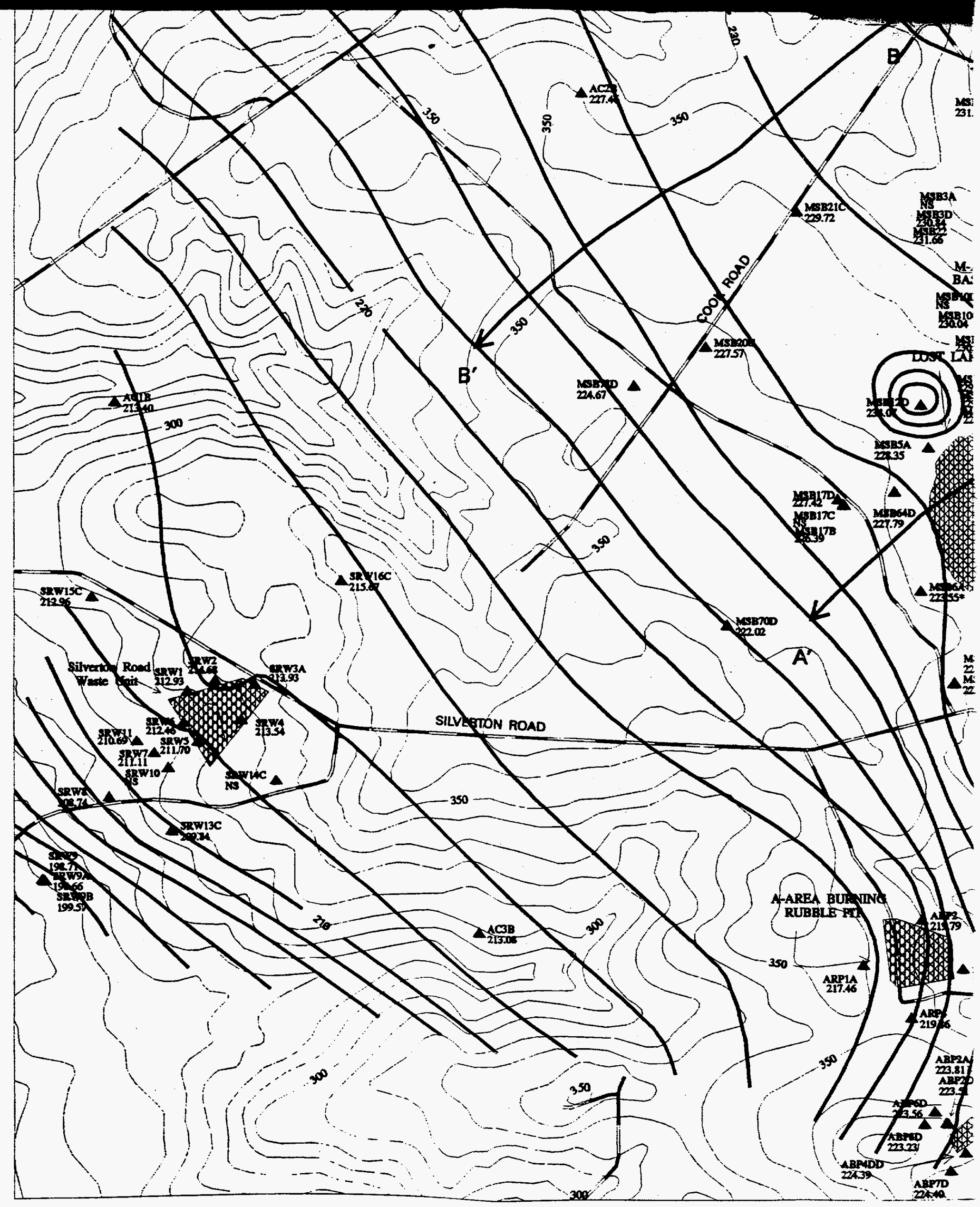




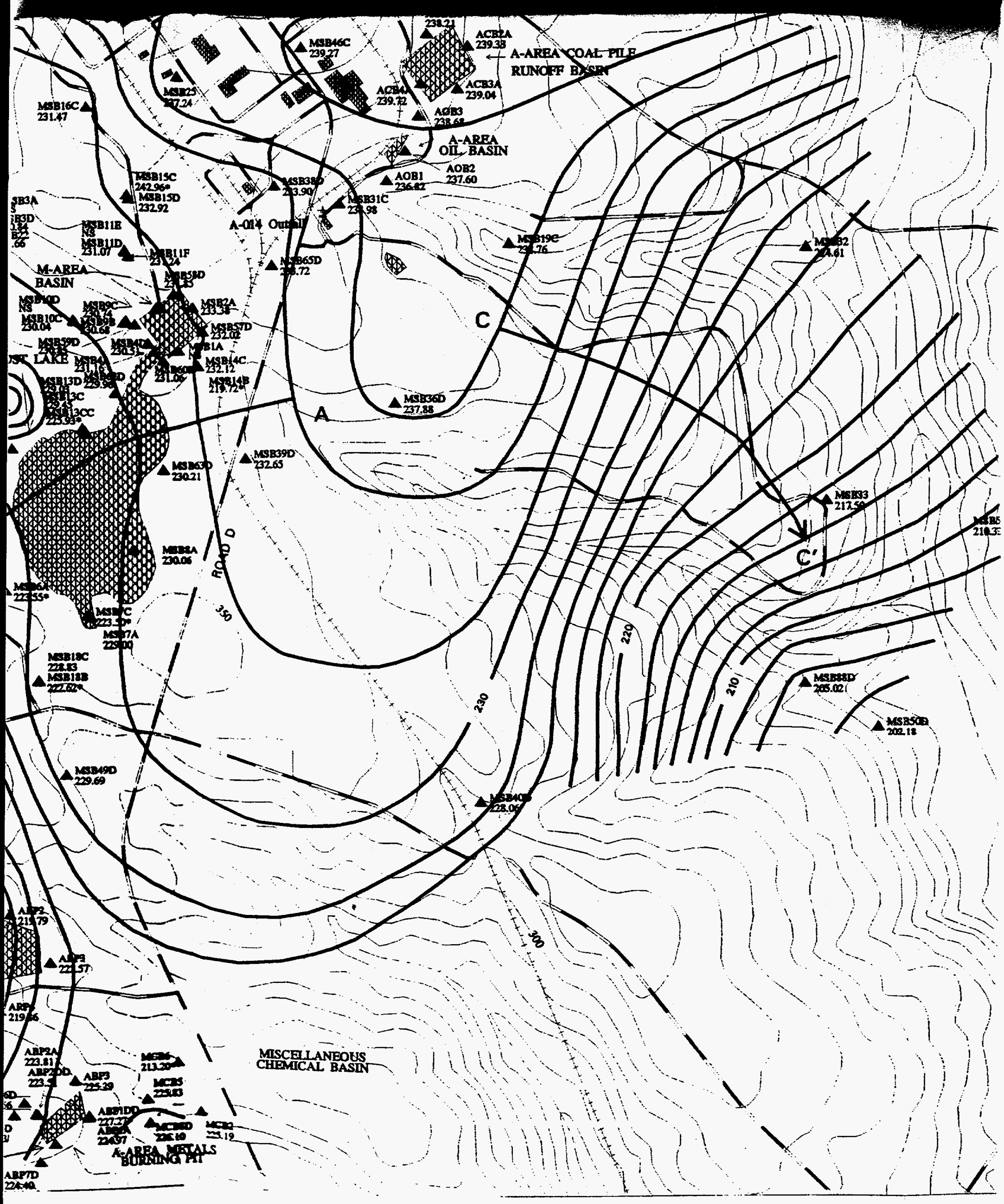




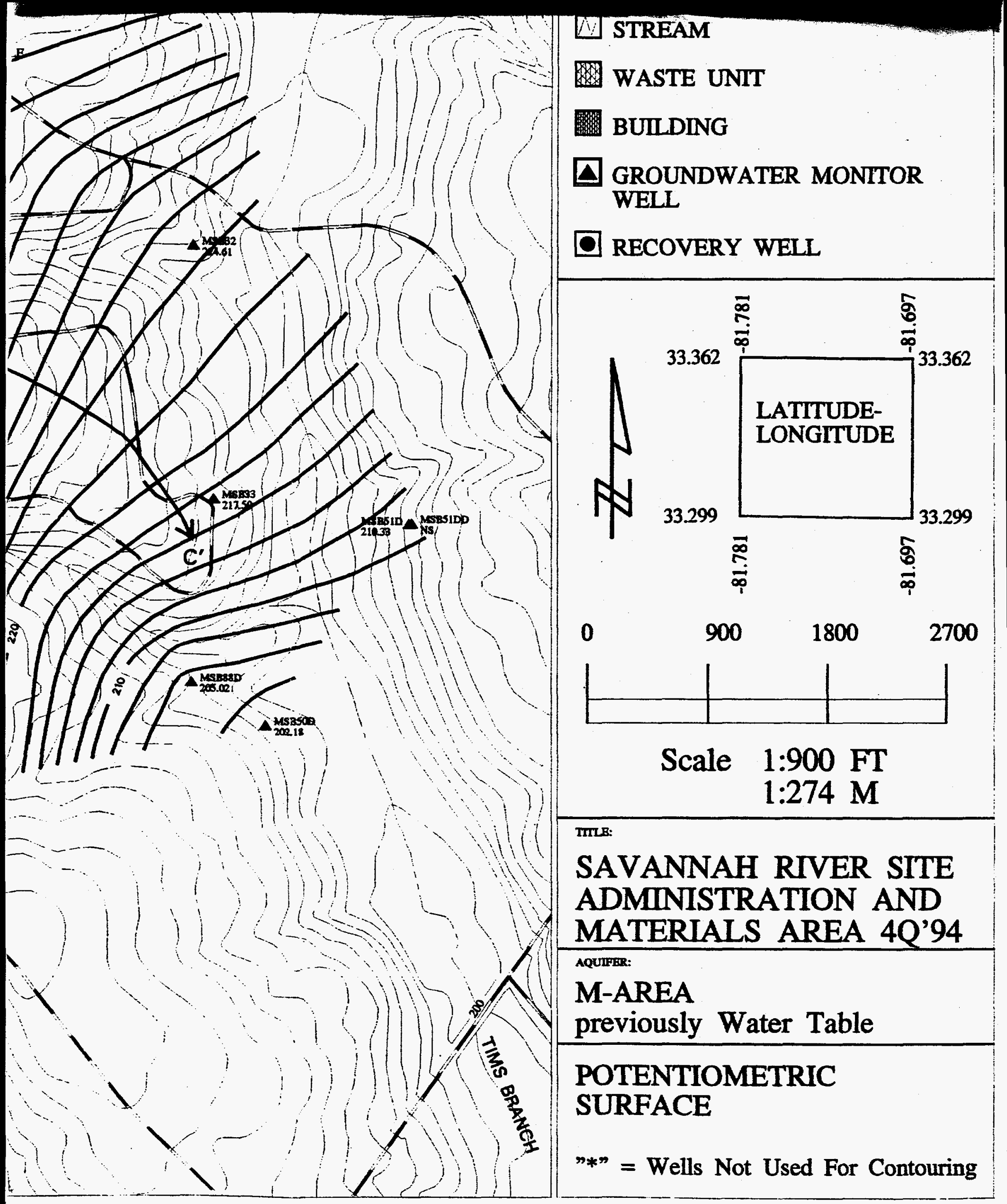




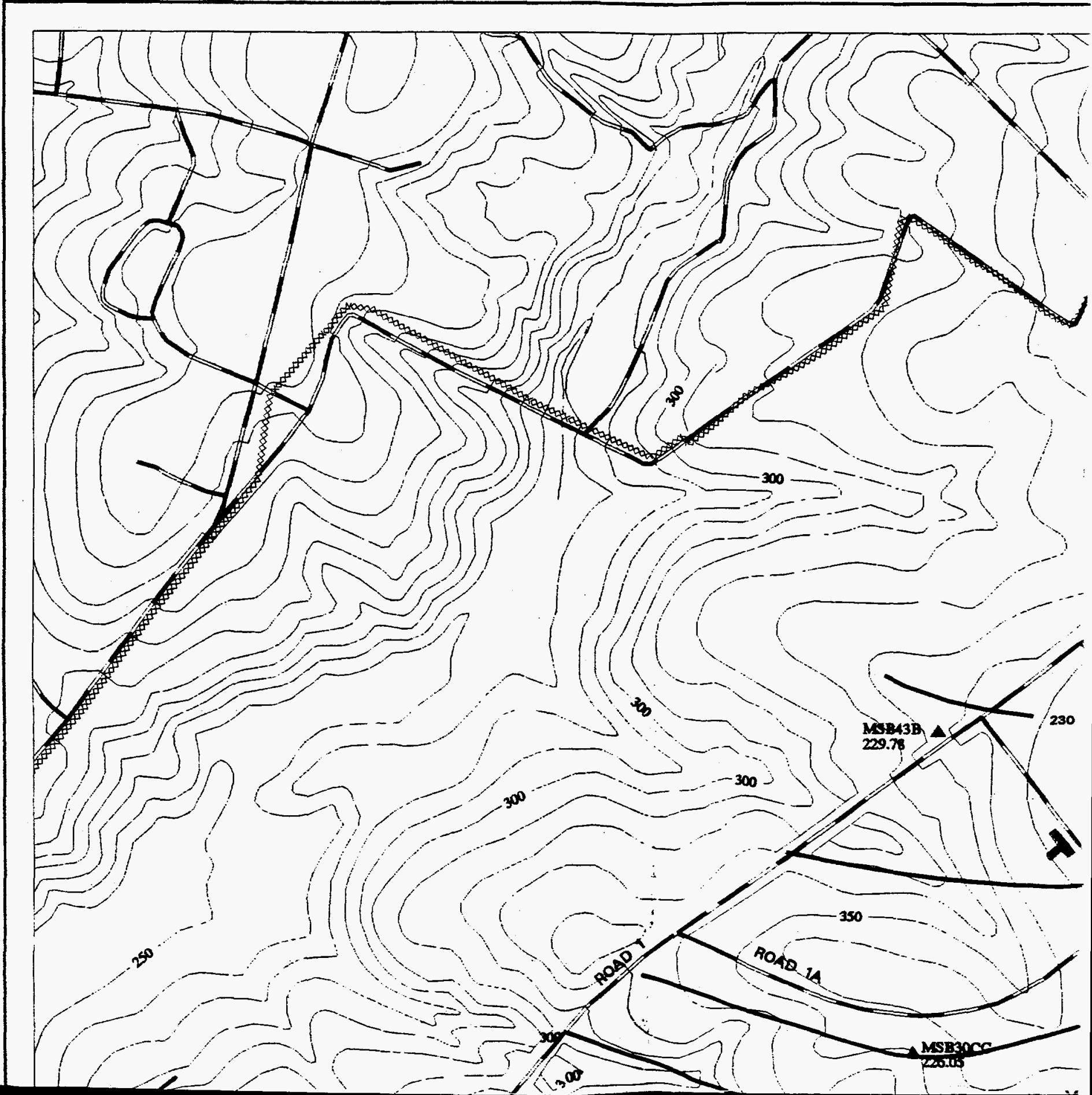




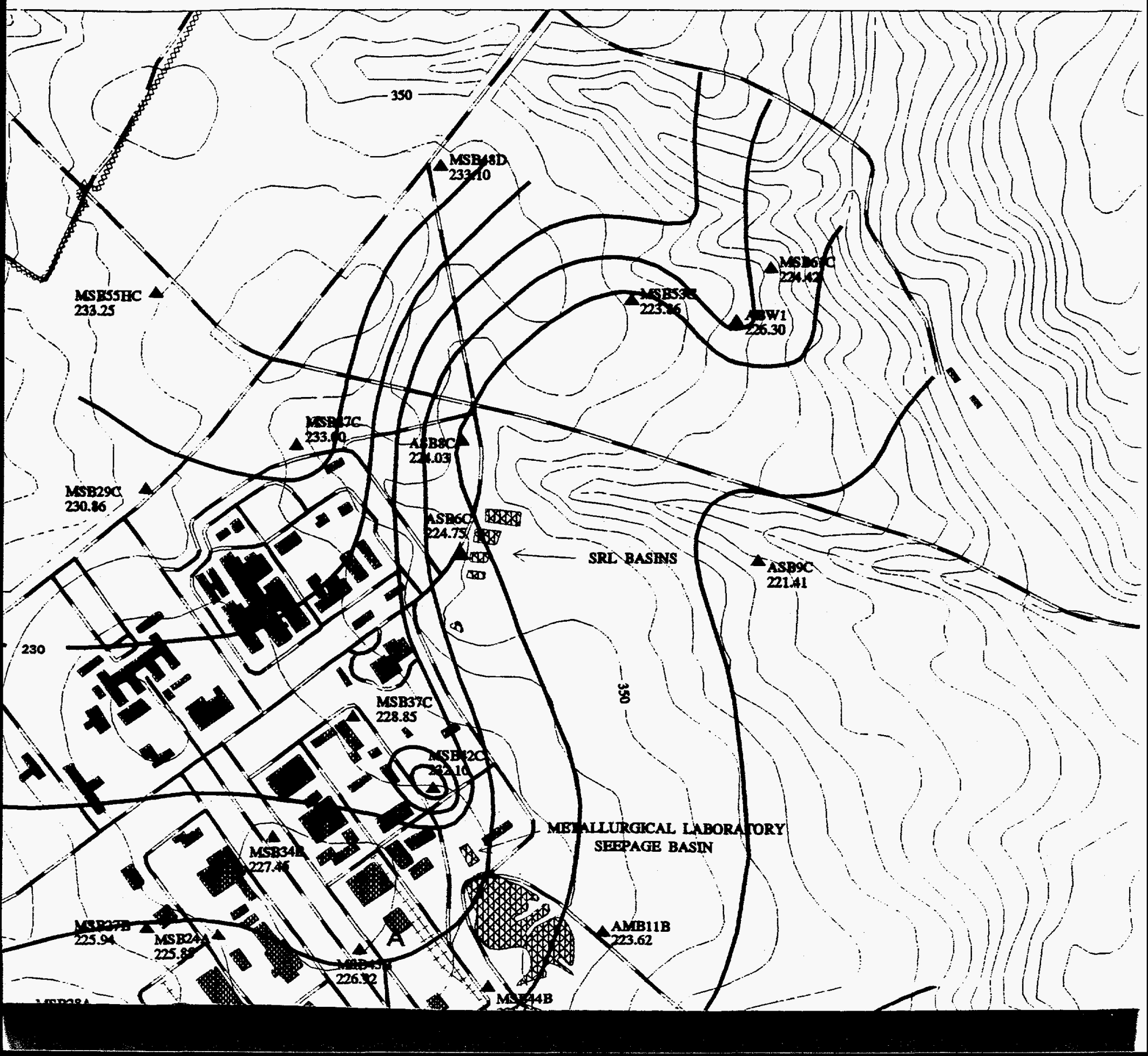




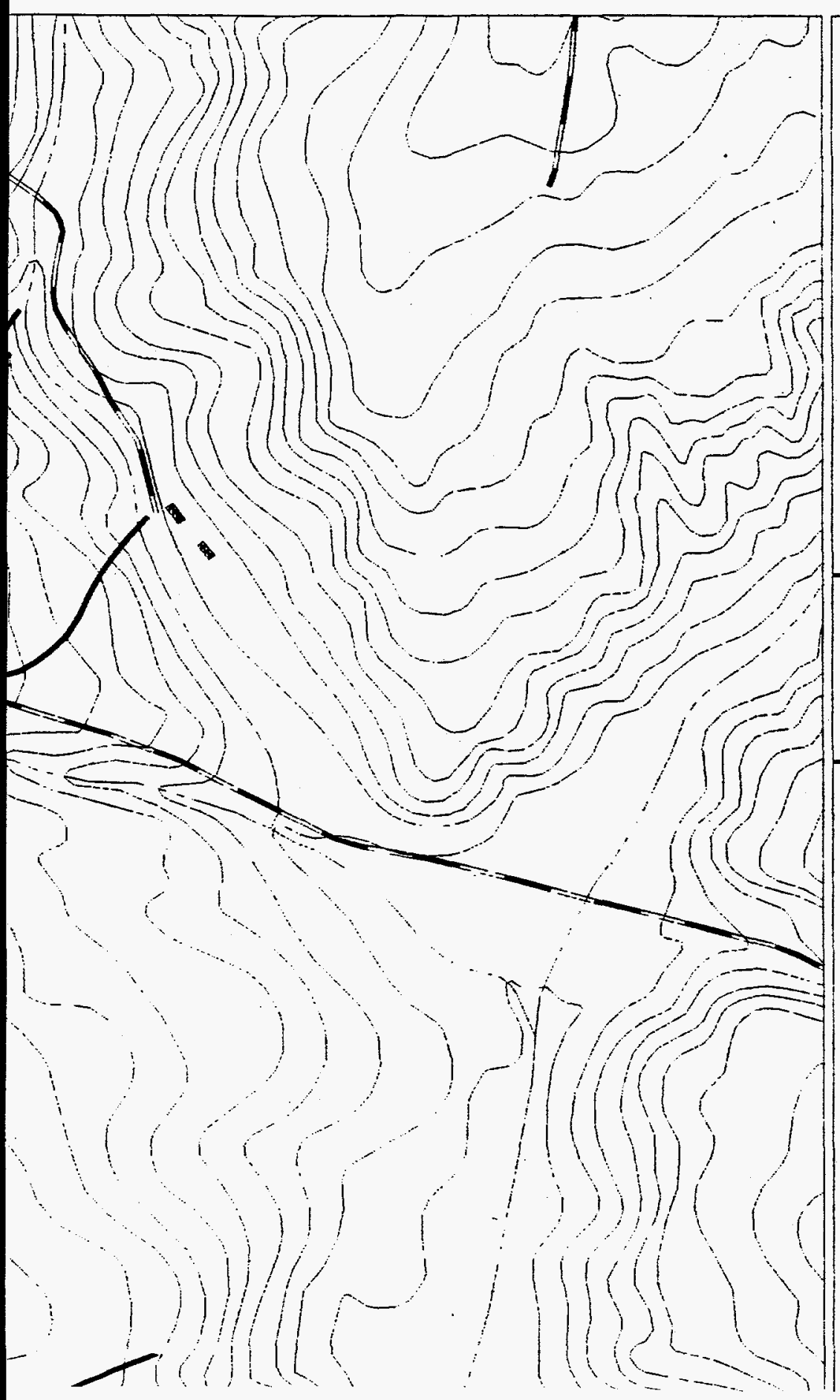

REFERENCE DOCUMENT \# WSRC-RP-94-67-4 for 4Q94 M-Area Hazardous Waste Management Facility Groundwater Monitoring Report

$\triangle$ PAVED ROAD

四 UNPAVED ROAD

图 SRS SITE BOUNDARY

RAIIROAD

TOPOGRAPHIC CONTOUR CONTOUR INTERVAL 10 FT

因 INFERRED ISOCONCENTRATION CONTOUR

$\triangle$ CONSTITUENT ISOCONCENTRATION CONTOUR 


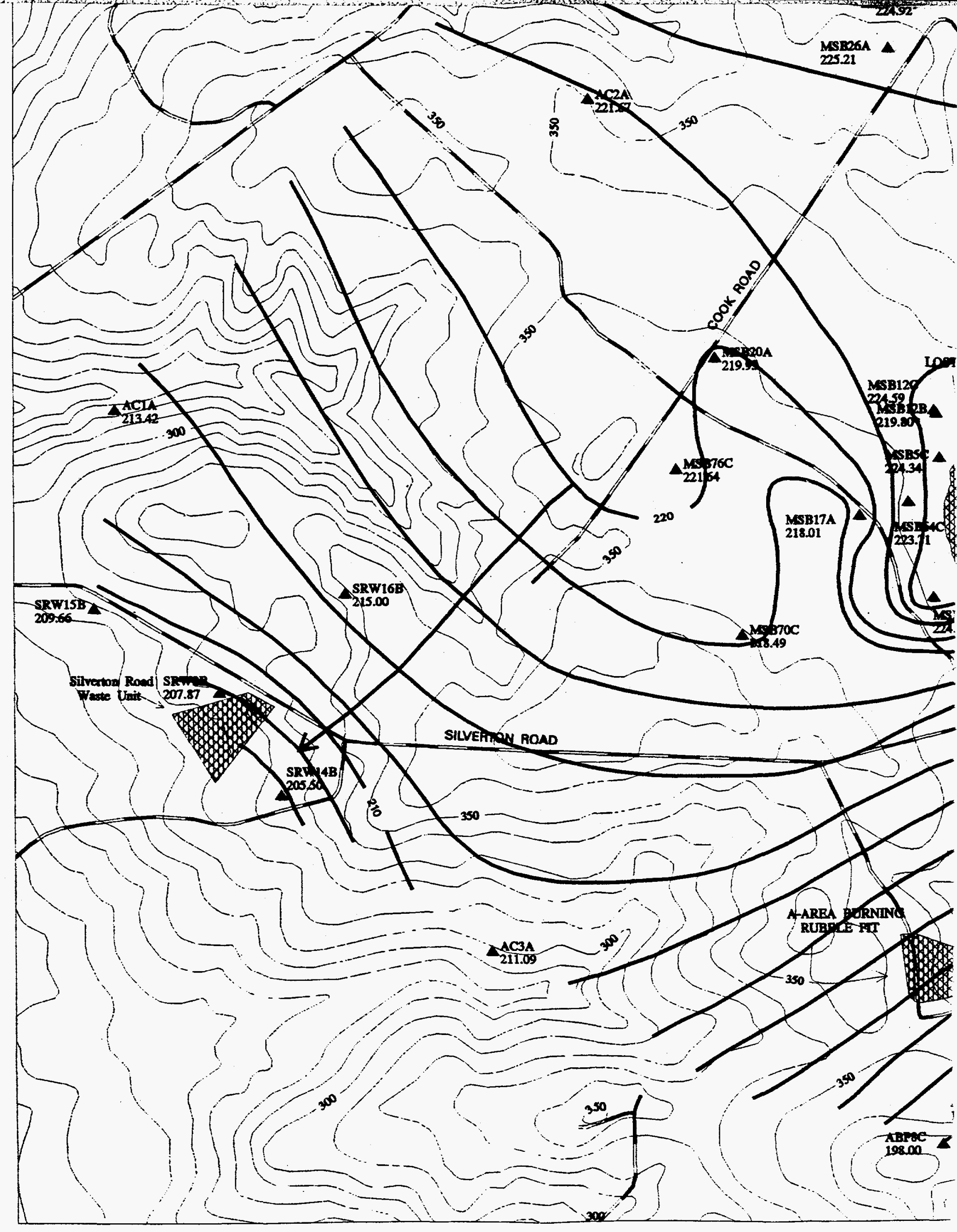




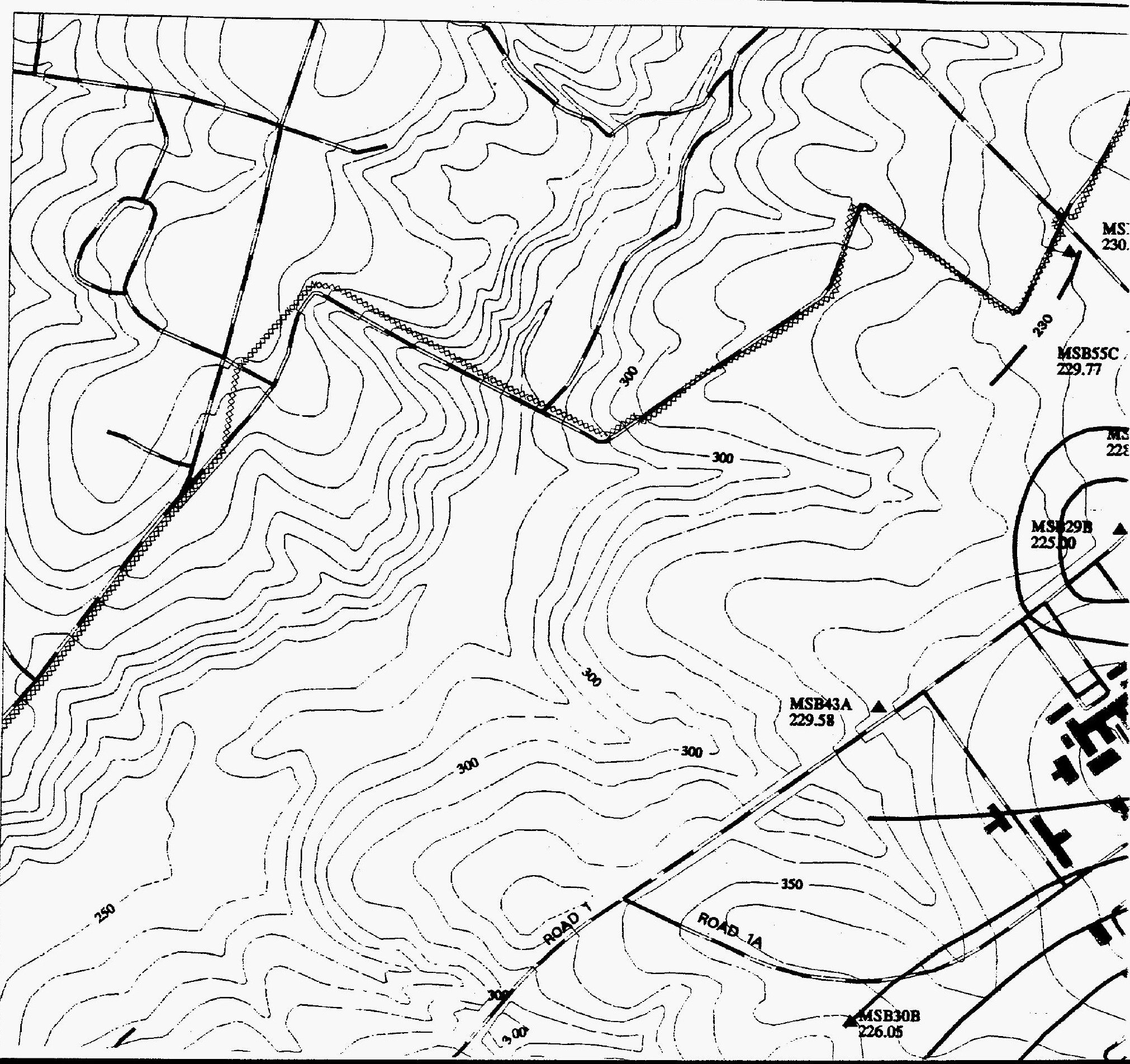




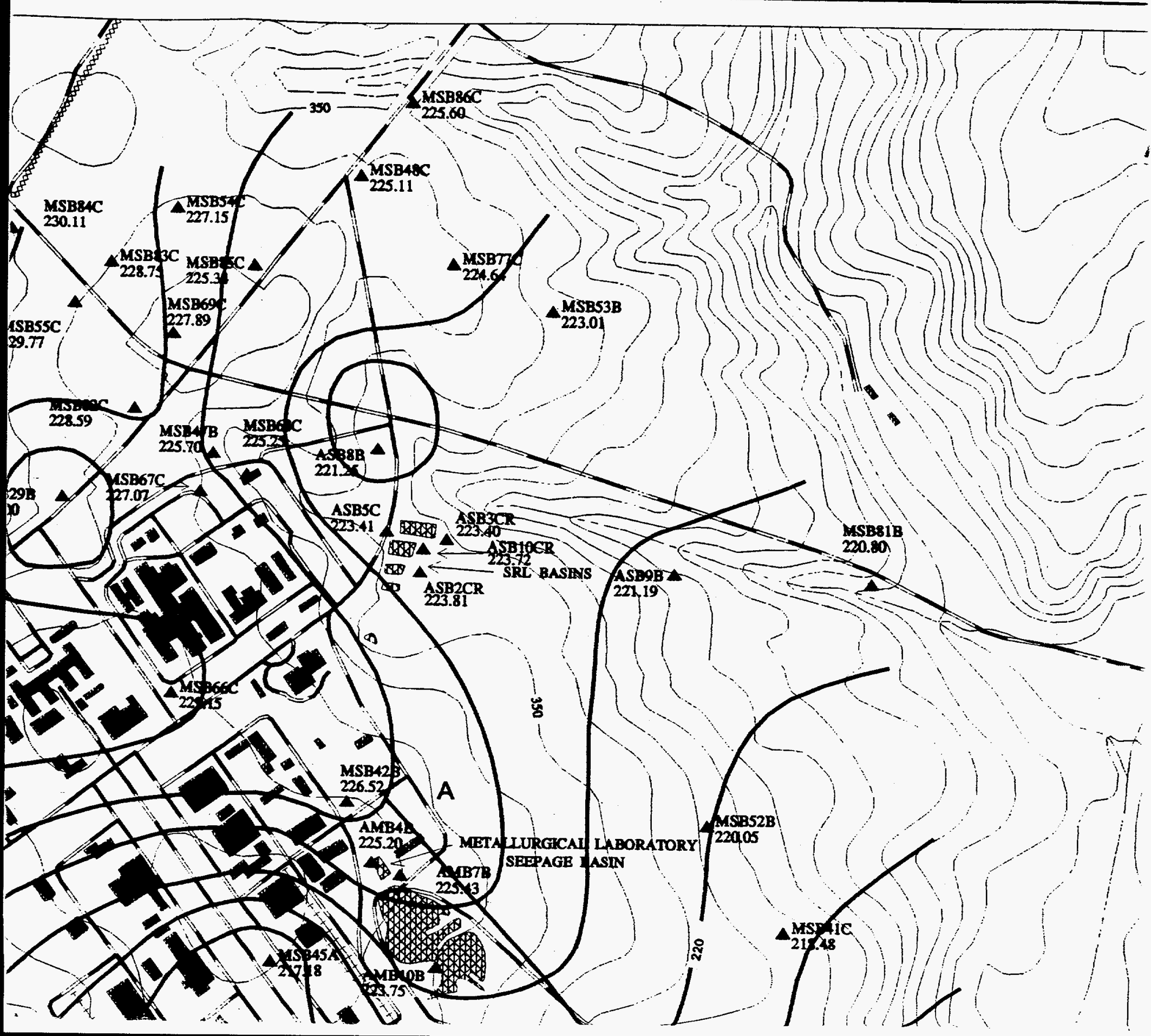



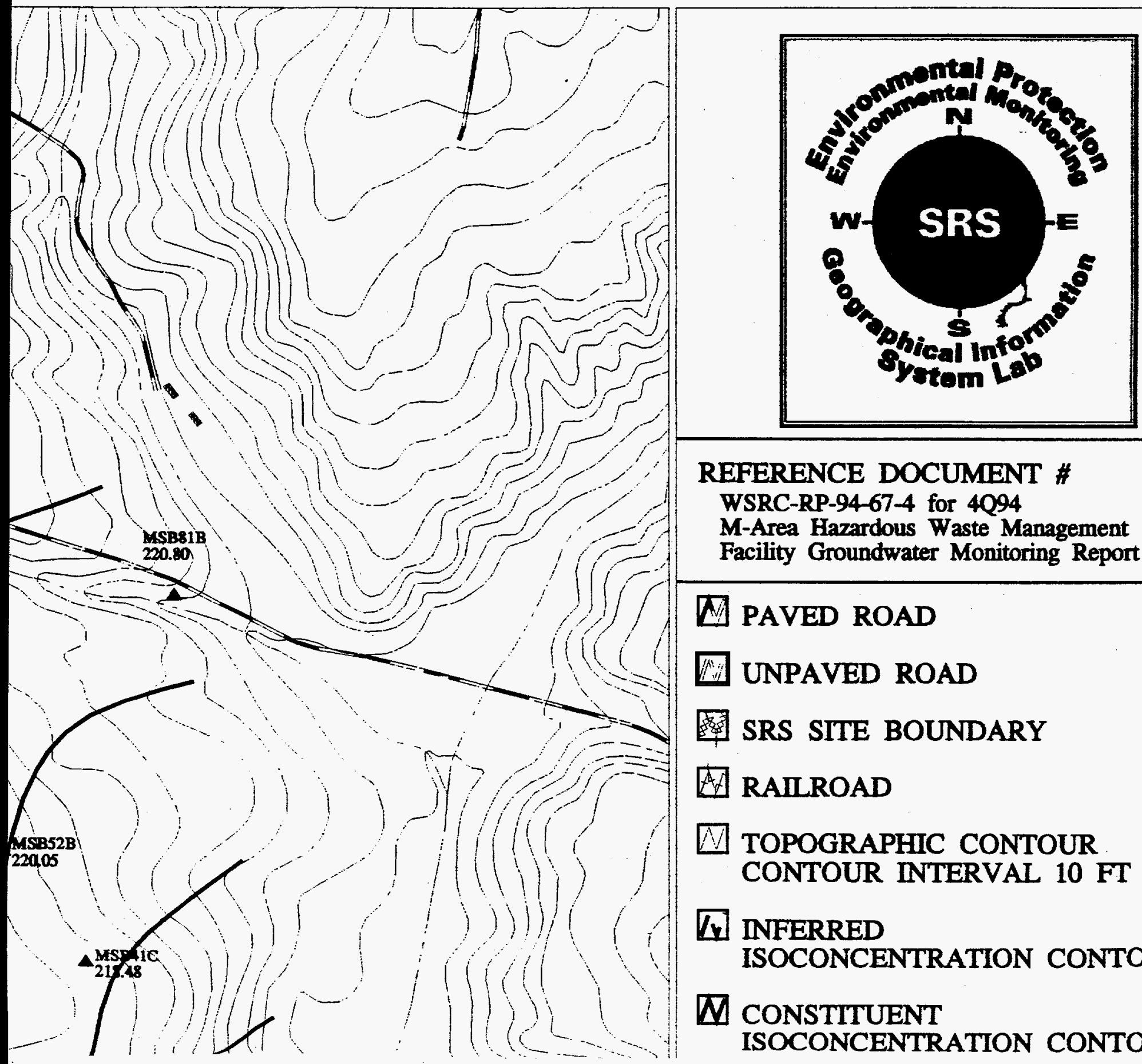

REFERENCE DOCUMENT \# WSRC-RP-94-67-4 for 4Q94 M-Area Hazardous Waste Management Facility Groundwater Monitoring Report

MAVED ROAD

UNPAVED ROAD

SRS SITE BOUNDARY

A RAILROAD

A] TOPOGRAPHIC CONTOUR CONTOUR INTERVAL 10 FT

1. INFERRED ISOCONCENTRATION CONTOUR

$\square$ CONSTITUENT ISOCONCENTRATION CONTOUR 


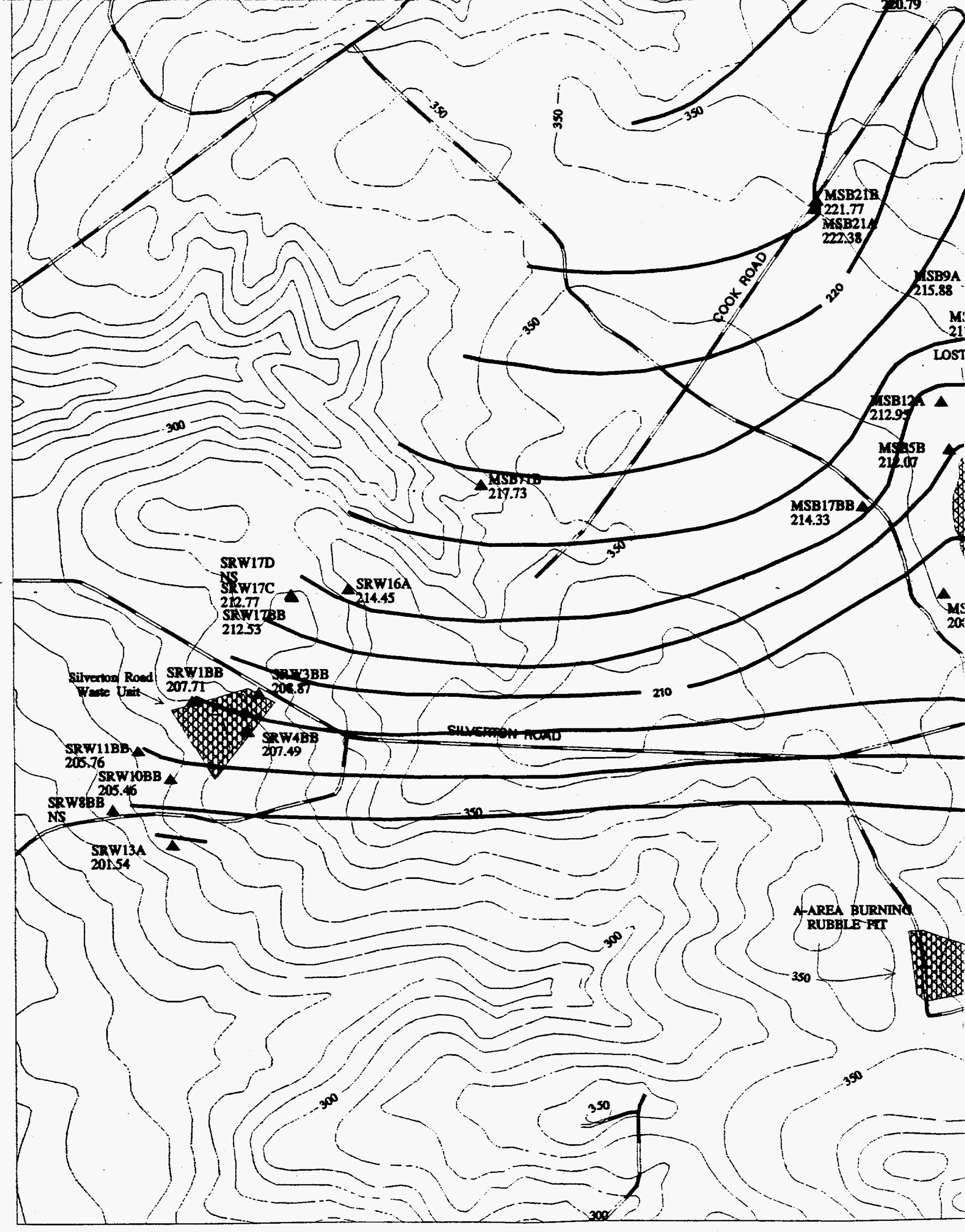




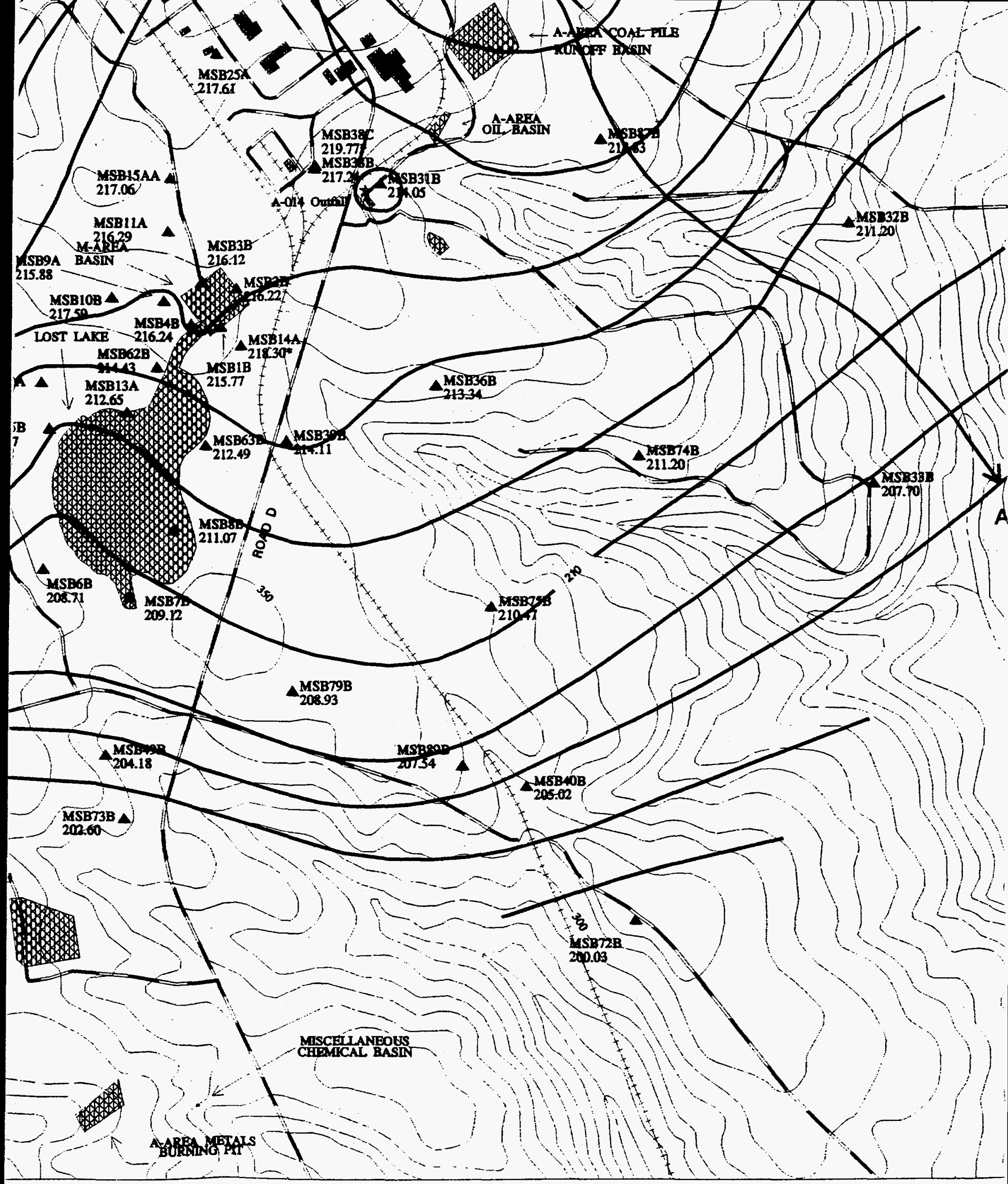




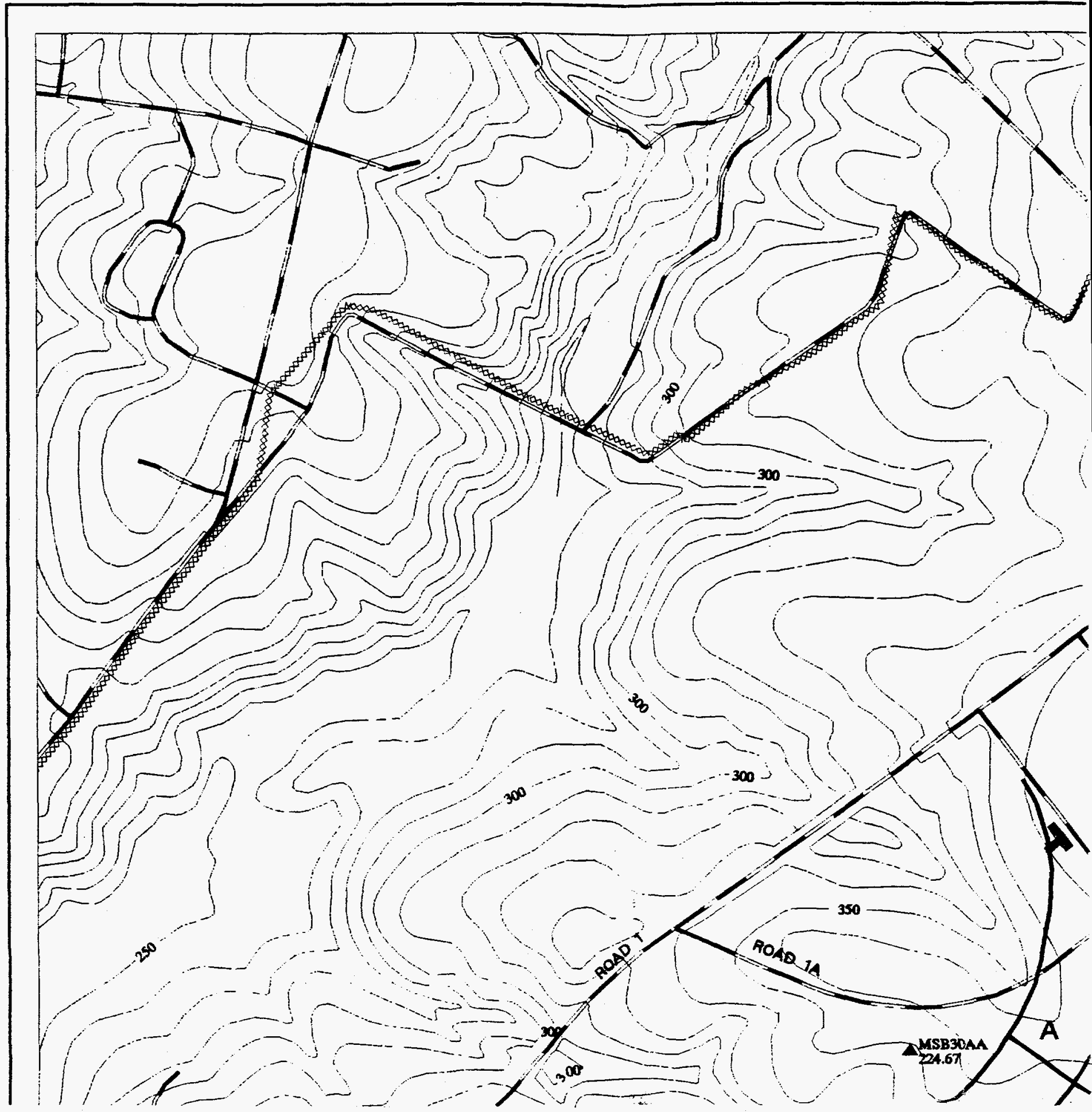




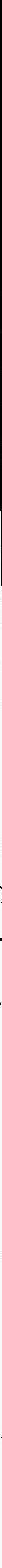




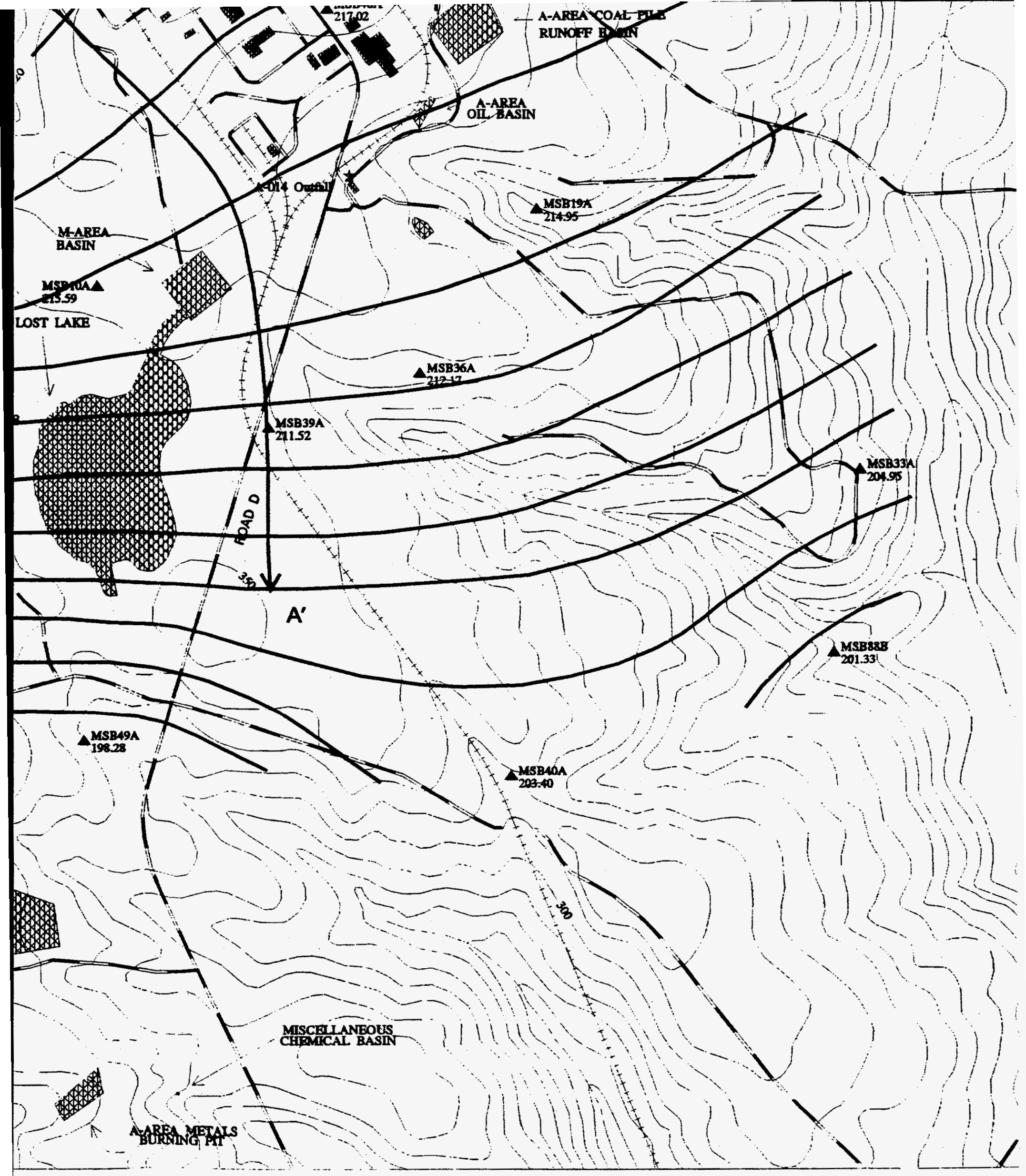




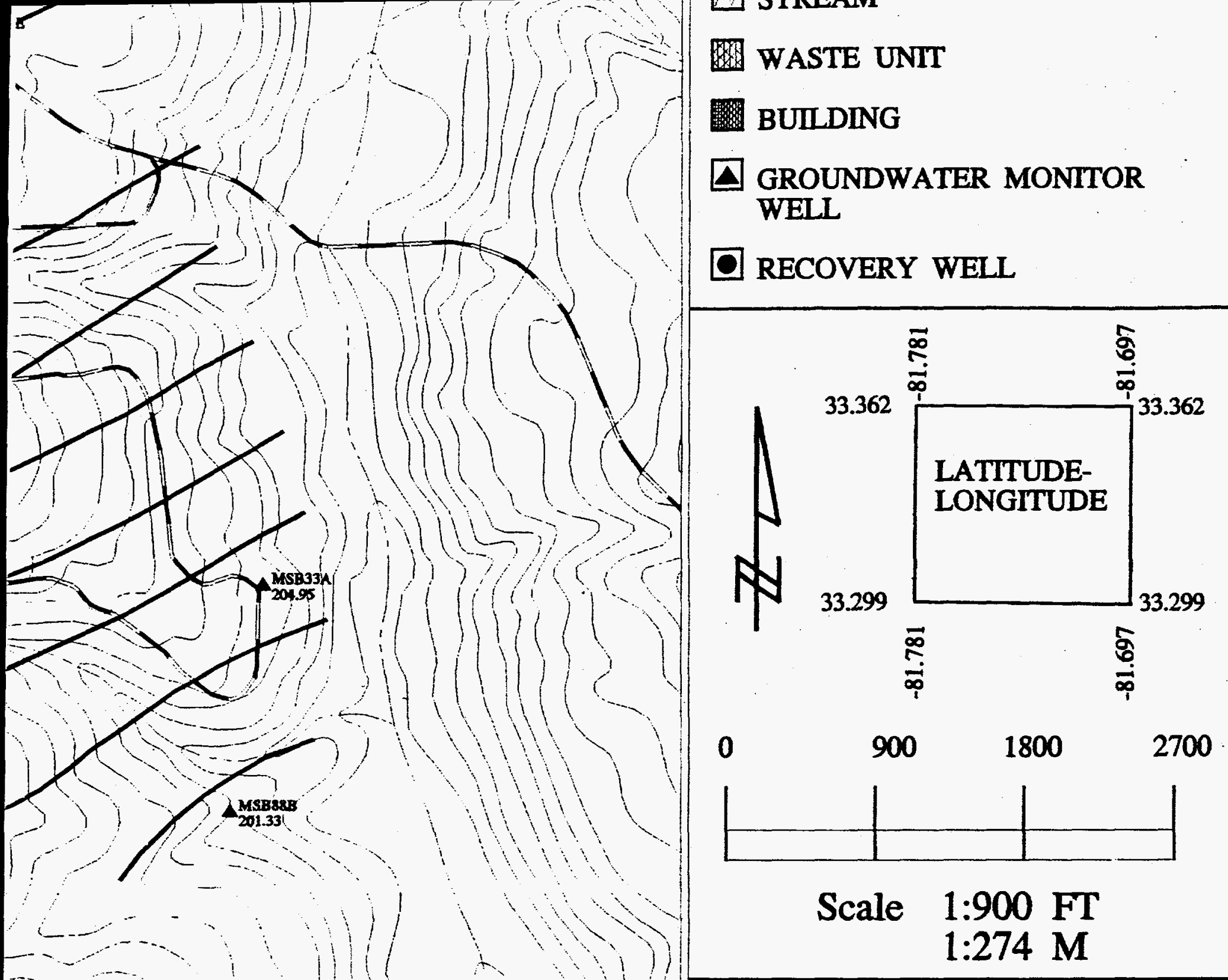

